\title{
Physics with Jets in Association with a $Z$ Boson in pp-collisions with the ATLAS Detector at the Large Hadron Collider
}

\author{
Dissertation
}

zur Erlangung des mathematisch-naturwissenschaftlichen

Doktorgrades

"Doctor rerum naturalium"

der Georg-August-Universität Göttingen

im Promotionsprogramm ProPhys

der Georg-August University School of Science (GAUSS)

vorgelegt von

Katharina Bierwagen

aus Preetz

Göttingen, 2013 
Prof. Dr. Arnulf Quadt, II. Physikalisches Institut, Georg-August-Universität Göttingen

Prof. Dr. Ariane Frey, II. Physikalisches Institut, Georg-August-Universität Göttingen

Dr. Ulrike Blumenschein, II. Physikalisches Institut, Georg-August-Universität Göttingen

Mitglieder der Prüfungskommission

Referent: Prof. Dr. Arnulf Quadt, II. Physikalisches Institut, Georg-August-Universität Göttingen

Koreferent: Prof. Dr. Jonathan Butterworth, Dept. of Physics and Astronomy, University College London

Weitere Mitglieder der Prüfungskommission:

Prof. Dr. Ariane Frey, II. Physikalisches Institut, Georg-August-Universität Göttingen PD. Dr. Jörn Große-Knetter, II. Physikalisches Institut, Georg-August-Universität Göttingen

Prof. Dr. Florentin Andreas Wörgötter, III. Physikalisches Institut, Georg-AugustUniversität Göttingen

Prof. Dr. Fred Wolf, Institut für Nichtlineare Dynamik, Max-Planck-Institut für Dynamik und Selbstorganisation

Tag der mündlichen Prüfung: 19.06.2013 


\title{
Physics with Jets in Association with a $Z$ Boson in pp-collisions with the ATLAS Detector at the Large Hadron Collider
}

\author{
by \\ Katharina Bierwagen
}

This thesis presents the measurements of the production cross section of jets in association with a $Z$ boson in pp-collisions at $\sqrt{s}=7 \mathrm{TeV}$ with an integrated luminosity of $\int L \mathrm{~d} t=36 \mathrm{pb}^{-1}$ and $\int L \mathrm{~d} t=4.6 \mathrm{fb}^{-1}$ recorded by the ATLAS experiment at the Large Hadron Collider in Geneva. Inclusive and differential $Z\left(\rightarrow e^{+} e^{-}\right)+$jets cross sections are measured for jets with a transverse momentum $p_{T}^{\text {jet }}>30 \mathrm{GeV}$ and rapidity $\left|y^{\text {jet }}\right|<4$.4. The datasets allow to exploit the data in kinematic regimes which were not accessible before and can be used to probe the $Z / \gamma^{*}+$ jets modelling in typical phase-space regions expected for the Higgs boson decay and searches for new physics. The results are unfolded to particle level and compared to predictions from different Monte Carlo generators and next-to-leading-order perturbative QCD calculations.

Post address:

Friedrich-Hund-Platz 1

37077 Göttingen

Germany
II.Physik-UniGö-Diss-2013/03

II. Physikalisches Institut Georg-August-Universität Göttingen

May 2013 



\section{Zusammenfassung}

Die Arbeit beschreibt die Messung des Wirkungsquerschnittes für die Produktion von Jets in Ereignissen mit $Z$-Bosonen in Proton-Proton Kollisionen bei einer Schwerpunktsenergie von $\sqrt{s}=7 \mathrm{TeV}$ mit einer integrierten Luminosität von $\int L \mathrm{~d} t=36 \mathrm{pb}^{-1}$ und $\int L \mathrm{~d} t=4.6 \mathrm{fb}^{-1}$ aufgenommen mit dem ATLAS Experiment am Large Hadron Collider in Genf. Die inklusiven und differenziellen Wirkungsquerschnitte für $Z\left(\rightarrow e^{+} e^{-}\right)+$jets werden für Jets mit einem Transversalimpuls von $p_{T}^{\text {jet }}>30 \mathrm{GeV}$ und einer Rapidität von $\left|y^{j e t}\right|<4.4$ gemessen. Die Datensätze erlauben Messungen in vorher nicht zugänglichen Phasenraumregionen und können genutzt werden, um die Modellierungen von $Z / \gamma^{*}+$ jets in typischen Phasenraumregionen, die vom Zerfall des Higgs Bosons oder Suchen nach neuer Physik erwarten werden, zu testen. Die Ergebnisse werden auf Partonlevel entfaltet und mit Vorhersagen verschiedener Monte-Carlo Generatoren und Vorhersagen der perturbativen QCD in nächst-führender Ordnung verglichen. 



\section{Contents}

1. Introduction 1

2. $Z$ Boson and Higgs Boson Production in the Context of the Standard Model 3

2.1. Introduction into the Standard Model of Particle Physics . . . . . . . . . . 3

2.1.1. Mathematical Description of the Standard Model . . . . . . . 6 6

2.2. Phenomenology of pp Collisions . . . . . . . . . . . . . 10

2.2.1. Parton Model . . . . . . . . . . . . . . . . 11

2.2.2. Parton Distribution Functions . . . . . . . . . . . . . . 11

2.2.3. Cross Section . . . . . . . . . . . . . . . 12

2.2.4. Higher Order Corrections . . . . . . . . . . . . . . . 13

2.2.5. Luminosity . . . . . . . . . . . . . . . . . 13

2.2.6. Multiple Proton Interactions . . . . . . . . . . . . . . . 14

2.2.7. Underlying Event . . . . . . . . . . . . . . . . . . . . 15

2.2.8. Modelling of QCD Radiation . . . . . . . . . . . . . . 15

2.2.9. Fragmentation . . . . . . . . . . . . . . 16

2.3. $Z / \gamma^{*}+$ jets Production at Hadron Colliders . . . . . . . . . . . . . . 16

2.3.1. Z Boson Production and Decay . . . . . . . . . . . . . 17

2.3.2. Jet Production in Association with a $Z$ Boson . . . . . . . . . . . 18

2.3.3. Background Processes for Dileptonic Final States . . . . . . . . . . 21

2.3.4. Experimental Measurements of $Z / \gamma^{*}+$ jets Production . . . . . . . 22

2.4. Higgs Boson Production at Hadron Colliders . . . . . . . . . . . . . . 23

2.4.1. Higgs Boson Mass Constraints . . . . . . . . . . . . . . . 23

2.4.2. Higgs Boson Decay . . . . . . . . . . . . . . . . . . . . 24

2.4.3. Higgs Boson Production . . . . . . . . . . . . . . . 25 25

2.4.4. Experimental Measurements of Higgs Boson Production . . . . . . . 27

3. Experimental Setup [29

3.1. The Large Hadron Collider . . . . . . . . . . . . . . . . . . . . . . . . . . . 29

3.2. The ATLAS Detector . . . . . . . . . . . . . . . . 31

3.2.1. Magnet System . . . . . . . . . . . . . . . . 32

3.2.2. Inner Detector System . . . . . . . . . . . . . . . 33

3.2.3. Calorimeter System . . . . . . . . . . . . . . . . . . . . . . . . . . . . . . . . .

3.2.4. Muon System . . . . . . . . . . . . . . . . . . 34

3.2.5. Data Acquisition and Trigger System . . . . . . . . . . . 35

4. Monte Carlo Simulation and Event Generators 37

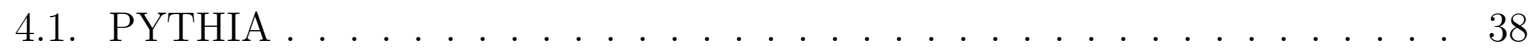

4.2. HERWIG . . . . . . . . . . . . . . . . . . . . 38

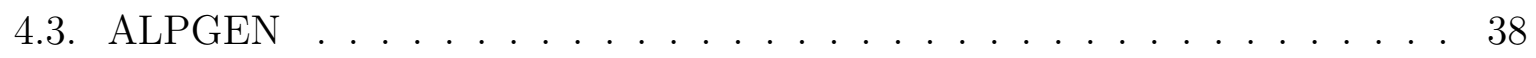


4.4. SHERPA . . . . . . . . . . . . . . . . . . . . . . 39

4.5. $\mathrm{MC} @ \mathrm{NLO} \ldots \ldots \ldots \ldots \ldots \ldots \ldots \ldots$

4.6. AcerMC . . . . . . . . . . . . . . . . . . . . . 39

5. Unfolding Methods 41

5.1. Iterative (Bayes) Method . . . . . . . . . . . . . . . . . . 41

5.1.1. The Toy Monte Carlo . . . . . . . . . . . . . . . . 43

5.1.2. Performance checks . . . . . . . . . . . . . . . 44

6. Object Definitions 47

6.1. Electrons . . . . . . . . . . . . . . . . . . . 47

6.1.1. Reconstruction Algorithm . . . . . . . . . . . . 47

6.1.2. Quality Criteria ................... 48

6.1.3. Performance . . . . . . . . . . . . . . . . 49

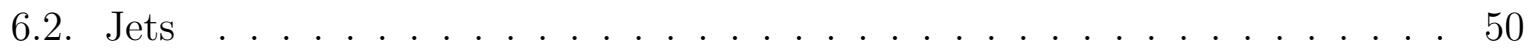

6.2.1. Reconstruction Algorithm . . . . . . . . . . . . 50

6.2.2. Calibration .................. 51

6.2.3. Quality Criteria ................. 51

6.2.4. Performance ...................... 55

7. General Analysis Approach

7.1. Data Samples and Event Selection . . . . . . . . . . . . . . 59

7.2. Modelling of Signal and Background Processes . . . . . . . . . . . . . 61 61

7.2.1. Signal and Background Monte Carlo Event Samples . . . . . . . . . 61

7.2.2. Data-Driven Estimate of the Multi-jet Background . . . . . . . . . 66

7.2.3. Data-Driven Estimate of the $t \bar{t}$ Background . . . . . . . . . . . 6 67

7.3. NLO pQCD Predictions . . . . . . . . . . . . . . . . 69

7.3.1. Study of Theoretical Uncertainties . . . . . . . . . . . . 69

8. Measurement with the Dataset of 2010

8.1. Uncorrected Distributions . . . . . . . . . . . . . . . 73

8.2. Corrections for Detector Effects . . . . . . . . . . . . . . . . 76

8.3. Study of Systematic Uncertainties . . . . . . . . . . . . . . . . . 799

8.3.1. Electron Selection . . . . . . . . . . . . . . . . . . 779

8.3.2. Jet Energy Scale . . . . . . . . . . . . . . . . . . . . . 80

8.3.3. Jet Energy Resolution . . . . . . . . . . . . . . . . . . 80

8.3.4. Background Modelling and Normalisation . . . . . . . . . . . . 80

8.3.5. Unfolding . . . . . . . . . . . . . . . . . . 80 80

8.3.6. Total Systematic Uncertainty . . . . . . . . . . . . . . 81

8.4. Theory Corrections . . . . . . . . . . . . . . . . 83

8.4.1. Corrections for Non-perturbative Effects . . . . . . . . . . . 83

8.4.2. Corrections for QED Final State Radiation . . . . . . . . . . . . . . 84

8.5. Results . . . . . . . . . . . . . . . . . . . . 85

8.5.1. Inclusive Jet Multiplicity and Jet Multiplicity Ratio . . . . . . . . . 86

8.5.2. Transverse Momentum Distributions . . . . . . . . . . 88

8.5.3. Rapidity Distributions . . . . . . . . . . . . . . . . . . 90

8.5.4. Invariant Dijet Mass . . . . . . . . . . . . . . . . . . . . 92

8.5.5. Angular Separation between the Leading Jets . . . . . . . . . . . . 92 


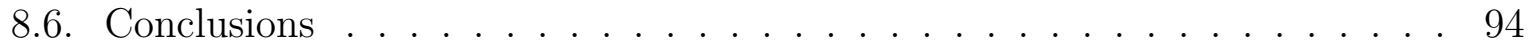

9. Measurement with the Dataset of 2011

9.1. Uncorrected Distributions . . . . . . . . . . . . . . . . 96

9.1.1. Double-parton Interactions . . . . . . . . . . . . . . . . . . . 100

9.1.2. Impact of Pile-up . . . . . . . . . . . . . . . . . . . . 101

9.2. Corrections for Detector Effects . . . . . . . . . . . . . 105

9.2.1. Method Implementation . . . . . . . . . . . . . . . . . 105

9.2.2. Optimal Number of Iterations . . . . . . . . . . . . . . . . 107

9.3. Study of Systematic Uncertainties . . . . . . . . . . . . . . . . . . . 109

9.3.1. Electron Selection . . . . . . . . . . . . . . . . . . . . 109

9.3.2. Jet Energy Scale . . . . . . . . . . . . . . . . . . . . . . 109

9.3.3. Jet Energy Resolution . . . . . . . . . . . . . . . . . . . . . . 112

9.3.4. Background Modelling and Normalisation . . . . . . . . . . . . . . 112

9.3.5. Unfolding . . . . . . . . . . . . . . . . . . . . 112

9.3.6. Total Systematic Uncertainty . . . . . . . . . . . . . . . 114

9.4. Theory Corrections . . . . . . . . . . . . . . . . . . . . 118

9.4.1. Corrections for Non-perturbative Effects . . . . . . . . . . . . . . 118

9.4.2. Corrections for QED Final State Radiation . . . . . . . . . . . . . . 122

9.5. Results . . . . . . . . . . . . . . . . . . . . . 124

9.5.1. Inclusive Jet Multiplicity . . . . . . . . . . . . . . . . . . . 124

9.5.2. Exclusive Jet Multiplicity and Jet Multiplicity Scaling . . . . . . 126

9.5.3. Transverse Momentum Distributions . . . . . . . . . . . . . . . . 129

9.5.4. Rapidity Distributions . . . . . . . . . . . . . . . . . 136

9.5.5. Invariant Dijet Mass . . . . . . . . . . . . . . . . . . . . 142

9.5.6. Angular Separation between the Leading Jets . . . . . . . . . . . . 142

9.5.7. Inclusive Quantities: $H_{\mathrm{T}}$ and $S_{\mathrm{T}} \ldots \ldots \ldots \ldots$. . . . . . . . . . .

9.6. Conclusions . . . . . . . . . . . . . . . . . . . . . 147

10.Higgs Boson Production via Weak Boson Fusion $\quad 149$

10.1. Weak Boson Fusion Preselection . . . . . . . . . . . . . . . . . . . . . . 151

10.2. Uncorrected Distributions . . . . . . . . . . . . . . . . . . 151

10.3. Results at Particle Level . . . . . . . . . . . . . . . . . . . . 155

10.4. Conclusions . . . . . . . . . . . . . . . . . . . 157

11. Overall Conclusion 159

12.Outlook 161

A. Measurement with the Dataset of 2010

A.1. Further Uncorrected Distributions . . . . . . . . . . . . . . . . . . . . 163

A.2. Further Uncorrected Distributions with $p_{T}^{\text {jet }}>20 \mathrm{GeV} \ldots \ldots . . . . . .165$

A.3. Cross Section Tables . . . . . . . . . . . . . . . . 167

B. Measurement with the Dataset of 2011

B.1. Further Uncorrected Distributions . . . . . . . . . . . . . . . . . 171

B.1.1. Impact of Pile-up . . . . . . . . . . . . . . . . . . . . . 178

B.2. Further Unfolding Distributions . . . . . . . . . . . . . . . . . . . . 179 
B.3. Unfolding Closure Tests . . . . . . . . . . . . . . . . . . . 185

B.3.1. ALPGEN+HERWIG Reweighting . . . . . . . . . . . . . . 185

B.3.2. Closure Tests . . . . . . . . . . . . . . . . . . . . . 186

B.4. Cross Section Tables . . . . . . . . . . . . . . . . . . 188

C. Higgs Boson Production via Weak Boson Fusion 207

C.1. Cross Section Tables . . . . . . . . . . . . . . . . . 207

Bibliography $\quad 209$

List of Figures

List of Tables 231

Acknowledgements 238 


\section{Introduction}

The question about the composition of matter is one of the oldest in history of mankind. Some of the ancient philosophers believed that matter was made of indivisible particles which they call átomos. In the early history of science, end of the 19th century, people like E. Rutherford and J. J. Thomson developed models of these particles, which include subatomic particles with negative and positive charge. In 1897, Thomson discovered the electron, which constitutes the basis of his atomic model. Based on the continuously improving methods of mass spectroscopy, Rutherford postulated in 1920 that the structure of atomic nuclei is made of positively charged protons and neutrally charged neutrons.

Since the 1950s, the technological and scientific progress allowed to develop new particle accelerators and particle detectors and therefore allowed to study atoms at even higher energies. With deep-inelastic scattering, it could be shown that also protons and neutrons are divisible and composed of quarks. This, together with the formulation of the electroweak theory by S. Glashow, built the first step towards the direction of the Standard Model (SM). The SM describes the constituents of matter and the interactions between them. The masses of these constituents are described by the Higgs mechanism, which predicts the existence of a new boson - the Higgs boson. The predictions of the SM have been tested at a high level of accuracy during the past years.

At the beginning of 2010, the Large Hadron Collider (LHC) at CERN, the European laboratory for particle physics in Geneva opened the door to a new era of particle physics. For the first time, proton-proton collisions ( $p p$-collisions) were taken at a centre of mass energy of $\sqrt{s}=7 \mathrm{TeV}$, which maybe opened the opportunity to shed light on some of the most fundamental questions of the SM, namely the process of mass generation.

The discovery of the Higgs boson and the measurement of its properties is a fundamental part of the physics programme at the LHC. The LHC has been designed to collide protons at a centre of mass energy of $14 \mathrm{GeV}$ with a collision rate of $40 \mathrm{MHz}$. Since the beginning of 2011, the LHC has already delivered an integrated luminosity of $5.6 \mathrm{fb}^{-1}$ of data at $\sqrt{s}=7 \mathrm{TeV}$ and $23.3 \mathrm{fb}^{-1}$ at $\sqrt{s}=8 \mathrm{TeV}$. On July 4th, 2012, the discovery of a new boson [1, 2] of mass near $125 \mathrm{GeV}$ was claimed. This boson is so far consistent with the SM Higgs boson, but the uncertainty on its properties is still very large. In order to confirm the consistency of the new resonance with the prediction more data is needed. So far the LHC shows only an evidence in the vector boson decay modes $H \rightarrow \gamma \gamma, H \rightarrow Z Z$ and $H \rightarrow W W$. From now on, the highest priority is to establish the nature of this boson, which also implies the observation of the Higgs boson decaying to fermions, e.g. $H \rightarrow \tau^{+} \tau^{-}$.

For the detailed investigation of the Higgs boson candidate, one of the main challenges is the understanding of the background contributions. With $25 \mathrm{fb}^{-1}$ of data, more production channels are accessible. One of the most promising channels is the Higgs boson production via weak boson fusion (WBF), since it provides a clean signature in the detector. From the WBF production two well separated forward jets and reduced activity in the central region are expected. The main background to this channel is the production 
of jets in association with a $Z$ boson, which has a similar signature in the detector.

The production of jets in association with a $Z$ boson constitutes not only an important background for studies of the Higgs boson candidate and searches for new physics, it provides also a stringent test of perturbative quantum chromodynamics (pQCD). The kinematic distributions of jets in association with a $Z$ boson can be predicted by NLO pQCD predictions and Monte Carlo (MC) predictions from matrix element generators accompanied by parton shower (ME+PS). The latter are affected by large scale uncertainties and need to be tuned and validated using comparisons with data.

The aim of the analysis presented in this thesis is to measure the inclusive and differential $Z / \gamma^{*}(\rightarrow e e)+$ jets cross section in $p p$-collisions with first ATLAS data at a centre-ofmass energy of $\sqrt{\mathrm{s}}=7 \mathrm{TeV}$. Similar measurements have been reported at moderate energy regimes and lower jet multiplicities in proton-antiproton collisions at $\sqrt{\mathrm{s}}=1.96 \mathrm{TeV}$ at the Tevatron [3, 4] and in $p p$-collisions at $\sqrt{\mathrm{s}}=7 \mathrm{TeV}$ at CMS [5]. At ATLAS the analysis has been performed for an integrated luminosity of $\int L \mathrm{~d} t=36 \mathrm{pb}^{-1}$ (full dataset of 2010) and $4.6 \mathrm{fb}^{-1}$ (full dataset of 2011) [6 8]. The former has the advantage of a relatively low collision rate and a low rate of multiple proton-proton interactions which allow for cross-section measurements at low jet transverse momentum. The latter provides the most accurate results and partly supersedes the measurement with the full dataset of 2010. This dataset allows to exploit the data in kinematic regions which were not accessible before and therefore can be used to probe the $Z / \gamma^{*}+$ jets modelling in typical phase space regions expected for the Higgs boson decay and searches for new physics. Inclusive and exclusive differential cross sections are accessible for higher jet multiplicities and energy regimes up to $1 \mathrm{TeV}$. For high energy regimes, large K-factors between the NLO and LO pQCD predictions are expected [9]. Typical phase space regions expected from the WBF Higgs boson decay and searches for new physics are characterised by large scales, often larger than the $Z$ boson mass, which results in large logarithmic corrections for higher order pQCD predictions [9, 10]. It is therefore probable that fixed-order NLO pQCD predictions fail to describe the data in such regimes [9]. The measurement menu contains the total inclusive and exclusive jet multiplicity cross sections and their ratios for different phase space constraints. Inclusive differential cross sections as a function of the jet transverse momentum and the jet rapidity are measured in $Z / \gamma^{*}$ events with at least one, two, three or four jets in the final state. For $Z / \gamma^{*}$ events with at least two jets in the final state, the inclusive differential cross section is also measured as a function of the dijet mass and the angular separation between the jets. The final results of these measurements are unfolded to particle level and compared to predictions from $\mathrm{ME}+\mathrm{PS}$ generators [11-13] and fixed-order NLO pQCD calculations [14-16].

This thesis is organized as follows: Starting with a brief description of the $Z$ boson and Higgs boson production in the context of the SM and the phenomenology of hadronic collisions in Chapter 2 . Chapter 3 proceeds with the description of the LHC and the ATLAS detector. Chapter 4 explains the MC simulation chain and the different generators used for the analyses. The different unfolding methods and the object definitions are detailed in Chapter 5 and Chapter 6, respectively. Chapter 7 presents the general analysis strategy, followed by three chapters explaining the different analyses. The first two, Chapter 8 and Chapter 9, are dedicated to the QCD measurements with the full dataset of 2010 and 2011 and Chapter 10 is dedicated to the studies on WBF Higgs boson production. Finally, an overall conclusion and an outlook are given in Chapter 11 and Chapter 12 . 


\section{2. $Z$ Boson and Higgs Boson Production in the Context of the Standard Model}

This chapter gives a brief introduction to the Standard Model (SM) and the Higgs mechanism. In particular, the formalisms of Quantum Chromodynamics (QCD) and electroweak (EW) theory are briefly reviewed.

\subsection{Introduction into the Standard Model of Particle Physics}

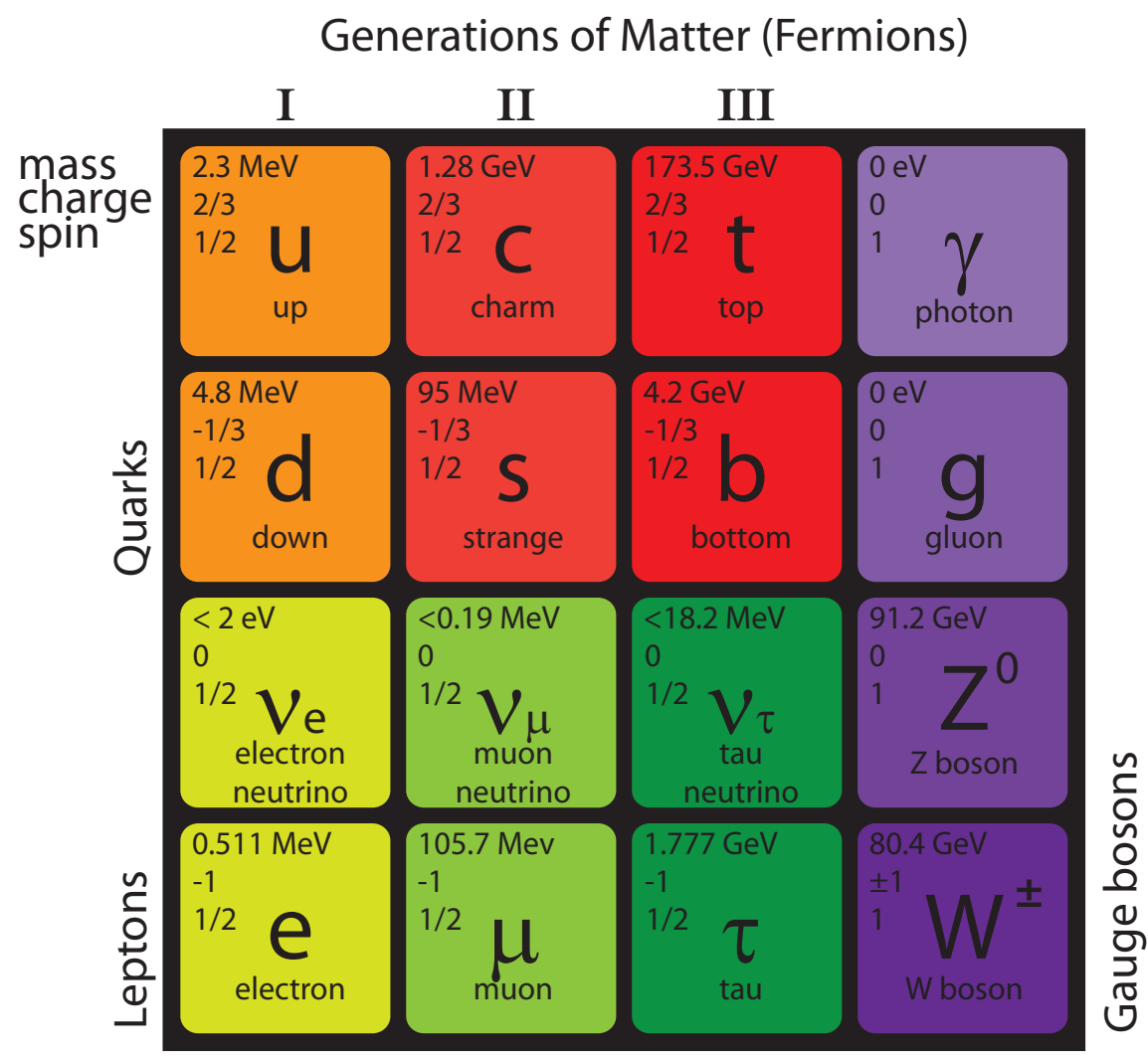

Figure 2.1.: Particle content of the Standard Model of particles physics.

Particle physics is the science of the constituents of matter and the interactions between them. The Standard Model (SM) [17-21] is a local Lorentz-invariant quantum field theory in which the properties of the interactions result from the requirement of local gauge 
invariance and can be described by group theory. The gauge group of the SM is the direct product

$$
\mathrm{SU}(3)_{C} \otimes \mathrm{SU}(2)_{L} \otimes \mathrm{U}(1)_{Y}
$$

where $C$ is colour, $Y$ is hypercharge and $L$ is left-handedness.

Within the SM, the known matter is ascribed to a few elementary particles, the fermions. The forces between those elementary particles are mediated by spin-1 gauge bosons: the strong force is carried by gluons $(\mathrm{g})$, the weak force by $W^{ \pm}$and $Z^{0}$ bosons and the electromagnetic force by photons $(\gamma)$. Figure 2.1 shows a representation of the known particles.

\begin{tabular}{|c|c|c|c|c|c|}
\hline \hline Gauge Boson & Mass & Charge & Spin & Force & Theory \\
\hline \hline gluon & 0 & 0 & 1 & strong & QCD \\
\hline$W^{ \pm}$ & $80.3850 \pm 0.0150 \mathrm{GeV}$ & \pm 1 & 1 & weak & EW \\
$Z^{0}$ & $91.1876 \pm 0.0021 \mathrm{GeV}$ & 0 & 1 & weak & EW \\
\hline$\gamma$ & $<1 \cdot 10^{-18} \mathrm{eV}$ & 0 & 1 & electromagnetic & EW \\
\hline \hline
\end{tabular}

Table 2.1.: Standard Model gauge bosons and their properties [22], along with their forces and the corresponding theory.

The properties and the quantum numbers of the gauge bosons, along with their forces are detailed in Tab. 2.1. The theories behind those forces are described in the next section. Gravity is the only known fundamental force which is not described by the SM.

The fermions are spin-1/2 particles and can be categorized into two groups of particles, the leptons and the quarks. Each group has three generations. Generally, from generation to generation the masses of the particles increase.

Leptons are classified according to their electric charge $(Q)$, the lepton flavour number (electron number $\left(\mathrm{L}_{e}\right)$, muon number $\left(\mathrm{L}_{\mu}\right)$ and tau number $\left(\mathrm{L}_{\tau}\right)$ ) and the third component of the weak isospin $\left(T_{3}\right)$. An additional quantity is the weak hypercharge $\left(Y_{W}\right)$, which is related to the charge and the third component of the weak isospin via:

$$
Y_{W}=2 \cdot\left(Q-T_{3}\right)
$$

The properties of the leptons are summarized in Tab. 2.2.

Each lepton generation forms a left-handed weak isospin doublet

$$
\left(\begin{array}{c}
T_{3}=+1 / 2 \\
T_{3}=-1 / 2
\end{array}\right)=\left(\begin{array}{c}
\nu_{e L} \\
e_{L}^{-}
\end{array}\right),\left(\begin{array}{c}
\nu_{\mu L} \\
\mu_{L}^{-}
\end{array}\right),\left(\begin{array}{c}
\nu_{\tau L} \\
\tau_{L}^{-}
\end{array}\right) .
$$

Since right-handed neutrinos are not in the SM, the charged right-handed leptons in each generation form a weak isospin singlet $\left(T_{3}=0\right): e_{R}^{-}, \mu_{R}^{-}$and $\tau_{R}^{-}$. The charged leptons interact via the weak and the electromagnetic force, whereas the neutral leptons are only allowed to interact via the weak force. 


\begin{tabular}{|c|c|r|c|c|c|c|}
\hline \hline Lepton Flavour & Mass & $\mathrm{Q}$ & $Y_{W}$ & $L_{e}$ & $L_{\mu}$ & $L_{\tau}$ \\
\hline \hline$\nu_{e}$ & $<2 \mathrm{eV}$ & 0 & -1 & 1 & 0 & 0 \\
\hline$e^{-}$ & $0.511 \mathrm{MeV}$ & -1 & -1 & 1 & 0 & 0 \\
\hline$\nu_{\mu}$ & $<0.19 \mathrm{MeV}$ & 0 & -1 & 0 & 1 & 0 \\
\hline$\mu^{-}$ & $105.7 \mathrm{MeV}$ & -1 & -1 & 0 & 1 & 0 \\
\hline$\nu_{\tau}$ & $<18.2 \mathrm{MeV}$ & 0 & -1 & 0 & 0 & 1 \\
\hline$\tau^{-}$ & $1776.8 \mathrm{MeV}$ & -1 & -1 & 0 & 0 & 1 \\
\hline \hline
\end{tabular}

Table 2.2.: Properties of the leptons [22].

Quarks are classified according to their charge (Q) and their flavour quantum numbers (baryon number (third component of the isospin $\left(I_{3}\right)$, strangeness $(\mathrm{S})$, charmness $(\mathrm{C})$, bottomness $(\mathrm{B})$ and topness $(\mathrm{T})$ ). The quarks are the only particles which are able to interact via all three fundamental interactions. The quark properties and quantum numbers are summarised in Tab. 2.3.

\begin{tabular}{|c|ccc|c|c|c|c|c|c|}
\hline \hline Quark Flavour & \multicolumn{3}{|c|}{ Mass } & $\mathrm{Q}$ & $I_{3}$ & $\mathrm{C}$ & $\mathrm{S}$ & $\mathrm{T}$ & $\mathrm{B}$ \\
\hline \hline $\mathrm{u}$ & 2.3 & +0.7 & $\mathrm{MeV}$ & $2 / 3$ & $1 / 2$ & 0 & 0 & 0 & 0 \\
\hline $\mathrm{d}$ & 4.8 & $\underset{-0.3}{+0.7}$ & $\mathrm{MeV}$ & $-1 / 3$ & $-1 / 2$ & 0 & 0 & 0 & 0 \\
\hline $\mathrm{c}$ & $1.275 \pm 0.025 \mathrm{GeV}$ & $2 / 3$ & 0 & 1 & 0 & 0 & 0 \\
\hline $\mathrm{s}$ & 95. & \pm 5 & $\mathrm{MeV}$ & $-1 / 3$ & 0 & 0 & -1 & 0 & 0 \\
\hline $\mathrm{t}$ & 173.2 & \pm 0.9 & $\mathrm{GeV}$ & $2 / 3$ & 0 & 0 & 0 & 1 & 0 \\
\hline $\mathrm{b}$ & 4.18 & \pm 0.03 & $\mathrm{GeV}$ & $-1 / 3$ & 0 & 0 & 0 & 0 & -1 \\
\hline \hline
\end{tabular}

Table 2.3.: Properties and quantum numbers of the different quarks [22]. The masses are given in the $\overline{M S}$ scheme, except for top mass, where the world average from direct measurements is quoted.

Each fermion has its own antiparticle with reversed signs of all quantum numbers. In addition, each quark has one additional quantum number which can take three values, called colour charge. The SM ends up with 12 leptons and 36 quarks in total.

The SM predictions have been tested to a high level of accuracy during the past years. It is one of the most successful theories in history. But at the same time, the electroweak (EW) theory imposes one of the most fundamental constraints on this theory. Within the EW theory masses for the gauge bosons of the weak interaction violate invariance under local gauge transformations. Nonetheless, the weak gauge bosons $W^{ \pm}$and $Z$ have large masses, as confirmed by experiment [22, 23]. This can be accommodated by the Higgs mechanism, which is described in Sect. 2.1.1. The Higgs mechanism results in an additional particle, the Higgs boson. Recently, a new boson with a mass around $125 \mathrm{GeV}$ has been discovered [1, 2], which is consistent with the SM Higgs boson.

Although the SM fits very well to the current measurements, it is not a complete theory. 
There are still some phenomena beyond the SM which need further understanding, e.g.:

- Gravity is the only of the four fundamental forces which is not described by the SM. This force is described by Einstein's macroscopic theory of general relativity.

- The SM in its current formulation only describes $\sim 4.9 \%$ of the mass content of the universe. The remaining $95.1 \%$ are described by dark matter $26.8 \%$ and dark energy $68.3 \%$ [24, 25]. The SM does not incorporate a candidate for dark matter or an explanation for dark energy.

- Within the SM no masses for neutrinos are foreseen. Nonetheless, the existence of neutrino flavour oscillations implies non-zero masses for the neutrinos [26-28].

- The SM contains a large number of free parameters (e.g the fermion masses, the mixing angles and the Higgs boson mass), which gives the impression that it is an effective low-energy approximation of a more fundamental theory.

- The Higgs boson mass receives corrections from quantum loop diagrams, which tend to increase the Higgs boson mass to an enormous value at the order of the Planck mass $M_{P} \sim 10^{19} \mathrm{GeV}$. But from electroweak precision measurements the SM Higgs boson mass is at the order of $100 \mathrm{GeV}$, which is significantly below $M_{P}$. Therefore a fine tuning of the bare Higgs mass is necessary, which is able to cancel the difference between the radiative corrections and the mass (fine tuning problem, hierarchy problem) [29].

\subsubsection{Mathematical Description of the Standard Model}

In the following sections the mathematical formulation of SM interactions is described.

\section{Quantum Chromodynamics}

Quantum Chromodynamics (QCD) [22, 30] is the non-Abelian gauge theory behind the strong interaction, which is based on the $\mathrm{SU}(3)$ symmetry group of colour. The eight generators of this group correspond to the eight massless gluons, which mediate the interaction of coloured quarks. The quarks are described by colour triplets

$$
q_{f}^{T} \equiv\left(q_{f}^{1}, q_{f}^{2}, q_{f}^{3}\right)
$$

with 1,2,3 representing the three colour states: red, green and blue.

The Lagrangian density of QCD is given by

$$
L_{Q C D}=\underbrace{\sum_{j=1}^{n_{f}} \bar{q}_{j}\left(i D_{\mu} \gamma^{\mu}-m_{j}\right) q_{j}}_{\text {quarks }} \underbrace{-\frac{1}{4} \sum_{A=1}^{8} F^{A \mu \nu} F_{\mu \nu}^{A}}_{\text {gluons }}
$$

with the quark-field spinors, $q_{j}$, and the quark masses $m_{j}$. The $\gamma^{\mu}$ represent the Dirac matrices and $D_{\mu}=\partial_{\mu}-i g_{s} T_{A} \mathcal{A}_{\mu}^{A}$ is the covariant derivative, where $\mathcal{A}_{\mu}^{A}$ correspond to the 
gluon fields and $T_{A}$ to the eight generators of the $\mathrm{SU}(3)$ symmetry group. Finally, $F_{\mu \nu}^{A}$ represents the field strength tensor based on the gluon field $\mathcal{A}_{\mu}^{A}$

$$
F_{\mu \nu}^{A}=\partial_{\mu} \mathcal{A}_{\nu}^{A}-\partial_{\nu} \mathcal{A}_{\mu}^{A}-g_{s} f_{A B C} \mathcal{A}_{\mu}^{B} \mathcal{A}_{\nu}^{C}
$$

with the structure constants of the $\mathrm{SU}(3)$ symmetry group, $f_{A B C}$, and the QCD coupling constant, $g_{s}=\sqrt{4 \pi \alpha_{s}}$.

Due to the fact that the $\mathrm{SU}(3)$ is a non-Abelian group, the 3rd term of Eq. 2.6 does not vanish and thus gluon fields are able to self-interact. Due to this self-interaction, the effective coupling constant of the strong interaction decreases with increasing energy, leading to asymptotic freedom. Here, for short distances the strong coupling constant converges asymptotically against zero, so that quarks and gluons can be treated as free and their interactions can be calculated within perturbation theory. On the other hand with increasing distance between two quarks, the quarks become bounded in hadrons through a process called confinement.

\section{Electroweak Theory}

The electroweak (EW) theory [17-19] is the gauge theory behind the electroweak interaction. It describes the unification of the weak interaction with the electromagnetic interaction under the $S U(2)_{L} \otimes U(1)_{Y}$ symmetry group. The $S U(2)$ group involves three gauge fields and the $U(1)$ group one gauge field. The corresponding gauge bosons are $W_{\mu}^{i}$, $i=1,2,3$ for $S U(2)$ and $B_{\mu}$ for $U(1)$. The EW theory, also known as GSW theory, was introduced by S. Glashow, A. Salam and S. Weinberg. The Lagrangian of the EW theory is

$$
\mathcal{L}_{E W}=\underbrace{\sum_{j=1}^{3} i \bar{\psi}_{j}(x) \gamma^{\mu} D_{\mu} \psi_{j}(x)}_{\text {part for the fermions }} \underbrace{-\frac{1}{4} B_{\mu \nu} B^{\mu \nu}-\frac{1}{4} W_{\mu \nu}^{j} W_{j}^{\mu \nu}}_{\text {part for the gauge field }} .
$$

$D_{\mu}$ describes the covariant derivative

$$
D_{\mu}=\partial_{\mu}-i g \frac{\sigma_{j}}{2} W_{\mu}^{j}(x)-i g^{\prime} \frac{Y}{2} B_{\mu}(x)
$$

with the coupling constants $g$ corresponding to $S U(2)_{L}$ and $g^{\prime}$ corresponding to $U(1)_{Y}$. The fermionic part of the Lagrangian describes the kinetic energy of the fermions and their interactions, while the covariant derivative describes the interaction with the gauge field. It is worth mentioning that no explicit mass term for the fermions is allowed. If there is an explicit mass term, there will be a mixture of left-handed multiplets with right-handed singlets. Therefore, the local gauge invariance would be violated, since the weak interaction only couples to left-handed fermions.

The second part of the Lagrangian describes the gauge fields. Again, there is a term for the kinetic energy and a term that describes the self interaction between the gauge fields. In this part of the Lagrangian no explicit mass term is included, in order to avoid a violation of the invariance of local gauge transformations. 
The four gauge bosons of the $S U(2)_{L} \otimes U(1)_{Y}$ symmetry group do not translate directly in $W^{ \pm}, Z$ and $\gamma$. $W^{ \pm}$are linear combinations of $W_{\mu}^{1}$ and $W_{\mu}^{2}$

$$
W_{\mu}^{ \pm}=(1 / \sqrt{2})\left(W_{\mu}^{1} \mp i W_{\mu}^{2}\right),
$$

representing the charged part of the interaction. The neutral part of the interaction, represented by $Z$ and $\gamma$, evolves from the mixing of the two neutral fields $W_{\mu}^{3}$ and $B_{\mu}$

$$
\left(\begin{array}{c}
A_{\mu} \\
Z_{\mu}
\end{array}\right)=\left(\begin{array}{cc}
\cos \theta_{W} & \sin \theta_{W} \\
-\sin \theta_{W} & \cos \theta_{W}
\end{array}\right)\left(\begin{array}{c}
B_{\mu} \\
W_{\mu}^{3}
\end{array}\right)
$$

with the weak mixing angle $\theta_{W}$, which has been experimentally determined to $\sin ^{2} \theta_{W}=0.23116 \pm 0.00012$ at the $Z$ scale [22].

\section{Higgs Mechanism}

The existence of massive gauge bosons within the EW theory requires an additional mechanism which is able to accommodate those masses in a gauge invariant and renormalisable way. The most popular and minimal solution of this problem is through the Higgs mechanism [31 36].

The Higgs mechanism leaves the fundamental symmetry of the EW theory unchanged and generates the masses by spontaneous symmetry breaking of the quantum vacuum ground state. Within the theory a complex scalar $\mathrm{SU}(2)$ doublet $\phi$ with a hypercharge $Y=1$ is introduced

$$
\phi(x)=\left(\begin{array}{l}
\phi^{(+)}(x) \\
\phi^{(0)}(x)
\end{array}\right)=\sqrt{\frac{1}{2}}\left(\begin{array}{c}
\phi_{1}(x)+i \phi_{2}(x) \\
\phi_{3}(x)+i \phi_{4}(x)
\end{array}\right) .
$$

A gauge invariant Lagrangian is obtained by coupling $\phi$ to the gauge bosons

$$
L_{\text {Higgs }}=\left(D_{\mu} \phi\right)^{\dagger} D^{\mu} \phi-V(\phi),
$$

using the covariant derivative $D_{\mu}$ defined in Eq. 2.8. Here, $V(\phi)$ describes the most general renormalisable potential, which is invariant under an $S U(2)_{L} \otimes U(1)_{Y}$ gauge transformation

$$
V(\phi)=\mu^{2} \phi^{\dagger} \phi+\lambda\left(\phi^{\dagger} \phi\right)^{2}
$$

The potential depends on the choice of $\mu$ and $\lambda$. For $\mu^{2}<0$ and $\lambda>0$ the potential is bounded from below, with a rotationally symmetric degenerate ground state

$$
-\frac{\mu^{2}}{2 \lambda}=\frac{v^{2}}{2}
$$

$v$ describes the vacuum expectation value, which is related to the Fermi constant $G_{F}[22]$ :

$$
v=\sqrt{\frac{1}{\sqrt{2} G_{F}}} \approx 246.22 \mathrm{GeV} .
$$

$\phi(x)$ is expanded using Eq. 2.14 by means of perturbation theory. Regarding a rotation in phase space, the choice of the ground state is arbitrary. Therefore it can be fixed to $\phi_{1}=\phi_{2}=\phi_{4}=0$ and $\phi_{3}=v$ at 


$$
\phi_{0}(x)=\frac{1}{\sqrt{2}}\left(\begin{array}{l}
0 \\
v
\end{array}\right) .
$$

The ground state is invariant with respect to a $U(1)_{e m}$ symmetry, which is a subgroup of $S U(2)_{L} \otimes U(1)_{Y}$. Then one expands $\phi(x)$ around the ground state $\phi_{0}(x)$, resulting in

$$
\phi(x)=\frac{1}{\sqrt{2}}\left(\begin{array}{c}
0 \\
v+H(x)
\end{array}\right)
$$

for the complex and scalar Higgs $S U(2)$ doublet.

Once the vacuum state of Eq. 2.16 is chosen, the underlying $S U(2)_{L} \otimes U(1)_{Y}$ symmetry is spontaneously broken. Only the $U(1)_{e m}$ symmetry remains, leaving the photon massless. The electroweak theory has four degrees of freedom, three of them are absorbed by the longitudinal polarization of the gauge bosons to form massive particles $\left(W^{ \pm}, Z^{0}\right)$. The remaining degree of freedom implies the existence of one additional neutral scalar particle, the so-called Higgs boson. Non-minimal models are based on a more complex Higgs sector and therefore predict additional neutral and charged Higgs bosons [37].

Summarising the considerations above, the Lagrangian of the Higgs field after spontaneous symmetry breaking is

$$
\begin{aligned}
& L_{\text {Higgs }}= \underbrace{\frac{1}{2} \partial_{\mu} H \partial^{\mu} H}_{\text {kinetic part }}+\text { const } \\
& \underbrace{+\frac{1}{4} g^{2} v^{2} W_{\mu}^{+} W^{-\mu}+\frac{1}{8}\left(g^{2}+g^{\prime 2}\right) v^{2} Z_{\mu} Z^{\mu}-\lambda v^{2} H^{2}}_{\text {mass terms }} \\
& \underbrace{+\frac{1}{2} g^{2} v H W_{\mu}^{+} W^{-\mu}+\frac{1}{4}\left(g^{2}+g^{\prime 2}\right) v H Z_{\mu} Z^{\mu}}_{\text {trilinear } H W^{+} W^{-} \text {and } H Z Z \text { coupling }} \\
& \underbrace{+\frac{1}{4} g^{2} H^{2} W_{\mu}^{+} W^{-\mu}+\frac{1}{8}\left(g^{2}+g^{\prime 2}\right) H^{2} Z_{\mu} Z^{\mu}}_{-\lambda v H^{3}-\frac{1}{4} \lambda H^{4}} \\
& \text { Suartic } H H W^{+} W^{-} \text {and } H H Z Z \text { coupling } \\
& \text { Self-coupling of the Higgs field }
\end{aligned}
$$

The mass terms of the gauge bosons at tree level can be determined directly from the Lagrangian

$$
\begin{aligned}
M_{W} & =\frac{1}{2} v g=\frac{e v}{2 \sin \theta_{W}}, \\
M_{Z} & =\frac{1}{2} \sqrt{g^{2}+g^{\prime 2}} v=\frac{e v}{2 \sin \theta_{W} \cos \theta_{W}}=\frac{M_{W}}{\cos \theta_{W}}, \\
M_{\gamma} & =0 \\
M_{H} & =v \sqrt{2 \lambda} .
\end{aligned}
$$


The masses of the $W$ and $Z$ boson depend directly on the vacuum expectation value, thus it could be determined by measuring both masses. But the Higgs boson mass cannot be calculated from the vacuum expectation value, since it also depends on $\lambda$, which is a free parameter in the SM.

Mass terms for the fermions have to be added via trilinear Yukawa couplings of the fermions to the Higgs fields, which results in extra terms for the Lagrangian. Fermion masses are given by

$$
m_{f}=\frac{1}{\sqrt{2}} g_{f} v
$$

with the coupling constants $g_{f}$ being free parameters of the SM.

\subsection{Phenomenology of pp Collisions}

The description of hadronic collisions requires a profound understanding of the proton structure, since the actual interaction takes place between the proton constituents, the partons. Figure 2.2 shows a schematic view of the evolution of a hadronic collision.

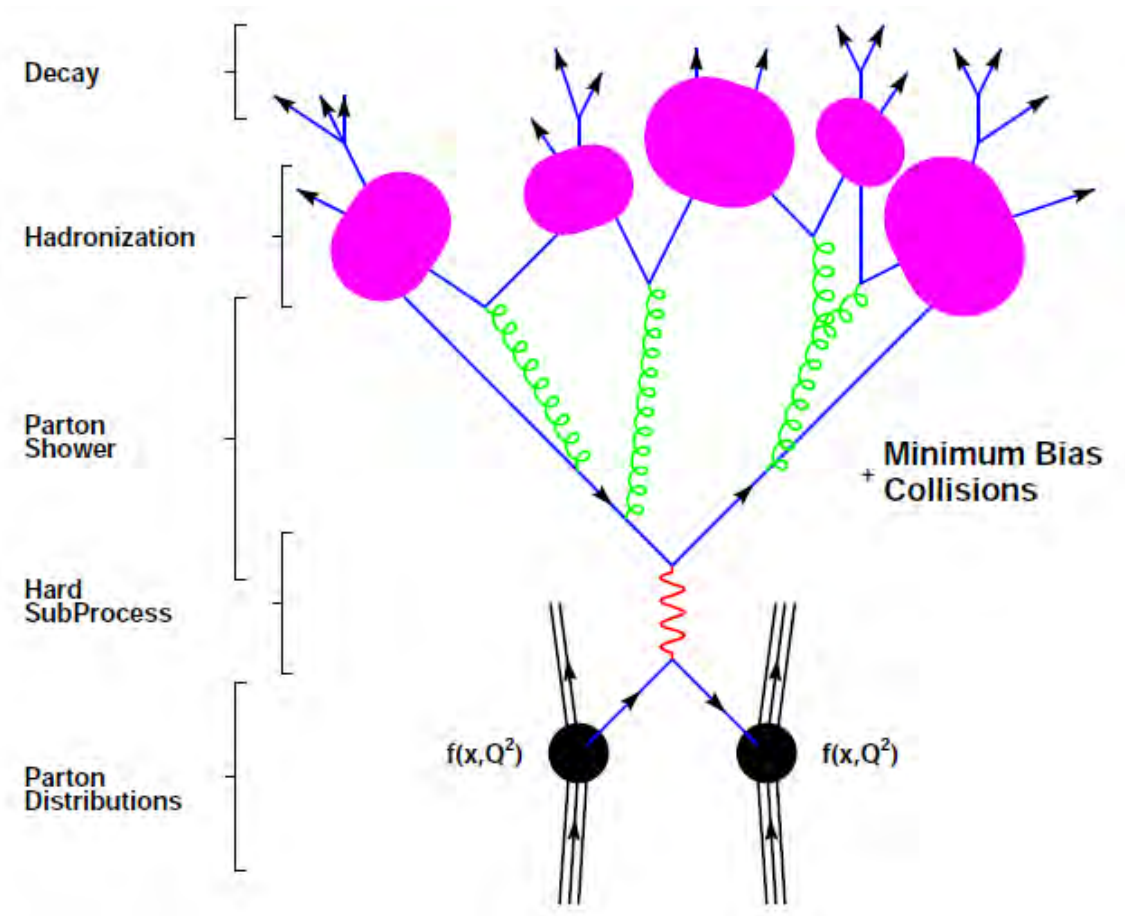

Figure 2.2.: Schematic view of a hadronic collision event [38].

Sub-processes in hadronic collisions can be classified into two types of processes, hard and soft. For both the underlying theory is QCD, but only the former can be described using fixed-order perturbation theory. In order to describe the latter, phenomenological models are needed, since the rates and properties of soft processes are dominated by nonperturbative QCD effects. In addition, fixed-order perturbation theory is able to provide parton level predictions at LO, NLO and NNLO for inclusive cross sections, but for the 
description of the full final state effects such as QCD radiation and fragmentation, play an essential role.

This chapter gives a brief overview on general features of hadronic collisions and the calculation of hadronic cross sections. In addition, it details some phenomenological QCD models, which are relevant for the following analysis.

\subsubsection{Parton Model}

The parton model in its original formulation was proposed by R. P. Feynman in 1969 [39] and was used to describe deep inelastic lepton-hadron scattering. Hadronic collisions at high energies are described by the QCD improved parton model [40]. In this model, each hadron with four-momentum $P$ is described by point-like constituents, the partons, which are moving collinear to the hadron, carrying a fraction of its momentum $p_{i}=x_{i} P_{i}$. The actual interaction of the hadronic collision, the hard scattering, takes place between the partons at the 4-momentum exchange $Q^{2}$, which could be e.g. the mass of the $Z$ boson or the transverse momentum of a jet. In order to provide an exact description of the hard scattering process, the momentum distributions of the partons $f\left(x, \mu^{2}\right)$, so called Parton Distribution Functions (PDFs), have to be well known. $\mu$ denotes the factorisation scale, which separates the two energy regimes of short and long distance physics. For inclusive measurements the factorisation scale is usually chosen to be at the same order as the renormalisation scale $Q$ of the hard process.

\subsubsection{Parton Distribution Functions}

PDFs are determined at LO and NLO by various collaborations. The approaches from the CTEQ [4] and MRST [2, 44] groups are mainly used at the ATLAS experiment. PDFs cannot be calculated from first principles using perturbation theory. Therefore, they are obtained from global fits to experimental data from deep inelastic scattering (DIS), Drell-Yan (DY) and jet production covering a wide range in $x$ and $Q^{2}$. Figure 2.3 shows as an example the PDFs from the CTEQ6M PDF set for $Q=100 \mathrm{GeV}$.

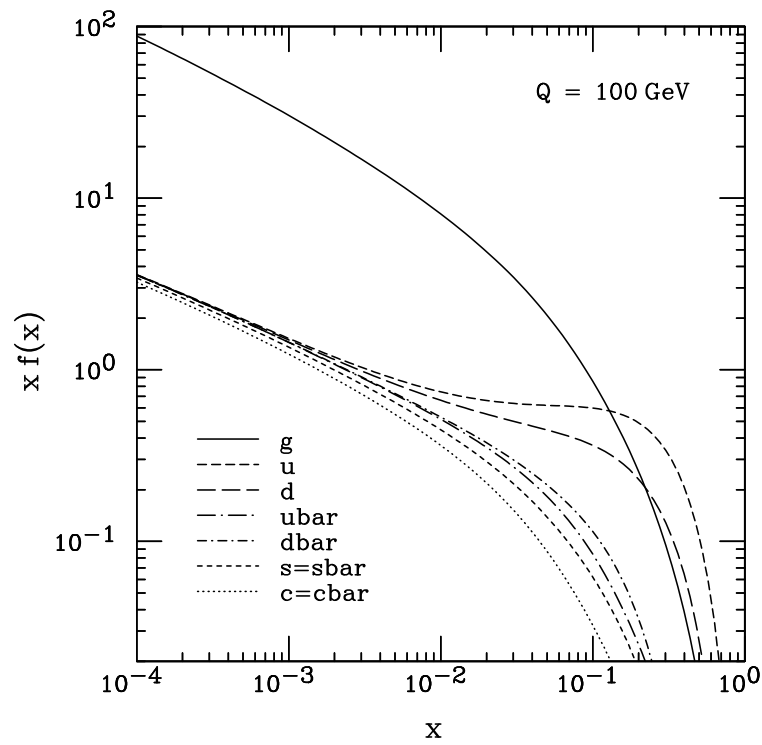

Figure 2.3: CTEQ6M parton distribution functions for a momentum scale of $Q=$ $100 \mathrm{GeV}$ [41].

In addition to a precise knowledge of the values of the PDFs, the uncertainties on 
these values are of great importance. The different sources of uncertainties are explained in Ref. [41, 45, 46]. The quark distributions are mainly determined from DIS and DY production, whereas the gluon distribution is determined indirectly from measurements of the quark distributions at low $x$, and from jet production measurements for high $x$. Thus, the largest uncertainty comes from the gluon distribution. For the evaluation of PDF uncertainties two approaches are used, the Lagrange Multiplier [47] and the Hessian technique [48]. Latest results from both the CTEQ and the MRST group use the Hessian technique. In the context of the Hessian technique, a matrix with dimensions defined by the number of free parameters $N_{p}$ of the global fit is diagonalized, which results in a set of eigenvectors, 20(26) for CTEQ and 15 for MRST. The set of eigenvectors provide the basis for the calculation of the PDF uncertainty on the cross section. From that, the final PDF error set is determined by varying up and down each eigenvector within the corresponding uncertainties, resulting in 40(52) PDF error sets for CTEQ and 30 PDF error sets for MRST.

\subsubsection{Cross Section}

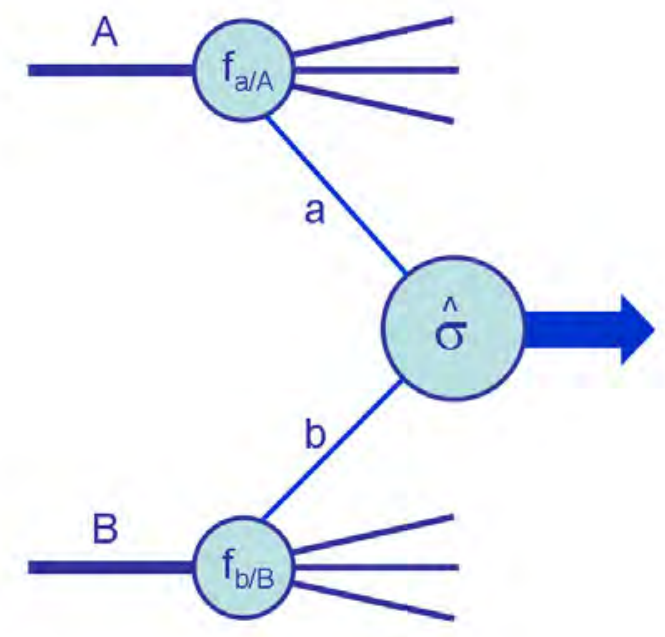

Figure 2.4.: Schematic view of a hard scattering process [49].

Using the factorization theorem [50], the cross section $\sigma_{A B}$ of a hard scattering process, schematically depicted in Fig. 2.4, is given by

$$
\sigma_{A B \rightarrow X}=\int \mathrm{d} x_{a} \mathrm{~d} x_{b} f_{a / A}\left(x_{a}, Q^{2}\right) f_{b / B}\left(x_{b}, Q^{2}\right) \hat{\sigma}_{a b \rightarrow X}
$$

where $\hat{\sigma}_{a b \rightarrow X}$ describes the short-distance cross section for the scattering of the partons $a$ and $b$, also called partonic cross section. The long-distance part is separated from the short-distance part and included in the PDFs. Therefore, the short-distance part does not depend on the incoming hadrons and can be calculated by a perturbative expansion in $\alpha_{s}$ 


$$
\sigma_{A B \rightarrow X}=\int \mathrm{d} x_{a} \mathrm{~d} x_{b} f_{a / A}\left(x_{a}, \mu_{F}^{2}\right) f_{b / B}\left(x_{b}, \mu_{F}^{2}\right) \times\left[\hat{\sigma}_{0}+\alpha_{s}\left(\mu_{R}^{2}\right) \hat{\sigma}_{1}+\ldots\right]_{a b \rightarrow X} .
$$

$\mu_{F}$ denotes the factorisation scale and $\mu_{R}$ the renormalisation scale, at which the QCD coupling constant is evaluated. $\mu_{R}$ is introduced due to renormalisation of the QCD expansion. At higher order of the perturbative expansion, the dependence of the cross section on $\mu_{F}$ and $\mu_{R}$ decreases. Typically, the factorisation scale and the renormalisation scale are set equal and chosen to be at the order of the momentum scale $Q$ of the hard scattering process.

\subsubsection{Higher Order Corrections}

The cross section of the hard scattering process, detailed in Sect. 2.2.3, is determined using a perturbative expansion. Leading order (LO) calculations provide a first estimate of the cross section, but for large parts of the phase-space this approximation is not sufficient. The scale uncertainty for LO calculations is quite large, since LO calculations are highly dependent on the choice of $\mu_{F}$ and $\mu_{R}$. In general, including higher orders of $\alpha_{s}$ in the calculation leads to more precise cross-section estimates. At each order in perturbation theory, the partonic cross section contains ultraviolet (UV) infinities, which have to be renormalised. In addition, higher order corrections consist of real and virtual corrections, both need to be included in the calculation to cancel infrared (IR) divergences [51, 52]. Higher-order calculations require a large effort, since all diagrams containing an additional factor of $\alpha_{s}$ have to be taken into account. An approximation of higher-order effects can be achieved by applying a so-called global $K$-factor, the ratio of the higher-order cross section to the LO cross section, e.g.

$$
\begin{gathered}
K_{N L O}=\frac{\sigma_{N L O}}{\sigma_{L O}} \\
K_{N N L O}=\frac{\sigma_{N N L O}}{\sigma_{L O}}
\end{gathered}
$$

Already, NLO calculations improve the cross-section estimate and uncertainty, but even NLO calculations are not appropriate in all phase space regions. For NLO calculations the higher-order corrections consist of one-loop virtual corrections and the real emission of one extra quark or gluon. The test of NLO perturbative QCD predictions is one of the main parts of this thesis.

\subsubsection{Luminosity}

The event rate, $R$, of a given process is related to its cross section $\sigma$ and the instantaneous luminosity $L$

$$
R=\sigma \cdot L
$$

The latter is proportional to the number of bunches, $n_{b}$, the numbers of particles per bunch in each beam, $n_{1}$ and $n_{2}$ and and the collision frequency, $f$. In addition, it is 
inversely proportional to the effective interaction area $A_{\text {eff }}$. These considerations are valid for a simplified model, in which the particles are assumed to be point-like.

$$
L=n_{b} \frac{n_{1} \cdot n_{2}}{A_{e f f}} f
$$

with

$$
A_{e f f}=4 \pi \sigma_{x} \sigma_{y},
$$

where $\sigma_{x}$ and $\sigma_{y}$ are the Gaussian beam width in horizontal and vertical direction. The expected event rates and cross sections of various physics processes in $p \bar{p}$-collisions and $p p$-collisions are shown in Fig. 2.5.

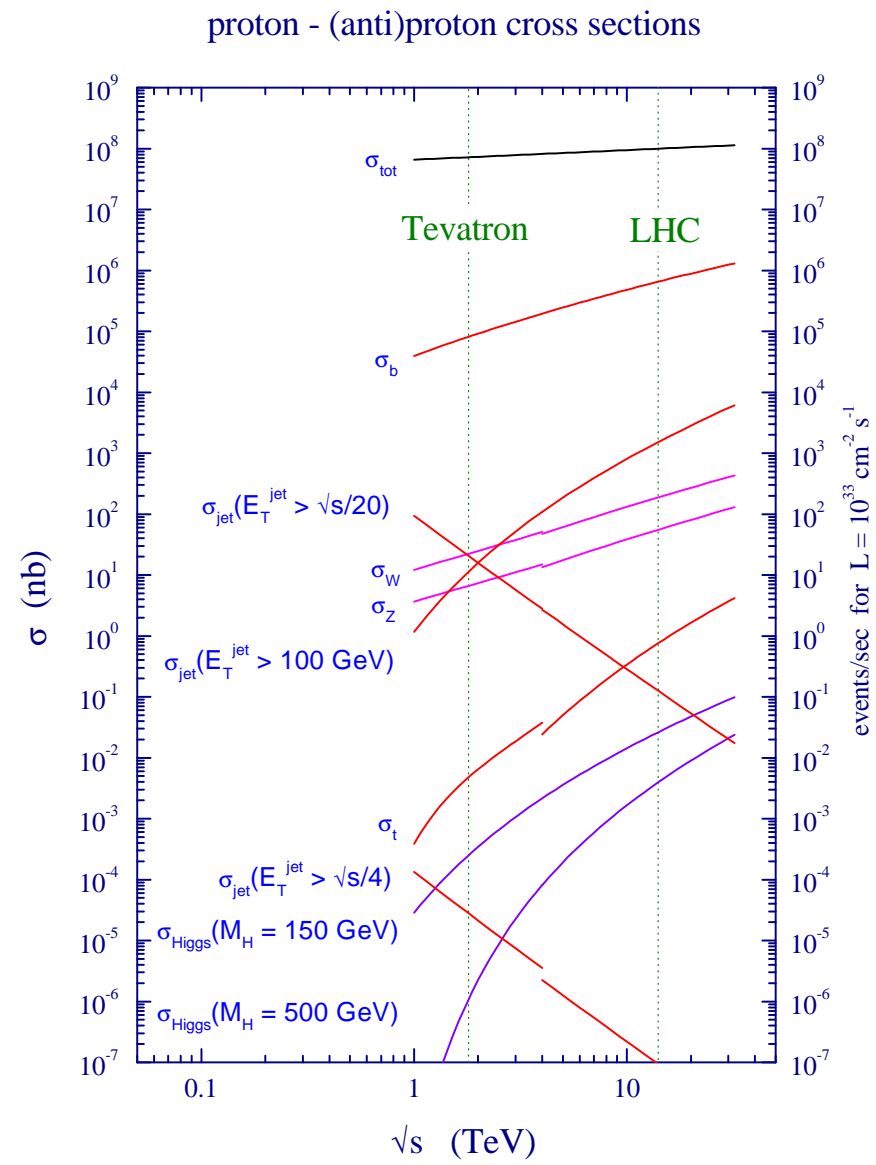

Figure 2.5.: Expected cross section and event rates for various physics processes in $p \bar{p}$-collisions and $p p$-collisions [49].

\subsubsection{Multiple Proton Interactions}

Hadronic collisions are characterized by the hard interaction and additional soft interactions. Figure 2.6 shows the luminosity-weighted distribution of the mean number of interactions per bunch crossing $\mu$ for the data taken in 2011 and 2012 with the ATLAS experiment at the LHC.

The soft interactions, are composed of single-diffractive (SD), double-diffractive (DD), non-diffractive (ND) and central-diffractive (CD) interactions. Minimum bias events are 


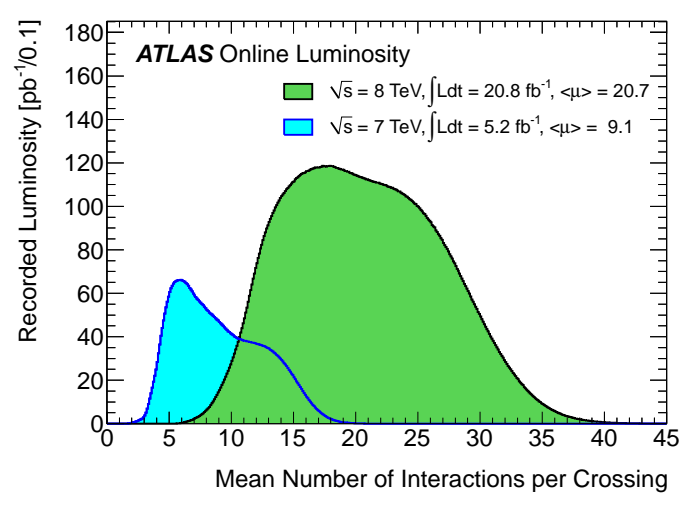

Figure 2.6: Luminosity-weighted distribution of the mean number of interactions per bunch crossing for the data taken in 2011 and 2012 with the ATLAS experiment at the LHC [53].

usually associated with events occurring from non-single-diffractive (NSD) interactions. They are characterised by a low transverse momentum and low multiplicity. The number of minimum bias events is luminosity dependent. To some extent, minimum bias events can be measured at low luminosities using random triggers.

Minimum bias interactions which take place at the same bunch crossing cause so-called in-time pile-up resulting in additional tracks and energy deposits in the different detector parts. In addition to in-time pile-up, Minimum bias interactions from previous bunch crossings could cause so-called out-of-time pile-up, which mainly leads to additional energy deposits in the calorimeter. The correct modelling of in-time and out-of-time pile-up is important for physics measurements at high transverse momentum.

\subsubsection{Underlying Event}

The underlying event (UE) is related to the hard interaction of the hadronic collision and consists of beam remnants and particles arising from soft multiple-parton interactions (MPI). The correct modelling of the UE is essential for precise physics measurements at high transverse momentum and large jet multiplicity, but it cannot be described by perturbation theory. Therefore, non-perturbative phenomenological models are needed to describe the UE. Currently, various approaches exist [54, 55], which are tuned to data. Multi-jet events are ideal to study the UE, since the transverse region with respect to the direction of the leading jet is really sensitive to the UE [56].

\subsubsection{Modelling of QCD Radiation}

As described in Sec. 2.2.4, coloured and/or charged particles can radiate quarks or gluons before they enter the hard interaction, called initial state radiation (ISR), or after the hard scattering process, called final state radiation (FSR). Preferably, the quarks and gluons are emitted collinearly and/or soft to the hard interacting partons, carrying a nonvanishing momentum. The radiated quarks and gluons are again able to radiate, which leads to the formation of partonic cascades.

The correct modelling of ISR and FSR is essential for a full and realistic description of the physics process. Current perturbative calculations in QCD are only performed at NLO or for a few cases at NNLO. Nevertheless, for some phase-space regions corrections for additional parton emissions cannot be neglected. These emissions can be be modelled by either the full matrix-element calculation or by parton shower [57]. 
The former is based on fixed-order pQCD calculations in different orders of $\alpha_{s}$. For higher orders these calculations become increasingly difficult, especially including virtual corrections. Therefore, LO and NLO calculations only exist for a limited number of multiple parton final states such that collinear and/or soft parton emissions are not included. Nevertheless, the hard large-angle emission of a few partons is well described.

The parton-shower method is based on a probabilistic approach to describe the partonic cascades. The probability of additional parton emissions is determined from the DGLAP evolution [58 60] using a virtuality scale $Q^{2}$. Partonic shower evolution is cut-off at a value $Q_{0}$, typically around $1 \mathrm{GeV}$. ISR is calculated using the backward-shower algorithm [61], whereas FSR is calculated using the forward-shower algorithm [62, 63].

Since hard large-angle emissions are better covered by the full matrix-element calculations, whereas soft collinear emissions are better described by the parton shower method, both methods can be combined to provide an accurate description of physics processes with a multiple parton final state at high energies.

\subsubsection{Fragmentation}

The formation of hadrons out of quarks and gluons produced in the collision is called fragmentation or hadronisation. Due to the fact that $\alpha_{s}$ grows to values larger than one for energies at the order of $1 \mathrm{GeV}$, fragmentation cannot be described by perturbation theory. Currently, various phenomenological models exist in order to provide an approximation of such processes. The most successful models are the string fragmentation (SF) [64, 65] and the cluster fragmentation (CF) [66 69]. The different fragmentation models are tuned to match the data. Since the process of fragmentation is approximately universal, already $e^{+} e^{-}$-collision data from LEP could be used for tuning.

\section{3. $Z / \gamma^{*}+$ jets Production at Hadron Colliders}

The introduction of the electroweak theory [22] in 1968 predicted not only the existence of the $W$ boson, responsible for the beta decay, but as well the existence of the $Z$ boson, which was discovered in $p \bar{p}$ collisions at the Super Proton Synchrotron (SPS) at CERN in 1983 [70]. The $Z$ boson is the heaviest of the gauge bosons, as shown in Tab. 2.1. mediating the neutral current part of the weak interaction.

As $Z$ boson properties are precisely known from $e^{+} e^{-}$collisions at the Large Electron Positron Collider (LEP) at CERN and the Stanford Linear Collider (SLC) at SLAC, the selection of $Z$ bosons in hadronic collisions serves as a precision benchmark of SM physics. The production cross section is quite large, see Fig. 2.5. In addition, the decay of the $Z$ boson in two oppositely charged leptons provides a very clean signature in the detector, which is easy to trigger on, since the backgrounds are under control. Furthermore, the selection of $Z$ bosons in association with jets provides a valuable test of pQCD predictions and constitutes an important background for studies of the Higgs boson properties and searches for new physics. Studying the production of $Z$ bosons in association with jets with a high level of accuracy is possible in data taken from the CDF and D $\varnothing$ experiment at the Tevatron and from the ATLAS and CMS experiment at the LHC. $Z$ boson production and an overview of recent experimental results, together with theoretical predictions and the challenges of higher order corrections in kinematic regimes accessible at hadron colliders 
are explained in the following.

\subsection{1. $Z$ Boson Production and Decay}

In hadronic collisions $Z$ bosons are dominantly produced via the Drell-Yan (DY) process, which is depicted in Fig. 2.7. A quark and an anti-quark from the interacting hadrons annihilate to create a pair of oppositely charged leptons, via the exchange of a virtual photon $\gamma^{*}$ or a $Z$ boson.

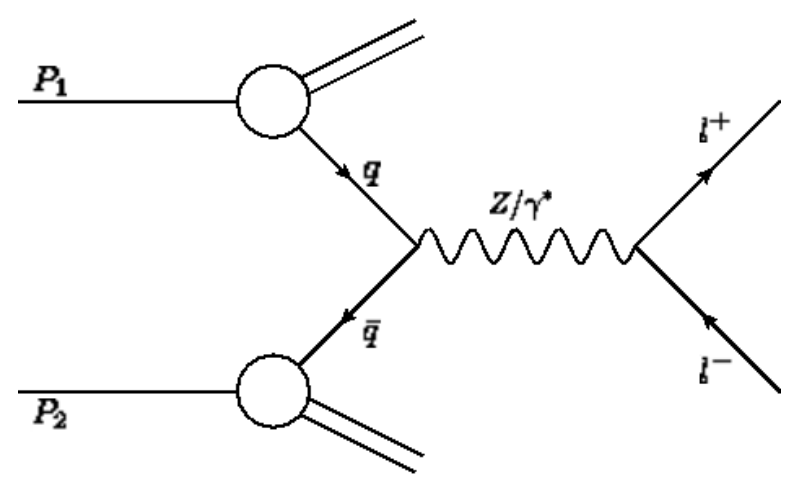

Figure 2.7.: Z boson production via the Drell-Yan process.

Theoretically, the inclusive DY cross section is known at next-to-next-to-leading order (NNLO) pQCD determined by the FEWZ program [71 73] using the MSTW2008 NNLO PDFs [42]. For the calculation, the QCD factorisation and renormalisation scales are set to the mass of the $Z$ boson. The NNLO pQCD prediction of the inclusive DY cross section reaches a precision of approximately $5 \%$ [74], accumulating the uncertainties from NNLO PDFs, the dependence on the factorisation and renormalisation scale and the choice of $\alpha_{s}$.

The decay width of the $Z$ boson is small $\Gamma_{Z}=2.4952 \pm 0.0023 \mathrm{GeV}$ [22] compared to its mass $m_{Z}=91.1876 \pm 0.0021 \mathrm{GeV}[22]$. $Z$ bosons decay leptonically or hadronically into a fermion-antifermion pair, except for the higher mass top and anti-top quark pair. The branching ratios for the different decay modes are summarised in Tab. 2.4.

\begin{tabular}{|c|c|}
\hline \hline Z decay modes & Fraction $\left(\Gamma_{i} / \Gamma\right)[\%]$ \\
\hline \hline$e^{+} e^{-}$ & $3.363 \pm 0.004$ \\
\hline$\mu^{+} \mu^{-}$ & $3.366 \pm 0.007$ \\
\hline$\tau^{+} \tau^{-}$ & $3.370 \pm 0.008$ \\
\hline invisible & $20.000 \pm 0.060$ \\
\hline hadrons & $69.910 \pm 0.060$ \\
\hline
\end{tabular}

Table 2.4.: Different decay modes of the $Z$ boson with their corresponding branching ratios [22]. 


\subsubsection{Jet Production in Association with a $Z$ Boson}

$Z$ bosons in association with a jet can be produced via the Compton process and via the $q \bar{q}$ annihilation. The LO Feynman diagrams for both production mechanisms are shown in Fig. 2.8 .
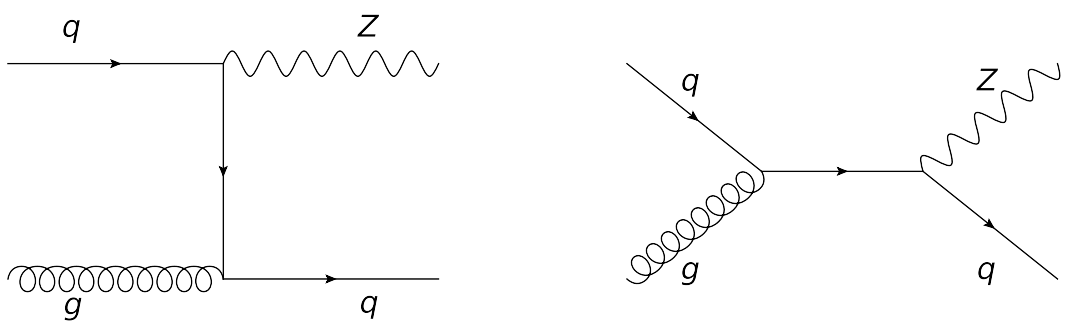

(a)
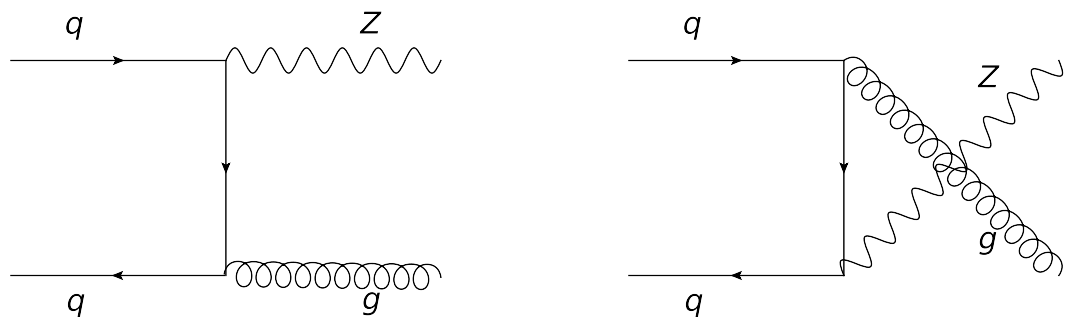

(b)

Figure 2.8.: Feynman diagrams for the production of a jet in association with a $Z$ boson via (a) the Compton process and (b) the $q \bar{q}$ annihilation.

At the LHC with $\sqrt{s}=7 \mathrm{TeV}$, the dominant production mechanism is the Compton process, whereas at the Tevatron with $\sqrt{s}=1.96 \mathrm{TeV}$ the production via $q \bar{q}$ annihilation dominates. A few NLO Feynman diagrams for the production of $Z$ bosons in association with multiple jets in the final state are depicted in Fig. 2.9.
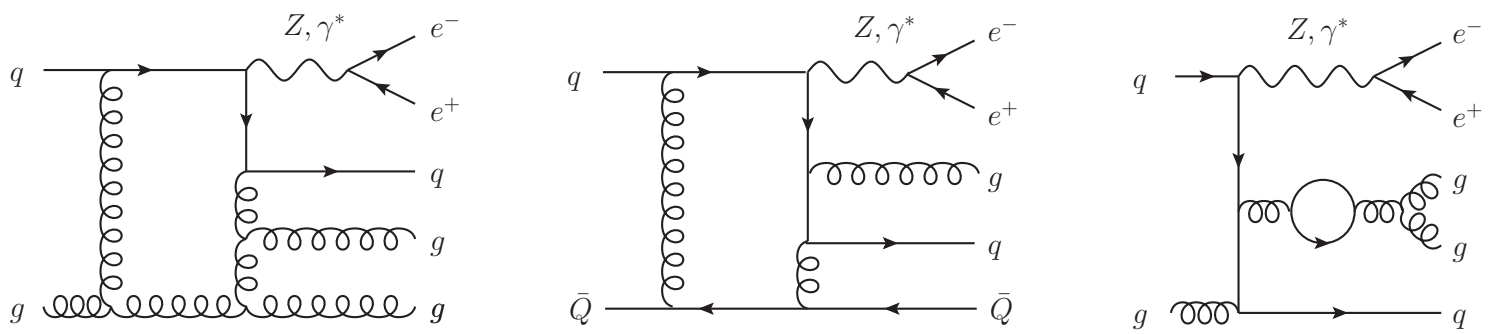

Figure 2.9.: A few NLO Feynman diagrams for the production of $Z$ bosons in association with three jets [15].

For high energy regimes, pQCD predictions are strongly affected by higher-order QCD and EW corrections [75]. For example for $p_{T}^{Z}$, higher-order EW corrections are expected to reduce the differential cross section by $10 \%-20 \%$ in the range $100 \mathrm{GeV}<p_{T}^{Z}<500 \mathrm{GeV}$ [75], as shown in Fig. 2.10, due to larger EW Sudakov logarithms [76]. 


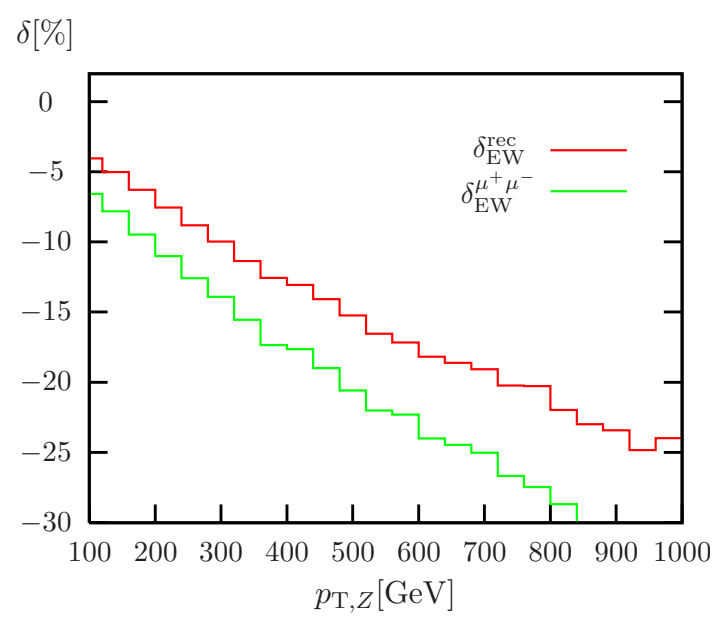

Figure 2.10: Relative electroweak corrections for $p_{T}^{Z}$ at the LHC [75]. The EW correction $\delta_{E W}^{\mu^{+} \mu^{-}}$ for bare muons (green) and $\delta_{E W}^{r e c}$ including leptonphoton recombination (red) are shown.

In addition, $K$-factors are expected to reach values of up to an order of 100 [9] for some phase-space regions. Figure 2.11 compares the LO and NLO distributions of $p_{T}^{Z}$, the $p_{T}$ of the leading jet and the scalar sum of the transverse momenta of all jets $H_{T, j e t s}$.
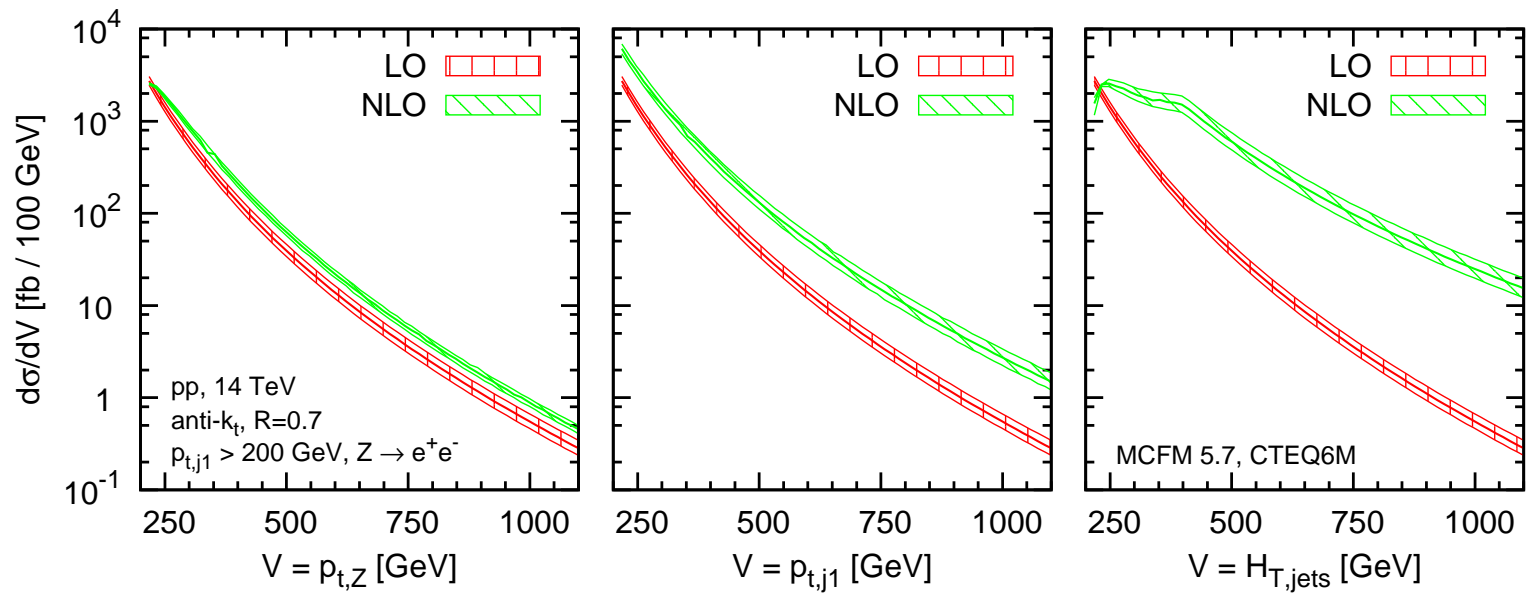

Figure 2.11.: The LO and NLO distributions for $p_{T}^{Z}$ (left), $p_{T}$ of the leading jet (middle) and the scalar sum of the transverse momenta of all jets $H_{T, j e t s}$ (right) obtained with MCFM [9].

The LO predictions of all three distributions are identical, whereas at NLO the predictions give very different results. Final states with high energetic jets, especially when the transverse momentum of the jet exceeds the scale given by the $Z$ boson mass, are expected to be no longer dominated by events with jets recoiling against the $Z$ boson. At these scales, large contributions from final states with a $Z$ boson radiated from a quark line are expected due to QCD corrections at the order of $\alpha_{s} \ln ^{2}\left(p_{T}^{j e t} / m_{Z}\right)$, resulting in large NLO $K$-factors.

For the cross section of the associate production of $Z$ bosons with up to four jets currently no fixed-order predictions higher than NLO exists [14-16]. A detailed discussion of the NLO pQCD predictions for the associate production, together with a comparison to recent measurements can be found in Chapter 8 to Chapter 9 of this thesis. 


\section{Jet Scaling Patterns}

As discussed in the previous sections, theoretical predictions for multi-jet events at a high level of accuracy are challenging. Therefore, the physics of multi-jet production has been studied since 1985 [77 79].

Poisson scaling of the exclusive jet multiplicity corresponds to a multiplicity ratio following

$$
R_{(n+1) / n}=\frac{\sigma_{n+1}}{\sigma_{n}} \sim \frac{\bar{n}}{n+1},
$$

where $n$ describes the number of jets in addition to the hard process and $\bar{n}$ describes the expectation value of the Poisson distribution. In the case of staircase scaling the multiplicity is expected to follow a linear function

$$
R_{(n+1) / n}=\frac{\sigma_{n+1}}{\sigma_{n}} \sim R_{0}+\frac{\mathrm{d} R}{\mathrm{~d} n} n .
$$

Current theoretical studies [10, 80, 81] predict a transition from staircase scaling to Poisson scaling for large scale differences. For small scales and high jet multiplicities additional jet emission is attributed to non-Abelian secondary splittings. For low jet multiplicities, where the scale of the hard process is not much larger compared to the scale of additional jet emissions, the non-Abelian secondary splittings are accompanied by a PDF suppression. The emission of the first parton is more suppressed than the additional parton emissions. For large scale differences between the leading jet and the additional radiated jets, the scaling behaviour is expected to change to a Poisson scaling, typical for an Abelian type of FSR resulting in subsequent soft parton emissions, in analogy to soft photon emissions in QED.

\section{Double-Parton Interactions}

Final states with a $Z$ boson in association with two jets can originate from a single-parton interaction (SPI) or a double-parton interaction (DPI). The LO Feynman diagrams for both processes are depicted in Fig. 2.12.

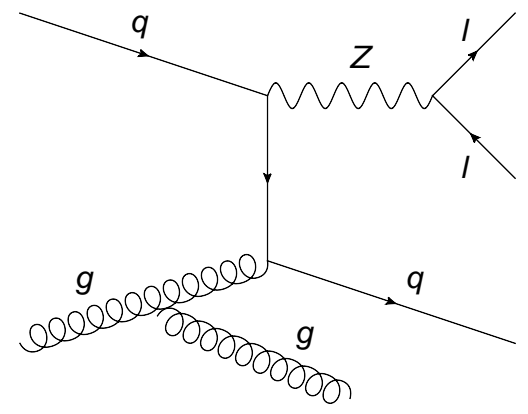

(a) SPI

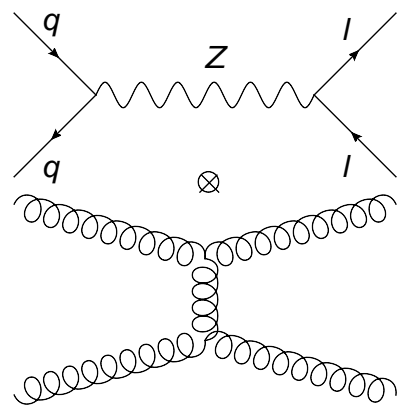

(b) DPI

Figure 2.12.: Leading order Feynman diagrams for the production of a two jets in association with a $Z$ boson originating from (a) single-parton interaction and (b) double-parton interaction.

The DPI part of the differential production cross section for a composite system $(\mathrm{Y}+\mathrm{Z})$ in hadronic collisions can be parametrised by the effective area parameter for DPI, $\sigma_{e f f}(s)$. 
Assuming no correlation at first order between the two production processes, the total cross section can be written as

$$
\mathrm{d} \hat{\sigma}_{Y+Z}^{(t o t)}(s)=\mathrm{d} \hat{\sigma}_{Y+Z}^{(S P I)}(s)+\mathrm{d} \hat{\sigma}_{Y+Z}^{(D P I)}(s)=\mathrm{d} \hat{\sigma}_{Y+Z}^{(S P I)}(s)+\frac{\mathrm{d} \hat{\sigma}_{Y}(s) \cdot \mathrm{d} \hat{\sigma}_{Z}(s)}{\sigma_{e f f}(s)}
$$

where $\mathrm{d} \hat{\sigma}_{Y+Z}^{(S P I)}(s)$ and $\mathrm{d} \hat{\sigma}_{Y+Z}^{(D P I)}(s)$ correspond to the differential cross section of the direct production and the production via DPI, respectively. $\mathrm{d} \hat{\sigma}_{Y}(s)$ and $\mathrm{d} \hat{\sigma}_{Z}(s)$ are the differential cross section of the individual production processes of $Y$ and $Z$. By integrating over the full phase space, $\sigma_{e f f}(s)$ can be determined by

$$
\sigma_{e f f}(s)=\frac{\hat{\sigma}_{Y}(s) \cdot \hat{\sigma}_{Z}(s)}{\hat{\sigma}_{Y+Z}^{(D P I)}(s)}=\frac{\hat{\sigma}_{Y}(s) \cdot \hat{\sigma}_{Z}(s)}{\hat{\sigma}_{Y+Z}^{(t o t)}(s)-\hat{\sigma}_{Y+Z}^{(S P I)}(s)} .
$$

$\sigma_{\text {eff }}$ is expected to be approximately independent of the process and the phase-space requirements.

Current predictions from NLO fixed-order pQCD calculations do not include contributions from DPI, but for $Z+2$ jets events the fraction of DPI events is expected to be rather small $\sim 3 \%$ [82. Therefore, the influence from DPI on the analysis presented in this thesis has been assumed to be negligible, compared to the large uncertainties on the theory predictions and on the measured cross sections.

\subsubsection{Background Processes for Dileptonic Final States}

The jet production in association with a $Z$ boson for dielectron and dimuon final states are characterised by two isolated oppositely charged leptons with additional well separated jets. Background contributions are expected from the production of $t \bar{t}$ pairs, single top quarks, pairs of $W$ bosons, $Z$ bosons and a $W$ and a $Z, Z / \gamma^{*}(\rightarrow \tau \tau), W(\rightarrow e \nu)$ and multi-jet events. At the LHC, pairs of top antitop quarks are mainly produced via gluon fusion. Single top quarks are produced in electroweak interactions via the s-channel, tchannel and the associate production with a $\mathrm{W}$ boson (Wt-channel). Pairs of $W$ bosons, $Z$ bosons and a $W$ boson and a $Z$ boson, in the following referred to as diboson, are mainly produced in quark-antiquark interactions.

The background processes can be split into two groups, the irreducible backgrounds and the reducible backgrounds. The former are characterised by an identical final state with two isolated leptons. This is the case for $t \bar{t}$, diboson and $Z / \gamma^{*} \rightarrow \tau^{+} \tau^{-}$production. The reducible backgrounds can be split again into two groups, the backgrounds with one isolated lepton and the backgrounds without isolated leptons. For both reducible backgrounds the additional leptons originate from misidentification of jets or additional activity in the detector. Events with only one isolated lepton in the final state are coming from jet production in association with a $W$ boson, where the $W$ boson is decaying leptonically, or from single-top-quark production. Events without isolated leptons originate from multi-jet processes. Even if the misidentification rate for jets faking an isolated lepton is rather low, the background from multi-jet processes has a non-negligible contribution due to the large cross section for multi-jet processes.

The individual background contributions at the Tevatron and the LHC vary because of the different production mechanisms due to the different centre-of-mass energies. The 
cross sections for the individual processes are shown in Fig. 2.5 in Sec. 2.2.5. At the Tevatron the dominant background contributions are multi-jet processes and the production of jets in association with a $W$ boson, whereas at the LHC multi-jet processes, $t \bar{t}$ and diboson production are the dominant contributions.

\subsubsection{Experimental Measurements of $Z / \gamma^{*}+$ jets Production}

Measurements of the jet production in association with a $Z$ boson have been performed at the Tevatron [3, 4] as well as at the LHC. At both colliders the analysis strategies are quite similar. The measured cross sections, determined by a cut and count method, are corrected for detector effects back to particle level and compared to pQCD predictions and predictions from various generators. Results from the Tevatron show a good agreement between NLO pQCD predictions and the measured cross sections, as shown in Fig. 2.13. The measurements have only been performed for jet multiplicities up to three jets in the final state and transverse momentum distributions up to $400 \mathrm{GeV}$ for events with one or two jets in the final state.

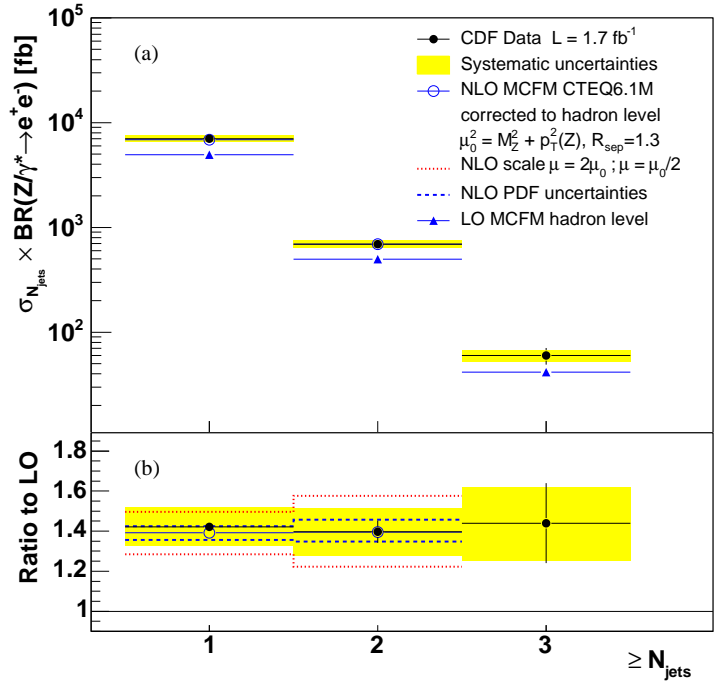

(a) Inclusive jet multiplicity

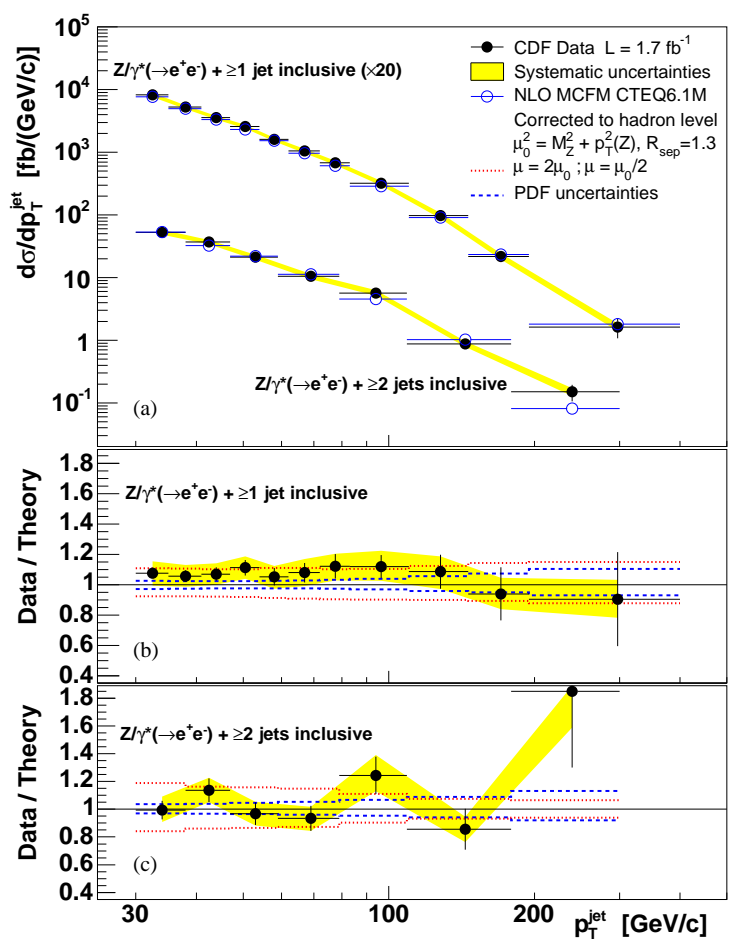

(b) Inclusive jet $p_{T}$

Figure 2.13.: (a) Measured cross section as a function of the inclusive jet multiplicity and (b) measured inclusive differential cross section as a function of the transverse momentum of the jets for $Z / \gamma^{*}\left(\rightarrow e^{+} e^{-}\right)+$jets $[3$.

The significantly higher center-of-mass energy and the increased production cross section at the LHC allow to explore completely new phase-space regions and provide more precise tests of $\mathrm{pQCD}$ predictions. 


\subsection{Higgs Boson Production at Hadron Colliders}

The Higgs mechanism, as described in Sec. 2.1.1, yields the most popular and minimal solution to solve the problem of mass generation. It predicts the existence of at least one single Higgs boson. The Higgs boson is a scalar massive particle, carrying no electric and colour charge. Within the SM, the Higgs boson mass is an unknown parameter. Only upper and lower limits can be set by theoretical arguments. However, previous experiments, such as LEP and the Tevatron, were able to set exclusion limits and shrink the possible Higgs mass range. At the LHC, the search for the Higgs boson and the measurement of its properties is a fundamental part of the physics program.

On July 4th, 2012, the discovery of a new boson of mass near $125 \mathrm{GeV}$ was claimed [1, 2], which is consistent with the SM Higgs boson. The properties of the SM Higgs boson and the different production mechanisms, as well as recent results are described in the remainder of this section. Non-minimal models with more Higgs bosons are not discussed in this thesis.

\subsubsection{Higgs Boson Mass Constraints}

Within the minimal SM no mass for the Higgs boson is predicted, but theoretical arguments suggest some constraints [83-89]. With increasing Higgs mass, the $W W$ scattering cross section via Higgs boson exchange also increases. In order to not violate the unitarity in $W W$ scattering, the Higgs bosons mass must be smaller than $m_{H} \sim 1 \mathrm{TeV}$, unitarity bound. An additional upper limit on the Higgs boson mass has been derived from the $\phi^{4}$ dependence of the Higgs potential of $m_{H}<700 \mathrm{GeV}$, triviality bound. In addition, the Higgs potential is bounded from below, since the vacuum has to remain stable, vacuum stability bound. With decreasing value of $\lambda$, the potential becomes flatter, which could cause an instability of the vacuum and therefore results in a lower bound on $\lambda$. As the Higgs mass is proportional to $\sqrt{\lambda}$, the Higgs mass is limited, as well, by $m_{H}>7 \mathrm{GeV}$. Both the upper and the lower limits on the Higgs boson mass are dependent on the cut-off scale $\Lambda$ up to which the SM is expected to be valid.

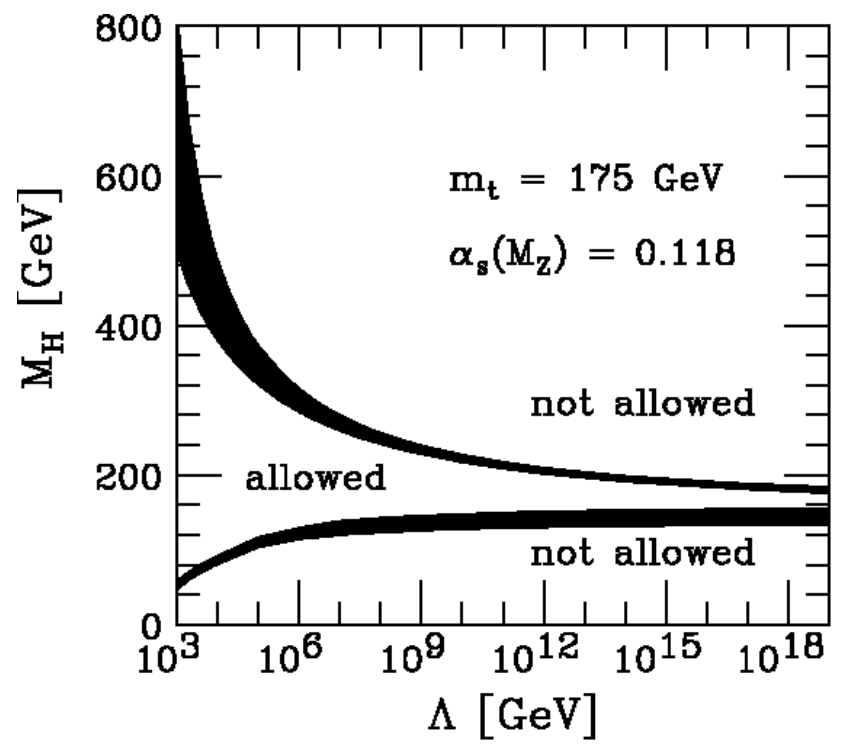

Figure 2.14: "Chimney plot": Upper and lower limits on the Higgs boson mass as a function of the cut-off scale $\Lambda$ up to which the SM is expected to be valid [90].

Figure 2.14 shows the Higgs boson mass as a function of $\Lambda$ taking the theoretical 
arguments from above into account. The upper limit is given by the trivality bound, while the lower limit has been determined from the vacuum stability bound.

Further constraints on the Higgs boson mass were set by indirect and direct measurements from the experiments. The former is determined from a global fit to data considering precision measurements of weak neutral currents and the $W$ and $Z$ masses. An essential part is the sensitivity of the $W$ mass on electroweak radiative corrections, since it is proportional to $\ln \left(m_{H}\right)$ but also proportional to $m_{t}^{2}$, which makes the determination quite complicated. The combination of electroweak precision measurements from LEP and the Tevatron results in an upper limit on the Higgs boson mass of about $152 \mathrm{GeV}$ at $95 \%$ confidence level (CL) [91]. Direct measurements from LEP have set a lower limit of $m_{H}=114.4 \mathrm{GeV}$ at $95 \% \mathrm{CL}$ [92. In addition, the combined results of the Tevatron experiments CDF and D $\varnothing$ could exclude the Higgs boson in the mass region $147 \mathrm{GeV}<m_{H}<180 \mathrm{GeV}$ at $95 \% \mathrm{CL}$ 93.

\subsubsection{Higgs Boson Decay}

The Higgs boson couples directly to massive particles, coupling to massless particles are realised via massive gauge boson or heavy quark loops. Within the SM, the possible Higgs boson decays are predicted for a given Higgs boson mass. The probability of the different decay channels is expressed by the individual branching ratios. Figure 2.15 shows the set of branching ratios for a Higgs boson mass in the range $90 \mathrm{GeV}<m_{H}<1000 \mathrm{GeV}$ with the corresponding theoretical uncertainties.

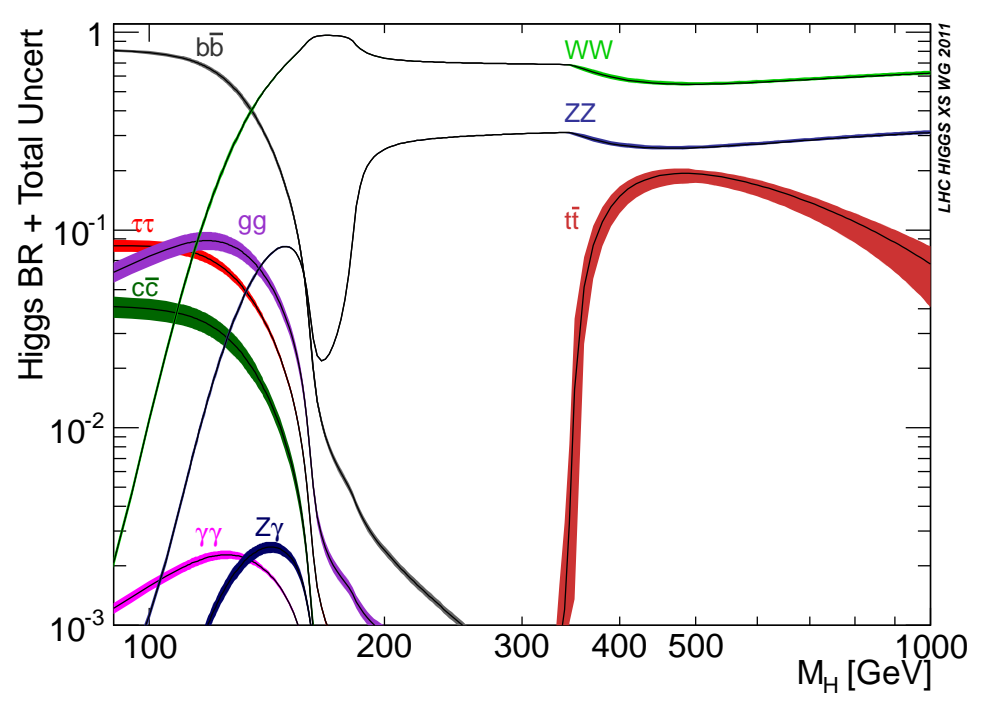

Figure 2.15: Branching ratios of the SM Higgs boson, together with their corresponding theoretical uncertainties [94].

For a light Higgs boson, below the $W W$ threshold, the dominant decay channel is the decay into a bottom-antibottom quark pair with a branching ratio of $\sim 90 \%$ followed by the decay into $\tau \tau$ with $\sim 10 \%$. This difference comes from the fact that the partial decay width for fermion decays is proportional to the fermion mass squared and the $b$-quark mass is a factor of three higher than the $\tau$ mass. Above the $W W$ threshold, the decays into $W W$ and $Z Z$ are dominant. For a heavy Higgs boson, $m_{H}>350 \mathrm{GeV}$, the Higgs boson can also decay into a top-antitop quark pair.

At hadron colliders, final states containing jets must compete with a large amount of multi-jet background, which make searches in these channels, e.g. $H \rightarrow b \bar{b}$, quite difficult. 
Therefore, searches with purely leptonic final states or photons are favoured. For a light Higgs boson the decays into an oppositely charged $\tau$ pair and into a pair of photons are the preferred ones.

\subsubsection{Higgs Boson Production}

The SM predicts various production mechanisms for the Higgs boson, but only a limited number of them is accessible at the LHC, due to their in general low production cross sections compared to other SM processes. Figure 2.16 shows the cross sections of the individual production mechanisms accessible at the $\mathrm{LHC}$ with their corresponding theoretical uncertainties at a centre-of-mass energy of $7 \mathrm{TeV}$ in $p p$-collisions.

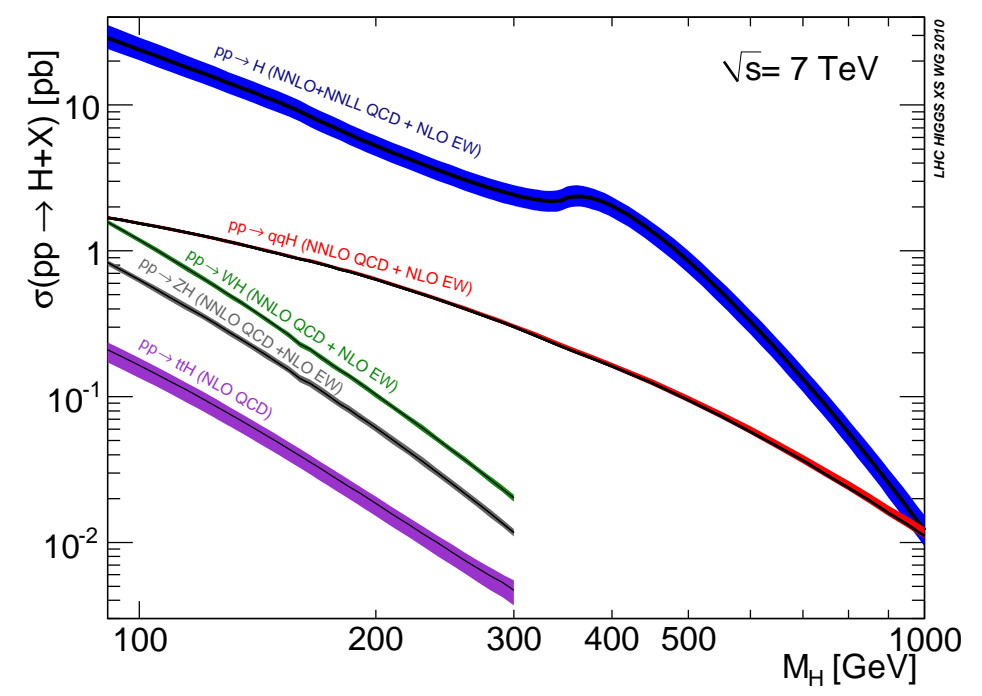

Figure 2.16: Production cross section for the SM Higgs boson as a function of its mass with its corresponding theoretical uncertainties at a centre-of-mass energy of $7 \mathrm{TeV}$ in $p p$-collisions [94].

At the LHC, the dominant production mechanism over the whole Higgs boson mass range is the gluon fusion via a virtual heavy quark loop. The second most important contribution to the overall production cross section is the weak boson fusion (WBF), which has a ten times smaller cross section than the gluon fusion. The WBF production is characterised by two high energetic, well separated forward jets and reduced central jet activity in the final state, which can be used for background suppression. Other possible production mechanisms are the associated Higgs boson production with weak bosons or top quarks. Figure 2.17 shows the LO Feynman diagrams of the dominant production mechanisms at the LHC. 


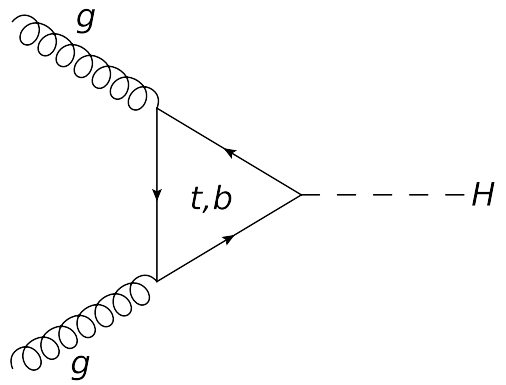

(a) Gluon fusion

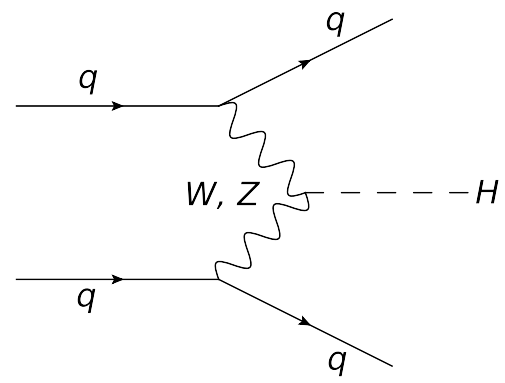

(b) Weak boson fusion

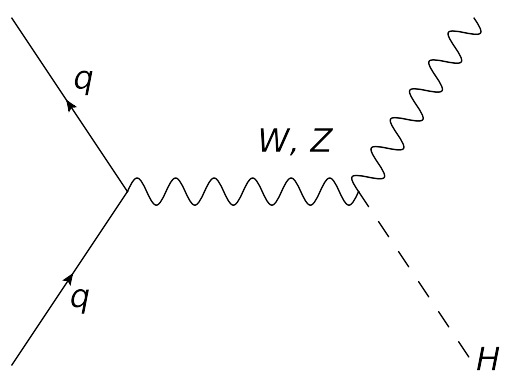

(c) Higgs-Strahlung

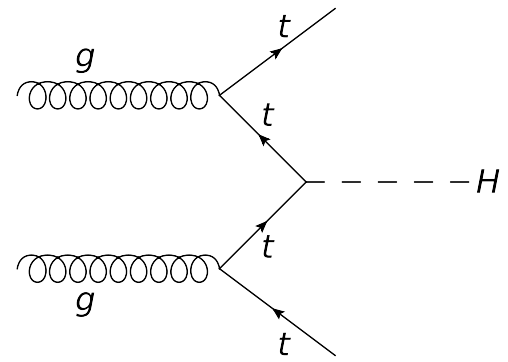

(d) Top fusion

Figure 2.17.: LO Feynman diagrams for the dominant Higgs boson production mechanisms at the LHC: (a) the gluon fusion, (b) the weak boson fusion, (c) the associated Higgs boson production with vector bosons and (d) top quarks.

By moving to higher centre-of-mass energies the cross sections will be significantly increased, as shown in Fig. 2.18.

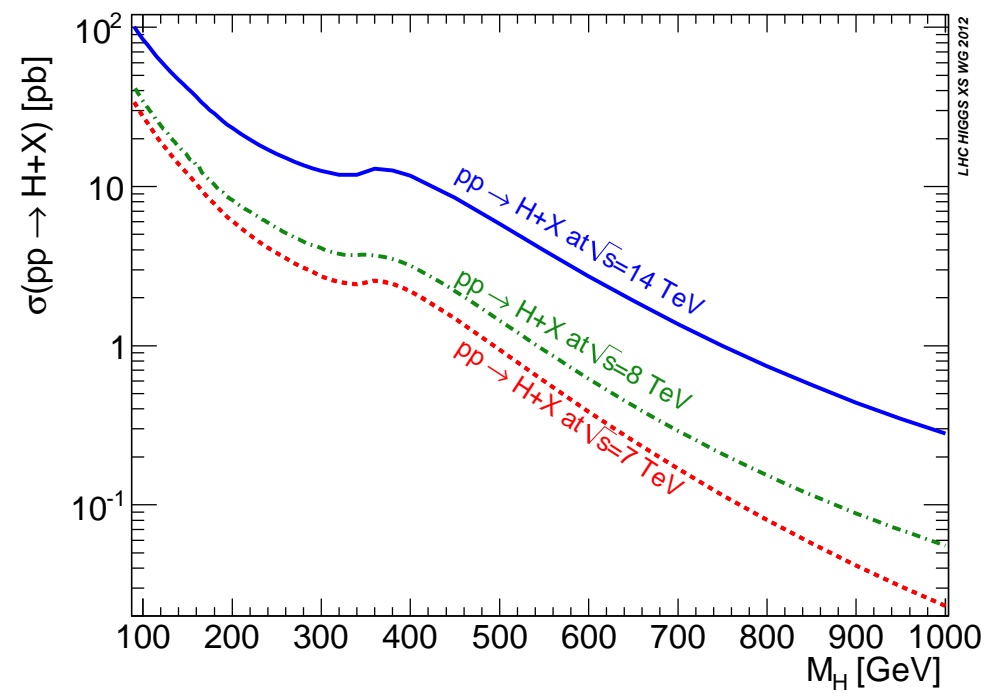

Figure 2.18: Total production cross section of the SM Higgs boson as a function of its mass for different centre-of-mass energies in $p p$-collisions [94]. 


\subsubsection{Experimental Measurements of Higgs Boson Production}

Searches at previous colliders, such as LEP and the Tevatron, were able to shrink the possible Higgs boson mass range, as discussed in Sec. 2.4.1. In addition, recent results from the Tevatron have shown an excess with a global significance of $\sim 2.5 \sigma$ in the mass range $115 \mathrm{GeV}<m_{H}<140 \mathrm{GeV} 93$.

The LHC has successfully started the quest for the Higgs boson. On July 4, 2012, both the ATLAS collaboration and the CMS collaboration claimed the discovery of a new boson with a mass of $126.0 \pm 0.4$ (stat) \pm 0.4 (syst) GeV (ATLAS) [1] and $125.3 \pm 0.4$ (stat) \pm 0.5 (syst) GeV (CMS) [2] with a local significance above $5 \sigma$. Recent results from ATLAS and CMS from the end of 2012 using a combined dataset of up to $4.8 \mathrm{fb}^{-1}$ at $\sqrt{s}=7 \mathrm{TeV}$ and $13 \mathrm{fb}^{-1}$ at $\sqrt{s}=8 \mathrm{TeV}$ (ATLAS) and up to $5.1 \mathrm{fb}^{-1}$ at $\sqrt{s}=7 \mathrm{TeV}$ and $12.2 \mathrm{fb}^{-1}$ at $\sqrt{s}=8 \mathrm{TeV}$ (CMS) have shown an increased local significance of the new boson of $7.0 \sigma$ (ATLAS) [95] and $6.9 \sigma$ (CMS) [96] for a Higgs boson mass hypothesis near $m_{H}=125 \mathrm{GeV}$. Figure 2.19 shows the observed local probability $p_{0}$ for both experiments for the individual channels and the combination.

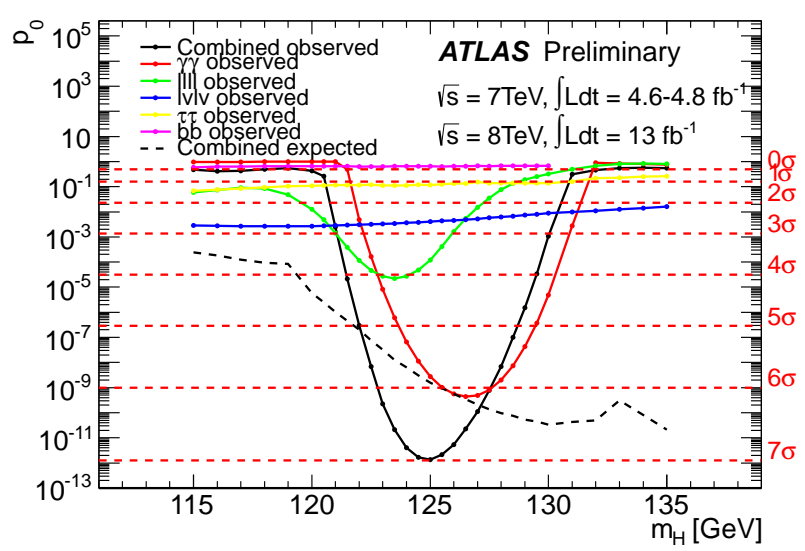

(a)

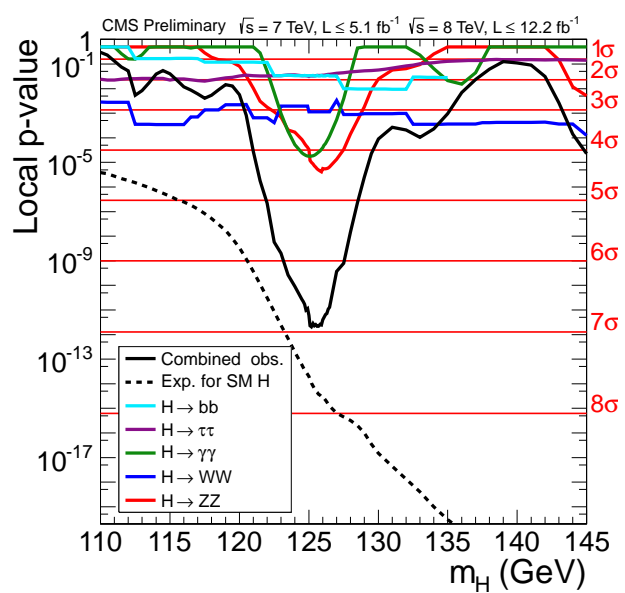

(b)

Figure 2.19.: Observed local probability $p_{0}$ for the individual channels and the combination for (a) the ATLAS experiment [95] and (b) the CMS experiment [96]. The dashed black line shows the expected local probability $p_{0}$ as a function of the Higgs boson mass.

The properties of this boson are consistent with the expectations of a SM Higgs boson, but the uncertainties on the properties are still very large. So far, the LHC shows evidence only in the vector boson decay modes $H \rightarrow \gamma \gamma, H \rightarrow Z Z$ and $H \rightarrow W W$.

From now on, the highest priority is to establish the nature of this new boson, which also implies the observation of the boson decaying to fermions, such as $H \rightarrow \tau \tau$. One of the most promising channels would be the WBF production, since it provides a very clean signature in the detector. 



\section{Experimental Setup}

The Large Hadron Collider (LHC) at CERN plays a decisive role in the investigation of the fundamental problems of the SM and beyond. This chapter describes the LHC accelerator complex and the ATLAS detector. The first section is dedicated to the description of the LHC and the second section gives an overview on the ATLAS detector with its main components.

\subsection{The Large Hadron Collider}

The LHC [97] is the highest energy particle accelerator ever built. It is located in the old tunnel of the Large Electron-Positron Collider at CERN. With its circumference of $27 \mathrm{~km}$, the LHC crosses the Franco-Swiss border twice. It has been designed to collide protons at a centre-of-mass energy of $14 \mathrm{TeV}$ with a collision rate of $40 \mathrm{MHz}$ and an instantaneous luminosity of $10 \mathrm{nb}^{-1} \mathrm{~s}^{-1}$. Before being injected into the main accelerator, the protons, produced by the ionisation of hydrogen atoms, have to be preaccelerated. Finally, the protons are injected in the main LHC ring in opposite directions, where they are accelerated up to the nominal beam energy, before they are brought to collision. By design, each proton beam includes 2808 bunches of about $10^{11}$ protons per bunch, which results on average in 23 interactions per bunch crossing.

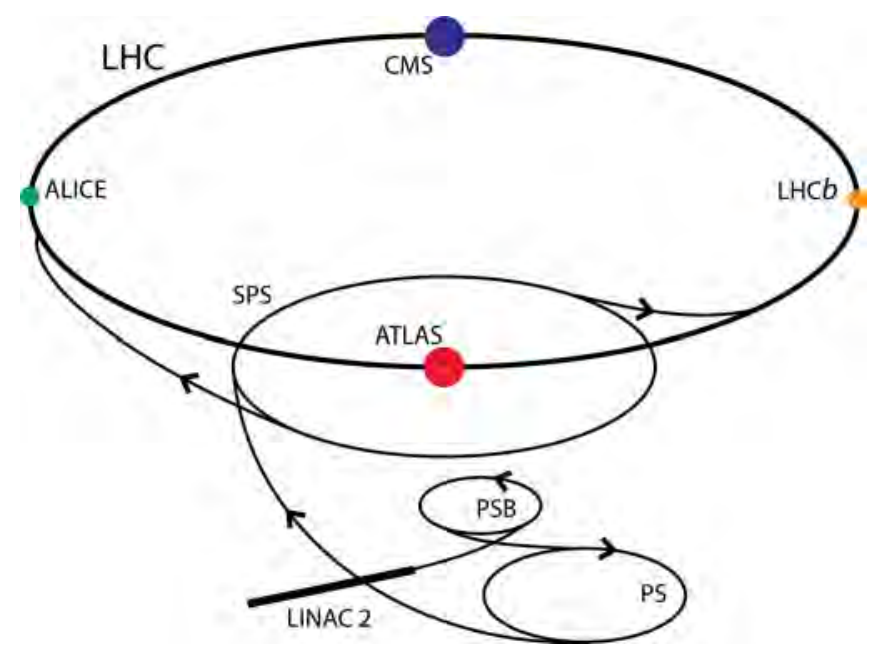

Figure 3.1.: The Large Hadron Collider accelerator with its main experiments.

Figure 3.1 shows a sketch of the LHC accelerator complex. The LHC has four main experiments, ATLAS, CMS, ALICE and LHCb, which are placed at four interaction points around the beam pipe. The two general-purpose detectors ATLAS, which is detailed in Sec. 3.2, and CMS [98] have been built to study many aspects of $p p$-collisions. Both were designed mainly for the discovery of the Higgs Boson, the measurement of its properties 
and searches for new physics. The ALICE [99] detector was built to investigate heavy ion collisions, especially with the purpose of studying the properties of matter at high energy densities. The LHCb [100] detector was also built to study pp collisions, but with a specialisation on physics with b-quarks.

The LHC has successfully started its operation in November 2009, after the first test in September 2008, which was stopped by a magnet quench caused by an electrical fault. On March 30, 2010, a new era of particle physics was heralded. The first collisions at a centre-of-mass energy of $7 \mathrm{TeV}$ were recorded, which set a new world energy record. The operational conditions of the LHC for the data taking of 2010 and 2011 are summarised in Tab. 3.1.

\begin{tabular}{|l|c|c|}
\hline \hline Parameter & 2010 & 2011 \\
\hline \hline Maximum number of bunch pairs colliding & 348 & 1331 \\
Minimum bunch spacing $(\mathrm{ns})$ & 150 & 50 \\
Typical number of protons per bunch $\left(10^{11}\right.$ protons $)$ & 0.9 & 1.2 \\
Peak luminosity $\left(10^{33} \mathrm{~cm}^{-2} \mathrm{~s}^{-1}\right)$ & 0.2 & 3.6 \\
Maximum number of interactions per bunch crossing & $\sim 5$ & $\sim 20$ \\
Total integrated luminosity delivered & $48.1 \mathrm{pb}^{-1}$ & $5.61 \mathrm{fb}^{-1}$ \\
\hline
\end{tabular}

Table 3.1.: Operational conditions of the LHC for 2010 and 2011 data taking in $p p$-collisions at $\sqrt{s}=7 \mathrm{TeV}[101]$.

From the beginning of 2010 until the end of 2012, ATLAS has collected an integrated luminosity of $45 \mathrm{pb}^{-1}$ in $2010,5.25 \mathrm{fb}^{-1}$ in 2011 at $\sqrt{s}=7 \mathrm{TeV}$ and $23.3 \mathrm{fb}^{-1}$ at $\sqrt{s}=$ $8 \mathrm{TeV}$. For 2013 and 2014, a long shutdown is planned to set up the machine for a long running period with its design parameters. Figure 3.2 shows the total integrated luminosity for 2010 and 2011 versus days in $p p$-collisions. For 2010 and 2011 the average data taking efficiencies were $93.6 \%$ and $93.5 \%$, respectively.

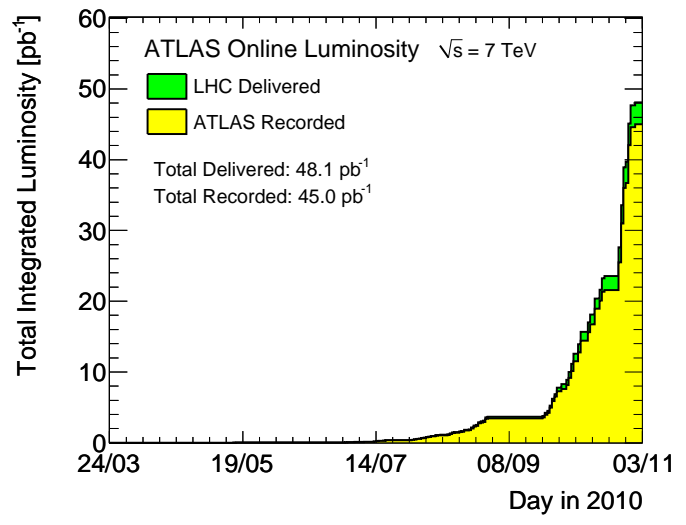

(a) 2010

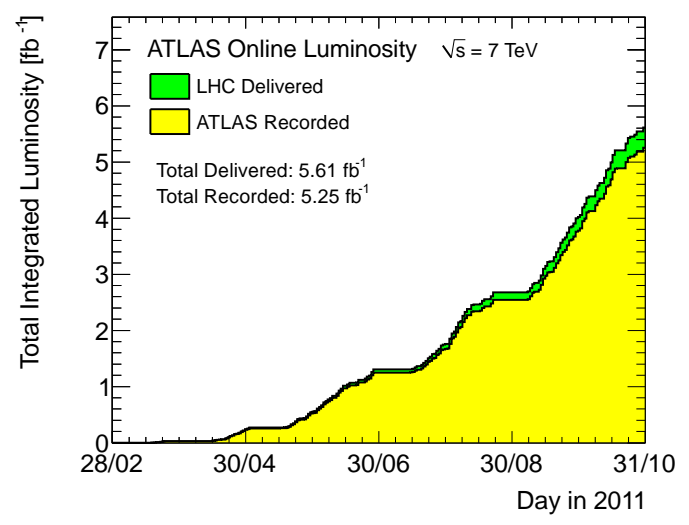

(b) 2011

Figure 3.2.: Total integrated luminosity in pp-collisions for (a) 2010 and (b) 2011 versus day, delivered by the LHC (green) and recorded by ATLAS (yellow) [102. 


\subsection{The ATLAS Detector}

The ATLAS detector [103, with a height of $22 \mathrm{~m}$ and a length of $45 \mathrm{~m}$, is the biggest of the four LHC experiments. Figure 3.3 shows a schematic view of the ATLAS detector with its main components.

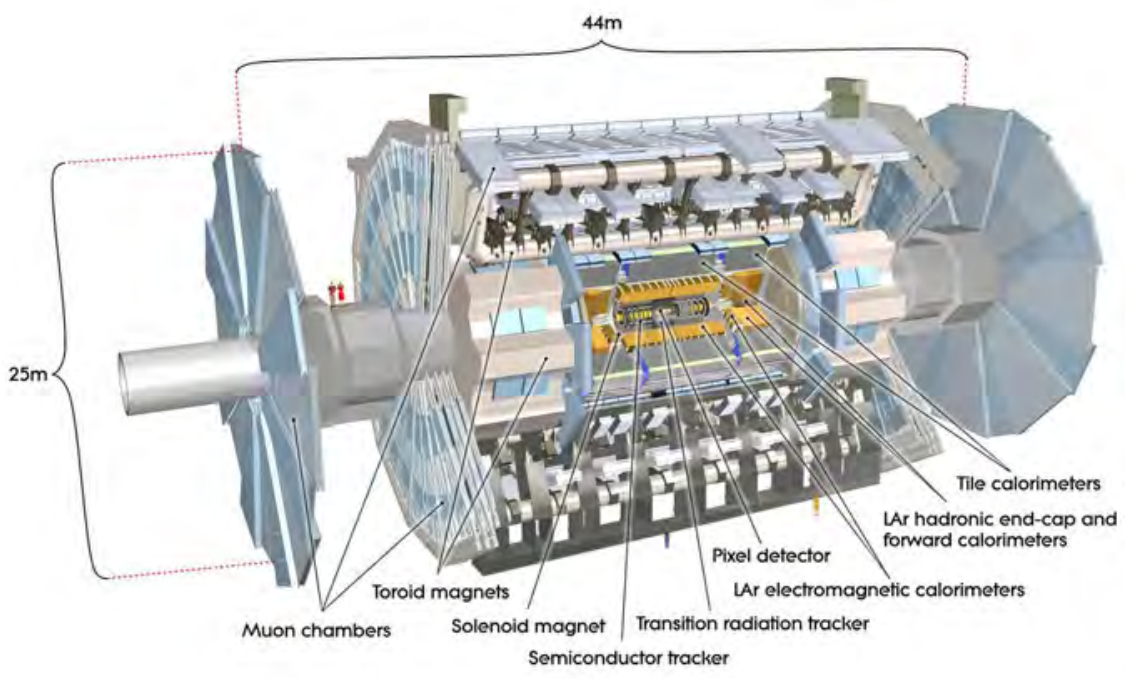

Figure 3.3.: Schematic view of the ATLAS detector [103].

Based on the large physics programme of the LHC, the ATLAS detector has to fulfil many requirements. Most of the interesting physics processes are expected to have a very low cross section, therefore a large integrated luminosity is needed to reach the significance for an observation or a discovery. Since the discovery of the Higgs boson and the measurement of its properties is a fundamental part of the LHC physics program, the ATLAS detector has been optimized for such measurements.

Many of the interesting physics processes are characterised by leptons, high energetic forward jets and a large amount of missing transverse energy (MET) in the final state. Therefore, a very good electromagnetic calorimeter for electron and photon identification and measurements, together with a hermetic hadronic calorimeter for jet and MET measurements is needed. In addition, a high performance of the muon chambers is needed to provide a good muon identification and resolution.

The ATLAS detector has a cylindrical shape and is centred around the beam pipe. It consists of five main components: the magnet system, the inner detector system, the calorimeter system, the muon system, and the data acquisition system. The innermost part of the detector is a silicon-based tracker plus a transition radiation tracker, surrounded by the electromagnetic calorimeter (ECAL) and the hadronic calorimeter (HCAL). The outermost part of the ATLAS detector is the muon spectrometer. In the following subsections, the different detector components are described in more detail.

The coordinate system of the ATLAS detector is defined such that its origin is identical with the nominal interaction point. The $z$-axis points in the direction of the beam axis and the $x$ - $y$ plane is transverse to it. The positive $x$-axis points in the direction of the centre of the LHC ring and the positive $y$-axis points upwards, building a right-handed coordinate system. In addition, cylindrical coordinates are used to parametrise the detector geometry. 
$\phi$ is the azimuthal angle perpendicular to the beam axis and $\theta$ is the polar angle from the beam axis perpendicular to the x-axis. In order to describe the direction of particles in the detector, the pseudo rapidity, defined as

$$
\eta=-\frac{1}{2}\left(\frac{|\vec{p}|+p_{z}}{|\vec{p}|-p_{z}}\right)=-\ln \left(\tan \frac{\theta}{2}\right)
$$

is used. For massive particles like jets, the rapidity is used

$$
y=\frac{1}{2} \ln \left[\left(E+p_{z}\right) /\left(E-p_{z}\right)\right] .
$$

\subsubsection{Magnet System}

The ATLAS detector has two large superconducting magnet systems: the solenoid and the toroid system. Both are used for the bending of charged tracks in order to measure their momentum. Figure 3.4 shows a schematic view of the magnet system.

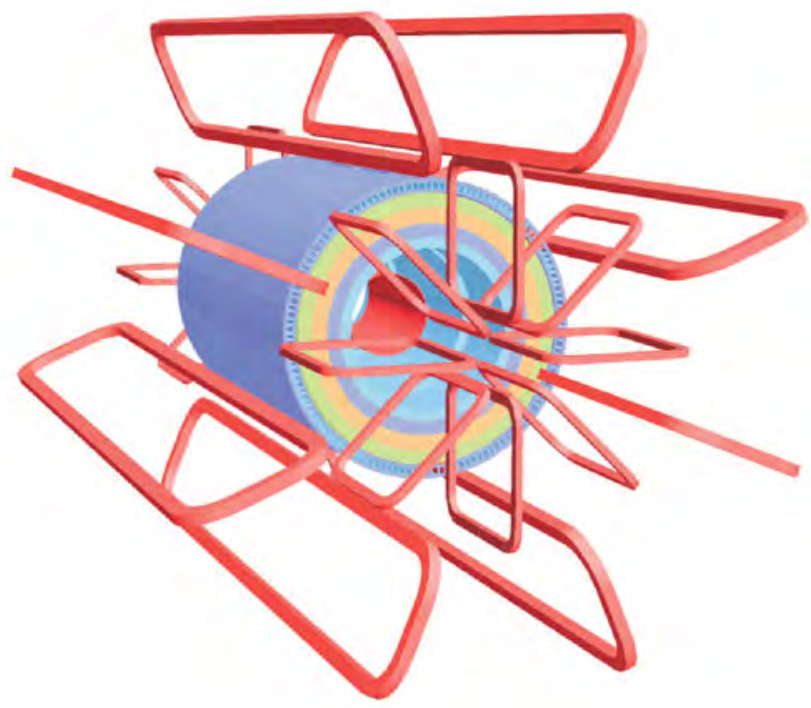

Figure 3.4.: Magnet system of the ATLAS detector including the solenoid and the air-core toroids [103].

Solenoid The solenoid system produces an axial magnetic field of about $2 \mathrm{~T}$ and surrounds the tracking system. It is used for the momentum measurement of charged particles in the inner detector. The solenoid system covers the pseudo rapidity range $|\eta|<2.7$.

Toroid The toroid system consists of three large air-core toroids, one barrel toroid and two end-cap toroids. Each of them has 8 air coils. The toroid system encloses the electromagnetic (EM) and hadronic calorimeter and is placed within the muon spectrometer. The barrel and the end-cap toroid magnets together cover a range of $|\eta|<2.7$, producing a magnetic field of up to $2.5 \mathrm{~T}$ in the barrel region and up to $3.5 \mathrm{~T}$ in the end-cap region. The bending power of the barrel toroid encompasses the range between 2 to $6 \mathrm{Tm}$. Both end-cap toroids provide a bending power of 4 to $8 \mathrm{Tm}$. 


\subsubsection{Inner Detector System}

The inner detector system has a full coverage in $\phi$ and covers a pseudorapidity range up to $|\eta|=2.5$. A schematic view of the inner detector is shown in Fig. 3.5 .

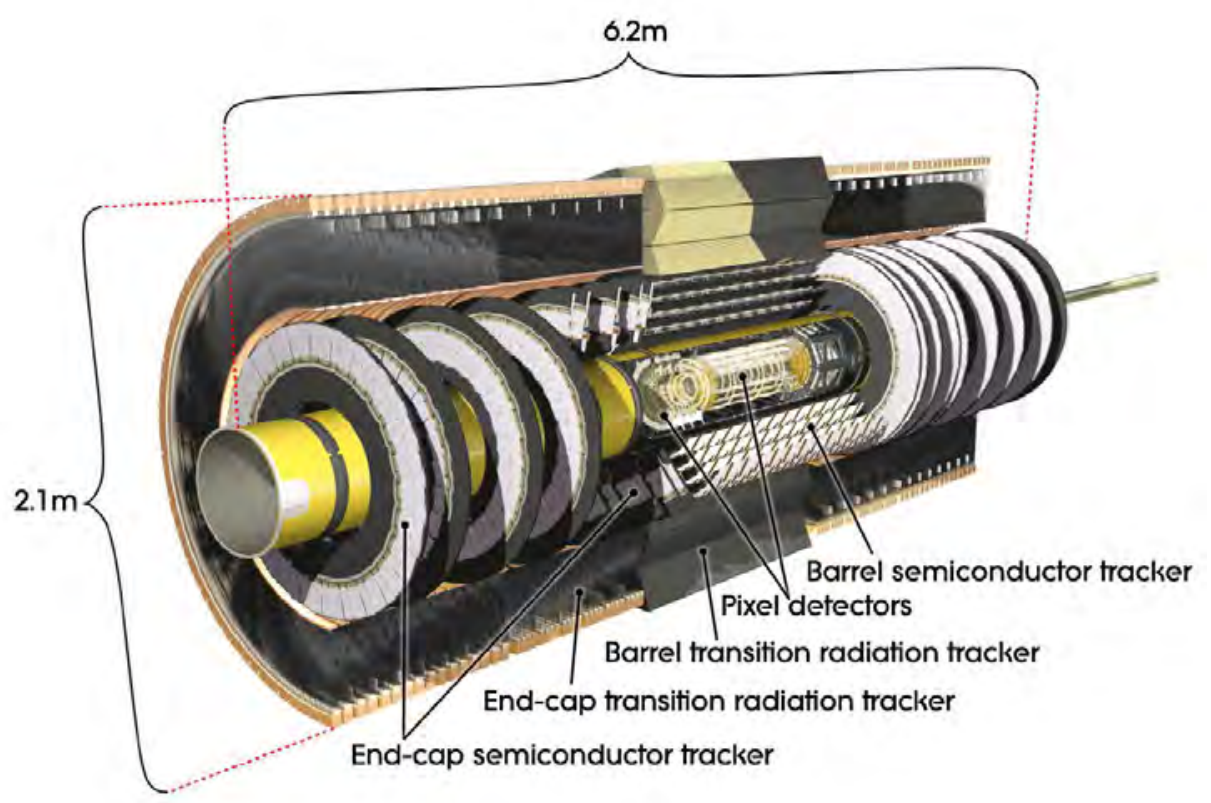

Figure 3.5.: Schematic view of the ATLAS inner detector [103].

It consists of three sub-detectors: the silicon pixel detector, the silicon micro-strip detector (SCT) and the transition radiation tracker (TRT). The inner detector is used for vertex and track reconstruction of charged particles with $p_{T}>0.5 \mathrm{GeV}$. It allows to identify secondary vertices, which is of great importance for the tagging of $b$ quarks.

Silicon Pixel Detector The pixel detector, the innermost part of the inner detector, provides high precision measurements very close to the interaction point. It provides the best resolution of the impact parameter due to its high granularity and its proximity to the beam pipe. The pixel detector consists of three barrel layers around the beam axis and three end-cap discs on each side. It typically provides three space points per track over the full acceptance region of the inner detector and reaches a resolution of up to $9-10 \mu \mathrm{m}$.

Silicon Micro-Strip Detector The SCT encloses the pixel detector and is composed of 8 layers of micro-strip sensors in the barrel region, which are grouped in pairs using a small stereo angle of $40 \mathrm{mrad}$ to provide measurements in z-direction. The barrel region covers a range up to $|\eta|=2.4$. The end-caps consists of 9 discs and cover a pseudo rapidity region between $|\eta|=1.4$ and $|\eta|=2.5$. The SCT typically provides four space points per track and reaches a resolution of up to $17 \mu \mathrm{m}$.

Transition Radiation Tracker The TRT, the outermost part of the inner detector, is, in contrast to the two other inner detector parts, a gaseous detector. It is composed of drift straw tubes, filled with Xenon gas, covered by a radiator foil. The TRT uses a 
combination of two effects for the detection of the charged particles: EM-ionisation and transition radiation. The production of transition radiation, which depends on the mass of the particle, its charge and momentum, can be used to distinguish between electrons and pions. The TRT typically provides 36 space points per track and reaches a resolution of up to $130 \mu \mathrm{m}$ per straw tube.

The combination of the pixel detector, the SCT and the TRT typically provides overall 43 space points per track and therefore delivers a very good pattern recognition and a high precision coordinate measurement.

\subsubsection{Calorimeter System}

The calorimeter encloses the solenoid system and the inner detector. It is composed of an inner EM calorimeter and an outer hadronic calorimeter. The purpose of the calorimeter systems is to measure the energy deposition of charged and neutral particles. The EM calorimeter absorbs most of the energy from the EM showers produced by pair production and bremsstrahlung, whereas hadronic showers range into the hadronic calorimeter, where most of their energy is absorbed. Both calorimeter systems have a full coverage in $\phi$.

Electromagnetic Calorimeter The EM calorimeter is a liquid-argon (LAr) sampling calorimeter, which has a high granularity and provides a very good energy and position resolution [104]. It consists of a barrel calorimeter and two end-cap calorimeters, one on each side. The EM calorimeter uses lead as absorber material and LAr as active material and covers a pseudo-rapidity range up to $|\eta|=3.2$. The absorber are accordeon shaped, which has the advantage of a full coverage in $\phi$ without azimuthal cracks. The resolution of the EM barrel calorimeter has been determined in electron test beam measurements and found to be $\sigma / E=10 \% / \sqrt{E} \oplus 2 \%$ [105].

Hadronic Calorimeter The hadronic calorimeter is composed of a scintillator-tile calorimeter and a LAr hadronic end-cap calorimeter (HEC). Both are also sampling calorimeters. The tile calorimeter uses steel as absorber material and scintillating tiles as active material, whereas the HEC uses copper as absorber material and LAr as active material. The tile calorimeter consists of a large central barrel and two smaller extended barrel cylinders and covers a range up to $|\eta|=1.7$. The region between $|\eta|=1.5$ and $|\eta|=3.2$ is covered by the HEC. The energy resolution of hadrons in the tile calorimeter has been measured to $\sigma / E=52.9 \% / \sqrt{E} \oplus 5.7 \%$ in test beam studies [106].

The region $3.1<|\eta|<4.9$ is covered by the LAr forward calorimeter (FCAL), which provides electromagnetic and hadronic energy measurements.

\subsubsection{Muon System}

The muon system, the outermost part of the ATLAS detector, is designed to measure the muon momentum with high resolution in the pseudo-rapidity range $|\eta| \leq 2.7$. The muon system is composed of four components: the monitored drift tubes (MDT), the cathode strip chambers (CSC), the resistive plate chambers (RPC) and the thin gap chambers 
(TGC). The MDT's are used as high precision tracking chambers in the full pseudorapidity range, except for the innermost end-cap layer in the region $2.0<|\eta|<2.7$, where the CSC's are used due to their higher granularity. The MDT's and CSC's reach a resolution of $80 \mu \mathrm{m}$ and $60 \mu \mathrm{m}$, respectively. The RPC's and TGC's are used for triggering with a response time of $15-25$ ns. The RPC's covers the barrel region $(|\eta|<1.05)$ and the TGC's cover the end-cap regions $(1.05<|\eta|<2.4)$.

\subsubsection{Data Acquisition and Trigger System}

Given the collision rate and average number of interactions per bunch crossing, the ATLAS detector has to deal with a very high event rate at the order of $1 \mathrm{GHz}$. By design, the maximum storage rate was restricted to less than $200 \mathrm{~Hz}$. Therefore, a highly efficient trigger system with a maximized physics coverage is needed. In addition, each event requires on average $1.5 \mathrm{MB}$ of storage space, which calls for a very efficient data acquisition system (DAQ).

The ATLAS trigger system [107, 108] consists of three levels: the level-1 trigger (LVL1), the level-2 trigger (LVL2) and the event filter (EF). The High-Level trigger (HLT) is represented by the combination of LVL2 and EF. The event rate is reduced consecutively on each level, to give sufficient time for data processing.

The different trigger signatures are summarised in the so-called trigger menu, which strongly depends on the data taking conditions. Some of the triggers need to be prescaled, since for triggers with high rates, only a fraction of events can be selected due to limited storage rate. These events are selected randomly. Figure 3.6 shows a functional overview of the ATLAS trigger system, together with a sketch of the event rates and processing times at the different decision stages.

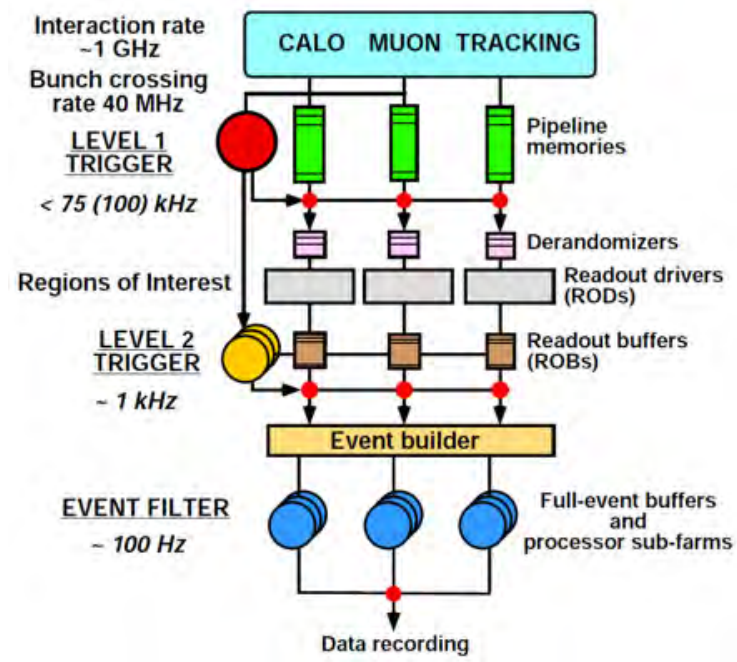

(a)

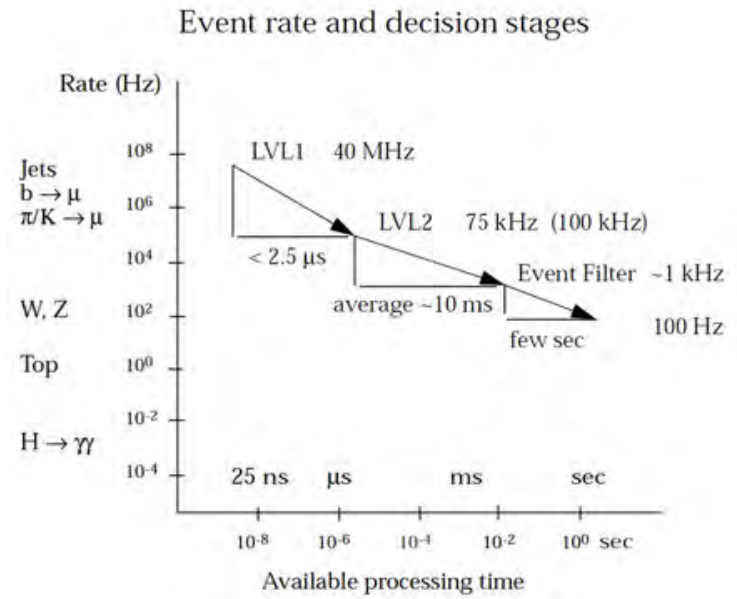

(b)

Figure 3.6.: (a) Functional overview of the ATLAS trigger/DAQ system and (b) the event rates and processing times at the different decision stages [108]. 
Level-1 Trigger The LVL1 is a hardware-based trigger, which uses information from the calorimeter system and the muon spectrometer. This level defines a region of interest and the first classification of particles is made. It reduces the initial event rate to less than $75 \mathrm{kHz}(100 \mathrm{kHz}$ by design).

Level-2 Trigger The LVL2 is a software-based trigger. Its decision is based on the region of interest defined by the LVL1, refining the object classification by taking the full detector information into account. This leads to an event rate of $1 \mathrm{kHz}$.

Event Filter Like the LVL2, the EF is a software-based trigger and is part of the DAQ system. The EF has access to the full event information. On this stage a refined and complex event selection is used to reduce the event rate furthermore to $O(100 \mathrm{~Hz})$. This selection is similar to the subsequent offline selection used for the final reconstruction. The event rate in 2011 was on average $400 \mathrm{~Hz}$. 


\section{Monte Carlo Simulation and Event Generators}

Monte Carlo (MC) simulation plays an essential role in modelling of particle physics processes, especially beyond fixed order perturbative QCD. Predictions from MC generators are supplemented with detector simulation in order to map theoretical predictions to experimental results.

In particle physics, three types of MC generators are used: multi-purpose LO hadronisation and shower generators, NLO generators and multi-leg LO generators. In addition, generators for specific final states exist.

Multi-purpose parton shower generators, such as HERWIG [109] or PYTHIA [57], are based on calculations of the leading order hard process. Higher order corrections which are enhanced by large kinematic logarithms are modelled by parton shower (PS). Parton shower implies the production of extra jets in the event, but mainly accounts for collinear and soft emissions and not for hard large angle emissions, as discussed in Sec. 2.2.8.

Multi-leg generators, such as ALPGEN [11, SHERPA [12] or MadGraph [110], are parton-level generators which use fixed-order matrix element (ME) calculations in lowest order in perturbation theory, not including virtual corrections. In addition, ALPGEN and MadGraph do not incorporate any kind of hadronisation, resulting in final states of bare quarks and gluons. Therefore, they need to be supplemented by hadronisation and showering programmes to obtain a full and realistic model of the physics process. The phase space at low $p_{T}$ and small angles is dominated by parton shower jets, whereas at high $p_{T}$ and large angles jets from the hard matrix element dominate, with a small overlap region. In order to avoid double counting, ME+PS matching algorithms, like CKKW [11] or MLM [112, 113], are needed, which allow the combination of tree-level matrix elements for hard large angle emissions and parton shower for soft collinear emissions.

NLO generators, such as MC@NLO [114] or POWHEG [115, 116], are parton-level generators which are based on hard matrix element calculations including the full NLO QCD corrections. They are expected to give a better description of the hard core $2 \rightarrow 1$ and $2 \rightarrow 2$ process and the overall rate compared to matched ME+PS generators and pure showering generators. In order to provide a full and realistic model for the physics process, NLO generators also have to be interfaced to hadronisation and showering programs.

The full production chain of simulated MC events at the ATLAS experiment consists of 5 steps, starting from the generation process up to the creation of Analysis Object Data (AOD). The intermediate steps are the event simulation, the digitization and the reconstruction. All these steps are performed within the Athena framework [117]. In the first step, particle four-vectors for specific physics processes are generated and saved as HepMC [38]. The MC generators which are used for the following analysis are described in more detail in the following sections. The four-vectors are then passed through the GEANT4 [118, 119] simulation or the ATLFAST II [120] simulation. The latter replaces the CPU intensive steps: the simulation, the digitisation, and the reconstruction by a fast 
simulation. This results in more statistics, but has the disadvantage that not all detector components are modelled precisely. The digitisation step is used to transform the output from the GEANT4 simulation into the actual output format of the detector, the Raw Data Objects (RDOs). Finally, in the reconstruction step, which is identical for digitised MC events and real data, the output from the readout electronics is reconstructed as tracks and energy depositions and saved as Event Summary Data (ESD). A reduced version of the ESDs, only containing a summary of the reconstructed events, are saved as AODs. For analysis purposes, D3PDs are created from the AODs, which can be easily accessed within ROOT [121.

\subsection{PYTHIA}

PYTHIA is a leading order multi-purpose hadronisation and showering generator, which can be used to simulate hard and soft interactions. It contains a large library of subprocesses around 240 different $2 \rightarrow n$ processes, which are mainly $2 \rightarrow 1$ or $2 \rightarrow 2$ processes. PYTHIA describes initial and final state radiation, multiple particle interactions, fragmentation and decays. Initial and final state radiation is simulated using $Q^{2}$-ordered or $p_{T}$-ordered parton showers depending on the tune while for the fragmentation the Lund string model [64, 65] is used. For multiple interactions PYTHIA uses a $p_{T}$-ordered parton shower and colour string fragmentation.

\subsection{HERWIG}

HERWIG, just as PYTHIA, is a leading order multi purpose hadronisation and showering generator, which can be used to simulate hadronic and leptonic collisions. It also contains a large library of $2 \rightarrow n$ hard scattering processes. HERWIG includes initial and final state radiation, as well as UE simulation, hadronisation and decays. In contrast to PYTHIA, HERWIG uses the angular-ordered parton shower model for the description of the initial and final state radiation and for hadronisation the cluster hadronisation model [66-69]. The UE simulation in HERWIG is based on high energy $\bar{p}$ p simulation program of the UA5 collaboration [122, incorporating the cluster fragmentation model. In addition, HERWIG can be interfaced to JIMMY [123, 124] for the UE simulation, which uses the multiple scattering model.

\subsection{ALPGEN}

ALPGEN is a multi-leg generator and has been designed to generate hard multi-parton processes in hadronic collisions. It provides fixed order matrix element calculations at tree-level in QCD and EW interactions for a large set of processes, such as $W / Z+$ jets for up to 6 jets. ALPGEN is interfaced to HERWIG or PYTHIA to evolve the event by parton shower and finally form hadrons from the partonic cascades. In order to avoid double counting, MLM matching is used for the merging of the tree-level matrix elements and the parton shower jets. For QED radiative corrections in massive boson decays ALPGEN is interfaced to PHOTOS [125]. 


\subsection{SHERPA}

SHERPA, just as ALPGEN, is a multi-leg generator, which in addition provides its own algorithms for initial and final state radiation [126, 127], multiple interactions [54], fragmentation and decays. It has been designed to generate hard scattering processes within the SM and beyond. In order to avoid double counting in the merging of tree-level matrix elements and parton shower jets, the CKKW approach is used. For the formation of hadrons, SHERPA uses a modified cluster hadronisation model [128], which is based on the model used in HERWIG. In addition, the YFS [129] approach is incorporated in SHERPA to describe the effect of virtual and real QED corrections.

\section{5. $\mathrm{MC} @ \mathrm{NLO}$}

MC@NLO is a NLO generator which has been designed to simulate hadronic processes. For the evolution of the events with parton shower, MC@NLO is interfaced to HERWIG. Due to the resummation technique used for the NLO corrections, a fraction of events is generated with negative weights.

\subsection{AcerMC}

AcerMC [130] is a parton level generator which has been designed to simulate hard interactions for various SM background processes in pp-collisions. It provides calculations of the hard matrix element with the help of the MadGraph package. For initial and final state radiation, UE simulation, hadronisation and decays AcerMC has to be interfaced to a multi-purpose hadronisation and showering generator such as PYTHIA or HERWIG. 



\section{Unfolding Methods}

This chapter gives an introduction into possible methods for the solution of inverse problems. Within particle physics the solution of such problems is better known as unfolding and many different unfolding methods are available, e.g. the iterative (Bayes) method [131] or the singular value decomposition (SVD) [132]. Measured distributions in particle physics are influenced by resolution effects, non linearities and the acceptance of the detector. Unfolding describes the transition from the measured distributions to the underlying truth distribution. Therefore, unfolded results have the advantage that they are detector independent and can be directly compared to theory predictions or results from other experiments. However, it is not always necessary to unfold the measurements, it depends on the problem. Sometimes it is easier to fold the theory predictions with the detector resolution and compare it directly to the measurement, since inverse problems are difficult to solve and the solution provides only an approximation of the problem.

The choice of the unfolding method depends on different parameters, e.g. the size of migration and the shape of the distribution. For the following analyses the iterative (Bayes) method and the bin-by-bin method [133 are used. The latter is a simple correction method and only includes migrations between bins to the extend to which they are modelled in MC. In the following, the iterative (Bayes) method is described in more detail. In addition, the performance of this method and its error calculations is tested using toy MC.

\subsection{Iterative (Bayes) Method}

The iterative (Bayes) method [131] was introduced in 1994 by G. D'Agostini and is implemented in the RooUnfold package [134].

The method can be explained using a picture of causes $\mathrm{C}$ and effects E. The causes represent the true distribution before smearing and the effects substitute the reconstructed values, including smearing, migration, reconstruction efficiencies and fakes. Each cause can produce different effects, but for a given effect, as it is the case in a measurement, the exact cause is not known. Figure 5.1 visualises the connections between the causes and effects. The node $T$ indicates the trash bin, representing the inefficiency of the reconstruction.

However, the probability for an effect produced from a defined cause $P\left(E_{j} \mid C_{i}\right)$ can be estimated assuming some knowledge about the migration, efficiency and resolution. This is usually achieved by using $\mathrm{MC}$ event samples. Now the goal is to estimate the probability $P\left(C_{i} \mid E_{j}\right)$ that different causes $C_{i}$ were responsible for the observed effect $E_{j}$. A simple inversion cannot be used to solve this problem, since most matrices are not invertible, but the Bayes theorem ${ }^{1}$ yields a solution.

${ }^{1} P(A \mid B)=\frac{P(B \mid A) P(A)}{P(B)}$ 


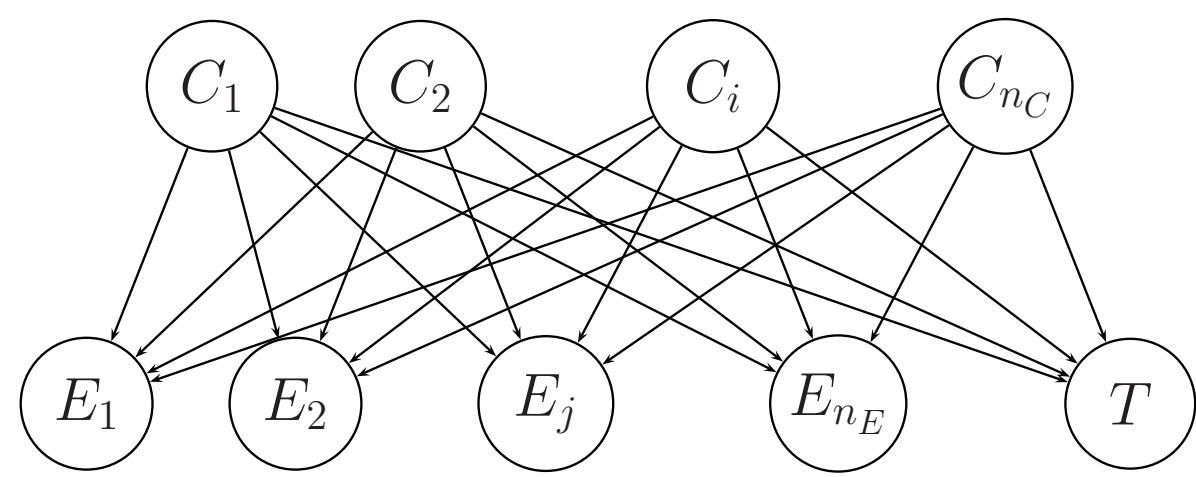

Figure 5.1.: Connections between causes and effects [135]. The node $T$ indicates the trash bin, representing the inefficiency of the reconstruction.

$$
\begin{aligned}
P\left(C_{i} \mid E_{j}\right) & =\frac{P\left(E_{j} \mid C_{i}\right) \cdot P_{0}\left(C_{i}\right)}{\sum_{l=1}^{n_{C}} P\left(E_{j} \mid C_{l}\right) \cdot P_{0}\left(C_{l}\right)}, \\
\hat{n}\left(C_{i}\right) & =\frac{1}{\epsilon_{i}} \sum_{j=1}^{n_{E}} n\left(E_{j}\right) \cdot P\left(C_{i} \mid E_{j}\right) \quad \epsilon_{i} \neq 0,
\end{aligned}
$$

with $\hat{n}\left(C_{i}\right)$ the expected number of events in the cause bin $i, n\left(E_{j}\right)$ the number of events in the effect bin $j, P_{0}\left(C_{i}\right)$ the initial probabilities and $\epsilon_{i}$ the efficiency that the cause $i$ has an effect. The expected number of events in the different cause bins can be used to calculate the total true number of events $\hat{N}_{\text {true }}$ and the probabilities of the causes $\hat{P}\left(C_{i}\right)$ :

$$
\begin{aligned}
\hat{N}_{\text {true }} & =\sum_{i=1}^{n_{c}} \hat{n}\left(C_{i}\right), \\
\hat{P}\left(C_{i}\right) & =P\left(C_{i} \mid n(E)\right)-\frac{\hat{n}\left(C_{i}\right)}{\hat{N}_{\text {true }}} .
\end{aligned}
$$

The unfolding is performed iteratively, starting from the initial probabilities for the first iteration. Further iterations are based on the Bayesian posterior as prior, therefore $P_{0}(C)$ needs to be substituted by $\hat{P}(C)$ and $n_{0}(C)$ by $\bar{n}(C)$.

Equation 5.2 can be rewritten in terms of the unfolding matrix $M$

$$
\begin{aligned}
\hat{n}\left(C_{i}\right) & =\sum_{j=1}^{n_{E}} M_{i j} \cdot n\left(E_{j}\right), \\
M_{i j} & =\frac{P\left(E_{j} \mid C_{i}\right) \cdot P_{0}\left(C_{i}\right)}{\left[\sum_{l=1}^{n_{E}} P\left(E_{l} \mid C_{i}\right)\right] \cdot\left[\sum_{l=1}^{n_{C}} P\left(E_{j} \mid C_{l}\right) \cdot P_{0}\left(C_{l}\right)\right]},
\end{aligned}
$$

which is clearly not equal to the inverse of the migration matrix. But with increasing number of iterations the result becomes close to the result which would be obtained with a matrix inversion.

For the calculation of the covariance matrix of $\hat{n}\left(C_{i}\right)$ two different sources are taken into account, an uncertainty on the distribution of the effects $n\left(E_{j}\right)$ 


$$
\begin{aligned}
V_{k l}(\underline{n}(E)) & =\sum_{j=1}^{n_{E}} M_{k j} \cdot M_{l j} \cdot n\left(E_{j}\right) \cdot\left(1-\frac{n\left(E_{j}\right)}{\hat{N}_{\text {true }}}\right) \\
& -\sum_{\substack{i, j=1 \\
i \neq j}}^{n_{E}} M_{k i} \cdot M_{l j} \cdot \frac{n\left(E_{i}\right) \cdot n\left(E_{j}\right)}{\hat{N}_{\text {true }}},
\end{aligned}
$$

with the true number of events $\hat{N}_{\text {true }}$ and an uncertainty on the migration probabilities $P\left(E_{j} \mid C_{i}\right)$

$$
V_{k l}(M)=\sum_{i, j=1}^{n_{E}} n\left(E_{i}\right) \cdot n\left(E_{j}\right) \cdot \operatorname{Cov}\left(M_{k i} M_{l j}\right)
$$

The total uncertainty is calculated from the sum of both covariance matrices

$$
V_{k l}=V_{k l}(\underline{n}(E))+V_{k l}(M) .
$$

In the following, the absolute values and uncertainties provided by this method are tested using toy MC.

\subsubsection{The Toy Monte Carlo}

This section describes a simple toy MC, which is used in the following to check the method.

Three different migration matrices with different migration (large migration $\left(S_{1}\right)$, medium migration $\left(S_{2}\right)$ and low migration $\left(S_{3}\right)$ ) for 3 bins without efficiency losses are defined

$$
S_{1}=\left(\begin{array}{ccc}
0 & 0.1 & 0.1 \\
0.2 & 0.3 & 0.5 \\
0.8 & 0.6 & 0.4
\end{array}\right), S_{2}=\left(\begin{array}{ccc}
0 & 0.1 & 0.8 \\
0.2 & 0.8 & 0.2 \\
0.8 & 0.1 & 0
\end{array}\right), S_{3}=\left(\begin{array}{ccc}
0 & 0.025 & 0.95 \\
0.05 & 0.95 & 0.05 \\
0.95 & 0.025 & 0
\end{array}\right)
$$

For the unfolding, two different sources of uncertainties are present: a finite amount of data and a finite number of events for the creation of the migration matrix. In order to study the different uncertainty components, two different cases are defined:

1. create randomly 2000 test distributions to simulate the finite amount of data events

2. create randomly 2000 migration matrices from fixed probabilities on top of the test distributions to simulate statistical fluctuations in the migration matrix due to a finite statistics in Monte Carlo 


\subsubsection{Performance checks}

For the performance checks of the method, a C++ implementation of Ref. [131] is used.

In order to quantify if the absolute values and the uncertainties are correct ensemble testing is used. The width of the Gaussian distribution from ensemble testing is compared to the uncertainties calculated by the programme using pull distributions:

$$
\text { pull }=\frac{y_{i}-\mu}{\sigma_{i}}
$$

with the unfolded values $y_{i}$, the truth value $\mu$ and the calculated uncertainties $\sigma_{i}$. For a correct treatment of the migration effect in the unfolding and a correct estimation of the unfolding uncertainties, the pull distributions are expected to be standard Gaussian (mean zero and unit variance).

Figure 5.2 shows the pull distributions for the three different migration matrices for the first bin and the width of the pull distributions for the different bins considering the randomly generated test distributions.

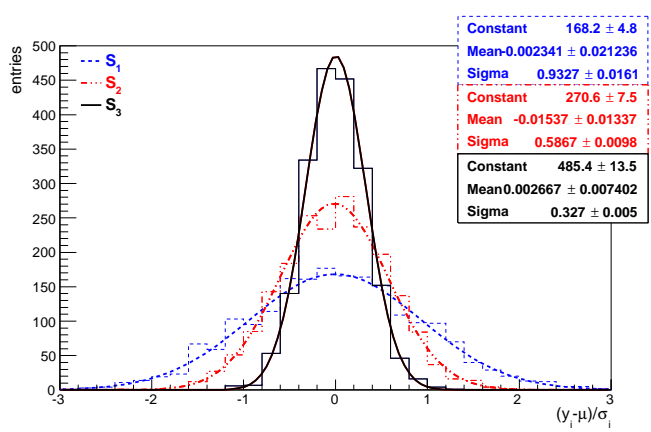

(a)

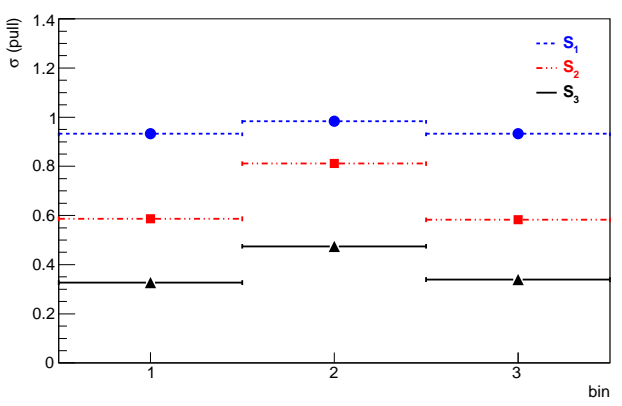

(b)

Figure 5.2.: Comparison of (a) the pull distributions for the first bin and (b) the width of the pull distributions for the different migration matrices with large migration $S_{1}$ (blue), medium migration $S_{2}$ (red) and low migration $S_{3}$ (black) for the randomly generated test distributions.

For all three migration matrices the pull distributions are centred around zero, but with decreasing migration in the migration matrix, the pull distributions become narrower. This leads to the conclusion that less migration leads to an overestimate of the uncertainties in the programme, and therefore the migration effect is not treated correctly in the uncertainty calculation.

This problem seems to come from the fact that the programme assumes a multinomial distribution ${ }^{2}$ for the data. But each data bin is multinomially distributed and the sum of multinomial distributions is only a multinomial distribution if all distributions are equal. In order to fulfil this requirement, the columns of the migration matrix have to be equal to get the correct estimate for the uncertainty from the programme, which is not the typical case in data analysis.

Due to the fact, that the data sample is a sum of multinomial distributions, the formula for the calculation of the covariance matrix $V_{k l}$ for the data sample $\underline{n}(E)$ is changed from Eq. 5.7 to

\footnotetext{
${ }^{2} \mathrm{~A}$ multinomial distribution is a generalisation of the binomial distribution with more than two possible outcomes.
} 


$$
\begin{aligned}
V_{k l}(\underline{n}(E) & =\sum_{j=1}^{n_{E}} M_{k j} \cdot M_{l j} \cdot \sum_{r=1}^{n_{C}} \hat{n}\left(C_{r}\right) \cdot P\left(E_{j} \mid C_{r}\right) \cdot\left(1-P\left(E_{j} \mid C_{r}\right)\right) \\
& -\sum_{\substack{i, j=1 \\
i \neq j}}^{n_{E}} M_{k i} \cdot M_{l j} \cdot \sum_{r=1}^{n_{C}} \hat{n}\left(C_{r}\right) P\left(E_{i} \mid C_{r}\right) \cdot P\left(E_{j} \mid C_{r}\right) .
\end{aligned}
$$

Figure 5.3 and Fig. 5.4 show the comparison of the pull distributions between the old and the new uncertainty calculation for $S_{1}$ and $S_{2}$ for the first bin and the width of the pull distributions for each of the three bins.

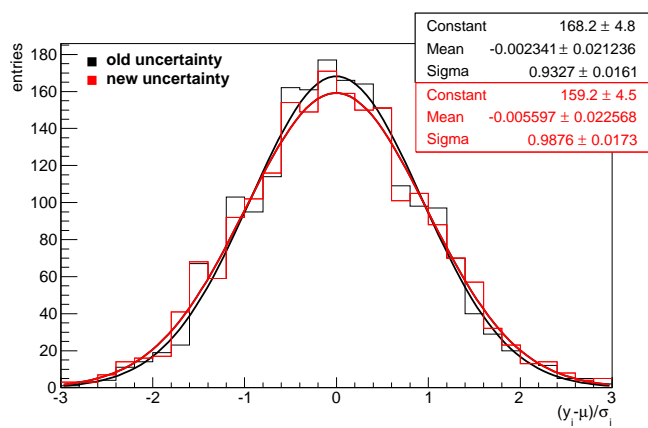

(a)

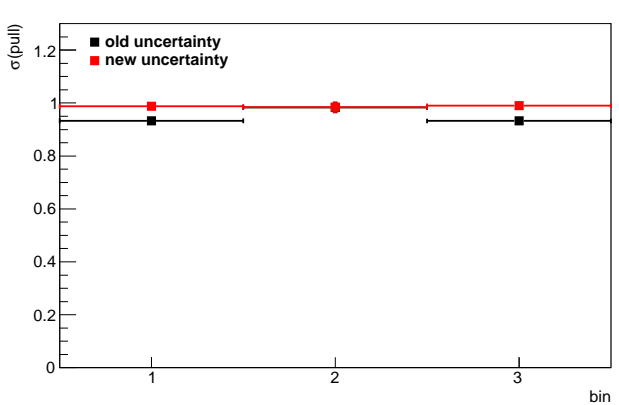

(b)

Figure 5.3.: Comparison of (a) the pull distributions and (b) the width of the pull distributions for the old (black) and the new (red) uncertainty calculation for the migration matrix with large migration $S_{1}$.

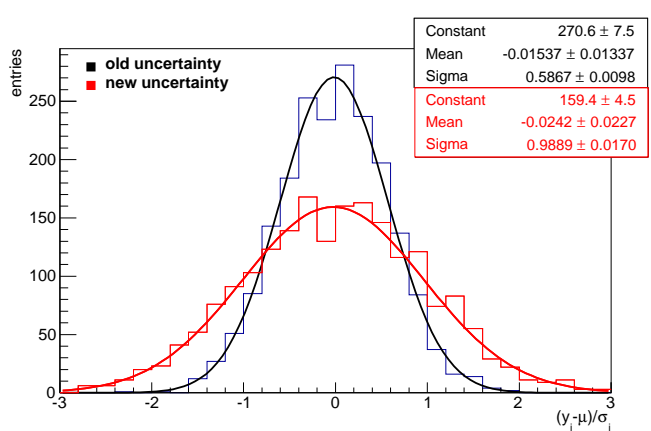

(a)

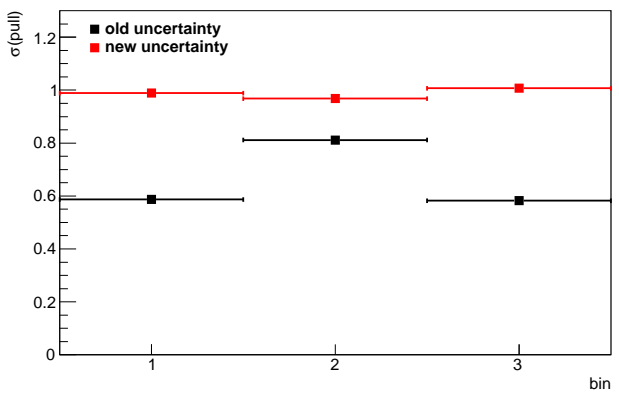

(b)

Figure 5.4.: Comparison of (a) the pull distributions and (b) the width of the pull distributions for the old (black) and the new (red) uncertainty calculation for the migration matrix with medium migration $S_{2}$.

As expected, for large migration, only a small improvement due to the new uncertainty calculation is visible, whereas for the migration matrix with medium migration a clear improvement is visible. For both cases with the new uncertainty calculation the width of the pull distribution is now compatible with unity. 
Figure 5.5 compares the width of the pull distributions for the fixed migration matrices and the randomly generated migration matrices for the old and the new uncertainty calculation.

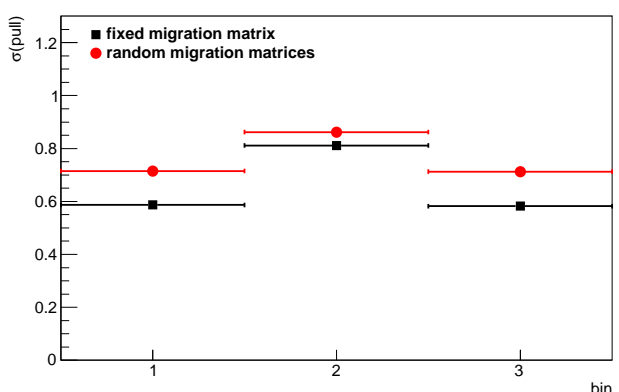

(a)

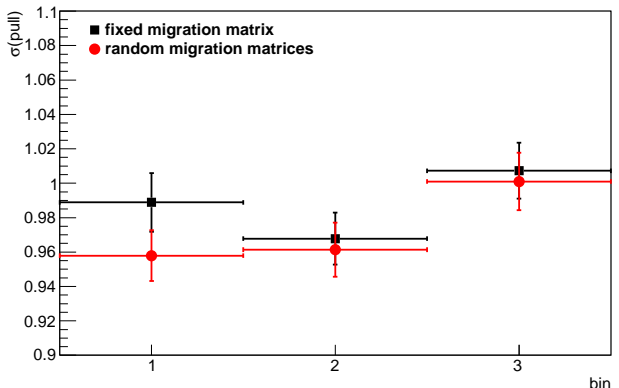

(b)

Figure 5.5.: Comparison of the width of the pull distributions for (a) the old uncertainty calculation and (b) the new uncertainty calculation for the fixed migration matrix (black) and the randomly generated migration matrices (red).

For the old uncertainty calculations the width of the pull distributions is broader for the randomly generated migration matrices than for the fixed migration matrices, while for the new uncertainty this difference nearly vanishes.

In summary, the new uncertainty calculation shows a clear improvement, but further checks in more complicated scenarios need to be performed, before it can be used for physics analyses. Since the iterative (Bayes) method shows problems concerning the error calculation, in the following analyses ensemble testing is used to estimate the statistical uncertainty including the intrinsic uncertainty on the method.

Recently, an improved Bayesian Unfolding method has become available, which was also described by G. D'Agostini in Ref. [135] and which is based on the previous method but the uncertainties are treated differently. In this method the quantities are described by probability density functions and the error propagation is done by sampling. A comparison between that method and the improvements for the old method is not performed yet. 


\section{Object Definitions}

The identification and reconstruction of physical objects are based on the information from the respective detector subsystems. This thesis focuses on final states with jets and electrons. The different analyses presented in this thesis are based on the data taken in 2010 and 2011. From 2010 to 2011, the object definitions and calibrations have been improved. In addition, the average number of interactions per bunch crossing have increased from 2010 to 2011, as shown in Fig. 2.6 in Sec. 2.2.6, which necessitate more refined methods to suppress events originating from pile-up.

In the following two subsections the different electron and jet algorithms are detailed.

\subsection{Electrons}

The electron reconstruction and identification is performed separately for electrons in the central region of $|\eta|<2.47$ and electrons in the forward region, $2.5<|\eta|<4.9$. For central electrons, the reconstruction and identification is based on information from the EM calorimeter and the tracking detectors, whereas for forward electrons only information from the EM calorimeter can be used, since tracking detectors only cover the region up to $|\eta|<2.5$. The following subsections detail the reconstruction and identification algorithms for central electrons, as well as the performance of the electron measurements. The algorithms for forward electrons will not be described in detail, since the analyses presented in this thesis are performed for central electrons only.

\subsubsection{Reconstruction Algorithm}

The reconstruction of central electrons is based on the calorimeter-seeded algorithm [136]. Starting with cluster information reconstructed from the energy deposits in the EM calorimeter, the clusters are then matched to reconstructed tracks of charged particles in the inner detector. In order to find and reconstruct the EM clusters, the sliding-window algorithm [137] is used. The size of the window has been set to $3 \times 5$ in units of the cell dimensions of the 2 nd EM calorimeter layer, $\Delta \eta \times \Delta \phi=0.025 \times 0.025$ and the total transverse energy of the EM clusters is required to exceed $2.5 \mathrm{GeV}$. The window is positioned to maximize the amount of energy. For each reconstructed cluster, the algorithm searches for matched tracks within a window of the size $\Delta \eta \times \Delta \phi=0.05 \times 0.10$. For the case in which more than one track matches the seed cluster, the closest in $\Delta R=\sqrt{\Delta \eta^{2}+\Delta \phi^{2}}$ to the seed cluster are selected. Tracks containing silicon hits are preferred.

Finally, the electron cluster is recomputed from a window with a size of $3 \times 7$ and $5 \times 5$ in units of the cell dimensions in the barrel and the endcaps, respectively. The energy of the electrons is obtained from the calorimeter, while the $\phi$ and $\eta$ direction are obtained from the inner detector information. In the cases where the tracks do not contain any 
silicon hits, the $\phi$ direction is determined from the track and the $\eta$ direction is determined from the cluster $\eta$-pointing.

\subsubsection{Quality Criteria}

For the identification of central electrons a cut-based selection is used, which is based on calorimeter and tracking information. For physics analyses three cut-based selections with different jet rejections are available: the loose, medium and tight selection [104, 138]. The jet rejections have been determined from $\mathrm{MC}$ simulation, which are at the order of 500, 5000 and 50000 [104]. The selection criteria of the loose and medium selections are summarised in Tab. 6.1. The loose selection includes cuts on the hadronic leakage, as well as on shower shape variables for the middle layer of the EM calorimeter. On top of the loose selection, the medium selection includes cuts on shower shape variables for the strip layer of the EM calorimeter and cuts on track quality requirements and track-cluster matching. The tight selection is not described in this thesis, since only loose and medium electrons are used for the analyses.

\begin{tabular}{|c|c|c|}
\hline Type & Description & Name \\
\hline \multicolumn{3}{|l|}{ Loose selection } \\
\hline Acceptance & 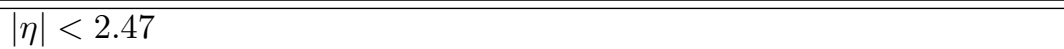 & \\
\hline \multirow[t]{2}{*}{ Hadronic leakage } & $\begin{array}{l}\text { Ratio of } E_{T} \text { in the first layer of the hadronic calorimeter to } E_{T} \text { of } \\
\text { the EM cluster (used over the range }|\eta|<0.8 \text { and }|\eta|>1.37 \text { ) }\end{array}$ & $R_{\text {had1 }}$ \\
\hline & $\begin{array}{l}\text { Ratio of } E_{T} \text { in the hadronic calorimeter to } E_{T} \text { of the EM cluster } \\
\text { (used over the range }|\eta|>0.8 \text { and }|\eta|<1.37 \text { ) }\end{array}$ & $R_{\text {had }}$ \\
\hline \multirow[t]{2}{*}{$\begin{array}{l}\text { Middle layer of } \\
\text { EM calorimeter }\end{array}$} & $\begin{array}{l}\text { Ratio of the energy in } 3 \times 7 \text { cells over the energy in } 7 \times 7 \text { cells } \\
\text { centred at the electron cluster position }\end{array}$ & $R_{\eta}$ \\
\hline & $\begin{array}{l}\text { Lateral shower width, } \sqrt{\left(\sum E_{i} \eta_{i}^{2}\right) /\left(\Sigma E_{i}\right)-\left(\left(\sum E_{i} \eta_{i}\right) /\left(\sum E_{i}\right)\right)^{2}} \text {, } \\
\text { where } E_{i} \text { is the energy and } \eta_{i} \text { is the pseudorapidity of cell } i \\
\text { and the sum is calculated within a window of } 3 \times 5 \text { cells }\end{array}$ & $w_{\eta 2}$ \\
\hline \multicolumn{3}{|c|}{ Medium selection (includes loose) } \\
\hline \multirow[t]{2}{*}{$\begin{array}{l}\text { Strip layer of } \\
\text { EM calorimeter }\end{array}$} & $\begin{array}{l}\text { Shower width, } \sqrt{\left(\Sigma E_{i}\left(i-i_{\max }\right)^{2}\right)\left(\Sigma E_{i}\right)} \text {, where } i \text { runs over all strips } \\
\text { in a window of } \Delta \eta \times \Delta \phi \approx 0.0625 \times 0.2 \text {, corresponding typically } \\
\text { to } 20 \text { strips in } \eta \text {, and } i_{\max } \text { is the index of the highest-energy strip }\end{array}$ & $w_{\text {stot }}$ \\
\hline & $\begin{array}{l}\text { Ratio of the energy difference between the largest and second largest } \\
\text { energy deposits in the cluster over the sum of these energies }\end{array}$ & $E_{\text {ratio }}$ \\
\hline \multirow[t]{3}{*}{ Track quality } & Number of hits in the pixel detector $(\geq 1)$ & $n_{\text {pixel }}$ \\
\hline & Number of total hits in the pixel and SCT detectors $(\geq 7)$ & $n_{\mathrm{Si}}$ \\
\hline & Transverse impact parameter $\left(\left|d_{0}\right|<5 \mathrm{~mm}\right)$ & $d_{0}$ \\
\hline $\begin{array}{l}\text { Track-cluster } \\
\text { matching }\end{array}$ & $\begin{array}{l}\Delta \eta \text { between the cluster position in the strip layer and the } \\
\text { extrapolated track }(|\Delta \eta|<0.01)\end{array}$ & $\Delta \eta$ \\
\hline
\end{tabular}

Table 6.1.: Definition of variables used for loose and medium electron identification cuts for the central region of the detector with $|\eta|<2.47$ [104].

For analyses with 2011 data, the selection criteria were improved to deal with higher pile-up environments, called loose ++ , medium ++ and tight ++ . These criteria have a slightly lower selection efficiency, but a higher background rejection. 


\subsubsection{Performance}

The electron energy scale and energy resolution have been determined using in-situ techniques in $Z \rightarrow e^{+} e^{-}$decays for central and forward electrons in 2010 and 2011 data [104, 139]. These measurements have been cross checked for central electrons using $J / \Psi \rightarrow e^{+} e^{-}$and $W \rightarrow e \nu$ decays. The electron calibration has been performed in bins of the energy $E$ for different rapidity regions. Figure 6.1 exemplarily shows the electron energy scale correction with the associated uncertainties in the region $|\eta|<0.6$ for 2010 .

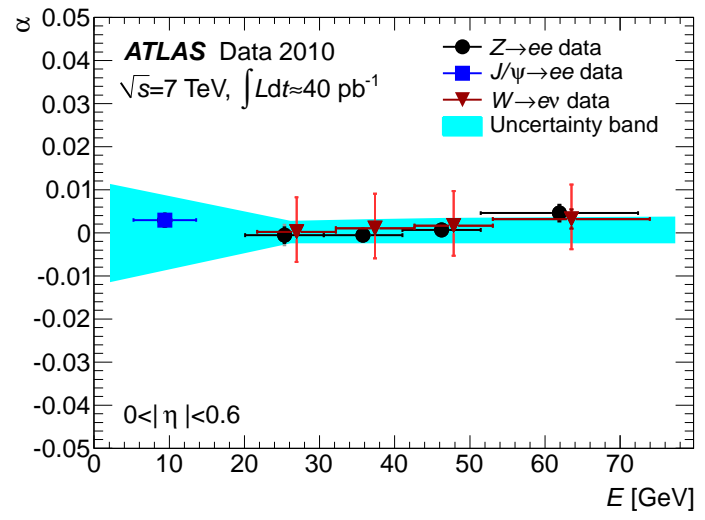

Figure 6.1: Electron energy scale correction with the associated uncertainties in the region $|\eta|<0.6$ for 2010 data, determined using insitu techniques in $Z \rightarrow e^{+} e^{-}$(black circles), $J / \Psi \rightarrow e^{+} e^{-}$(blue squares) and $W \rightarrow e \nu$ (red triangles) decays [104].

Figure 6.2 shows the reconstructed invariant dielectron mass for $Z \rightarrow e^{+} e^{-}$decays in 2010 and 2011 data. The width of the invariant dielectron mass gives an estimate of the electron energy resolution.

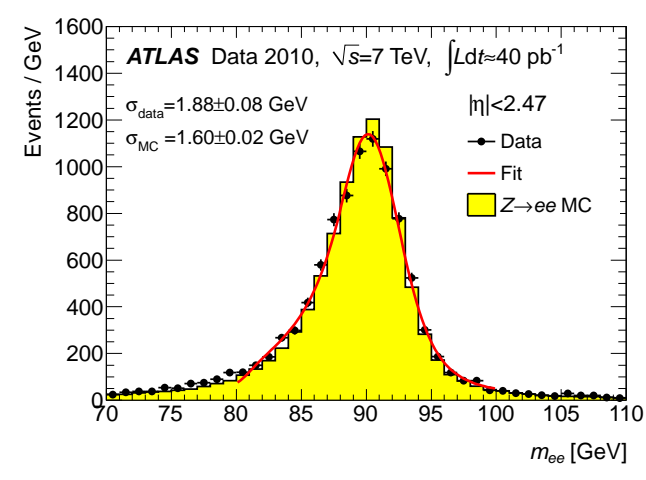

(a) Electron energy resolution 2010

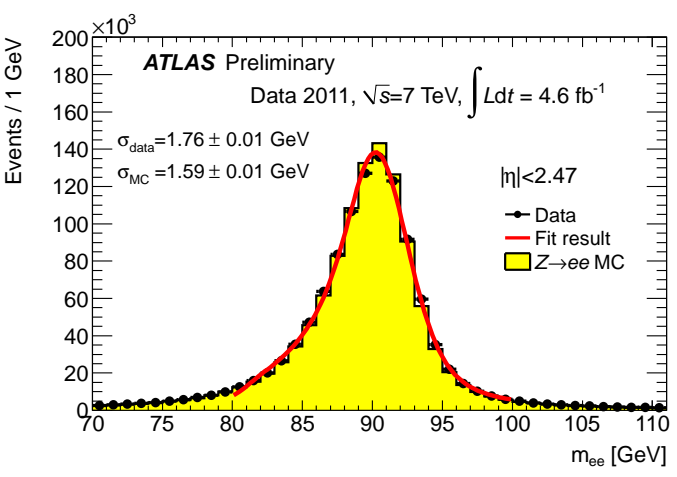

(b) Electron energy resolution 2011

Figure 6.2.: Reconstructed invariant dielectron mass for $Z \rightarrow e^{+} e^{-}$decays in (a) 2010 data [104] and (b) 2011 data [139].

The distribution in data is well reproduced by MC simulation, except for some small residual differences that results in the mass peak being slightly broader in data than in the MC simulation. Therefore, smearing factors have been determined to account for those residual differences, that have to be applied on the MC events.

Electron trigger, reconstruction and identification efficiencies have been measured in data and MC using tag-and-probe methods [140, 141] in $Z \rightarrow e^{+} e^{-}, W \rightarrow e \nu$ and $J / \Psi \rightarrow e^{+} e^{-}$events. For $Z \rightarrow e^{+} e^{-}$and $J / \Psi \rightarrow e^{+} e^{-}$events, one electron has been used 
to define the event (tag) and the other electron has been used to measure the efficiency (probe), whereas for $W \rightarrow e \nu$ events the missing transverse energy has been used as tag. From those measurements, scale factors are provided to account for the differences in data and MC. For 2011 data, the scale factors and their corresponding uncertainties are slightly improved, due to the larger statistics and a better understanding of the differences between the results from tag-and-probe in $W$ and $Z$ events. Figure 6.3 shows as an example the loose ++ identification efficiency as a function of the transverse energy.

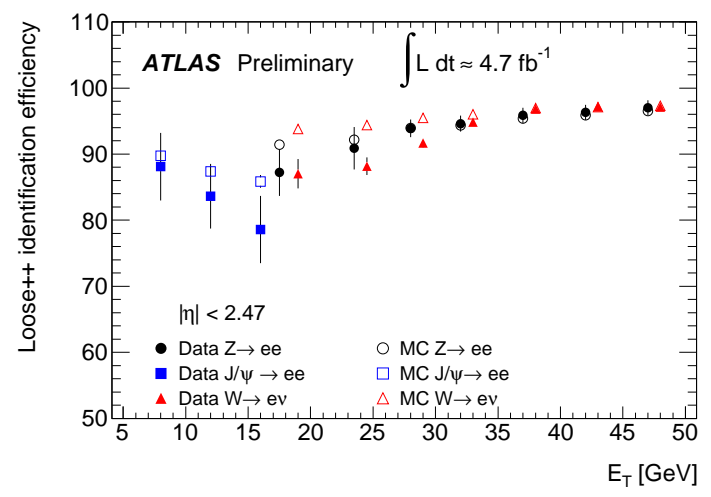

Figure 6.3: Identification efficiency for electrons passing the loose++ criteria in 2011 data with a transverse energy between $7 \mathrm{GeV}$ and $50 \mathrm{GeV}[142$.

\subsection{Jets}

Jets are ensembles of hadrons and other particles defined in a given cone, originating from the hadronisation of a parton. For the reconstruction of jets various jet finding algorithms exist. At ATLAS, the anti- $k_{t}$ algorithm [143] with a distance parameter of 0.4 is used as default. This algorithm is infrared and collinear safe. The following subsections detail the reconstruction and calibration of jets, as well as the jet quality selection and the performance of the jet energy measurements.

\subsubsection{Reconstruction Algorithm}

Jet finding algorithms can use simulated partons (parton level jets), stable simulated particles (particle level jets), reconstructed tracks in the inner detector (track jets) or energy depositions in the calorimeter (calorimeter jets) as input. Calorimeter jets can be reconstructed from various different inputs, e.g. calorimeter towers or topological calorimeter clusters (topo-clusters) [137, 144].

For the analyses presented in this thesis, topo-clusters are used as inputs to the jet finding algorithm, which are reconstructed at the electromagnetic (EM) scale, correctly measuring the energy deposition by electromagnetic showers in the calorimeter. The formation of topo-clusters follows a 4-2-0 algorithm, starting with the cells which have an energy above four times the electronic plus pile-up noise as seed. The algorithm proceeds with the recursive aggregation of all neighbouring cells, which have a signal-to-noise ratio above two. Finally, all remaining cells are added to the clusters. Figure 6.4 shows an overview of the jet reconstruction in ATLAS. 


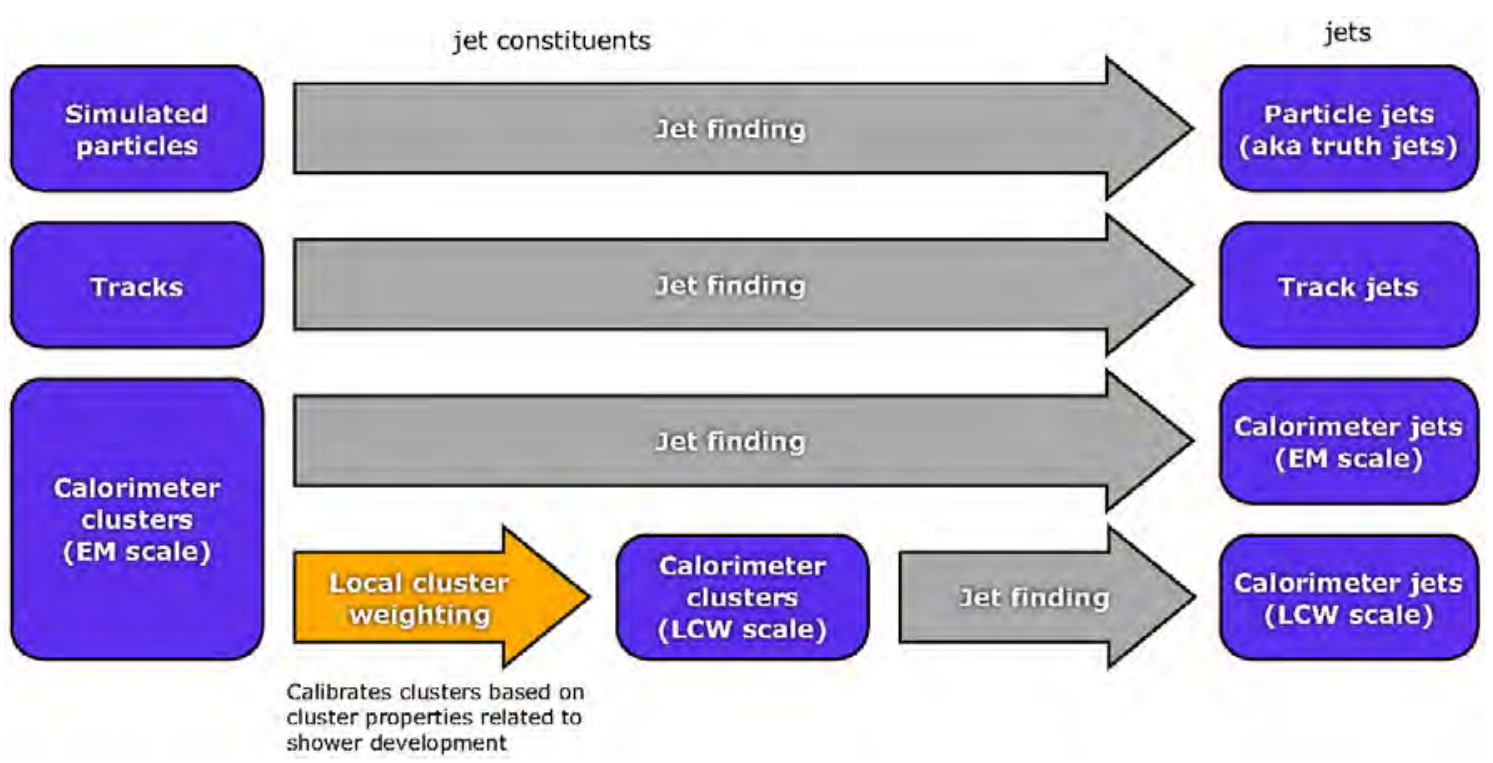

Figure 6.4.: Overview of the jet reconstruction in ATLAS. After the jet finding, the jet four momentum is defined as the four momentum sum of its constituents [145].

\subsubsection{Calibration}

In ATLAS various jet calibration schemes are available [146], which all use jets reconstructed at EM scale as starting point. The calibration corrects for various detector effects, such as calorimeter non-compensation, since the calorimeter response is lower for hadrons than for electrons or photons. In addition, it corrects for dead detector material, energy leakage due to particles outside the calorimeter, energy deposits outside the calorimeter jet cone from particles inside the cone and inefficiencies due to noise threshold and particle reconstruction.

For the following analyses, jets are calibrated to the hadronic scale using the EM+JES scheme [146]. The calibration is performed in bins of the jet energy and jet rapidity and restores the jet energy scale (JES) to the one for jets reconstructed on particle level. For the 2010 dataset, the calibration consists of three steps [147]: a pile-up correction, a vertex correction and a jet energy and direction correction derived from MC simulation. This approach assumes implicitly that GEANT4 perfectly models the detector. For the 2011 dataset, due to its larger statistics, a further step correcting for the remaining difference between data and MC could be added to the jet calibration scheme. This correction has been determined using a combination of in-situ measurements based on the transversemomentum balance between a jet and well-calibrated reference object in $Z+$ jet [148], $\gamma+$ jet [149], multi-jet [150] and dijet [151] events. A full overview of the ATLAS jet calibration scheme used for the 2011 dataset is depicted in Fig. 6.5.

\subsubsection{Quality Criteria}

Jets used for physics analysis have to fulfil several quality requirements, in order to distinguish them from jets not originating from the hard interactions of the pp-collision (fake jets). Those fake jets refer to beam-gas events, beam-halo events, cosmic ray muons or calorimeter noise. For physics analysis with 2010 data two quality selections are defined [147], the loose and medium selection, while for 2011 data two additional selections are 


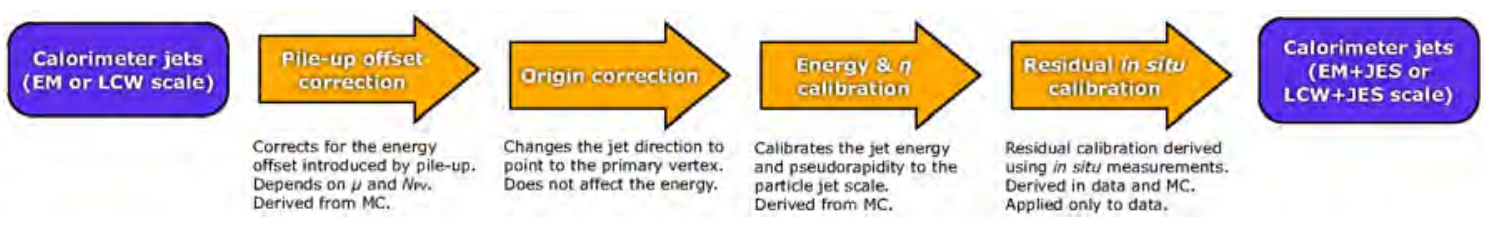

Figure 6.5.: Overview of the ATLAS jet calibration scheme used for the 2011 dataset. The pile-up, absolute JES and the residual in situ corrections calibrate the scale of the jet, while the origin and the eta corrections affect the direction of the jet [145].

introduced [152, the looser and tight selection. Figure 6.6 shows exemplarily the distribution of fake jets before and after applying the different jet quality criteria for the transverse momentum and the $\phi$ distribution of jets.

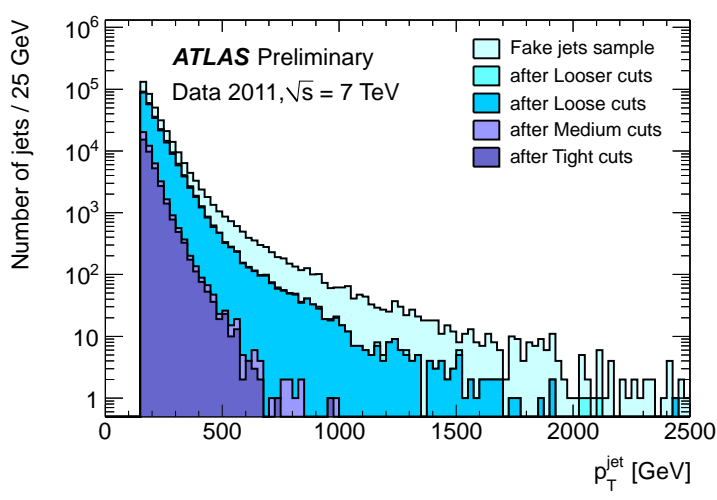

(a) Jet $p_{T}$

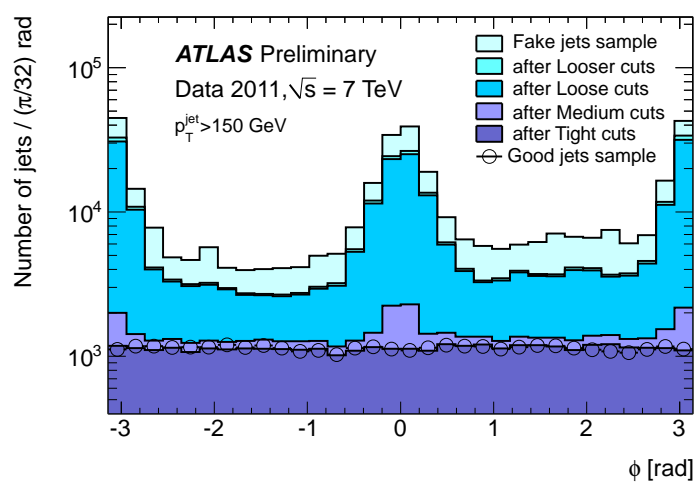

(b) Jet $\phi$

Figure 6.6.: (a) Jet transverse momentum distribution and (b) $\phi$ distribution of fake jets before and after applying the different jet quality criteria for 2011 data [152]. Fake jets are selected from events with a single hard jet in opposite direction to large missing transverse momentum.

The efficiency of the different quality selections have been measured in data using tagand-probe methods in dijet events [152]. Figure 6.7 shows exemplarily the jet quality selection efficiency for anti- $k_{T}$ jets with $R=0.4$ in the region $|\eta|<0.3$.

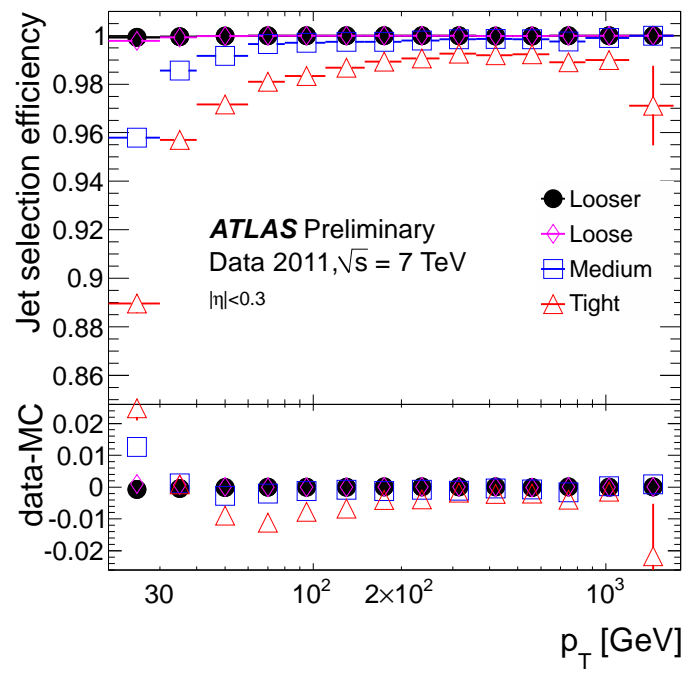

Figure 6.7: Jet quality selection efficiency for anti- $k_{T}$ jets with $R=0.4$ in the region $|\eta|<0.3$ [152. 
For the analysis with the 2010 data presented in this thesis the medium quality selection for jets is used, since it was optimised for high transverse momentum jets. For the analysis with 2011 data the looser selection is applied, which has a larger jet selection efficiency than medium $\left(>99.8 \%\right.$ for $p_{T}^{\text {jet }}>20 \mathrm{GeV}$ and the full $\eta$ region) and is able to reject most of the calorimeter noise.

Furthermore, jets can originate from additional pp-interactions per bunch crossing, as described in Sec. 2.2.6. In order to reject those jets, the jet vertex fraction (JVF) is defined as the ratio of the $p_{T}$ sum of its associated tracks belonging to the vertex of the hard interaction to the $p_{T}$ sum of all its associated tracks. Since the JVF requires tracking information, it is only defined within the tracker acceptance region. Jets originating from the primary vertex are expected to have a value close to 1 for the JVF, whereas the value for jets from additional pp-interactions are expected to be close to 0 . For the following analyses the absolute value of the JVF is required to be greater than 0.75 for the jets which are identified in the acceptance region of the tracker $(|y|<2.4)$.

\subsubsection{Performance}

The jet energy scale and energy resolution has been studied in data and MC using insitu techniques. In the following, the performance of those methods is explained for the dataset of 2010 and 2011.

\section{Performance in 2010 Data}

In 2010, jets are calibrated using MC simulation, as described in Sec. 6.2.2. Systematic uncertainties of the JES account for uncertainties on the JES calibration method, the calorimeter response, the detector simulation, the noise threshold of the calorimeter cells, the knowledge of additional detector material and the relative calibration of jets with $\eta>0.8$ ( $\eta$-intercalibration). Additional uncertainties account for the presence of pileup and close-by jets in the final state. Since the JES is derived from multi-jet events, which have a different relative quark- and gluon-jet population compared to $Z / \gamma^{*}+$ jets events, an additional uncertainty has been calculated to cover this difference. Figure 6.8 exemplarily shows the fractional JES uncertainty for the 2010 dataset as a function of the jet transverse momentum in the region $0.3 \geq|\eta| \geq 0.8$.

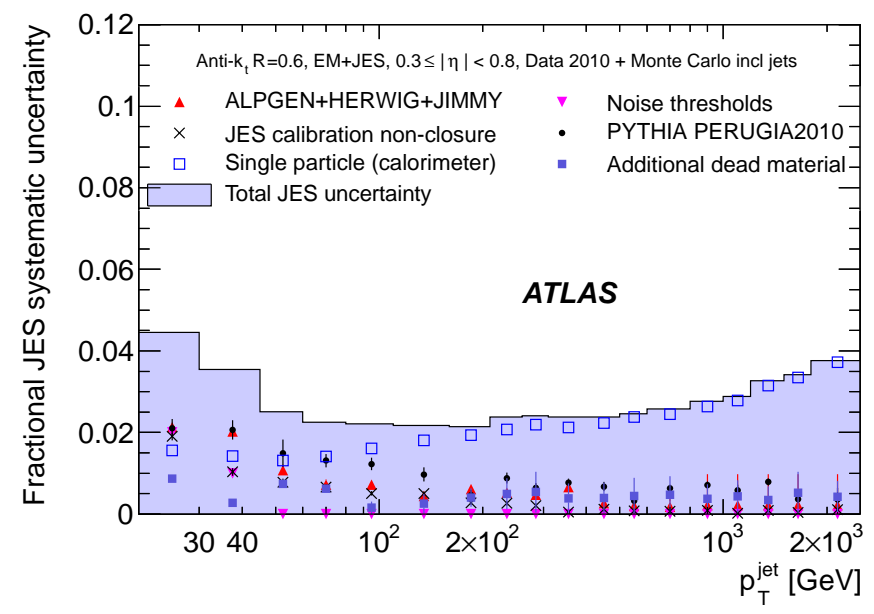

Figure 6.8: Fractional JES uncertainty for jets reconstructed with the anti- $k_{t}$ algorithm with $R=0.6$ and calibrated using the EM+JES scheme as a function of the jet transverse momentum in the region $0.3 \geq|\eta| \geq 0.8$ [147]. The total uncertainty as well as the different components are shown. The uncertainties have been derived from data taken in 2010 and MC simulation. 
The jet calibration in 2010 data has been tested using in-situ techniques in dijet and $\gamma+$ jet events and is able to restore the jet energy for the full kinematic range to the extend of $2 \%$ [147].

The jet energy resolution (JER) has been studied using dijet-events in data and MC event samples, generated by PYTHIA, based on two in-situ techniques, the dijet balance and the bisector techniques [153] in the range $30 \mathrm{GeV}<p_{T}<500 \mathrm{GeV}$ and $|y|<2.8$. Figure 6.9 shows the fractional jet transverse momentum resolution.

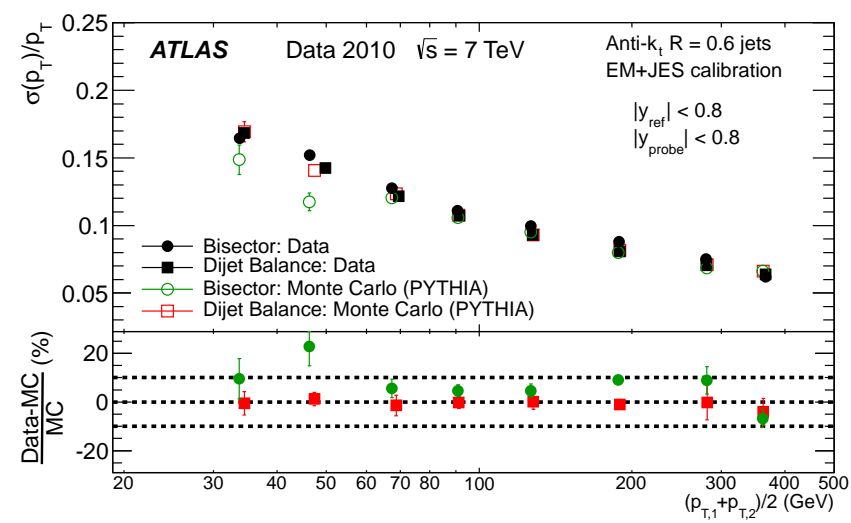

Figure 6.9: Fractional jet transverse momentum resolution measured in data taken in 2010 and MC simulation using two different in-situ techniques, the dijet balance and bisector method [154].

The resolution measured in MC simulation and data have been found to be in agreement within $10 \%$ [154]. The relative difference is used to calculate the systematic uncertainty. The extrapolation of the systematic uncertainty beyond the region mentioned above, has been done by smoothly increasing the uncertainty up to a factor of three for $p_{T}=10 \mathrm{GeV}$ and up to a factor of two for $p_{T}=1000 \mathrm{GeV}$. In the forward region $(2.8<|y|<4.5)$ the JER uncertainty from the region $2.1<|y|<2.8$ has been taken.

\section{Performance in 2011 Data}

In 2011 data, jets are calibrated using MC simulation plus an additional correction derived from in-situ measurements to correct for the remaining differences between data and MC, as described in Sec. 6.2.2. Systematic uncertainties of the JES account for uncertainties on the different in-situ measurements entering in the jet calibration, uncertainties on the pile-up correction, uncertainties due to close-by jets and uncertainties due to the different flavour composition and response between the MC event sample used for the calibration and the samples used for the analysis. The different systematic uncertainties have been evaluated as a function of $\eta$ and $p_{T}$ of the jet. In addition, the uncertainty due to the pile-up correction is determined as a function of $N_{P V}$ and $\mu$. In total the JES uncertainty in 2011 is decomposed into 64 uncertainty components in order to fully take into account the correlations. 54 of these components belong to the in-situ analysis. Figure 6.10 shows the fractional JES uncertainty for the 2011 dataset as a function of the jet transverse momentum for two different rapidity values. 


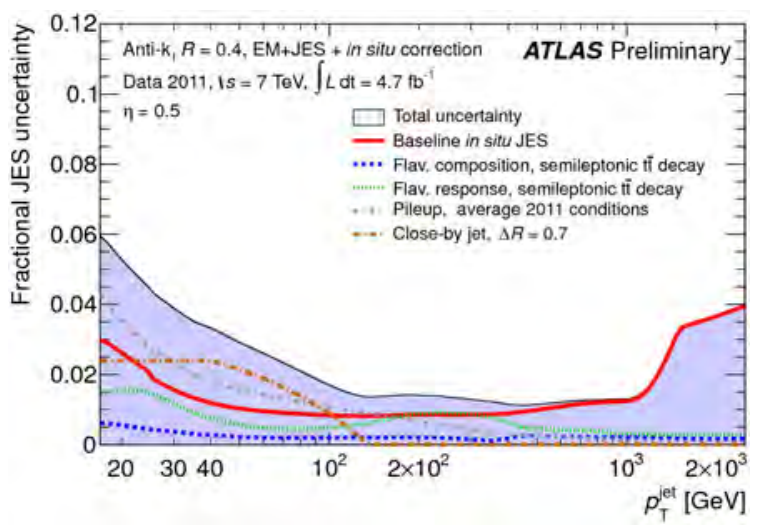

(a) jet $p_{T}, \eta=0.5$

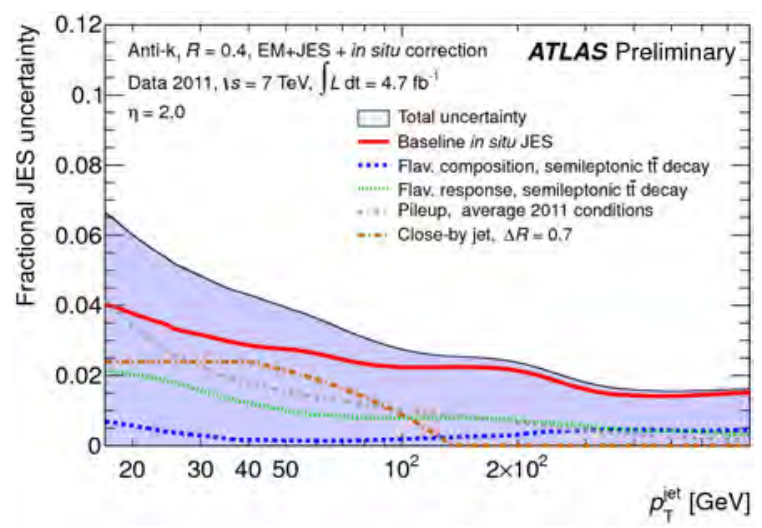

(b) jet $p_{T}, \eta=2.0$

Figure 6.10.: Fractional JES uncertainty for jets reconstructed with the anti- $k_{t}$ algorithm with $R=0.4$ and calibrated using the EM+JES scheme as a function of the jet transverse momentum for (a) $\eta=0.5$ and (b) $\eta=2.0$ [145]. The total uncertainty as well as the different components are shown. The uncertainties have been derived from data taken in 2011 and MC simulation.

Compared to 2010 data, the JES uncertainty has significantly been reduced by approximately $50 \%$ up to $1 \mathrm{TeV}$, due to the in-situ based calibration, as shown in Fig. 6.11.

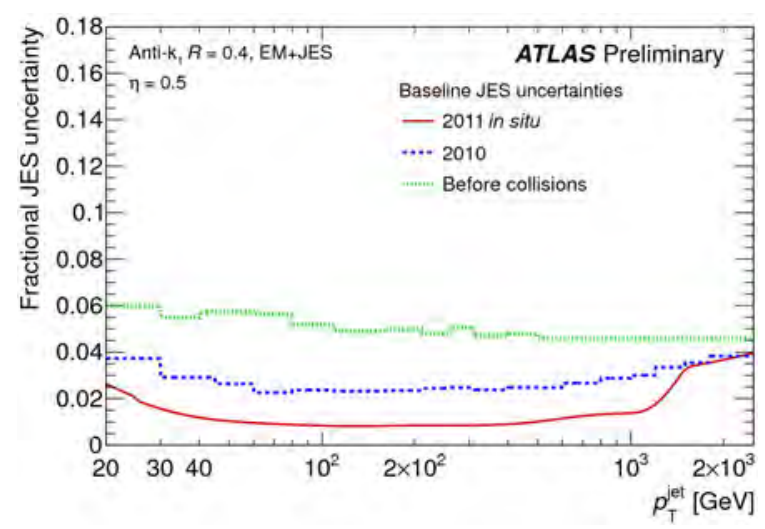

Figure 6.11: Fractional JES uncertainty for jets reconstructed with the anti- $k_{t}$ algorithm with $R=0.4$ and calibrated using the EM+JES scheme as a function of the jet transverse momentum for $\eta=0.5$ for the data taken in 2010 and 2011 [145].

The JER in 2011 data has as well been studied using dijet-events in data and MC event samples, generated by PYTHIA, based on the same two in-situ techniques [153]. In contrast to the studies with the 2010 dataset, the measurements have been extended to the forward region and to larger $p_{T}$ regimes. The resolution measured in $\mathrm{MC}$ simulation and data have been found to be in agreement within $6 \%$ to $15 \%$ depending on the $|\eta|$ region. Similar to 2010, the relative difference is used to calculate the systematic uncertainty. 



\section{General Analysis Approach}

A proper understanding of the jet production in association with a $\mathrm{Z} / \gamma^{*}$ boson is a fundamental part of the LHC physics programme. It provides a stringent test of pQCD predictions and can be used to test NLO EW corrections. In addition, it constitutes an important background for studies of the Higgs boson properties and searches of new physics.

The kinematic distributions of jets in association with a $Z$ boson can be predicted by NLO pQCD calculation and MC predictions from matrix element generators accompanied by parton shower (ME+PS). The latter are affected by large scale uncertainties and need to be tuned and validated using comparisons with data.

The aim of the following analysis is to measure the inclusive and differential $Z / \gamma^{*}(\rightarrow e e)+$ jets cross section in pp-collisions with first ATLAS data at a centre of mass energy of $\sqrt{s}=7 \mathrm{TeV}$. At ATLAS the analysis has been performed for three different integrated luminosities: $\int L \mathrm{~d} t=1.3 \mathrm{pb}^{-1}, 36 \mathrm{pb}^{-1}$ (full dataset of 2010) and $4.6 \mathrm{fb}^{-1}$ (full dataset of 2011). Since the measurement with the full dataset of 2010 supersedes the measurement with $\int L \mathrm{~d} t=1.3 \mathrm{pb}^{-1}$, only the analyses with the full dataset of 2010 and 2011 are detailed in this thesis. The full dataset of 2010 has the advantage of a relatively low collision rate and a low rate of multiple proton-proton interactions $(\langle\mu\rangle=2)$, due to a moderate instantaneous luminosity of $2.1 \times 10^{32}$ and a bunch spacing of $150 \mathrm{~ns}$, which allow for cross-section measurements at low jet transverse momentum. The measurement with the full dataset of 2011 provides the most accurate results and partly supersedes the measurement with the full dataset of 2010. This dataset allows to exploit the data in kinematic regions which were not accessible before and can be used to probe the $Z / \gamma^{*}+$ jets modelling in typical phase space regions expected for the Higgs boson decay and searches for new physics.

Cross sections can be quoted on three different levels: detector level, particle level (hadron level) and parton level.

- Detector level: Cross sections on detector level correspond to electrons and jets reconstructed in the detector.

- Particle level: Cross sections on particle level correspond to dressed electrons, which are defined to include the contributions from photons within a cone of 0.1 around the electron direction. Jets are clustered around all final state particles (decay length $c \tau>10 \mathrm{~mm}$ ) except for the dressed decay products of the $Z$ boson and identified with the anti-kt $\mathrm{k}_{t}$ algorithm with a cone of $R=0.4$.

- Parton level: Cross sections on parton level correspond to Born level electrons before QED FSR. Jets are clustered around all partons before fragmentation using the anti- $\mathrm{k}_{t}$ algorithm with a cone of $R=0.4$.

For a first comparison, the measurements are performed on detector level and compared to predictions from MC event generators. In order to provide results which are 


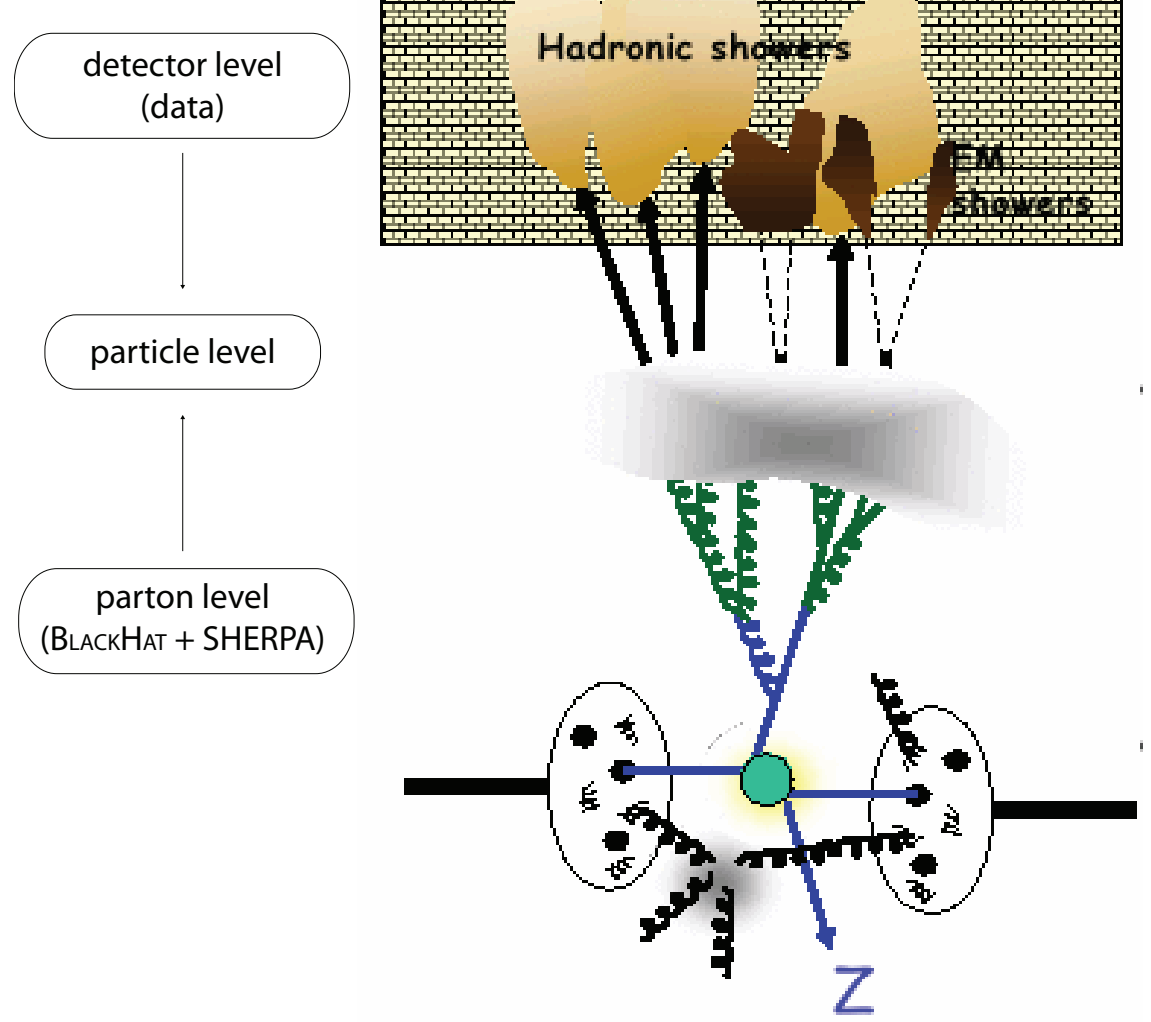

Figure 7.1.: Schematic view of jet production and reconstruction. The different levels are shown; parton level, particle level and detector level.

independent of the detector setup, the final results of the measurements are presented on particle level, as shown in Fig. 7.1. Thus, the background subtracted data distributions have to be corrected for detector effects back to particle level. The correction for detector effects is performed using two different methods: the iterative (Bayes) method and the bin-by-bin method, as described in Chapter 5. The corrected measurements refer to distributions with exactly two dressed electrons and additional jets, which fulfil the phase space requirements detailed in Tab. 7.1 (fiducial phase space).

\begin{tabular}{|l|c|}
\hline \hline Variable & Criteria \\
\hline \hline electron $p_{T}$ & $p_{T}>20 \mathrm{GeV}$ \\
electron $|\eta|$ & $|\eta|<1.37$ or $1.52<|\eta|<2.47$ \\
\hline electron charges & opposite charge \\
electron separation $\Delta R^{e e}$ & $\Delta R^{e e}>0.2($ only 2011$)$ \\
electron invariant mass $m^{e e}$ & $66 \mathrm{GeV} \leq m^{e e} \leq 116 \mathrm{GeV}$ \\
\hline jet $p_{T}^{\text {jet }}$ & $p_{T}^{\text {jet }}>30 \mathrm{GeV}$ \\
jet rapidity $y^{\text {jet }}$ & $\left|y^{j e t}\right|<4.4$ \\
electron-jet separation $\Delta R^{e j}$ & $\Delta R^{e j}>0.5$ \\
\hline \hline
\end{tabular}

Table 7.1.: Summary of $Z / \gamma^{*}(\rightarrow e e)$ and jet selection criteria.

Cross sections are only determined for bins with at least 10 observed events on detector level. Systematic uncertainties on the measurements can be split in three main 
groups: detector and reconstruction related uncertainties (jet energy scale, jet energy resolution, electron triggering, energy scale, resolution, reconstruction and identification), uncertainties due to background modelling and normalisation and uncertainties related to the unfolding method. In order to reduce the uncertainties on the measured differential cross sections, related to the luminosity estimate and the electron reconstruction and identification, the cross sections are divided by the inclusive $Z / \gamma^{*}(\rightarrow e e)$ cross section, respectively. This results in a relative uncertainty which is clearly reduced in comparison with the uncertainty on the absolute cross sections.

The final results are compared to theory predictions from various generators and to NLO fixed-order perturbative QCD predictions from BLACKHAT+SHERPA [14 16], corrected for non-perturbative effects, such as UE and fragmentation, and electroweak final state radiation (QED FSR).

\subsection{Data Samples and Event Selection}

The datasets used for these measurements were collected in pp-collisions at a centre-ofmass energy of $\sqrt{s}=7 \mathrm{TeV}$ in 2010 and 2011 with the ATLAS detector.

For both analyses, the events were selected online using a set of triggers. In 2010 single electron triggers [155] have been used, while for 2011 dielectron triggers [156] have been used in order to be able to keep the electron $p_{T}$ threshold for the offline selection.

- 2010: Events in early data periods (A-E3) are selected with a hardware-based L1 trigger (L1_EM_14), requiring at least an electromagnetic cluster with a minimum $E_{T}$ threshold of $E_{T}=14 \mathrm{GeV}$, whereas events in later data periods (E4-I2) are selected with a software-based higher-level trigger (EF_e15_medium) requiring at least one electron with a minimum $E_{T}$ threshold of $E_{T}=15 \mathrm{GeV}$ in the region $|\eta|<2.5$. The trigger efficiency per electron is above $99 \%$ [155].

- 2011: Events are selected using variations of a software-based higher-level dielectron trigger (EF_2e12_medium, EF_2e12T_medium and EF_2e12Tvh_medium) with a minimum $E_{T}$ threshold of $E_{T}=12 \mathrm{GeV}$ in the region $|\eta|<2.5$. The $T$ in the trigger name indicates that a tighter trigger requirement on L1 has been used. For EF_2e12_medium L1_2EM7 has been used, while the other two use L1_2EM10. The additional $v h$ in the trigger name indicates that an additional cut on the hadronic core isolation at $\mathrm{L} 1$ of $\leq 1 \mathrm{GeV}$ has been applied. The efficiency plateau for e12_medium is reached at $20 \mathrm{GeV}$, while both $2 \mathrm{e} 12 \mathrm{~T} \_$medium and e12Tvh_medium reaches the plateau at $25 \mathrm{GeV}$. For all three triggers the trigger efficiency with respect to the offline electron $p_{T}$ is between $95 \%$ and $99 \%$ for $p_{T}>20 \mathrm{GeV}$ [156].

The electron identification for these triggers is similar to the subsequent offline identification. All these triggers are unprescaled. Only events which have been collected with a fully operational inner detector, calorimeters, muon chambers and magnets are considered by applying the Good Run List (GRL). The selected data samples correspond to an integrated luminosity of $\int L \mathrm{~d} t=36.2 \pm 1.2 \mathrm{pb}^{-1}\left[101\right.$, 157] for 2010 and $4.64 \pm 0.08 \mathrm{fb}^{-1}$ [101] for 2011. Table 7.2 illustrates the data collected with the respective triggers for the different periods of data taking and their corresponding integrated luminosities. 


\begin{tabular}{|c|c|c|c|c|}
\hline \hline \multicolumn{5}{|c|}{2010} \\
\hline \hline Data Period & L1 & L2 & EF & Integrated Luminosity \\
\hline \hline A-E3 & EM14 & & & $0.7 \mathrm{pb}^{-1}$ \\
\hline E4-I2 & EM10 & e15_medium & e15_medium & $35.5 \mathrm{pb}^{-1}$ \\
\hline \hline \multicolumn{5}{|c|}{2011} \\
\hline \hline Data Period & L1 & L2 & EF & Integrated Luminosity \\
\hline \hline D-H & 2EM7 & 2e12_medium & 2e12_medium & $1.10 \mathrm{fb}^{-1}$ \\
I-K & 2EM10 & 2e12T_medium & 2e12T_medium & $1.14 \mathrm{fb}^{-1}$ \\
L-M & 2EM10VH & 2e12Tvh_medium & 2e12Tvh_medium & $2.40 \mathrm{fb}^{-1}$ \\
\hline \hline
\end{tabular}

Table 7.2.: Trigger chains for the various data taking periods in 2010 and 2011 with their corresponding integrated luminosities.

Each selected event is required to have at least one vertex with a minimum of three associated tracks. In addition, events where the first vertex is not a good vertex are removed.

In 2010, $Z$ bosons are selected by requiring the events to have at least two oppositely charged reconstructed electrons, which fulfil the medium quality requirement, as described in Sec. 6.1, with a minimum transverse momentum of $p_{T}=20 \mathrm{GeV}$ and a pseudorapidity in the region $|\eta|<2.47$, excluding the transition regions between the calorimeter sections $1.37<|\eta|<1.52$. The dielectron invariant mass, $m_{e^{+} e^{-}}$, is required to be within a mass window of $66 \mathrm{GeV}<m_{e^{+} e^{-}}<116 \mathrm{GeV}$ around the mass of the $\mathrm{Z}$ boson to optimise the signal purity.

For 2011 the electron definition has been updated, as described in Sec. 6.1. The events are required to have exactly two oppositely charged reconstructed electrons, which fulfil the medium ++ quality requirement, as explained in Sec.6.1.2. In addition, the selected electrons are required to be well separated in the $\eta-\phi$ plane, $\Delta R=\sqrt{(\Delta \eta)^{2}+(\Delta \phi)^{2}}>0.2$, which gives a clean electron definition in particular for highly boosted $Z$ bosons. A veto on events with more than two electrons is used to further suppress background contributions from diboson production. It has been checked that this veto has negligible influence on the signal process.

Associated jets are build from calorimeter clusters using the anti- $\mathrm{k}_{t}$ algorithm with a distance parameter of $R=0.4$ and calibrated with the EM+JES calibration, as described in Sec. 6.2. Furthermore, the energies of reconstructed jets in the data taken in 2011 are corrected using a combination of in-situ measurements, as detailed in Sec. 6.2.2. Jets are selected in the region $\left|y^{\text {jet }}\right|<4.4$ and required to have a transverse momentum $p_{T}^{\text {jet }}>30 \mathrm{GeV}$. In addition, the absolute value of the JVF has to be greater than 0.75 . For the analysis with the full dataset of 2010, the JVF cut is applied on all selected jets, while for 2011 it is only applied on jets, which have been identified in the acceptance region of the tracker $(|y|<2.4)$. In order to account for beam-gas events, beam-halo events, cosmic ray muons or calorimeter noise, further jet cleaning procedures are applied, as detailed in Sec. 6.2.3. Studies in Ref. 158, have shown that the reconstruction efficiency for jets decreases in the proximity of electrons $(\Delta R<0.5)$, which directly translates into a bias on the measured jet multiplicity. Therefore, jets closer in $\Delta R$ than 0.5 to the two selected electrons are removed. 


\subsection{Modelling of Signal and Background Processes}

MC event samples are used to model signal and background processes. A good modelling especially of the signal process is essential, since it is used to determine the corrections for detector effects and study the different sources of systematic uncertainties on the measurement. In addition, MC event samples are used to correct the fixed-order NLO predictions for non-perturbative effects and QED radiation effects.

Background contributions to the jet production in association with a $Z$ boson are composed of EW and multi-jet processes, as detailed in Sec. 2.3.3. The dominant contributions at the LHC originate from multi-jet processes and $t \bar{t}$ production. For the analysis with the dataset of 2010 the background contribution originating from multi-jet processes has been estimated using a data-driven method, while for the analysis with the dataset of 2011 , both the background contribution from multi-jet processes and the $t \bar{t}$ production have been estimated using data-driven methods.

This section details the different MC event samples used for the analyses and introduces the methods for the data-driven background estimations.

\subsubsection{Signal and Background Monte Carlo Event Samples}

The nominal $Z / \gamma^{*}(\rightarrow e e)+$ jets MC event sample is modelled with ALPGEN v2.13 using CTEQ6L1 PDFs and $\sqrt{m_{Z}^{2}+p_{T, Z}^{2}}$ as QCD factorisation and renormalisation scale. ALPGEN is interfaced to HERWIG v6.510 (v.6.520) ${ }^{1}$ for parton shower evolution and hadronisation and to JIMMY v4.31 for UE simulation, using the AUET1-CTEQ6L1 [159] (AUET2-CTEQ6L1 [160]) tune. The MLM matching cut is at $20 \mathrm{GeV}$. Similar samples are generated with ALPGEN v2.14, interfaced to PYTHIA v.6425 using the PERUGIA2011c tune (only 2011) and SHERPA 1.2.3 (1.4.1). The latter uses CTEQ6.6 (CT10) PDFs. SHERPA 1.4.1 has been improved compared to SHERPA 1.2.3 in terms of shower kinematics, which are relevant for the clustering of the ME events in the matching. In addition SHERPA 1.4.1 includes a new tune and the MC event samples are generated using the MENLOPS approach [13. Both, the ALPGEN and SHERPA samples are generated with an invariant dilepton mass above $40 \mathrm{GeV}$, including LO matrix elements with up to five partons. For ALPGEN, PHOTOS is used to describe QED radiation, whereas SHERPA incorporates the YFS approach to describe virtual and real QED corrections. Both, the ALPGEN and SHERPA samples are normalised globally to the NNLO pQCD inclusive DY cross section of $1.07 \pm 0.054 \mathrm{nb}[72$. The $\mathrm{MC}$ event samples generated with ALPGEN+HERWIG only includes heavy flavour jets due to additional gluon splittings in the parton shower, therefore for 2011 the production of $Z$ bosons in association with jets originating from $b$ quarks is modelled separately using ALPGEN+HERWIG including LO matrix elements with up to three additional partons. The overlap between both ALPGEN+HERWIG samples is removed. In contrast, the predictions from SHERPA already includes LO matrix elements containing $b$ quarks in the nominal samples, but in this samples the $b$ quarks are assumed to be massless. For 2010 it was not necessary to include additional samples for the production of $Z$ bosons in association with heavy flavour jets, since the expected precision was much lower.

\footnotetext{
${ }^{1}$ The numbers in brackets correspond to the settings for 2011.
} 
Additional samples for the production of $Z / \gamma^{*}(\rightarrow e e)+$ jets events with an invariant dilepton mass above $60 \mathrm{GeV}$ are simulated with PYTHIA v6.423, using the processes $q \bar{q} \rightarrow Z / \gamma^{*} g$ and $q g \rightarrow Z / \gamma^{*} q$ for the 2010 analysis and with MC@NLO, interfaced to HERWIG and JIMMY, for the 2011 analysis. For the PYTHIA MC event sample, MRST2007LO* 161] PDFs are used and the LO pQCD predictions are normalised to the inclusive $Z / \gamma^{*}(\rightarrow e e)+\geq 1$ jet cross section measured in data for the comparisons on hadron level. The resulting global scale factor is 1.19 \pm 0.02 [7]. The MC@NLO MC event sample uses CT10 PDFs and the predictions are normalised globally to the NNLO pQCD inclusive DY cross section for $m^{e e}>60 \mathrm{GeV}$ of $0.989 \pm 0.049 \mathrm{nb}$ [72]. The signal MC event samples do not include contributions from the WBF production of $Z / \gamma^{*}(\rightarrow e e)+$ jets, but it has been shown in generator-level studies that the expected contribution is at per-mille to per-cent level for the selections and kinematic ranges studied in this thesis.

Background MC event samples for $W(\rightarrow e \nu)+$ jets, $Z / \gamma^{*}(\rightarrow \tau \tau)+$ jets and diboson processes $(W W, W Z$ and $Z Z)$ are modelled with ALPGEN, interfaced to HERWIG and JIMMY, using CTEQ6L1 PDFs, similarly to the signal MC event samples. For the analysis with the dataset of 2011 diboson processes are generated only with HERWIG, interfaced to JIMMY, using the AUET2-LO* [160] tune. The $W(\rightarrow e \nu)+$ jets and $Z / \gamma^{*}(\rightarrow \tau \tau)+$ jets samples are normalised globally to the NNLO pQCD predictions of $10.46 \pm 0.52 \mathrm{nb}\left[73\right.$. for $W(\rightarrow e \nu)+$ jets and $1.07 \pm 0.05 \mathrm{nb}\left[72\right.$ for $Z / \gamma^{*}(\rightarrow \tau \tau)+$ jets whereas diboson processes are normalised globally to NLO [14] pQCD predictions. For $Z / \gamma^{*}(\rightarrow \tau \tau)+$ jets TAUOLA v1.0.2 [162] is used to simulate tau decays. $t \bar{t}$ events are simulated with MC@NLO using CTEQ6.6 (CT10) PDFs. Since MC predictions of the multi-jet production have large uncertainties, the contributions are estimated using a data-driven method, as described in Sec. 7.2.2. In addition, for the analysis with the dataset of 2011, $t \bar{t}$ production is estimated using a data-driven method, as detailed in Sec. 7.2.3.

In order to account for the presence of pile-up, the predictions from the different MC generators are overlaid with minimum-bias (MB) interactions, simulated by PYTHIA. The amount of pile-up for 2011 is much larger than in 2010. In addition, the pile-up and detector conditions have been changed during the 2011 running period. Therefore for 2011 a more sophisticated method has been used to correct for this effect.

- 2010: The number of additional MB interactions follows a Poisson distribution, with an average of $2 \mathrm{MB}$ interactions per bunch crossing, in order to match the distribution in data. Remaining residual differences between the data and MC predictions are corrected by reweighting the primary vertex distribution in MC, such that it follows the distribution observed in data. The corresponding reweighting factors are detailed in Tab. 7.3 .

- 2011: Due to different pile-up and detector conditions in the 2011 data, the MC event samples are divided into four subsamples to account for these differences. The relative size of the subsamples corresponds to the relative size of the different data taking periods. Remaining residual differences between the data and the MC predictions are corrected by reweighting the $\mathrm{MC}$ predictions to the average number of interactions per bunch crossing in data. Figure 7.2 shows the number of primary vertices $N_{P V}$ and the average number of interactions per bunch crossing $\mu$ in data and ALPGEN+HERWIG event samples after re-weighting and applying the inclusive $Z / \gamma^{*}(\rightarrow e e)$ selection, as described in Sec. 7.1. 


\begin{tabular}{|l|c|c|c|c|c|c|c|c|c|c|}
\hline \hline \# Vertices & 1 & 2 & 3 & 4 & 5 & 6 & 7 & 8 & 9 & $\geq 10$ \\
\hline Reweighting Factors & 1.97 & 1.23 & 0.85 & 0.65 & 0.52 & 0.45 & 0.42 & 0.39 & 0.37 & 0.42 \\
\hline \hline
\end{tabular}

Table 7.3.: Primary vertex reweighting factors for MC event samples in 2010.

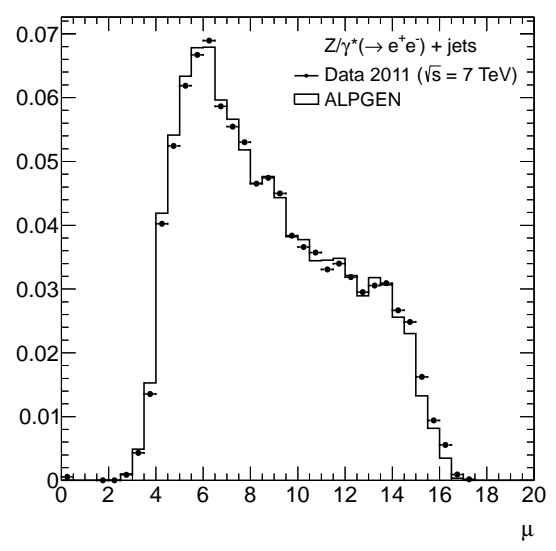

(a) Interactions per bunch crossing

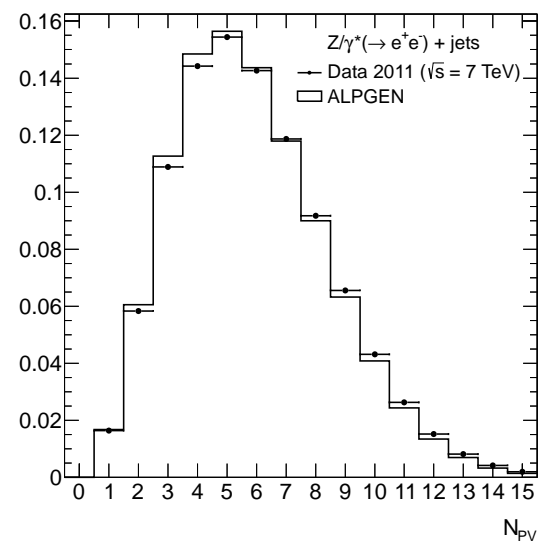

(b) Primary vertices

Figure 7.2.: (a) Average number of interactions per bunch crossing and (b) number of primary vertices in data taken in 2011 and ALPGEN+HERWIG after reweighting and applying the inclusive $Z / \gamma^{*}(\rightarrow e e)$ selection, as described in Sec. 7.1. The distributions are normalised to unity.

Finally, all MC event samples are passed through the GEANT4 simulation of the ATLAS detector and trigger system, as described in Chapter 4.

Further signal MC event samples without full detector simulation, used to determine the correction factors for non-perturbative effects for the analysis with the full dataset of 2010, have been generated with PYTHIA and HERWIG, interfaced to JIMMY. The generation is performed for events with an invariant dilepton mass above $40 \mathrm{GeV}$ using MRST2007LO* PDFs and different parton shower, fragmentation models and UE settings. For PYTHIA variations of the AMBT1 [163] tune are used, while HERWIG+JIMMY uses the AUET1 tune.

The details and settings of the different MC event samples are summarised in Tab. 7.4 and Tab. 7.5 for 2010 and Tab. 7.6 for 2011. 


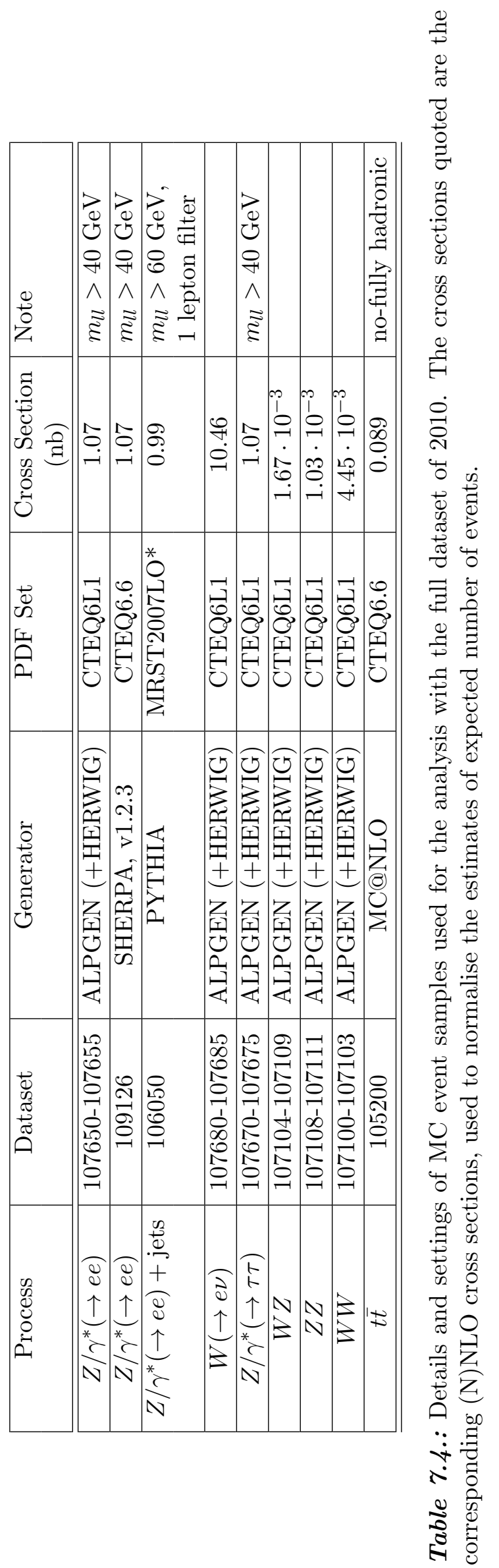

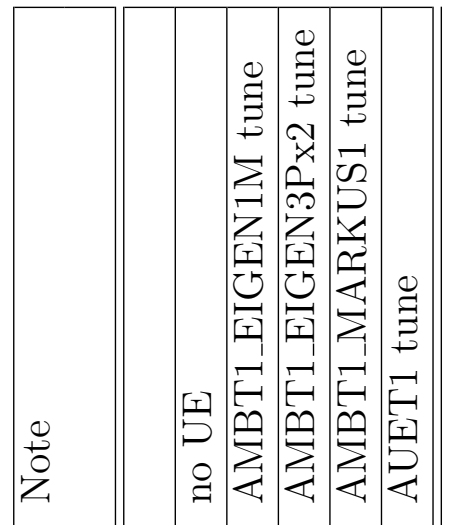

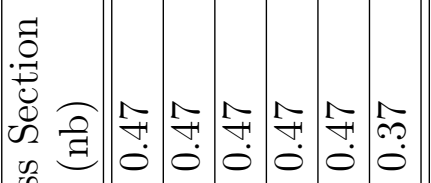

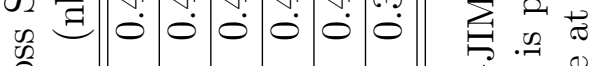

ปี

****** * :

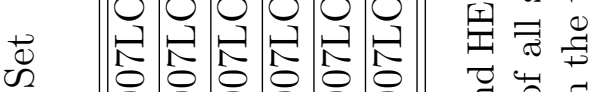

U

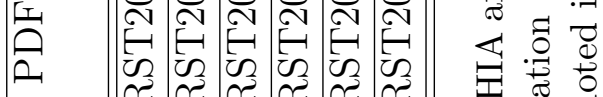

1.

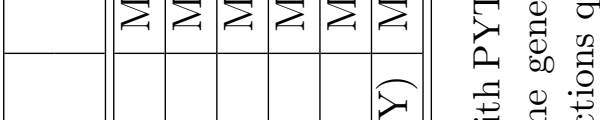

营焉尊

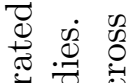

.

$\mathbb{D}_{0}$ is

幽

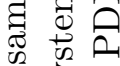

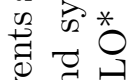

要表

O

$\sum_{0}$ 迎

远

$+2$

बै

$\uparrow$

*

N

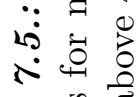

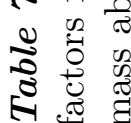




\begin{tabular}{|c|c|c|c|c|c|c|c|c|c|c|c|c|c|c|c|c|}
\hline $\begin{array}{l}0 \\
\text { 苟 } \\
Z^{2}\end{array}$ & $\mid \begin{array}{c} \\
z \\
0 \\
0 \\
0 \\
0 \\
\stackrel{1}{1} \\
\Lambda \\
\vec{\Xi}\end{array}$ & 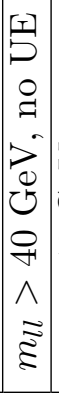 & 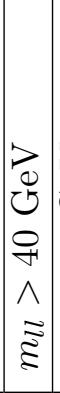 & 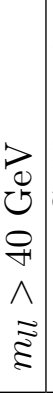 & 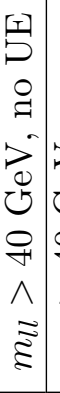 & 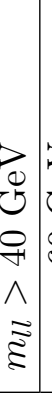 & $\begin{array}{l}\overrightarrow{0} \\
0 \\
0 \\
0 \\
1 \\
\vec{\Xi}\end{array}$ & & 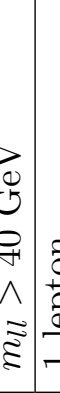 & 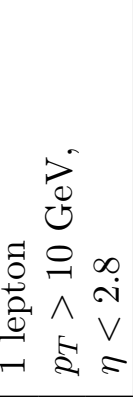 & 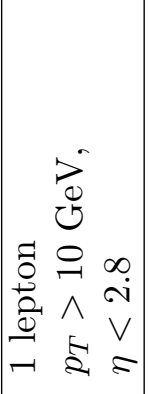 & 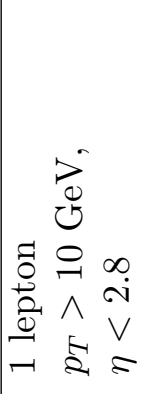 & 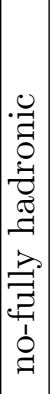 & & & \\
\hline 离 & $\stackrel{0}{-1}$ & $\stackrel{0}{-}$ & $\stackrel{\circ}{-}$ & $\stackrel{0}{-}$ & $\stackrel{0}{\circ}$ & $\stackrel{0}{-}$ & 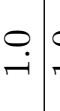 & $\begin{array}{lll}0 & 0 \\
-i & .\end{array}$ & $\dot{-}$ & שֶ. & $\overrightarrow{\widehat{N}}$ & ஜि? & \begin{tabular}{c|}
0 \\
10 \\
0 \\
0
\end{tabular} & $\stackrel{0}{-}$ & & $\stackrel{0}{\circ}$ \\
\hline 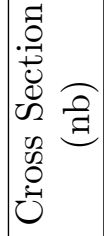 & $\stackrel{1}{\circ}$ & $\begin{array}{c}\hat{0} \\
-i \\
-1\end{array}$ & $\left|\begin{array}{c}m \\
0 \\
0 \\
0\end{array}\right|$ & 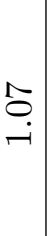 & 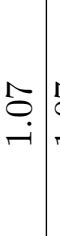 & 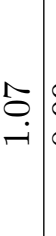 & 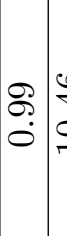 & $\begin{array}{ll}0 \\
0 \\
\stackrel{0}{0} \\
\stackrel{1}{1}\end{array}$ & 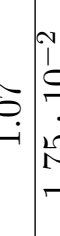 & 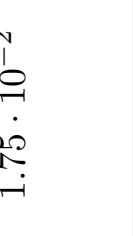 & $\begin{array}{l}\stackrel{0}{\dashv} \\
\dot{\sigma} \\
\underset{+}{+} \\
\dot{0}\end{array}$ & 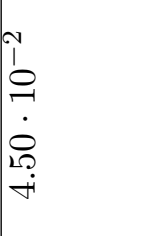 & $\begin{array}{l}\infty \\
0 \\
0 \\
-1 \\
0 \\
0\end{array}$ & \begin{tabular}{l}
$i$ \\
$i$ \\
0 \\
\hdashline \\
$\dot{\alpha}$ \\
0 \\
$\dot{0}$
\end{tabular} & & 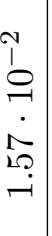 \\
\hline 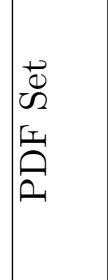 & $\begin{array}{l}-1 \\
\overrightarrow{0} \\
0 \\
0 \\
1 \\
0 \\
0 \\
0\end{array}$ & 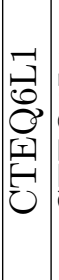 & 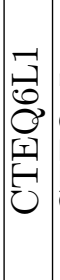 & 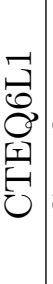 & 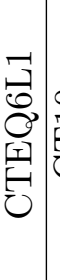 & 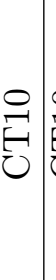 & 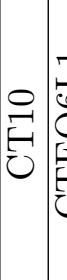 & 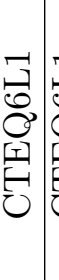 & 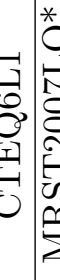 & 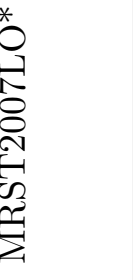 & 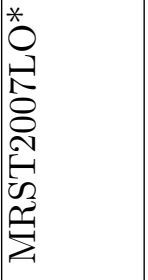 & 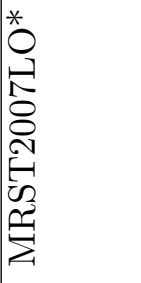 & $\begin{array}{l}\stackrel{0}{0} \\
\stackrel{1}{0} \\
\end{array}$ & 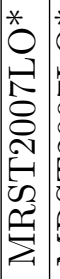 & 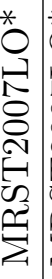 & 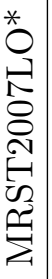 \\
\hline 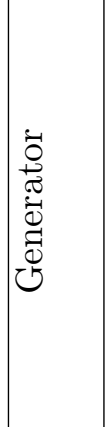 & 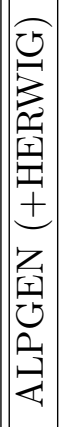 & 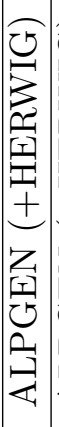 & 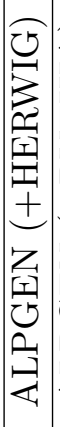 & 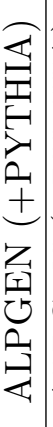 & 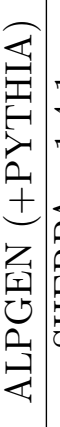 & 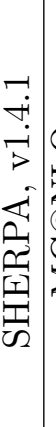 & 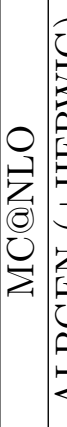 & 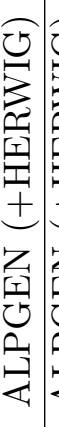 & 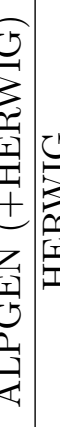 & $\frac{\pi}{c}$ & 党 & 芳 & 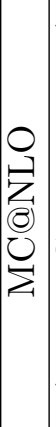 & 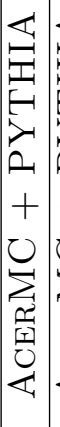 & 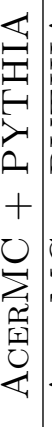 & 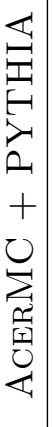 \\
\hline 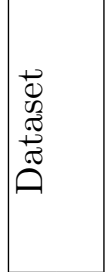 & $\begin{array}{c}20 \\
10 \\
0 \\
0 \\
0 \\
1 \\
0 \\
1 \\
0 \\
0 \\
0 \\
0 \\
-1\end{array}$ & $\begin{array}{l}0 \\
0 \\
0 \\
0 \\
0 \\
-1 \\
-1 \\
1 \\
1 \\
120 \\
0 \\
0 \\
-1\end{array}$ & 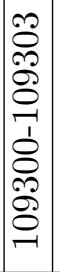 & $\begin{array}{l}20 \\
10 \\
0 \\
1 \\
=1 \\
0 \\
0 \\
0 \\
0 \\
0 \\
=1\end{array}$ & 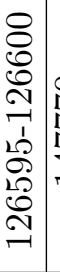 & 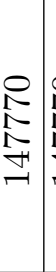 & 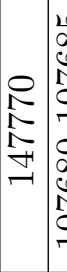 & $\begin{array}{ll}1 & 1 \\
2 & 1 \\
0 & 1 \\
0 & 1 \\
0 & 1 \\
-1 & 1 \\
1 & 1 \\
0 & 1 \\
0 & 0 \\
0 & 1 \\
0 & 1\end{array}$ & 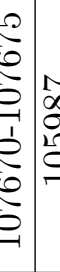 & 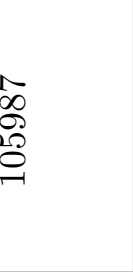 & $\begin{array}{l}0 \\
0 \\
0 \\
2 \\
0 \\
0 \\
-1\end{array}$ & $\begin{array}{l}20 \\
0 \\
0 \\
20 \\
0 \\
0 \\
-1\end{array}$ & $\begin{array}{l}0 \\
0 \\
1 \\
0 \\
0 \\
0 \\
-1\end{array}$ & 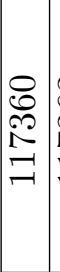 & & 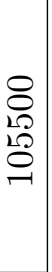 \\
\hline $\begin{array}{l}0 \\
0 \\
0 \\
0 \\
0 \\
0 \\
0\end{array}$ & 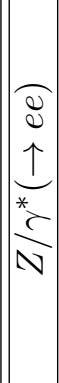 & 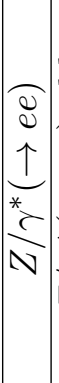 & 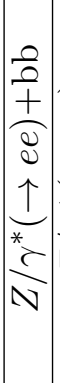 & 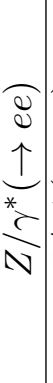 & 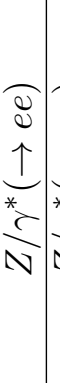 & 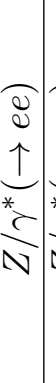 & 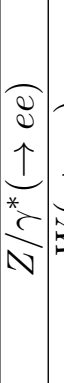 & $\begin{array}{l}3 \\
0 \\
\uparrow \\
3\end{array}$ & 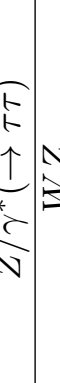 & $\hat{N}$ & $N$ & $\vec{z}$ & 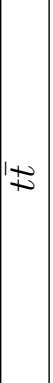 & 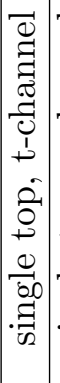 & 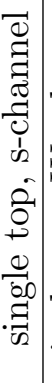 & 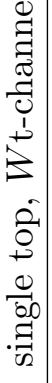 \\
\hline
\end{tabular}

$\stackrel{g}{\ddagger}$

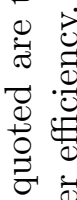

허엄

萬

足

. ?

E.

궁

पे

प्ष

䒕

寻

용

衰造

.

त्ञ

$\underset{+}{ \pm}$

क्षَ

ర్త

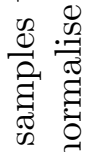

붕요

ठ ]

$\circlearrowright \stackrel{\partial}{=}$

पै

क्ष

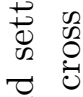

สี 0

柰合

$\stackrel{2}{\square}$

$\because: \exists$

$r$.

जै 


\subsubsection{Data-Driven Estimate of the Multi-jet Background}

The multi-jet background constitutes the major background to $Z / \gamma^{*}(\rightarrow e e)+$ jets for low jet multiplicities. In addition, $\mathrm{MC}$ predictions of the multi-jet background have large uncertainties, due to limited available MC statistics. Using a data-driven estimate provides a more reliable shape for each of the measured distributions and results in a significantly lower uncertainty.

\section{Estimate for the Measurement with the Dataset of 2010}

Multi-jet events are selected similarly to the $Z / \gamma^{*}(\rightarrow e e)+$ jets selection, except for the ID and trigger requirement. The electron candidates are required to pass the loose criterion, but fail the medium one. It is assumed that the shape of multi-jet events passing this selection is similar to the shape of multi-jet events passing the standard selection.

The normalisation of the multi-jet contribution is extracted from a maximum likelihood fit to the measured dielectron mass distribution in the range $50 \mathrm{GeV}<m_{e^{+} e^{-}}<120 \mathrm{GeV}$ for events passing the standard $Z / \gamma^{*}(\rightarrow e e)+$ jets selection, using two template distributions. The first template is created from the selected multi-jet events in data, and the second one originates from the combined simulated signal events plus non-multi-jet background events. The normalisation of both templates are free parameters of the fit.

Systematic uncertainties account for the choice of fit parameters, by varying the bin width and the fit range, the shape of the multi-jet template and the impact of the electron energy scale, by shifting the $\mathrm{Z}$ boson mass peak. The maximum deviations upwards and downwards are taken as final systematic uncertainty.

\section{Estimate for the Measurement with the Dataset of 2011}

For analysis with the dataset of 2011 the method to estimate the multi-jet background has been improved. The template of multi-jet events is created similarly to the one for the analysis with dataset of 2010, but using different trigger requirements. The trigger selects events with two clusters in the calorimeter with a $p_{T}$ above $20 \mathrm{GeV}$. Contamination from other backgrounds are subtracted using MC predictions.

The normalisation of the multi-jet contribution is also extracted from a maximum likelihood fit to the invariant dielectron mass using the two template distributions, defined above. But due to the larger available data statistics, a finer binning could be used and the mass range for the fit could be extended to $50 \mathrm{GeV}<m_{e^{+} e^{-}}<150 \mathrm{GeV}$. Figure 7.3 represents the result of the maximum likelihood fit.

In addition, the treatment of systematic uncertainties has been refined. The different sources of uncertainties are grouped into four more or less uncorrelated categories: the choice of fit parameters, the choice of the signal MC, the shape of the multi-jet template and the impact of electron energy scale and electron energy resolution. In order to obtain the total systematic uncertainty, the maximum deviations in each category are taken and added in quadrature. 


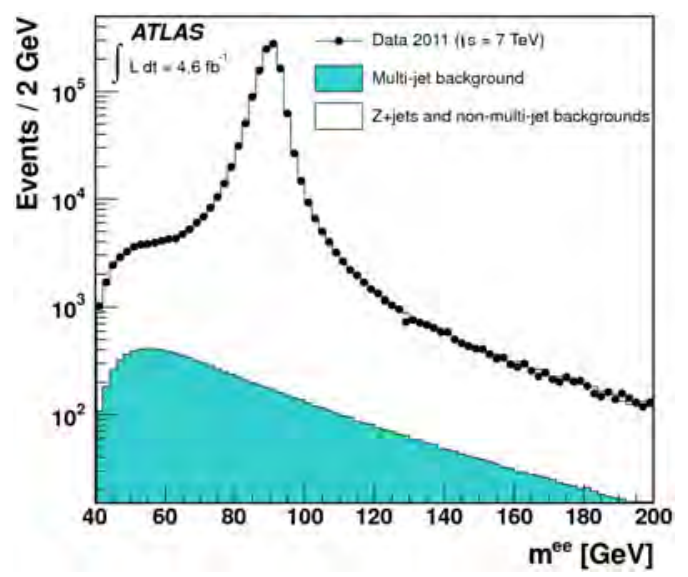

Figure 7.3: Result of the maximum likelihood fit to the dielectron mass distribution in an extended mass range using two templates, the selected multijet events in data and the combined simulated signal events plus non-multi-jet background events. The normalisation of both templates are free parameters of the fit [8].

\subsubsection{Data-Driven Estimate of the $t \bar{t}$ Background}

The $t \bar{t}$ background constitutes the major background to $Z / \gamma^{*}(\rightarrow e e)+\geq 1$ jet, dominated by events where both $W$ bosons decay leptonically. For the analysis with the dataset of 2010 measurements are performed for jet multiplicities up to four jets in the final state, whereas the dataset of 2011 allows to measure cross sections for up to seven jets in the final state. MC event generators, such as MC@NLO and POWHEG, use parton showers to model final states with more than four jets, which leads to an underestimation of the cross section. Therefore, it is necessary to use a data-driven estimate to describe higher jet multiplicities. Due to lepton universality, no difference in final state kinematics for the $e e$ and $e \mu$ channel are expected. In addition, no overlap with $Z / \gamma^{*}(\rightarrow e e)$ events is expected. Thus, $e \mu$ events from the $t \bar{t}$ decay can be used to provide a data-driven estimate of the $t \bar{t}$ background. The event selection for the $t \bar{t}$-enriched sample in $e \mu$ final states is performed similarly to the $Z / \gamma^{*}(\rightarrow e e)+$ jets selection, but requiring exactly one electron and exactly one muon, passing a combined electron and muon trigger with a minimum $p_{T}$ threshold of $10 \mathrm{GeV}$ for the electron and $6 \mathrm{GeV}$ for the muon.

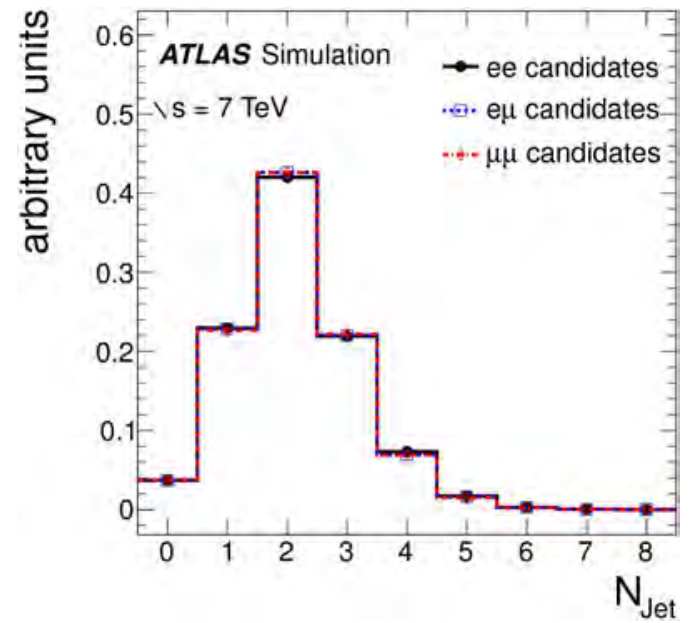

(a) Exclusive jet multiplicity

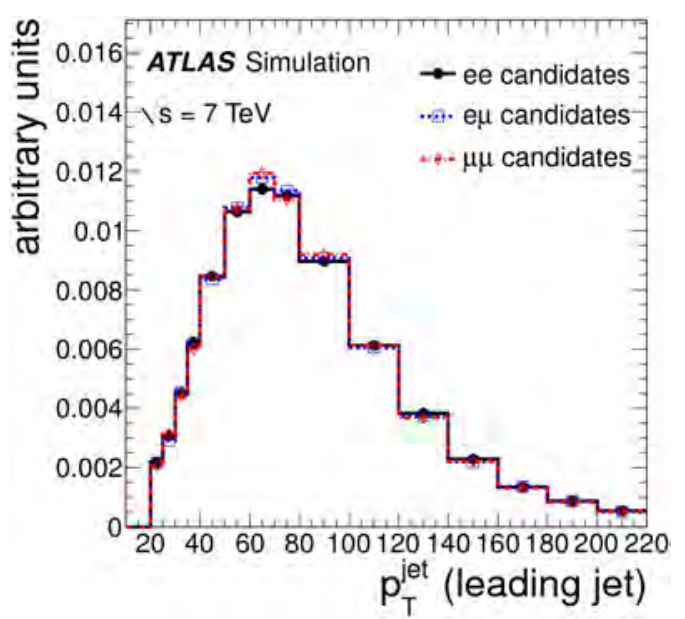

(b) Leading jet $p_{T}$

Figure 7.4.: Shape comparisons of (a) the exclusive jet multiplicity distribution and (b) the transverse momentum distribution of the leading jet for the $e e, e \mu$ and $\mu \mu$ channel in $t \bar{t}$ production simulated with MC@NLO [8]. 
Figure 7.4 shows the shape comparisons of the exclusive jet multiplicity and the transverse momentum of the leading jet for the $e e, e \mu$ and $\mu \mu$ channels in $t \bar{t}$ production simulated with MC@NLO.

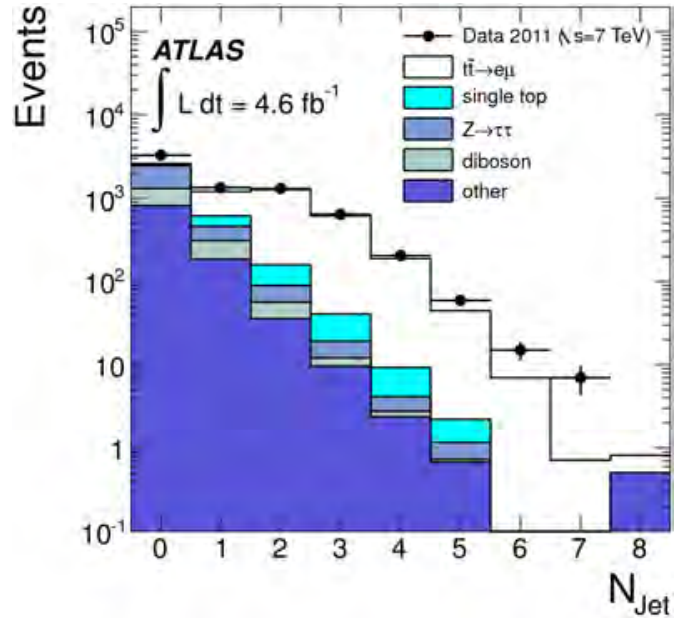

(a) Exclusive jet multiplicity

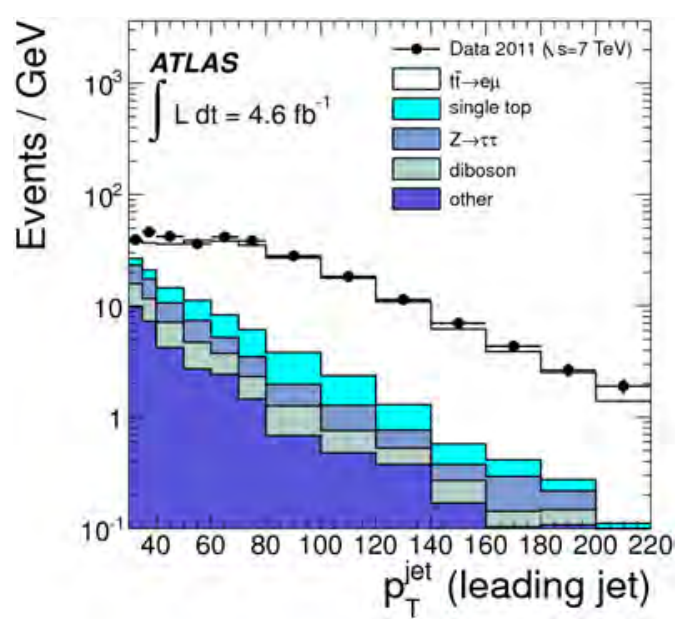

(b) Leading jet $p_{T}$

Figure 7.5.: Comparison between data and MC predictions for (a) the exclusive jet multiplicity distribution and (b) the transverse momentum distribution of the leading jet in the $e \mu$ channel [8].

Figure 7.5 shows the comparison between data and MC predictions for the exclusive jet multiplicity and the transverse momentum of the leading jet in the $e \mu$ channel. Up to $N_{\text {jet }}=4$, both plots show a good agreement. For higher jet multiplicities the predictions from MC@NLO underestimates the data, which reveals that a data-driven estimate is necessary for jet multiplicities above four. Top, diboson and $Z / \gamma^{*}(\rightarrow \tau \tau)+$ jets are subtracted. From the dileptonic $t \bar{t}$ decay twice as many $e \mu$ events compared to $e e$ events are expected. The exact ratio of rates varies a bit, due to different reconstruction efficiencies of electrons and muons and different trigger requirements. Therefore, the data-driven shape and cross-section estimates are normalised to ee using a scale factor $S F_{e \mu \rightarrow e e}=N_{\text {events }}^{e e} / N_{\text {events }}^{e \mu}$ derived from NLO pQCD predictions by MC@NLO+HERWIG and POWHEG+PYTHIA. Systematic uncertainties on the data-driven estimate result from the choice of the $\mathrm{MC}$ generator used to calculate $S F_{e \mu \rightarrow e e}$, the electron trigger, reconstruction and identification efficiencies and the subtraction of EW backgrounds. 


\subsection{NLO pQCD Predictions}

NLO fixed-order ${ }^{2}$ pQCD predictions are determined by the BLACKHAT+SHERPA programme [14 16] for $Z / \gamma^{*}+\geq 0$ jets up to $Z / \gamma^{*}+\geq 4$ jets using NLO PDFs. In this programme BLACKHAT is used for the numerical calculation of the on-shell one loop amplitudes, while SHERPA delivers the remaining things of the calculation, e.g. the integration of the phase space.

For the analysis with the full dataset of 2010, CTEQ6.6 PDFs are used, while for the analysis with the full dataset of 2011 CT10 PDFs are used. For the calculation the QCD factorisation and renormalisation scales are set to $\mu=H_{T} / 2$, where $H_{T}$ describes the scalar $p_{T}$ sum of all particles and partons in the final state. The jet reconstruction on parton level is performed similar to the one in data using the anti- $\mathrm{k}_{t}$ algorithm with a cone of 0.4 .

\subsubsection{Study of Theoretical Uncertainties}

The systematic uncertainties on the predictions account for the uncertainties from the NLO PDFs, the choice of $\alpha_{s}$ and the dependence on the QCD factorisation and renormalisation scales.

\section{Uncertainties for 2010 Settings}

The systematic uncertainty related to the NLO PDFs is computed from the complete CTEQ6.6 PDF error sets using the Hessian method at 90\% CL, as described in Sec. 2.2.2. This corresponds to 41 different PDF sets: the central fit $S_{0}$ and 40 eigenvector uncertainty sets, splitted in $S_{i}^{+}$and $S_{i}^{-}$, which can be used to calculate the PDF uncertainty for each desired distribution using Eq. 7.1.

$$
\begin{aligned}
& (\Delta X)_{+}=\left(\sum_{i}^{N_{P}} \max \left(X\left(S_{i}^{+}\right)-X\left(S_{0}\right), X\left(S_{i}^{-}\right)-X\left(S_{0}\right), 0\right)^{2}\right)^{1 / 2} \\
& (\Delta X)_{-}=\left(\sum_{i}^{N_{P}} \max \left(X\left(S_{0}\right)-X\left(S_{i}^{+}\right), X\left(S_{0}\right)-X\left(S_{i}^{-}\right), 0\right)^{2}\right)^{1 / 2} .
\end{aligned}
$$

This results in an uncertainty of $2 \%$ to $5 \%$ on the inclusive jet multiplicity for $N_{\text {jet }} \geq 1$ to $N_{\text {jet }} \geq 4$. Additional changes on the PDF, due to variations of the input $\alpha_{s}$ at the $Z$ boson scale around its nominal value of $\alpha_{s}\left(m_{Z}\right)=0.118$ by \pm 0.002 , which represents the $90 \% \mathrm{CL}$, result in an uncertainty of $2 \%$ to $7 \%$ on the inclusive jet multiplicity for $N_{\text {jet }} \geq 1$ to $N_{\text {jet }} \geq 4$. Both, the PDF uncertainty and the uncertainty due to the variation of the input $\alpha_{s}$ are added in quadrature.

Systematic uncertainties related to the size of QCD higher-order effects are approximated by varying the factorisation and renormalisation scale simultaneously by a factor of 2 , assuming that the jet multiplicities are correlated. This results in an uncertainty of $4 \%$ to $14 \%$ on the inclusive jet multiplicity for $N_{\text {jet }} \geq 1$ to $N_{\text {jet }} \geq 4$.

\footnotetext{
${ }^{2}$ NLO fixed-order calculations are performed inclusively for a specific final state at NLO including the matrix element for one additional real parton emission.
} 


\section{Uncertainties for 2011 Settings}

The systematic uncertainty related to the NLO PDFs is computed from the complete CT10 PDF error sets using the Hessian method at 68\% CL, as described in Sec. 2.2.2, corresponding to 52 eigenvectors. This results in an uncertainty of $1 \%$ to $3 \%$ on the inclusive jet multiplicity for $N_{\text {jet }} \geq 1$ to $N_{\text {jet }} \geq 4$ and of $1 \%$ to $5 \%$ as $p_{T}$ of the leading jet increases in the range $30 \mathrm{GeV}<p_{T}^{\text {jet }}<500 \mathrm{GeV}$. Additional changes on the PDF, due to variations of the input $\alpha_{s}$ at the $Z$ boson scale around its nominal value of $\alpha_{s}\left(m_{Z}\right)=0.118$ by \pm 0.0012 , which represents the $68 \% \mathrm{CL}$, result in an uncertainty of $1 \%$ to $3 \%$ on the inclusive jet multiplicity for $N_{\text {jet }} \geq 1$ to $N_{\text {jet }} \geq 4$ and of $1 \%$ on the transverse momentum of the leading jet. Both, the PDF uncertainty and the uncertainty due to the variation of the input $\alpha_{s}$, are added in quadrature.

Systematic uncertainties related to the size of QCD higher-order effects are approximated by varying the factorisation and renormalisation scale simultaneously by a factor of 2, using two different approaches, assuming that the different jet multiplicities are uncorrelated. The nominal approach is used for the inclusive distributions. This results in an uncertainty of $4 \%$ to $13 \%$ on the inclusive jet multiplicity for $N_{\text {jet }} \geq 1$ to $N_{\text {jet }} \geq 4$ and of $2 \%$ to $18 \%$ as $p_{T}$ of the leading jet increases in the range $30 \mathrm{GeV}<p_{T}^{\text {jet }}<500 \mathrm{GeV}$. For exclusive final states, the approach from Ref. [164] is used, where inclusive scale variations are used, assuming the scale uncertainty to be uncorrelated between the jet multiplicities. In addition, for the comparison of NLO pQCD predictions and data in Sec. 9.5 and Sec. 10.3 the naive approach of calculating the scale uncertainties, which is identical to the one used for the 2010 analysis and the theoretical uncertainty excluding the scale uncertainty are shown.

For both analyses the scale uncertainty constitutes the dominant contribution to the total uncertainty in most phase-space regions. Figure 7.6 shows exemplarily the relative uncertainties on the theory predictions for a few selected observables using the 2011 settings. 


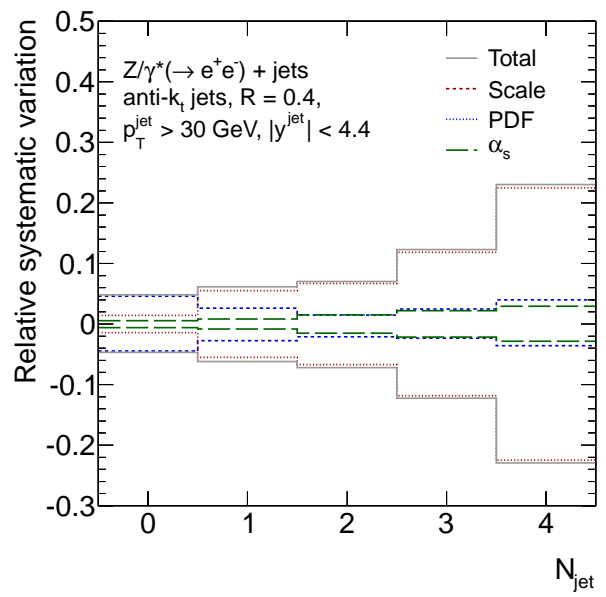

(a) Exclusive jet multiplicity

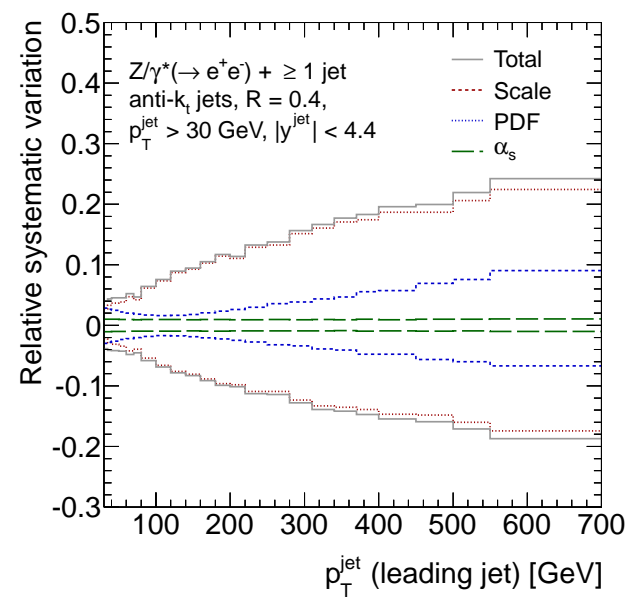

(b) Leading jet $p_{T}$

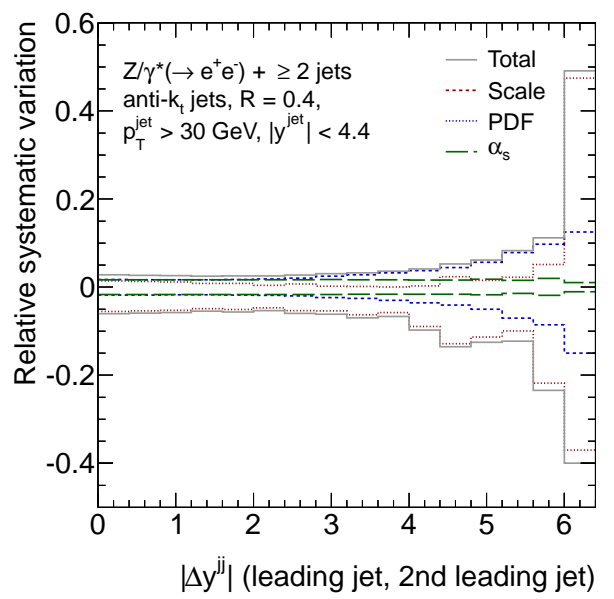

(d) Absolute rapidity difference

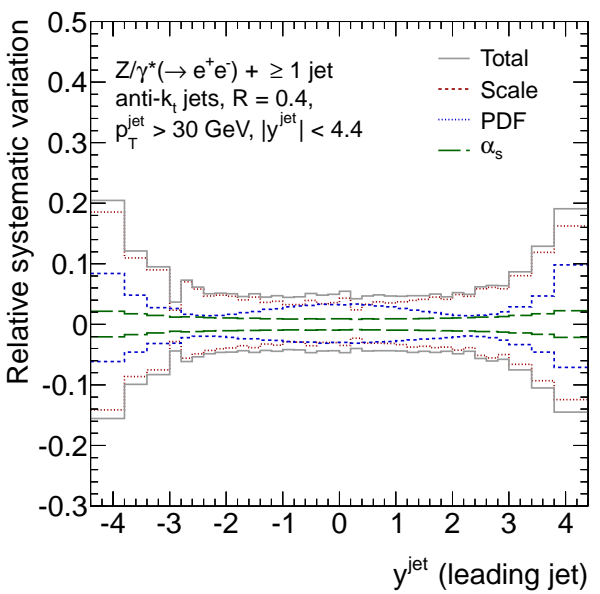

(c) Leading jet $y$

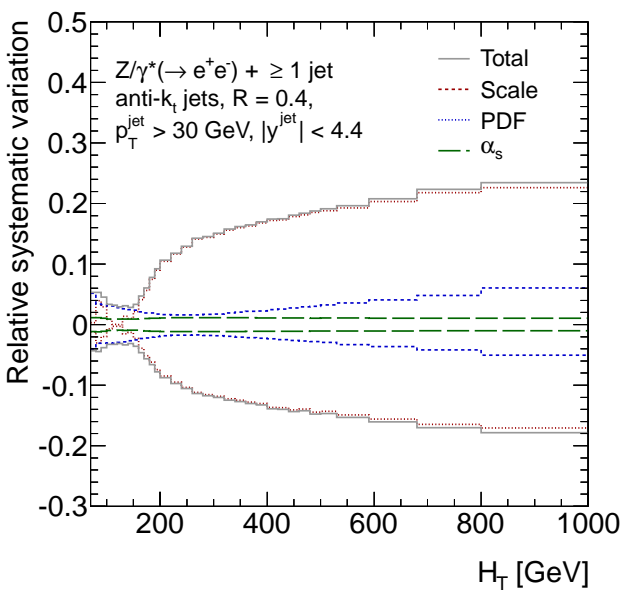

(e) $H_{T}$

Figure 7.6.: Theoretical uncertainties on the NLO fixed-order pQCD predictions for (a) the exclusive jet multiplicity, (b) the transverse momentum of the leading jet for events with at least one jet in the final state, (c) the rapidity of the leading jet for events with at least one jet in the final state, (d) the absolute rapidity difference of the two leading jets $\left|\Delta y^{j j}\right|$ for events with at least two jets in the final state and (e) $H_{T}$ for events with at least one jet in the final state. 



\section{Measurement with the Dataset of 2010}

This chapter describes the inclusive and differential $Z / \gamma^{*}(\rightarrow e e)+$ jets cross-section measurement in pp-collisions at a centre-of-mass energy of $\sqrt{s}=7 \mathrm{TeV}$ with an integrated luminosity of $\int L \mathrm{~d} t=36 \mathrm{pb}^{-1}$ (full dataset of 2010). This dataset has the advantage of a relatively low collision rate and a low rate of multiple proton-proton interactions, due to a moderate instantaneous luminosity of $2.1 \times 10^{32}$ and bunch spacing of $150 \mathrm{~ns}$, which allow for cross-section measurements at low jet transverse momentum.

Total inclusive cross sections and their ratios are measured as a function of the jet multiplicity, $N_{\text {jet }}$. Additionally, inclusive differential cross sections are measured as a function of the jet transverse momentum, $p_{T}^{j e t}$, the jet rapidity, $y^{j e t}$, the dijet mass, $m^{j j}$, and the angular separation between the jets for at least two jets in the final state.

This chapter is organised as follows: Starting with the description of the uncorrected distributions comparing MC predictions to the measurements on detector level in Sec. 8.1, Sec. 8.2 proceeds with the explanation of the method to correct for detector effects. The different sources of systematic uncertainties on the measurement are discussed in Sec. 8.3. followed by the theoretical corrections for non-perturbative effects and QED FSR in Sec. 8.4. Finally, Sec. 8.5 discusses the results at particle level.

\subsection{Uncorrected Distributions}

The uncorrected data distributions for $Z / \gamma^{*}(\rightarrow e e)+$ jets are compared to the expected signal and background distributions. For signal expectations two different ME+PS generators have been used, ALPGEN+HERWIG and SHERPA 1.2.3. Electron trigger, efficiency and smearing corrections, as well as jet energy corrections are applied on $\mathrm{MC}$, as described in Chapter 6. Table 8.1 summarises the events in data and the expectations from $\mathrm{MC}$ for both ALPGEN+HERWIG and SHERPA as signal MC. Only statistical uncertainties are shown.

The main backgrounds for $Z / \gamma^{*}(\rightarrow e e)+$ jets are multi-jets with $2 \%-4 \%$ for $N_{\text {jet }} \geq 0$ to $N_{\text {jet }} \geq 2$, diboson with $6.9 \%$ for $N_{\text {jet }} \geq 3$ and for higher jet multiplicities $t \bar{t}$ with $8.6 \%$. The multi-jet background has been estimated using a data-driven method as explained in Sec. 7.2.2. The other two backgrounds, together with $Z / \gamma^{*}(\rightarrow \tau \tau)+$ jets, $W(\rightarrow e \nu)+$ jets are estimated by $\mathrm{MC}$ and normalized to the integrated luminosity in data using the respective (N)NLO cross sections [14, 72, 73]. 


\begin{tabular}{|c|c|c|c|c|c|}
\hline \hline & $\geq 0$ jets & $\geq 1$ jet & $\geq 2$ jets & $\geq 3$ jets & $\geq 4$ jets \\
\hline \hline$Z / \gamma^{*}(\rightarrow e e)$ & $8925 \pm 7$ & $1357 \pm 3$ & $307 \pm 1$ & $64.4 \pm 0.6$ & $12.7 \pm 0.2$ \\
$($ ALPGEN) & & & & & \\
$Z / \gamma^{*}(\rightarrow e e)$ & $9103 \pm 17$ & $1289 \pm 4$ & $262 \pm 2$ & $52.0 \pm 0.6$ & $10.8 \pm 0.3$ \\
$($ SHERPA $)$ & & & & & \\
$W(\rightarrow e \nu)$ & $14 \pm 1$ & $4.3 \pm 0.3$ & $1.0 \pm 0.2$ & $0.31 \pm 0.09$ & $0.11 \pm 0.06$ \\
$Z / \gamma^{*}(\rightarrow \tau \tau)$ & $5.0 \pm 0.2$ & $0.9 \pm 0.1$ & $0.25 \pm 0.04$ & $0.03 \pm 0.01$ & $0.005 \pm 0.003$ \\
$W W, W Z, Z Z$ & $13.6 \pm 0.2$ & $9.6 \pm 0.1$ & $4.8 \pm 0.1$ & $1.66 \pm 0.05$ & $0.45 \pm 0.03$ \\
$t \bar{t}$ & $12.0 \pm 0.3$ & $11.7 \pm 0.3$ & $9.20 \pm 0.24$ & $4.25 \pm 0.16$ & $1.29 \pm 0.09$ \\
Multi-jets & $197 \pm 4$ & $49 \pm 2$ & $12.6 \pm 0.9$ & $2.22 \pm 0.36$ & $0.7 \pm 0.2$ \\
\hline \hline Total predicted & $9166 \pm 8$ & $1432 \pm 3$ & $334 \pm 2$ & $72.9 \pm 0.7$ & $15.2 \pm 0.3$ \\
$($ ALPGEN $)$ & & & & & \\
Total predicted & $9344 \pm 17$ & $1364 \pm 5$ & $290 \pm 2$ & $60.5 \pm 0.7$ & $13.3 \pm 0.4$ \\
(SHERPA) & & & & & \\
Data observed & 9705 & 1514 & 333 & 62 & 15 \\
\hline \hline
\end{tabular}

Table 8.1.: Number of events expected from MC event sample and observed in data for several inclusive jet multiplicities. Only statistical uncertainties are included. The multi-jet background has been extracted from data, as described in Sec. 7.2 .2

Figure 8.1 shows the uncorrected invariant dielectron mass for $Z$ boson candidates, $m_{e^{+} e^{-}}$, in events with at least one associated jet in the final state, as well as the uncorrected inclusive jet multiplicity.

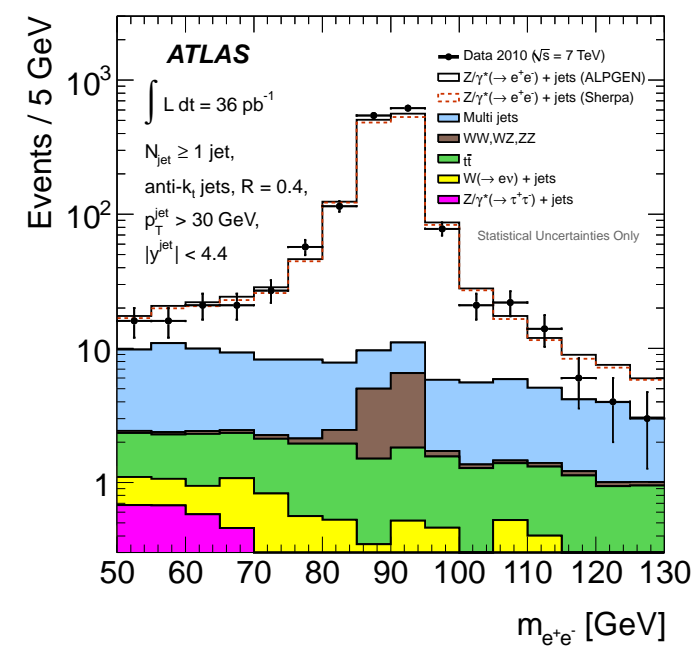

(a) Invariant dielectron mass

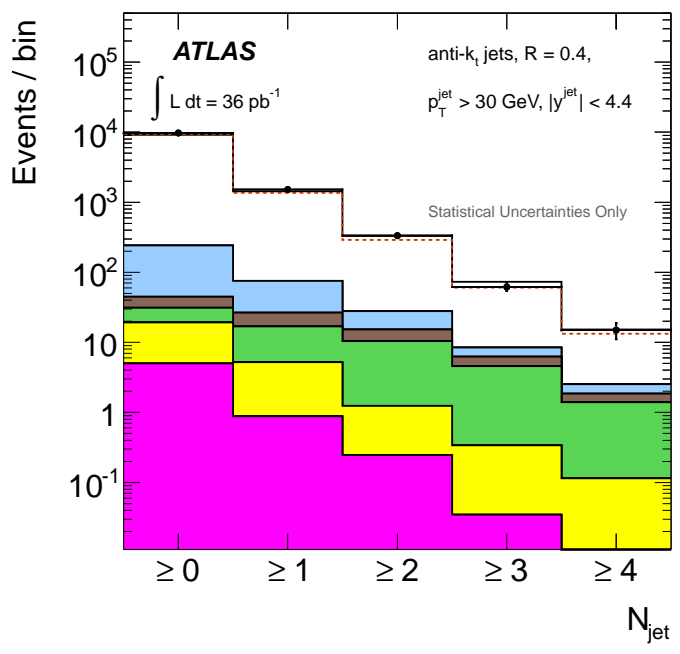

(b) Inclusive jet multiplicity

Figure 8.1.: (a) Dielectron mass for $Z$ candidates with at least one jet in the event and (b) inclusive jet multiplicity in data and simulation. Only statistical uncertainties are shown. The multi-jet background has been estimated from data.

The uncorrected invariant dielectron mass is shown for a wider range than the one which is selected $\left(66 \mathrm{GeV}<m_{e^{+} e^{-}}<116 \mathrm{GeV}\right)$. For both ALPGEN+HERWIG and SHERPA, Data and MC are consistent for the final selection and in the side bands. 
Figure 8.2 shows the uncorrected inclusive jet $p_{T}$ and $y$ distributions in events with at least one jet in the final state.

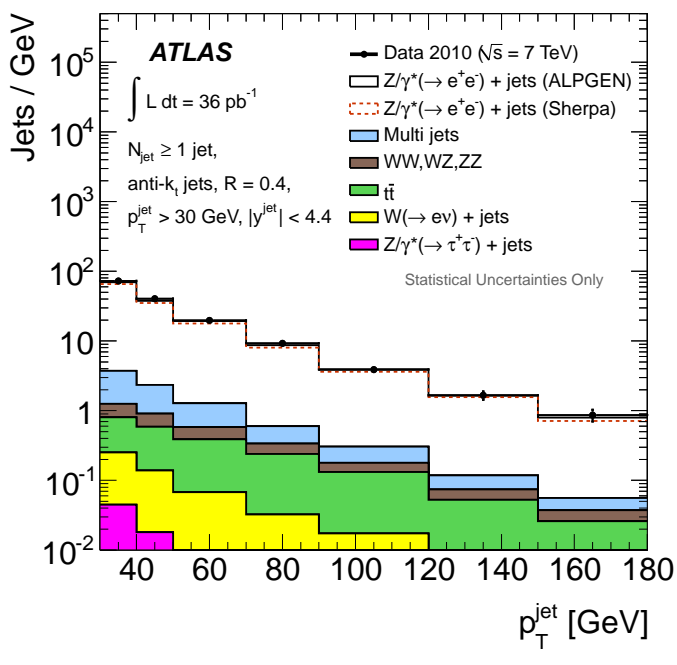

(a) Inclusive jet $p_{T}$

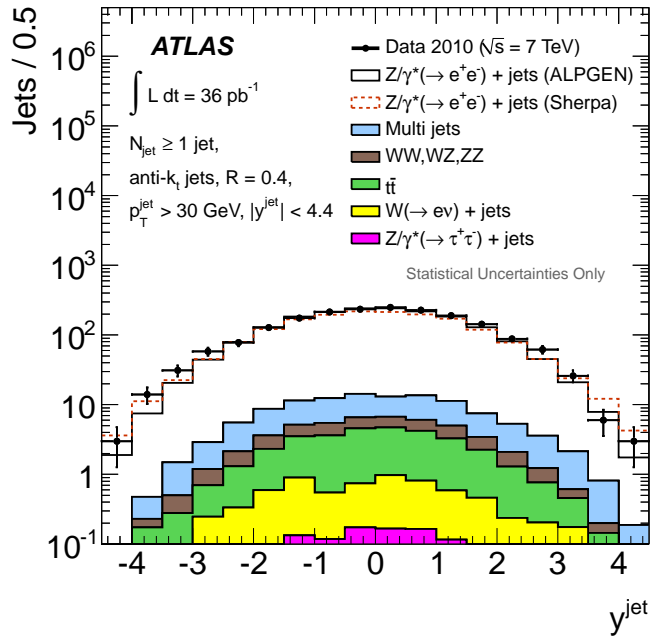

(b) Inclusive jet $y$

Figure 8.2.: (a) Transverse momentum distribution and (b) rapidity distribution of all jets in data and simulation. Only statistical uncertainties are shown. The multi-jet background has been estimated from data.

Further uncorrected distributions can be found in Appendix A.1.

All plots illustrated in this section and in Appendix A.1 are shown only with the statistical uncertainty. In general, the predictions from ALPGEN+HERWIG and SHERPA are consistent with the data. In addition, it has been checked that the migration around the $p_{T}^{\text {jet }}$ threshold at $30 \mathrm{GeV}$ is correctly taken into account by looking at the data-MC comparisons for $p_{T}^{\text {jet }}>20 \mathrm{GeV}$ and $\left|y^{\text {jet }}\right|<4$.4. Figure 8.3 to Fig. 8.4 show the uncorrected inclusive jet multiplicity, jet $p_{T}$ and jet $y$ distribution for jets with $p_{T}^{\text {jet }}>20 \mathrm{GeV}$ and $\left|y^{j e t}\right|<4$ 4.4. Further uncorrected distributions can be found in Appendix A.2. Unfolded cross checks for jets with $p_{T}^{\text {jet }}>20 \mathrm{GeV}$ and $\left|y^{\text {jet }}\right|<4.4$ have been performed in $W+$ jets events [165].

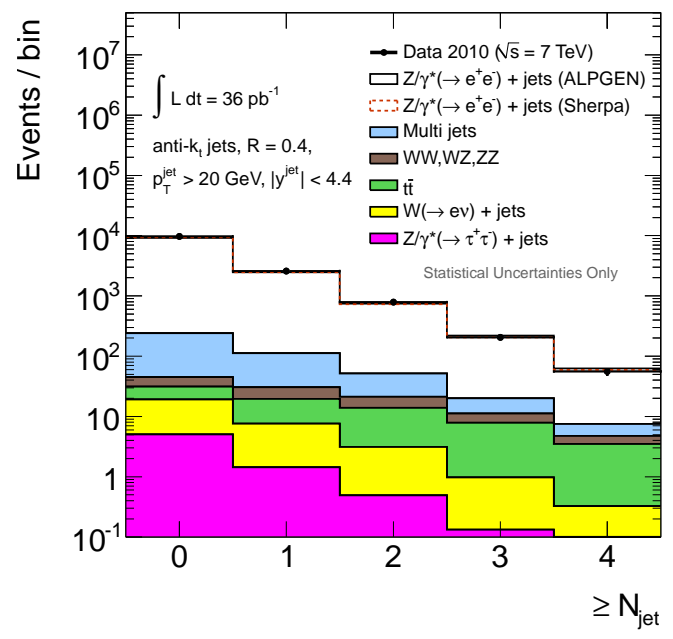

Figure 8.3: Inclusive jet multiplicity in data and simulation. Only statistical uncertainties are shown. Jets are required to have a transverse momentum of $p_{T}^{\text {jet }}>20 \mathrm{GeV}$ and $\left|y^{\text {jet }}\right|<4$.4. The multi-jet background has been estimated from data. 


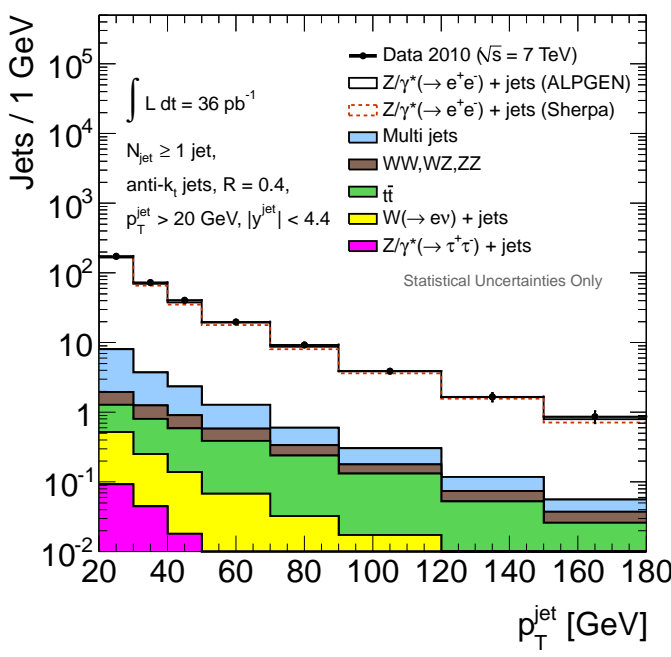

(a) Inclusive jet $p_{T}$

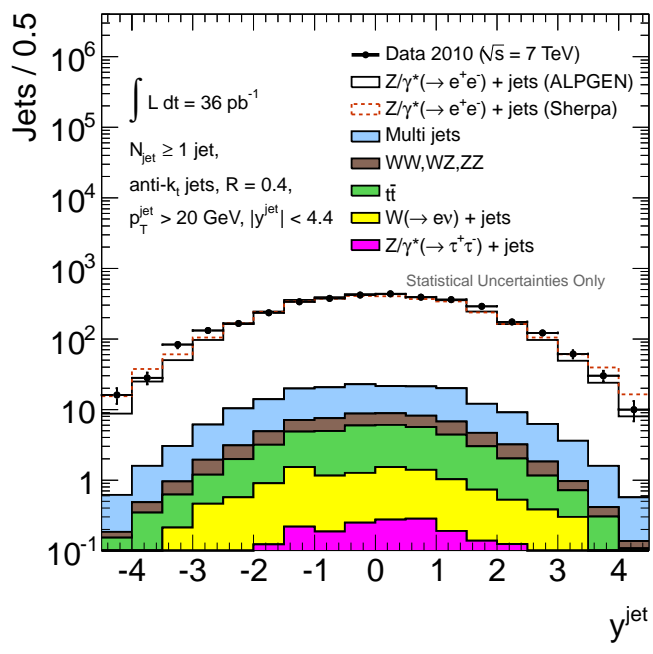

(b) Inclusive jet $y$

Figure 8.4.: (a) Transverse momentum distribution and (b) rapidity distribution of all jets in data and simulation. Only statistical uncertainties are shown. Jets are required to have a transverse momentum of $p_{T}^{\text {jet }}>20 \mathrm{GeV}$ and $\left|y^{\text {jet }}\right|<4$.4. The multi-jet background has been estimated from data.

Both together give confidence that these MC event samples provide a reasonable description of the event kinematics and the detector response, which is necessary since they are used as input sample for the unfolding. A detailed discussion of all distributions is presented in Sec. 8.5 together with the comparison on particle level.

\subsection{Corrections for Detector Effects}

The cross sections are quoted on particle level to facilitate the comparison with pQCD predictions and with measurements from other experiments. Therefore, the measurements are corrected for detector effects back to particle level. This correction accounts for resolution effects, non linearities and efficiencies of the $Z / \gamma^{*}$ boson and jet identification and reconstruction.

The corrected measurements refer to distributions with exactly two dressed electrons and additional jets, which fulfil the phase-space requirements detailed in Chapter 7.

The differential cross sections are defined as a function of a variable $\xi$ in the given fiducial region

$$
\frac{\mathrm{d} \sigma}{\mathrm{d} \xi}=\frac{1}{\mathcal{L}} \frac{1}{\Delta \xi}\left(N_{\text {data }}-N_{b k g}\right) \times U(\xi)
$$

where $N_{d a t a}$ and $N_{b k g}$ describe the number of observed events and the number of expected background events in bin $\xi, \Delta \xi$ describes the bin widths and $U(\xi)$ the corresponding correction factor of bin $\xi$. Finally, $\mathcal{L}$ is the total integrated luminosity.

The correction factor $U(\xi)$ back to particle level is determined separately for each observable using the bin-by-bin method [133] based on MC event samples. Since the bin-by-bin method only includes migrations between bins to the extend to which they are 
modelled in MC, the bin width is chosen such that the purity of each bin is above $70 \%$, which substantially reduces the bin-by-bin migration. In order to further reduce biases from this method, the MC event samples used for the calculation of the correction are corrected for residual differences observed between the predictions and the data.

Since the correction is MC based, systematic uncertainties have to account for the wrong modelling of jet kinematics and jet composition, such as UE tunes, fragmentation model and the heavy flavour fraction within the jets. Thus, the correction factor is determined twice, once with the ALPGEN+HERWIG signal MC event sample as nominal correction and once with the SHERPA signal MC event sample for systematic studies. As an example, Fig. 8.5 depicts the different bin-by-bin correction factors for the inclusive jet multiplicity distribution and the inclusive jet transverse momentum distribution for events with at least one jet in the final state.

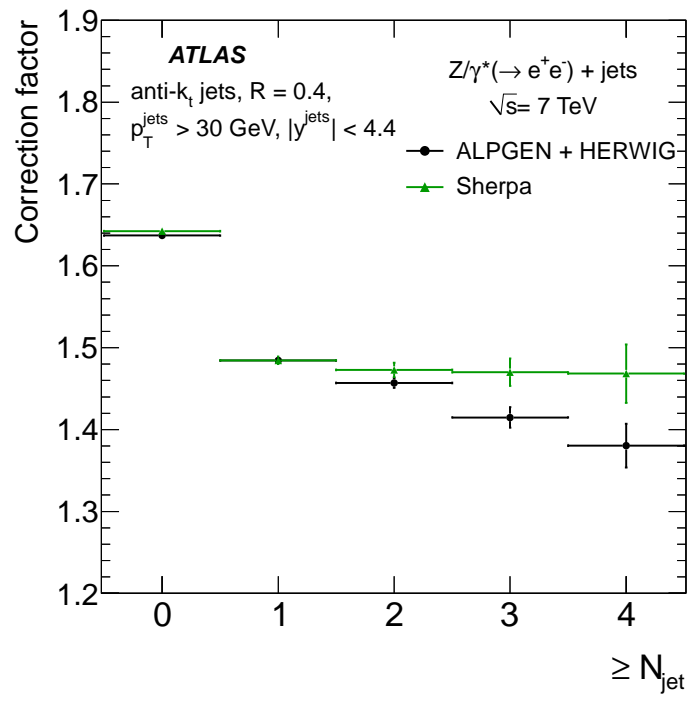

(a) Inclusive jet multiplicity

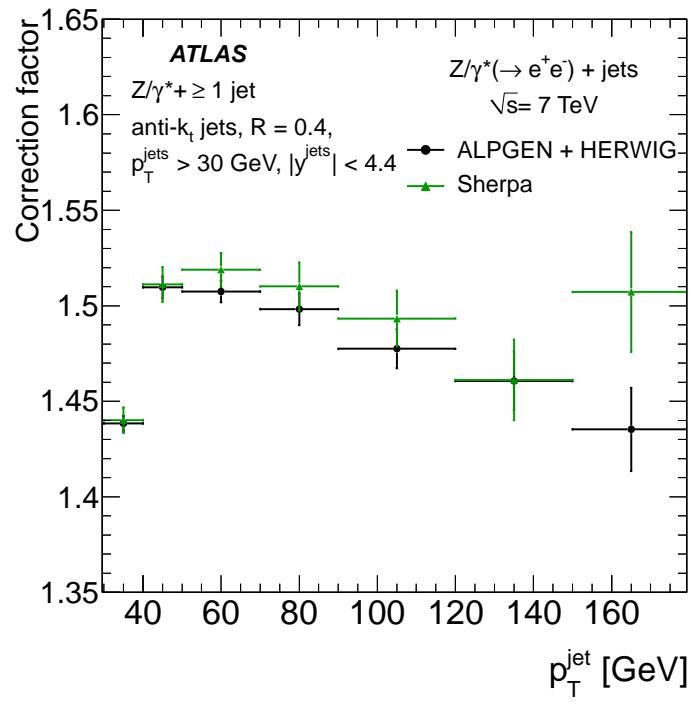

(b) Inclusive jet $p_{T}$

Figure 8.5.: Bin-by-bin correction factors for (a) the inclusive jet multiplicity and (b) the jet transverse momentum distribution for events with at least one jet in the final state. The correction factors are calculated using ALPGEN+HERWIG and SHERPA MC. The error bars indicate the statistical uncertainties.

This results in differences between both corrections of $0.3 \%$ to $6 \%$ on the inclusive jet multiplicity for $N_{j e t} \geq 0$ to $N_{j e t} \geq 4$. Additional systematics are taken from the comparison of the bin-by-bin method with the iterative (Bayes) method, which is described in Sec. 5.1 and implemented in the RooUnfold package [134]. The method starts from an initial prior for the first iteration, further iterations are afterwards based on the Bayesian posterior as prior. Depending on the choice of the initial prior, the optimal number of iterations can vary a lot.

The migration matrix is created by matching each particle level jet to its corresponding jet on detector level within a cone of $\Delta R=0.4$. In addition, each reconstructed event has to fulfil the $Z$ selection on particle level and on detector level. Events which do not fulfil these requirements at particle or detector level, are put in the correction of not-matched reconstructed events or counted as inefficiency, respectively. As an example, the resulting 
correction for not-matched events and the migration matrix for the inclusive jet transverse momentum distribution for events with at least one jet in the final state are shown in Fig. 8.6 and Fig. 8.7. The migration matrix is normalised to the inclusive jet transverse momentum distribution on particle level.

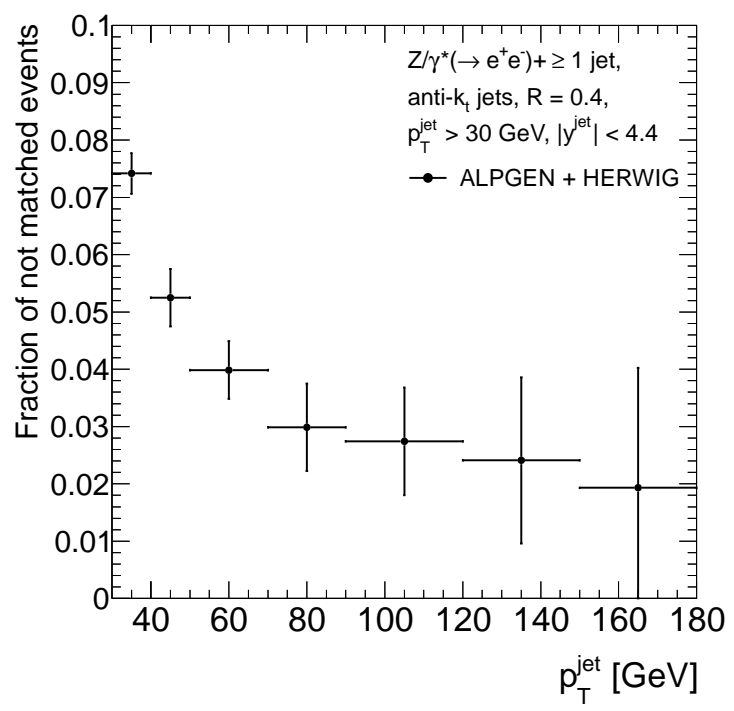

Figure 8.6: Correction for not-matched events for the inclusive jet transverse momentum distribution for events with at least one jet in the final state. The correction has been determined using ALPGEN+HERWIG MC event samples.

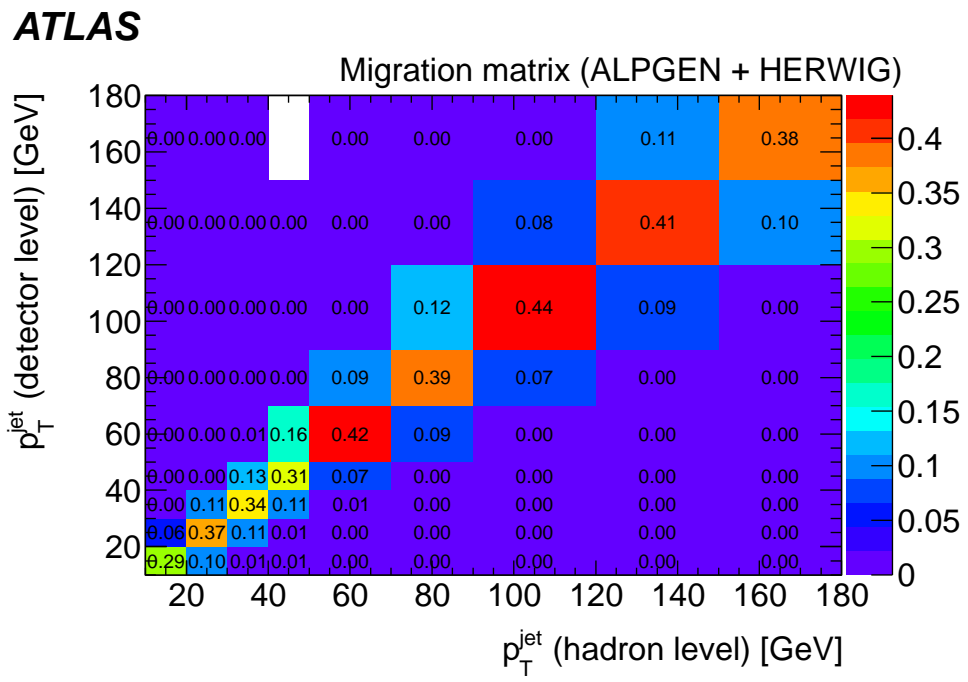

Figure 8.7.: Migration matrix for the inclusive jet transverse momentum distribution for events with at least one jet in the final state. The migration matrix has been determined using ALPGEN+HERWIG MC event samples.

Before unfolding, the background subtracted measurements are corrected for the notmatched events. The number of iterations has been estimated from MC, separately for each observable. Typical numbers are between one and three.

Figure 8.8 shows the comparison between the results obtained with the iterative (Bayes) method and with the bin-by-bin method for the inclusive jet transverse momentum distribution for events with at least one jet in the final state. The ratio between both results has been fitted with a 2 nd grade polynomial and the fit result is taken as a relative systematic uncertainty on the cross section, which is mainly at the percentage level. 


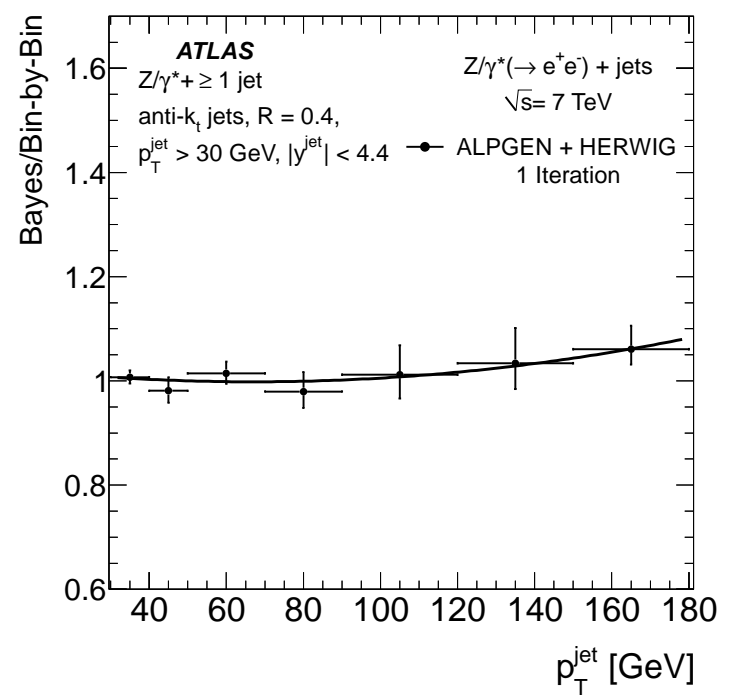

Figure 8.8: Comparison between the results obtained with the iterative (Bayes) method and with the bin-by-bin method for the inclusive jet transverse momentum distribution for events with at least one jet in the final state.

Finally, the MC event samples used to calculate the correction factors have limited statistics. Therefore, an additional uncertainty is propagated on the final cross section to account for this fact.

\subsection{Study of Systematic Uncertainties}

The different sources of systematic uncertainties have been studied in detail for each observable presented in this analysis. The different systematic shifts, corresponding to $1 \sigma$, are applied on the predictions from MC event samples and the data-driven background estimate. Uncertainties related to a given background prediction or estimation are only applied to this specific background contribution. The modified background distributions are then subtracted from the data distribution and corrected for detector effects, using the modified correction factors. The difference between the nominal unfolded result and the modified unfolded distributions has been taken as systematic uncertainty. Finally, the different sources of systematic uncertainties are added in quadrature.

\subsubsection{Electron Selection}

Electron trigger, identification and reconstruction efficiencies have been studied in data and MC event samples using tag and probe methods. Remaining residual differences between the data and the MC predictions are corrected by applying the corresponding scale factors on the MC event samples, as described in Sec. 6.1.3. The individual systematic uncertainties on these scale factors are propagated into the uncertainty on the cross section by increasing or decreasing the scale factors by $\pm 1 \sigma$.

Additional uncertainties are coming from the electron energy scale and resolution, as detailed in Sec. 6.1.3. The systematic uncertainty due to the electron energy scale is propagated into the uncertainty on the cross section in a correlated manner by increasing or decreasing the electron energy simultaneously for both electrons within $\pm 1 \sigma$. The electron energy resolution is not reproduced by the resolution in data. In order to account for this difference the MC is smeared to match the distribution in data. The corresponding 
uncertainty is taken into account by smearing each electron within $\pm 1 \sigma$.

The different source of uncertainties are added in quadrature, resulting in an uncertainty of approximately $4 \%$ independent of the jet multiplicity, the jet $p_{T}$ and the jet $y$. The dominant contributions are coming from the uncertainty of the electron identification.

\subsubsection{Jet Energy Scale}

The different components of the JES uncertainty, as described in Sec. 6.2, are added in quadrature and propagated to the final cross section. This results in an uncertainty of $7 \%$ to $22 \%$ for $N_{\text {jet }} \geq 1$ to $N_{\text {jet }} \geq 4$.

\subsubsection{Jet Energy Resolution}

The JER uncertainty is propagated to the final cross section by over-smearing the jet energy in $\mathrm{MC}$ within $1 \sigma$ using random numbers before performing the unfolding. Given the nature of stochastic processes, the procedure has been repeated six times by modifying the random seed. It has been checked that further repetitions do no yield an improvement. Finally, the resulting uncertainties are symmetrised and the average is taken as a systematic uncertainty. This translates into an uncertainty of $1 \%$ to $2 \%$ on the inclusive jet multiplicity.

\subsubsection{Background Modelling and Normalisation}

The uncertainties on the background predictions are related to normalisation and statistical uncertainties. These two types of uncertainties are treated differently. On the one hand, the normalisation uncertainty is assumed to be correlated between the bins, whereas the statistical uncertainty is assumed to be uncorrelated.

The normalisation uncertainty is propagated to the final cross section by shifting the different background contributions up and down. The dominant contribution comes from the data-driven estimate of the multi-jets background. The uncertainty on the multi-jet background, as described in Sec. 7.2.2, translates into an uncertainty of $0.6 \%$ to $2 \%$ on the inclusive jet multiplicity for $N_{\text {jet }} \geq 1$ to $N_{\text {jet }} \geq 4$. The background predictions from $t \bar{t}$ and EW are varied within their corresponding normalisation uncertainty of $+7 \% /-9.6 \%$ [166] and 5\% [72, 73], respectively. The normalisation uncertainty accounts for PDF uncertainties, uncertainties due to the choice of $\alpha_{s}$ and scale uncertainties. Since the impact from these background contributions is rather small, the resulting uncertainty on the final cross section is less than $1 \%$.

The statistical uncertainties from the background predictions are added in quadrature to the statistical uncertainty in data. They are propagated simultaneously to the final cross section.

\subsubsection{Unfolding}

The systematic uncertainty due to unfolding includes three components, the choice of the MC event sample, the choice of the unfolding method and the uncertainty due to the limited statistics of the MC event sample, as discussed in Sec. 8.2. The different sources 
of systematic uncertainties are added in quadrature separately for each observable and propagated to the final systematic uncertainty on the cross section measurement.

\subsubsection{Total Systematic Uncertainty}

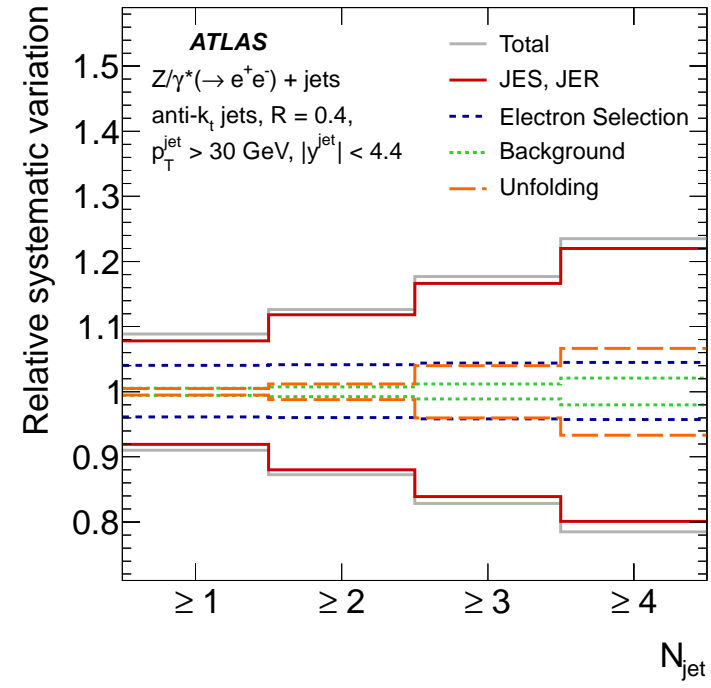

(a) Inclusive jet multiplicity

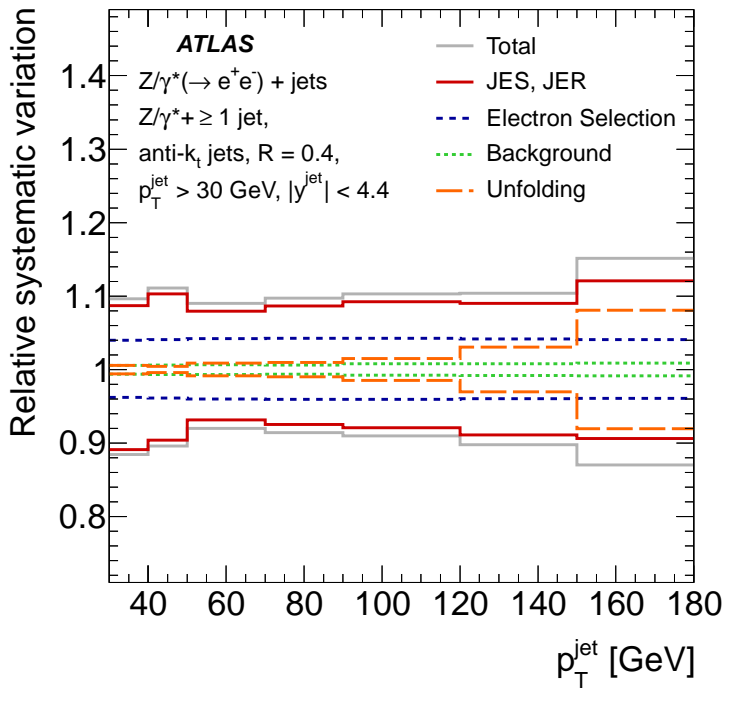

(b) Inclusive jet $p_{T}$

Figure 8.9.: Different contributions to the combined relative systematic uncertainty for the measured cross section as a function of (a) the inclusive jet multiplicity and (b) the inclusive jet transverse momentum distribution with at least one jet in the final state. The total systematic uncertainty is determined by summing up the different contributions in quadrature.

Figure 8.9 exemplarily presents the results of the detailed study of the systematic uncertainties for the inclusive cross section as a function of $N_{\text {jet }}$ and the inclusive jet cross section as a function of the transverse momentum $p_{T}^{\text {jet }}$ in events with at least one jet in the final state. In addition, Tab. 8.2 summarises the different components of the total systematic uncertainty on the measured cross section for several inclusive jet multiplicities. 


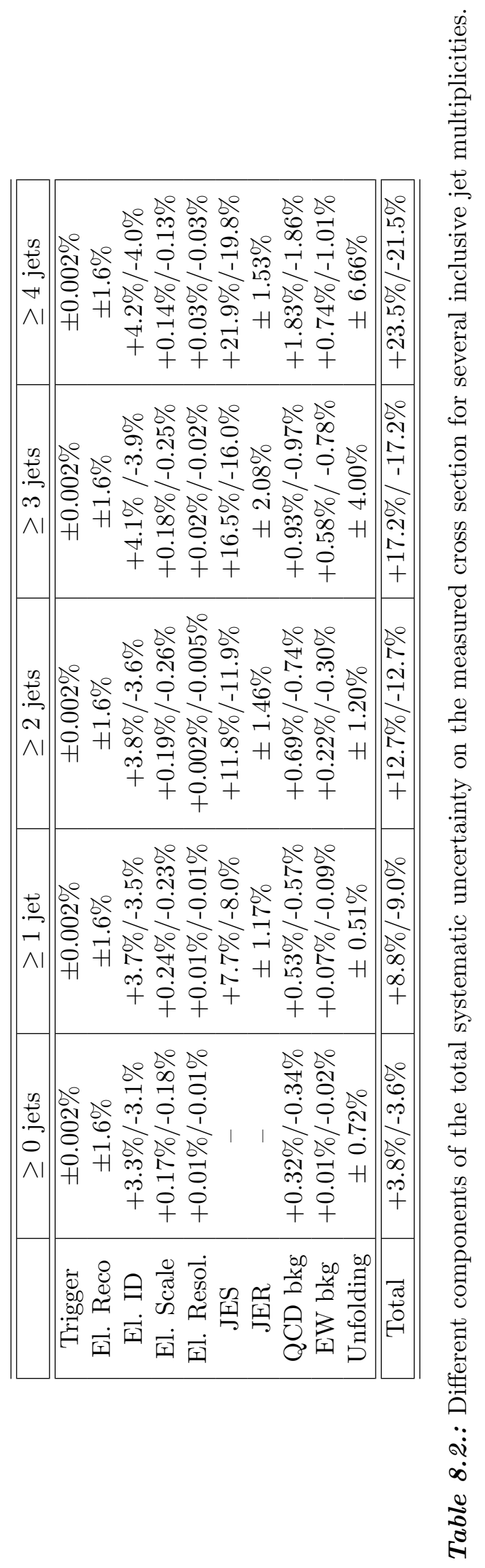


The total systematic uncertainty is determined by summing up the different contributions in quadrature. This results in an uncertainty of $9 \%$ to $24 \%$ on the inclusive jet multiplicity for $N_{\text {jet }} \geq 1$ to $N_{\text {jet }} \geq 4$ and of $10 \%$ to $13 \%$ on the inclusive jet transverse momentum for $30 \mathrm{GeV}<p_{T}^{\text {jet }}<180 \mathrm{GeV}$. The dominant uncertainty contribution for the inclusive $Z / \gamma^{*}(\rightarrow e e)$ selection comes from the uncertainty on the electron identification, whereas the measurement of the production cross section of $Z / \gamma^{*}(\rightarrow e e)$ associated with jets is dominated by the uncertainty on the JER and JES.

Finally, the total systematic uncertainty is added in quadrature to the statistical uncertainty and to the uncertainty on the total integrated luminosity of $3.4 \%$ [101, 157].

\subsection{Theory Corrections}

For the extraction from parton level to particle level, the NLO fixed-order pQCD predictions are corrected for QED FSR and non-perturbative effects, such as UE and fragmentation. The UE correction also accounts for events originating from DPI. Both corrections are determined using MC event samples. The corresponding uncertainties are added in quadrature to the other theoretical uncertainties. A detailed description of both corrections can be found in the following two subsections.

\subsubsection{Corrections for Non-perturbative Effects}

The nominal correction is determined using HERWIG+JIMMY MC event samples with the HERWIG cluster fragmentation and the AUET1-CTEQ6L1 tune. The correction is performed on a bin-by-bin basis, therefore it has to be calculated for each desired variable separately. In order to obtain the correction, two different MC event samples have been generated: one using the standard tune and one with both fragmentation and UE switched off. The bin-by-bin ratio of the final correction is built between the nominal distribution and the distribution obtained from the MC event sample with fragmentation and UE switched off separately for each observable.

Figure 8.10 illustrates the different components combining to the final correction for the transverse momentum distribution of the jets in events with at least one jet in the final state. The ratios presented in Fig. 8.10(a) and Fig. 8.10(b) represent only approximations of the different components since the correction does not factorise. The first ratio has been built between the nominal distribution and the distribution without fragmentation. The second ratio has been built between the nominal distribution and the distribution without UE.

Fragmentation leads to an energy loss in the jet reconstruction due to increased outof-cone showering, which results in a decreased number of jets passing the $p_{T}$ threshold. In contrast, UE increases the average jet energy, resulting in an increased number of jets passing the $p_{T}$ threshold. The influence from both effects is larger at low $p_{T}$, since low energetic jets are wider and the fraction of jet energy provided by the UE is larger. The combination of both effects leads to a nominal correction factor of 0.99 for the inclusive jet multiplicity and transverse momentum distribution, which shows a negligible dependence on $N_{\text {jet }}$ and $p_{T}^{\text {jet }}$.

Systematic uncertainties on this correction are obtained from the comparison with PYTHIA MC event samples using the Lund string model [65] and different variations of 


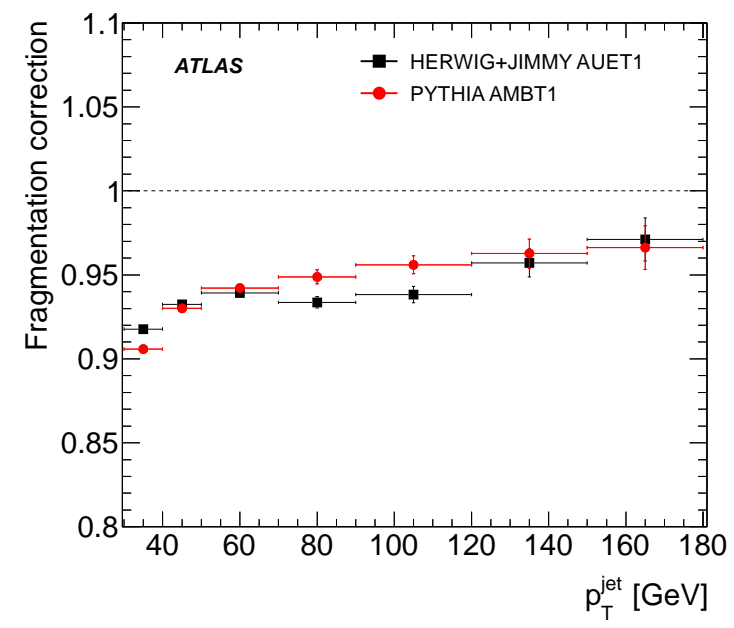

(a) Fragmentation correction

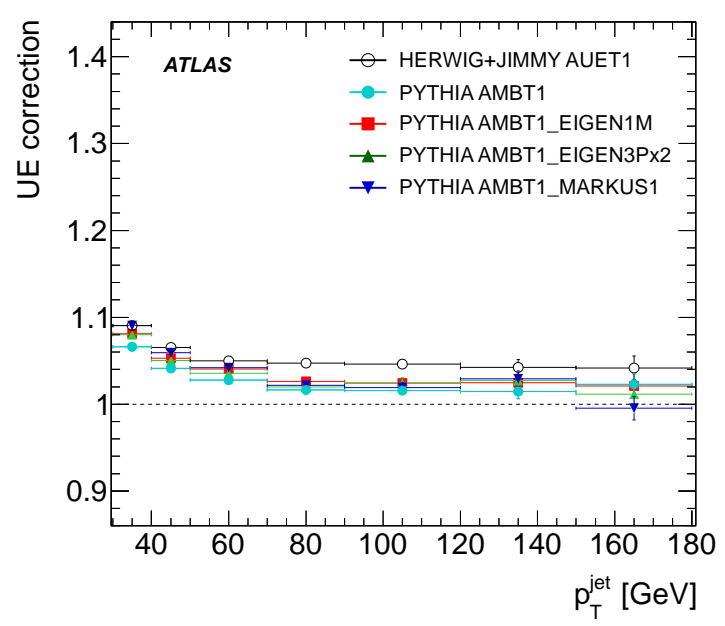

(b) UE correction

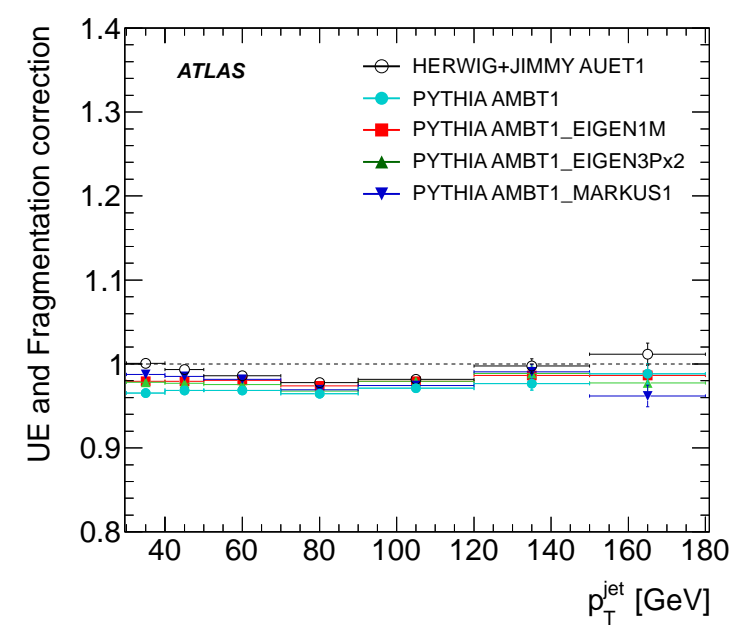

(c) Combined correction

Figure 8.10.: (a) Fragmentation correction, (b) UE correction and (c) combined correction for $p_{T}^{j e t}$ in events with at least one jet in the final state.

the AMBT1 UE tunes [163. The maximum deviation from the different tunes to the reference tune is taken as systematic uncertainty, which varies between $2 \%$ and $5 \%$.

\subsubsection{Corrections for QED Final State Radiation}

Lepton kinematics on particle level in the MC event samples are defined to include the contributions from photon radiation within a cone of 0.1 around the lepton direction (dressed leptons), whereas the predictions from NLO fixed-order calculations only include initial state leptons ${ }^{1}$ (Born level). Therefore, MC event samples are used to obtain correction factors for QED FSR on a bin-by-bin basis for each desired variable. The nominal correction is derived from ALPGEN+HERWIG MC event samples with and without photon radiation in the final state. For photon radiation ALPGEN is interfaced to PHOTOS,

\footnotetext{
${ }^{1}$ The term initial state leptons refers to leptons prior to radiation.
} 
as described in Sec. 4.3. The correction is approximately 0.98 and is nearly independent of the $N_{\text {jet }}$ and $p_{T}^{\text {jet }}$ distributions. Figure 8.11 shows the resulting QED FSR corrections for the $p_{T}^{\text {jet }}$ distribution. In addition, the correction from Born level to the level of bare final-state leptons is shown, which demonstrates that the dressing of leptons with photons brings the final state leptons closer to the initial state leptons.

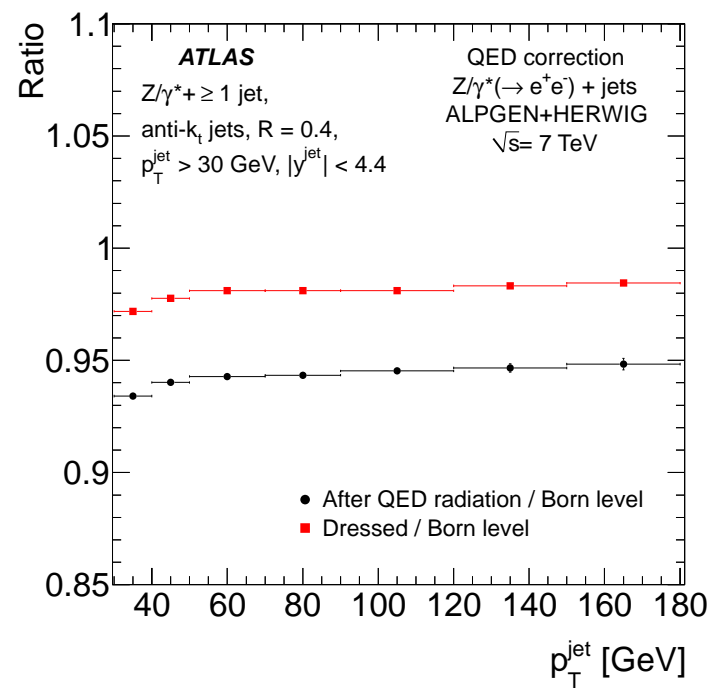

Figure 8.11: QED FSR correction for $p_{T}^{\text {jet }}$ in events with at least one jet in the final state.

For systematic studies, the corrections obtained with ALPGEN+HERWIG MC event samples are compared to the corrections derived with SHERPA MC event samples, which includes a different approach for modelling QED FSR, as described in Sec. 4.4. The differences between the corrections obtained with ALPGEN+HERWIG and SHERPA are at the per-mille level.

\subsection{Results}

This section presents the final results of the measurement with the full dataset of 2010 . The measured distributions depicted in Sec. 8.1 are now unfolded to particle level taking into account the systematic uncertainties discussed in Sec. 8.3. Predictions from NLO fixed-order pQCD predictions, corrected for non-perturbative effects and QED radiation effects, as described in Sec. 8.4, as well as predictions from ALPGEN+HERWIG, SHERPA and PYTHIA using the ATLAS configurations, as detailed in Sec. 7.2, are compared to the measured cross sections. ALPGEN+HERWIG and SHERPA include LO matrix elements with up to five partons, while higher jet multiplicities are modelled by parton shower, as detailed in Sec. 7.2.1. In contrast, PYTHIA only includes the tree-level matrix element for $Z / \gamma^{*}+\geq 1$ jet, additional jet emission is modelled by parton shower. Inclusive and differential cross sections for $Z / \gamma^{*}(\rightarrow e e)+\geq \mathrm{n}$ jets are compared to the corresponding NLO fixed-order pQCD calculation for $Z+\geq n$ jets from BLACKHAT+SHERPA.

Except for the cross sections as a function of the jet multiplicity, the results presented in this chapter are divided by their respective inclusive $Z / \gamma^{*}(\rightarrow e e)$ cross section. This has the advantage of cancelling part of the systematic uncertainties, related to the luminosity estimate and the electron reconstruction and identification.

The figures in this section are organized such that they show the absolute or normalised cross sections in the upper pad, together with the ratios data/BLACKHAT+SHERPA, 
data/ALPGEN and data/SHERPA in the lower three pads. The predictions from ALPGEN+HERWIG and SHERPA have been normalised to the inclusive NNLO cross section with global $K$-factors, whereas PYTHIA has been normalised to the inclusive one jet cross section in data, which brings PYTHIA close to the data. Theoretical uncertainties are shown separately from the total combined statistical and systematic uncertainty on the measurement. The hatched uncertainty bands in the figures correspond to the total systematic and statistical uncertainty on the measurement, added in quadrature, whereas the shaded bands represent the theoretical uncertainties on the respective predictions.

\subsubsection{Inclusive Jet Multiplicity and Jet Multiplicity Ratio}

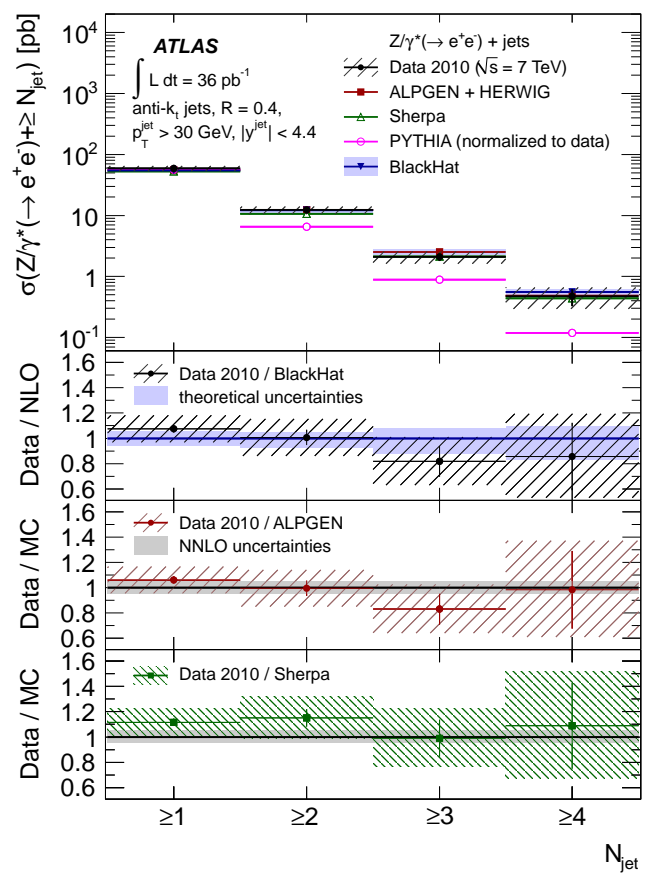

(a) Inclusive jet multiplicity

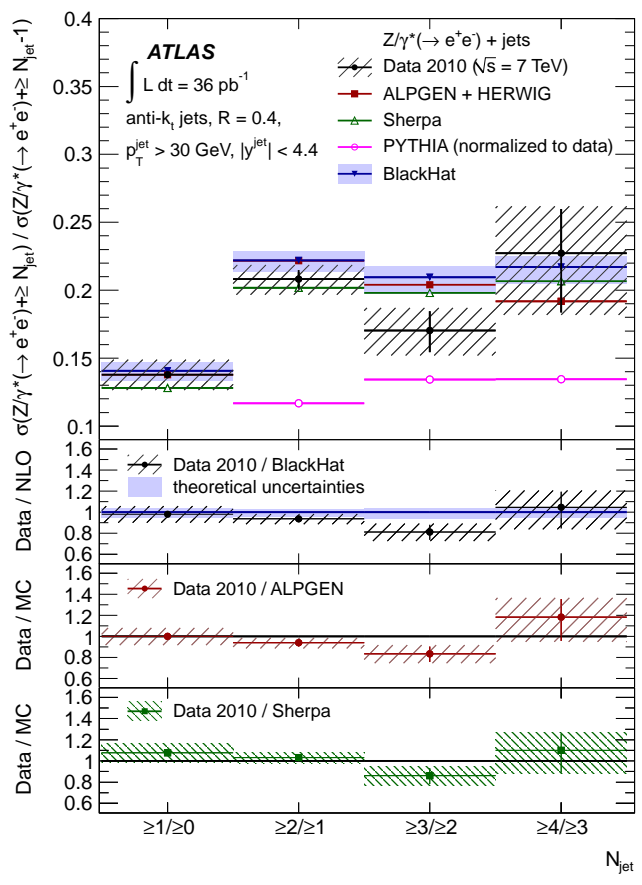

(b) Inclusive jet multiplicity ratio

Figure 8.12.: (a) Measured cross section as a function of the inclusive jet multiplicity and (b) the ratio of cross sections for successive inclusive jet multiplicities. The measured values are represented by the black dots, the error bars indicate the statistical uncertainty and the hatched band incorporates the total statistical and systematic uncertainty added in quadrature. The measurements are compared to predictions from ALPGEN+HERWIG, SHERPA and PYTHIA event generators, as well as to NLO pQCD predictions from BLACKHAT+SHERPA. The shaded bands represent the theoretical uncertainties.

Figure 8.12(a) shows the inclusive cross section for up to four jets. The predictions from ALPGEN + HERWIG and SHERPA include a $5 \%$ uncertainty from the normalisation to the NNLO pQCD inclusive DY cross section, as detailed in Sec. 7.2.1, and the theoretical uncertainties on the prediction from BLACKHAT+SHERPA are described in Sec. 7.3. The prediction from BLACKHAT+SHERPA uses for each inclusive jet multiplicity bin, the respective NLO fixed-order calculation for $Z / \gamma^{*}+\geq n$ jets. As expected the measured cross section decreases with increasing jet multiplicity. The predictions from 
ALPGEN+HERWIG and SHERPA, as well as the NLO fixed-order pQCD prediction from BLACKHAT+SHERPA show a good description of the measured distribution in data, whereas PYTHIA underestimates the measured cross section for larger jet multiplicities in spite of the additional normalisation. This can be attributed to the fact that PYTHIA only includes the tree-level matrix element for $Z / \gamma^{*}+\geq 1$ jet, additional jet emission are done by parton shower. Detailed values of the measured cross section with respect to the fiducial region, as well as NLO pQCD predictions from BLACKHAT+SHERPA are listed in Tab. 8.3.

\begin{tabular}{|c|c|c|}
\hline \hline$N_{\text {jet }}$ & Data Cross Section $(\mathrm{pb})$ & NLO Cross Section $(\mathrm{pb})$ \\
\hline \hline$\geq 0$ jets & $[4.277 \pm 0.045 \text { (stat) })_{-0.153}^{+0.159}$ (syst) $)_{-0.141}^{+0.151}$ (lumi) $] \times 10^{2}$ & $3.903_{-0.164}^{+0.170} \times 10^{2}$ \\
$\geq 1$ jets & $[5.896 \pm 0.160 \text { (stat) })_{-0.529}^{+0.522}$ (syst) $)_{-0.197}^{+0.211}$ (lumi) $] \times 10^{1}$ & $5.489_{-0.312}^{+0.318} \times 10^{1}$ \\
$\geq 2$ jets & $[1.227 \pm 0.074 \text { (stat) })_{-0.156}^{+0.155}$ (syst) $)_{-0.042}^{+0.045}$ (lumi) $] \times 10^{1}$ & $1.219_{-0.086}^{+0.060} \times 10^{1}$ \\
$\geq 3$ jets & $[2.091 \pm 0.308 \text { (stat) })_{-0.358}^{+0.370}$ (syst) $)_{-0.077}^{+0.082}$ (lumi) $] \times 10^{0}$ & $2.554_{-0.313}^{+0.202} \times 10^{0}$ \\
$\geq 4$ jets & $[4.751 \pm 1.479 \text { (stat) })_{-1.023}^{+1.117}$ (syst) $)_{-0.179}^{+0.192}$ (lumi) $] \times 10^{-1}$ & $5.545_{-0.912}^{+0.552} \times 10^{-1}$ \\
\hline \hline
\end{tabular}

Table 8.3.: Cross sections as a function of the inclusive jet multiplicity with respect to the fiducial region measured in data and predicted from NLO fixed-order pQCD calculations from BLACKHAT+SHERPA.

The ratios $\sigma_{N_{\text {jet }}+1} / \sigma_{N_{\text {jet }}}$ of cross sections for two successive jet multiplicities are shown in Fig. 8.12(b), This observable has a higher experimental precision than the inclusive jet multiplicity distribution, due to the fact that part of the systematics related to the luminosity estimate, the electron reconstruction and identification, the JES and JER cancel. It provides an improved test of the SM, since it is sensitive to the value of the strong coupling, the implementation of higher order matrix elements and soft gluon contributions in the theoretical predictions. The measurement in data indicates that the cross section decreases by a factor of five for increasing jet multiplicity. The predictions from ALPGEN+HERWIG and SHERPA, as well as the NLO fixed-order pQCD prediction from BLACKHAT+SHERPA show a good description of the measured ratio. PYTHIA fails to describe the ratio. Detailed values of the cross section ratio, as well as NLO pQCD predictions from BLACKHAT+SHERPA with respect to the fiducial region are listed in Tab. 8.4,

\begin{tabular}{|c|c|c|}
\hline \hline$N_{\text {jet }}+1 / N_{\text {jet }}$ & Data Cross Section Ratio & NLO Cross Section Ratio \\
\hline \hline$\geq 1$ jet $/ \geq 0$ jets & $0.138 \pm 0.002$ (stat) ${ }_{-0.011}^{+0.011}$ (syst) & $0.140_{-0.007}^{+0.006}$ \\
$\geq 2$ jets $/ \geq 1$ jets & $0.208 \pm 0.007$ (stat) ${ }_{-0.009}^{+0.008}$ (syst) & $0.222_{-0.009}^{+0.006}$ \\
$\geq 3$ jets $/ \geq 2$ jets & $0.170 \pm 0.016$ (stat) ${ }_{-0.009}^{+0.009}$ (syst) & $0.209_{-0.012}^{+0.008}$ \\
$\geq 4$ jets $/ \geq 3$ jets & $0.227 \pm 0.044$ (stat) ${ }_{-0.012}^{+0.012}$ (syst) & $0.217_{-0.012}^{+0.008}$ \\
\hline \hline
\end{tabular}

Table 8.4.: Ratio of cross sections for successive inclusive jet multiplicities $\sigma\left(Z / \gamma^{*}+\geq N+1\right.$ jets $) / \sigma\left(Z / \gamma^{*}+\geq N\right.$ jets $)$ measured with respect to the fiducial region measured in data and predicted from NLO fixed-order pQCD calculations from BLACKHAT+SHERPA. 


\subsubsection{Transverse Momentum Distributions}

The differential cross section as a function of the transverse momentum $p_{\mathrm{T}}^{\text {jet }}$ in the range $30 \mathrm{GeV}<p_{\mathrm{T}}^{\text {jet }}<180 \mathrm{GeV}$ for events with at least one jet in the final state is depicted in Fig. 8.13(a), The cross sections are normalised to the inclusive $Z / \gamma^{*}(\rightarrow e e)$ cross section, which clearly reduces the relative uncertainty in comparison to the absolute cross sections. For the prediction from BLACKHAT+SHERPA, the NLO fixed order pQCD calculation for $Z+1$ parton is used. As expected, the measured cross section decreases with increasing transverse momentum. The predictions from ALPGEN+HERWIG, SHERPA and the NLO fixed-order pQCD prediction from BLACKHAT+SHERPA are consistent with the data, whereas PYTHIA shows a too soft transverse momentum spectrum. Similar conclusions can be drawn from the differential cross section as a function of the transverse momentum of the leading jet for events with at least one jet in the final state, which is shown in Fig. 8.13(b),

Figure 8.13(c) shows the differential cross section as a function of the transverse momentum of the 2nd leading jet in the range $30 \mathrm{GeV}<p_{\mathrm{T}}^{\text {jet }}<120 \mathrm{GeV}$ for events with at least two jets in the final state. The cross section is also normalised to the inclusive $Z / \gamma^{*}(\rightarrow e e)$ cross section and decreases with increasing transverse momentum. The measurements are again well described by the predictions from ALPGEN+HERWIG, SHERPA and the NLO fixed-order pQCD predictions from BLACKHAT+SHERPA. PYTHIA fails to describe the data. This can be attributed to the fact that the emission of a second jet in PYTHIA is modelled by parton shower.

Detailed values of the measured cross sections, as well as NLO pQCD predictions from BLACKHAT+SHERPA with respect to the fiducial region are listed in Appendix A.3. For low $p_{\mathrm{T}}^{\text {jet }}$ the measurements are limited by systematics, mainly JES and JER, whereas as for large $p_{\mathrm{T}}^{\text {jet }}$ the measurements are still limited by statistics. 


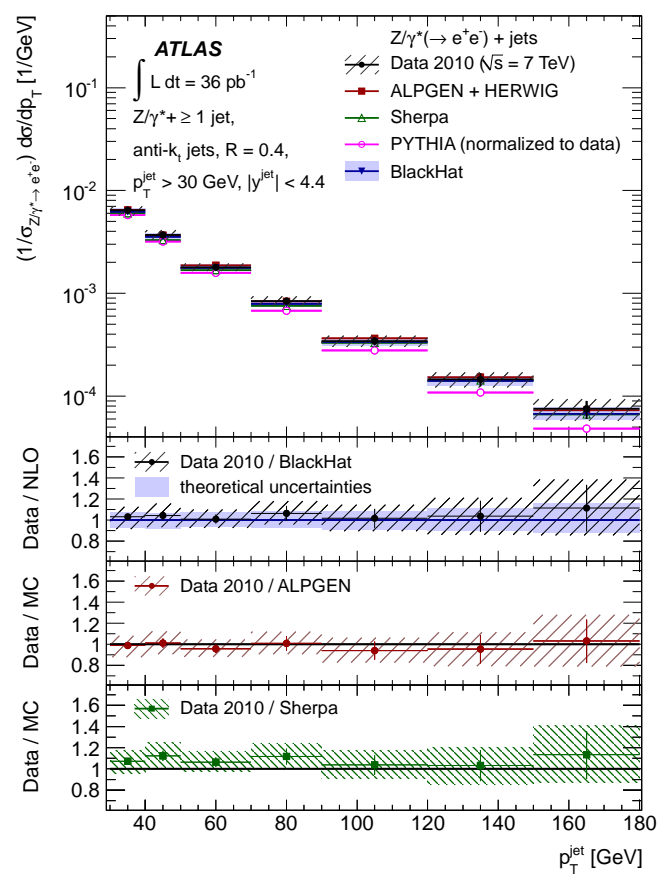

(a) Inclusive jet $p_{T}$

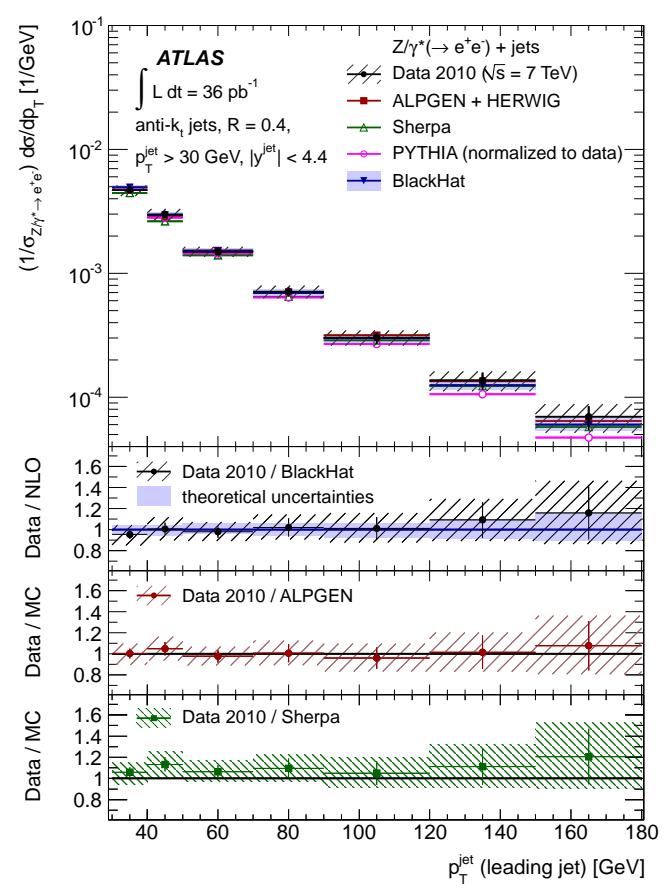

(b) Leading jet $p_{T}$

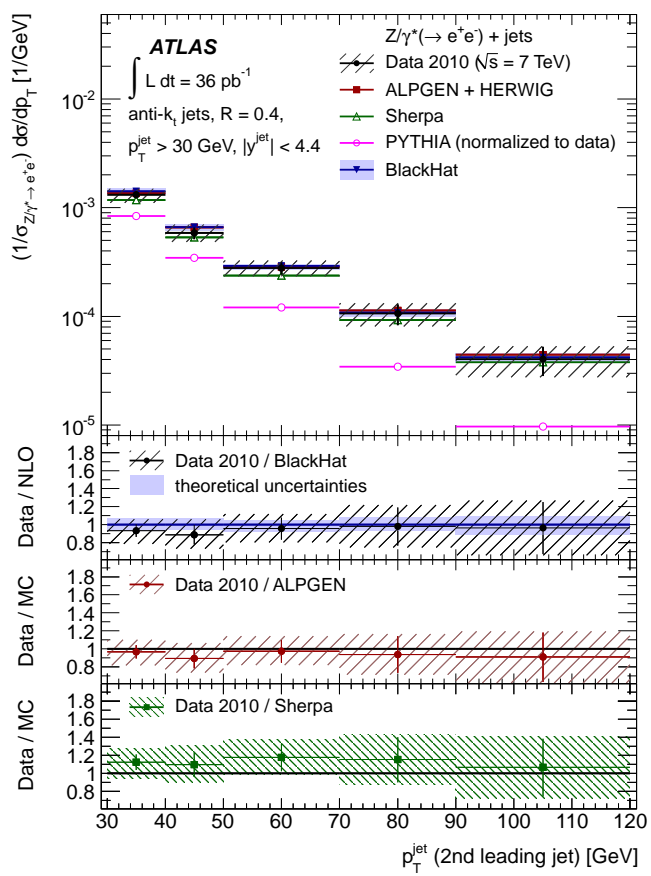

(c) 2nd leading jet $p_{T}$

Figure 8.13.: Differential cross section as a function of (a) the transverse momentum $p_{\mathrm{T}}^{\text {jet }}$ for events with at least one jet in the final state, (b) $p_{\mathrm{T}}^{\text {jet }}$ of the leading jet for events with at least one jet in the final state and (c) $p_{\mathrm{T}}^{\text {jet }}$ of the 2 nd leading jet for events with at least two jets in the final state. The cross sections are normalised to the inclusive $Z / \gamma^{*}(\rightarrow e e)$ cross section. The other details are as in Fig. 8.12 . 


\subsubsection{Rapidity Distributions}

The normalised differential cross sections as a function of the absolute rapidity $\left|y^{\text {jet }}\right|$ and as a function of $\left|y^{j e t}\right|$ of the leading jet for events with at least one jet with $p_{\mathrm{T}}^{\text {jet }}>30 \mathrm{GeV}$ in the final state are depicted in Fig. 8.14(a) and in Fig. 8.14(b). The measured cross sections decrease with increasing $\left|y^{\text {jet }}\right|$. The predictions from ALPGEN+HERWIG, SHERPA and the NLO fixed-order pQCD calculations from BLACKHAT+SHERPA are consistent with the data. Both measurements are limited by systematics over the whole rapidity range, the dominant contribution comes from JES, especially in the forward region. PYTHIA also shows a good description of the shape in the region up to $\left|y^{j e t}\right|=2.5$, but underestimates the measured cross section in the forward region.

Figure $8.14(\mathrm{c})$ shows the normalised differential cross section as a function of $\left|y^{\text {jet }}\right|$ of the 2nd leading jet for events with at least two jets in the final state. The cross section also decreases with increasing $\left|y^{j e t}\right|$. The predictions from ALPGEN+HERWIG, SHERPA and the NLO fixed-order pQCD calculations from BLACKHAT+SHERPA are consistent with the data, whereas PYTHIA is characterised by a general offset, which is consistent with the offset observed for the inclusive jet multiplicity depicted in Fig. 8.12(a).

Detailed values of the measured cross sections, as well as NLO pQCD predictions from BLACKHAT+SHERPA with respect to the fiducial region are listed in Appendix A.3. 


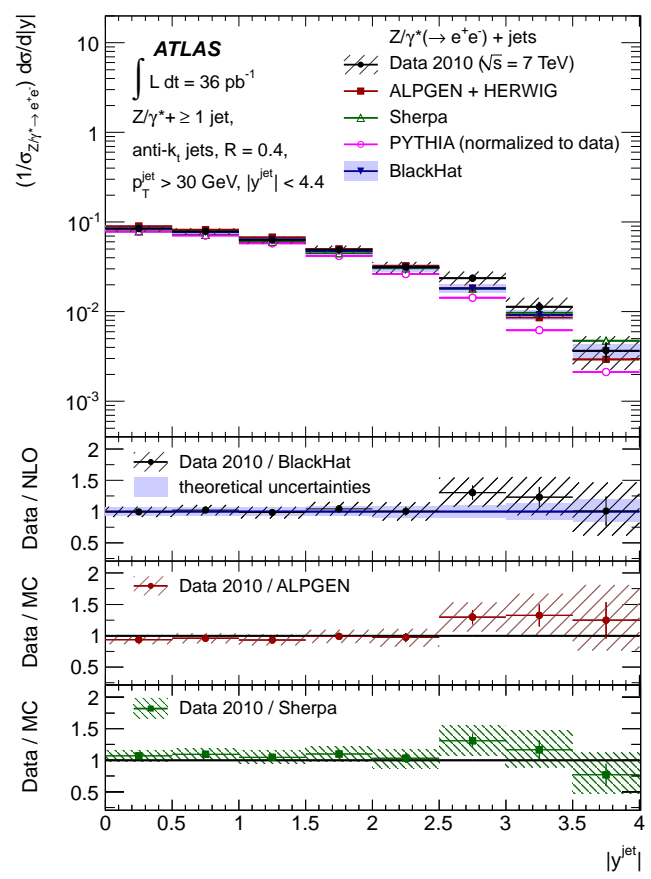

(a) Inclusive jet $y$

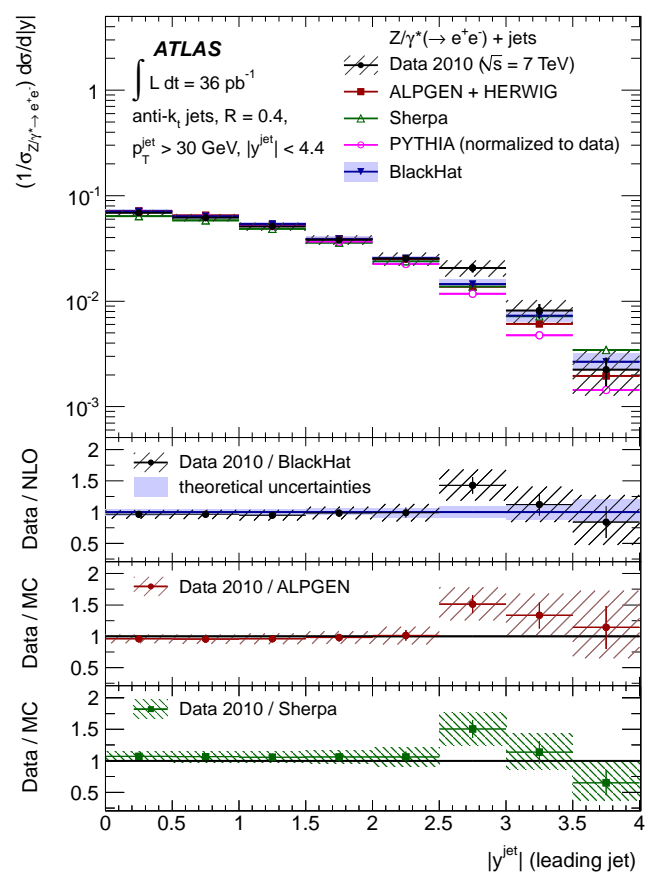

(b) Leading jet $y$

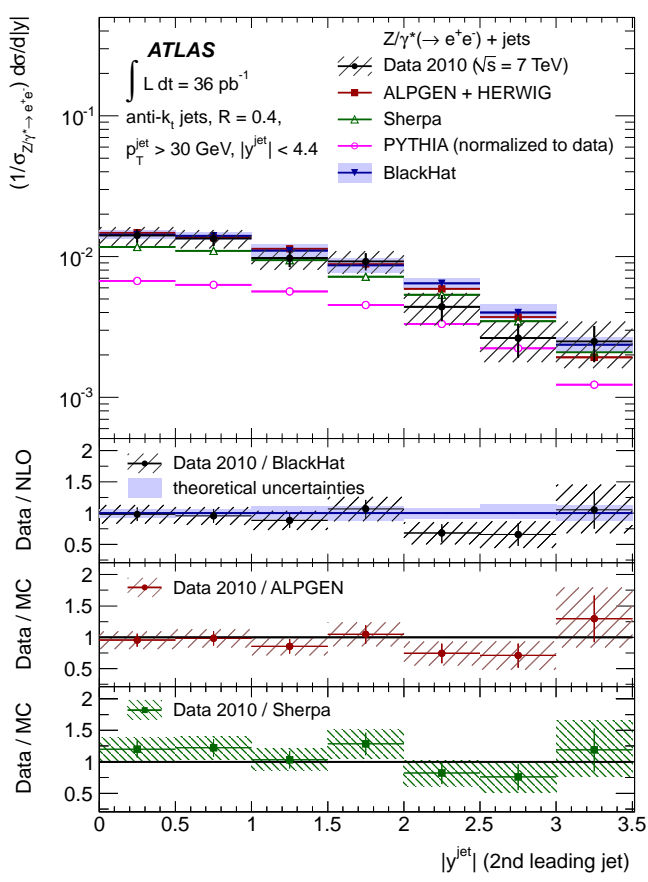

(c) 2nd leading jet $y$

Figure 8.14.: Differential cross section as a function of (a) the absolute rapidity $\left|y^{\text {jet }}\right|$ for events with at least one jet in the final state, (b) $\left|y^{j e t}\right|$ of the leading jet for events with at least one jet in the final state and (c) $p_{\mathrm{T}}^{\text {jet }}$ of the 2 nd leading jet for events with at least two jets in the final state. The cross sections are normalised to the inclusive $Z / \gamma^{*}(\rightarrow e e)$ cross section. The other details are as in Fig. 8.12 . 


\subsubsection{Invariant Dijet Mass}

The normalised differential cross section as a function of the invariant mass $m^{j j}$ of the two leading jets for events with at least two jets in the final state in the range $60 \mathrm{GeV}<m^{j j}<300 \mathrm{GeV}$ is shown in Fig. 8.15. The correct modelling of this distribution is essential, since it is often used to separate signal from background in searches for new physics or studies of the Higgs boson.

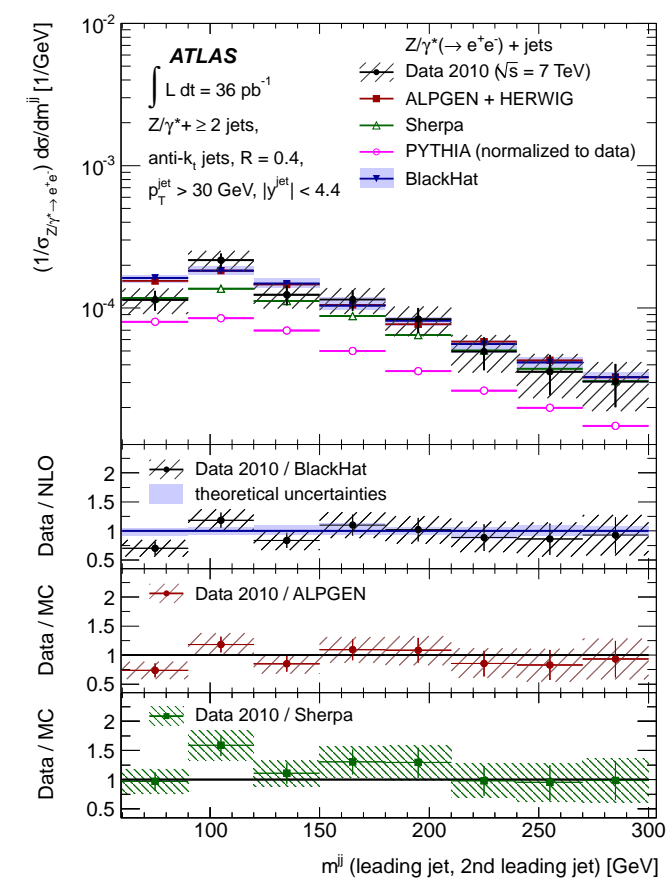

Figure 8.15: Differential cross section as a function of the invariant mass $m^{j j}$ of the two leading jets for events with at least two jets in the final state. The cross sections are normalised to the inclusive $Z / \gamma^{*}(\rightarrow e e)$ cross section. The other details are as in Fig. 8.12 .

For $m^{j j}>100 \mathrm{GeV}$ the measured cross section decreases with increasing $m^{j j}$. The predictions from ALPGEN+HERWIG, SHERPA and the NLO fixed-order pQCD calculations from BLACKHAT+SHERPA are consistent with the data, while PYTHIA only reproduces the shape, but underestimates the cross section.

Detailed values of the measured cross sections, as well as NLO pQCD predictions from BLACKHAT+SHERPA with respect to the fiducial region are listed in Appendix A.3.

\subsubsection{Angular Separation between the Leading Jets}

The normalised differential dijet cross sections as a function of the spatial separation of the two leading jets for events with at least two jets are shown in Fig 8.16.

The measurements are well described by the predictions from ALPGEN+HERWIG, SHERPA and the NLO fixed-order pQCD predictions from BLACKHAT+SHERPA, while PYTHIA underestimates the measured cross section for $\left|\Delta y^{j j}\right|$, large $\left|\Delta \phi^{j j}\right|$ and large $\Delta R^{j j}$.

Detailed values of the measured cross sections, as well as NLO pQCD predictions from BLACKHAT+SHERPA with respect to the fiducial region are listed in Appendix A.3. 


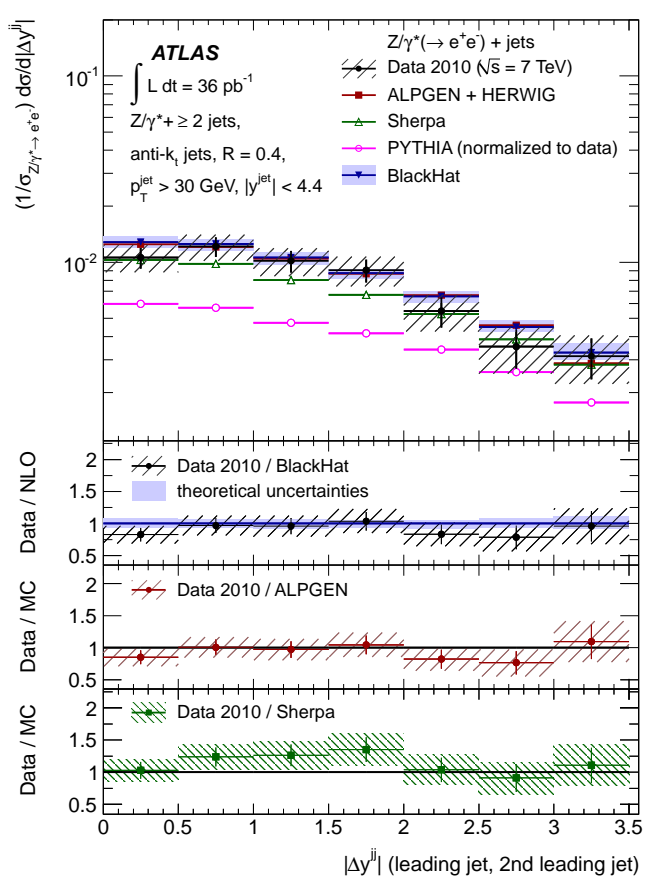

(a) Absolute rapidity difference

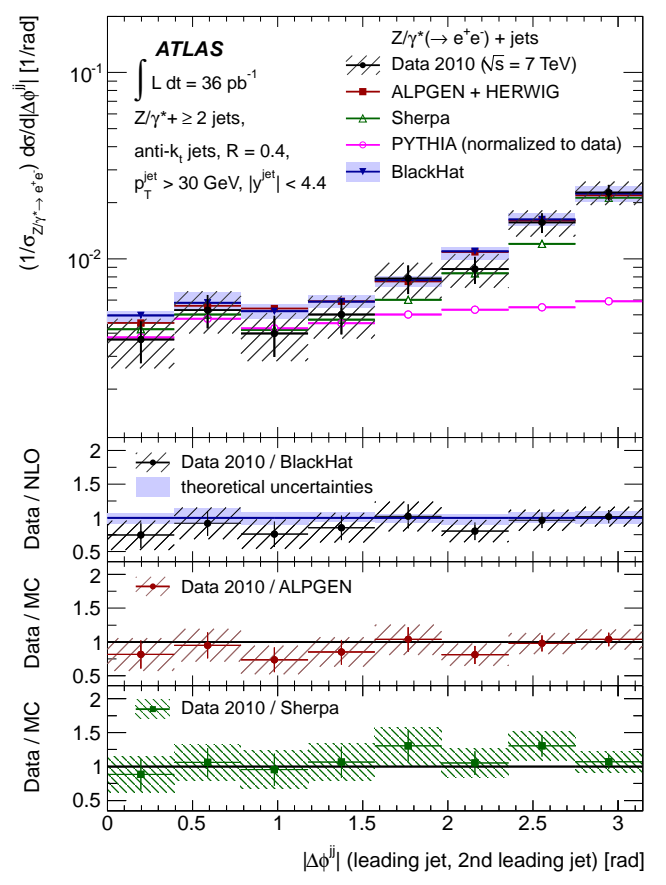

(b) Absolute azimuthal separation

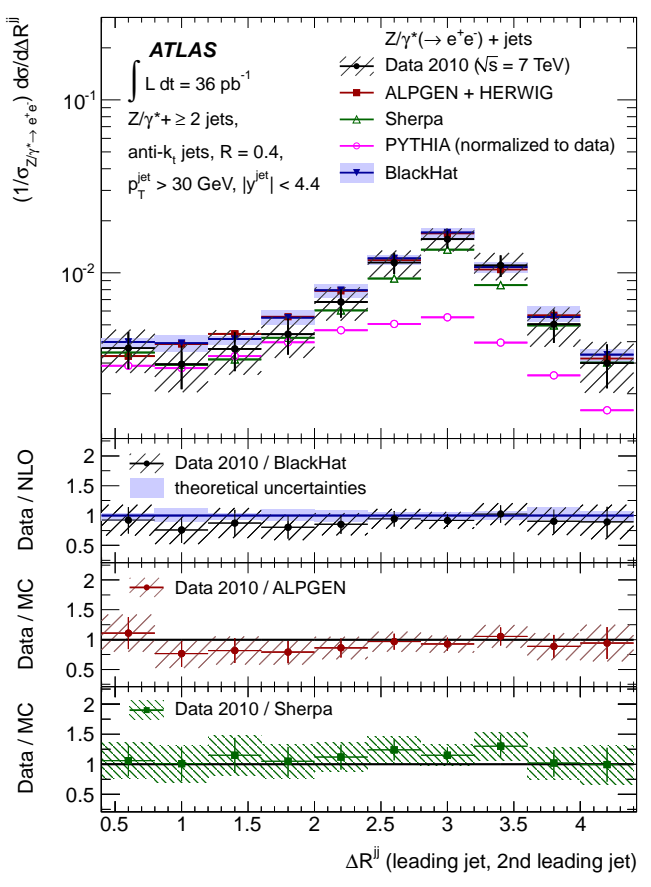

(c) Angular separation in $y-\phi$ space

Figure 8.16.: Differential dijet cross section as a function of (a) the absolute rapidity difference of the two leading jets $\left|\Delta y^{j j}\right|$, (b) the absolute azimuthal separation of the two leading jets $\left|\Delta \phi^{j j}\right|$ and (c) the angular separation in $y$ - $\phi$ space of the two leading jets $\Delta R^{j j}$ for events with at least two jets in the final state. The cross sections are normalised to the inclusive $Z / \gamma^{*}(\rightarrow e e)$ cross section. The other details are as in Fig. 8.12, 


\subsection{Conclusions}

The inclusive and differential $Z / \gamma^{*}(\rightarrow e e)+$ jets cross section has been measured in ppcollisions at a centre-of-mass energy of $\sqrt{s}=7 \mathrm{TeV}$ with the full dataset of 2010. Total inclusive cross sections and their ratios are measured as a function of jet multiplicity, as well as inclusive differential cross sections as a function of the jet transverse momentum, the jet rapidity, the dijet mass and the angular separation between the two leading jets.

Systematic and statistical uncertainties have been evaluated and the systematic uncertainty has been reduced by normalising the cross sections to the inclusive $Z / \gamma^{*}(\rightarrow e e)$ cross section. Within uncertainties the predictions from ALPGEN+HERWIG, SHERPA and the NLO fixed-order pQCD calculations from BLACKHAT+SHERPA are consistent with the data, whereas PYTHIA fails to describe the data in large parts of the phase space.

The measurements are already limited by systematics, mainly due to JES and JER, except for the high transverse momentum and forward regions, in which the statistical uncertainty is comparable to the total systematic uncertainty.

The increased dataset of 2011 on the one hand allows for more precise measurements and measurements in new regions of phase space, on the other hand measurements have to deal with increased pile-up. 


\section{Measurement with the Dataset of 2011}

This chapter describes the inclusive and differential $Z / \gamma^{*}(\rightarrow e e)+$ jets cross-section measurement in pp-collisions at a centre of mass energy of $\sqrt{s}=7 \mathrm{TeV}$ with an integrated luminosity of $\int L \mathrm{~d} t=4.6 \mathrm{fb}^{-1}$. This corresponds to the full dataset of 2011 . This measurement provides the most accurate results and partly supersedes the measurement with the full dataset of 2010. It allows to exploit the data in kinematic regions which were not accessible before, due to statistical limitations. It can be used to probe the $Z / \gamma^{*}+$ jets modelling in typical phase space regions expected for the Higgs boson decay and searches for new physics.

Total inclusive and exclusive cross sections are measured as a function of the jet multiplicity, $N_{\text {jet }}$. Additionally, the inclusive and exclusive jet multiplicity ratios are measured for different values of the transverse momentum of the leading jet to test the jet multiplicity scaling. Furthermore, inclusive differential cross sections are measured as a function of the jet transverse momentum, $p_{T}^{\text {jet }}$, the jet rapidity, $y^{\text {jet }}$, the transverse momentum of the $Z$ boson $p_{T}^{e e}$, the invariant mass of the two leading jets, $m^{j j}$, and the angular separation between the two leading jets. Finally, inclusive differential cross sections as a function of $H_{T}$ and $S_{T}$ are measured. $H_{T}$ is defined as the scalar $p_{T}$ sum of all final state objects, while $S_{T}$ is defined as the scalar $p_{T}$ sum of all hadronic jets in the final state.

The different steps of the analysis are exemplarily presented for a few key observables: the exclusive jet multiplicity, the transverse momentum and the rapidity of the leading jet for events with at least one jet in the final state, the absolute rapidity difference of the two leading jets for events with at least two jets in the final state and $H_{T}$ for events with at least on jet in the final state. The distributions for the other observables can be found in Ref. [167].

This chapter is organised in the same way as the previous chapter: Starting with the description of the uncorrected distributions comparing MC predictions to the measurements on detector level in Sec. 9.1. Sec. 9.2 proceeds with the explanation of the method to correct for detector effects. The different sources of systematic uncertainties on the measurement are discussed in Sec. 9.3 , followed by the theoretical corrections for nonperturbative effects and QED FSR in Sec. 9.4. Finally, Sec. 9.5 discusses the results at particle level. 


\subsection{Uncorrected Distributions}

The uncorrected data distributions for $Z / \gamma^{*}(\rightarrow e e)+$ jets are compared to the expected signal and background distributions. For signal expectations two different ME+PS generators have been used, ALPGEN+HERWIG and SHERPA 1.4.1. Electron trigger, efficiency and smearing corrections, as well as jet energy corrections are applied on MC, as described in Chapter 6. Since the electron identification efficiency has been determined from a MC event sample with a too wide beamspot $\sigma_{z}$ compared to the one used for the analysis, a global correction factor of $0.9860 \pm 0.0013$ has been determined by the comparison of the $Z$ boson reconstruction efficiency in both $\mathrm{MC}$ event samples and is applied to the predictions from the MC event generators.

Figure 9.1 depicts the different rapidity distributions predicted by ALPGEN+HERWIG and SHERPA and in data normalised to unity.

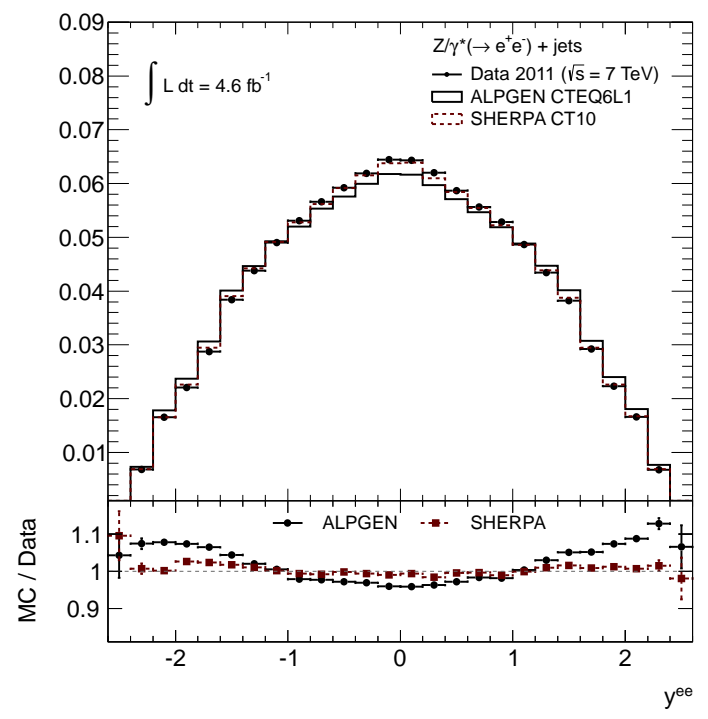

Figure 9.1: $Z$ boson rapidity distribution in data and MC event samples. The distributions are normalised to unity.

ALPGEN+HERWIG, which uses a LO PDF, models a too wide rapidity distribution of the $Z$ boson, whereas SHERPA, which uses an NLO PDF, shows good agreement. A too wide rapidity distribution of the $Z$ boson results in a too small acceptance for the lepton fiducial selection. Therefore, the expectations from ALPGEN+HERWIG in this subsection are reweighted by a global scale factor of 1.062 on an event-by-event base. This scale factor has been derived from the comparison of the nominal ALPGEN+HERWIG prediction with the prediction reweighted to NLO PDFs [167] and has only been used for the results presented in this subsection.

Table 9.1 summarises the events in data and the expectations from MC for both ALPGEN+HERWIG and SHERPA as signal MC. The uncertainties incorporates the combined statistical and systematic uncertainty, as detailed in Sec. 9.3 . 


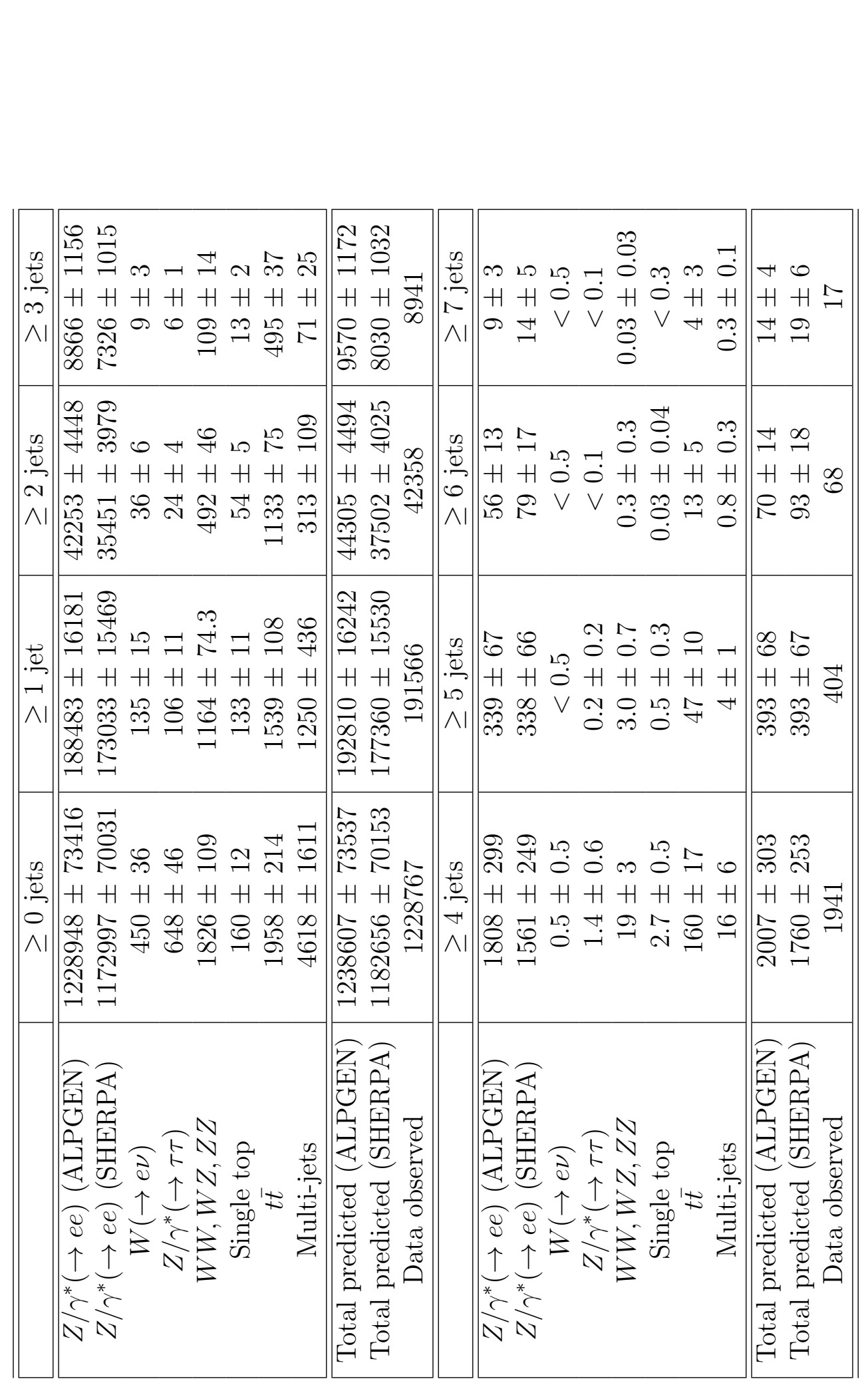

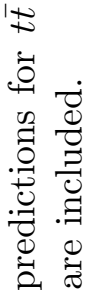

宛

$\dot{\vec{D}} \dot{\mathcal{D}}$

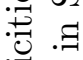

. 국

奇

.

령

氖.

\&

:

䒕

䆑

$\exists$

D

离

령

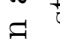

.

学

苜

용

$\because$

류

袍

늉

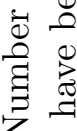

乙

$\because: 0$

ஸे

$\stackrel{\circ}{\circ}$

0 
The expected background fraction for $Z / \gamma^{*}(\rightarrow e e)+$ jets increases with increasing jet multiplicity from $0.8 \%$ for $N_{\text {jet }} \geq 0$ to $20 \%$ for $N_{\text {jet }} \geq 6$. The main contributions come from QCD multi-jet, $t \bar{t}$ and diboson events for $Z / \gamma^{*} \leq 1$ jet, and for higher jet multiplicities from $t \bar{t}$ events. The fraction of the multi-jet background varies between $(0.38 \pm 0.13) \%$ for $N_{j e t} \geq 0$ and $(1.20 \pm 0.44) \%$ for $N_{j e t} \geq 6$, while the $t \bar{t}$ fractions vary between $(0.16 \pm 0.02) \%$ for $N_{\text {jet }} \geq 0$ to $(18.6 \pm 7.0) \%$ for $N_{\text {jet }} \geq 6$. Both backgrounds are estimated using a data-driven method as explained in Sec. 7.2. The other backgrounds, such as $Z / \gamma^{*}(\rightarrow \tau \tau)+$ jets, $W(\rightarrow e \nu)+$ jets, diboson and single top are estimated by $\mathrm{MC}$ and normalised to the integrated luminosity in data using the respective (N)NLO cross sections. For the following figures, background contributions from $W(\rightarrow e \nu)+$ jets, $Z / \gamma^{*}(\rightarrow \tau \tau)+$ jets and diboson processes are summarised as electroweak background and contributions from $t \bar{t}$ and single top are labelled as top background.

Figure 9.2 shows the uncorrected invariant dielectron mass for $Z$ boson candidates, $m_{e^{+} e^{-}}$, in events with at least one associated jet in the final state, as well as the uncorrected inclusive jet multiplicity. The uncorrected invariant dielectron mass is shown for a wider range than the one which is selected $\left(66 \mathrm{GeV}<m_{e^{+} e^{-}}<116 \mathrm{GeV}\right)$.

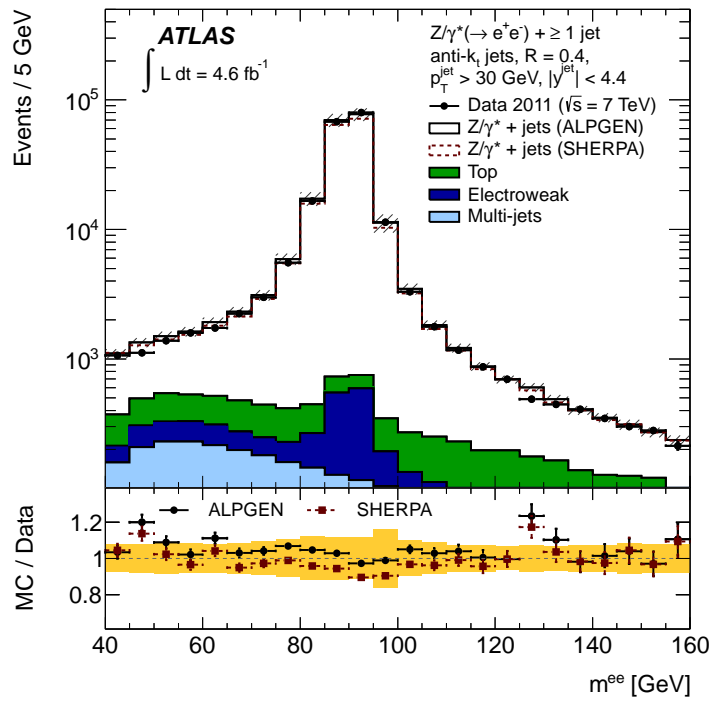

(a) Dielectron mass

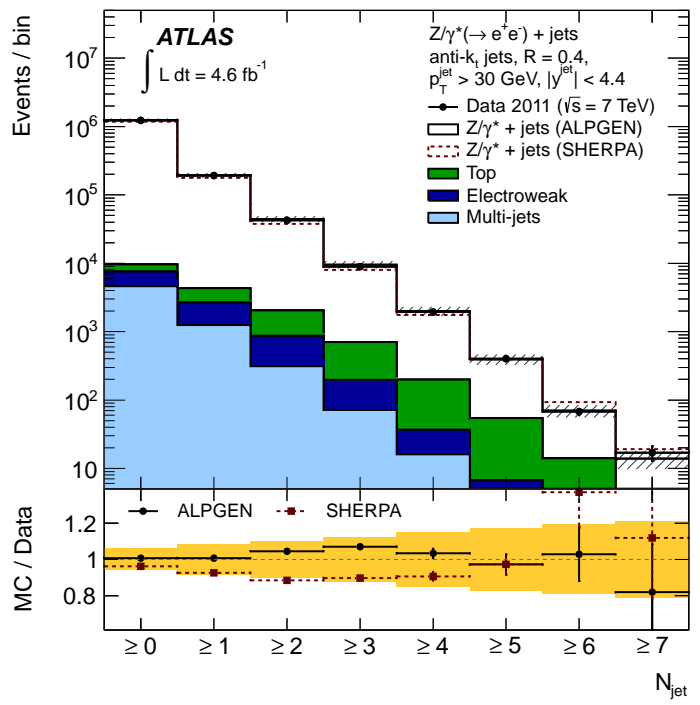

(b) Inclusive jet multiplicity

Figure 9.2.: (a) Dielectron mass for $Z$ candidates with at least one jet in the event and (b) inclusive jet multiplicity in data and simulation. Multi-jets and $t \bar{t}$ backgrounds have been estimated from data. The hatched band incorporates the total statistical and systematic uncertainty on the predictions. The yellow band in the ratio plots reflects the total systematic uncertainty.

For both ALPGEN+HERWIG and SHERPA, Data and MC are consistent for the final selection and in the side bands. The current statistics in data allows for the first time to gain access to the seventh jet bin in the inclusive and exclusive jet multiplicity. But the MC expectation has to be taken with care in this region, since both ALPGEN+HERWIG and SHERPA only uses matrix elements with up to five partons and the rest is done by parton shower. As already known from the 2010 analysis, parton shower does not fully describe the jet kinematics which results in an offset in the jet multiplicity.

Figure 9.3 shows the uncorrected $p_{T}^{j e t}$ and $y^{\text {jet }}$ distributions for the leading jet in events with at least one jet in the final state, as well as the uncorrected absolute rapidity dif- 


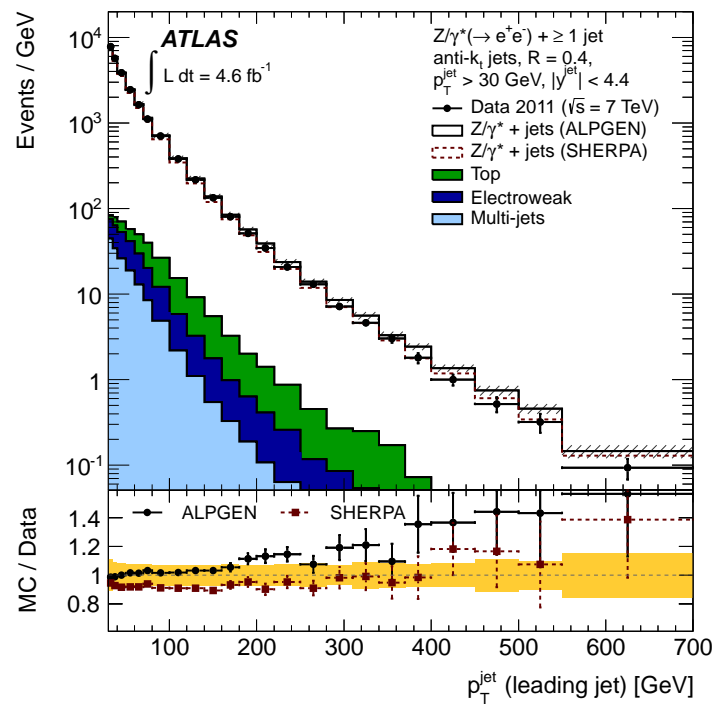

(a) Leading jet $p_{T}$

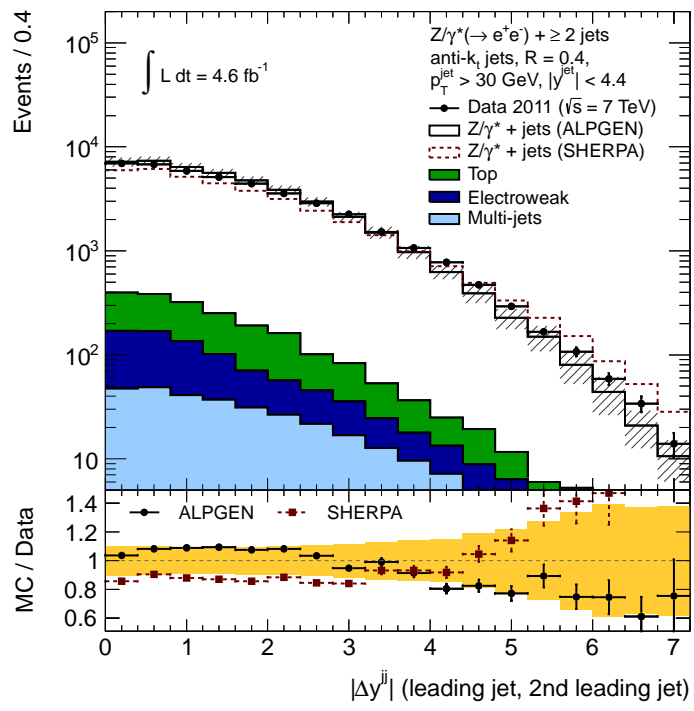

(c) Absolute rapidity difference

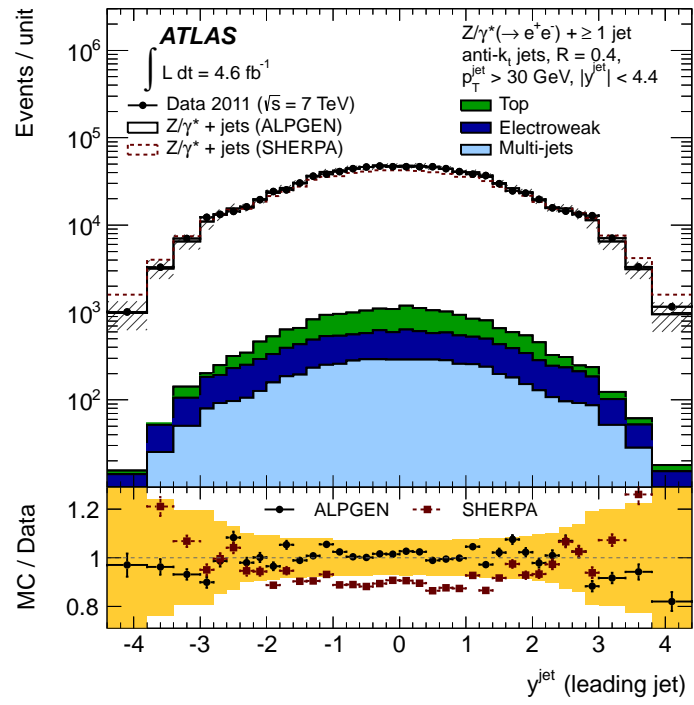

(b) Leading jet $y$

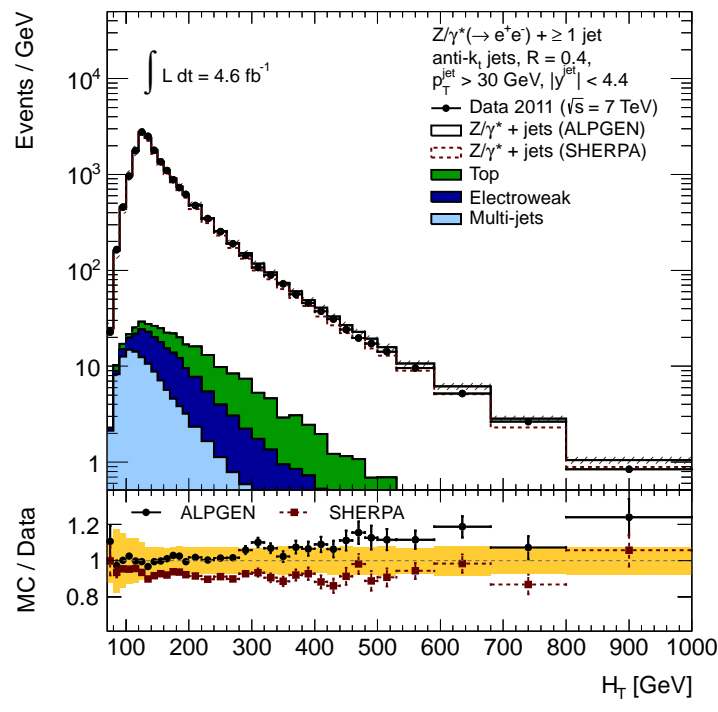

(d) $H_{T}$

Figure 9.3.: (a) Transverse momentum distribution of the leading jet in events with at least one jet in the final state, (b) rapidity distribution of the leading jet in events with at least one jet in the final state, (c) $\left|\Delta y^{j j}\right|$ of the two leading jets in events with at least two jets in the final state and (d) $H_{\mathrm{T}}$ in events with at least one jet in the final state in data and simulation. Multi-jets and $t \bar{t}$ backgrounds have been estimated from data. The hatched band incorporates the total statistical and systematic uncertainty on the predictions. The yellow band in the ratio plots reflects the total systematic uncertainty. 
ference, $\left|\Delta y^{j j}\right|$, of the two leading jets in events with at least two jets in the final state and the uncorrected $H_{\mathrm{T}}$ distribution in events with at least one jet in the final state. The predictions from ALPGEN+HERWIG model a too hard $p_{T}$ distribution for the leading jet, while they are consistent with the data for the other distributions. SHERPA models a two broad rapidity distributions, which is reflected in large values of $\left|\Delta y^{j j}\right|$. Further uncorrected distributions can be found in Appendix B.1.

All plots illustrated in this section and in Appendix B.1 are shown with the combined statistical and systematic uncertainty, as described in Sec. 9.3. Since there are no systematic uncertainties coming from the unfolding process, they provide a valuable reference for a comparison between generator predictions and data. In general, the predictions from ALPGEN+HERWIG and SHERPA are consistent with the data. This gives confidence that these $\mathrm{MC}$ event samples provide a reasonable description of the event kinematics and the detector response, which is necessary since they are used as input sample for the unfolding. A detailed discussion of all distributions is given in Sec. 9.5 together with the comparison on particle level.

\subsubsection{Double-parton Interactions}

The production of $Z / \gamma^{*}(\rightarrow e e)+$ jets events includes a fraction of events originating from DPI, which is expected from MC predictions to be small, at the order of $3 \%$. This contribution is not included in the NLO fixed-order calculations from BLACKHAT+SHERPA, therefore the correction for non-perturbative effects has to cover this effect. Due to the fact that this correction is based on MC predictions from ALPGEN+HERWIG, the fraction of expected DPI events has to be validated with data.

In order to study DPI, the production of $Z+2$ jets can be used. As described in Sec. 2.3.2, $\sigma_{\text {eff }}$ is expected to be approximately independent of the process and the phase space requirements, thus $Z / \gamma^{*}+$ jets events can lead to an improved understanding of DPI. For $Z+2$ jets events originating from DPI, the two jets are more likely back-to-back in the transverse plane, whereas for events originating from SPI the two jets are expected to recoil against the $Z$ boson. Therefore, the transverse momentum balance between the two jets $\Delta_{\text {jets }}$ provides a good observable to separate DPI from SPI events, following the approach from Ref. [168]. The observable $\Delta_{\text {jets }}$ is defined as

$$
\Delta_{\text {jets }}=\left|\vec{p}_{T}^{j e t_{1}}+\vec{p}_{T}^{j e t_{2}}\right|
$$

In order to reduce the dependence of $\Delta_{\text {jets }}$ on the jet energy scale, $\Delta_{\text {jets }}$ has been normalised to the sum of the individual transverse momenta

$$
\Delta_{\text {jets }}^{n}=\frac{\left|\vec{p}_{T}^{j e t_{1}}+\vec{p}_{T}^{j e t_{2}}\right|}{\left|\vec{p}_{T}^{j e t_{1}}\right|+\left|\vec{p}_{T}^{j e t_{2}}\right|} .
$$

Figure 9.4 shows the uncorrected distribution of $\Delta_{\text {jets }}^{n}$ and $\Delta_{\text {jets }}$. Effects from DPI are mainly expected in the region $0 \mathrm{GeV}<\Delta_{\text {jets }}<50 \mathrm{GeV}$ and $0<\Delta_{\text {jets }}^{n}<0.5[168$ ]. The predictions from ALPGEN+HERWIG are consistent with the data for $\Delta_{\text {jets }}^{n}$ and $\Delta_{\text {jets }}$. The predictions from SHERPA show a constant offset, which can be attributed to the mismodelling of the number of two jet events. This gives confidence that the MC events samples provide a reasonable description of DPI events and no coarse mismodelling exists. For a clearer statement, further studies using template fits are necessary. 


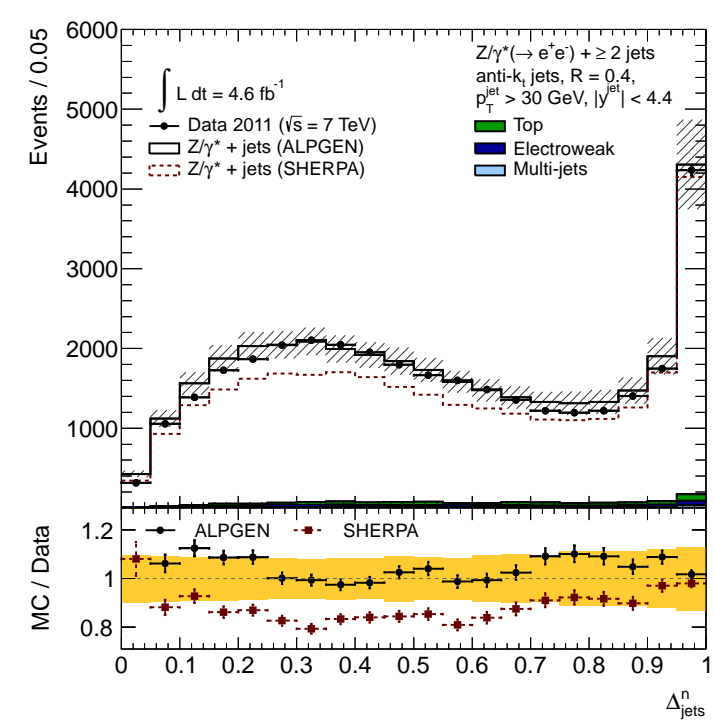

(a) $\Delta_{\text {jets }}^{n}$

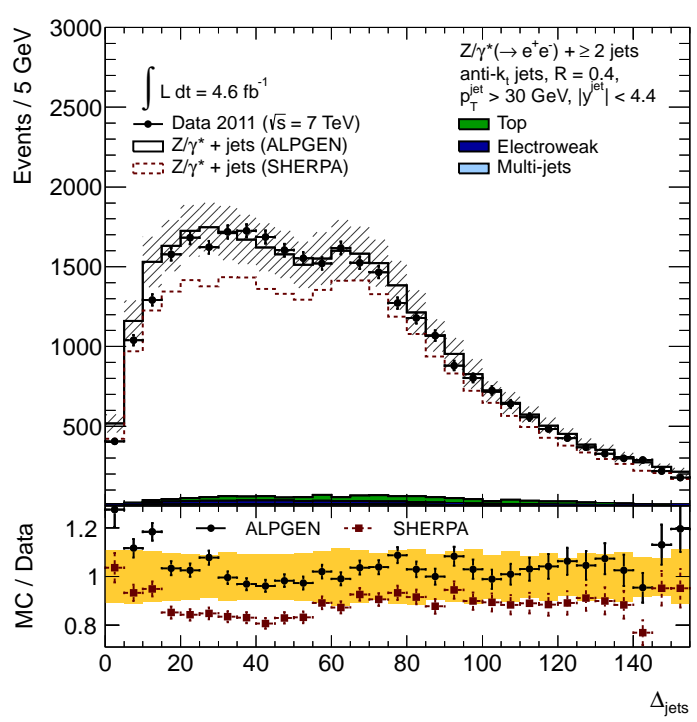

(b) $\Delta_{\text {jets }}$

Figure 9.4.: (a) $\Delta_{\text {jets }}^{n}$ and (b) $\Delta_{\text {jets }}$ distribution in events with exactly two jets in the final state in data and simulation. Multi-jets and $t \bar{t}$ backgrounds have been estimated from data. The hatched band incorporates the total statistical and systematic uncertainty on the predictions. The yellow band in the ratio plots reflects the total systematic uncertainty.

\subsubsection{Impact of Pile-up}

From 2010 to 2011 the amount of pile-up has significantly increased. During the 2010 data taking the average number of interactions per bunch crossing was two, while at the end of 2011 it increased to up to 20. Therefore for the analysis with 2010 data the dependence on pile-up could be neglected. In contrast, for the analysis with 2011 data the measured quantities on detector level are expected to be affected by pile-up, even if the cut on the JVF rejects jets coming from pile-up in the central region and the jet energies are corrected for an offset depending on the number of primary vertices $N_{P V}$ and the number of interactions per bunch crossing $\mu$. This can be ascribed to the fact that no algorithm exists to reject jets originating from pile-up outside the acceptance region of the inner detector. If the pile-up observed in data after applying the inclusive $Z / \gamma^{*}(\rightarrow e e)$ selection is correctly modelled by the MC event samples, the measured cross sections are expected to be independent from the amount of pile-up, since its impact is correctly taken into account in the unfolding.

A first check is performed by comparing the average number of interactions per bunch crossing and the number of primary vertices predicted by ALPGEN+HERWIG with the data, as shown in Fig. 7.2. For both distributions, the predictions from ALPGEN+HERWIG are consistent with the data, which indicates that the re-weighting procedure, as described in Sec. 7.2, works correctly.

Further tests are performed by comparing the predictions from ALPGEN+HERWIG with the data for a few key distributions after applying the inclusive $Z / \gamma^{*}(\rightarrow e e)$ selection for different pile-up scenarios. Three regions (low, medium and high) for the average number of interactions, as well as for the number of primary vertices are defined:

- Low $\mu$ : $\mu<6.5$ 
- Medium $\mu: 6.5 \leq \mu<10.5$

- High $\mu: \mu \geq 10.5$

- Low $N_{P V}: N_{P V}<5$

- Medium $N_{P V}: 5 \leq N_{P V}<8$

- High $N_{P V}: N_{P V} \geq 8$

At first order, it is expected that the number of primary vertices is mainly influenced by in-time pile-up whereas $\mu$ is influenced by out-of-time pile-up. In order to disentangle both effects, four regions have been defined: low $\mu$ or high $\mu$ together with medium $N_{P V}$ to study the impact of out-of-time pile-up and medium $\mu$ together with low $N_{P V}$ or high $N_{P V}$ for in-time pile-up.

In order to compare the different pile-up scenarios, the ratio between the distribution with requirements on $\mu$ and $N_{P V}$ and the inclusive distribution without requirements on $\mu$ and $N_{P V}$ is taken. The impact coming from electrons are removed by dividing each distribution by its respective number of inclusive $Z / \gamma^{*}(\rightarrow e e)$ events before calculating the ratio. Figure 9.5 shows the ratios to test the impact of in-time and out-of-time pile-up for the transverse momentum and the rapidity distribution of all jets. As expected, the largest impact of pile-up is found in the low $p_{T}^{\text {jet }}$ region and in the forward region beyond the acceptance region of the tracker, where no cut on the JVF is applied. In addition the impact of in-time pile-up is much larger on the measured quantities. In general, a good agreement between the predictions from ALPGEN+HERWIG and the data are found, except for some phase-space regions (low $p_{T}$ and very high $|y|$ ). In order to provide more precise tests of the $\mathrm{MC}$ data agreement, the double ratio between the ratios from ALPGEN+HERWIG and data is built and shown in Fig. 9.6. The hatched bands reflect the pile-up component of the JES uncertainty.

As seen before the impact of pile-up on the measured quantities is well described by the predictions from ALPGEN+HERWIG, except for the region $20 \mathrm{GeV}<p_{T}^{\text {jet }}<30 \mathrm{GeV}$, which supports the choice to perform the measurements only for jets with $p_{T}>30 \mathrm{GeV}$. But the deviations in this region are still covered by the pile-up component of the JES uncertainty. Also in the forward region the predictions from ALPGEN+HERWIG are consistent with the data within the large statistical and systematic uncertainties. Results of similar pile-up studies for the inclusive jet multiplicity are shown in Appendix B.1.1. 


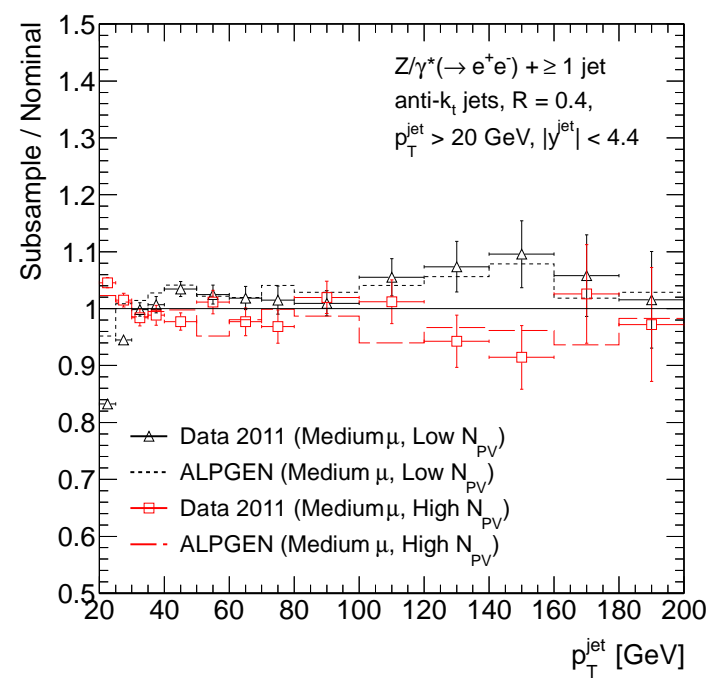

(a) Inclusive jet $p_{T}$, impact of in-time pile-up

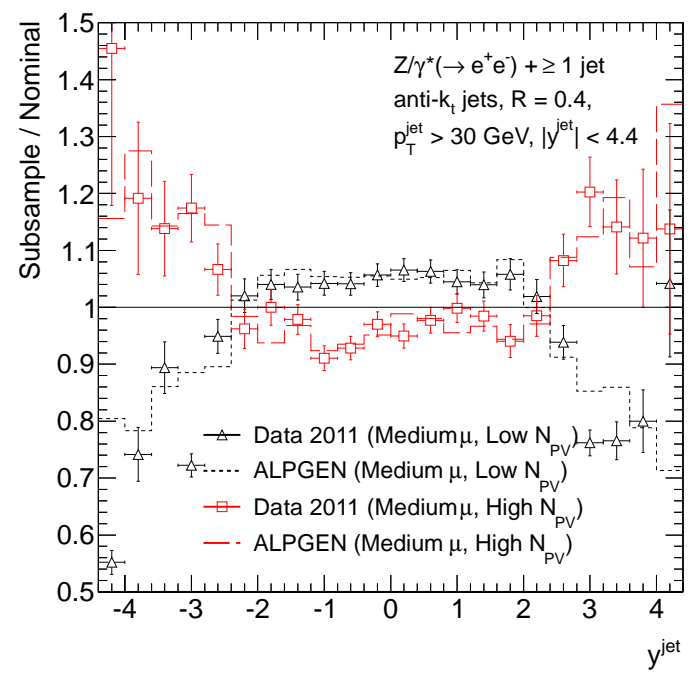

(c) Inclusive jet $y$, impact of in-time pile-up

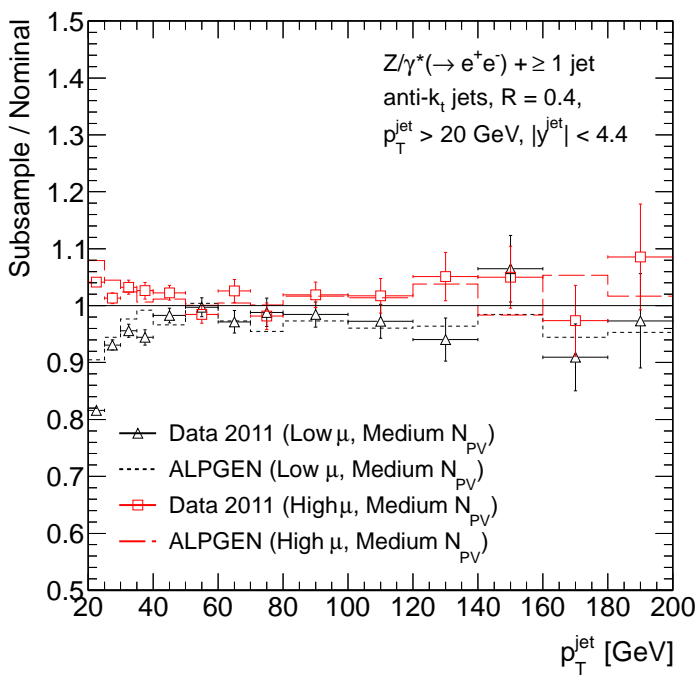

(b) Inclusive jet $p_{T}$, impact of out-of-time pile-up

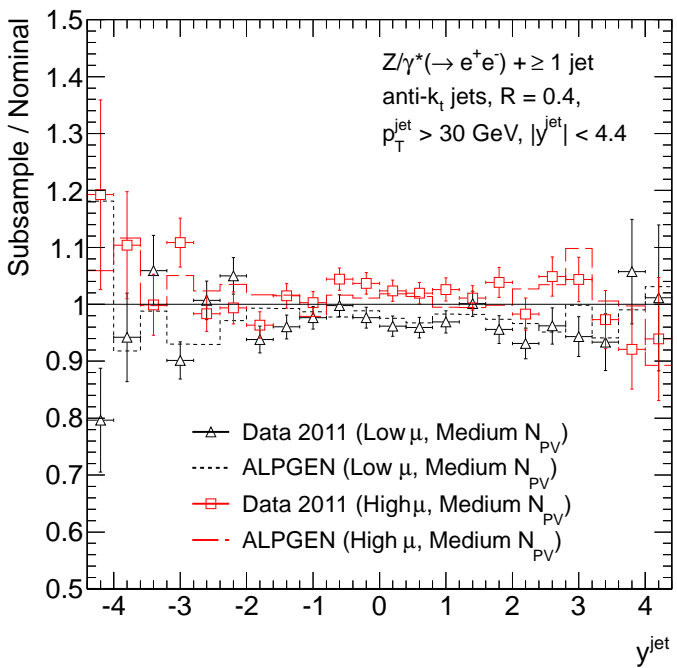

(d) Inclusive jet $y$, impact of out-of-time pile-up

Figure 9.5.: Ratios of (a),(b) the transverse momentum and (c),(d) the rapidity distributions of all jets with and without requirements on $\mu$ and $N_{P V}$ in data and simulation to test the impact of (a),(c) in-time pile-up and (b),(d) out-of-time pile-up. The distributions are divided by the respective number of inclusive $Z / \gamma^{*}(\rightarrow e e)$ events before calculating the ratios. 

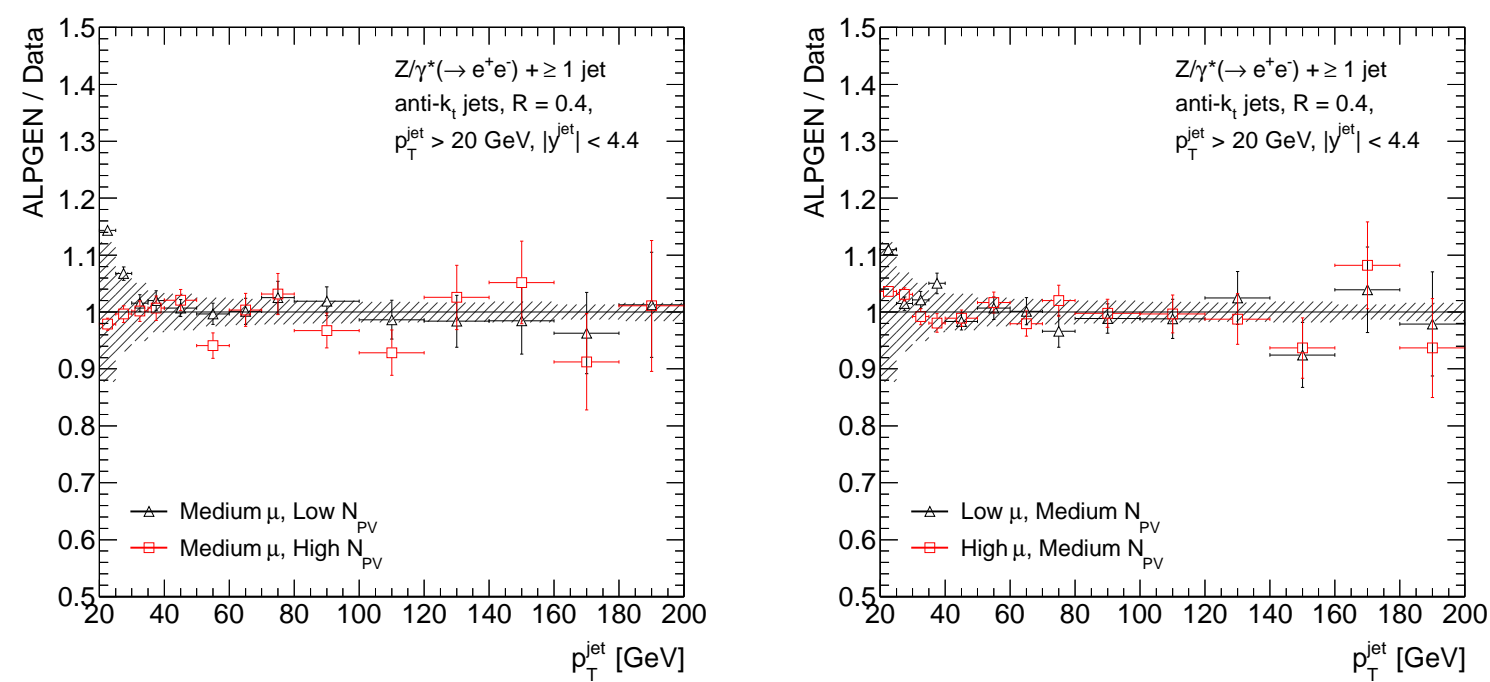

(a) Inclusive jet $p_{T}$, impact of in-time pile-up

(b) Inclusive jet $p_{T}$, impact of out-of-time pile-up

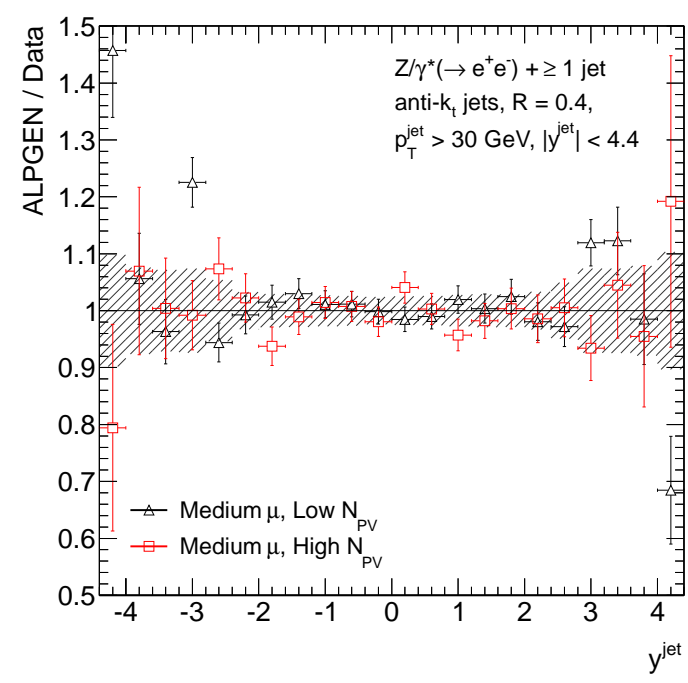

(c) Inclusive jet $y$, impact of in-time pile-up

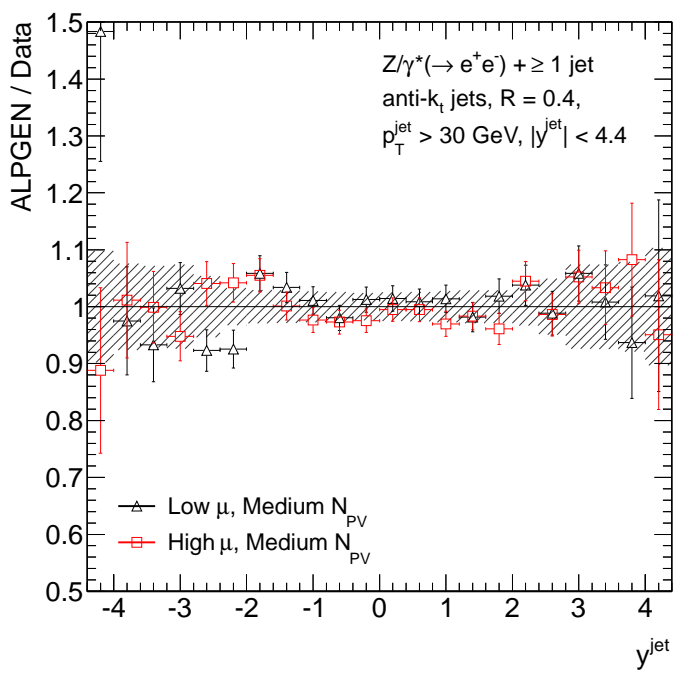

(d) Inclusive jet $y$, impact of out-of-time pile-up

Figure 9.6.: Double ratio between the ratios from ALPGEN+HERWIG and data for (a),(b) the transverse momentum and (c),(d) the rapidity distributions of all jets to test the impact of (a),(c) in-time pile-up and (b),(d) out-of-time pile-up. The hatched bands reflect the pile-up component of the JES uncertainty. 


\subsection{Corrections for Detector Effects}

Similar to the 2010 analysis, the final cross sections are quoted on particle level to facilitate the comparison with pQCD predictions and with measurements from other experiments. Therefore, the measurements are corrected for detector effects back to particle level. This correction accounts for resolution effects, non linearities and efficiencies of the $Z / \gamma^{*}$ and jet identification and reconstruction. In contrast to the 2010 analysis, the nominal correction is done using the iterative (Bayes) method [131] based on the ALPGEN+HERWIG signal MC event sample. The iterative (Bayes) method has been optimised using more refined corrections and a better method to choose the optimal number of iterations. In addition, the available MC statistics has significantly increased, which allows for measurements with a higher level of accuracy.

In the following subsections the implementation of the iterative (Bayes) method and the method to evaluate the optimal number of iterations are presented. The systematic uncertainties due to the unfolding procedure are discussed in Sec. 9.3.5.

\subsubsection{Method Implementation}

The correction for detector effects is done using the iterative (Bayes) method following in general the approach from the analysis with the dataset of 2010, as described in Sec. 8.2. The correction is performed for each distribution separately, due to the fact that the statistics of the MC event samples is limited. With more statistics a multi-dimensional unfolding would be preferred to correctly take into account the full event kinematics.

The jet hierarchy on particle level, defined by $p_{T}^{\text {jet }}$ in decreasing order, is preserved on detector level in the matched events. This results in a different jet hierarchy for the matched distribution compared to the nominal distribution on detector level. In order to better understand this effect, the correction of not-matched events used in 2010, is split into two separate corrections for 2011, a pure correction for not-matched events and a jet hierarchy correction. Both corrections are based on bin-by-bin correction factors, which introduces a dependence of the MC event samples in the unfolding procedure. Hence, it is attempted to keep these corrections small.

The binning of the different distributions is chosen such that the bin width commensurates with the resolution and the bin purity is $\sim 70 \%$. The $\mathrm{MC}$ distribution on particle level is used as initial prior. A sanity check of this method is performed using SHERPA MC event samples and ALPGEN + HERWIG reweighted to data. The results are presented in Appendix B.3. Finally, the optimal number of iterations has been studied, as described in Sec. 9.2.2.

Figure. 9.7 - Fig. 9.9 exemplarily show the migration matrices, the correction for notmatched events and the jet hierarchy for the transverse momentum and the absolute rapidity distribution of the leading jet for events with at least one jet in the final state. Further distributions can be found in Appendix B.2 and Ref. [167]. 


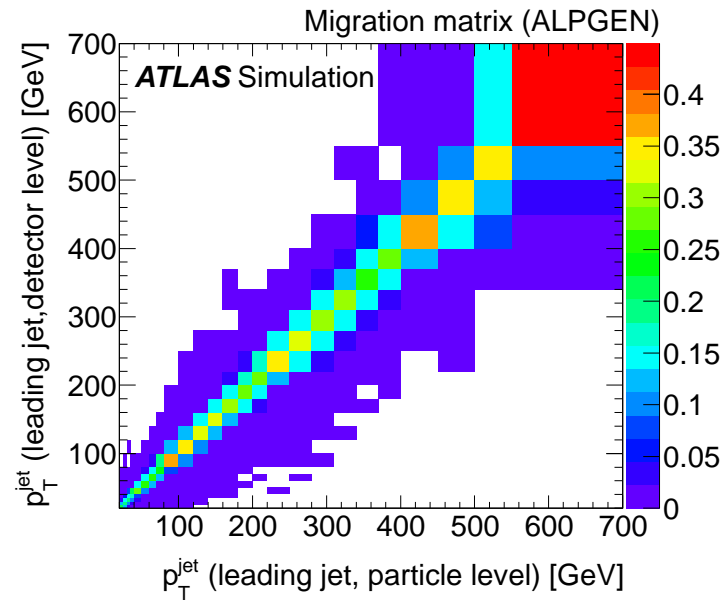

(a) Leading jet $p_{T}$

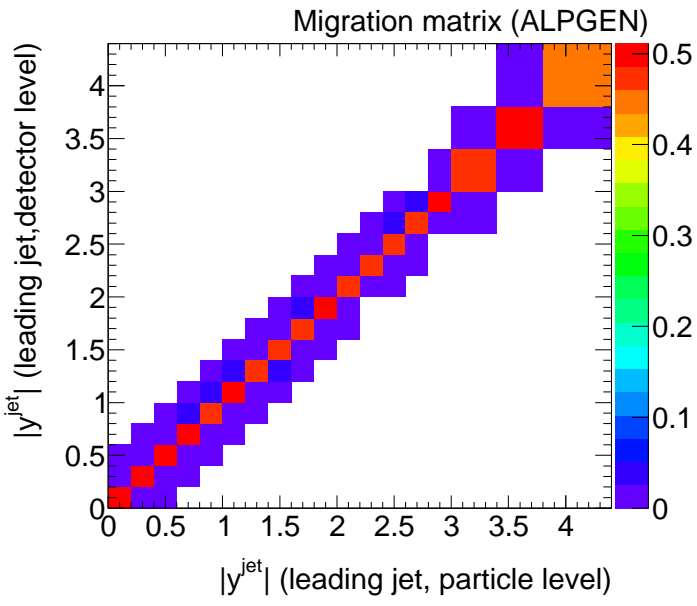

(b) Leading jet $|y|$

Figure 9.7.: Migration matrices for (a) the transverse momentum of the leading jet and (b) the absolute rapidity of the leading jet for events with at least one jet in the final state. The migration matrices are determined using ALPGEN+HERWIG MC event samples.

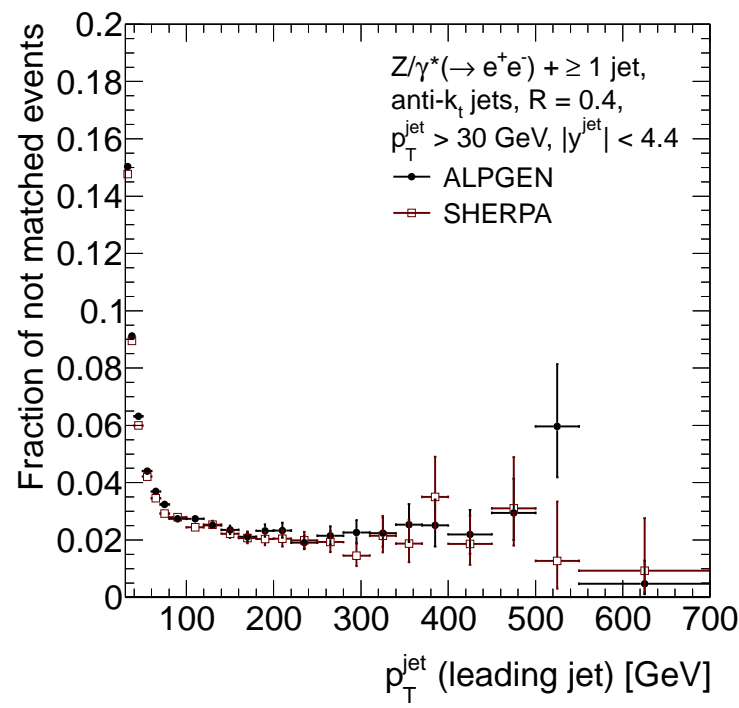

(a) Leading jet $p_{T}$

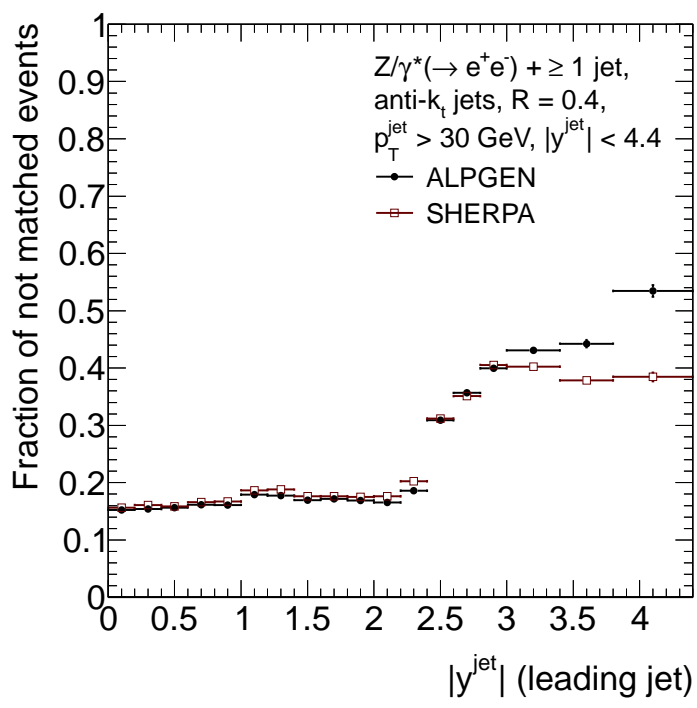

(b) Leading jet $|y|$

Figure 9.8.: Correction for not-matched events for (a) the transverse momentum of the leading jet and (b) the absolute rapidity of the leading jet for events with at least one jet in the final state. The correction factors are shown for ALPGEN+HERWIG and SHERPA MC event samples. 


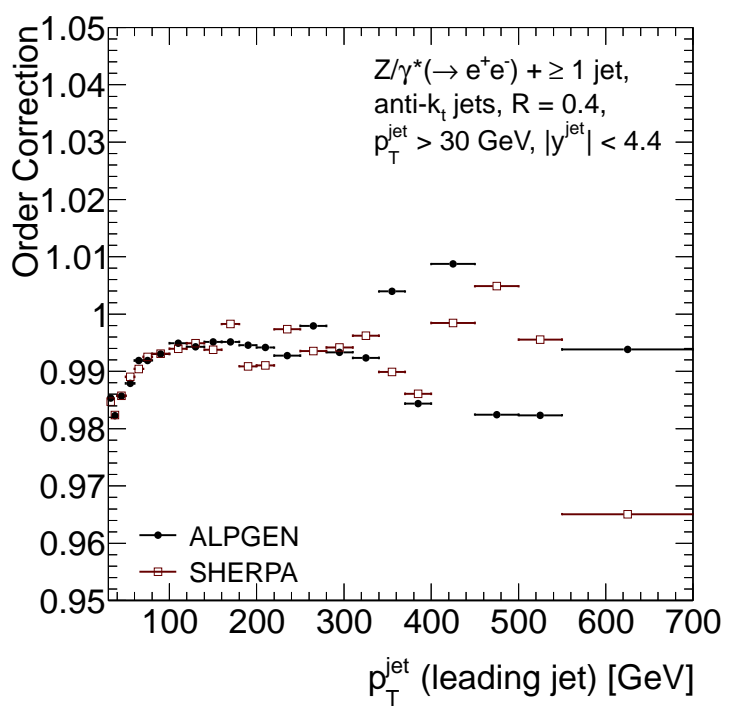

(a) Leading jet $p_{T}$

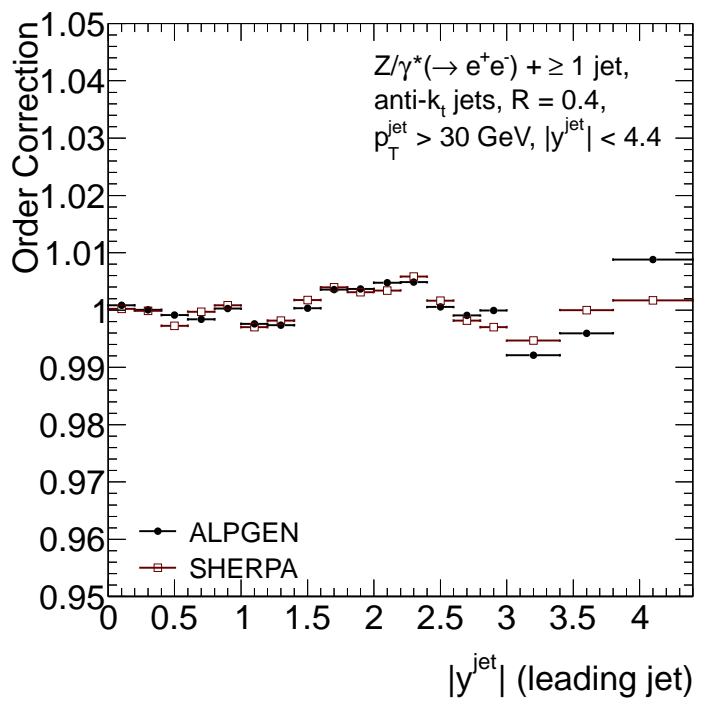

(b) Leading jet $|y|$

Figure 9.9.: Order correction for (a) the transverse momentum of the leading jet and (b) the absolute rapidity of the leading jet for events with at least one jet in the final state. The correction factors are shown for ALPGEN+HERWIG and SHERPA MC event samples.

Whereas substantial migration is observed for the transverse momentum of the jets, the migration matrix for the absolute rapidity distribution is approximately diagonal. In order to account for the correct migration around the $p_{T}^{\text {jet }}$ threshold of $30 \mathrm{GeV}$, the $p_{T}^{\text {jet }}$ distributions are expanded to $20 \mathrm{GeV}$. For the rapidity distribution, a cross check using a two-dimensional unfolding is performed to account for the migration around the $p_{T}^{\text {jet }}$ threshold, as described in Sec. 9.5.4. For some phase-space regions, the correction for not-matched events is rather large, e.g. in the forward region. In addition, in this region the predictions from ALPGEN+HERWIG and SHERPA diverges, which leads to large systematic uncertainties on the final measurements. In the future with increasing precision of the measurement and more MC statistics this method needs to be optimised. As expected, the jet hierarchy correction is rather small over the whole phase-space region at the order of $1 \%$, only for the low $p_{T}^{\text {jet }}$ the correction slightly increases. This is due to the fact that the $p_{T}$ distribution is steeply falling and therefore it is more likely for low $p_{T}$ jets to change their ordering due to resolution effects.

\subsubsection{Optimal Number of Iterations}

In order to determine the optimal number of iterations for the unfolding, the detector level distributions obtained with SHERPA are unfolded to particle level using ALPGEN+HERWIG event samples separately for each observable, similar to the unfolding performed for the data distributions. In addition, an MC@NLO MC event sample is used to perform a cross check. The statistics of the SHERPA and MC@NLO MC event samples are at the same order as the statistics in data, such that statistical fluctuations are taken into account. The unfolded results are then compared to the truth distributions of the different MC event samples, respectively. The optimal number of iterations is quantified 
by minimising the variable

$$
\chi^{2}=\sum_{i} \frac{\left(U_{i}-T_{i}\right)^{2}}{\left(\delta T_{i}\right)^{2}},
$$

Here, $U_{i}$ and $T_{i}$ describe the unfolded number of events and the true number of events in bin $i$, respectively. $\delta T_{i}$ is the statistical uncertainty on $T_{i}$. The sum runs over all bins of the distributions.

Figure 9.10 exemplarily shows the $\chi^{2}$ values divided by the number of bins for the transverse momentum and the absolute rapidity distribution of the leading jet for events with at least one jet in the final state. Zero iterations in these figures correspond to the result from the bin-by-bin method. Further distributions can be found in Appendix B.2 and Ref. [167.

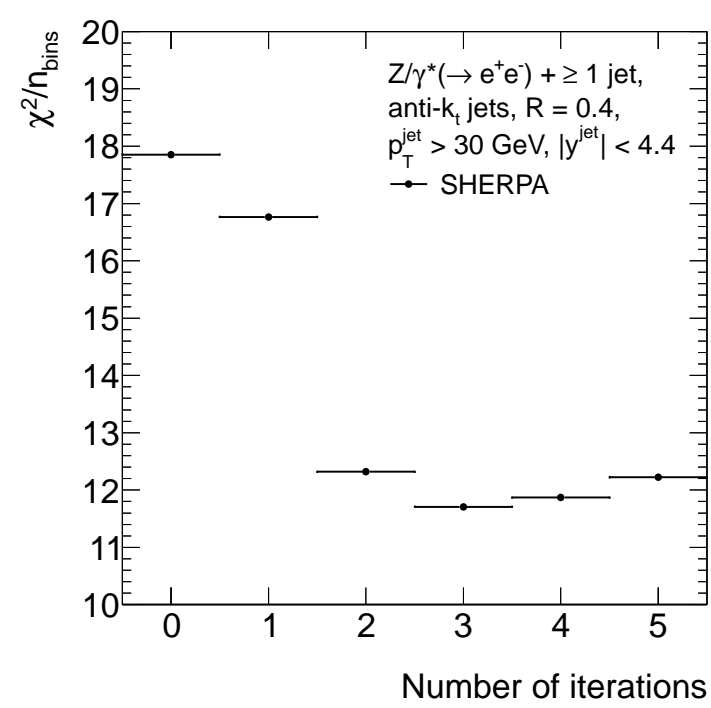

(a) Leading jet $p_{T}$

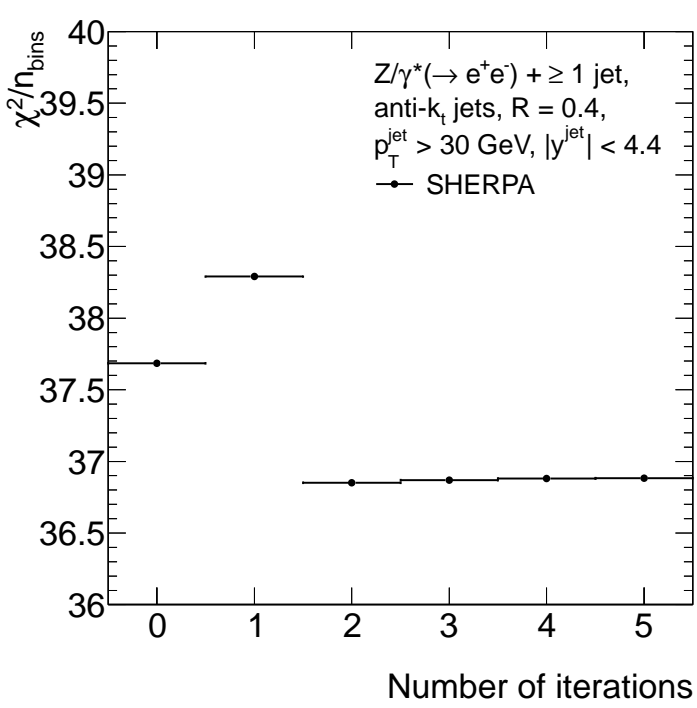

(b) Leading jet $|y|$

Figure 9.10.: $\chi^{2}$ divided by the number of bins as a function of the number of iterations for (a) the transverse momentum of the leading jet and (b) the absolute rapidity of the leading jet for events with at least one jet in the final state. The values have been obtained with SHERPA $\mathrm{MC}$ event samples. Zero iterations correspond to the result from the bin-by-bin method.

In general, the optimal number of iterations obtained with SHERPA and MC@NLO are quite similar, although the generators predict very different kinematics. For the few cases where both generators give different results, the smallest number of both is chosen, in order to reduce the impact from statistical fluctuations. For the transverse momentum and the absolute rapidity distribution of the leading jet the optimal numbers of iterations are chosen to three and two, respectively. 


\subsection{Study of Systematic Uncertainties}

The different sources of systematic uncertainties are studied in detail for each observable presented in this analysis, similar to the analysis with the full dataset of 2010. The different systematic shifts, corresponding to $1 \sigma$, are applied on the predictions from MC event samples and the data-driven background estimate. Uncertainties related to a given background prediction or estimation are only applied to this specific background contribution. The modified background distributions are then subtracted from the data distribution. The resulting background-subtracted data distributions are unfolded to particle level, using the modified migration matrices and correction factors. The difference between the nominal unfolded result and the modified unfolded distributions is taken as systematic uncertainty. The experimental uncertainties are expected to be mainly symmetric for plus and minus $1 \sigma$, therefore the systematic uncertainties are symmetrised in order to mitigate artificial fluctuations due to a lack of statistics in the $\mathrm{MC}$ event samples. The symmetrisation is performed by taking the average of the up and down variation of the different components on the unfolded result. Finally, the different sources of systematic uncertainties are added in quadrature.

\subsubsection{Electron Selection}

The systematic uncertainties coming from the electron selection are estimated in the same way as for the analysis with the full dataset of 2010, as described in Sec. 8.3. Compared to the analysis with the full dataset of 2010 , the uncertainty due to electron identification has been significantly reduced, as described in Sec. 6.1.3. It is now at the same order of magnitude as the uncertainty due to electron reconstruction of $2 \%$.

The different sources of uncertainties are symmetrised and added in quadrature, resulting in an uncertainty of $\sim 2.8 \%$ approximately independent of the jet multiplicity, the jet $p_{T}$ and the jet $y$. The dominant contributions are coming from the electron identification and reconstruction.

\subsubsection{Jet Energy Scale}

As already seen for the measurement with the full dataset of 2010, the measurement is quite sensitive to the systematic uncertainty of the JES.

For the analysis presented in this chapter, the set of 64 uncertainty components, as described in Sec. 6.2.4, has been reduced to 14, by diagonalising the total covariance matrix for the uncertainties related to the different in-situ measurements, separating out the five eigenvectors with the largest contribution and combining the others to a sixth component. It has been checked that a decomposition into more components does not make a difference on the final results [167]. In addition, the uncertainty due to b-jets can be neglected, since the expected fraction of b-jets in this analysis is small. The remaining different uncertainty components are:

- six from the different in-situ measurements

- one from the $\eta$-intercalibration

- one for high $p_{T}$ jets larger than $\sim 1 \mathrm{TeV}$, derived from the single hadron response 
- one for the MC non-closure of the calibration between $\mathrm{MC} 11 \mathrm{c}$ relative to $\mathrm{MC11b}$.

- two from the pile-up offset correction, one for $N_{P V}$ and one for $\mu$

- one for the presence of close-by jets

- two from the flavour composition and response of the samples

The different components are propagated separately for each observable to the final cross section. The total JES uncertainty has been determined by symmetrizing each component and adding them in quadrature. Figure 9.11 shows the different contributions to the total JES uncertainty exemplarily for a few distributions. The leading systematic component of the first six coming from the different in-situ measurements is shown separately, called effective 1, the other five components are shown combined as effective rest. The uncertainty on the JES translates into an uncertainty on the final cross-section measurement of $6.7 \%$ to $16 \%$ for $N_{\text {jet }} \geq 1$ to $N_{\text {jet }} \geq 4$ and of up to $30 \%$ for large values for the jet rapidity and the absolute rapidity difference. The dominant contribution to the JES uncertainty for forward jets is coming from the uncertainty on the $\eta$-intercalibration, while the uncertainty on the high $p_{T}$ jets are dominated by effective1 and the uncertainty on the flavour composition and response. The JES uncertainty still constitutes the dominant contribution to the total systematic uncertainty. 


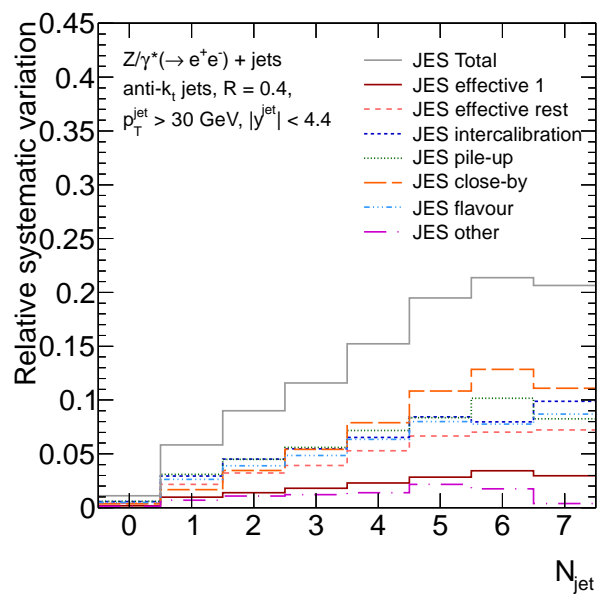

(a) Exclusive jet multiplicity

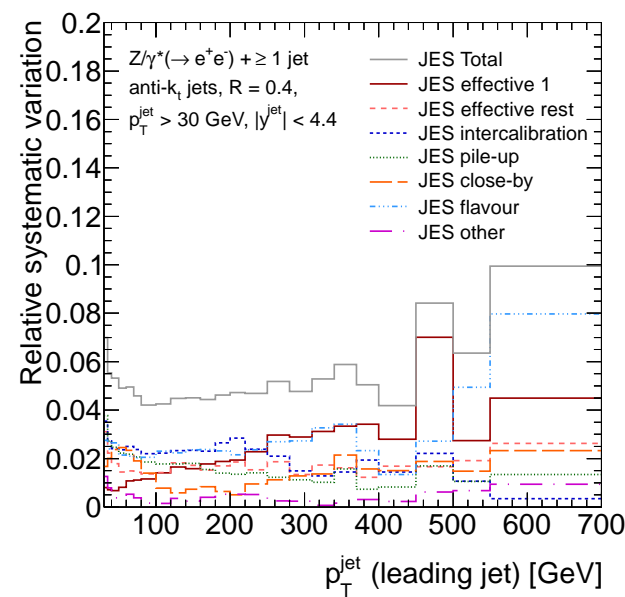

(b) Leading jet $p_{T}$

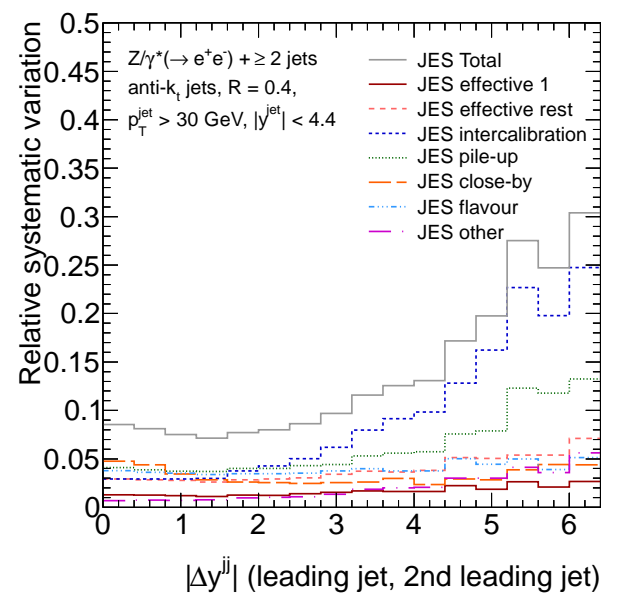

(d) Absolute rapidity difference

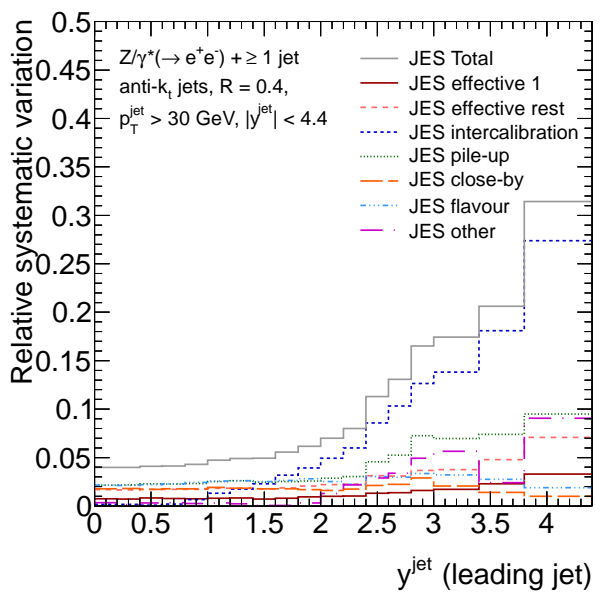

(c) Leading jet $|y|$

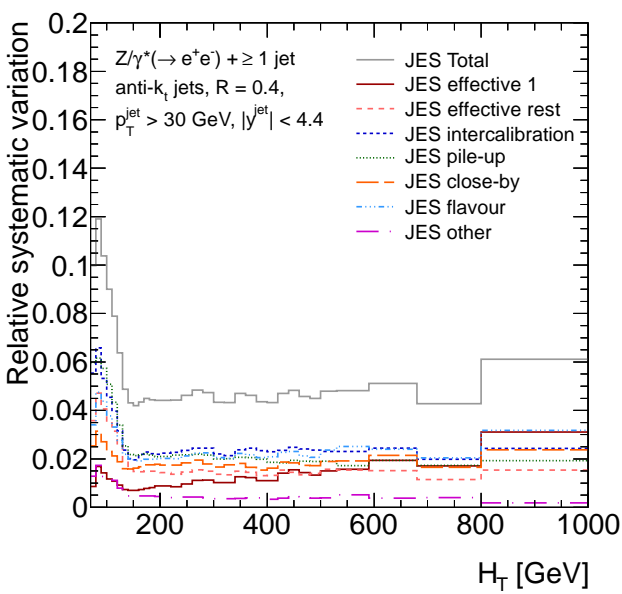

(e) $H_{T}$

Figure 9.11.: Different contributions to the combined relative JES uncertainty for the measured cross section as a function of (a) the exclusive jet multiplicity, (b) the transverse momentum of the leading jet for events with at least one jet in the final state, (c) the absolute rapidity of the leading jet for events with at least one jet in the final state, (d) the absolute rapidity difference of the two leading jets $\left|\Delta y^{j j}\right|$ for events with at least two jets in the final state and (e) $H_{T}$ for events with at least one jet in the final state. The total uncertainty is determined by summing up the different contributions in quadrature. 


\subsubsection{Jet Energy Resolution}

The uncertainty on the JER is propagated to the final cross section by over-smearing the jet energy in the MC event samples within $1 \sigma$ using random numbers. The procedure has been repeated 5 times by modifying the random seed. Finally, the resulting uncertainties are symmetrised and the average is taken as a systematic uncertainty. This translates into an uncertainty at the order of a few percent $(3 \%-4 \%)$ for the inclusive jet multiplicity.

\subsubsection{Background Modelling and Normalisation}

The uncertainties on the background predictions related to background modelling and normalisation are treated similarly to the ones in the analysis with the full dataset of 2010, as described in Sec. 8.3 .

For this analysis the multi-jets and the $t \bar{t}$ background have been estimated from data, as described in Sec. 7.2. The uncertainty on the normalisation of the data-driven estimates of the $t \bar{t}$ and the multi-jets background translates into an uncertainty of $0.015 \%$ to $0.37 \%$ and of $0.13 \%$ to $0.33 \%$ on the inclusive jet multiplicity for $N_{\text {jet }} \geq 1$ to $N_{\text {jet }} \geq 4$, respectively. The cross section of the single top and the EW backgrounds are varied within their corresponding normalisation uncertainties, accounting for PDF uncertainties, uncertainties due to the choice of $\alpha_{s}$ and scale uncertainties on the NNLO predictions. Since the fractions from these background contributions are rather small, the resulting uncertainty on the final cross section is less than $1 \%$.

The statistical uncertainties from the background predictions are added in quadrature to the statistical uncertainty in data. They are propagated simultaneously to the final cross section.

\subsubsection{Unfolding}

For the systematic uncertainty on the unfolding three uncorrelated components are considered, similar to the analysis with the full dataset of 2010 .

The unfolding has been repeated twice to account for the potentially insufficient modelling of jet kinematics and composition and to account for variations of the final results due to different unfolding techniques and method implementations, once using the iterative (Bayes) method based on the SHERPA signal MC event sample and once using the bin-by-bin method based on the ALPGEN+HERWIG signal MC event sample.

The third component of the systematic uncertainty accounts for the limited statistics of the MC event samples. This uncertainty is propagated to the particle level distribution using toy MC. Each bin of the migration matrices is fluctuated independently according to a Gaussian distribution 1000 times and the correction factors are recalculated. The RMS of the different unfolded results are taken as systematic uncertainty.

The first two components are highly influenced by statistical fluctuations in some phasespace regions, therefore the difference between the nominal unfolded results and the modified results $\mathcal{D}^{k}\left(\mathcal{O}_{i}\right)$ with respect to the nominal results are smoothed using a Gaussian 
kernel separately for each observable $\mathcal{O}$

$$
\begin{aligned}
\tilde{\mathcal{D}}^{k}\left(\mathcal{O}_{i}\right) & =\frac{\sum_{j=1}^{N_{\text {bins }}} \mathcal{D}^{k}\left(\mathcal{O}_{j}\right) \cdot \mathcal{W}\left(\mathcal{O}_{i}, \mathcal{O}_{j}\right)}{\sum_{j=1}^{N_{\text {bins }}} \mathcal{W}\left(\mathcal{O}_{i}, \mathcal{O}_{j}\right)} \\
\mathcal{W}\left(\mathcal{O}_{i}, \mathcal{O}_{j}\right) & =w\left(\mathcal{O}_{j}\right) \times \operatorname{Gauss}\left(\frac{S\left(\mathcal{O}_{i}\right)-S\left(\mathcal{O}_{j}\right)}{\sigma_{\mathcal{O}}}\right) .
\end{aligned}
$$

Here, $\tilde{\mathcal{D}}^{k}\left(\mathcal{O}_{i}\right)$ is the smoothed result of the systematic source $k$ for bin $i$. The width of the Gaussian kernel is $\sigma_{\mathcal{O}}$, which has been chosen such that the smoothed curve provides a good and stable description of the shape with respect to the statistical fluctuations. $\sigma_{\mathcal{O}}$ is typically between 0.2 and 0.8 . The function $S$ is used to change the scale on which $\mathcal{O}$ is evaluated. For $p_{T}$-like observables $S$ is the natural logarithm of $\mathcal{O}$, whereas for the other observables $S$ is the identity. The $w\left(\mathcal{O}_{j}\right)$ describe a set of weights with $\sum_{j=1}^{N_{\text {bins }}} w\left(\mathcal{O}_{j}\right)=1$, which are calculated from the statistical uncertainties $\delta \sigma\left(\mathcal{O}_{j}\right)$ on the nominal unfolded results:

$$
w\left(\mathcal{O}_{j}\right)=\frac{1 /\left(\delta \sigma\left(\mathcal{O}_{j}\right)\right)^{2}}{\sum_{j=1}^{N_{\text {bins }}} 1 /\left(\delta \sigma\left(\mathcal{O}_{j}\right)\right)^{2}} .
$$

Figure 9.12 and Fig. 9.13 exemplarily show the smoothed results for the transverse momentum and the absolute rapidity distribution of the leading jet for events with at least one jet in the final state.

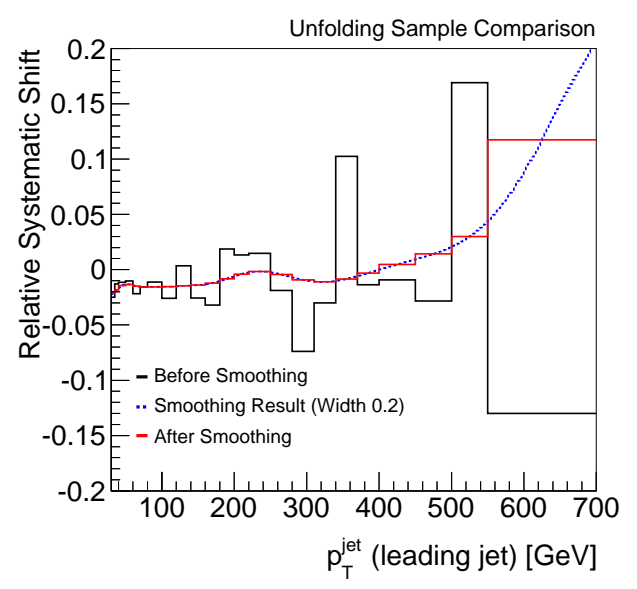

(a) Leading jet $p_{T}$

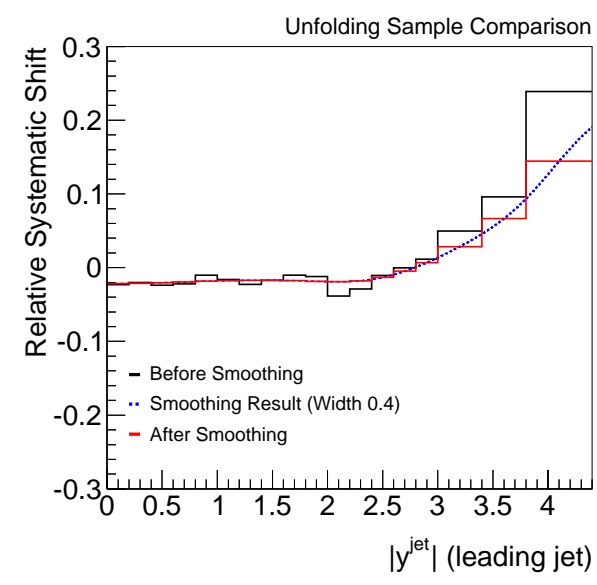

(b) Leading jet $|y|$

Figure 9.12.: Relative systematic shifts on the unfolded cross section obtained by comparing the unfolding procedure using ALPGEN+HERWIG or SHERPA for (a) the transverse momentum of the leading jet and (b) the absolute rapidity of the leading jet for events with at least one jet in the final state. The relative shifts are smoothed using a Gaussian kernel.

Finally, the three different sources of systematic uncertainties are symmetrised and added in quadrature, resulting in an uncertainty of $0.22 \%$ to $1.9 \%$ on the inclusive jet multiplicity for $N_{\text {jet }} \geq 1$ to $N_{\text {jet }} \geq 4$ and up to $6.8 \%$ as $p_{T}^{\text {jet }}$ increases. The dominant contribution to this uncertainty comes from the choice of the $\mathrm{MC}$ event sample. The uncertainty coming from the choice of the unfolding method, even if it is not dominant, is expected to be too conservative, since the bin-by-bin method fully depends on the MC 


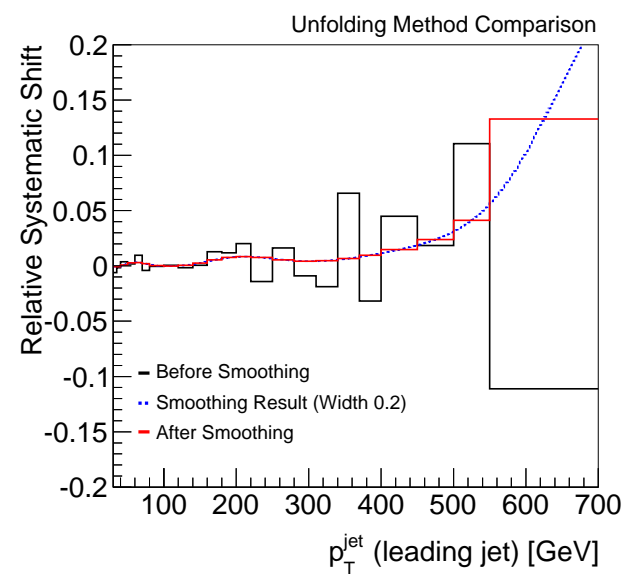

(a) Leading jet $p_{T}$

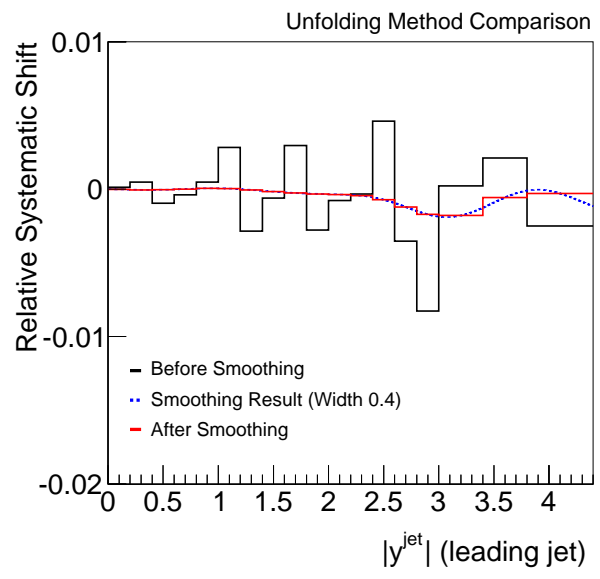

(b) Leading jet $|y|$

Figure 9.13.: Relative systematic shifts on the unfolded cross section obtained by comparing the results obtained with iterative (Bayes) method and bin-by-bin method for (a) the transverse momentum of the leading jet and (b) the absolute rapidity of the leading jet for events with at least one jet in the final state. The relative shifts are smoothed using a Gaussian kernel.

predictions and therefore does not provide a valuable systematic uncertainty. For future analyses this contribution will be neglected and more refined methods will be used. A comparison with the bin-by-bin method will only be used as a cross check.

\subsubsection{Total Systematic Uncertainty}

Table 9.2 summarises the different components of the total systematic uncertainty on the measured cross section for several inclusive jet multiplicities.

\begin{tabular}{|c|c|c|c|c|c|}
\hline \hline & $\geq 0$ jets & $\geq 1$ jet & $\geq 2$ jets & $\geq 3$ jets & $\geq 4$ jets \\
\hline \hline Electron Trigger & $0.28 \%$ & $0.40 \%$ & $0.45 \%$ & $0.50 \%$ & $0.54 \%$ \\
Electron Energy Scale & $0.14 \%$ & $0.22 \%$ & $0.21 \%$ & $0.21 \%$ & $0.25 \%$ \\
Electron Energy Resolution & $0.0073 \%$ & $0.011 \%$ & $0.015 \%$ & $0.012 \%$ & $0.030 \%$ \\
Electron Reconstruction & $1.6 \%$ & $1.6 \%$ & $1.6 \%$ & $1.6 \%$ & $1.6 \%$ \\
Electron ID & $2.2 \%$ & $2.3 \%$ & $2.3 \%$ & $2.3 \%$ & $2.2 \%$ \\
Jet Energy Scale & - & $6.7 \%$ & $9.7 \%$ & $13 \%$ & $16 \%$ \\
Jet Energy Resolution & - & $3.1 \%$ & $2.6 \%$ & $3.3 \%$ & $3.7 \%$ \\
Multi-jets Background & $0.13 \%$ & $0.25 \%$ & $0.29 \%$ & $0.31 \%$ & $0.33 \%$ \\
$t \bar{t}$ Background & $0.015 \%$ & $0.053 \%$ & $0.17 \%$ & $0.29 \%$ & $0.37 \%$ \\
EW Background & $0.0087 \%$ & $0.036 \%$ & $0.070 \%$ & $0.072 \%$ & $0.051 \%$ \\
Single Top Background & $0.0009 \%$ & $0.0055 \%$ & $0.010 \%$ & $0.012 \%$ & $0.010 \%$ \\
Unfolding Method & $0.0043 \%$ & $0.012 \%$ & $0.34 \%$ & $0.89 \%$ & $0.38 \%$ \\
Unfolding Sample & $0.49 \%$ & $0.16 \%$ & $0.85 \%$ & $0.77 \%$ & $1.7 \%$ \\
Unfolding Stat & $0.49 \%$ & $0.14 \%$ & $0.21 \%$ & $0.42 \%$ & $0.84 \%$ \\
\hline \hline Total & $2.8 \%$ & $7.9 \%$ & $10.5 \%$ & $13.4 \%$ & $16.9 \%$ \\
\hline \hline
\end{tabular}

Table 9.2.: Different components of the total systematic uncertainty on the measured cross section for several inclusive jet multiplicities. 
The dominant systematic uncertainty on the inclusive $Z / \gamma^{*}(\rightarrow e e)$ selection comes from the electron identification and reconstruction, while the measurement of the production cross section of $Z / \gamma^{*}(\rightarrow e e)$ associated with jets is dominated by JER and JES.

In addition, Fig. 9.14 presents the detailed study of the systematic uncertainties for the cross section as a function of the exclusive jet multiplicity, the transverse momentum and the absolute rapidity of the leading jet for events with at least one jet in the final state, the absolute rapidity difference of the two leading jets $\left|\Delta y^{j j}\right|$ for events with at least two jets in the final state and $H_{T}$ for events with at least one jet in the final state. The dominant contribution to the total systematic uncertainty comes from JES, except for a few regions, e.g. high $p_{T}$ or large values of $\left|\Delta y^{j j}\right|$ where the systematic contribution from the unfolding is compatible with the JES uncertainty or is even larger. Further distributions for the study of the systematic uncertainties can be found in Ref. [167].

Furthermore, the correlations between bins for the different sources of systematic uncertainties are computed, which are of great importance especially for the combination of the measurements from the electron and muon channel. The results of the combination can be found in Ref. [8]. The correlations are calculated using the following formula:

$$
\rho(i, j)=\frac{\operatorname{cov}(i, j)}{\sigma_{i} \sigma_{j}}
$$

with

$$
\operatorname{cov}(i, j)=\sum_{k} s_{i}^{k} \times s_{j}^{k}
$$

Here, $\rho(i, j)$ describes the correlation coefficient between bin $i$ and $j, \operatorname{cov}(i, j)$ describes the entry of the covariance matrix for bin $i$ and $j$ and $\sigma_{i, j}$ are the total uncertainties of bin $i$ and $j$. The covariance matrix is calculated by summing over all systematic sources $k$, except for the systematic uncertainty coming from the limited statistics of the MC event samples used for unfolding, which is assumed to be fully uncorrelated between bins. The $s_{i, j}^{k}$ describe the relative systematic shifts of the component $k$ for bin $i$ and $j$. Figure 9.15 exemplarily shows the correlation matrices for a few selected observables. The values of the correlation coefficients are driven by the relative size and the shape of each systematic component. For example, the fact that the JES uncertainty increases with increasing exclusive jet multiplicity starting from zero for the zero jet bin anti-correlates the zero jet bin to the other jet bins and correlates all the other jet bins.

Similar to the systematic uncertainties, the uncertainty on the total integrated luminosity of $1.8 \%$ [101] is propagated through the unfolding procedure.

The total systematic uncertainty is added in quadrature to the statistical uncertainty of the measurement and to the uncertainty on the total integrated luminosity. 


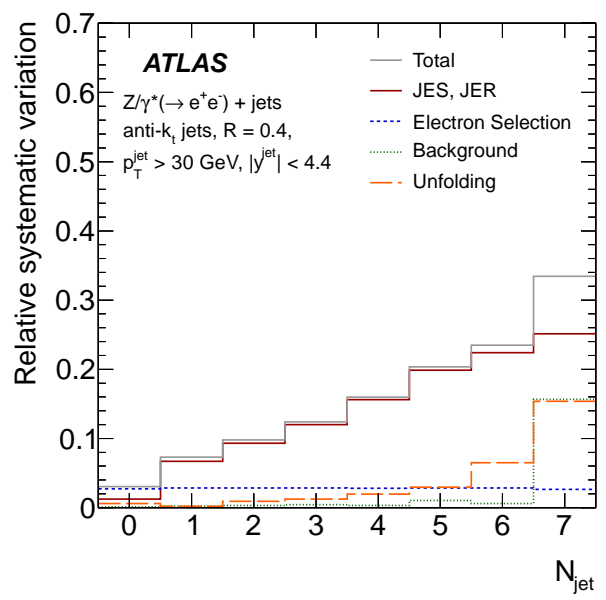

(a) Exclusive jet multiplicity

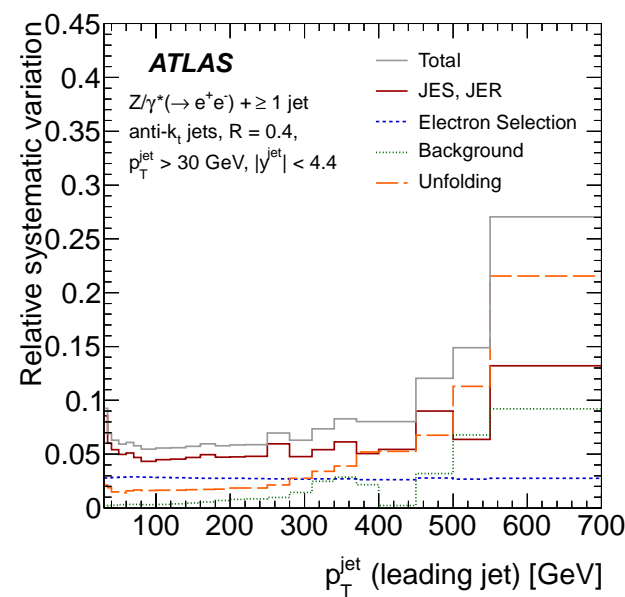

(b) Leading jet $p_{T}$

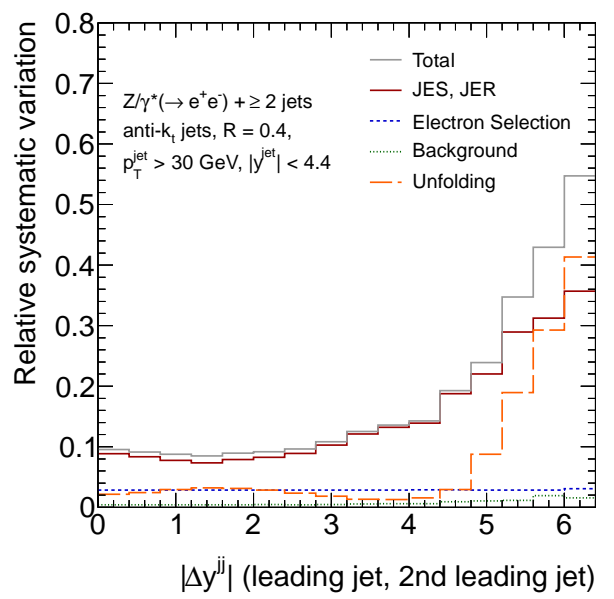

(d) Absolute rapidity difference

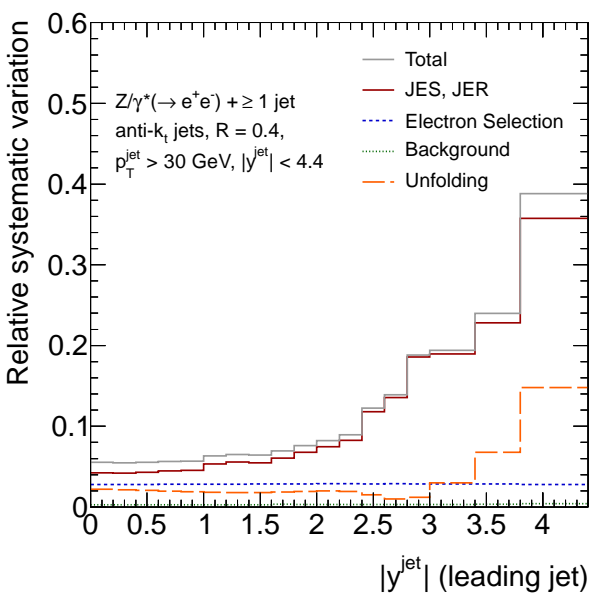

(c) Leading jet $|y|$

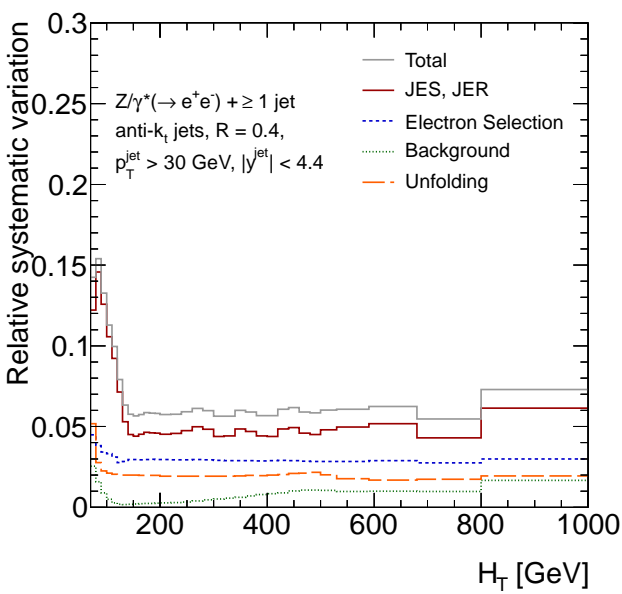

(e) $H_{T}$

Figure 9.14.: Different contributions to the combined relative systematic uncertainty for the measured cross section as a function of (a) the exclusive jet multiplicity, (b) the transverse momentum of the leading jet for events with at least one jet in the final state, (c) the absolute rapidity of the leading jet for events with at least one jet in the final state, (d) the absolute rapidity difference of the two leading jets $\left|\Delta y^{j j}\right|$ for events with at least two jets in the final state and (e) $H_{T}$ for events with at least one jet in the final state. The total systematic uncertainty is determined by summing up the different contributions in quadrature. 


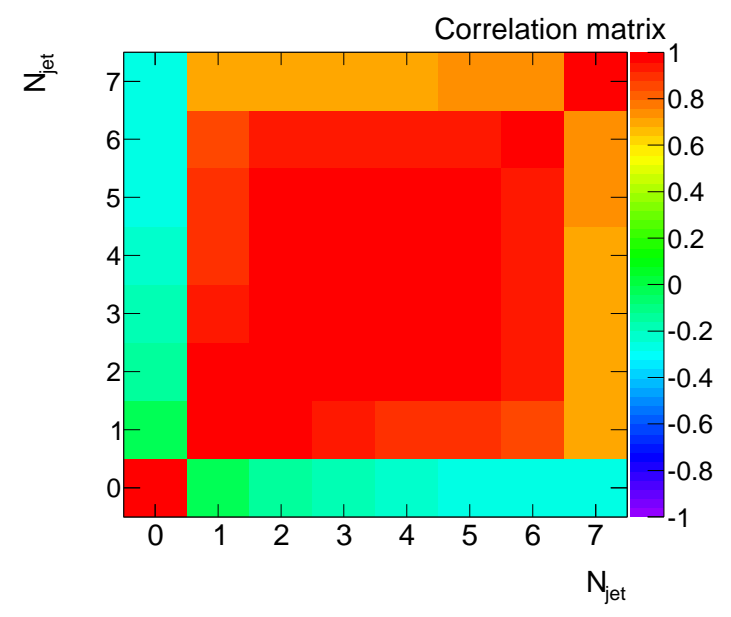

(a) Exclusive jet multiplicity

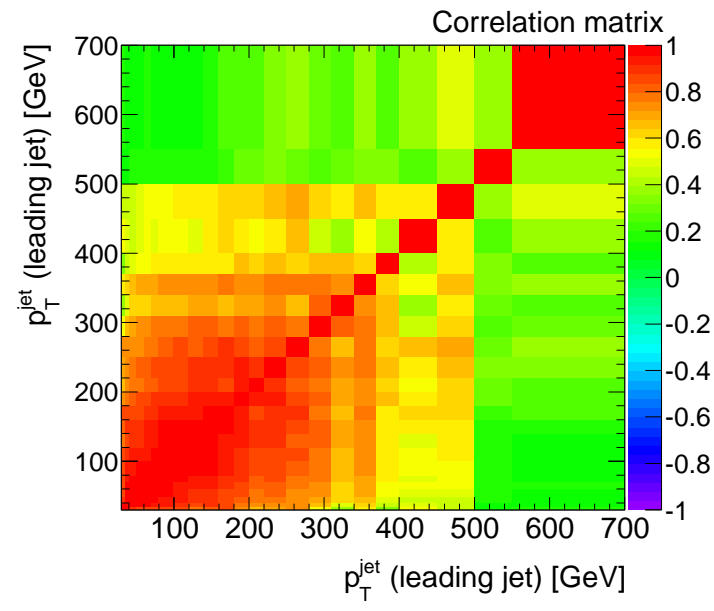

(b) Leading jet $p_{T}$

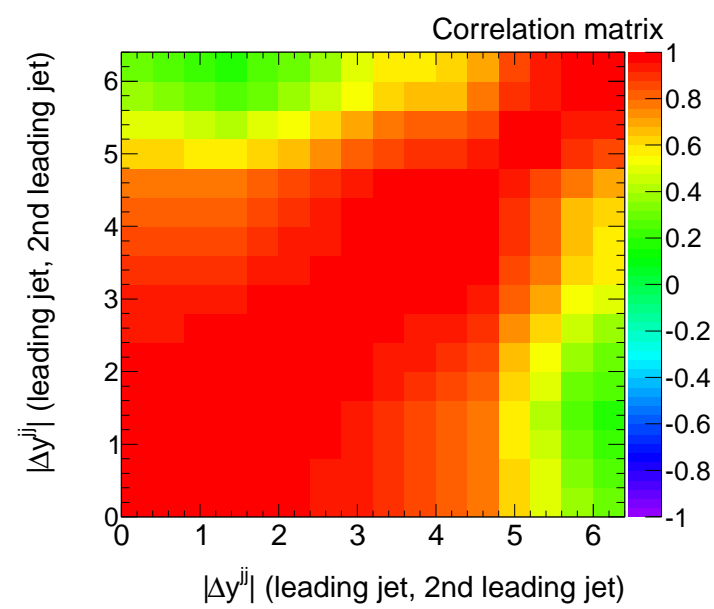

(d) Absolute rapidity difference

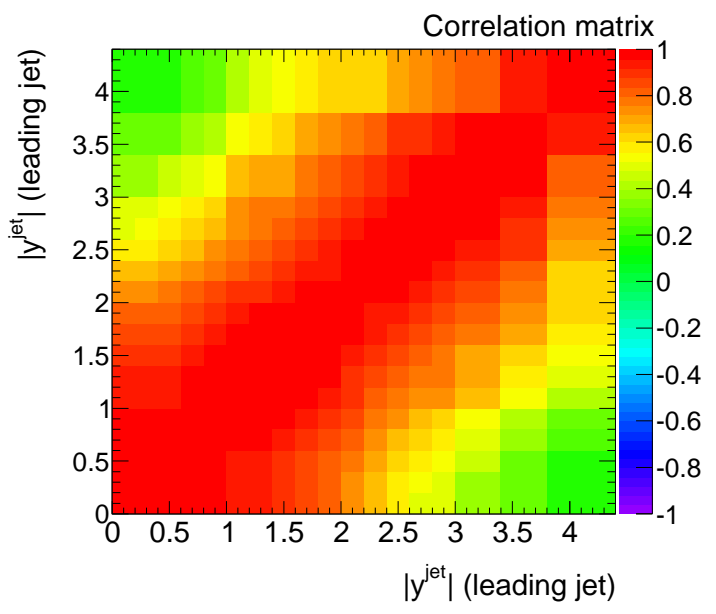

(c) Leading jet $|y|$

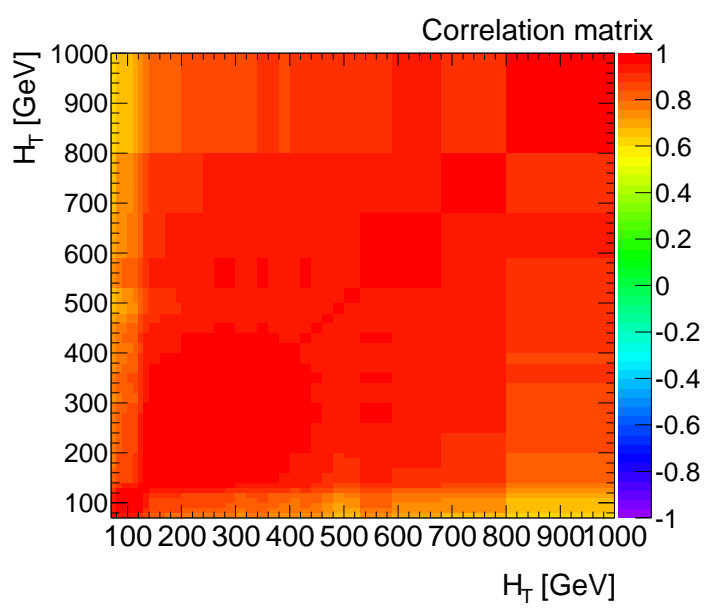

(e) $H_{T}$

Figure 9.15.: Correlation matrices for the measured cross section as a function of (a) the exclusive jet multiplicity, (b) the transverse momentum of the leading jet for events with at least one jet in the final state, (c) the absolute rapidity of the leading jet for events with at least one jet in the final state, (d) the absolute rapidity difference of the two leading jets $\left|\Delta y^{j j}\right|$ for events with at least two jets in the final state and (e) $H_{T}$ for events with at least one jet in the final state. 


\subsection{Theory Corrections}

For the extraction from parton level to particle level, the NLO fixed-order pQCD predictions are corrected for QED FSR and non-perturbative effects, such as UE and fragmentation. The UE correction also accounts for events originating from DPI. Both corrections are determined using MC event samples. The corresponding uncertainties are added in quadrature to the other theoretical uncertainties. A detailed description of both corrections can be found in the following two subsections.

\subsubsection{Corrections for Non-perturbative Effects}

The correction for non-perturbative effects is performed on a bin-by-bin basis separately for each observable similarly to the one for the 2010 analysis, as described in Sec. 8.4.1. For 2011, the statistics of the MC event sample used to determine the corrections are significantly increased. In addition, new UE tunes are used.

The nominal correction is determined using ALPGEN+HERWIG+JIMMY MC event samples with the HERWIG cluster fragmentation and the AUET2-CTEQ6L1 tune.

Systematic uncertainties on this correction are obtained from the comparison with ALPGEN+PYTHIA MC event samples using string fragmentation and the PERUGIA2011 tune.

Figure 9.16 to Fig. 9.18 show the correction for the exclusive jet multiplicity, the transverse momentum and the rapidity of the leading jet for events with at least one jet in the final state, illustrating the different components, which are combined in the final correction. Similar to the 2010 analysis, the separate correction factors for fragmentation and UE are only presented to approximately show the effects of the different components, for the final correction the combined correction factors are used. Fragmentation leads to an energy loss in the jet reconstruction due to increased out-of-cone showering, which results in a decreased number of jets passing the $p_{T}$ threshold. In contrast, UE increases the average jet energy, resulting in an increased number of jets passing the $p_{T}$ threshold. The influence from both effects is larger at low $p_{T}$ and high $y$, since low energetic jets are wider and the fraction of jet energy provided by the UE is larger.

The combined fragmentation and UE correction decreases with increasing $p_{T}$, starting with a correction of $7 \%$ towards no correction for the high- $p_{T}$ regime. The correction determined with ALPGEN+PYTHIA compared to ALPGEN+HERWIG+JIMMY is slightly larger for low $p_{T}$. This can be attributed to the fact that PYTHIA predicts a larger amount of UE compared to HERWIG+JIMMY as shown in Fig. 9.18(b). For large values of $p_{T}^{\text {jet }}$ the correction factors are highly influenced by statistical fluctuations due to the limited statistics of the MC event samples, therefore a linear fit in this regime is performed to determine the final correction.

The correction with respect to the jet rapidity is flat in the region $-3.0<y<3.0$ at $\sim 5 \%$ for both ALPGEN+HERWIG and ALPGEN+PYTHIA MC event samples, whereas for large values of $y$ the predictions differ. The correction determined with ALPGEN+HERWIG increases to $\sim 35 \%$, while the correction determined using ALPGEN+PYTHIA remains flat. This difference is fully propagated into the systematic uncertainty of this correction and can be explained by the fact that HERWIG + JIMMY with the AUET2 tune predicts larger UE corrections compared to PYTHIA with the PERUGIA2011c tune. 


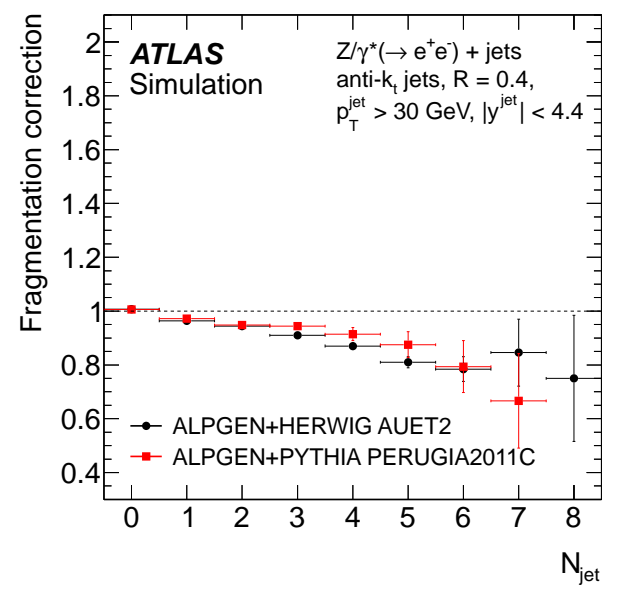

(a) Fragmentation correction

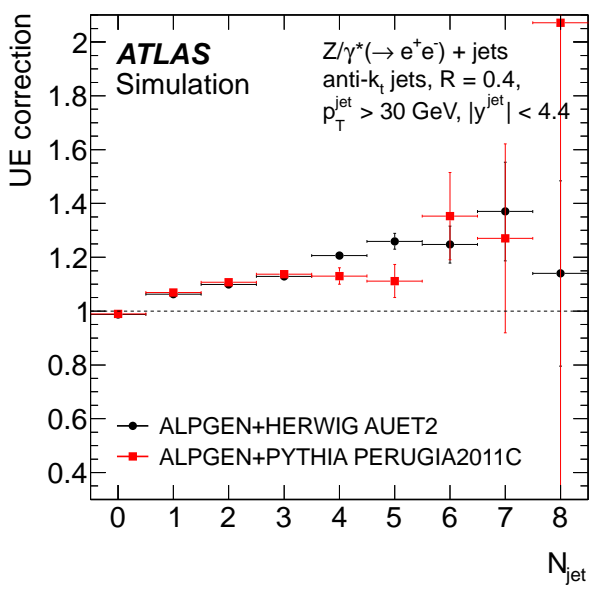

(b) UE correction

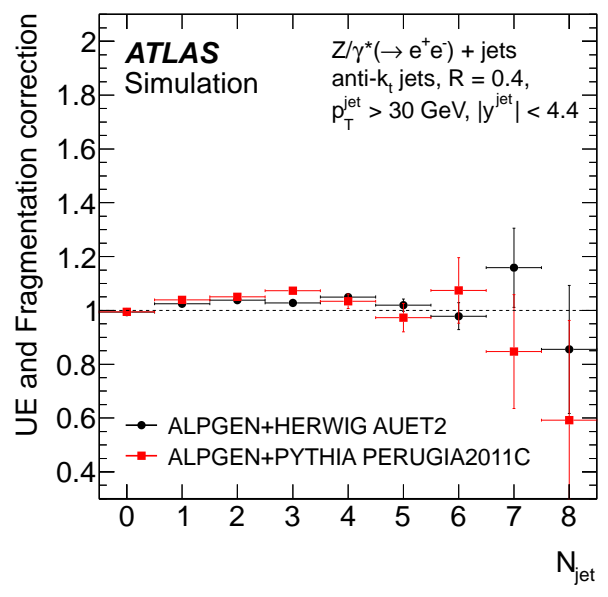

(c) Combined correction

Figure 9.16.: (a) Fragmentation correction, (b) UE correction and (c) combined fragmentation and UE correction for the exclusive jet multiplicity. 


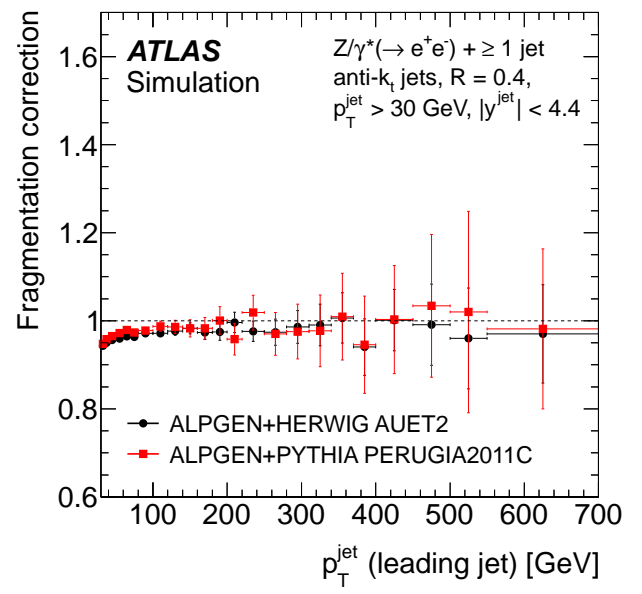

(a) Fragmentation correction

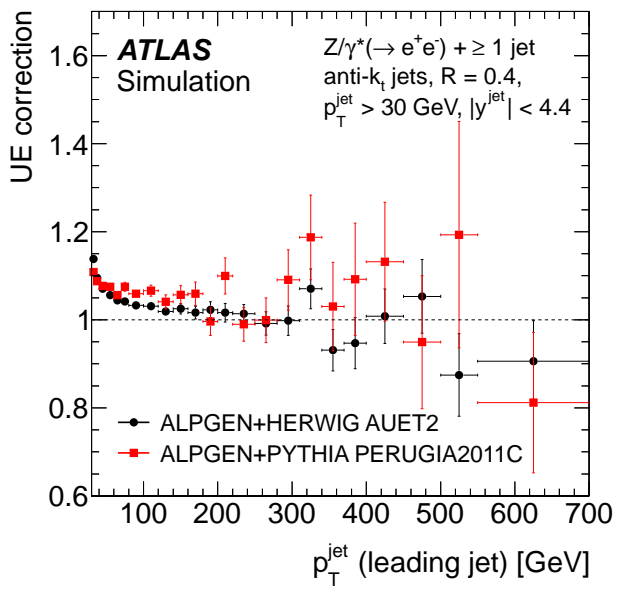

(b) UE correction

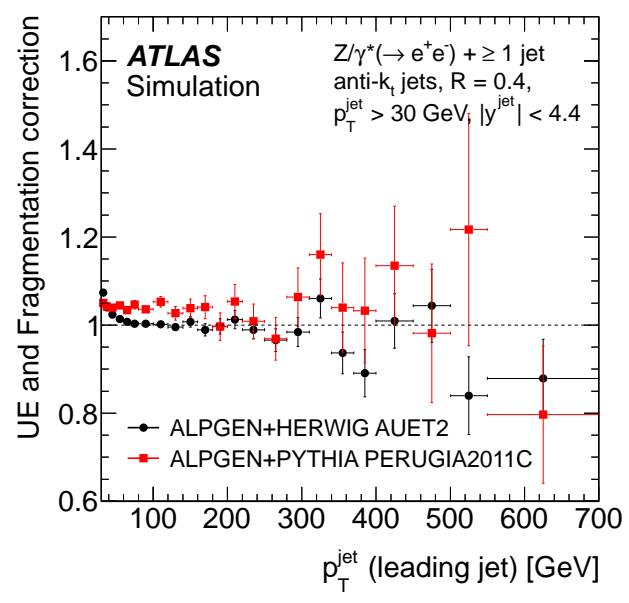

(c) Combined correction

Figure 9.17.: (a) Fragmentation correction, (b) UE correction and (c) combined fragmentation and UE correction for the transverse momentum of the leading jet for events with at least one jet in the final state. 


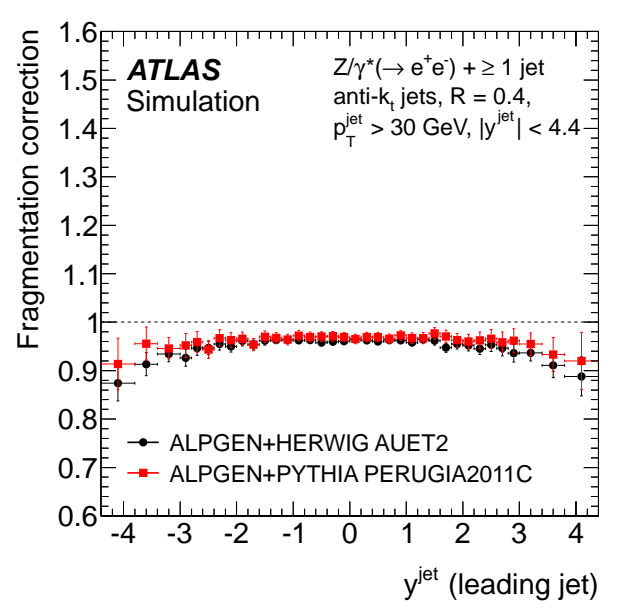

(a) Fragmentation correction

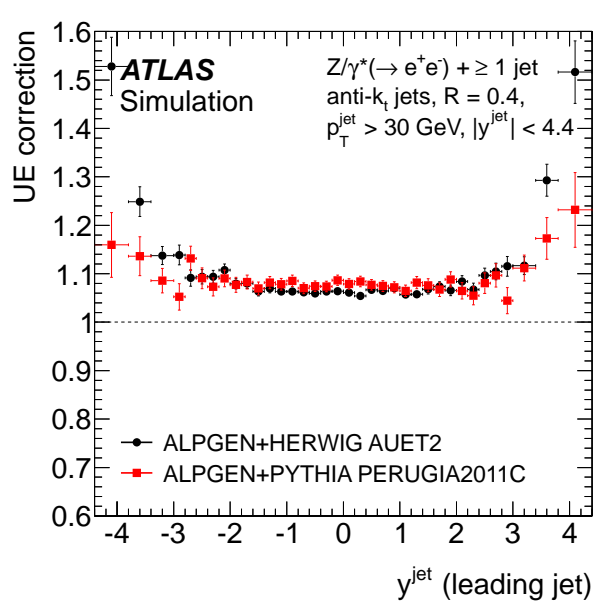

(b) UE correction

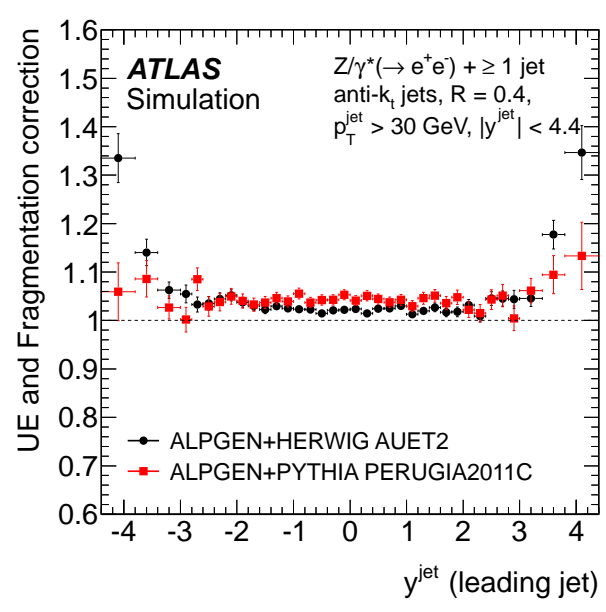

(c) Combined correction

Figure 9.18.: (a) Fragmentation correction, (b) UE correction and (c) combined fragmentation and UE correction for the rapidity of the leading jet for events with at least one jet in the final state. 
Figure 9.19 shows the combined fragmentation and UE correction for the absolute rapidity difference of the two leading jets for events with at least two jets in the final state and $H_{T}$ in events with at least one jet in the final state.

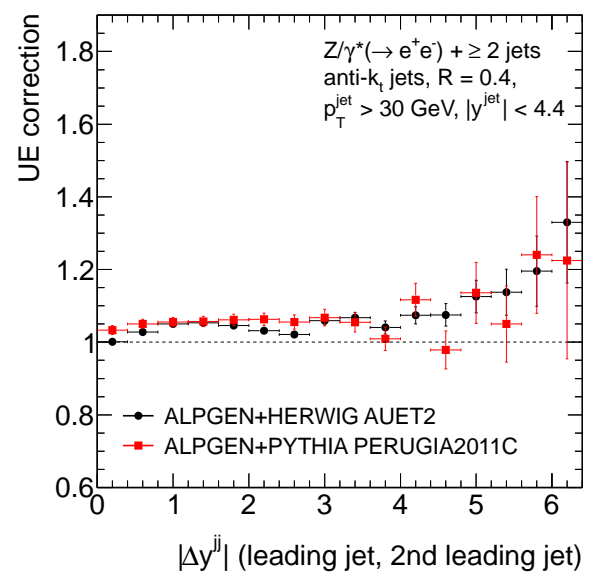

(a) Absolute rapidity difference

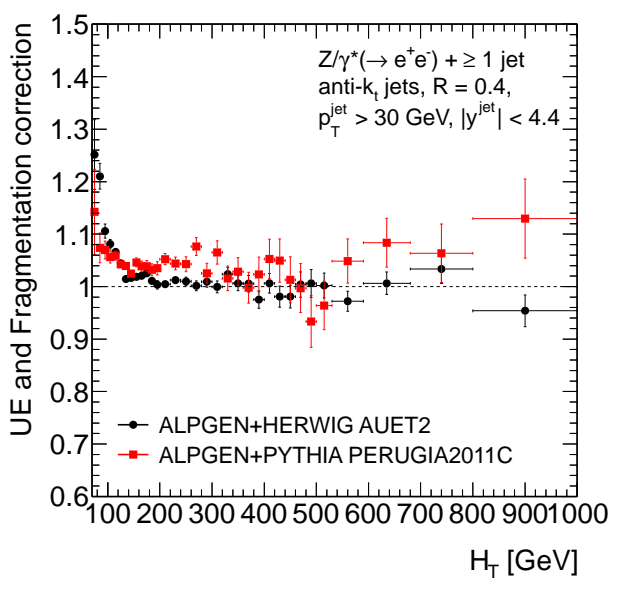

(b) $H_{T}$

Figure 9.19.: Combined fragmentation and UE correction for (a) the absolute rapidity difference of the two leading jets for events with at least two jets in the final state and (b) $H_{T}$ in events with at least one jet in the final state.

While the corrections determined from ALPGEN+HERWIG and ALPGEN+PYTHIA clearly differ for the rapidity distribution, both generators predict similar corrections for the absolute rapidity distance between the two leading jets. Fragmentation and UE lead to a migration of the jets in $p_{T}$, therefore the number of jets included in $H_{T}$ varies. Large values of $H_{T}$ can result from a large number of jets or a small number of very hard jets, for both the influence of UE and fragmentation is expected to be small as confirmed in Fig. 9.19(b), For large values of $H_{T}$ the correction factors are highly influenced by statistical fluctuations similar to $p_{T}$ of the leading jet. Therefore, the final correction is also determined from a linear fit in this regime.

\subsubsection{Corrections for QED Final State Radiation}

The correction for QED FSR is performed in the same way as for the analysis with the full dataset of 2010, as described in Sec. 8.4.2. The nominal correction is derived using ALPGEN+HERWIG MC event samples interfaced to PHOTOS for photon radiation. Figure 9.20 exemplarily shows the QED FSR correction for a few observables. The correction is approximately 0.98 and is nearly independent of the jet multiplicity and jet kinematics. In addition, the correction from Born level to the level of bare final-state leptons is shown, which demonstrates that the dressing of leptons with photons brings the final state leptons closer to the initial state leptons. The size of the correction for low values of $H_{T}$ refers to the influence from QED radiation on the electron kinematics.

For systematic studies, the corrections obtained with ALPGEN+HERWIG MC event samples are compared to the corrections derived with SHERPA MC event samples, which uses the YFS [129] approach to model QED FSR, as described in Sec. 4.4. The differences 


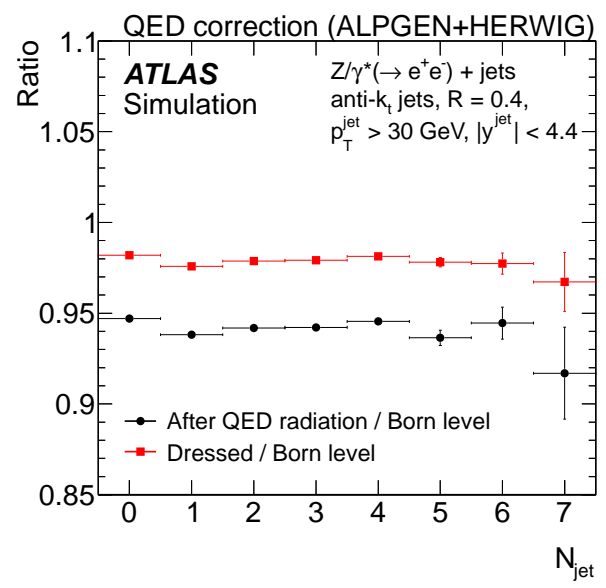

(a) Exclusive jet multiplicity

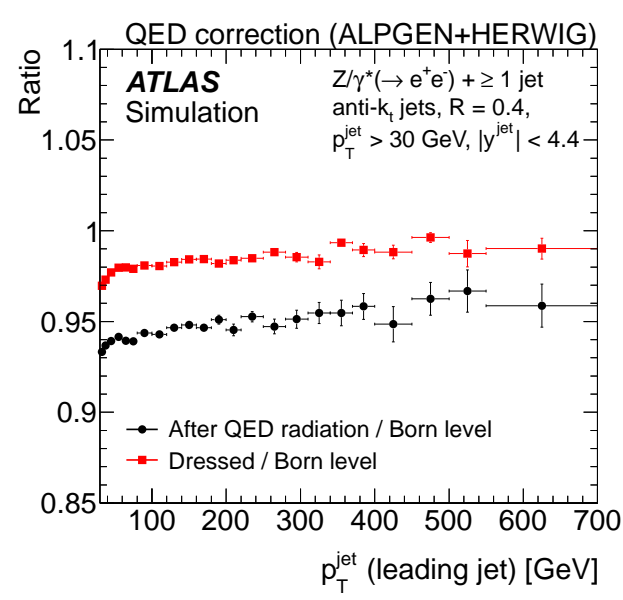

(b) Leading jet $p_{T}$

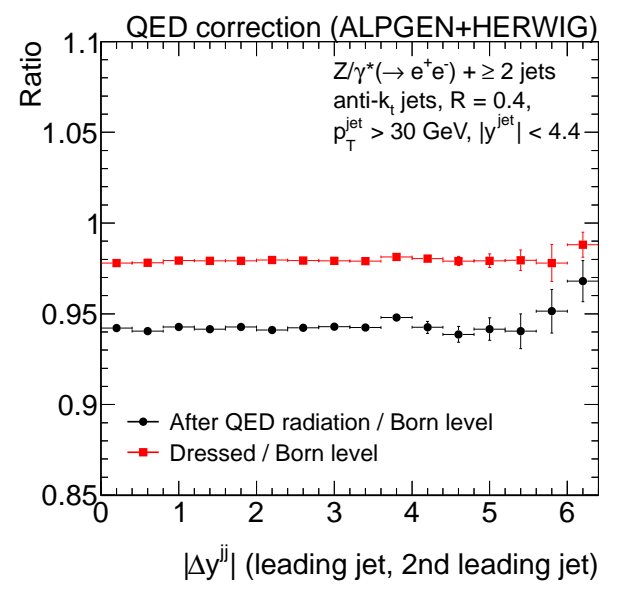

(d) Absolute rapidity difference

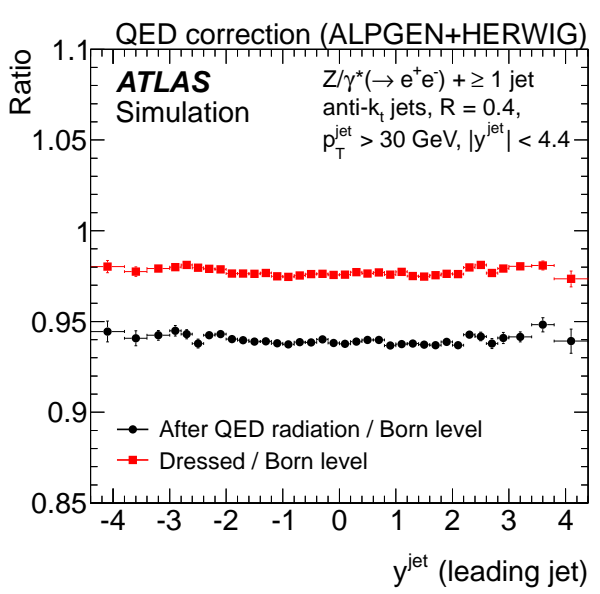

(c) Leading jet $y$

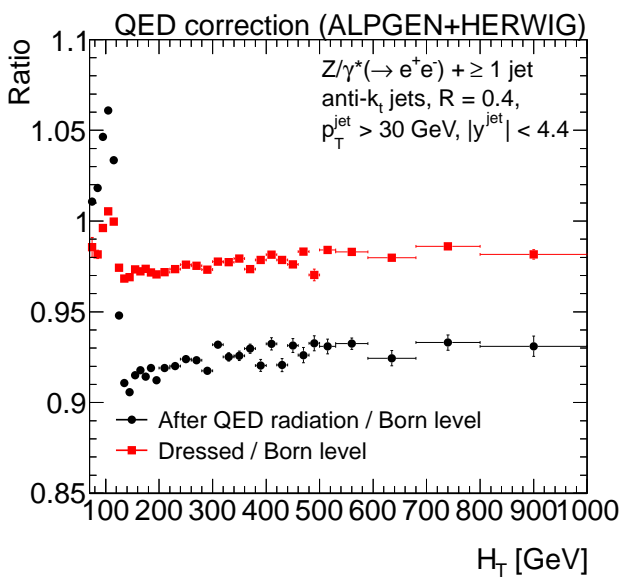

(e) $H_{T}$

Figure 9.20.: QED FSR correction as a function of (a) the exclusive jet multiplicity, (b) the transverse momentum of the leading jet for events with at least one jet in the final state, (c) the rapidity of the leading jet for events with at least one jet in the final state, (d) the absolute rapidity difference of the two leading jets $\left|\Delta y^{j j}\right|$ for events with at least two jets in the final state and (e) $H_{T}$ for events with at least one jet in the final state. 
between the corrections obtained with ALPGEN+HERWIG and SHERPA are at the permille level.

\subsection{Results}

This section presents the final results of the measurement with the full dataset of 2011 . Similar to the analysis with the full dataset of 2010, the measured distributions depicted in Sec. 9.1 are unfolded to particle level taking into account the systematic uncertainties discussed in Sec. 9.3. Predictions from NLO fixed-order pQCD calculations, corrected for non-perturbative effects and QED radiation effects, as described in Sec. 9.4, as well as predictions from ALPGEN+HERWIG, SHERPA and MC@NLO using the ATLAS configurations, as detailed in Sec. 7.2, are compared to the measured cross sections. In addition, the predictions from ALPGEN+PYTHIA are compared to the measured cross section for a few selected observables. ALPGEN+HERWIG, ALPGEN+PYTHIA and SHERPA include LO matrix elements with up to five partons, while higher jet multiplicities are modelled by parton shower, as detailed in Sec. 7.2.1. In contrast, MC@NLO generates the DY process at NLO, which includes the real emission of one additional parton. Events with more than one jet in the final state are modelled by parton shower. Inclusive and differential cross sections for $Z / \gamma^{*}(\rightarrow e e)+\geq \mathrm{n}$ jets are compared to the corresponding NLO fixed-order pQCD calculation for $Z+\geq n$ jets from BLACKHAT+SHERPA.

The figures in this section are organized such that they show the absolute or normalised cross sections in the upper part, together with the ratios BLACKHAT+SHERPA/data, ALPGEN/data and SHERPA/data in the lower three parts. The predictions from ALPGEN+HERWIG, SHERPA and MC@NLO have been normalised to the inclusive NNLO cross section with global $K$-factors. Theoretical uncertainties are shown separately from the total combined statistical and systematic uncertainty on the measurement. The hatched uncertainty bands in the figures correspond to the total systematic and statistical uncertainty, added in quadrature, whereas the shaded bands represent the theoretical uncertainties on the respective predictions.

\subsubsection{Inclusive Jet Multiplicity}

Figure 9.21(a) shows the inclusive cross section as a function of the jet multiplicity up to seven jets. Final states with more than five jets are accessible for the first time. The predictions from ALPGEN+HERWIG and SHERPA include a 5\% uncertainty from the normalisation to the NNLO pQCD inclusive DY cross section, as detailed in Sec. 7.2.1, and the theoretical uncertainties on the predictions from BLACKHAT+SHERPA are described in Sec. 7.3. As expected the measured cross section decreases with increasing jet multiplicity. The NLO fixed-order pQCD predictions from BLACKHAT+SHERPA show a good description of the measured distribution in data. Predictions from ALPGEN+HERWIG and SHERPA are consistent with the measured distribution in data, whereas MC@NLO fails to describe the data beyond the first jet emission. This mismodelling is attributed to the HERWIG parton shower, since for MC@NLO the associated first jet corresponds to the real emission term of the NLO calculation and events with more than one jet in the final state are modelled by parton shower. Therefore, MC@NLO is only shown for a few example distributions in the following. Detailed values of the measured cross section, as 


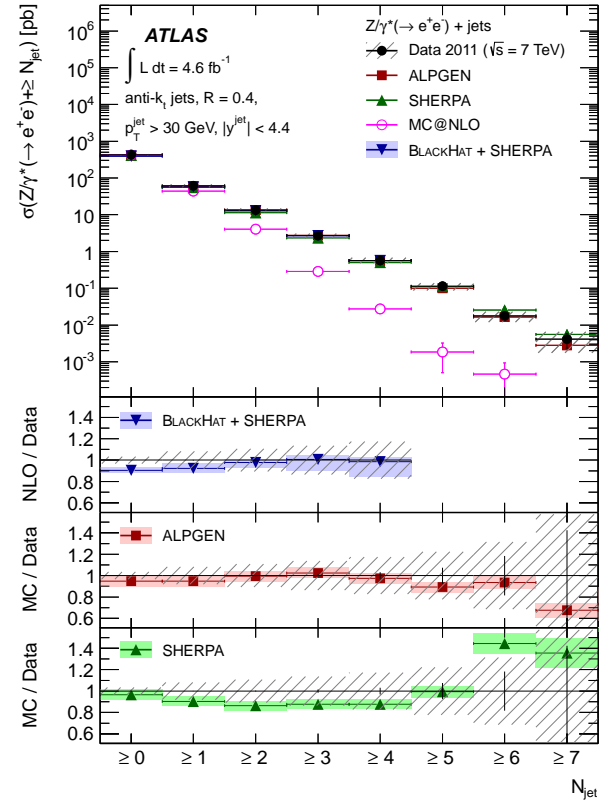

(a) Inclusive jet multiplicity

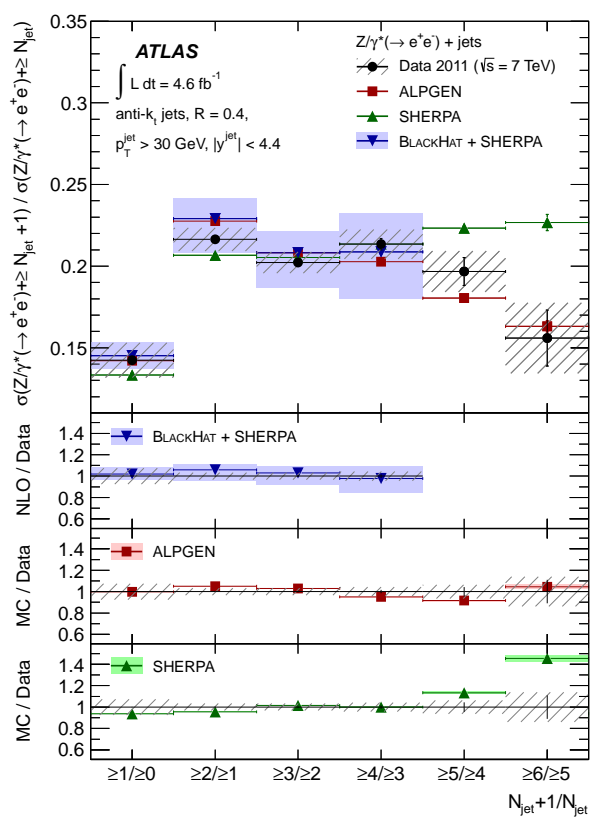

(b) Inclusive jet multiplicity ratio

Figure 9.21.: (a) Measured cross section as a function of the inclusive jet multiplicity and (b) the ratio of cross sections for successive inclusive jet multiplicities. The measured values are represented by the black dots, the error bars indicate the statistical uncertainty and the hatched band incorporates the total statistical and systematic uncertainty added in quadrature. The measurements are compared to predictions from ALPGEN+HERWIG and SHERPA event generators, as well as to NLO pQCD calculations from BLACKHAT+SHERPA. The shaded bands represent the theoretical uncertainties.

well as NLO pQCD predictions from BLACKHAT+SHERPA with respect to the fiducial region are listed in Tab. 9.3 .

\begin{tabular}{|c|c|c|}
\hline \hline$N_{\text {jet }}$ & Data Cross Section $(\mathrm{pb})$ & NLO Cross Section $(\mathrm{pb})$ \\
\hline \hline$\geq 0$ jets & {$\left[4.314 \pm 0.004\right.$ (stat) \pm 0.120 (syst) $\pm 0.078($ lumi) $] \times 10^{2}$} & $3.906_{-0.12}^{+0.119} \times 10^{2}$ \\
$\geq 1$ jets & {$\left[6.142 \pm 0.018\right.$ (stat) \pm 0.486 (syst) $\pm 0.112($ lumi) $] \times 10^{1}$} & $5.672_{-0.253}^{+0.278} \times 10^{1}$ \\
$\geq 2$ jets & {$[1.329 \pm 0.008$ (stat) \pm 0.139 (syst) \pm 0.024 (lumi) $] \times 10^{1}$} & $1.300_{-0.075}^{+0.037} \times 10^{1}$ \\
$\geq 3$ jets & {$[2.688 \pm 0.039$ (stat) \pm 0.359 (syst) \pm 0.049 (lumi) $] \times 10^{0}$} & $2.707_{-0.275}^{+0.120} \times 10^{0}$ \\
$\geq 4$ jets & {$[5.738 \pm 0.178$ (stat) \pm 0.969 (syst) \pm 0.105 (lumi) $] \times 10^{-1}$} & $5.650_{-0.773}^{+0.214} \times 10^{-1}$ \\
$\geq 5$ jets & {$[1.129 \pm 0.084$ (stat) \pm 0.235 (syst) \pm 0.020 (lumi) $] \times 10^{-1}$} & - \\
$\geq 6$ jets & {$[1.760 \pm 0.324$ (stat) \pm 0.435 (syst) \pm 0.032 (lumi) $] \times 10^{-2}$} & - \\
$\geq 7$ jets & {$[4.133 \pm 1.755$ (stat) \pm 1.532 (syst) \pm 0.074 (lumi) $] \times 10^{-3}$} & - \\
\hline \hline
\end{tabular}

Table 9.3.: Cross sections as a function of the inclusive jet multiplicities with respect to the fiducial region measured in data and predicted from NLO fixed-order pQCD calculations from BLACKHAT +SHERPA.

Additional jet emissions for larger jet multiplicities are dominated by successive gluon radiation. As already discussed for the 2010 analysis, by calculating the ratio of cross 
sections of successive jet multiplicities, which is depicted in Fig. 9.21(b), an improved test of the SM is provided, since part of the systematics related to the luminosity estimate, the electron reconstruction and identification, the JES and JER cancel. Starting from $R_{2 / 1}$ the ratio is expected to be constant, representing a staircase scaling of the jet multiplicities, which is attributed to the non-Abelian type of FSR accompanied by a PDF suppression, as discussed in Sec. 2.3.4. The predictions from ALPGEN+HERWIG and SHERPA, as well as the NLO fixed-order pQCD predictions from BLACKHAT+SHERPA show a good description of the measured ratio. Detailed values of the cross section ratio, as well as NLO pQCD predictions from BLACKHAT+SHERPA with respect to the fiducial region are listed in Tab. 9.4.

\begin{tabular}{|c|c|c|}
\hline \hline$N_{\text {jet }}+1 / N_{\text {jet }}$ & Data Cross Section Ratio & NLO Cross Section Ratio \\
\hline \hline$\geq 1$ jet $/ \geq 0$ jets & {$[1.424 \pm 0.004$ (stat) \pm 0.106 (syst) $] \times 10^{-1}$} & $1.452_{-0.082}^{+0.084} \times 10^{-1}$ \\
$\geq 2$ jets $/ \geq 1$ jets & {$[2.164 \pm 0.012$ (stat) \pm 0.071 (syst) $] \times 10^{-1}$} & $2.292_{-0.212}^{+0.120} \times 10^{-1}$ \\
$\geq 3$ jets $/ \geq 2$ jets & {$[2.022 \pm 0.026$ (stat) \pm 0.064 (syst) $] \times 10^{-1}$} & $2.083_{-0.214}^{+0.135} \times 10^{-1}$ \\
$\geq 4$ jets $/ \geq 3$ jets & {$[2.135 \pm 0.059$ (stat) \pm 0.083 (syst) $] \times 10^{-1}$} & $2.087_{-0.290}^{+0.236} \times 10^{-1}$ \\
$\geq 5$ jets $/ \geq 4$ jets & {$[1.967 \pm 0.130$ (stat) \pm 0.092 (syst) $] \times 10^{-1}$} & - \\
$\geq 6$ jets $/ \geq 5$ jets & {$[1.559 \pm 0.265$ (stat) \pm 0.130 (syst) $] \times 10^{-1}$} & - \\
\hline \hline
\end{tabular}

Table 9.4.: Ratio of cross sections for successive inclusive jet multiplicities $\sigma\left(Z / \gamma^{*}+\geq N+1\right.$ jets $) / \sigma\left(Z / \gamma^{*}+\geq N\right.$ jets $)$ with respect to the fiducial region measured in data and predicted from NLO fixed-order pQCD calculations from BLACKHAT+SHERPA.

\subsubsection{Exclusive Jet Multiplicity and Jet Multiplicity Scaling}

Multiple-jet final states in association with leptons are typical signatures of Higgs boson production and new physics phenomena. Analysis categories are split into different jet multiplicities, effectively enforcing a jet veto. This is quite common to distinguish signal from background, such that a proper description of these quantities in SM processes is really important. In the 2010 analysis the focus was set to inclusive jet multiplicities, while the analysis with the full dataset of 2011 also includes cross-section measurements for exclusive jet multiplicities and their ratios. Inclusive multiple-jet final states are less problematic from the theoretical point of view [81] and the uncertainties from the choice of QCD renormalisation and factorisation scale are smaller.

Exclusive jet multiplicities are described by two benchmark patterns, staircase scaling or Poisson scaling [10], depending on the scale difference between the leading jet and the additional radiated jets, as detailed in Sec. 2.3.4. In order to test the two benchmark scenarios, two selections are used: (a) the standard $Z / \gamma^{*}+$ jets selection and (b) the standard $Z / \gamma^{*}+$ jets selection with an additional requirement on the transverse momentum of the leading jet of $p_{T}^{\text {jet }}$ (leading jet) $>150 \mathrm{GeV}$. The cross section as a function of the exclusive jet multiplicity for these two benchmark scenarios is shown in Fig. 9.22, the corresponding ratios are shown in Fig. 9.23.

The relatively large theoretical uncertainty on the NLO pQCD predictions are dominated by the scale uncertainty, coming from the approach of Ref. [164], where the scale variations are assumed to be uncorrelated between the jet multiplicities. For comparisons, 


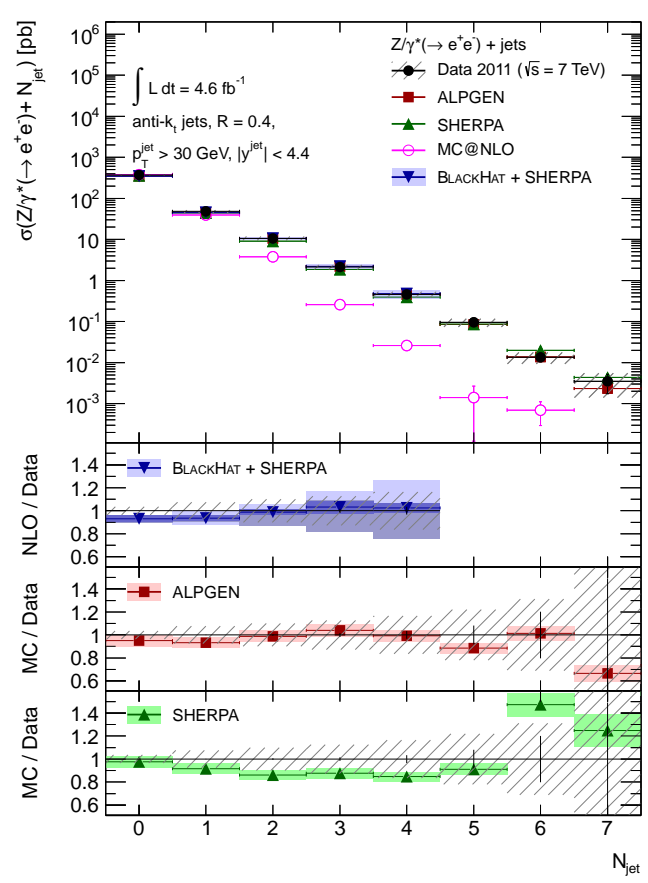

(a) Exclusive jet multiplicity

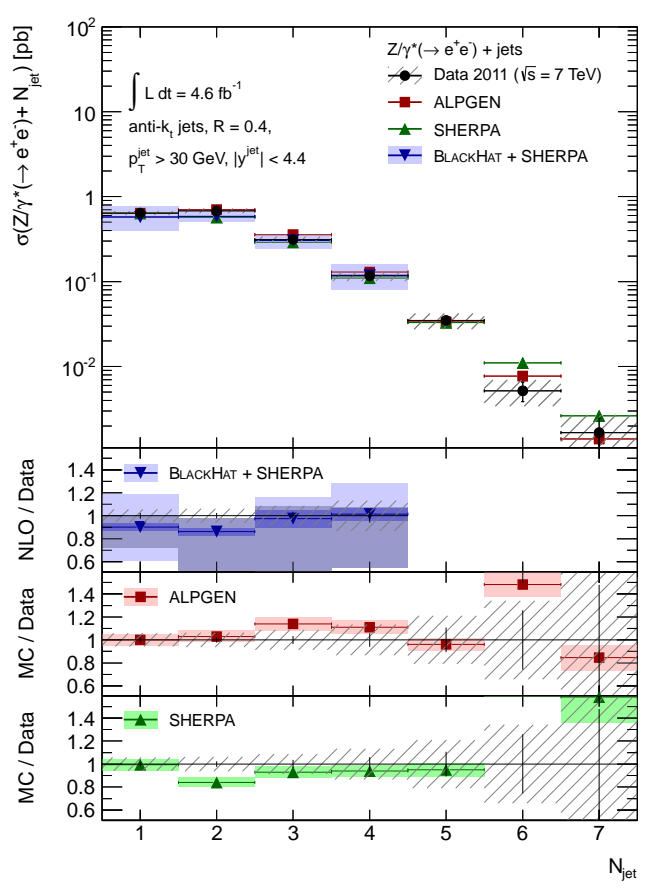

(b) Exclusive jet multiplicity, $p_{T}^{\text {jet }}$ (leading jet) $>150 \mathrm{GeV}$

Figure 9.22.: Measured cross section as a function of the exclusive jet multiplicity for events selected with (a) the standard $Z / \gamma^{*}(\rightarrow e e)+$ jets selection and (b) with an additional requirement on the transverse momentum of the leading jet of $p_{T}^{\text {jet }}>150 \mathrm{GeV}$. The measured values are represented by the black dots, the error bars indicate the statistical uncertainty and the hatched band incorporates the total statistical and systematic uncertainty added in quadrature. The measurements are compared to predictions from ALPGEN+HERWIG and SHERPA event generators, as well as to NLO pQCD calculations from BLACKHAT+SHERPA. The shaded bands represent the theoretical uncertainties excluding the scale uncertainties (dark blue) and the total uncertainty using the naive approach (medium) blue and the nominal approach (light blue) to calculate the scale uncertainty, as described in Sec. 7.3 .

two additional bands are shown, once with the total uncertainty using the naive approach to calculate the scale uncertainty and once without the scale uncertainty, as described in Sec. 9.4. The central values of the NLO fixed-order pQCD calculations from BLACKHAT+SHERPA, as well as the predictions from ALPGEN+HERWIG and SHERPA are consistent with the measurements. The precision in data already exceeds the precision of the fixed-order pQCD predictions. MC@NLO fails to describe the exclusive jet multiplicity, similarly to the inclusive jet multiplicity.

As predicted, the cross-section ratio depicted in Fig. 9.23(a) shows an approximately linear dependence with a small slope above $R_{2 / 1}$. The measurement has been fitted linearly with the function $R_{(n+1) / n}=R_{0}+\frac{\mathrm{d} R}{\mathrm{~d} n} n$ in the range $R_{2 / 1} \leq R_{(n+1) / n} \leq R_{5 / 4}$, the result is shown in Tab. 9.5. The systematic uncertainty of the fit result has been determined by fitting each systematic variation of the ratio separately and adding the differences in quadrature. The $\chi^{2} /$ DoF is computed using only the statistical uncertainties.

The exclusive cross-section ratio for events with an additional requirement on the transverse momentum of the leading jet of $p_{T}^{\text {jet }}>150 \mathrm{GeV}$ is shown in Fig. 9.23(b). The mea- 


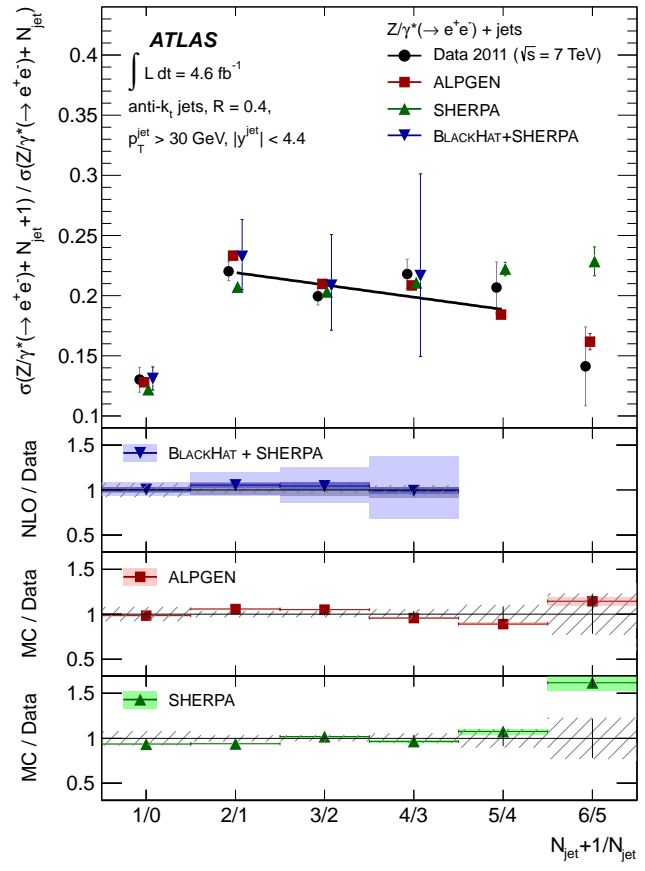

(a) Exclusive jet multiplicity

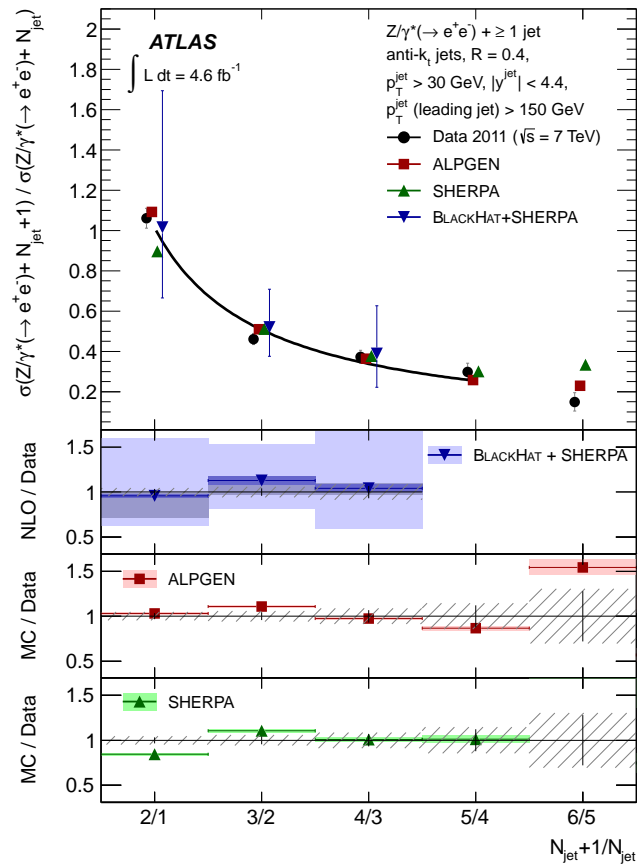

(b) Exclusive jet multiplicity, $p_{T}^{j e t}($ leading jet $)>150 \mathrm{GeV}$

Figure 9.23.: Ratios of cross sections for successive exclusive jet multiplicities for events selected with (a) the standard $Z / \gamma^{*}(\rightarrow e e)+$ jets selection and (b) with an additional requirement on the transverse momentum of the leading jet of $p_{T}^{\text {jet }}>150 \mathrm{GeV}$. The figures include (a) a linear fit $R_{(n+1) / n}=R_{0}+\frac{\mathrm{d} R}{\mathrm{~d} n} n$ and (b) a Poisson fit $R_{(n+1) / n}=\frac{\bar{n}}{n}$ of the data in the range $R_{2 / 1} \leq R_{(n+1) / n} \leq R_{5 / 4}$. The other details are as in Fig. 9.22 .

surement has been fitted with the function $R_{(n+1) / n}=\frac{\bar{n}}{n}$ in the range $R_{2 / 1} \leq R_{(n+1) / n} \leq R_{5 / 4}$, assuming that the exclusive jet multiplicity follows a Poisson distribution. The results of the fit are also shown in Tab. 9.5. The predicted Poisson scaling pattern provides a good overall description of the measurement.

\begin{tabular}{|c|c|c|}
\hline \multicolumn{3}{|c|}{ Linear Fit } \\
\hline$R_{0}$ & $\mathrm{~d} R / \mathrm{d} n$ & $\chi^{2} / \mathrm{DoF}$ \\
\hline $0.229 \pm 0.004$ (stat) \pm 0.009 (sys) & $-0.0102 \pm 0.0029$ (stat) \pm 0.0031 (sys) & $12.9 / 2$ \\
\hline \multicolumn{3}{|c|}{ Poisson Fit } \\
\hline & $\bar{n}$ & $\chi^{2} / \mathrm{DoF}$ \\
\hline $1.015 \pm 0.02$ & $($ stat $) \pm 0.036$ (sys) & $9.7 / 3$ \\
\hline
\end{tabular}

Table 9.5.: Results of a linear fit of the exclusive cross-section ratio $R_{(n+1) / n}$ with the standard selection, and results of a fit of the exclusive cross-section ratio $R_{(n+1) / n}$ with a pattern expected for Poisson distributed exclusive jet multiplicity for events with an additional requirement on the transverse momentum of the leading jet of $p_{T}^{\text {jet }}>150 \mathrm{GeV}$. The systematic uncertainty of the fit result has been determined by fitting each systematic variation of the ratio separately and adding the differences in quadrature. The $\chi^{2} / \mathrm{DoF}$ is computed using only the statistical uncertainties. 
The detailed values of the measured cross sections, as well as NLO pQCD predictions from BLACKHAT+SHERPA with respect to the fiducial region are listed in Appendix B.4.

\subsubsection{Transverse Momentum Distributions}

The correct description of multiple-jet rates in association with a $Z$ boson does not automatically imply that the kinematics of these jets are well described. For final states with high energetic jets, especially when the transverse momentum of the jet exceeds the scale given by the $Z$ boson mass, large $K$-factors are expected, as described in Sec. 2.3.2. This results in large scale uncertainties for the NLO fixed-order pQCD calculations [9], which is in-line with the fact that half of the events where the leading jet has a transverse momentum above $150 \mathrm{GeV}$ has at least two jets with $p_{T}>30 \mathrm{GeV}$ in the final state, as shown in Fig. 9.22(b), In addition, a large impact from higher-order EW corrections is expected, which reduce the cross section by $10-20 \%$ in the range $100 \mathrm{GeV}<p_{T}^{Z}<500 \mathrm{GeV}$ [75], due to larger EW Sudakov logarithms. Currently, these corrections are neither included in the predictions from ALPGEN+HERWIG nor in the NLO pQCD calculations. Only the predictions from SHERPA partly include higher-order EW corrections [129], due to its implementation of additional photon radiation. Higher order QCD corrections and NLO EW corrections are expected to factorise for the transverse momentum distribution of the leading jet in final states with exactly one jet and the $Z$ boson in final states with at least one jet and exactly one jet [75].

Figure 9.24 depicts the normalised differential cross section as a function of the transverse momentum of the leading, 2nd leading, 3rd leading and 4th leading jet in events with one, two, three and four jets in the final state. The predictions from NLO fixed-order pQCD calculations are consistent for all jet multiplicities.

For the transverse momentum of the leading jet in events with at least one jet in the final state, the predictions from SHERPA are consistent with the data, while the predictions from ALPGEN+HERWIG overestimate the cross section for large $p_{T}$. The deviation decreases for events with exactly one jet, as shown in Fig. 9.25(a), which leads to the conclusion that the deviation is mainly due to higher order QCD effects. The predictions from NLO fixed-order calculations from BLACKHAT + SHERPA are consistent with data, even if they do not include NLO EW corrections. MC@NLO fails to describe the cross section of the transverse momentum of the leading jet for events with at least one jet in the final state, whereas it provides a better description of the exclusive one jet final state, as expected. With increasing transverse momentum of the leading jet, the possibility of a secondary jet emission increases, which for MC@NLO is modelled by parton showers.

The difference observed for the predictions from ALPGEN+HERWIG for the transverse momentum of the leading jet for events with at least one jet is reflected in low values of the transverse momentum ratio of the 2 nd leading jet to the leading jet for events with at least two jets in the final state, as shown in Fig. 9.25(b). This variable has a higher experimental precision than the individual transverse momentum distribution, due to the fact that part of the systematics related to the JES and JER cancel. Therefore, the predictions can be tested to a higher level of accuracy. The predictions from SHERPA reproduce the shape of the ratio, but adopts the offset from its too low estimate of $R_{1 / 2}$. The fixed-order pQCD calculations for $Z / \gamma^{*}+\geq 2$ jets from BLACKHAT+SHERPA are consistent with data. 


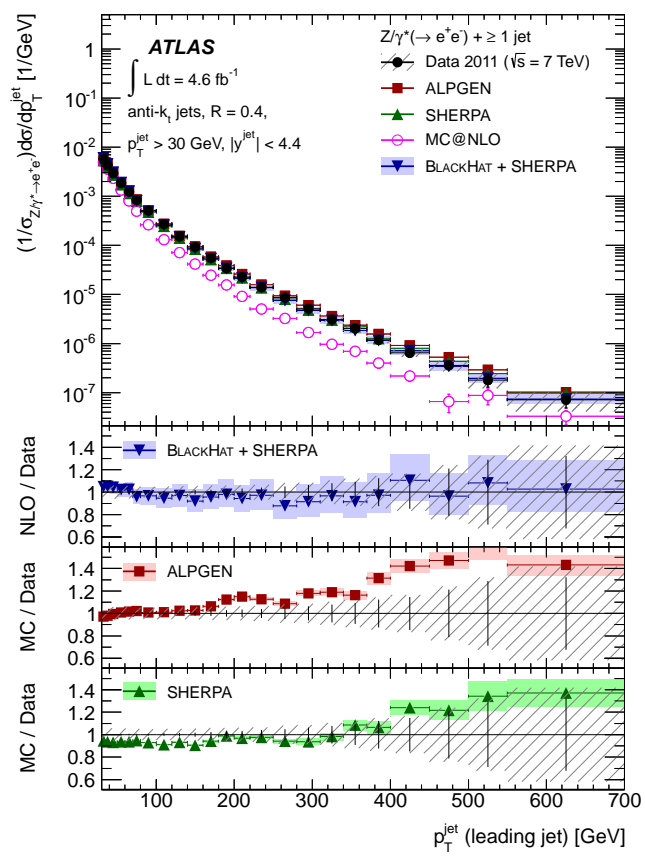

(a) Leading jet $p_{T}$

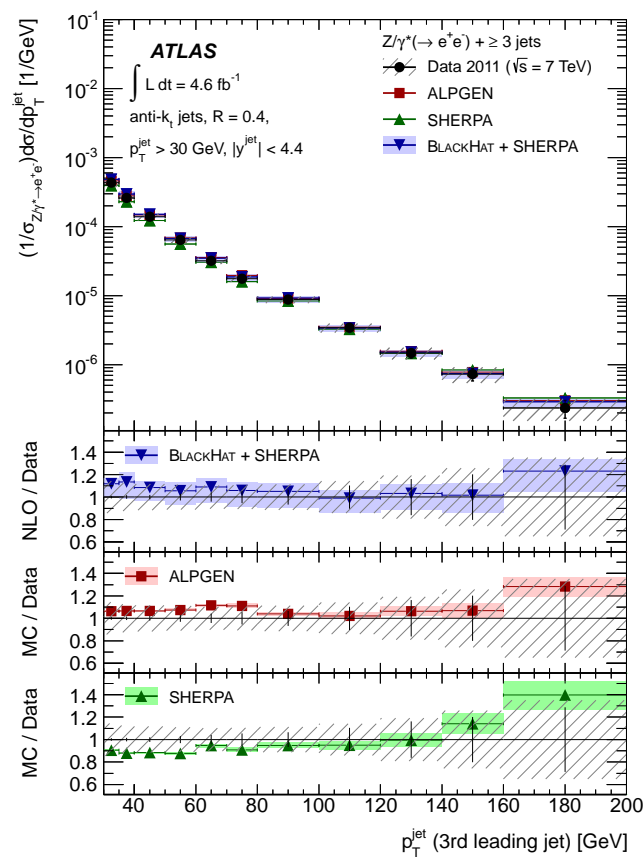

(c) 3rd leading jet $p_{T}$

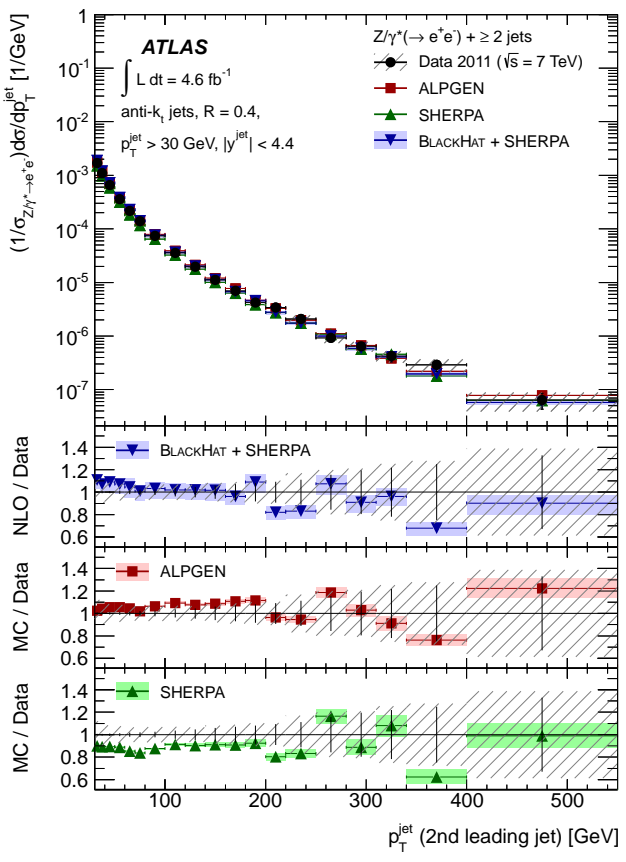

(b) 2nd leading jet $p_{T}$

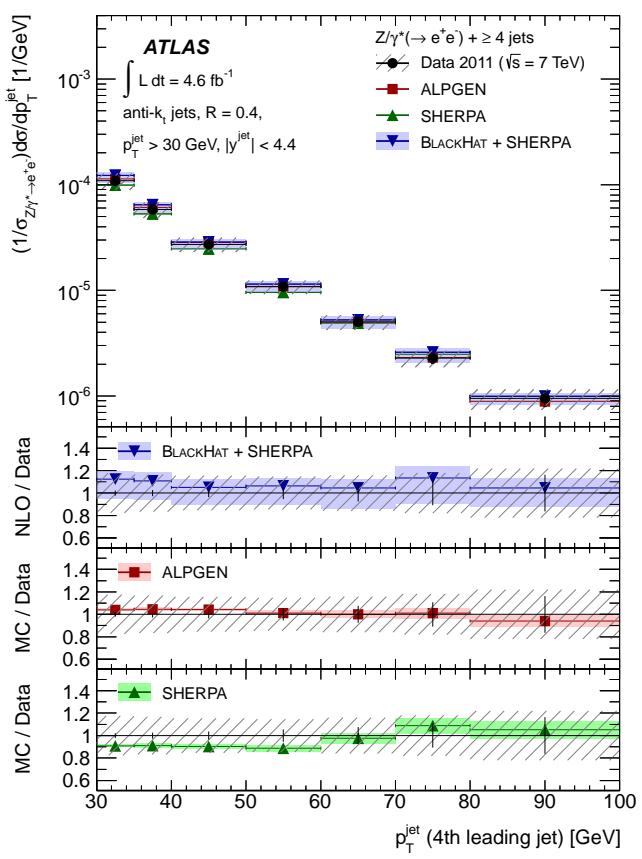

(d) 4th leading jet $p_{T}$

Figure 9.24.: Differential cross section as a function of (a) the transverse momentum $p_{\mathrm{T}}^{\text {jet }}$ of the leading jet for events with at least one jet in the final state, (b) $p_{\mathrm{T}}^{\text {jet }}$ of the 2 nd leading jet for events with at least two jets in the final state, (c) $p_{\mathrm{T}}^{\text {jet }}$ of the $3 \mathrm{rd}$ leading jet for events with at least three jets in the final state and (d) $p_{\mathrm{T}}^{\text {jet }}$ of the 4 th leading jet for events with at least four jets in the final state. The cross sections are normalised to the inclusive $Z / \gamma^{*}(\rightarrow e e)$ cross section. The other details are as in Fig. 9.21. 


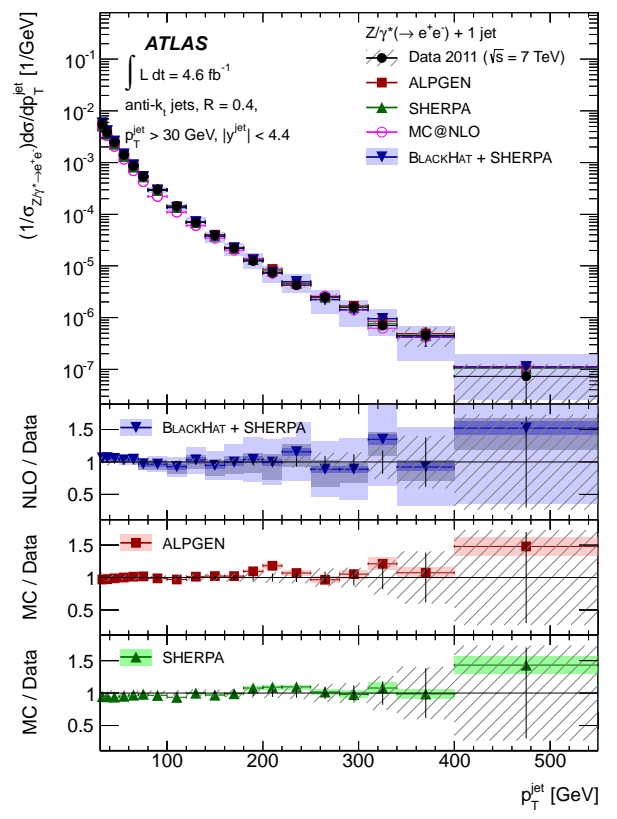

(a) Jet $p_{T}$

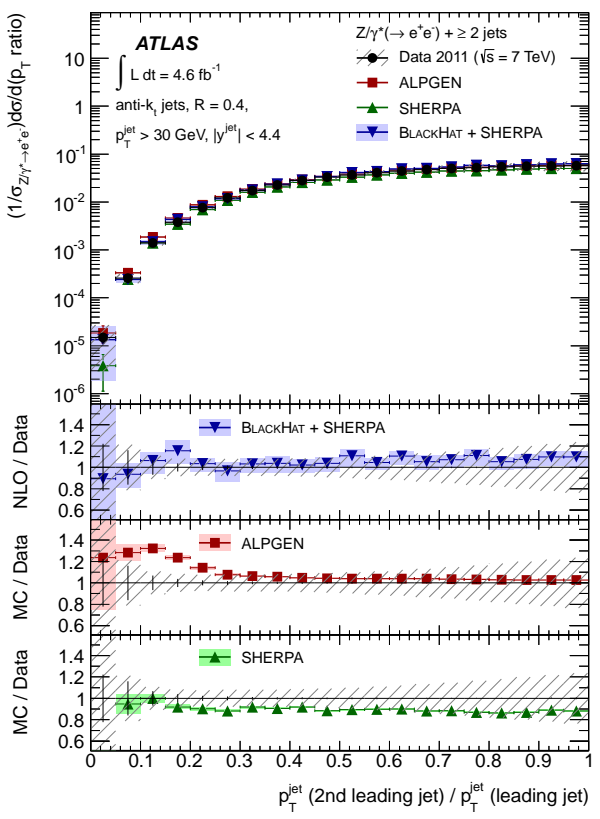

(b) $p_{T}$ ratio

Figure 9.25.: Differential cross section as a function of (a) $p_{\mathrm{T}}^{\text {jet }}$ for events with exactly one jet in the final state and (b) the ratio of the transverse momentum of the 2nd leading jet to that of the leading jet for events with at least two jets in the final state. The cross sections are normalised to the inclusive $Z / \gamma^{*}(\rightarrow e e)$ cross section. The other details are as in Fig. 9.22 .

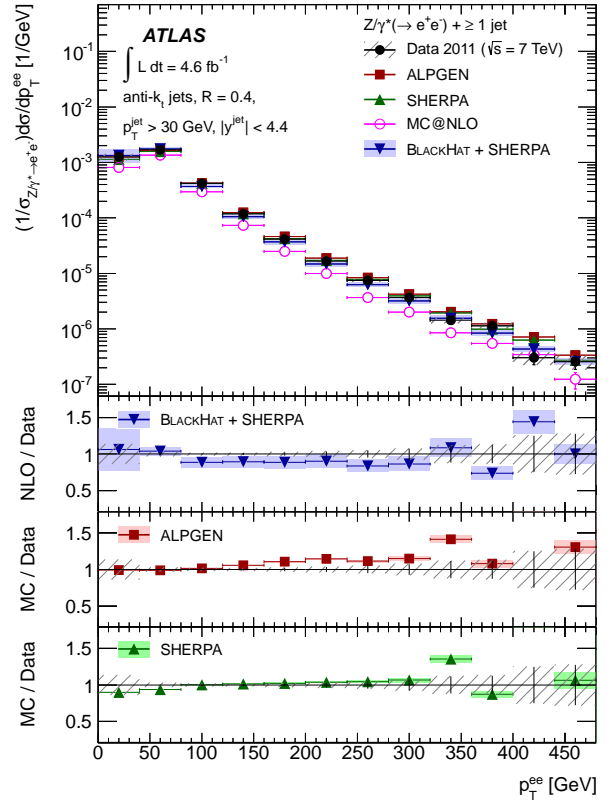

(a) $Z$ boson $p_{T}, N_{j e t} \geq 1$

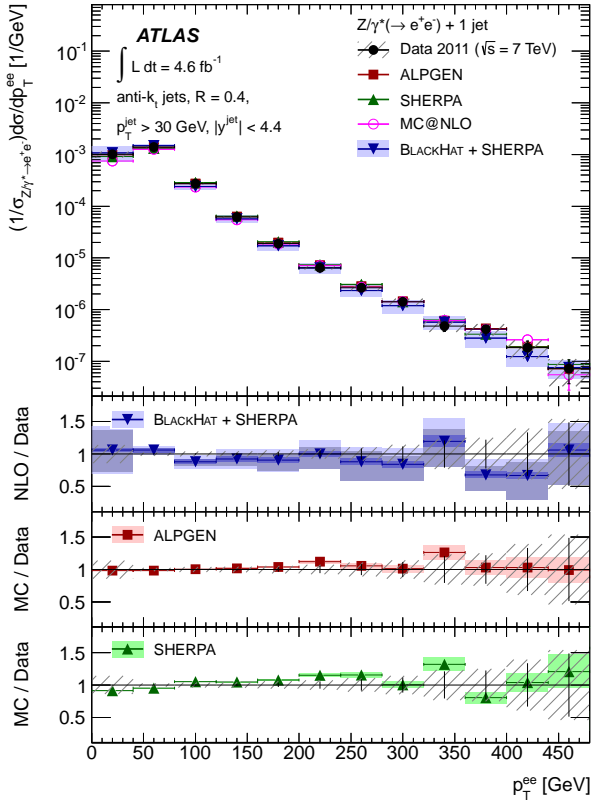

(b) $Z$ boson $p_{T}, N_{\text {jet }}=1$

Figure 9.26.: Differential cross section as a function of the $Z$ boson transverse momentum for events with (a) at least one jet in the final state and (b) exactly one jet in the final state. The cross sections are normalised to the inclusive $Z / \gamma^{*}(\rightarrow e e)$ cross section. The other details are as in Fig. 9.22 . 
Figure 9.26 shows the differential cross section as a function of the $Z$ boson transverse momentum for events with at least one jet in the final state and with exactly one jet in the final state. Higher order QCD corrections and NLO EW corrections are expected to factorise for the transverse momentum of the $Z$ boson, similar to the transverse momentum of the leading jet with exactly one jet in the final state. Predictions from ALPGEN+HERWIG overestimate the cross section for large transverse momenta of the $Z$ boson for events with at least one jet in the final state, which is consistent with the pure NLO EW correction. However, the predictions from ALPGEN+HERWIG are consistent with the data for the transverse momentum of the $Z$ boson for events with exactly one jet in the final state, similar to the case for the transverse momentum of the leading jet. This supports the conclusion that the deviation is mainly due to higher-order QCD effects for both large transverse momenta of the $Z$ boson and the leading jet. NLO fixed order $Z / \gamma^{*}+\geq 1$ jet calculations underestimate the cross sections for $p_{T}^{e e}$ above $p_{T}^{e e} \approx 80 \mathrm{GeV}$ by $10-20 \%$. This can be attributed to missing higher orders in QCD: Figure 9.27 shows the average number of jets as a function of $p_{T}^{e e}$ at detector level.

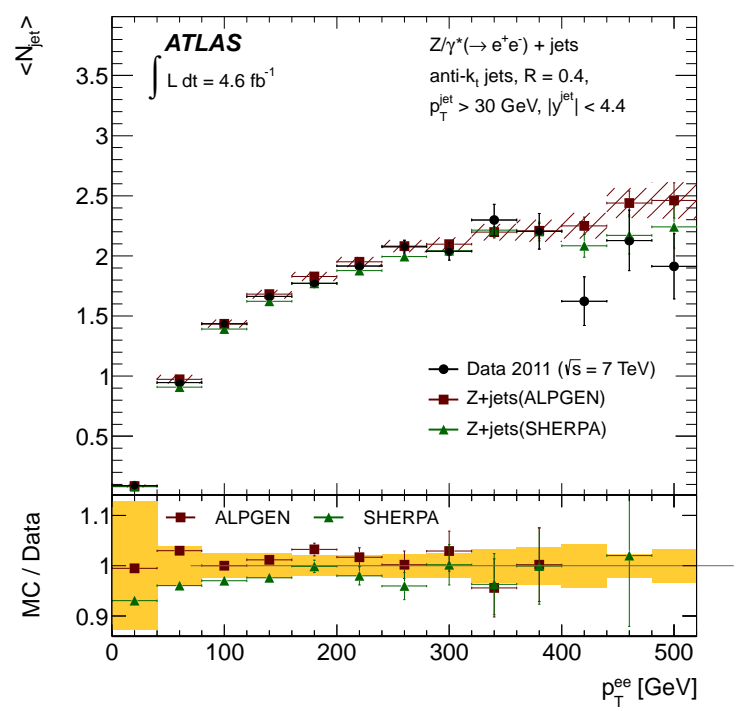

Figure 9.27: Average number of jets as a function the transverse momentum of the $Z$ boson in data and predicted by the generators ALPGEN+HERWIG and SHERPA. The measured values after background subtraction are represented by the black dots, the error bars indicate the combined statistical and systematic uncertainty on the data. The hatched band incorporates the total statistical and systematic uncertainty on the predictions, modelled with ALPGEN+HERWIG. The shaded band reflects the total systematic uncertainty.

The average jet multiplicity is modelled well within uncertainties for both $\mathrm{ME}+\mathrm{PS}$ generators. Already for $p_{T}^{e e} \approx 160 \mathrm{GeV}$ the average number of jets is close to two. The NLO fixed-order pQCD predictions are calculated inclusively for events with a $Z$ boson and at least one jet in the final state. Due to this fact, matrix elements for 3 or more real parton emissions are not included in the final state, while the predictions from ALPGEN+HERWIG and SHERPA include matrix elements for up to 5 partons in the simulation.

In the following, the event shapes in the subset of $Z / \gamma^{*}+\geq 2$ jets events are studied in more detail. Figure 9.28 shows $\Delta \phi$ and the ratio $R\left(p_{T} 2 / p_{T} 1\right)$ of the two leading jets for two regimes of $p_{T}^{e e}$. Whereas events with low $p_{T}^{e e}$ typically have two jets of similar $p_{\mathrm{T}}^{\text {jet }}$ back-to-back in the transverse plane, for large $p_{T}^{e e}$, the events are characterised by a small $\Delta \phi$ and small $R\left(p_{T} 2 / p_{T} 1\right)$, thus introducing a large scale difference between the two leading jets.

Apparently, NLO fixed-order $Z / \gamma^{*}+\geq 1$ jet calculations are not able to describe two jet final states with large scale differences. Discrepancies between NLO fixed-order pQCD calculations and data have already been reported for other observables, such as $H_{T}$, in 


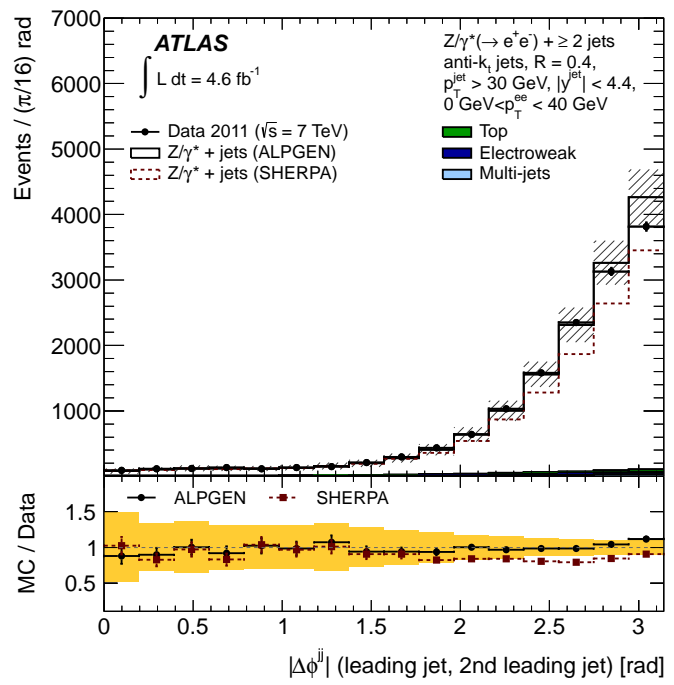

(a) Absolute azimuthal separation, $0 \mathrm{GeV}<p_{T}^{e e}<40 \mathrm{GeV}$

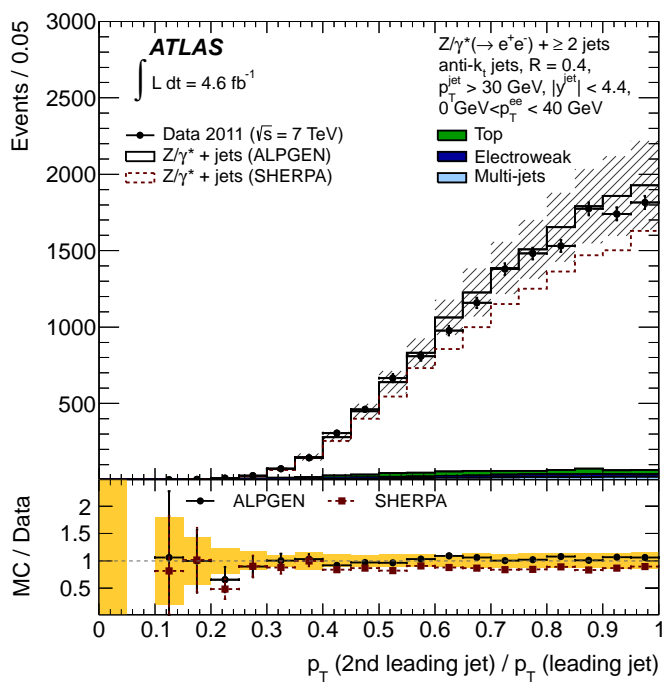

(c) $p_{T}$ ratio, $0 \mathrm{GeV}<p_{T}^{e e}<40 \mathrm{GeV}$

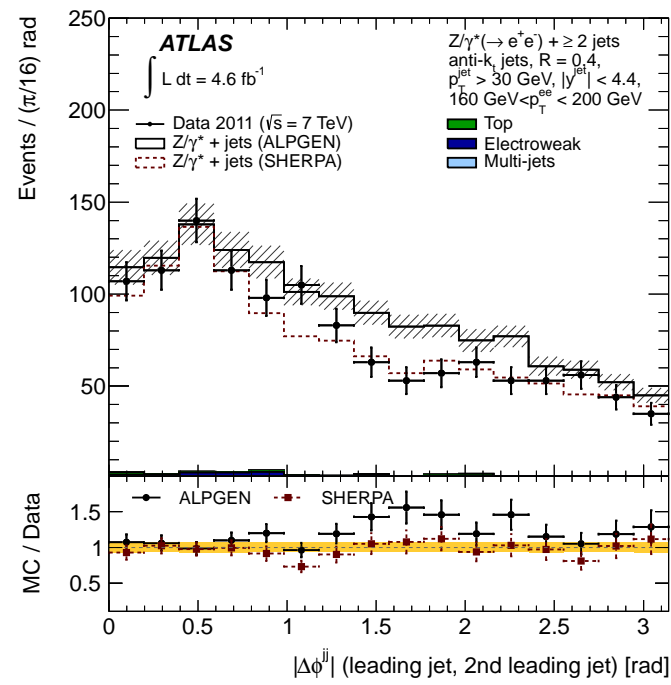

(b) Absolute azimuthal separation, $160 \mathrm{GeV}<p_{T}^{e e}<200 \mathrm{GeV}$

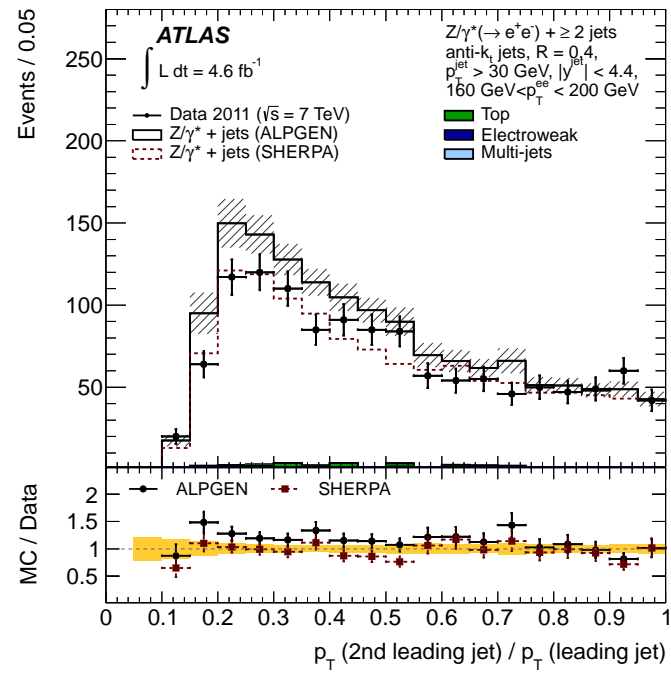

(d) $p_{T}$ ratio, $160 \mathrm{GeV}<p_{T}^{e e}<200 \mathrm{GeV}$

Figure 9.28.: Absolute azimuthal separation $\left|\Delta \phi^{j j}\right|$ of the two leading jets for different bins of $p_{T}^{e e}$, (a) $0 \mathrm{GeV}<p_{T}^{e e}<40 \mathrm{GeV}$ and (b) $160 \mathrm{GeV}<p_{T}^{e e}<200 \mathrm{GeV}$ and the ratio of the transverse momentum of the 2 nd leading jet to that of the leading jet for different bins of $p_{T}^{e e}$, (c) $0 \mathrm{GeV}<p_{T}^{e e}<40 \mathrm{GeV}$ and (d) $160 \mathrm{GeV}<p_{T}^{e e}<200 \mathrm{GeV}$ in events with at least two jets in the final state in data and simulation. Multi-jets and $t \bar{t}$ backgrounds have been estimated from data. The hatched band incorporates the total statistical and systematic uncertainty on the predictions. The yellow band reflects the total systematic uncertainty. 
$W+$ jets events, where they can be mitigated by including higher jet multiplicities in the calculation using exclusive sums [165]: the sum of the exclusive $Z / \gamma^{*}+1$ jet calculation and the inclusive $Z / \gamma^{*}+2$ jets calculation. Figure 9.29 shows the comparison of the exclusive sum to the measurement in data for the normalised differential cross section as a function of the $Z$ boson transverse momentum for events with at least one jet in the final state. The exclusive sum yields a much better performance and is able to restore the agreement with the data.

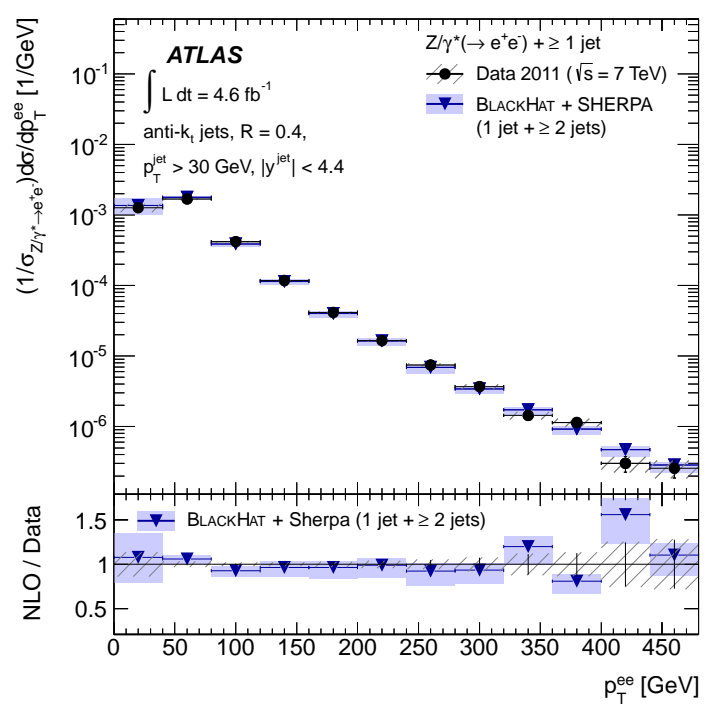

Figure 9.29: Differential cross section as a function of $Z$ boson transverse momentum for events with at least one jet in the final state. The cross sections are normalised to the inclusive $Z / \gamma^{*}(\rightarrow e e)$ cross section. The measured values are represented by the black dots, the error bars indicate the statistical uncertainty and the hatched band incorporates the total statistical and systematic uncertainty added in quadrature. The measurements are compared to NLO pQCD predictions from BLACKHAT+SHERPA, obtained by adding the exclusive $Z / \gamma^{*}(\rightarrow e e)+1$ jet and the inclusive $Z / \gamma^{*}(\rightarrow e e)+2$ jets calculations. The shaded bands represent the theoretical uncertainties.

Finally, predictions from ALPGEN+PYTHIA are compared to the measured cross section for the transverse momentum distribution of the leading jet and the $Z$ boson, shown in Fig. 9.30, The predictions from ALPGEN+PYTHIA compared to the predictions from ALPGEN+HERWIG are characterised by a different parton shower, fragmentation and UE modelling.

The predictions from ALPGEN+PYTHIA also overestimate the measured cross section for large values of $p_{T}^{\text {jet }}$ and $p_{T}^{e e}$, similar to the predictions from ALPGEN+HERWIG, which leads to the conclusion that the observed discrepancies between the predictions and the measurements actually refer to NLO or EW NLO corrections and not to parton shower. In contrast, the effect of parton shower is clearly visible for the transverse momentum of the 4th leading jet, as shown in Fig. 9.31, for which the fraction of higher jet multiplicity contributions is much larger.

In general, effects from both higher-order EW and QCD corrections are expected, which are assumed to factorise for the transverse momentum distribution of the leading jet in final states with exactly one jet and the $Z$ boson in final states with exactly one jet and with at least one jet. But for the observed differences between the prediction from ALPGEN+HERWIG and the data higher-order QCD corrections seem to play a mayor role. The prediction from NLO fixed-order pQCD calculations are consistent with the data.

The detailed values of the measured cross sections, as well as NLO pQCD predictions from BLACKHAT+SHERPA with respect to the fiducial region are listed in Appendix B.4. 


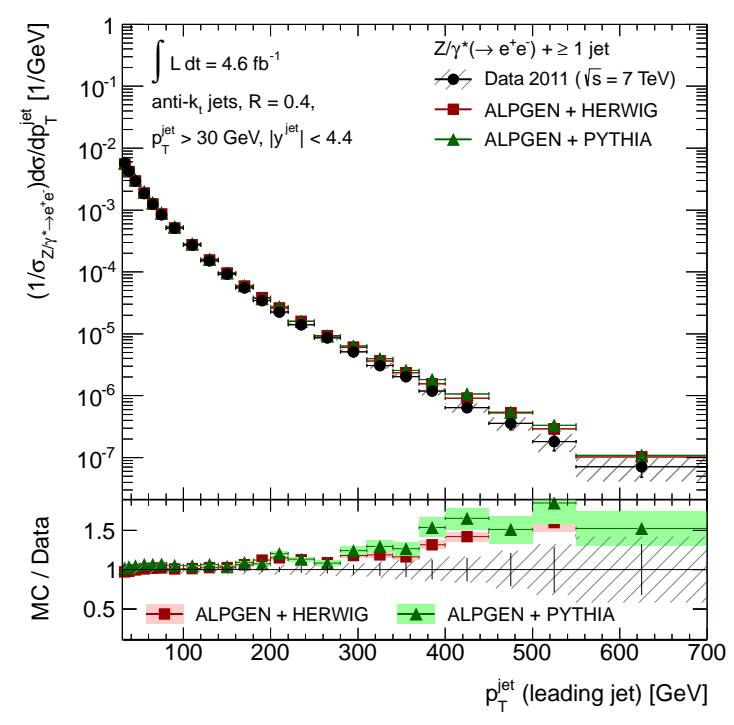

(a) Leading jet $p_{T}$

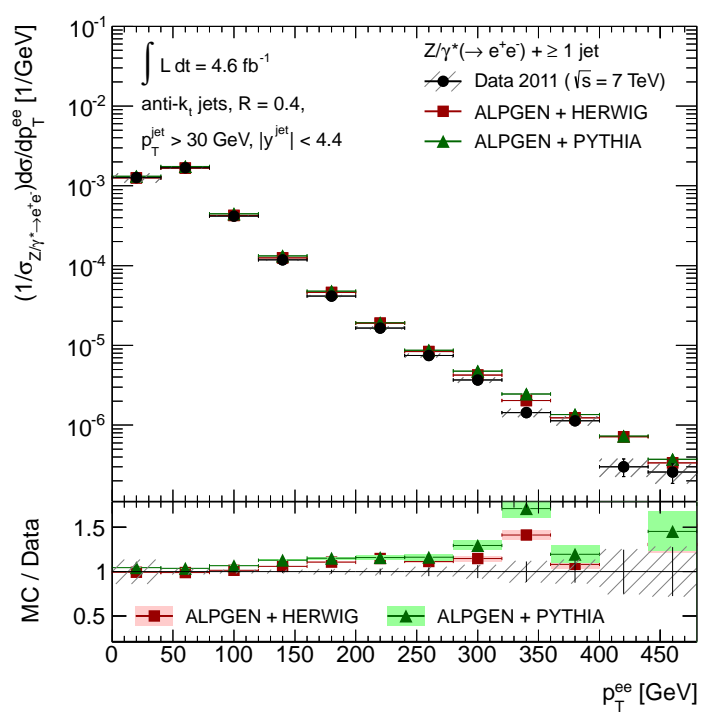

(b) $Z$ boson $p_{T}$

Figure 9.30.: Differential cross section as a function of the transverse momentum of (a) the leading jet and (b) the $Z$ boson for events with at least one jet in the final state. The cross sections are normalised to the inclusive $Z / \gamma^{*}(\rightarrow e e)$ cross section. The measured values are represented by the black dots, the error bars indicate the statistical uncertainty and the hatched band incorporates the total statistical and systematic uncertainty added in quadrature. The measurements are compared to predictions from ALPGEN+HERWIG and ALPGEN+PYTHIA. The shaded bands represent the theoretical uncertainties.

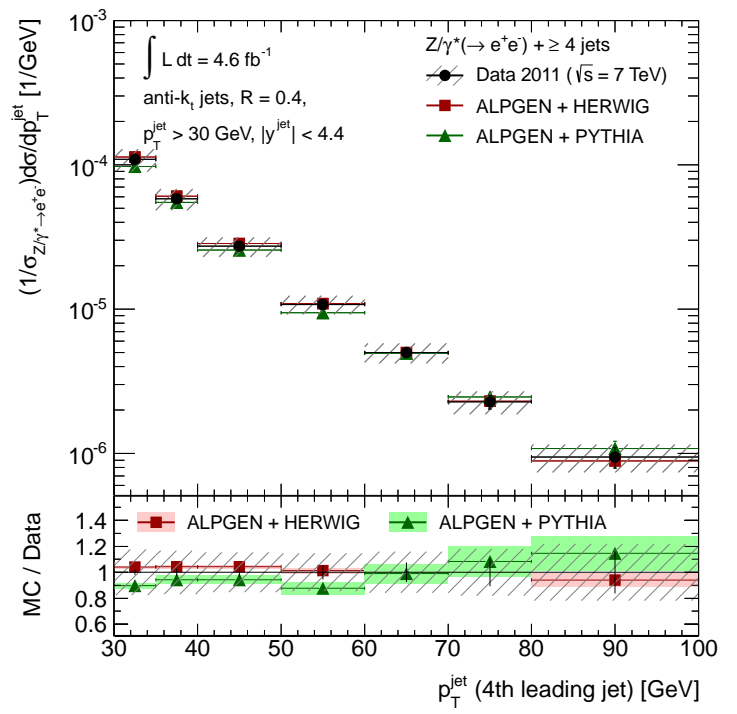

Figure 9.31: Differential cross section as a function of $p_{\mathrm{T}}^{\text {jet }}$ of the 4 th leading jet for events with at least four jets in the final state. The cross sections are normalised to the inclusive $Z / \gamma^{*}(\rightarrow e e)$ cross section. The other details are as in Fig. 9.30 


\subsubsection{Rapidity Distributions}

The correct modelling of the rapidity distribution of the jets, especially in the forward region, is essential, since this quantity plays an important role in searches for new physics and studies of the Higgs boson. Such processes are characterised by high energetic wellseparated forward jets. Therefore, an excellent knowledge of the rapidity distribution can be used to distinguish signal from background.

The normalised differential cross section as a function of the absolute rapidity $\left|y^{\text {jet }}\right|$ for the leading, 2nd leading, 3rd leading and 4th leading jet in events with at least one, two, three and four jets in the final state are shown in Fig. 9.32. The predictions from ALPGEN+HERWIG are consistent with the data for $\left|y^{j e t}\right|$ of the leading jet, whereas the predictions from SHERPA and the NLO fixed-order pQCD calculation overestimate the cross section in the forward region. MC@NLO predicts a too broad rapidity distribution, which is off by several orders of magnitude from the observation. The predictions from ALPGEN+HERWIG, SHERPA and the NLO fixed-order pQCD calculations from BLACKHAT+SHERPA for the absolute rapidity of the 2nd leading, 3rd leading and 4th leading jet are consistent with the data.

The precision of measurements in the forward region is strongly limited by systematic uncertainties, especially due to JES, but also the contribution of the unfolding has a large impact. The latter could be optimised using multi-dimensional unfolding. Multidimensional unfolding is expected to reduce the model dependence, since the full event kinematics is taken into account. In the remainder of this section the measurement of the normalised differential cross section as a function of the absolute rapidity $\left|y^{\text {jet }}\right|$ for the leading jet is studied using two-dimensional unfolding. 


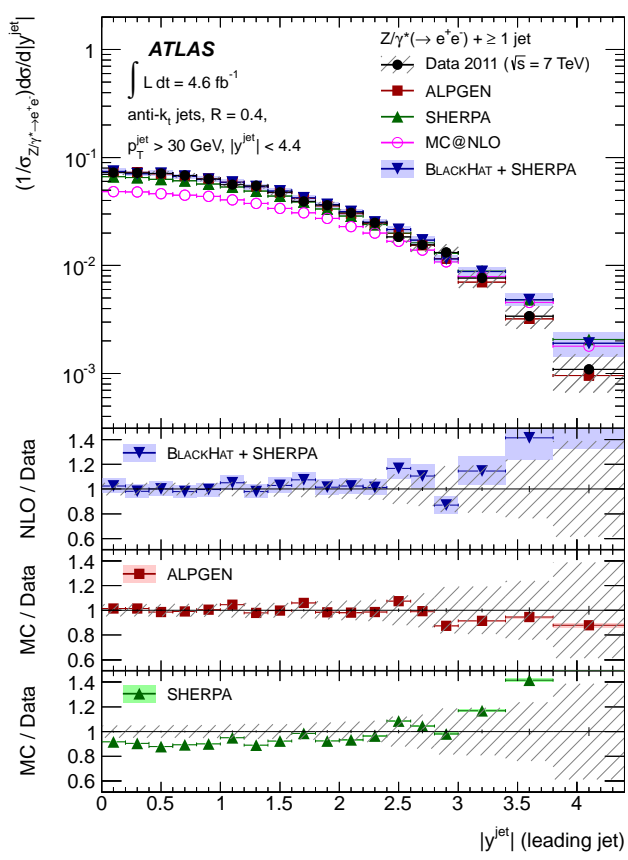

(a) Leading jet $|y|$

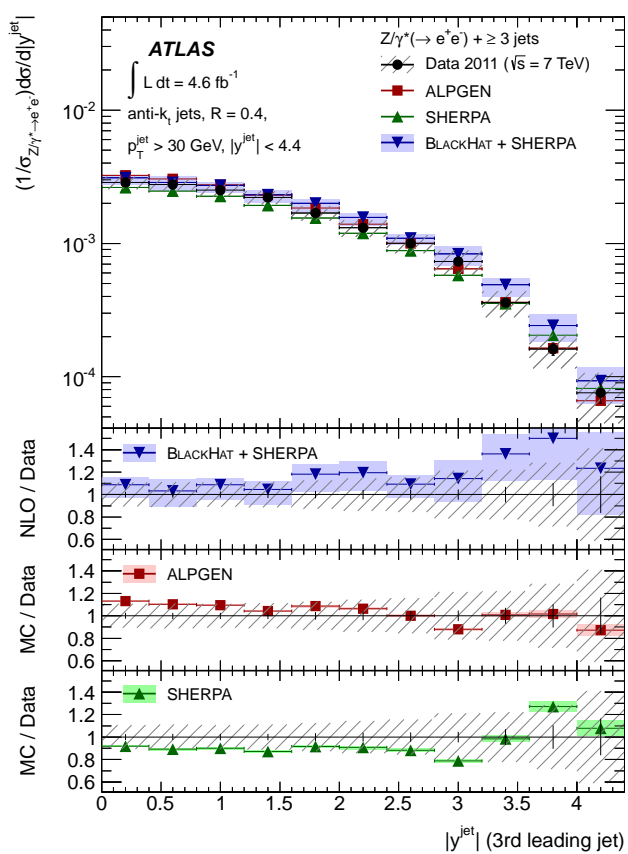

(c) 3rd leading jet $|y|$

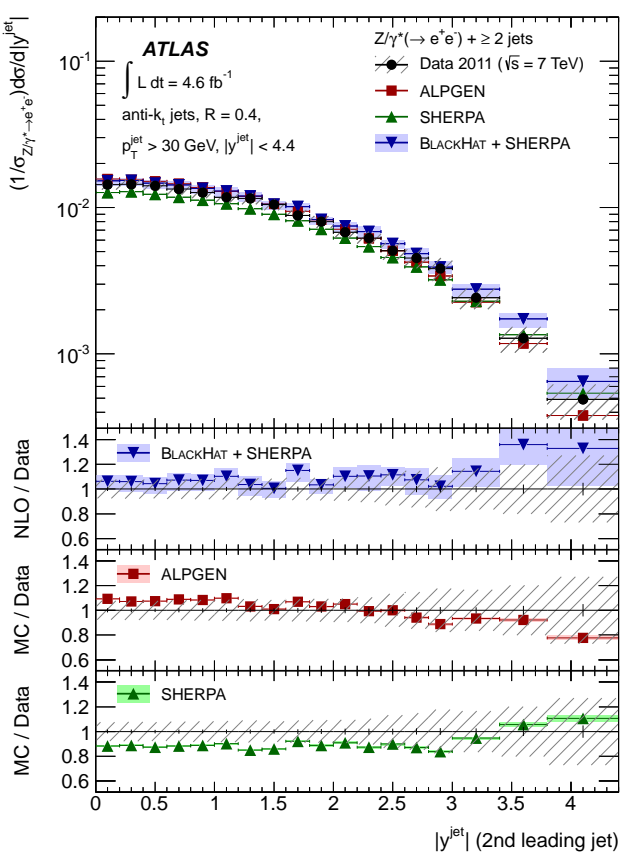

(b) 2nd leading jet $|y|$

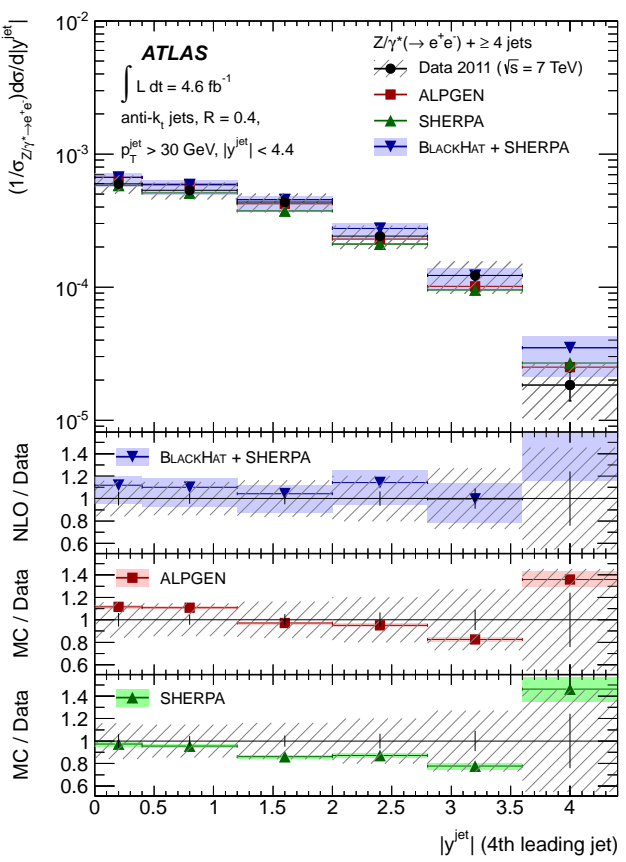

(d) 4th leading jet $|y|$

Figure 9.32.: Differential cross section as a function of (a) the absolute rapidity $\left|y^{\text {jet }}\right|$ of the leading jet for events with at least one jet in the final state, (b) $\left|y^{\text {jet }}\right|$ of the 2nd leading jet for events with at least two jets in the final state, (c) $\left|y^{\text {jet }}\right|$ of the 3rd leading jet for events with at least three jets in the final state and (d) $\left|y^{\text {jet }}\right|$ of the 4th leading jet for events with at least four jets in the final state. The cross sections are normalised to the inclusive $Z / \gamma^{*}(\rightarrow e e)$ cross section. The other details are as in Fig. 9.21. 


\section{Two-dimensional Unfolding}

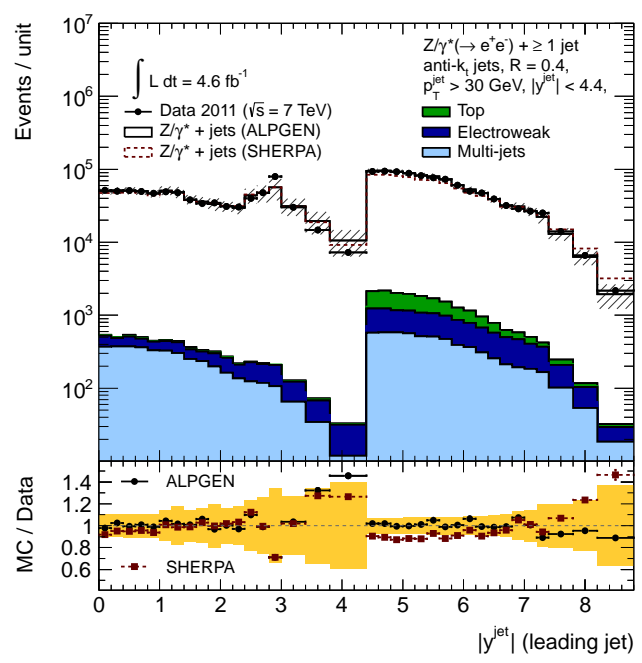

Figure 9.33: Absolute rapidity distribution of the leading jet in data and simulation for two $p_{T}^{j e t}$ bins: $20 \mathrm{GeV} \leq p_{T}^{\text {jet }} \leq 30 \mathrm{GeV}$ and $p_{T}^{\text {jet }}>30 \mathrm{GeV}$. Multi-jets and $t \bar{t}$ backgrounds have been estimated from data. The hatched band incorporates the total statistical and systematic uncertainty on the predictions. The yellow band reflects the total systematic uncertainty.

Figure 9.33 shows the uncorrected absolute rapidity distribution of the leading jet for two $p_{T}^{\text {jet }}$ bins: $20 \mathrm{GeV} \leq p_{T}^{\text {jet }} \leq 30 \mathrm{GeV}$ and $p_{T}^{\text {jet }}>30 \mathrm{GeV}$. The $p_{T}$-region between $20 \mathrm{GeV}$ and $30 \mathrm{GeV}$ is included to correctly account for the migration around the $p_{T}^{\text {jet }}$ threshold of $30 \mathrm{GeV}$. The distribution shown in the range $0<|y| \leq 4.4$ corresponds to the rapidity distributions of jets with $20 \mathrm{GeV} \leq p_{T}^{\text {jet }} \leq 30 \mathrm{GeV}$ and $\left|y^{\text {jet }}\right|<4.4$, while the distribution in the range $4.4<|y| \leq 8.8$ corresponds to the distribution for jets with $p_{T}^{\text {jet }}>30 \mathrm{GeV}$ and $\left|y^{j e t}\right|<4.4$. The predictions from SHERPA models a too broad rapidity distribution for both $p_{T}$-regions, while ALPGEN+HERWIG only predicts a too broad distribution for the $p_{T}$-region between $20 \mathrm{GeV}$ and $30 \mathrm{GeV}$. For $p_{T}^{\text {jet }}>30 \mathrm{GeV}$ the predictions from ALPGEN+HERWIG agree with the data. In order to account for the exact $p_{T}^{\text {jet }}$ distribution in each rapidity bin more $p_{T}^{\text {jet }}$ bins need to be included, which is currently not possible due to the limited MC statistics.

Figure 9.34 to Fig. 9.35 show the corresponding migration matrix and the correction for not-matched events and the jet hierarchy.

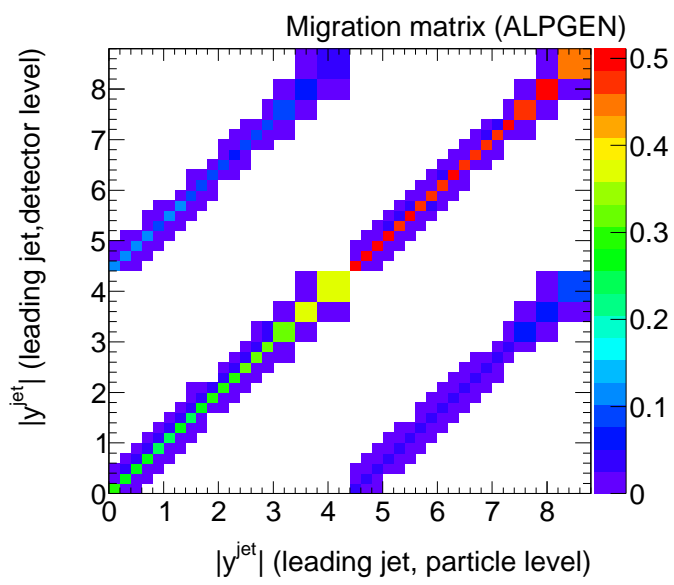

Figure 9.34: Two-dimensional migration matrix for the absolute rapidity of the leading jet for events with at least one jet in the final state. The migration matrix is determined using ALPGEN+HERWIG MC event samples.

As expected, the migration matrix shows that the migration between the two $p_{T}^{\text {jet }}$ bins is not negligible. The additional $p_{T}^{\text {jet }}$ bin leads to a higher matching probability of the jets, resulting in a significantly lower correction for not-matched events in the region of interest 


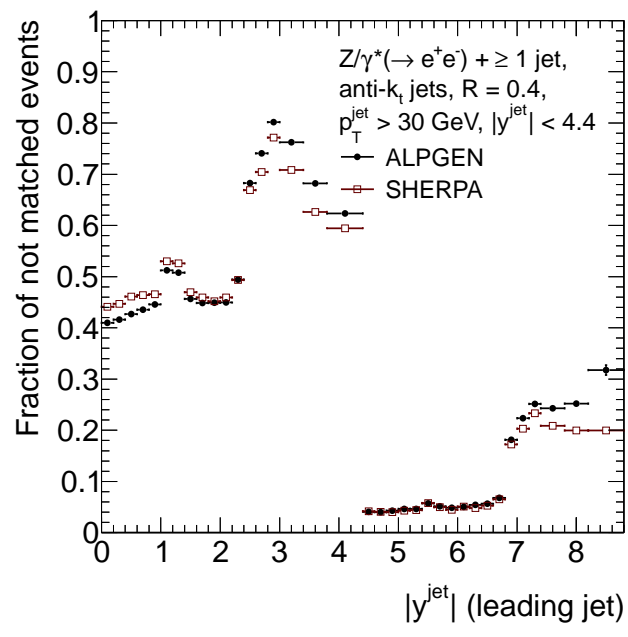

(a) Correction for not-matched events

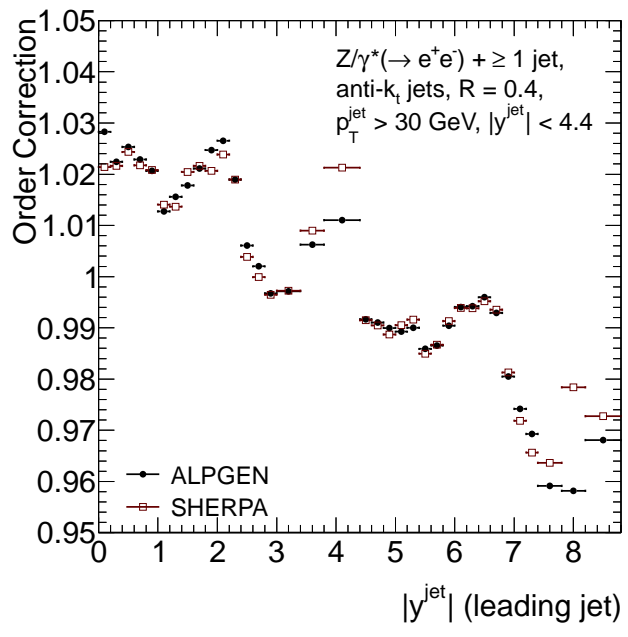

(b) Jet hierarchy correction

Figure 9.35.: (a) Correction for not-matched events and (b) order correction for the absolute rapidity of the leading jet for events with at least one jet in the final state. The correction factors are shown for ALPGEN+HERWIG and SHERPA MC event samples.

$\left(p_{T}^{\text {jet }}>30 \mathrm{GeV}\right.$ and $\left.|y|<4.4\right)$, but slightly larger jet hierarchy correction compared to the one-dimensional unfolding corrections, as shown in Fig. 9.8 and Fig. 9.9 in Sec. 9.2 . Also for the two-dimensional unfolding a sanity check is performed using closure tests. The detector level predictions from SHERPA are unfolded with ALPGEN+HERWIG and compared to the particle level predictions from SHERPA. Figure 9.36 shows the progression of the one-dimensional and two-dimensional unfolding with increasing number of iterations, indicated by the coloured lines.

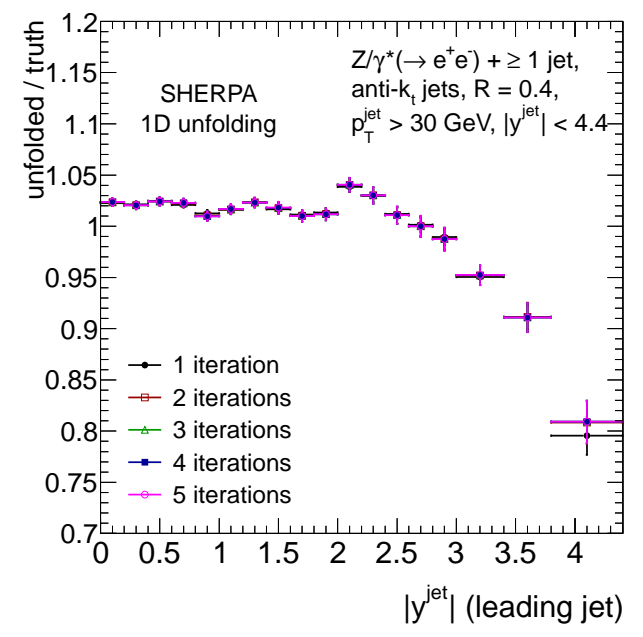

(a) one-dimensional unfolding

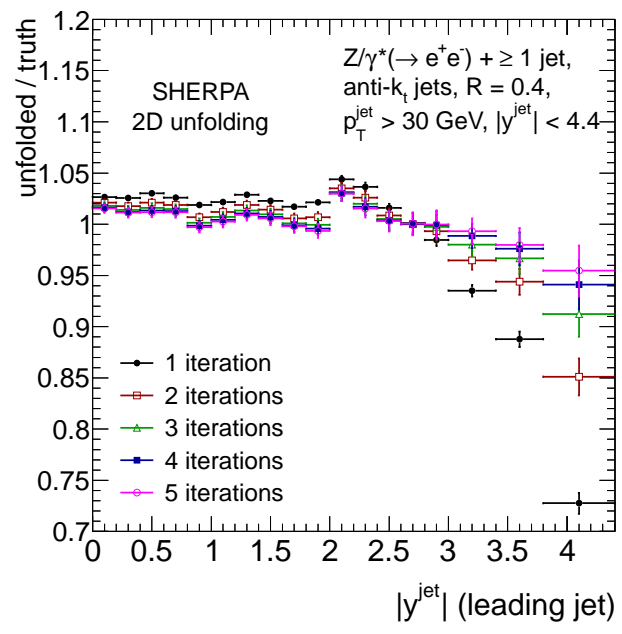

(b) two-dimensional unfolding

Figure 9.36.: Closure tests using (a) one-dimensional unfolding and (b) two-dimensional unfolding for the absolute rapidity of the leading jet for events with at least one jet in the final state. The detector level predictions from SHERPA have been unfolded with ALPGEN+HERWIG and compared to the particle level predictions from SHERPA. 
The bias in the forward region observed for the one-dimensional unfolding can be resolved using two-dimensional unfolding. The general offset observed in the tests can be ascribed to the fact that part of the correction is still based on bin-by-bin correction factors. The optimal number of iterations is determined from $\chi^{2}$ values divided by the number of bins for the different number of iterations, as described in Sec. 9.2.2 and shown in Fig. 9.37. In order to have a good balance between the unfolding bias and the statistical uncertainties the optimal number of iterations is chosen to five.

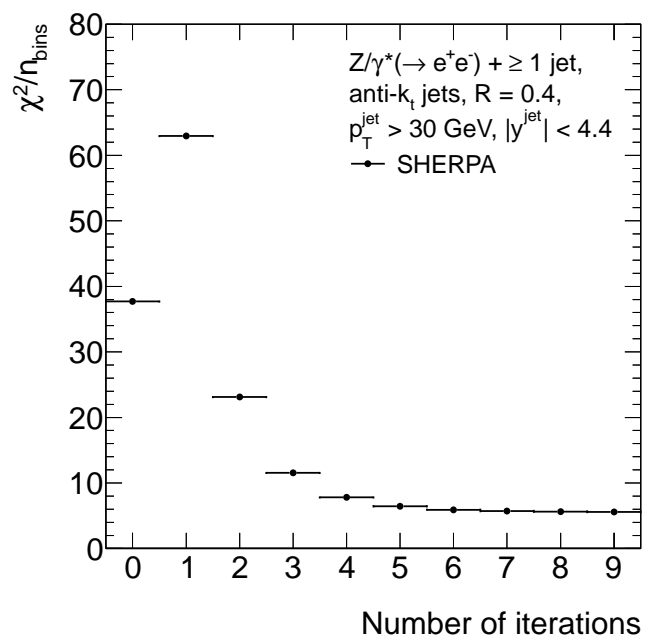

Figure 9.37: $\chi^{2}$ divided by the number of bins as a function of the number of iterations for the absolute rapidity of the leading jet for events with at least one jet in the final state. The values have been obtained with SHERPA MC event samples. Zero iterations correspond to the result from the Bin-by-Bin method.

For the systematic uncertainties on the two-dimensional unfolding two uncorrelated components are considered: the choice of the MC events sample used for unfolding and the limited statistics of the MC event sample. Figure 9.38 shows the smoothed results for the first component.

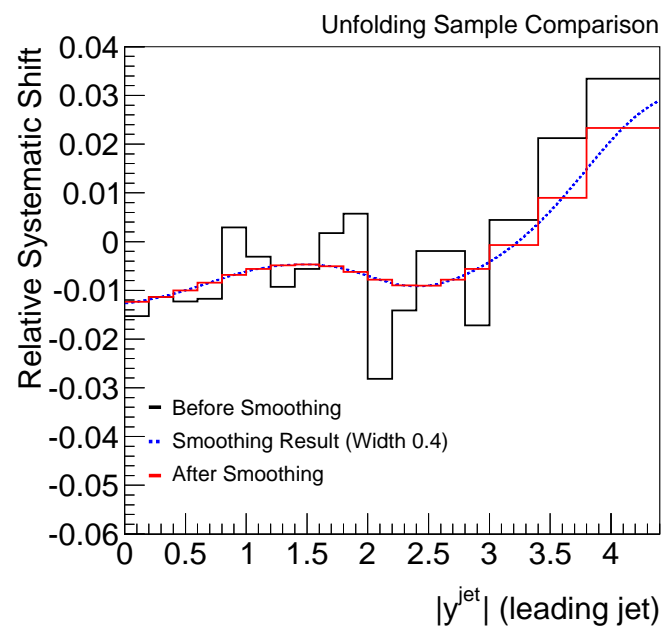

Figure 9.38: Relative systematic shifts on the unfolded cross section obtained by comparing the unfolding procedure using ALPGEN+HERWIG or SHERPA for the absolute rapidity of the leading jet for events with at least one jet in the final state. The unfolding has been performed in two dimensions. The relative shifts (black) are smoothed using a Gaussian kernel.

As expected, the dependence on the MC event sample is significantly reduced using two-dimensional unfolding compared to one-dimensional unfolding, as shown in Fig. 9.12 in Sec. 9.3. The total uncertainty due to unfolding has been decreased from up to $14.8 \%$ for the one-dimensional unfolding, as shown in Fig. 9.14(c) in Sec. 9.3 , to up to $3.7 \%$ for the two-dimensional unfolding. 
A detailed study of all components of the total systematic uncertainty is shown in Fig. 9.39. The total systematic uncertainty on the measured cross section in the forward region is significantly reduced due to the two-dimensional unfolding. In addition to the unfolding uncertainty, also the JES uncertainty is reduced. But this cannot be explained by the method itself, it refers to the rapidity distributions of the different $p_{T}^{\text {jet }}$ bins in the MC event samples. As shown in Fig. 9.33, ALPGEN+HERWIG predicts a broader rapidity distribution as the data for low $p_{T}$ jets, while for high $p_{T}$ jets ALPGEN+HERWIG tends to predict a narrower rapidity distribution than observed in the data.

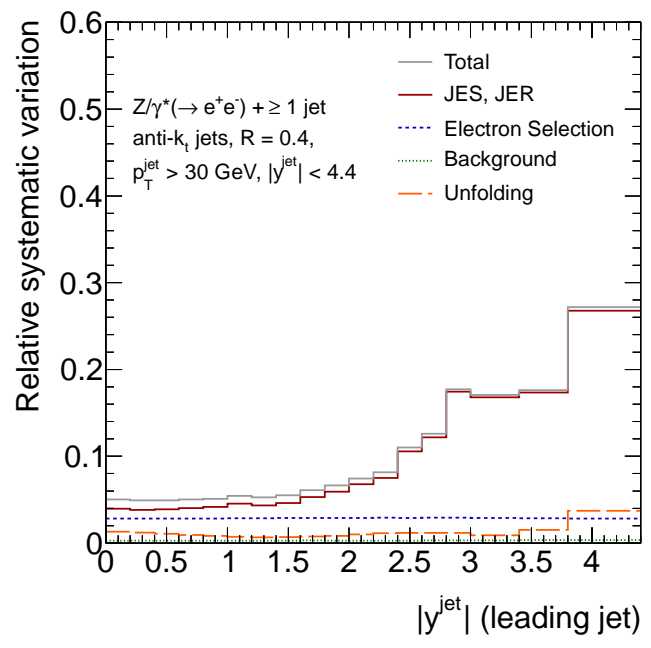

Figure 9.39: Different contributions to the combined relative systematic uncertainty for the measured cross section as a function of the absolute rapidity of the leading jet for events with at least one jet in the final state. The total systematic uncertainty is determined by summing up the different contributions in quadrature.

The result obtained with the two-dimensional unfolding, shown in Fig. 9.40, is consistent with the previous result obtained with the one-dimensional unfolding, which is shown in Fig. 9.32.

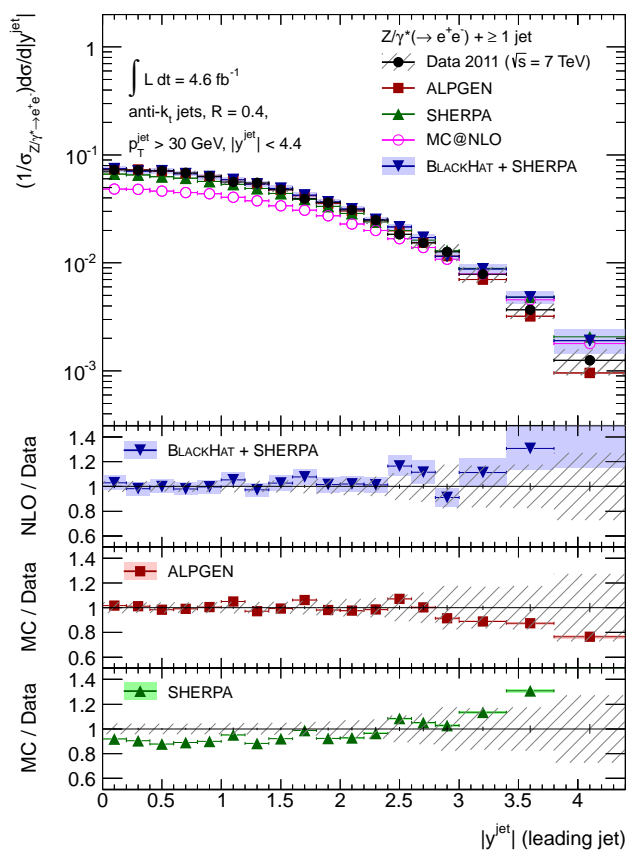

Figure 9.40: Differential cross section as a function of the absolute rapidity of the leading jet for events with at least one jet in the final state, determined using two-dimensional unfolding. The cross sections are normalised to the inclusive $Z / \gamma^{*}(\rightarrow e e)$ cross section. The other details are as in Fig. 9.21 .

The predictions from SHERPA and the NLO fixed-order pQCD calculations still overestimate the cross section in the forward region, while the predictions from ALPGEN+HERWIG now tend to model a too narrow rapidity distribution but are still consistent with the data. 
The current measurements are dominated by the JES uncertainty. In the future with a reduced JES systematics and a larger MC statistics, the uncertainty coming from the unfolding procedure might become dominant, which requires the usage of multi-dimensional unfolding in order to further reduce the systematic uncertainty.

\subsubsection{Invariant Dijet Mass}

The normalised differential cross section as a function of the invariant mass $m^{j j}$ of the two leading jets for events with at least two jets in the final state is depicted in Fig. 9.41. The correct modelling of this distribution is essential, since it is often used to separate signal from background in searches for new physics or studies of the Higgs boson.

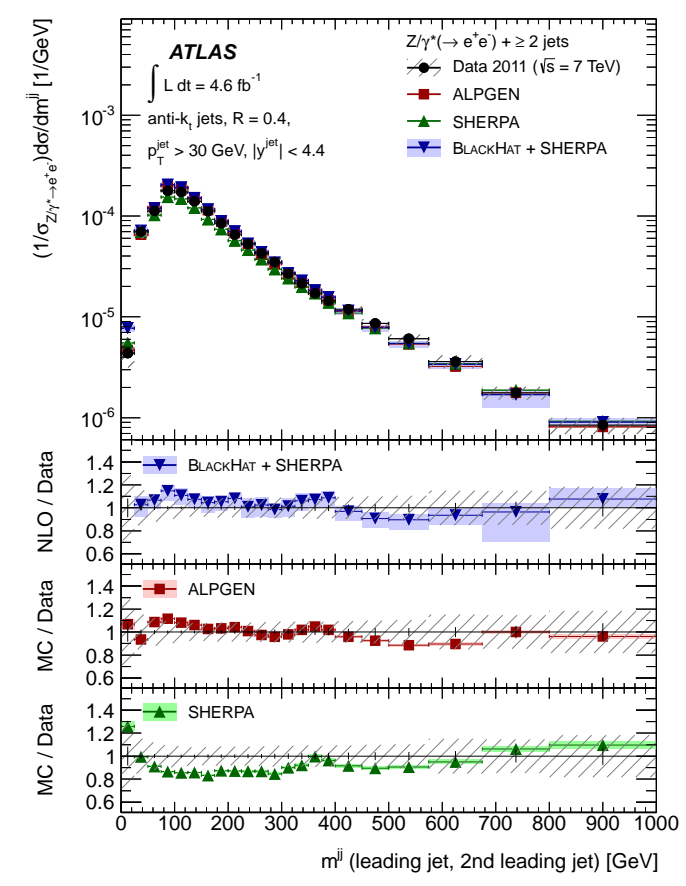

Figure 9.41: Differential cross section as a function of the invariant mass $m^{j j}$ of the two leading jets for events with at least two jets in the final state. The cross sections are normalised to the inclusive $Z / \gamma^{*}(\rightarrow e e)$ cross section. The other details are as in Fig. 9.21 .

For $m^{j j}>100 \mathrm{GeV}$, the measured cross section decreases with increasing $m^{j j}$. The predictions from ALPGEN+HERWIG, SHERPA and the NLO fixed-order pQCD predictions from BLACKHAT+SHERPA are consistent with the data. Detailed values of the measured cross section, together with the NLO fixed-order pQCD predictions, can be found in Appendix B.4.

\subsubsection{Angular Separation between the Leading Jets}

The normalised differential dijet cross sections as a function of the spatial separation between the two leading jets for events with at least two jets in the final state are shown in Fig. 9.42. The predictions from NLO fixed-order pQCD calculations from BLACKHAT+SHERPA are consistent with the data, although they predict a too broad rapidity distribution, as shown in Fig. 9.32. ALPGEN+HERWIG tends to underestimate the cross section for large values of $\left|\Delta y^{j j}\right|$ and $\Delta R^{j j}$, but the predictions are still consistent with the data. The tendencies observed in the rapidity distributions for the predictions from SHERPA are reflected in $\left|\Delta y^{j j}\right|$ and $\Delta R^{j j}$. In addition, the predictions from SHERPA 


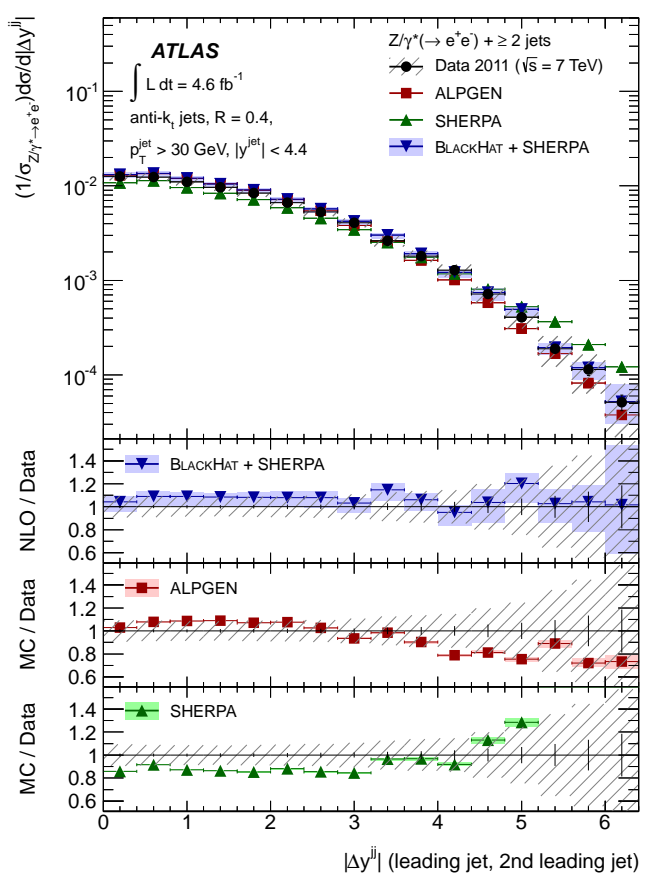

(a) Absolute rapidity difference

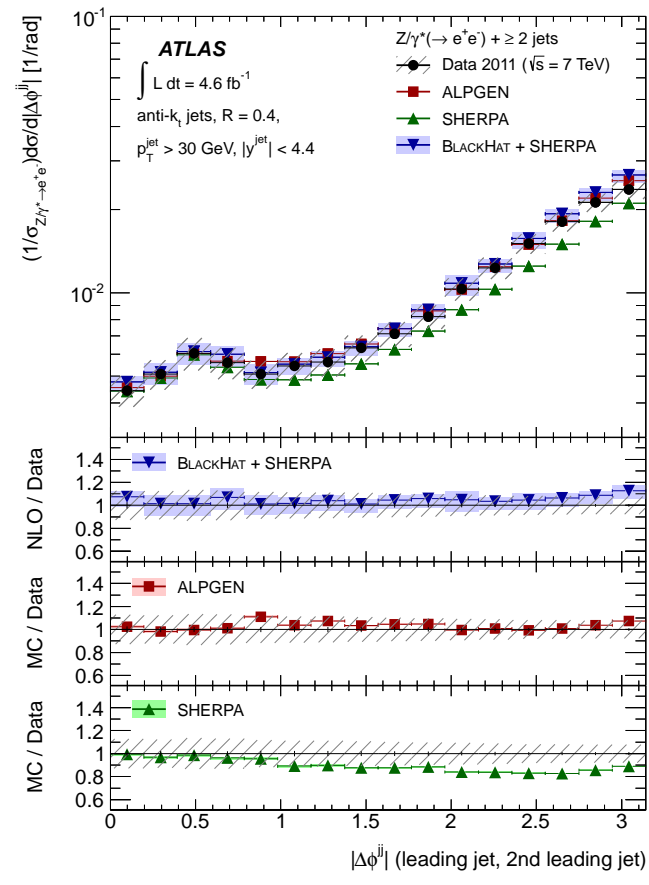

(b) Absolute azimuthal separation

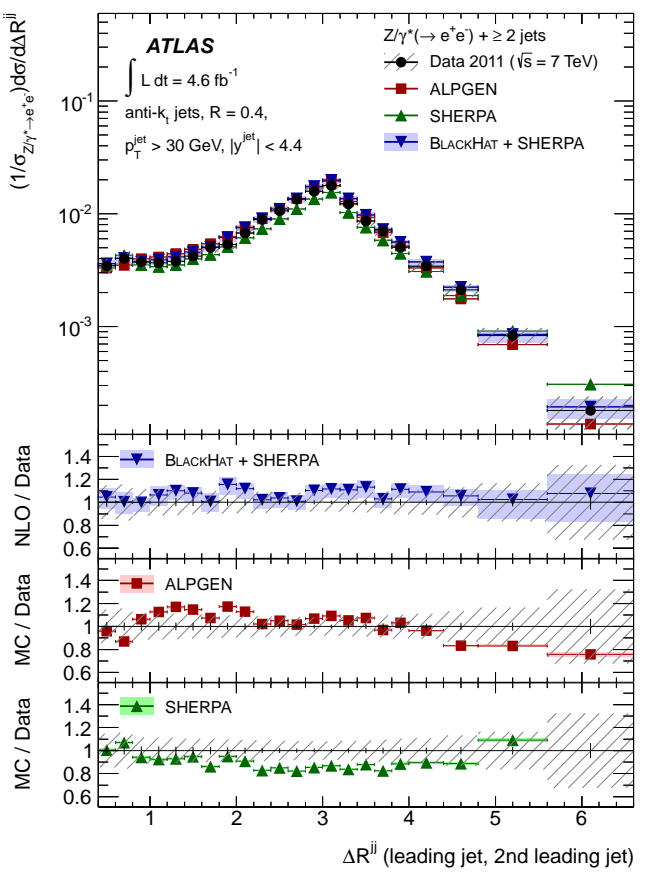

(c) Angular separation in $y-\phi$ space

Figure 9.42.: Differential dijet cross section as a function of (a) the absolute rapidity difference of the two leading jets $\left|\Delta y^{j j}\right|$, (b) the absolute azimuthal separation of the two leading jets $\left|\Delta \phi^{j j}\right|$ and (c) the angular separation in $y$ - $\phi$ space of the two leading jets $\Delta R^{j j}$ for events with at least two jets in the final state. The cross sections are normalised to the inclusive $Z / \gamma^{*}(\rightarrow e e)$ cross section. The other details are as in Fig. 9.21. 
model a too flat distribution for $\left|\Delta \phi^{j j}\right|$ and $\Delta R^{j j}$. The offset of the SHERPA prediction from the measured cross section is consistent with the observed offset of $15 \%$ presented in Fig. 9.21(a) for the inclusive $Z / \gamma^{*}(\rightarrow e e)+\geq 2$ jets cross section. Detailed values of the measured cross section, together with the NLO fixed-order pQCD predictions can be found in Appendix B.4.

\subsubsection{Inclusive Quantities: $H_{\mathrm{T}}$ and $S_{\mathrm{T}}$}

Inclusive quantities based on the $p_{T}$ sum of final state objects, such as $H_{T}$ and $S_{T}$, are of particular interests for searches of new physics. In addition, $H_{T}$ is of interest, since it is often used as the QCD renormalisation and factorisation scale for calculations of NLO fixed-order pQCD predictions. Large values of both $H_{T}$ and $S_{T}$, could result either from a small number of high energetic jets or from a large number of jets with a low energy. The latter are statistically favoured, but both require a good description of the $p_{T}^{j e t}$ and the jet multiplicity distribution.

Figure 9.43 shows the differential cross section as a function of $H_{T}$ and $S_{T}$. The cross sections are normalised to the inclusive $Z / \gamma^{*}(\rightarrow e e)$ cross section. For the predictions from BLACKHAT+SHERPA, the NLO fixed-order pQCD calculation for $Z / \gamma^{*}+\geq 1$ jet is used.

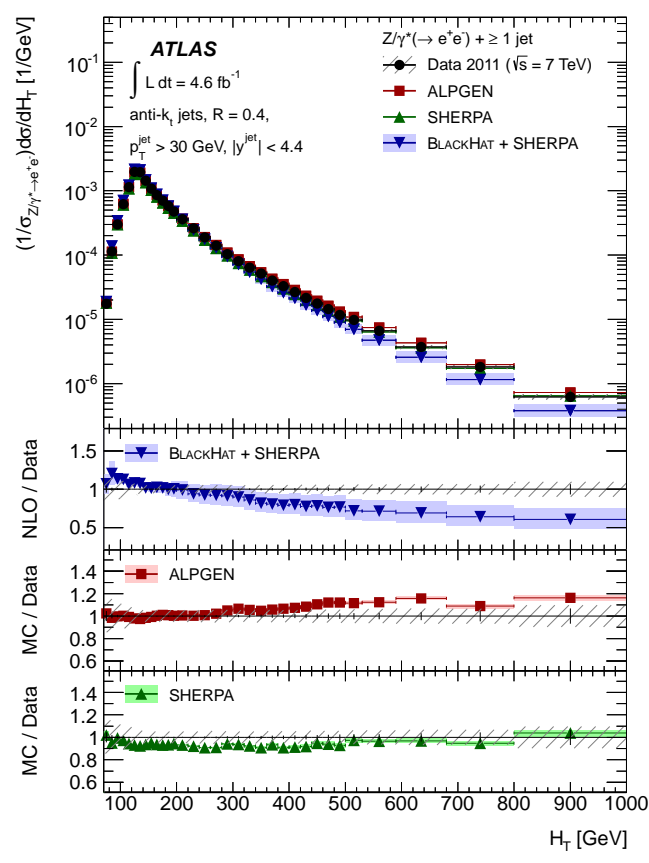

(a) $H_{T}$

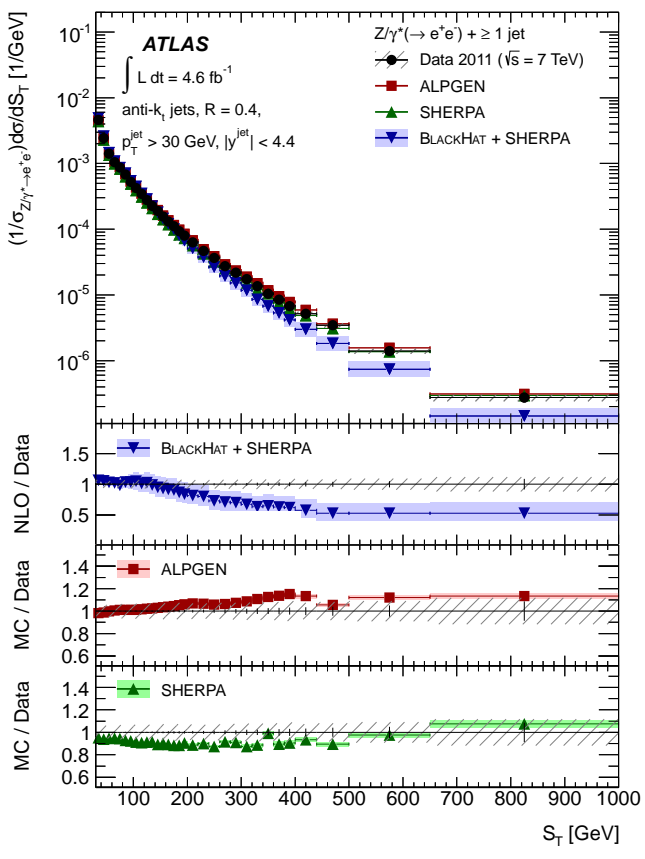

(b) $S_{T}$

Figure 9.43.: Differential cross section as a function of (a) $H_{T}$ and (b) $S_{T}$ for events with at least one jet in the final state. The cross sections are normalised to the inclusive $Z / \gamma^{*}(\rightarrow e e)$ cross section. The other details are as in Fig. 9.21.

The measurements agree well with the predictions from ALPGEN+HERWIG and SHERPA, while the predictions from NLO fixed-order pQCD calculations underestimate the cross section for large values of $H_{T}$ and $S_{T}$. These differences are attributed to a 
truncated number of jet emissions. The NLO fixed-order pQCD predictions are calculated inclusively for events with a $Z$ boson and at least one jet in the final state. Due to this fact, matrix elements for three or more real parton emissions are not included in the final state, while the predictions from ALPGEN+HERWIG and SHERPA include matrix elements for up to five partons in the simulation.

Figure 9.44 shows the average number of jets as a function of $H_{T}$ at detector level.

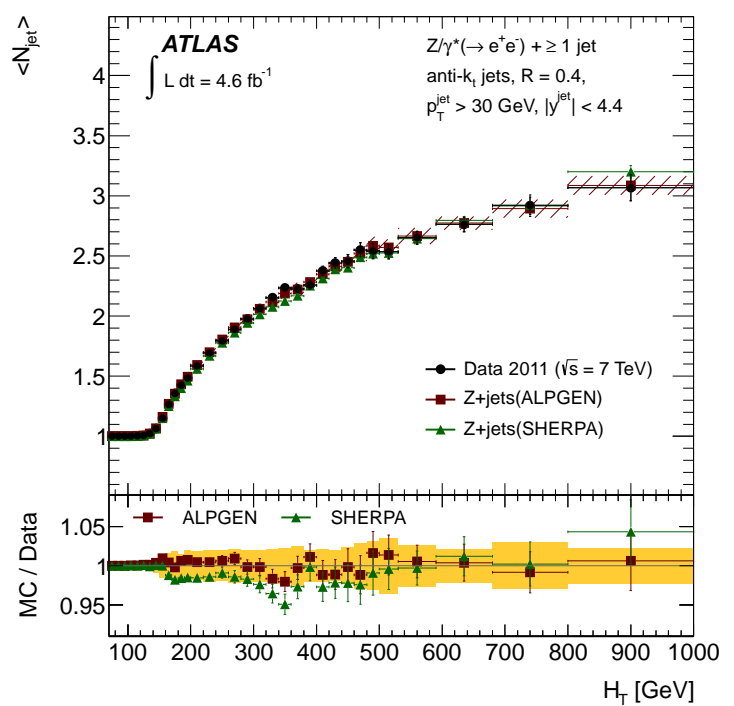

Figure 9.44: Average number of jets as a function of $H_{T}$ in data and predicted by the generators ALPGEN+HERWIG and SHERPA. The measured values after background subtraction are represented by the black dots, the error bars indicate the combined statistical and systematic uncertainty on the data. The hatched band incorporates the total statistical and systematic uncertainty on the predictions, modelled with ALPGEN+HERWIG. The shaded band reflects the total systematic uncertainty.

The average jet multiplicity is modelled well within uncertainties for both ME+PS generators. Already for $H_{T} \approx 300 \mathrm{GeV}$ the average number of jets is close to two and for $H_{T} \approx 600 \mathrm{GeV}$ close to three. In the following, the event shapes in the subset of $Z / \gamma^{*}+\geq 2$ jets events are studied in more detail, similar to the case for $p_{T}^{e e}$. Figure 9.45 shows $\Delta \phi$ and the ratio $R\left(p_{T} 2 / p_{T} 1\right)$ of the two leading jets for two regimes of $H_{T}$. While events with low $H_{T}$ are dominated by events with two jets of similar $p_{T}$, for large $H_{T}$, the events are characterized by a small $R\left(p_{T} 2 / p_{T} 1\right)$, thus introducing a large scale difference between the two leading jets. But for both cases the two jets are typically back-to-back, in contrast to the cases for $p_{T}^{e e}$, where only the events with the $Z$ boson at rest are dominated by events with two back-to-back jets.

Apparently, NLO fixed-order $Z / \gamma^{*}+\geq 1$ jet calculations are not able to describe two jet final states with large scale differences, as already seen in Sec. 9.5.3 for the transverse momentum of the $Z$ boson. In addition, events with three jets in the final state play an important role in the description of $H_{T}$, which are not included in the NLO fixedorder $Z / \gamma^{*}+\geq 1$ jet calculations, as described above. Discrepancies in the description of $H_{T}$ between NLO fixed-order pQCD calculations and data have already been reported in $W+$ jets events, where they can be mitigated by including higher jet multiplicities in the calculation using exclusive sums [165]. Figure 9.46 shows the comparison of the exclusive sum to the measurement in data for the normalised differential cross section as a function of $H_{T}$ and $S_{T}$ for events with at least one jet in the final state. Here, the exclusive sum is obtained by adding the exclusive $Z / \gamma^{*}(\rightarrow e e)+1$ jet and the inclusive $Z / \gamma^{*}(\rightarrow e e)+2$ jets calculations from BLACKHAT+SHERPA, similar to the case for the transverse momentum distribution of the $Z$ boson described in Sec. 9.5.3. As expected, the exclusive sum yields a much better performance. However, the discrepancy for low 


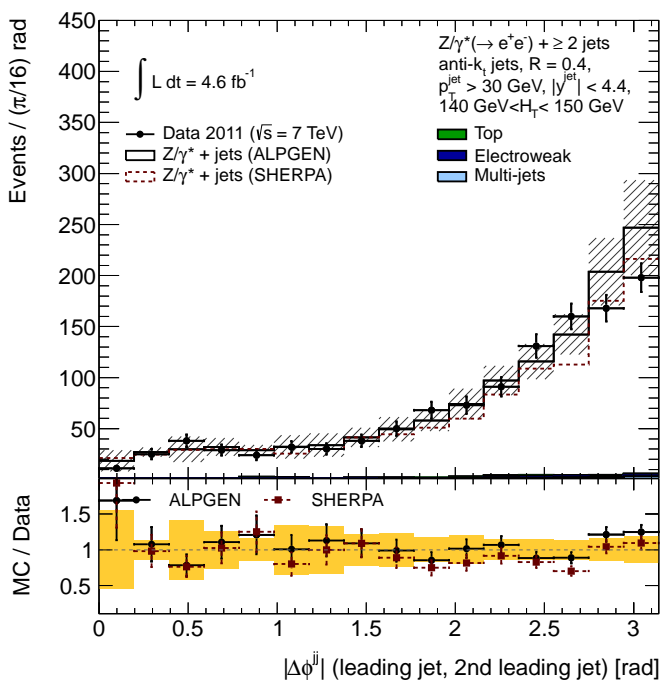

(a) Absolute azimuthal separation, $140 \mathrm{GeV}<H_{T}<150 \mathrm{GeV}$

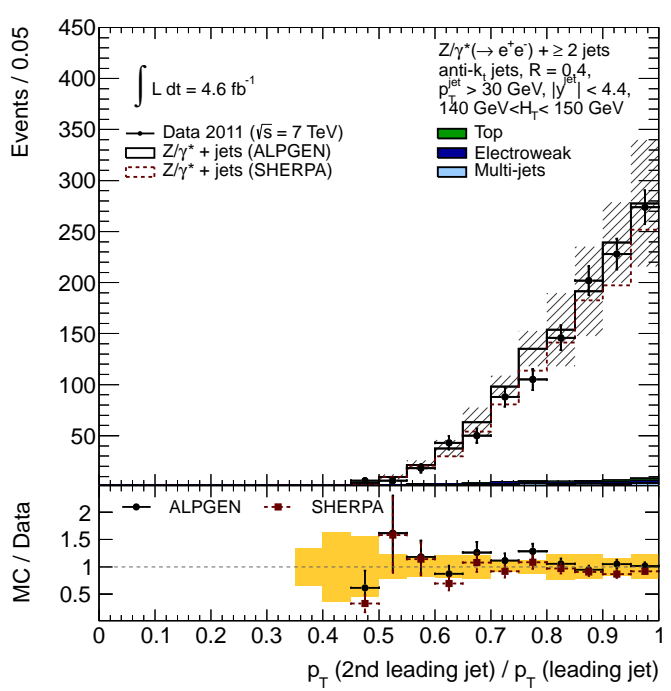

(c) $p_{T}$ ratio,

$140 \mathrm{GeV}<H_{T}<150 \mathrm{GeV}$

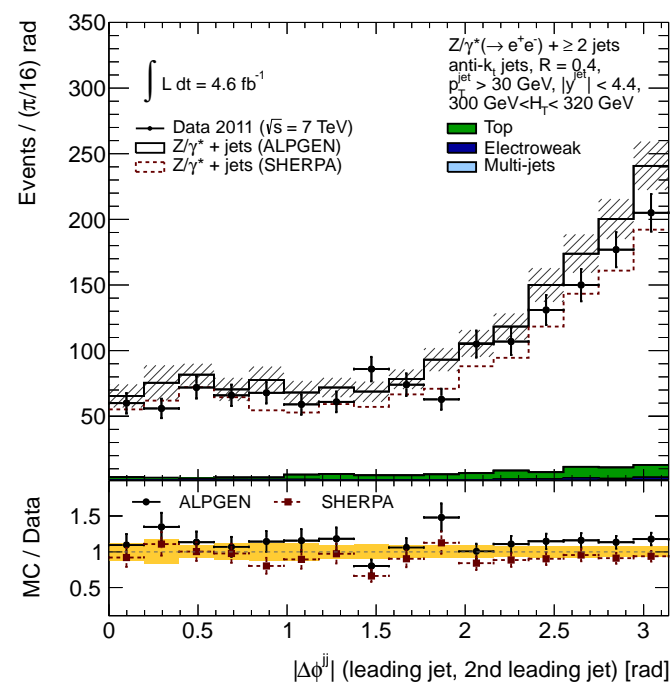

(b) Absolute azimuthal separation, $300 \mathrm{GeV}<H_{T}<320 \mathrm{GeV}$

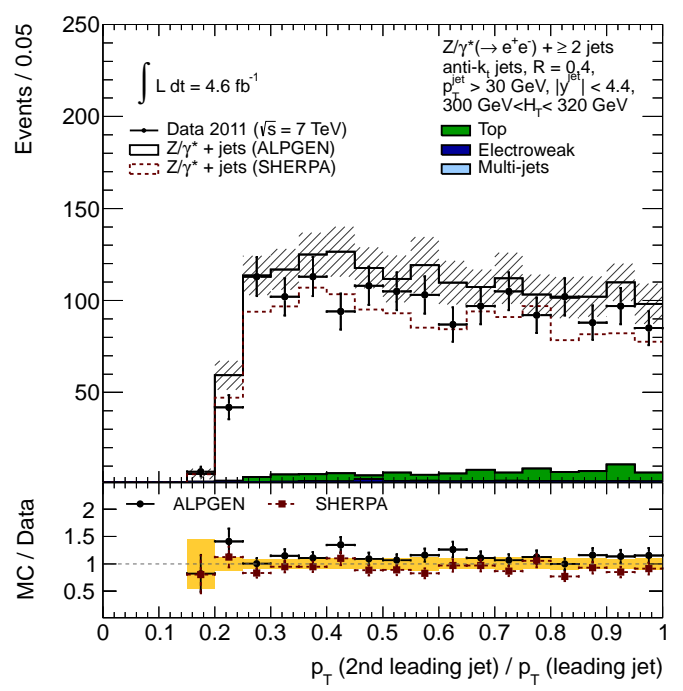

(d) $p_{T}$ ratio, $300 \mathrm{GeV}<H_{T}<320 \mathrm{GeV}$

Figure 9.45.: Absolute azimuthal separation $\left|\Delta \phi^{j j}\right|$ of the two leading jets for different bins of $H_{T}$, (a) $140 \mathrm{GeV}<H_{T}<150 \mathrm{GeV}$ and (b) $300 \mathrm{GeV}<H_{T}<320 \mathrm{GeV}$ and ratio of the transverse momentum of the 2nd leading jet to that of the leading jet for different bins of $p_{\mathrm{T}}^{Z}$, (c) $140 \mathrm{GeV}<H_{T}<150 \mathrm{GeV}$ and (d) $300 \mathrm{GeV}<H_{T}<320 \mathrm{GeV}$ in events with at least two jets in the final state in data and simulation. Multi-jets and $t \bar{t}$ backgrounds have been estimated from data. The hatched band incorporates the total statistical and systematic uncertainty on the predictions. The yellow band reflects the total systematic uncertainty. 
values of $H_{T}$ does not vanish and for very high values of $H_{T}$ and $S_{T}$ the NLO pQCD predictions still tend to underestimate the measured cross section. These differences are expected to be solved by either using nNLO predictions [9] or using the exclusive sum of the exclusive $Z / \gamma^{*}(\rightarrow e e)+1$ jet, the exclusive $Z / \gamma^{*}(\rightarrow e e)+2$ jets and the inclusive $Z / \gamma^{*}(\rightarrow e e)+3$ jets calculation [165].

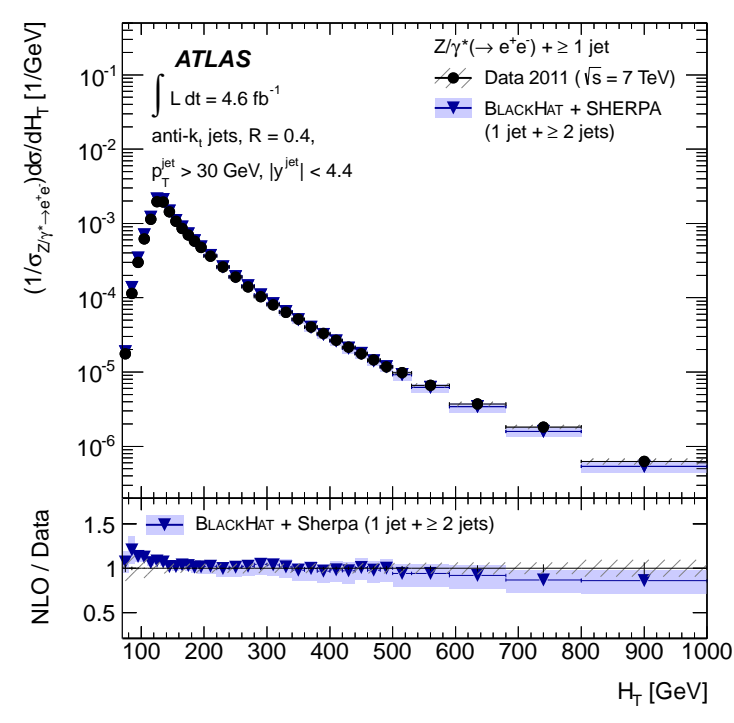

(a) $H_{T}$

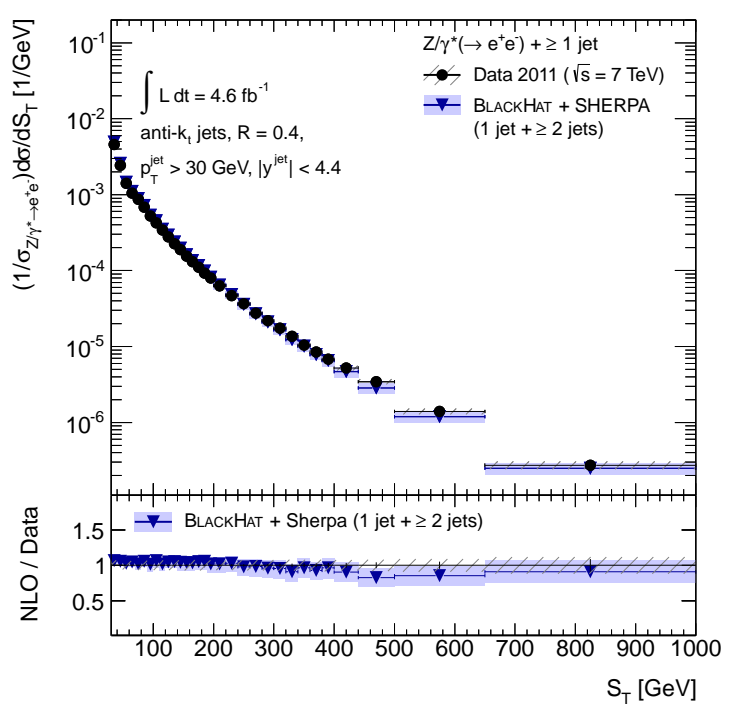

(b) $S_{T}$

Figure 9.46.: Differential cross section as a function of (a) $H_{T}$ and (b) $S_{T}$ for events with at least one jet in the final state. The cross sections are normalised to the inclusive $Z / \gamma^{*}(\rightarrow e e)$ cross section. The measured values are represented by the black dots, the error bars indicate the statistical uncertainty and the hatched band incorporates the total statistical and systematic uncertainty added in quadrature. The measurements are compared to NLO pQCD predictions from BLACKHAT+SHERPA, obtained by adding the exclusive $Z / \gamma^{*}(\rightarrow e e)+1$ jet and the inclusive $Z / \gamma^{*}(\rightarrow e e)+\geq 2$ jets calculations. The shaded bands represent the theoretical uncertainties.

The detailed values of the measured cross sections, as well as NLO pQCD predictions from BLACKHAT+SHERPA with respect to the fiducial region are listed in Appendix B.4.

\subsection{Conclusions}

The inclusive and differential $Z / \gamma^{*}(\rightarrow e e)+$ jets cross section has been measured in ppcollisions at a centre-of-mass energy of $\sqrt{s}=7 \mathrm{TeV}$ with the full dataset of 2011. Total inclusive and exclusive cross sections have been measured as a function of the jet multiplicity. In addition, the ratios of cross sections of successive jet multiplicities have been measured for different values of the transverse momentum of the leading jet to test the scaling behaviour. The inclusive differential cross sections have been measured as a function of the jet transverse momentum, the jet rapidity, the transverse momentum of the $Z$ boson, the invariant mass of the two leading jets, the angular separation between the two leading jets and the inclusive quantities $H_{T}$ and $S_{T}$. 
Systematic and statistical uncertainties have been evaluated and the systematic uncertainty has been reduced by normalising the cross sections to the inclusive $Z / \gamma^{*}(\rightarrow e e)$ cross section. Compared to the measurement with the full dataset of 2010 the sensitivity has been extended to higher jet multiplicities, larger transverse momentum and rapidity.

In general, a good agreement within uncertainties between predictions from ALPGEN+HERWIG, SHERPA, NLO fixed-order pQCD calculations and the data have been found, while MC@NLO fails to describe the data in large phase space regions. MC@NLO is not able to describe higher jet multiplicities, predicts a too soft $p_{T}$ spectrum and a too broad rapidity distribution. For exclusive jet multiplicities, the transition from staircase scaling to Poisson scaling for large scale differences between the leading jet and the additional radiated jets is confirmed by the measurement.

For some phase-space regions discrepancies between the predictions from the ME+PS generators and the measurements have been observed. The jet multiplicity is well described by ALPGEN+HERWIG and SHERPA up to five jets, but additional jet emissions are modelled by parton shower, which fail to describe the data. Furthermore, ALPGEN+HERWIG overestimates the cross section for high energetic jets or highly boosted $Z$ bosons, which is mainly due to higher-order QCD effects. Finally, SHERPA predicts a too wide rapidity distribution, which translates into the dijet mass, the absolute rapidity difference and the angular separation in $y$ - $\phi$ space. In addition, SHERPA models a too flat spectrum for the absolute azimuthal separation of the two leading jets.

The NLO fixed-order pQCD calculations from BLACKHAT+SHERPA underestimate the cross section for large $H_{T}$ and $S_{T}$ by several standard deviations, which is attributed to missing higher parton multiplicities in the calculation. Similar deviations have been found for large $p_{T}^{e e}$. Exclusive sums of the NLO fixed-oder pQCD calculations for $Z / \gamma^{*}+1$ jet and $Z / \gamma^{*}+\geq 2$ jets show a much better description of the hard $H_{T}, S_{T}$ and $p_{T}^{e e}$ regime. 


\section{Higgs Boson Production via Weak Boson Fusion}

On July 4th, 2012, the discovery of a new boson [1, 2] of mass near $125 \mathrm{GeV}$ was announced. This boson is consistent with the expectations of a Higgs boson but it still has to be proven that it is the same particle as predicted by the SM. It is now the highest priority of the LHC physics programme to explore the nature of this boson. This requires the precise measurement of its properties. As of today, only evidence of the Higgs boson in the vector boson decay modes was found. Interactions of the new particle with fermions, i.e. $H \rightarrow \tau^{+} \tau^{-}$, as predicted by the SM, have not been discovered yet.

For the detailed investigation of the Higgs boson candidate, one of the main challenges is the understanding of the background contributions. With $25 \mathrm{fb}^{-1}$ of data, more production channels are accessible. One of the most promising channels is the Higgs boson production via weak boson fusion (WBF), since it provides a clear signature in the detector, which can be easily used to distinguish signal from background. Figure 10.1 depicts the LO Feynman diagram for the Higgs boson production via WBF.

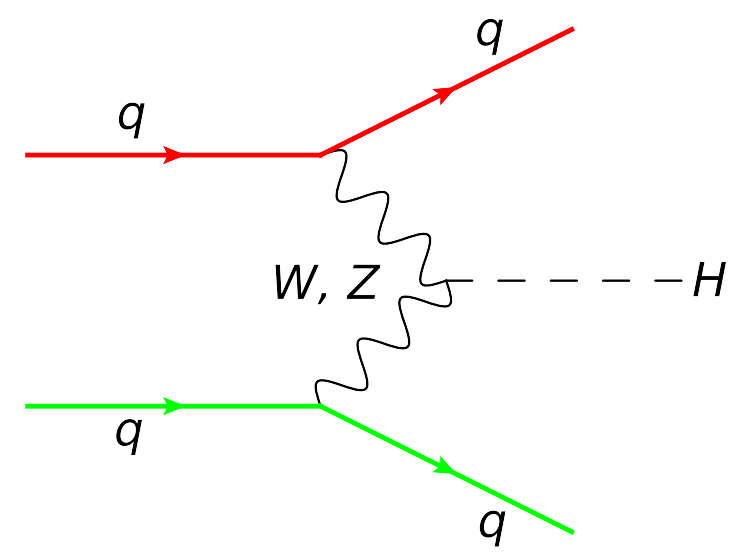

Figure 10.1.: Leading-order Feynman diagram for the Higgs boson production via vector boson fusion. The colour flow is indicated with the red and green lines.

From the WBF production two well separated high energetic forward jets are expected. Due to the colour coherence of those jets, indicated by the red and green line, central activity between them is suppressed. Several SM processes show a similar signature in the detector, e.g. $Z / \gamma^{*} \rightarrow \ell^{+} \ell^{-}$with $\ell=e, \mu, \tau, t \bar{t}$ production and diboson processes. Figure 10.2 shows the collinear mass distribution for events passing the $H \rightarrow \tau \tau$ selection in the WBF category, where both taus decay leptonically, as detailed in Ref. [169]. 


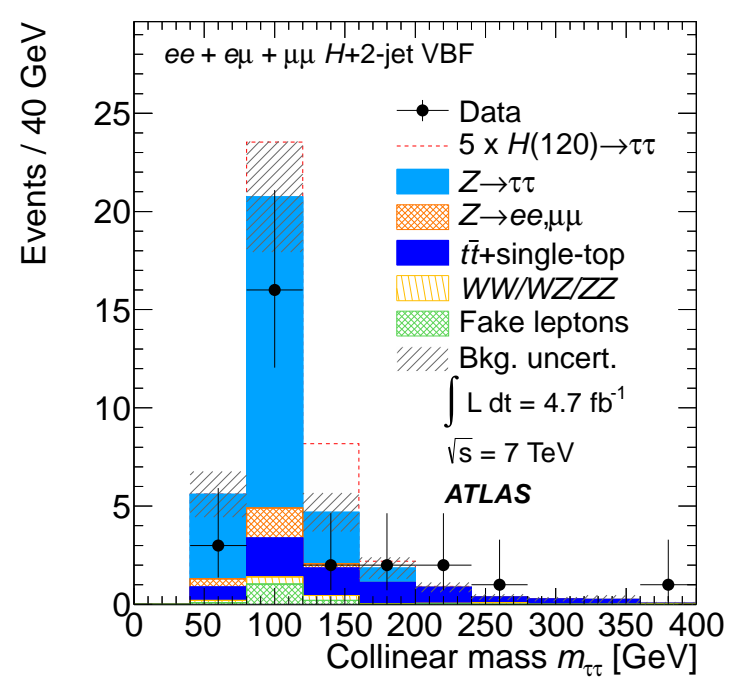

Figure 10.2: Collinear mass distribution for events passing $H \rightarrow \tau^{+} \tau^{-}$selection in the WBF category. The simulated samples have been scaled to the integrated luminosity in data [169].

In the mass range between $50 \mathrm{GeV}$ and $200 \mathrm{GeV}$, the production of $Z$ bosons in association with jets, where the $Z$ boson decays to two taus, constitutes the dominant background. Therefore it is essential that the jet kinematics in $Z / \gamma^{*}+$ jets events is well understood. The production of $Z / \gamma^{*}+$ jets events can be either estimated from data or modelled by MC event samples. Each serves as a cross check for the other. In order to probe the $Z / \gamma^{*}+$ jets modelling in typical phase-space regions expected for the decay of the Higgs boson, produced via WBF, the production of $Z / \gamma^{*}(\rightarrow e e)+$ jets events provides a valuable control region, since no contribution from the Higgs boson production is expected in this region.

The LO Feynman diagrams for the QCD and the EW production of $Z+2$ jets events are shown in Fig. 10.3 .

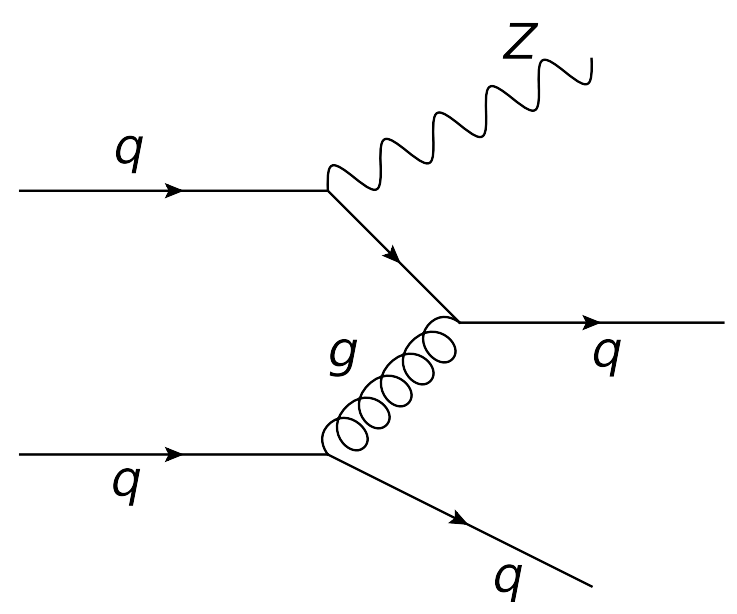

(a) QCD $Z+2$ jets production

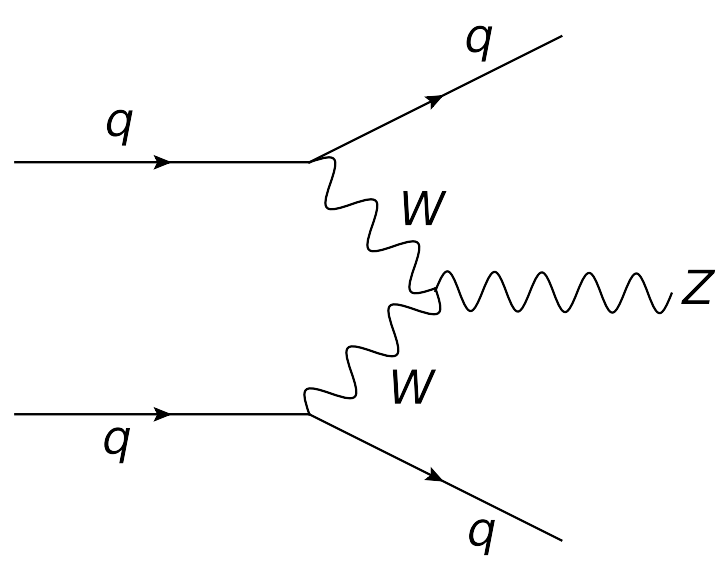

(b) EW $Z+2$ jets production

Figure 10.3.: Leading-order Feynman diagrams for QCD $Z+2$ jets production (a) and the $\mathrm{EW} Z+2$ jets production (b).

The QCD production of $Z+$ jets provides a reducible background, due to its larger probability for QCD radiation in the central region, while the EW production is an irreducible background, since it has the same colour coherence between the final state jets. 
In order to reduce the background contribution in the WBF selection, a veto against additional jets provides a good tool to distinguish signal from background. But a simple veto on all jets is expected to also cut on the signal, therefore more elaborated methods are needed.

This chapter describes the probe of the $Z / \gamma^{*}+$ jets modelling in typical phase-space regions expected for the decay of the Higgs boson, produced via WBF, in pp-collisions at a centre of mass energy of $\sqrt{s}=7 \mathrm{TeV}$ with an integrated luminosity of $\int L \mathrm{~d} t=4.6 \mathrm{fb}^{-1}$. The main focus has been placed on the kinematics of the 3rd jet after applying the WBF preselection, since a veto against a 3rd central jet (CJV) provides a good tool to distinguish signal from background. In this regions the expected contribution from the EW production of $Z / \gamma^{*}(\rightarrow e e)+$ jets events is still at the per-mille to the per-cent level, therefore it is not included in the following studies, as already described in Sec. 7.2.1. A good modelling of the data by the $\mathrm{MC}$ predictions is essential, especially for multivariate analyses, which use MC predictions for the training.

This chapter is organised as follows: Starting with a brief description of the WBF preselection in Sec. 10.1, Sec. 10.2 proceeds with the uncorrected distributions comparing MC predictions to the measurements on detector level, focussing on observables which can be used to optimise the central jet veto. Finally, Sec. 10.3 discusses the unfolded results of the jet multiplicity distribution, as well as the kinematic distributions of the 3rd jet after WBF preselection. The method for the correction of detector effects, the sources of systematic uncertainties and the NLO fixed-order pQCD predictions have already been discussed in Chapter 9 .

\subsection{Weak Boson Fusion Preselection}

This measurement uses the full dataset of 2011, which was collected in pp-collisions at a centre of mass energy of $\sqrt{s}=7 \mathrm{TeV}$ with the ATLAS detector.

The baseline of the selection used for this analysis is identical to the standard $Z / \gamma^{*}(\rightarrow e e)$ selection for 2011 described in Sec. 7.1 to provide a clean control region, in which no signal from the Higgs boson production is expected. As discussed earlier, from the WBF production of the Higgs boson, two well separated high energetic forward jets are expected. Therefore on top of the standard selection, events are required to have at least two jets, which are well separated in rapidity $\left|\Delta y^{j j}\right|>3.0$ and have a dijet mass of $m^{j j}>350 \mathrm{GeV}$ to enhance WBF-like signatures. Before performing these cuts, the modelling of these distributions has been checked, as shown in Fig. 9.42 of Sec. 9.5.6. In the following, the two leading jets are called tagging jets.

\subsection{Uncorrected Distributions}

The uncorrected data distributions for $Z / \gamma^{*}(\rightarrow e e)+\geq 2$ jets after passing the WBF preselection have been compared to the expected signal and background distributions. For signal expectations two different ME+PS generators have been used, ALPGEN+HERWIG and SHERPA 1.4.1. The corrections applied to these samples are identical to the ones described in Sec. 9.1. Table 10.1 summarises the events passing the WBF preselection in data and the expectations from MC for both ALPGEN+HERWIG and SHERPA as signal 
MC. The uncertainties incorporate the combined statistical and systematic uncertainty, as detailed in Sec. 9.3.

\begin{tabular}{|c|c|c|c|c|}
\hline \hline & $\geq 2$ jets & $\geq 3$ jets & $\geq 4$ jets & $\geq 5$ jets \\
\hline \hline$Z / \gamma^{*}(\rightarrow e e)($ ALPGEN $)$ & $2373 \pm 390$ & $697 \pm 116$ & $166 \pm 36$ & $32 \pm 7$ \\
$Z / \gamma^{*}(\rightarrow e e)($ SHERPA $)$ & $2701 \pm 444$ & $657 \pm 109$ & $134 \pm 29$ & $31 \pm 7$ \\
$W(\rightarrow e \nu)$ & $3 \pm 1$ & $0.6 \pm 0.6$ & $<0.6$ & $<0.6$ \\
$Z / \gamma^{*}(\rightarrow \tau \tau)$ & $1.9 \pm 0.7$ & $0.3 \pm 0.2$ & $<0.2$ & $<0.2$ \\
$W W, W Z, Z Z$ & $21 \pm 3$ & $8 \pm 2$ & $1.8 \pm 0.6$ & $0.4 \pm 0.1$ \\
Single top & $4.0 \pm 0.7$ & $1.5 \pm 0.5$ & $0.3 \pm 0.2$ & $0.2 \pm 0.2$ \\
$t \bar{t}$ & $59 \pm 13$ & $35 \pm 9$ & $13 \pm 5$ & $4 \pm 3$ \\
Multi-jets & $25 \pm 9$ & $6 \pm 2$ & $1.8 \pm 0.7$ & $0.5 \pm 0.2$ \\
\hline Total predicted (ALPGEN) & $2487 \pm 394$ & $748 \pm 117$ & $183 \pm 36$ & $37 \pm 7$ \\
Total predicted (SHERPA) & $2815 \pm 446$ & $709 \pm 111$ & $151 \pm 30$ & $36 \pm 7$ \\
Data observed & 2753 & 715 & 146 & 37 \\
\hline \hline
\end{tabular}

Table 10.1.: Number of events predicted from MC simulation and observed in data for the different jet multiplicities for events with at least two jets in the final state passing the WBF preselection $\left(m^{j j}>350 \mathrm{GeV}\right.$ and $\left.\left|\Delta y^{j j}\right|>3.0\right)$. The predictions for $t \bar{t}$ and multi-jets have been estimated from data. The combined statistical and systematic uncertainty, as described in Sec. 9.3, are included.

Figure 10.4 shows the inclusive and exclusive jet multiplicity distribution after WBF preselection.

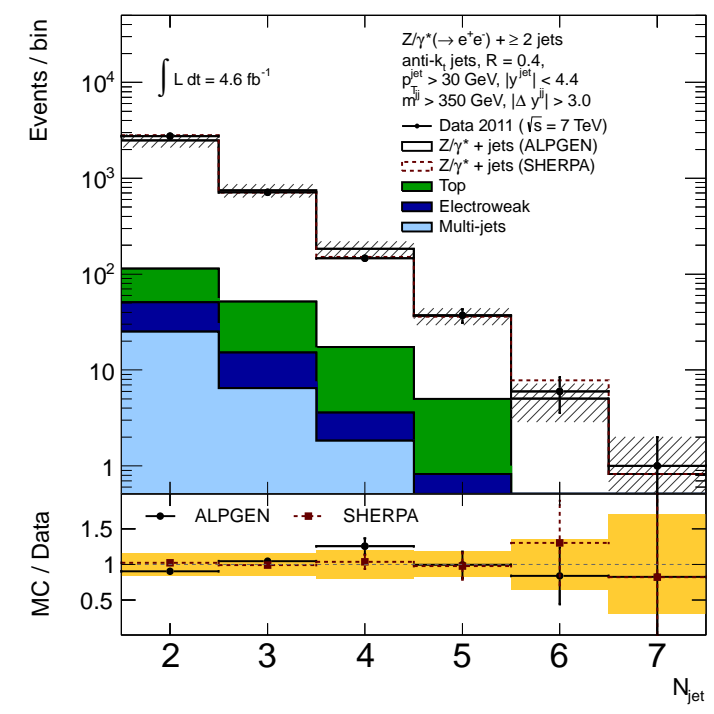

(a) Inclusive jet multiplicity

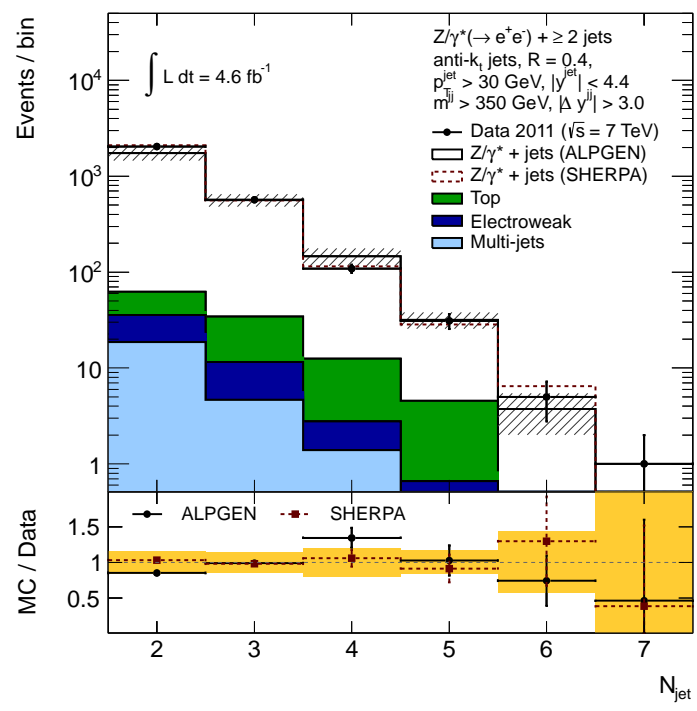

(b) Exclusive jet multiplicity

Figure 10.4.: (a) Inclusive jet multiplicity and (b) exclusive jet multiplicity for events with at least two jets in the final state passing the WBF preselection $\left(m^{j j}>350 \mathrm{GeV}\right.$ and $\left.\left|\Delta y^{j j}\right|>3.0\right)$ in data and simulation. Multi-jets and $t \bar{t}$ backgrounds have been estimated from data. The hatched band incorporates the total statistical and systematic uncertainty on the predictions. The yellow band reflects the total systematic uncertainty.

The predictions from SHERPA are in agreement with the data, while ALPGEN+HERWIG 
underestimates the number of events with two jets in the final state and overestimates the ratio $R_{3 / 2}$. The good modelling of the fraction of two jet events which have an additional third jet is essential, since a veto on a 3rd jet provides a powerful tool to distinguish signal from background for WBF Higgs boson searches.

In addition, an excellent modelling of the 3rd jet kinematics is required. Figure 10.5 shows the transverse momentum and the rapidity distribution of the 3rd jet after WBF preselection. The predictions from both generators are consistent with the data.

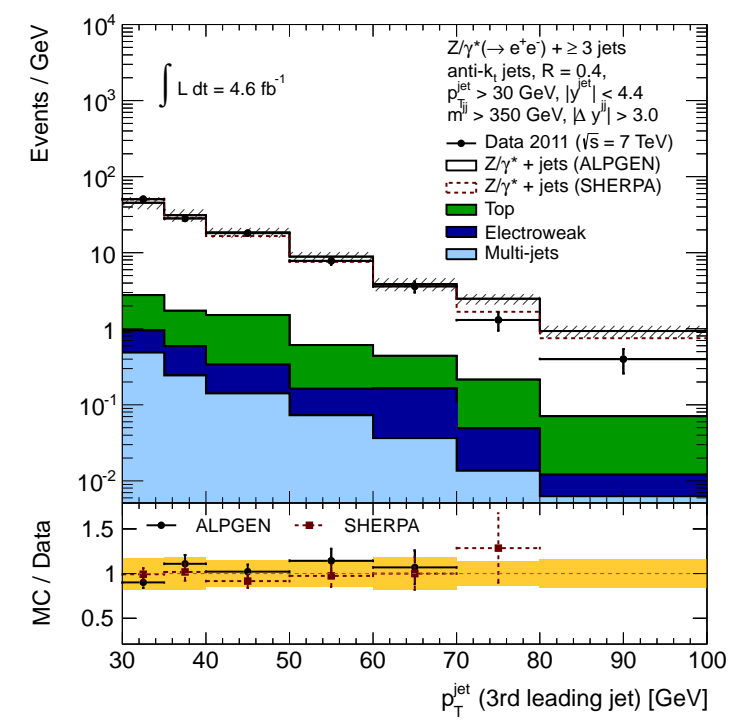

(a) 3rd leading jet $p_{T}$

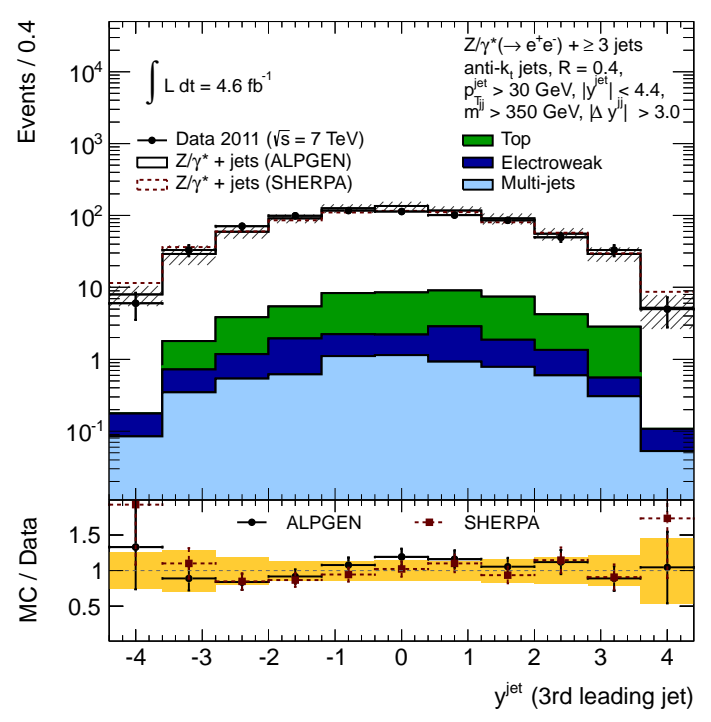

(b) 3rd leading jet $|y|$

Figure 10.5.: (a) Transverse momentum distribution and (b) rapidity distribution of the 3rd leading jet for $Z / \gamma^{*}(\rightarrow e e)+$ jets events with at least two jets in the final state passing the WBF preselection $\left(m^{j j}>350 \mathrm{GeV}\right.$ and $\left.\left|\Delta y^{j j}\right|>3.0\right)$ in data and simulation. Multi-jets and $t \bar{t}$ backgrounds have been estimated from data. The hatched band incorporates the total statistical and systematic uncertainty on the predictions. The yellow band reflects the total systematic uncertainty.

The probability to survive a veto on a 3rd jet above a given $p_{T}$ threshold for $Z / \gamma^{*}(\rightarrow e e)+$ jets events with at least two jets in the final state passing the WBF preselection $\left(m^{j j}>350 \mathrm{GeV}\right.$ and $\left.\left|\Delta y^{j j}\right|>3.0\right)$ is shown in Fig. 10.6, called jet veto efficiency in the following. Figure 10.5(a) shows the jet veto efficiency of a third jet with $\left|y^{\text {jet }}\right|<4.4$, while Fig. $10.5(\mathrm{~b})$ shows the jet veto efficiency of a central third jet with $\left|y^{\text {jet }}\right|<2.4$. The predictions from SHERPA agree well with the jet veto efficiency found in data, whereas the overestimation of $R_{3 / 2}$ for ALPGEN+HERWIG translates into an underestimation of the jet veto efficiency. 


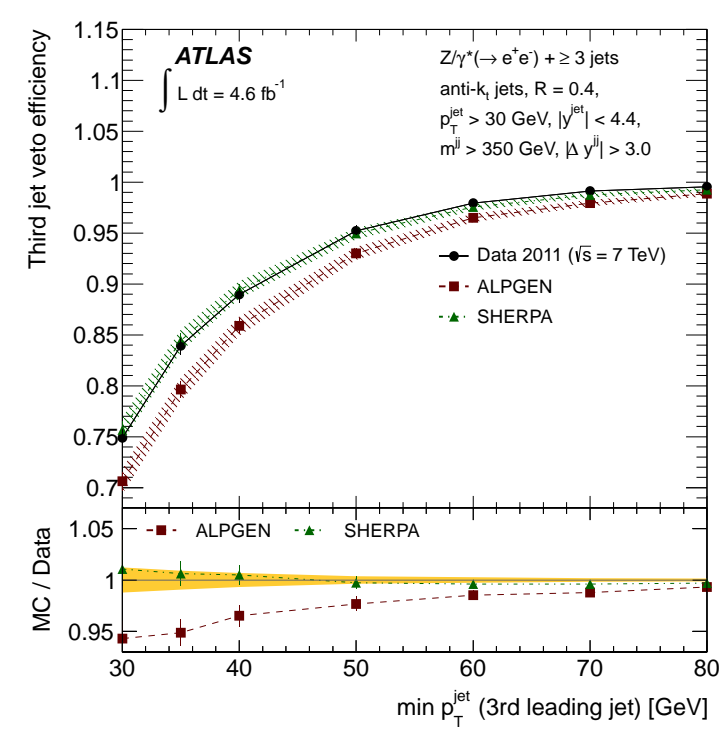

(a) 3rd jet veto efficiency

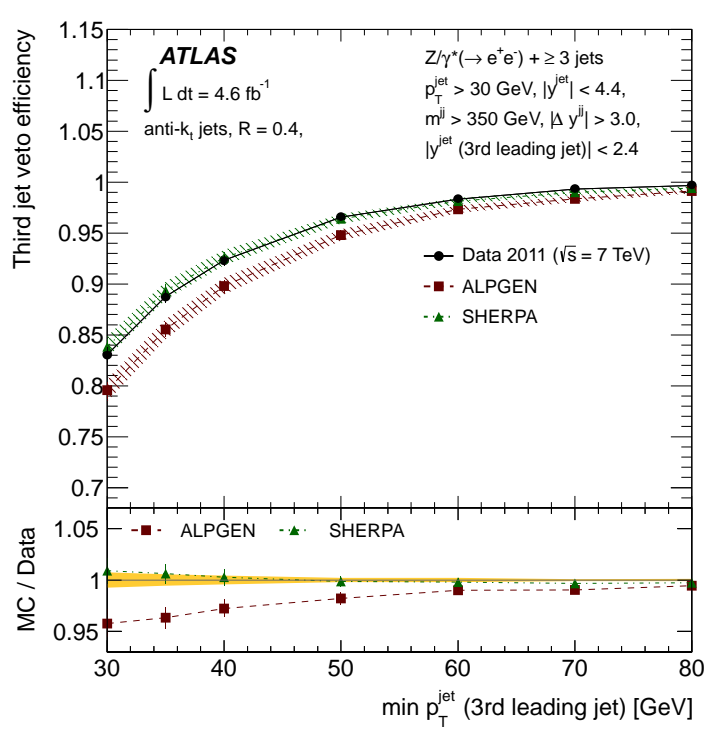

(b) 3rd central jet veto efficiency

Figure 10.6.: (a) 3rd jet veto efficiency and (b) 3rd central jet veto efficiency for $Z / \gamma^{*}(\rightarrow e e)+$ jets events with at least two jets in the final state passing the WBF preselection $\left(m^{j j}>350 \mathrm{GeV}\right.$ and $\left.\left|\Delta y^{j j}\right|>3.0\right)$ in data and predicted by the generators ALPGEN+HERWIG and SHERPA. The efficiency is shown as a function of the third jet $p_{T}$ threshold. The measured values after background subtraction are represented by the black dots, the error bars indicate the combined statistical and systematic uncertainty on the data. The hatched band incorporates the total statistical and systematic uncertainty on the predictions, modelled with ALPGEN+HERWIG. The shaded band reflects the total systematic uncertainty.

A simple veto against a third jet already provides a good tool to distinguish signal from background. But the cross section for WBF Higgs boson production is quite low and this veto also rejects parts of the signal. This is why a more sophisticated method is required. More elaborated observables are for example $\eta^{*}$ and $Z^{*}$. The variable $\eta^{*}$ is defined as the rapidity difference between the third jet and the mean value of the rapidities of the two tagging jets

$$
\eta^{*}=\eta_{j 3}-\frac{\eta_{j 2}+\eta_{j 1}}{2}
$$

$Z^{*}$ can be calculated from $\eta^{*}$ by dividing it by the absolute rapidity difference between the tagging jets.

$$
Z^{*}=\frac{\eta^{*}}{\left|\eta_{j 2}-\eta_{j 1}\right|}
$$

For a 3rd jet which is in between the two tagging jets $\left|\eta_{j 1, j 2}\right|<\left|\eta_{j 3}\right|<\left|\eta_{j 2, j 1}\right|, \eta^{*}$ is expected to be small and $\left|Z^{*}\right|<0.5$. Both the predictions from ALPGEN+HERWIG and SHERPA are consistent with the data, as shown in Fig. 10.7. 


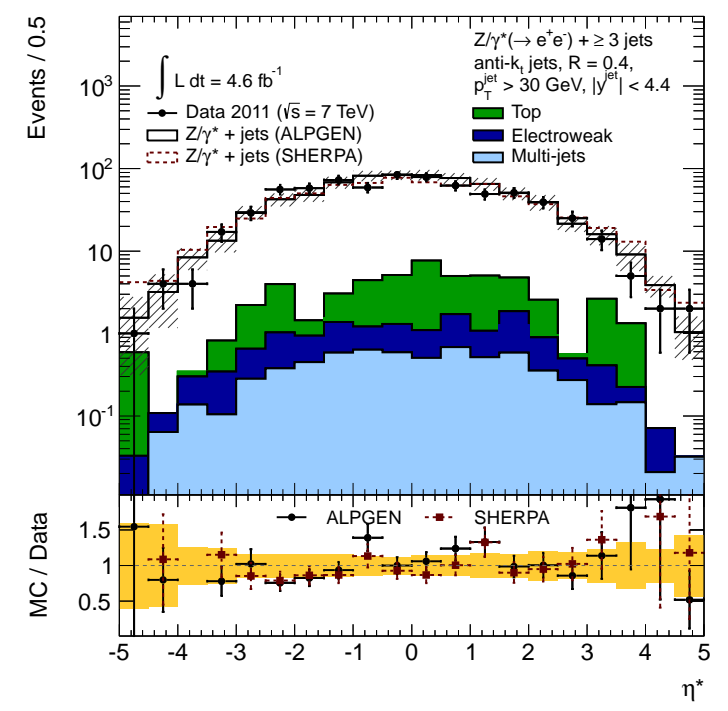

(a) $\eta^{*}$

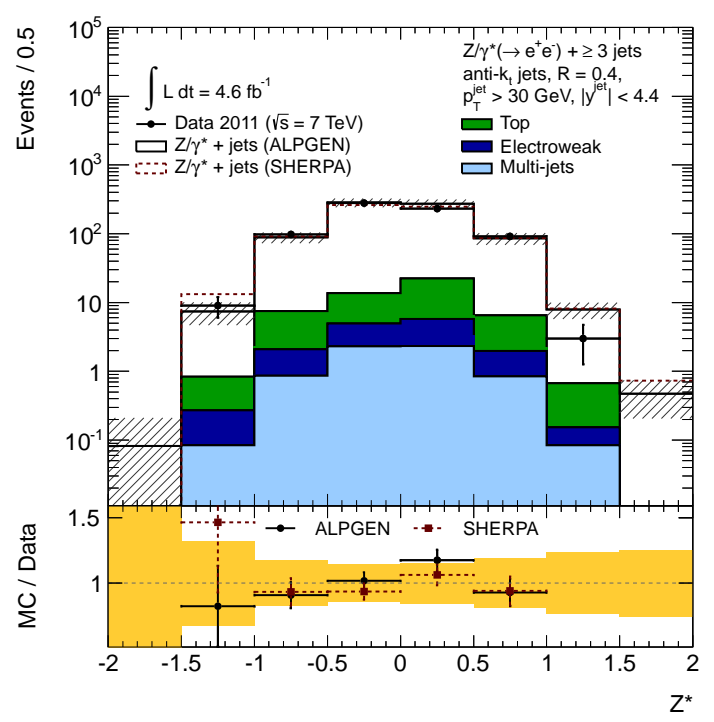

(b) $Z^{*}$

Figure 10.7.: (a) $\eta^{*}$ distribution and (b) $Z^{*}$ distribution for $Z / \gamma^{*}(\rightarrow e e)+$ jets events with at least three jets in the final state passing the WBF preselection $\left(m^{j j}>350 \mathrm{GeV}\right.$ and $\left.\left|\Delta y^{j j}\right|>3.0\right)$ in data and simulation. Multi-jets and $t \bar{t}$ backgrounds have been estimated from data. The hatched band incorporates the total statistical and systematic uncertainty on the predictions. The yellow band reflects the total systematic uncertainty.

\subsection{Results at Particle Level}

This section presents the results of the $Z / \gamma^{*}(\rightarrow e e)+$ jets cross-section measurement at particle level with the full dataset of 2011 after WBF preselection. In order to provide results which are independent of the detector setup, the results are presented on particle level. Therefore, the measured multiplicity distributions and the kinematic distributions of the 3rd jet depicted in Sec. 10.2 are unfolded to particle level, taking into account the systematic uncertainties as discussed in Sec. 9.3. Predictions from NLO fixed-order pQCD predictions, are corrected for non-perturbative effects and QED radiation effects, as described in Sec. 9.4. Finally, both the predictions from NLO fixed-order pQCD calculations and from ALPGEN+HERWIG and SHERPA using the ATLAS configurations, as detailed in Sec. 7.2, are compared to the measured cross sections.

The figures in this section are organised such that they show the absolute or normalised cross sections in the upper part, together with the ratios BLACKHAT+SHERPA/data, ALPGEN/data and SHERPA/data in the lower three parts. The predictions from ALPGEN+HERWIG and SHERPA have been normalised to the inclusive NNLO cross section with global $K$-factors. Theoretical uncertainties are shown separately from the total combined statistical and systematic uncertainty on the measurement.

A typical WBF preselection, requiring a large rapidity distance between the two tagging jets and a large dijet mass, is expected to have a similar effect on the jet multiplicity scaling as a hard cut on the transverse momentum of the leading jet [81]. Figure 10.8 shows the measured cross section as a function of the exclusive jet multiplicity and the ratio of cross 
sections for successive exclusive jet multiplicities for events with at least two jets in the final state passing the WBF preselection $\left(m^{j j}>350 \mathrm{GeV}\right.$ and $\left.\left|\Delta y^{j j}\right|>3.0\right)$.

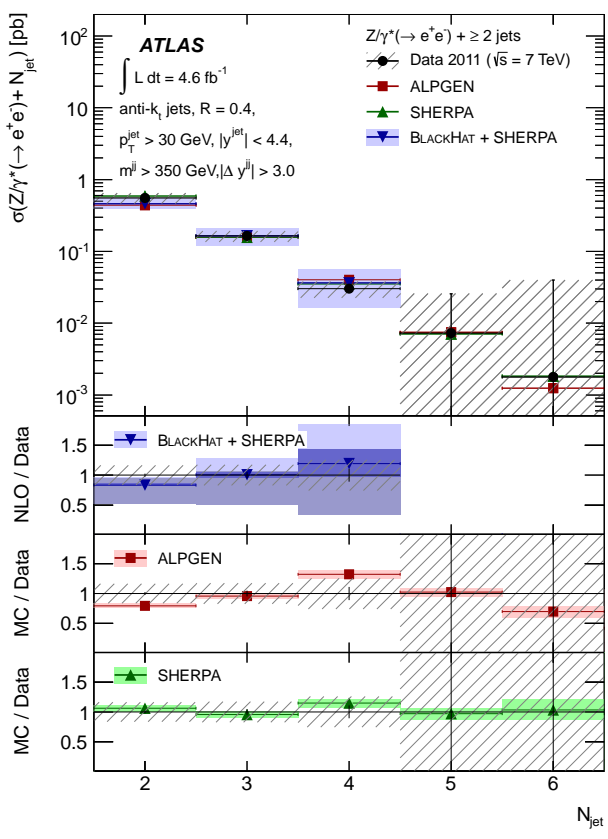

(a) Exclusive jet multiplicity

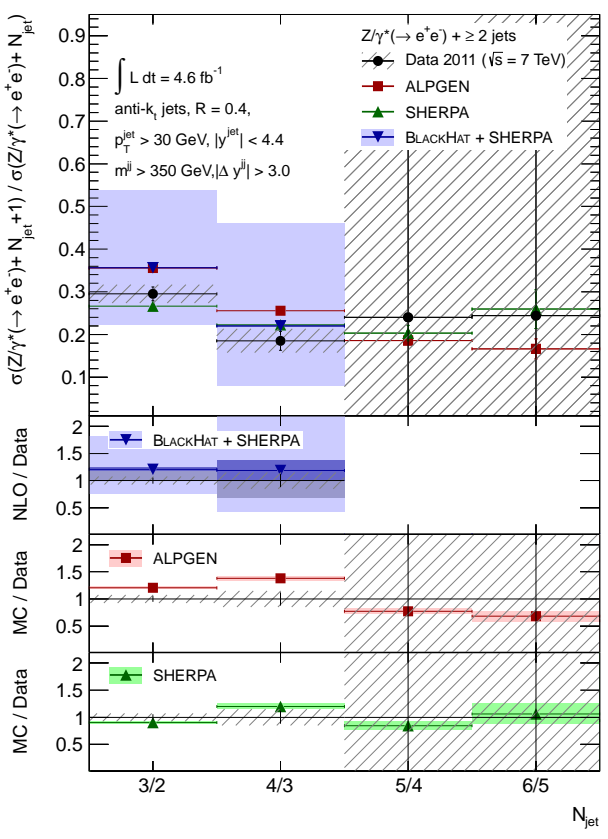

(b) Exclusive jet multiplicity ratio

Figure 10.8.: (a) Measured cross section as a function of the exclusive jet multiplicity and (b) ratio of cross sections for successive exclusive jet multiplicities for events with at least two jets in the final state passing the WBF preselection $\left(m^{j j}>350 \mathrm{GeV}\right.$ and $\left.\left|\Delta y^{j j}\right|>3.0\right)$. The cross sections are normalised to the inclusive $Z / \gamma^{*}(\rightarrow e e)$ cross section. The other details are as in Fig. 9.22 ,

The hypothesis of Poisson scaling is found to be consistent with the measured cross sections, at least in the first two ratios, but a larger cut on the dijet mass is expected to strengthen the effect of Poisson scaling. The predictions from BLACKHAT+SHERPA are consistent with the data, while the precision of the measurement exceeds the theory precision. In addition, the measurements are well described by the predictions from SHERPA, whereas ALPGEN+HERWIG predicts a too low number of events passing the $\mathrm{WBF}$ preselection, which is in line with the tendencies observed for large values of the absolute rapidity difference between the leading jets described in Sec. 9.5.6. In addition, the predictions from ALPGEN+HERWIG overestimates $R_{3 / 2}$.

Figure 10.9 shows the measured cross section as a function of the transverse momentum and the absolute rapidity distribution of the 3rd jet after WBF preselection. The NLO fixed order predictions from BLACKHAT+SHERPA, as well as the predictions from ALPGEN+HERWIG and SHERPA agree well with the data, except for the high $p_{T}^{\text {jet }}$ regime above $70 \mathrm{GeV}$, where a lack of data is observed.

Detailed values of the measured cross sections, as well as NLO pQCD predictions from BLACKHAT+SHERPA with respect to the fiducial region are listed in Appendix C.1. 


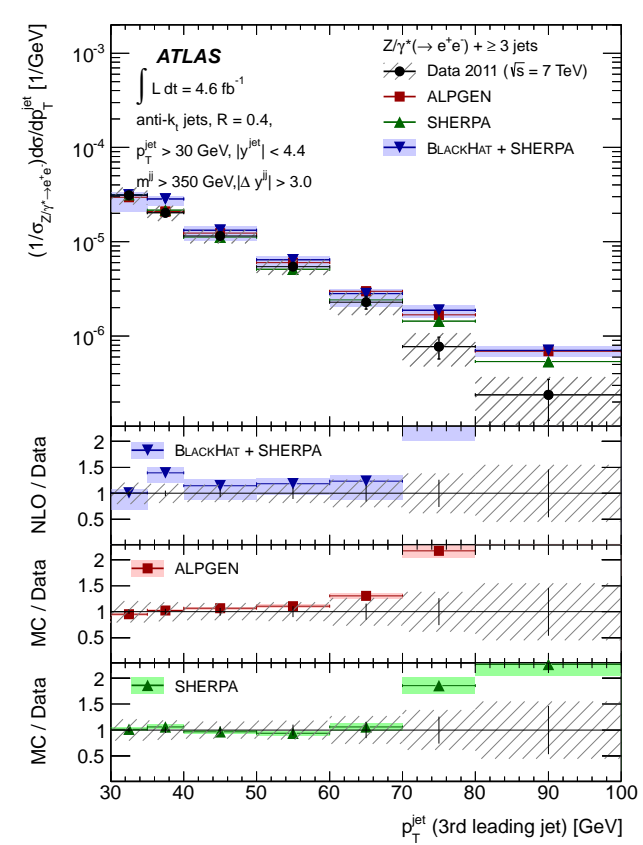

(a) 3rd leading jet $p_{T}$

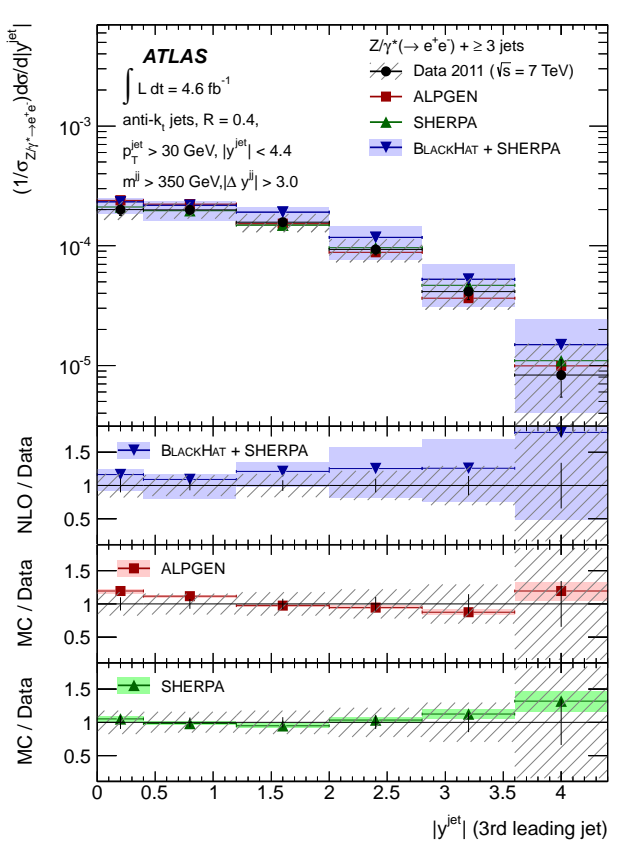

(b) 3rd leading jet $|y|$

Figure 10.9.: Differential cross section as a function of (a) the transverse momentum $p_{\mathrm{T}}^{\text {jet }}$ and (b) the absolute rapidity $\left|y^{\text {jet }}\right|$ of the 3rd leading jet for events with at least three jets in the final state passing the WBF preselection $\left(m^{j j}>350 \mathrm{GeV}\right.$ and $\left.\left|\Delta y^{j j}\right|>3.0\right)$. The cross sections are normalised to the inclusive $Z / \gamma^{*}(\rightarrow e e)$ cross section. The other details are as in Fig. 9.21 .

\subsection{Conclusions}

The $Z / \gamma^{*}+$ jets modelling has been tested in typical phase space regions expected for the decay of the Higgs boson, produced via WBF, in pp-collisions at a centre of mass energy of $\sqrt{s}=7 \mathrm{TeV}$ with the full dataset of 2011. In Higgs boson events produced via WBF two well separated high energetic jets and reduced central activity are expected. A veto on a 3rd jet can be used to distinguish signal from background.

Different observables which can be used for a central jet veto and 3rd jet veto efficiencies have been studied on detector level. In addition, total exclusive cross sections as a function of the jet multiplicity and their ratios, as well as the differential cross section as a function of the 3rd jet $p_{T}$ and $|y|$ have been measured.

Systematic and statistical uncertainties have been evaluated and the systematic uncertainty has been reduced by normalising the cross sections to the inclusive $Z / \gamma^{*}(\rightarrow e e)$ cross section.

The predictions from SHERPA and NLO fixed order pQCD calculations are consistent with the data, whereas ALPGEN+HERWIG predicts a too low number of events passing the WBF preselection. In addition, ALPGEN+HERWIG underestimates the jet veto efficiency.

This measurement can be used to tune MC generators, but for the mean time different reweighting procedures need to be studied. For the analysis with the full dataset of 2011 [169] a reweighting in bins of $p_{T}^{e e}$ has been used as a cross check. 



\section{Overall Conclusion}

The inclusive and differential $Z / \gamma^{*}(\rightarrow e e)+$ jets cross section has been measured in ppcollisions at a centre-of-mass energy of $\sqrt{s}=7 \mathrm{TeV}$ with integrated luminosities of $\int L \mathrm{~d} t=36 \mathrm{pb}^{-1}$ (full dataset of 2010) and $4.6 \mathrm{fb}^{-1}$ (full dataset of 2011). The full dataset of 2010 has the advantage of a relatively low collision rate and a low rate of multiple proton-proton interactions which allow for cross-section measurements at low jet transverse momentum, while the analysis with the full dataset of 2011 provides the most accurate results. In addition, the $Z / \gamma^{*}+$ jets modelling has been tested in typical phase space regions expected for the decay of the Higgs boson, produced via WBF, in pp-collisions at a centre of mass energy of $\sqrt{s}=7 \mathrm{TeV}$ with the full dataset of 2011 .

The measurements have been corrected for detector effects back to particle level and compared to predictions from the matrix element plus parton shower generators ALPGEN+HERWIG and SHERPA, $Z / \gamma^{*}+\geq 1$ jet production generated with PYTHIA and the inclusive Drell-Yan process modelled at NLO with MC@NLO using the ATLAS configurations. In addition, predictions from NLO fixed-order pQCD calculations are compared to the measured cross sections. Electron kinematics on particle level in the MC event samples have been defined to include the contributions from photons within a cone of 0.1 around the electron direction. Jets have been clustered around all final state particles except for the dressed decay products of the $Z$ boson and have been identified with the anti- $\mathrm{k}_{t}$ algorithm with a cone of $R=0.4$ in the region $p_{T}>30 \mathrm{GeV}$ and $|y|<4.4$.

Total inclusive and exclusive cross sections have been measured as a function of jet multiplicity. Additionally, the inclusive and exclusive jet multiplicity ratios have been extracted for different values of the transverse momentum of the leading jet in order to test the jet multiplicity scaling. Furthermore, inclusive differential cross sections have been measured as a function of the jet transverse momentum, the jet rapidity, the transverse momentum of the $Z$ boson, $p_{T}^{e e}$, the invariant mass of the two leading jets and the angular separation between the two leading jets. Finally, inclusive differential cross sections as a function of $H_{T}$, the scalar $p_{T}$ sum of all final state objects, and $S_{T}$, the scalar $p_{T}$ sum of all hadronic jets in the final state, have been measured.

Systematic and statistical uncertainties have been evaluated and the systematic uncertainty has been reduced by normalising the cross sections to the inclusive $Z / \gamma^{*}(\rightarrow e e)$ cross section.

In general, the data is well described by the predictions from NLO fixed order pQCD calculations and from ALPGEN+HERWIG and SHERPA, whereas PYTHIA and MC@NLO fail to describe the data in large phase space regions. PYTHIA only includes the tree-level matrix element for $Z / \gamma^{*}+\geq 1$ jet, additional jet emission are done by parton shower. For MC@NLO the associated first jet corresponds to the real emission term of the NLO calculation and events with more than one jet in the final state are modelled by parton shower. Therefore, the mismodelling can be attributed to the parton shower.

For exclusive jet multiplicities, the transition from staircase scaling to Poisson scaling for large scale differences between the leading jet and the additional radiated jets is 
confirmed by the measurement.

For some phase space regions discrepancies between the predictions from the ME+PS generators and the measurements have been evaluated. The jet multiplicity is well described by ALPGEN+HERWIG and SHERPA up to five jets, but additional jet emissions are modelled by parton shower, which fail to describe the data. Furthermore, ALPGEN+HERWIG overestimates the cross section for high energetic jets or highly boosted $Z$ bosons, which is mainly due to higher-order QCD effects. SHERPA predicts a too wide rapidity distribution, which translates into the absolute rapidity difference, the angular separation in $y-\phi$ space and the invariant mass of the two leading jets. Finally, for events passing the WBF preselection, the predictions from ALPGEN+HERWIG overestimate the measured cross sections for higher jet multiplicities, which leads to a small underestimation of the probability to survive a veto on a $3 r d$ jet in the low $p_{T}^{\text {jet }}$ regime.

The NLO fixed-order pQCD calculations from BLACKHAT + SHERPA underestimate the cross section for large $H_{T}$ and $S_{T}$ by several standard deviations, which is attributed to missing higher parton multiplicities in the calculation. Similar deviations have been found for large values of $p_{T}^{e e}$. Exclusive sums of the NLO fixed-order pQCD calculations for $Z / \gamma^{*}+1$ jet and $Z / \gamma^{*}+\geq 2$ jets show a much better description of the hard $H_{T}, S_{T}$ and $p_{T}^{e e}$ regime.

The analysis with the full dataset of 2011 provides the most precise measurements of this kind to date. 


\section{Outlook}

The analysis presented in this thesis covers a large spectrum of physics with jets in association with a $Z$ boson, which provide a valuable input for the tuning of Monte Carlo (MC) event generators.

In addition, part of the measurements exceeds the precision of the theory predictions. The largest contribution to the theoretical uncertainty comes from the impact of QCD higher-order effects. This could be reduced by either using nNLO calculations [9] or by optimising the method to evaluate this uncertainty.

The analysis also leaves the possibility for optimisations and extensions. For most phase-space regions the measurements are already limited by systematics, mainly due to the JES, especially in the forward region. The dominant component of the JES uncertainty in the forward region comes from the $\eta$-intercalibration. This can be reduced by using more sophisticated methods to estimate the uncertainties due to generator modelling.

For a reduced JES systematic uncertainty, the uncertainty coming from the unfolding process becomes dominant. It has been shown in this thesis that multi-dimensional unfolding is able to reduce this uncertainty, due to the fact that the model dependence for multi-dimensional unfolding is lower.

As shown in this thesis the event topology changes for extrem phase-space regimes, e.g. large transverse momentum of the $Z$ boson, large jet transverse momentum and large $H_{T}$, but measurements in this regions are limited by statistics. With more data, these kinematic regimes become accessible and allow to study the event shapes in more detail. Potential observables to characterise the event shapes are for example: the absolute azimuthal separation, the minimal azimuthal difference between a $Z$ boson and a jet, the transverse momentum ratio of the two leading jets [8] or the central trust [170]. The probe of the $Z / \gamma^{*}+$ jets modelling in these regimes is of particular interest for searches of new physics and for further tests of NLO fixed order pQCD predictions.

The fraction of $Z / \gamma^{*}+$ jets events produced via double-parton interactions (DPI) is rather small, but for other analyses, such as the measurement of $W+b$ jets production, the fraction of events originating from DPI is expected to be much larger, at the order of 30\% [171]. Since the effective area parameter for DPI is expected to be approximately independent of the process and the phase space requirements, the measurement in $Z / \gamma^{*}+$ jets events can contribute to a better understanding of DPI. 



\section{A. Measurement with the Dataset of 2010}

\section{A.1. Further Uncorrected Distributions}

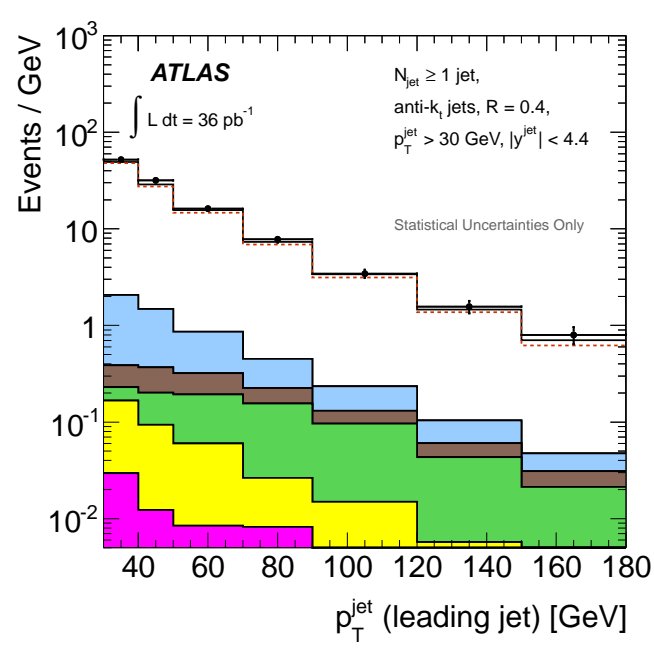

(a) Leading jet $p_{T}$

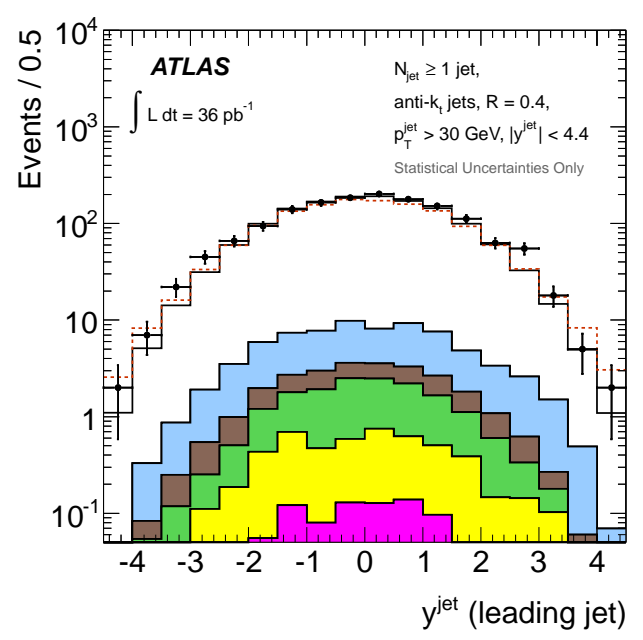

(c) Leading jet $y$

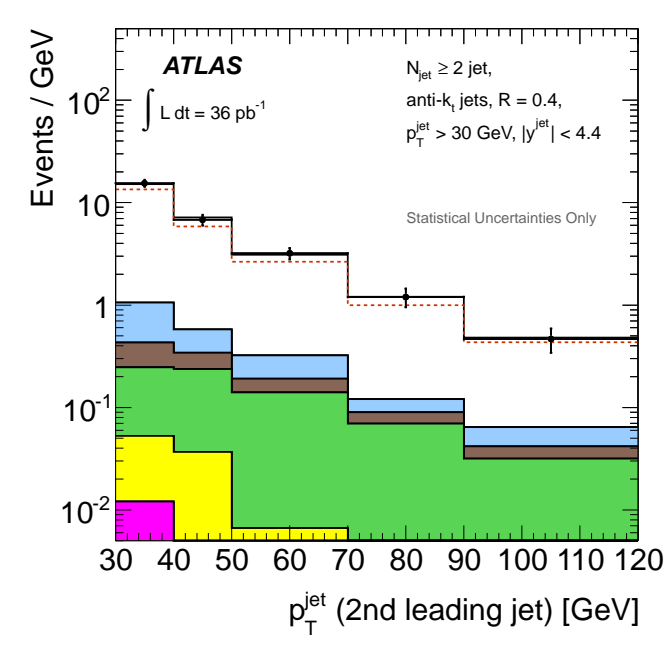

(b) 2nd leading jet $p_{T}$

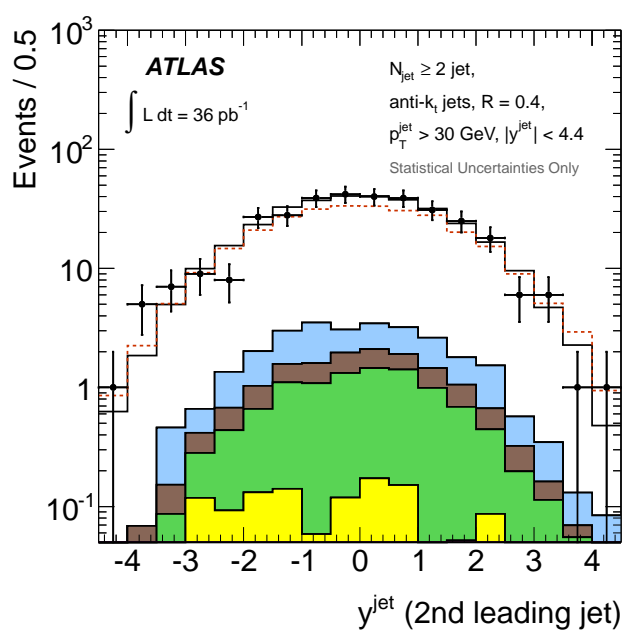

(d) 2nd leading jet $y$

Figure A.1.: Transverse momentum distribution of (a) the leading jet and (b) the 2nd leading jet and rapidity distribution of (c) the leading jet and (d) the 2nd leading jet in data and simulation. Only statistical uncertainties are shown. Multi-jets background have been estimated from data. 


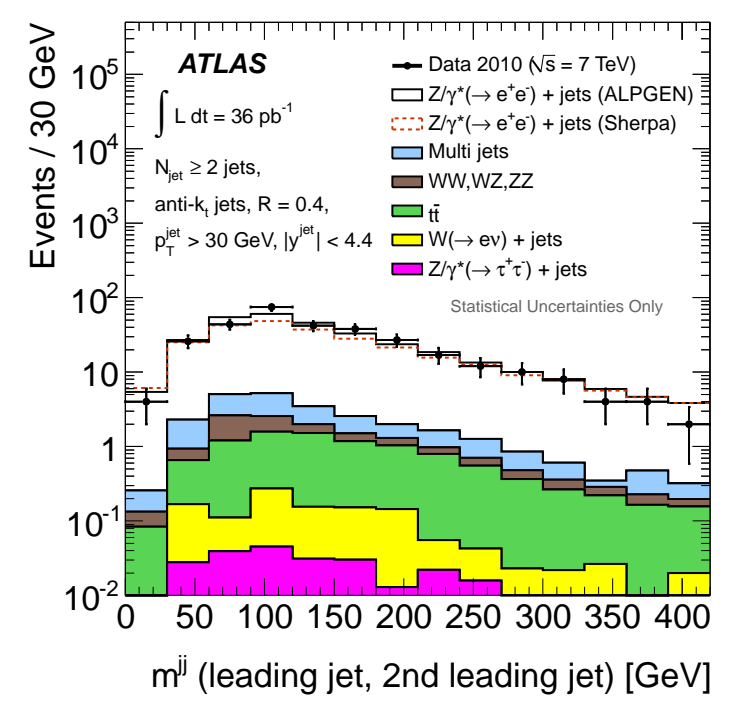

(a) Invariant dijet mass

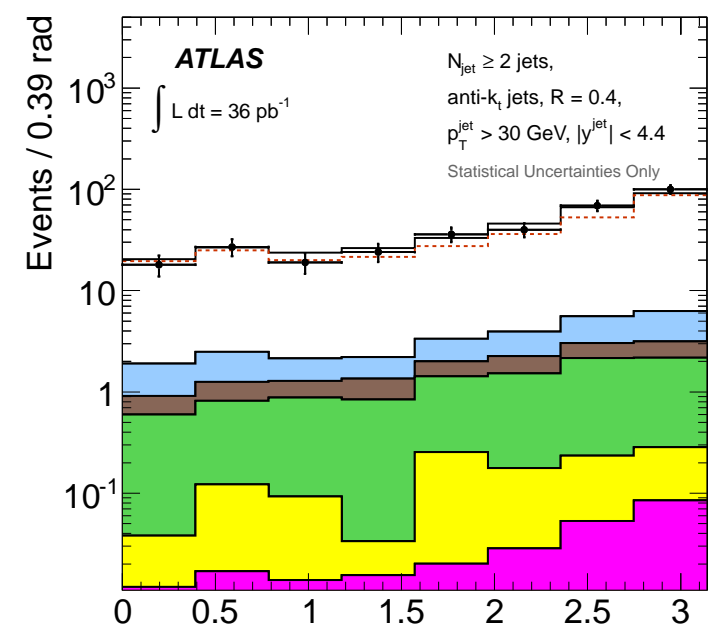

$\left|\Delta \phi^{\mathrm{ij}}\right|$ (leading jet, 2nd leading jet) [rad]

(c) Absolute azimuthal separation

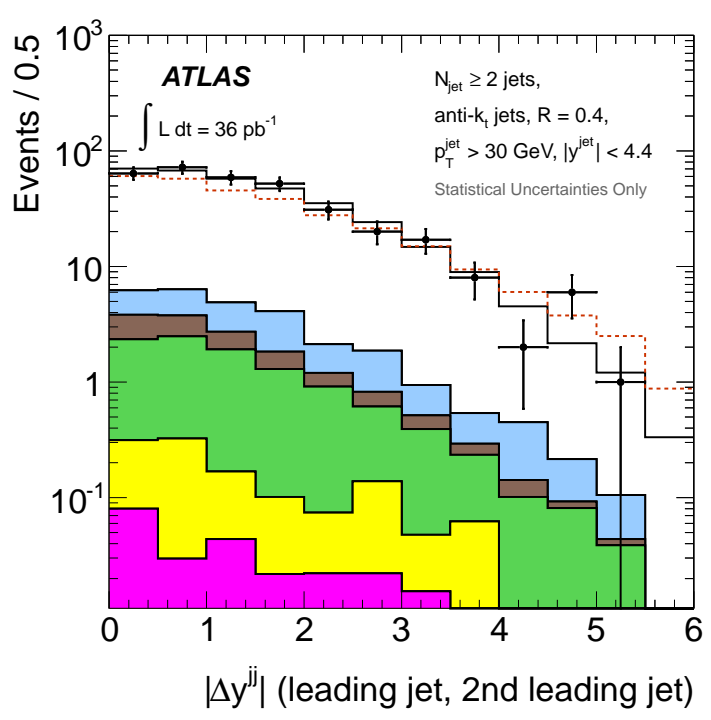

(b) Absolute rapidity difference

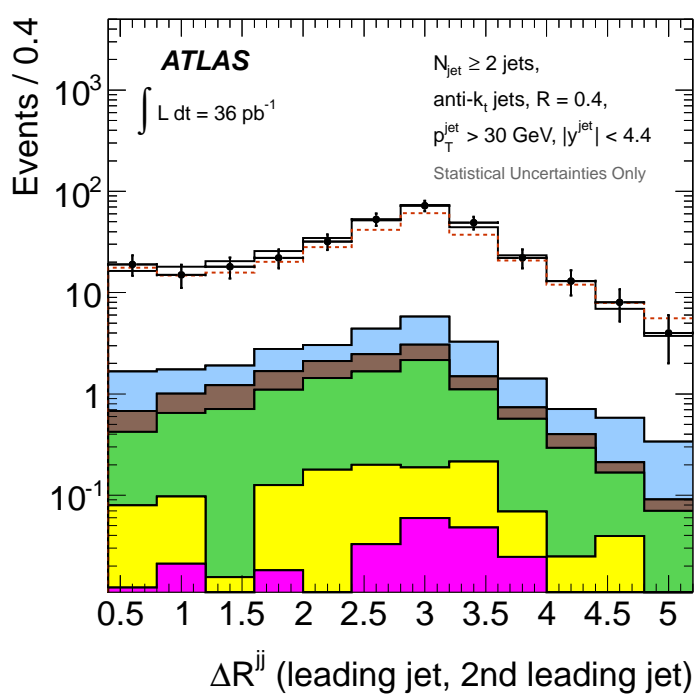

(d) Angular separation in $y-\phi$ space

Figure A.2.: (a) Invariant mass $m^{j j}$ and the angular separations (b) $\left|\Delta y^{j j}\right|$, (c) $\left|\Delta \phi^{j j}\right|$ and (d) $\Delta R^{j j}$ of the two leading jets in events with at least two jets in the final state in data and simulation. Only statistical uncertainties are shown. Multi-jets background have been estimated from data. 


\section{A.2. Further Uncorrected Distributions with $p_{T}^{\text {jet }}>20 \mathrm{GeV}$}

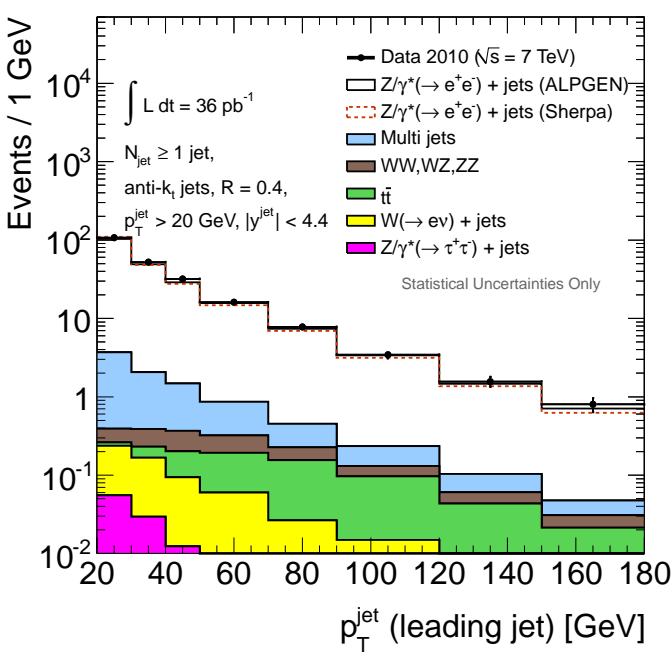

(a) Leading jet $p_{T}$

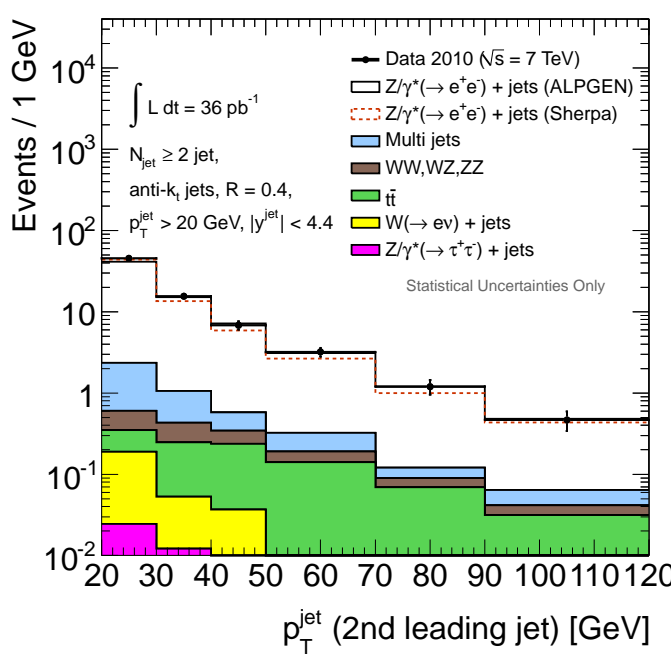

(b) 2nd leading jet $p_{T}$

Figure A.3.: Transverse momentum distribution of (a) the leading jet and (b) the 2nd leading jet in data and simulation. Only statistical uncertainties are shown. Multi-jets background have been estimated from data.

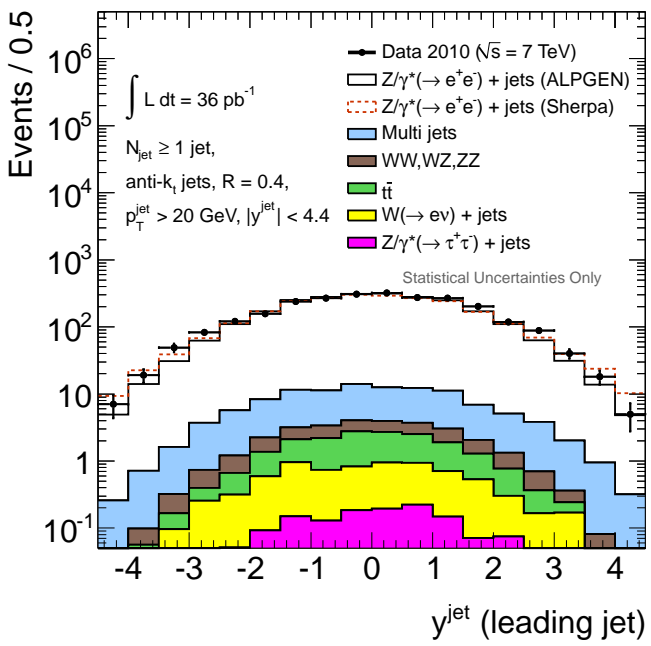

(a) Leading jet $y$

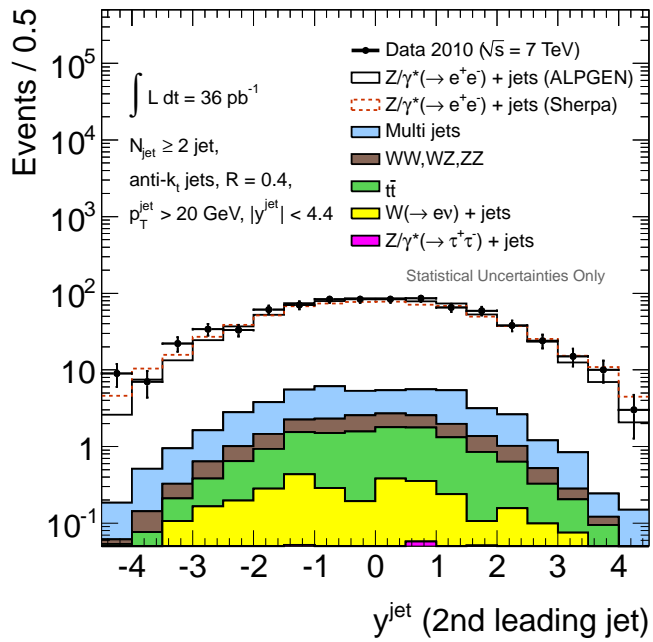

(b) 2nd leading jet $y$

Figure A.4.: Rapidity distribution of (a) the leading jet and (b) the 2nd leading jet in data and simulation. Only statistical uncertainties are shown. Multi-jets background have been estimated from data. 


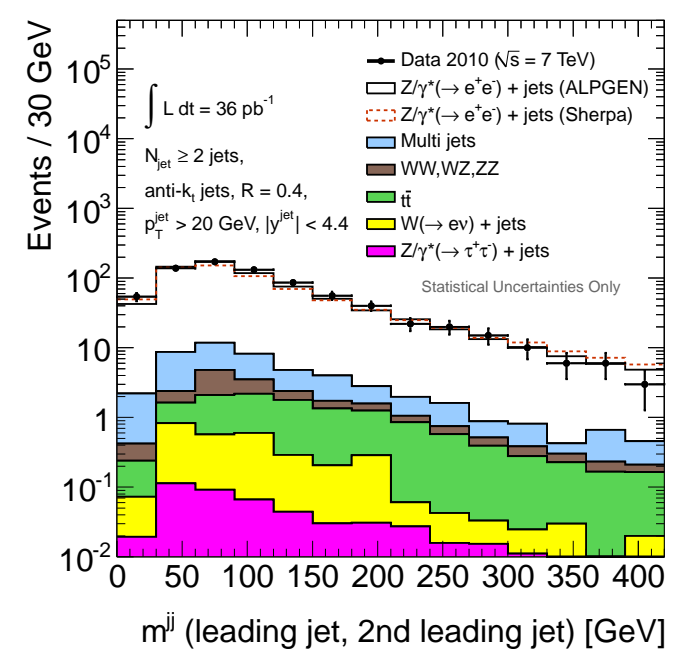

(a) Invariant dijet mass

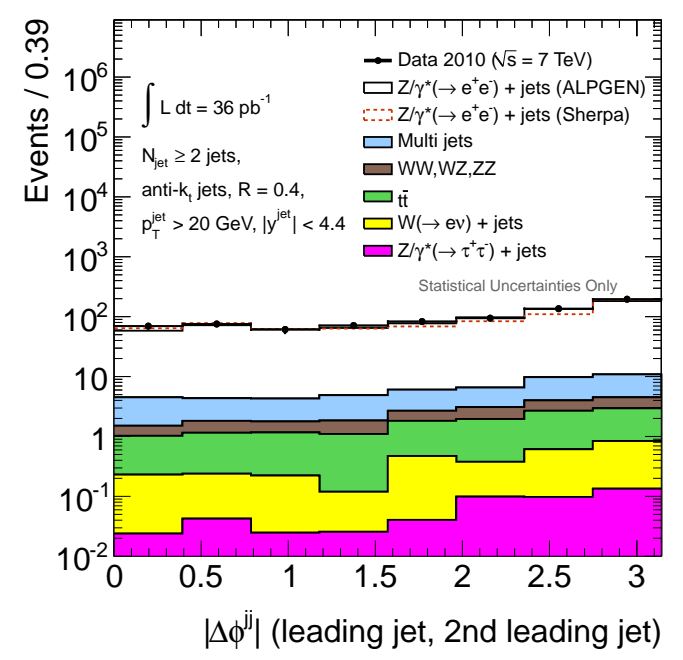

(c) Absolute azimuthal separation

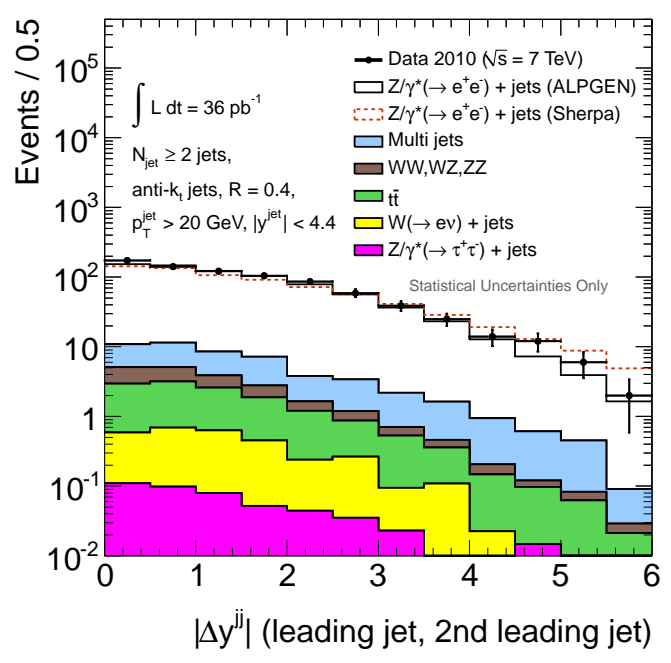

(b) Absolute rapidity difference

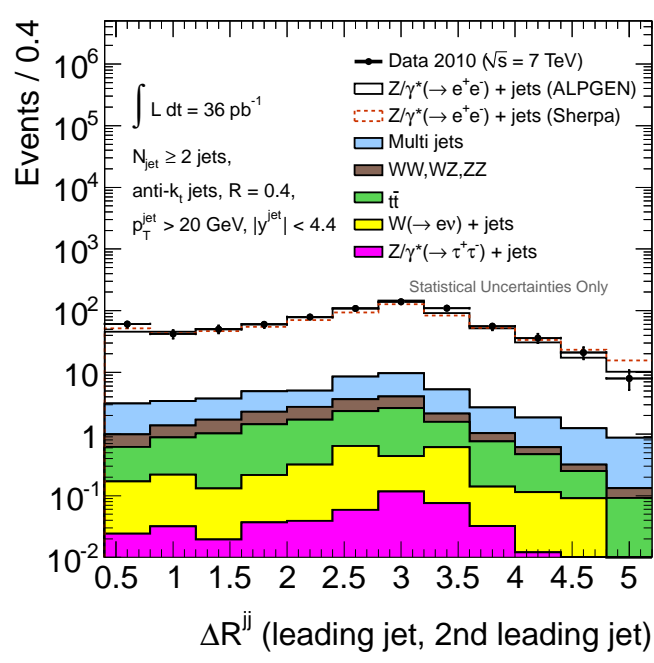

(d) Angular separation in $y$ - $\phi$ space

Figure A.5.: (a) Invariant mass $m^{j j}$ and the angular separations (b) $\left|\Delta y^{j j}\right|$, (c) $\left|\Delta \phi^{j j}\right|$ and (d) $\Delta R^{j j}$ of the two leading jets in events with at least two jets in the final state in data and simulation. Only statistical uncertainties are shown. Multi-jets background have been estimated from data. 


\section{A.3. Cross Section Tables}

\begin{tabular}{|c|c|c|}
\hline \hline$p_{T}^{\text {jet }}[\mathrm{GeV}]$ & Data cross section $[\mathrm{pb} / \mathrm{GeV}]$ & NLO cross section $[\mathrm{pb} / \mathrm{GeV}]$ \\
\hline \hline $30-40$ & {$\left[2.748 \pm 0.107\right.$ (stat) ${ }_{-0.318}^{+0.264}$ (syst) ${ }_{-0.092}^{+0.098}$ (lumi) $] \times 10^{0}$} & $2.444_{-0.200}^{+0.214} \times 10^{0}$ \\
$40-50$ & {$\left[1.586 \pm 0.084\right.$ (stat) ${ }_{-0.165}^{+0.176}$ (syst) ${ }_{-0.053}^{+0.057}$ (lumi) $] \times 10^{0}$} & $1.395_{-0.108}^{+0.117} \times 10^{0}$ \\
$50-70$ & {$\left[7.664 \pm 0.414\right.$ (stat) ${ }_{-0.615}^{+0.692}$ (syst) ${ }_{-0.260}^{+0.278}($ lumi) $] \times 10^{-1}$} & $6.960_{-0.509}^{+0.561} \times 10^{-1}$ \\
$70-90$ & {$\left[3.599 \pm 0.282\right.$ (stat) ${ }_{-0.309}^{+0.350}$ (syst) ${ }_{-0.123}^{+0.132}($ lumi) $] \times 10^{-1}$} & $3.101_{-0.246}^{+0.272} \times 10^{-1}$ \\
$90-120$ & {$\left[1.466 \pm 0.147\right.$ (stat) ${ }_{-0.132}^{+0.151}$ (syst) ${ }_{-0.051}^{+0.054}($ lumi) $] \times 10^{-1}$} & $1.323_{-0.117}^{+0.130} \times 10^{-1}$ \\
$120-150$ & {$\left[6.247 \pm 0.951\right.$ (stat) ${ }_{-0.639}^{+0.650}$ (syst) ${ }_{-0.215}^{+0.230}$ (lumi) $] \times 10^{-2}$} & $5.526_{-0.553}^{+0.621} \times 10^{-2}$ \\
$150-180$ & {$\left[3.213 \pm 0.674\right.$ (stat) ${ }_{-0.418}^{+0.486}$ (syst) ${ }_{-0.111}^{+0.118}$ (lumi) $] \times 10^{-2}$} & $2.644_{-0.318}^{+0.352} \times 10^{-2}$ \\
\hline \hline
\end{tabular}

Table A.1.: Cross section as a function of $p_{T}^{\text {jet }}$ for events with at least one jet in the final state with respect to the fiducial region measured in data and predicted from NLO fixed-order pQCD calculations from BLACKHAT+SHERPA.

\begin{tabular}{|c|c|c|}
\hline \hline$p_{T}^{j e t}[\mathrm{GeV}]$ & Data cross section $[\mathrm{pb} / \mathrm{GeV}]$ & NLO cross section $[\mathrm{pb} / \mathrm{GeV}]$ \\
\hline \hline $30-40$ & {$\left[2.014 \pm 0.092\right.$ (stat) ${ }_{-0.213}^{+0.181}$ (syst) ${ }_{-0.067}^{+0.071}$ (lumi) $] \times 10^{0}$} & $1.936_{-0.112}^{+0.110} \times 10^{0}$ \\
$40-50$ & {$\left[1.275 \pm 0.075\right.$ (stat) ${ }_{-0.127}^{+0.130}$ (syst) ${ }_{-0.042}^{+0.045}$ (lumi) $] \times 10^{0}$} & $1.162_{-0.065}^{+0.065} \times 10^{0}$ \\
$50-70$ & {$\left[6.369 \pm 0.375\right.$ (stat) ${ }_{-0.492}^{+0.544}$ (syst) ${ }_{-0.214}^{+0.229}$ (lumi) $] \times 10^{-1}$} & $5.962_{-0.317}^{+0.320} \times 10^{-1}$ \\
$70-90$ & {$\left[3.033 \pm 0.258\right.$ (stat) ${ }_{-0.256}^{+0.288}$ (syst) ${ }_{-0.103}^{+0.110}$ (lumi) $] \times 10^{-1}$} & $2.724_{-0.167}^{+0.172} \times 10^{-1}$ \\
$90-120$ & {$\left[1.294 \pm 0.137\right.$ (stat) ${ }_{-0.118}^{+0.135}$ (syst) ${ }_{-0.044}^{+0.047}$ (lumi) $] \times 10^{-1}$} & $1.171_{-0.084}^{+0.088} \times 10^{-1}$ \\
$120-150$ & {$\left[5.843 \pm 0.914\right.$ (stat) ${ }_{-0.606}^{+0.615}$ (syst) ${ }_{-0.200}^{+0.214}$ (lumi) $] \times 10^{-2}$} & $4.911_{-0.417}^{+0.444} \times 10^{-2}$ \\
$150-180$ & {$\left[2.969 \pm 0.645\right.$ (stat) ${ }_{-0.407}^{+0.466}$ (syst) ${ }_{-0.102}^{+0.109}$ (lumi) $] \times 10^{-2}$} & $2.354_{-0.253}^{+0.268} \times 10^{-2}$ \\
\hline \hline
\end{tabular}

Table A.2.: Cross section as a function of the leading jet $p_{T}$ for events with at least one jet in the final state with respect to the fiducial region measured in data and predicted from NLO fixed-order pQCD calculations from BLACKHAT+SHERPA.

\begin{tabular}{|c|c|c|}
\hline \hline$p_{T}^{j e t}[\mathrm{GeV}]$ & Data cross section $[\mathrm{pb} / \mathrm{GeV}]$ & NLO cross section $[\mathrm{pb} / \mathrm{GeV}]$ \\
\hline \hline $30-40$ & {$\left[5.636 \pm 0.487\right.$ (stat) ${ }_{-0.818}^{+0.683}$ (syst) ${ }_{-0.191}^{+0.204}$ (lumi) $] \times 10^{-1}$} & $5.531_{-0.418}^{+0.328} \times 10^{-1}$ \\
$40-50$ & {$\left[2.508 \pm 0.333\right.$ (stat) ${ }_{-0.336}^{+0.401}$ (syst) ${ }_{-0.087}^{+0.093}$ (lumi) $] \times 10^{-1}$} & $2.592_{-0.178}^{+0.119} \times 10^{-1}$ \\
$50-70$ & {$\left[1.191 \pm 0.166\right.$ (stat) ${ }_{-0.121}^{+0.144}$ (syst) ${ }_{-0.042}^{+0.045}$ (lumi) $] \times 10^{-1}$} & $1.143_{-0.083}^{+0.051} \times 10^{-1}$ \\
$70-90$ & {$\left[4.546 \pm 1.033\right.$ (stat) ${ }_{-0.496}^{+0.554}$ (syst) ${ }_{-0.162}^{+0.173}$ (lumi) $] \times 10^{-2}$} & $4.252_{-0.313}^{+0.206} \times 10^{-2}$ \\
$90-120$ & {$\left[1.729 \pm 0.537\right.$ (stat) ${ }_{-0.186}^{+0.201}$ (syst) ${ }_{-0.063}^{+0.067}$ (lumi) $] \times 10^{-2}$} & $1.644_{-0.162}^{+0.133} \times 10^{-2}$ \\
\hline \hline
\end{tabular}

Table A.3.: Cross section as a function of the 2 nd leading jet $p_{T}$ for events with at least two jets in the final state with respect to the fiducial region measured in data and predicted from NLO fixed-order pQCD calculations from BLACKHAT+SHERPA. 


\begin{tabular}{|c|c|c|}
\hline \hline$\left|y^{\text {jet }}\right|$ & Data cross section [pb/unit] & NLO cross section [pb/unit] \\
\hline \hline $0.0-0.5$ & {$\left[3.610 \pm 0.177\right.$ (stat) ${ }_{-0.294}^{+0.294}$ (syst) ${ }_{-0.122}^{+0.131}$ (lumi) $] \times 10^{1}$} & $3.328_{-0.259}^{+0.276} \times 10^{1}$ \\
$0.5-1.0$ & {$\left[3.371 \pm 0.172\right.$ (stat) ${ }_{-0.281}^{+0.272}$ (syst) ${ }_{-0.114}^{+0.122}$ (lumi) $] \times 10^{1}$} & $3.015_{-0.230}^{+0.247} \times 10^{1}$ \\
$1.0-1.5$ & {$\left[2.696 \pm 0.153\right.$ (stat) ${ }_{-0.247}^{+0.252}$ (syst) ${ }_{-0.091}^{+0.098}$ (lumi) $] \times 10^{1}$} & $2.513_{-0.197}^{+0.213} \times 10^{1}$ \\
$1.5-2.0$ & {$\left[2.114 \pm 0.138\right.$ (stat) ${ }_{-0.206}^{+0.211}$ (syst) ${ }_{-0.071}^{+0.076}$ (lumi) $] \times 10^{1}$} & $1.857_{-0.150}^{+0.163} \times 10^{1}$ \\
$2.0-2.5$ & {$\left[1.346 \pm 0.113\right.$ (stat) ${ }_{-0.183}^{+0.173}$ (syst) ${ }_{-0.045}^{+0.049}$ (lumi) $] \times 10^{1}$} & $1.227_{-0.105}^{+0.116} \times 10^{1}$ \\
$2.5-3.0$ & {$\left[1.014 \pm 0.099\right.$ (stat) ${ }_{-0.162}^{+0.169}$ (syst) ${ }_{-0.034}^{+0.036}$ (lumi) $] \times 10^{1}$} & $7.139_{-0.701}^{+0.774} \times 10^{0}$ \\
$3.0-3.5$ & {$\left[4.864 \pm 0.696\right.$ (stat) ${ }_{-0.971}^{+1.114}$ (syst) ${ }_{-0.163}^{+0.175}$ (lumi) $] \times 10^{0}$} & $3.629_{-0.450}^{+0.495} \times 10^{0}$ \\
$3.5-4.0$ & {$\left[1.569 \pm 0.380\right.$ (stat) ${ }_{-0.493}^{+0.612}$ (syst) ${ }_{-0.053}^{+0.056}$ (lumi) $] \times 10^{0}$} & $1.426_{-0.232}^{+0.261} \times 10^{0}$ \\
\hline \hline
\end{tabular}

Table A.4.: Cross section as a function of $\left|y^{j e t}\right|$ for events with at least one jet in the final state with respect to the fiducial region measured in data and predicted from NLO fixed-order pQCD calculations from BLACKHAT+SHERPA.

\begin{tabular}{|c|c|c|}
\hline \hline$\left|y^{\text {jet }}\right|$ & Data cross section [pb/unit] & NLO cross section [pb/unit] \\
\hline \hline $0.0-0.5$ & {$\left[2.932 \pm 0.156\right.$ (stat) ${ }_{-0.211}^{+0.212}$ (syst) ${ }_{-0.098}^{+0.105}$ (lumi) $] \times 10^{1}$} & $2.787_{-0.173}^{+0.171} \times 10^{1}$ \\
$0.5-1.0$ & {$\left[2.652 \pm 0.151\right.$ (stat) ${ }_{-0.199}^{+0.193}$ (syst) ${ }_{-0.089}^{+0.095}$ (lumi) $] \times 10^{1}$} & $2.515_{-0.148}^{+0.148} \times 10^{1}$ \\
$1.0-1.5$ & {$\left[2.190 \pm 0.135\right.$ (stat) ${ }_{-0.181}^{+0.185}$ (syst) ${ }_{-0.073}^{+0.079}$ (lumi) $] \times 10^{1}$} & $2.110_{-0.120}^{+0.120} \times 10^{1}$ \\
$1.5-2.0$ & {$\left[1.630 \pm 0.120\right.$ (stat) ${ }_{-0.143}^{+0.148}$ (syst) ${ }_{-0.055}^{+0.058}$ (lumi) $] \times 10^{1}$} & $1.520_{-0.084}^{+0.086} \times 10^{1}$ \\
$2.0-2.5$ & {$\left[1.082 \pm 0.101\right.$ (stat) ${ }_{-0.136}^{+0.129}$ (syst) ${ }_{-0.036}^{+0.039}$ (lumi) $] \times 10^{1}$} & $1.000_{-0.064}^{+0.066} \times 10^{1}$ \\
$2.5-3.0$ & {$\left[8.847 \pm 0.927\right.$ (stat) ${ }_{-1.337}^{+1.399}$ (syst) ${ }_{-0.294}^{+0.315}$ (lumi) $] \times 10^{0}$} & $5.681_{-0.478}^{+0.489} \times 10^{0}$ \\
$3.0-3.5$ & {$\left[3.483 \pm 0.586\right.$ (stat) ${ }_{-0.678}^{+0.735}$ (syst) ${ }_{-0.116}^{+0.124}$ (lumi) $] \times 10^{0}$} & $2.857_{-0.296}^{+0.304} \times 10^{0}$ \\
$3.5-4.0$ & {$\left[9.607 \pm 2.982\right.$ (stat) ${ }_{-3.023}^{+4.006}$ (syst) ${ }_{-0.320}^{+0.342}$ (lumi) $] \times 10^{-1}$} & $1.044_{-0.151}^{+0.161} \times 10^{0}$ \\
\hline \hline
\end{tabular}

Table A.5.: Cross section as a function of the leading jet $|y|$ for events with at least one jet in the final state with respect to the fiducial region measured in data and predicted from NLO fixed-order pQCD calculations from BLACKHAT+SHERPA.

\begin{tabular}{|c|c|c|}
\hline \hline$\left|y^{\text {jet }}\right|$ & Data cross section [pb/unit] & NLO cross section [pb/unit] \\
\hline \hline $0.0-0.5$ & $6.025 \pm 0.724$ (stat) ${ }_{-0.668}^{+0.674}$ (syst) ${ }_{-0.209}^{+0.223}$ (lumi) & $5.627_{-0.441}^{+0.333} \times 10^{0}$ \\
$0.5-1.0$ & $5.748 \pm 0.713$ (stat) ${ }_{-0.619}^{+0.604}$ (syst) ${ }_{-0.198}^{+0.212}$ (lumi) & $5.509_{-0.408}^{+0.276} \times 10^{0}$ \\
$1.0-1.5$ & $4.164 \pm 0.600$ (stat) ${ }_{-0.472}^{+0.507}$ (syst) ${ }_{-0.145}^{+0.155}$ (lumi) & $4.323_{-0.459}^{+0.416} \times 10^{0}$ \\
$1.5-2.0$ & $3.960 \pm 0.593$ (stat) ${ }_{-0.496}^{+0.494}$ (syst) ${ }_{-0.136}^{+0.145}$ (lumi) & $3.410_{-0.412}^{+0.374} \times 10^{0}$ \\
$2.0-2.5$ & $1.883 \pm 0.416$ (stat) ${ }_{-0.302}^{+0.278}$ (syst) ${ }_{-0.066}^{+0.070}$ (lumi) & $2.532_{-0.215}^{+0.165} \times 10^{0}$ \\
$2.5-3.0$ & $1.127 \pm 0.317$ (stat) ${ }_{-0.201}^{+0.206}$ (syst) ${ }_{-0.039}^{+0.042}$ (lumi) & $1.570_{-0.154}^{+0.137} \times 10^{0}$ \\
$3.0-3.5$ & $1.069 \pm 0.317$ (stat) ${ }_{-0.227}^{+0.281}$ (syst) ${ }_{-0.036}^{+0.039}$ (lumi) & $9.308_{-1.158}^{+1.069} \times 10^{-1}$ \\
\hline \hline
\end{tabular}

Table A.6.: Cross section as a function of the 2nd leading jet $|y|$ for events with at least one jet in the final state with respect to the fiducial region measured in data and predicted from NLO fixed-order pQCD calculations from BLACKHAT+SHERPA. 


\begin{tabular}{|c|c|c|}
\hline \hline$m^{j j}[\mathrm{GeV}]$ & Data cross section $[\mathrm{pb} / \mathrm{GeV}]$ & NLO cross section $[\mathrm{pb} / \mathrm{GeV}]$ \\
\hline \hline $60-90$ & {$\left[4.889 \pm 0.834\right.$ (stat) ${ }_{-0.660}^{+0.665}$ (syst) ${ }_{-0.172}^{+0.184}$ (lumi) $] \times 10^{-2}$} & $6.392_{-0.485}^{+0.351} \times 10^{-2}$ \\
$90-120$ & {$\left[9.265 \pm 1.151\right.$ (stat) ${ }_{-1.149}^{+1.181}$ (syst) ${ }_{-0.316}^{+0.338}$ (lumi) $] \times 10^{-2}$} & $7.184_{-0.543}^{+0.412} \times 10^{-2}$ \\
$120-150$ & {$\left[5.318 \pm 0.896\right.$ (stat) ${ }_{-0.699}^{+0.642}$ (syst) ${ }_{-0.184}^{+0.197}$ (lumi) $] \times 10^{-2}$} & $5.821_{-0.447}^{+0.305} \times 10^{-2}$ \\
$150-180$ & {$\left[4.903 \pm 0.854\left(\right.\right.$ stat) ${ }_{-0.643}^{+0.644}$ (syst) ${ }_{-0.168}^{+0.180}$ (lumi) $] \times 10^{-2}$} & $4.084_{-0.305}^{+0.238} \times 10^{-2}$ \\
$180-210$ & {$\left[3.579 \pm 0.744\right.$ (stat) ${ }_{-0.517}^{+0.430}$ (syst) ${ }_{-0.124}^{+0.133}$ (lumi) $] \times 10^{-2}$} & $3.206_{-0.245}^{+0.153} \times 10^{-2}$ \\
$210-240$ & {$\left[2.119 \pm 0.570\right.$ (stat) ${ }_{-0.288}^{+0.379}$ (syst) ${ }_{-0.074}^{+0.079}$ (lumi) $] \times 10^{-2}$} & $2.187_{-0.172}^{+0.134} \times 10^{-2}$ \\
$240-270$ & {$\left[1.529 \pm 0.494\right.$ (stat) ${ }_{-0.244}^{+0.200}$ (syst) ${ }_{-0.054}^{+0.057}$ (lumi) $] \times 10^{-2}$} & $1.633_{-0.154}^{+0.126} \times 10^{-2}$ \\
$270-300$ & {$\left[1.299 \pm 0.450\right.$ (stat) ${ }_{-0.232}^{+0.242}$ (syst) ${ }_{-0.045}^{+0.048}$ (lumi) $] \times 10^{-2}$} & $1.284_{-0.109}^{+0.087} \times 10^{-2}$ \\
\hline
\end{tabular}

Table A.7.: Cross section as a function of the invariant mass between the two leading jets for events with at least two jets in the final state with respect to the fiducial region measured in data and predicted from NLO fixed-order pQCD calculations from BLACKHAT+SHERPA.

\begin{tabular}{|c|c|c|}
\hline \hline$\left|\Delta y^{j j}\right|[\mathrm{GeV}]$ & Data cross section $[\mathrm{pb} /$ unit] & NLO cross section [pb/unit] \\
\hline \hline $0.0-0.5$ & $4.543 \pm 0.630$ (stat) ${ }_{-0.522}^{+0.519}$ (syst) ${ }_{-0.159}^{+0.170}$ (lumi) & $5.034_{-0.395}^{+0.296}$ \\
$0.5-1.0$ & $5.202 \pm 0.673$ (stat) ${ }_{-0.597}^{+0.584}$ (syst) ${ }_{-0.181}^{+0.194}$ (lumi) & $4.921_{-0.400}^{+0.292}$ \\
$1.0-1.5$ & $4.353 \pm 0.619$ (stat) ${ }_{-0.508}^{+0.505}$ (syst) ${ }_{-0.150}^{+0.161}$ (lumi) & $4.159_{-0.348}^{+0.271}$ \\
$1.5-2.0$ & $3.882 \pm 0.585$ (stat) ${ }_{-0.464}^{+0.493}$ (syst) ${ }_{-0.133}^{+0.142}$ (lumi) & $3.435_{-0.244}^{+0.168}$ \\
$2.0-2.5$ & $2.341 \pm 0.452$ (stat) ${ }_{-0.312}^{+0.332}$ (syst) ${ }_{-0.080}^{+0.086}$ (lumi) & $2.583_{-0.193}^{+0.141}$ \\
$2.5-3.0$ & $1.508 \pm 0.373$ (stat) ${ }_{-0.239}^{+0.210}$ (syst) ${ }_{-0.052}^{+0.055}$ (lumi) & $1.762_{-0.108}^{+0.082}$ \\
$3.0-3.5$ & $1.343 \pm 0.345$ (stat) ${ }_{-0.211}^{+0.215}$ (syst) ${ }_{-0.046}^{+0.049}$ (lumi) & $1.286_{-0.114}^{+0.076}$ \\
\hline
\end{tabular}

Table A.8.: Cross section as a function of the absolute rapidity distance between the two leading jets for events with at least two jets in the final state with respect to the fiducial region measured in data and predicted from NLO fixed-order pQCD calculations from BLACKHAT+SHERPA.

\begin{tabular}{|c|c|c|}
\hline \hline$\left|\Delta \phi^{j j}\right|[\mathrm{rad}]$ & Data cross section $[\mathrm{pb} / \mathrm{rad}]$ & NLO cross section $[\mathrm{pb} / \mathrm{rad}]$ \\
\hline \hline $0-\pi / 8$ & $1.581 \pm 0.417$ (stat) ${ }_{-0.257}^{+0.243}$ (syst) ${ }_{-0.055}^{+0.059}$ (lumi) & $1.948_{-0.173}^{+0.126}$ \\
$\pi / 8-\pi / 4$ & $2.278 \pm 0.484$ (stat) ${ }_{-0.355}^{+0.378}$ (syst) ${ }_{-0.079}^{+0.084}$ (lumi) & $2.278_{-0.199}^{+0.144}$ \\
$\pi / 4-3 \pi / 8$ & $1.703 \pm 0.441$ (stat) ${ }_{-0.262}^{+0.261}$ (syst) ${ }_{-0.060}^{+0.064}$ (lumi) & $2.057_{-0.198}^{+0.164}$ \\
$3 \pi / 8-\pi / 2$ & $2.152 \pm 0.484$ (stat) ${ }_{-0.309}^{+0.323}$ (syst) ${ }_{-0.075}^{+0.080}$ (lumi) & $2.311_{-0.173}^{+0.116}$ \\
$\pi / 2-5 \pi / 8$ & $3.359 \pm 0.618$ (stat) ${ }_{-0.468}^{+0.45}$ (syst) ${ }_{-0.117}^{+0.125}$ (lumi) & $3.014_{-0.224}^{+0.146}$ \\
$5 \pi / 8-3 \pi / 4$ & $3.767 \pm 0.661$ (stat) ${ }_{-0.476}^{+0.487}$ (syst) ${ }_{-0.132}^{+0.141}$ (lumi) & $4.304_{-0.348}^{+0.258}$ \\
$3 \pi / 4-7 \pi / 8$ & $6.714 \pm 0.880$ (stat) ${ }_{-0.758}^{+0.742}$ (syst) ${ }_{-0.231}^{+0.248}$ (lumi) & $6.379_{-0.469}^{+0.343}$ \\
$7 \pi / 8-\pi$ & $9.736 \pm 1.040$ (stat) ${ }_{-1.132}^{+1.121}$ (syst) ${ }_{-0.331}^{+0.354}$ (lumi) & $8.778_{-0.860}^{+0.787}$ \\
\hline \hline
\end{tabular}

Table A.9.: Cross section as a function of the absolute azimuthal separation between the two leading jets for events with at least two jets in the final state with respect to the fiducial region measured in data and predicted from NLO fixed-order pQCD calculations from BLACKHAT+SHERPA. 


\begin{tabular}{|c|c|c|}
\hline \hline$\Delta R^{j j}$ & Data cross section [pb/unit] & NLO cross section [pb/unit] \\
\hline \hline $0.4-0.8$ & $1.568 \pm 0.395$ (stat) ${ }_{-0.232}^{+0.238}$ (syst) ${ }_{-0.054}^{+0.057}$ (lumi) & $1.559_{-0.126}^{+0.086}$ \\
$0.8-1.2$ & $1.268 \pm 0.371$ (stat) ${ }_{-0.175}^{+0.175}$ (syst) ${ }_{-0.045}^{+0.048}$ (lumi) & $1.537_{-0.177}^{+0.154}$ \\
$1.2-1.6$ & $1.551 \pm 0.409$ (stat) ${ }_{-0.224}^{+0.223}$ (syst) ${ }_{-0.055}^{+0.059}$ (lumi) & $1.626_{-0.111}^{+0.075}$ \\
$1.6-2.0$ & $1.886 \pm 0.461$ (stat) ${ }_{-0.256}^{+0.253}$ (syst) ${ }_{-0.067}^{+0.072}$ (lumi) & $2.156_{-0.204}^{+0.171}$ \\
$2.0-2.4$ & $2.898 \pm 0.567$ (stat) ${ }_{-0.361}^{+0.369}$ (syst) ${ }_{-0.102}^{+0.109}$ (lumi) & $3.113_{-0.310}^{+0.258}$ \\
$2.4-2.8$ & $4.901 \pm 0.735$ (stat) ${ }_{-0.572}^{+0.564}$ (syst) ${ }_{-0.169}^{+0.181}$ (lumi) & $4.762_{-0.361}^{+0.239}$ \\
$2.8-3.2$ & $6.708 \pm 0.861$ (stat) ${ }_{-0.712}^{+0.719}$ (syst) ${ }_{-0.231}^{+0.247}$ (lumi) & $6.701_{-0.467}^{+0.314}$ \\
$3.2-3.6$ & $4.723 \pm 0.724$ (stat) ${ }_{-0.556}^{+0.550}$ (syst) ${ }_{-0.160}^{+0.172}$ (lumi) & $4.230_{-0.301}^{+0.218}$ \\
$3.6-4.0$ & $2.154 \pm 0.491$ (stat) ${ }_{-0.306}^{+0.298}$ (syst) ${ }_{-0.073}^{+0.079}$ (lumi) & $2.181_{-0.143}^{+0.121}$ \\
$4.0-4.4$ & $1.288 \pm 0.378$ (stat) ${ }_{-0.216}^{+0.215}$ (syst) ${ }_{-0.044}^{+0.047}$ (lumi) & $1.321_{-0.102}^{+0.088}$ \\
\hline
\end{tabular}

Table A.10.: Cross section as a function of the angular separation in $y$ - $\phi$ space between the two leading jets for events with at least two jets in the final state with respect to the fiducial region measured in data and predicted from NLO fixed-order pQCD calculations from BLACKHAT+SHERPA. 


\section{B. Measurement with the Dataset of 2011}

\section{B.1. Further Uncorrected Distributions}

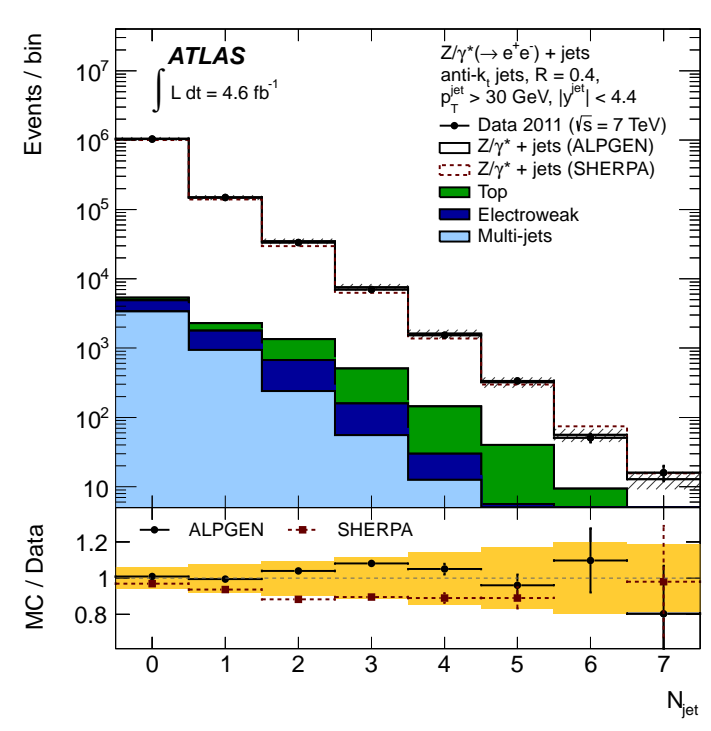

(a) Exclusive jet multiplicity

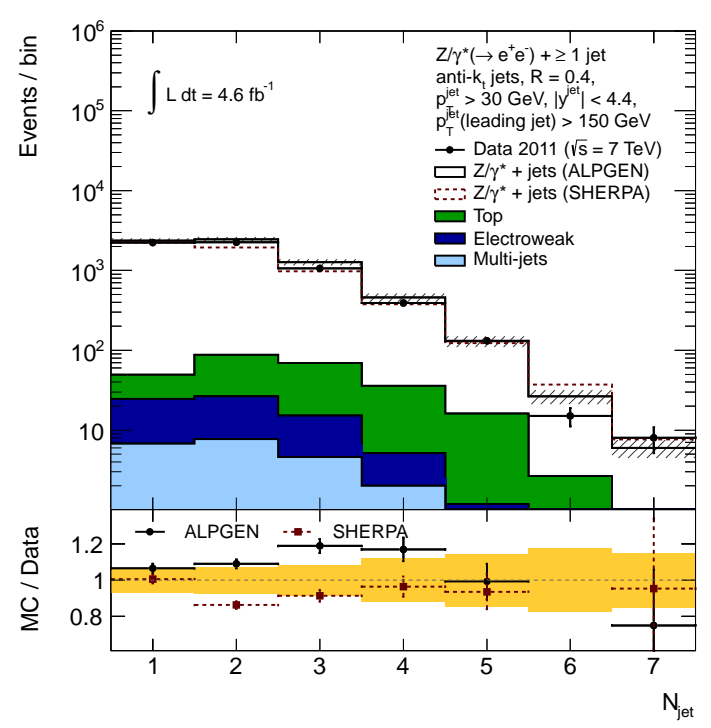

(b) Exclusive jet multiplicity, $p_{T}^{j e t}($ leading jet $)>150 \mathrm{GeV}$

Figure B.1.: Exclusive jet multiplicity with (a) the standard selection and (b) with an additional requirement on the transverse momentum of the leading jet of $p_{T}^{\text {jet }}>150 \mathrm{GeV}$ in data and simulation. Multi-jets and $t \bar{t}$ backgrounds have been estimated from data. The hatched band incorporates the total statistical and systematic uncertainty on the predictions. The yellow band in the ratio plots reflects the total systematic uncertainty. 


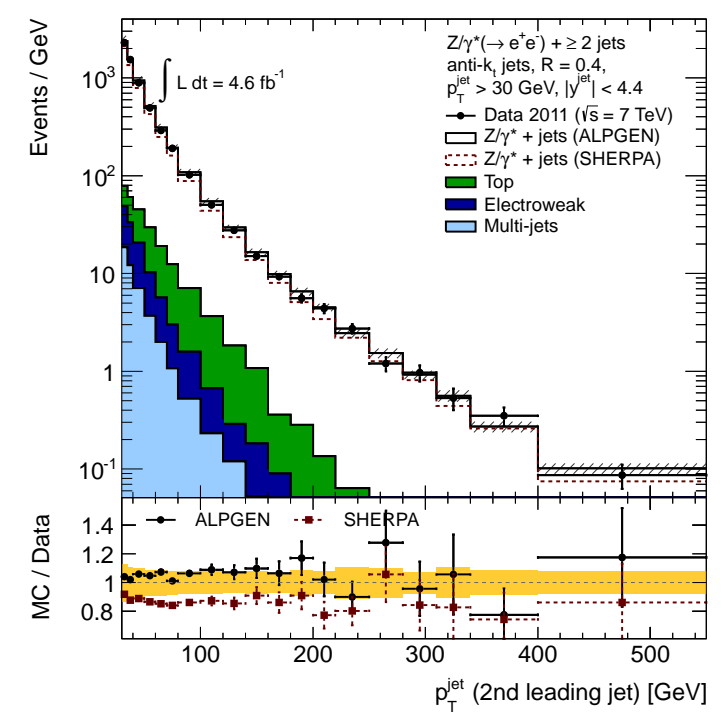

(a) 2nd leading jet $p_{T}$

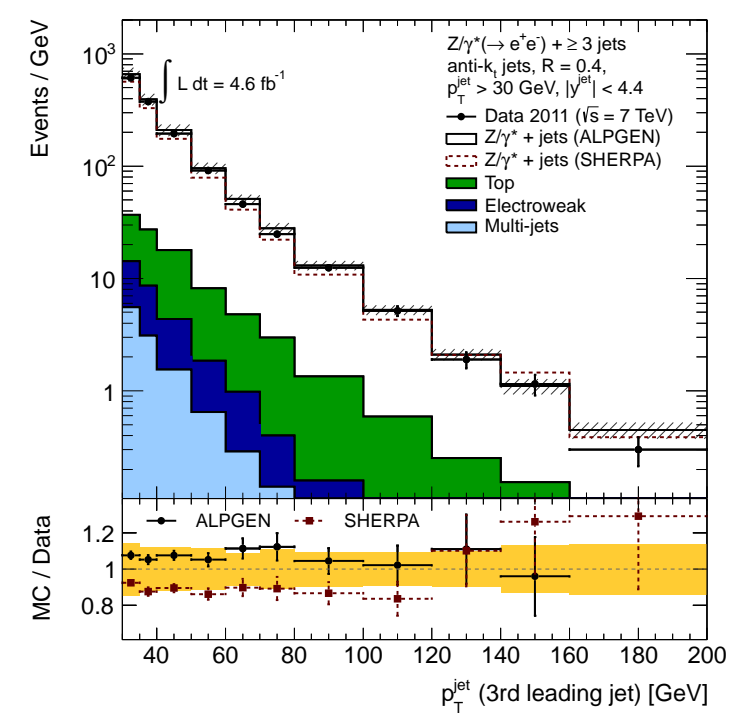

(b) 3rd leading jet $p_{T}$

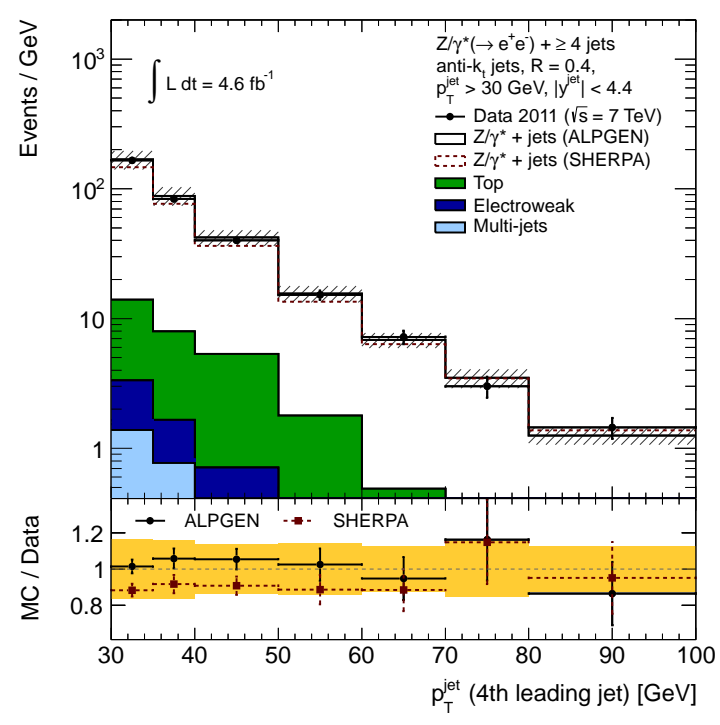

(c) 4th leading jet $p_{T}$

Figure B.2.: Transverse momentum distribution of (a) the 2nd leading jet, (b) the 3rd leading jet and (c) the 4th leading jet in data and simulation. Multi-jets and $t \bar{t}$ backgrounds have been estimated from data. The hatched band incorporates the total statistical and systematic uncertainty on the predictions. The yellow band in the ratio plots reflects the total systematic uncertainty. 


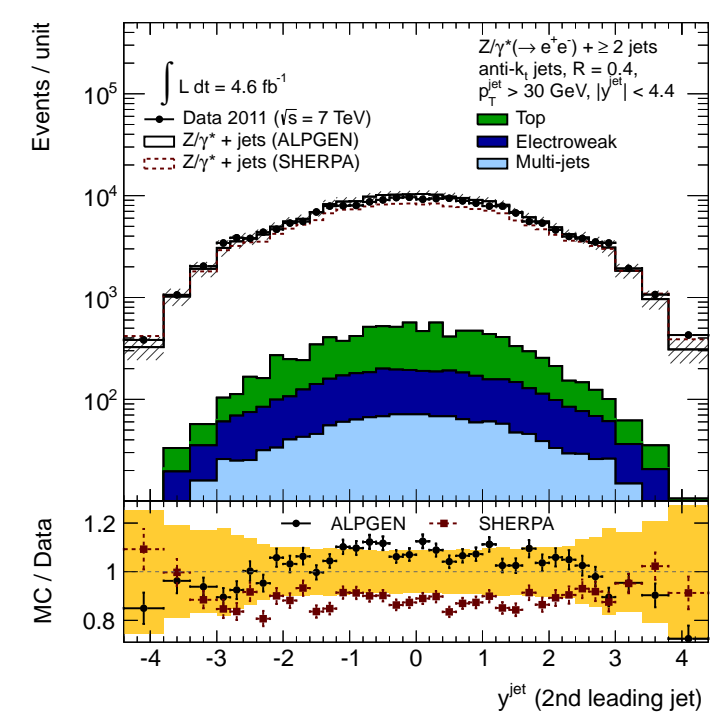

(a) 2nd leading jet $y$

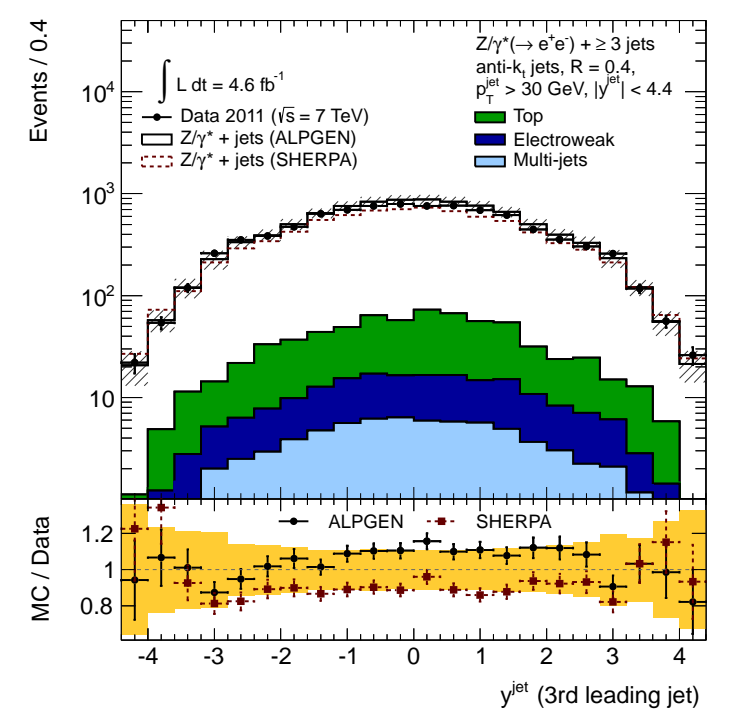

(b) 3rd leading jet $y$

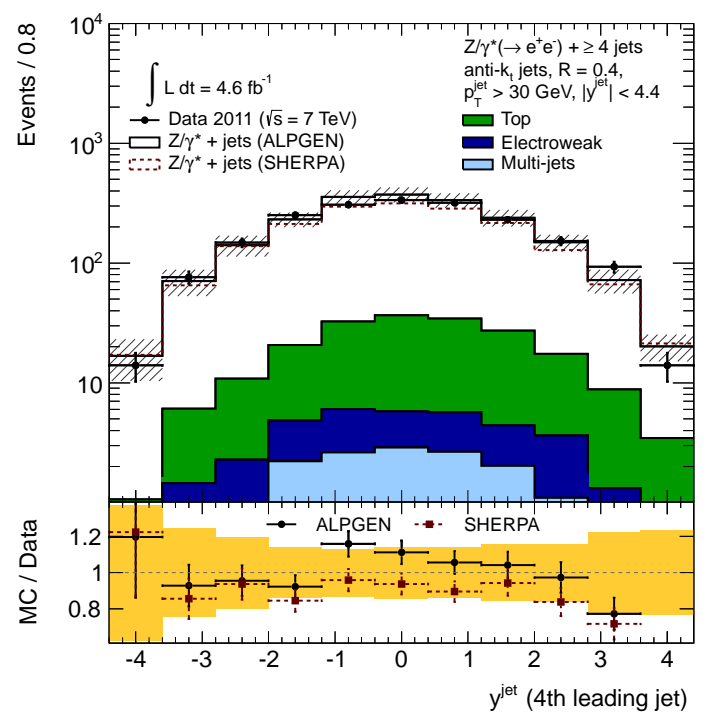

(c) 4th leading jet $y$

Figure B.3.: Rapidity distribution of (a) the 2nd leading jet, (b) the 3rd leading jet and (c) the 4th leading jet in data and simulation. Multi-jets and $t \bar{t}$ backgrounds have been estimated from data. The hatched band incorporates the total statistical and systematic uncertainty on the predictions. The yellow band in the ratio plots reflects the total systematic uncertainty. 


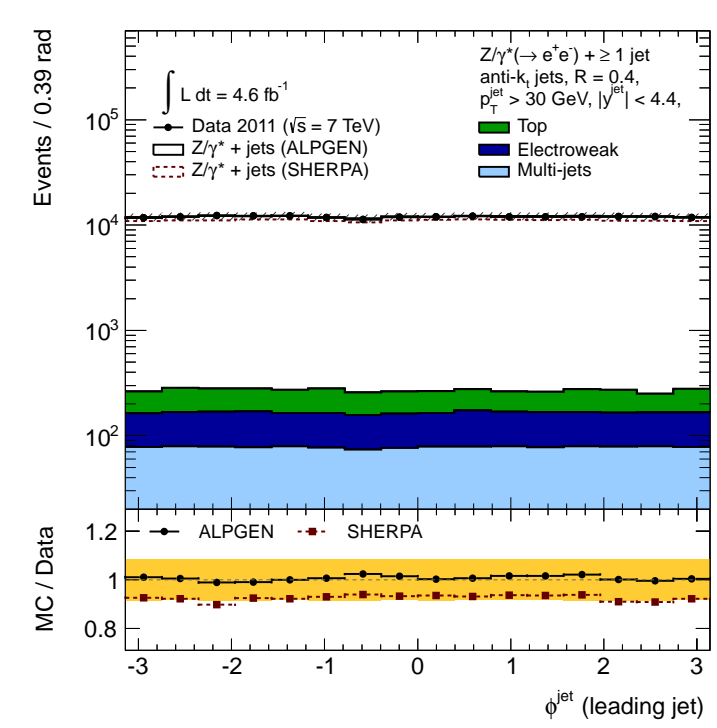

(a) Leading jet $\phi$

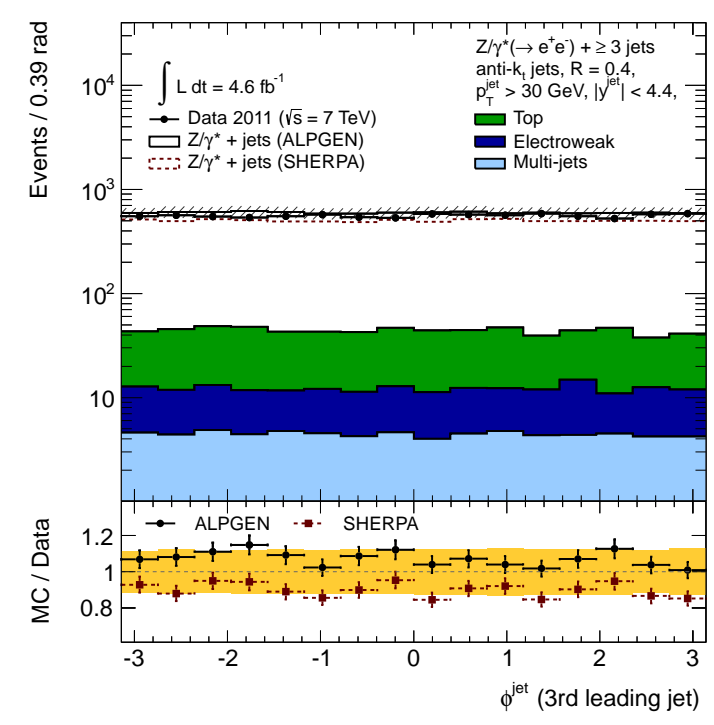

(c) 3rd leading jet $\phi$

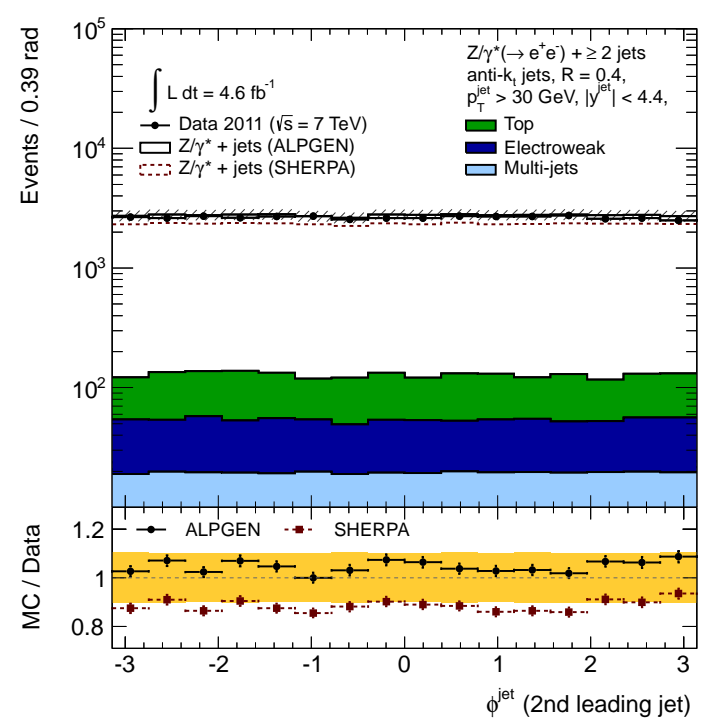

(b) 2nd leading jet $\phi$

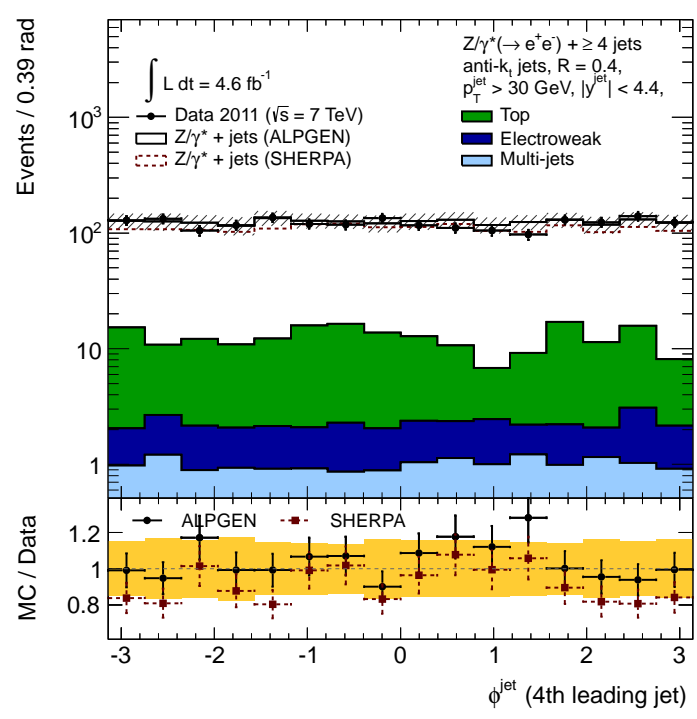

(d) 4th leading jet $\phi$

Figure B.4.: $\phi$ distribution of (a) the leading jet, (b) the 2nd leading jet, (c) the 3rd leading jet and $(\mathrm{d})$ the 4 th leading jet in data and simulation. Multi-jets and $t \bar{t}$ backgrounds have been estimated from data. The hatched band incorporates the total statistical and systematic uncertainty on the predictions. The yellow band in the ratio plots reflects the total systematic uncertainty. 


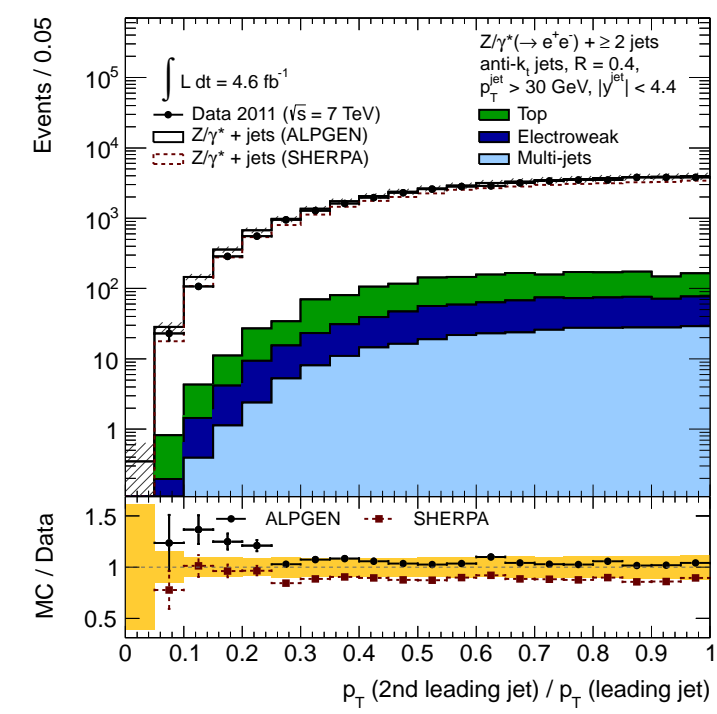

(a) $p_{T}$ ratio

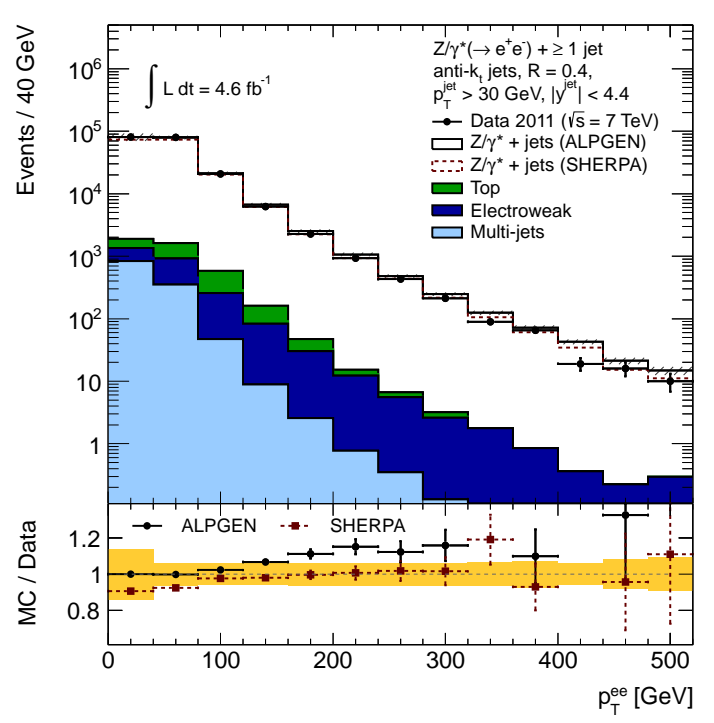

(b) $Z$ boson $p_{T}$

Figure B.5.: (a) $R\left(p_{T} 2 / p_{T} 1\right)$ distribution of the leading and 2 nd leading jet in events with at least two jets in the final state and (b) transverse momentum distribution of the $Z$ boson in events with at least one jet in the final state in data and simulation. Multi-jets and $t \bar{t}$ backgrounds have been estimated from data. The hatched band incorporates the total statistical and systematic uncertainty on the predictions. The yellow band in the ratio plots reflects the total systematic uncertainty.

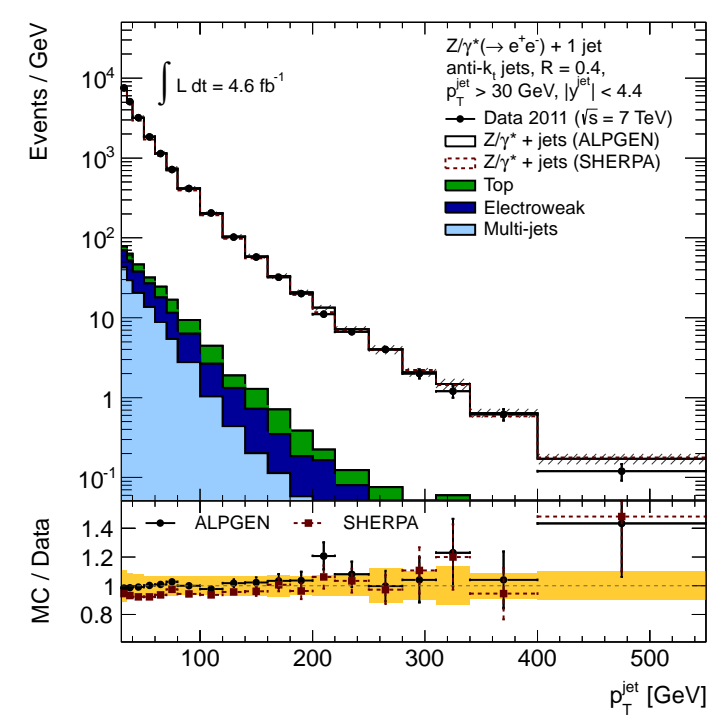

(a) Jet $p_{T}$

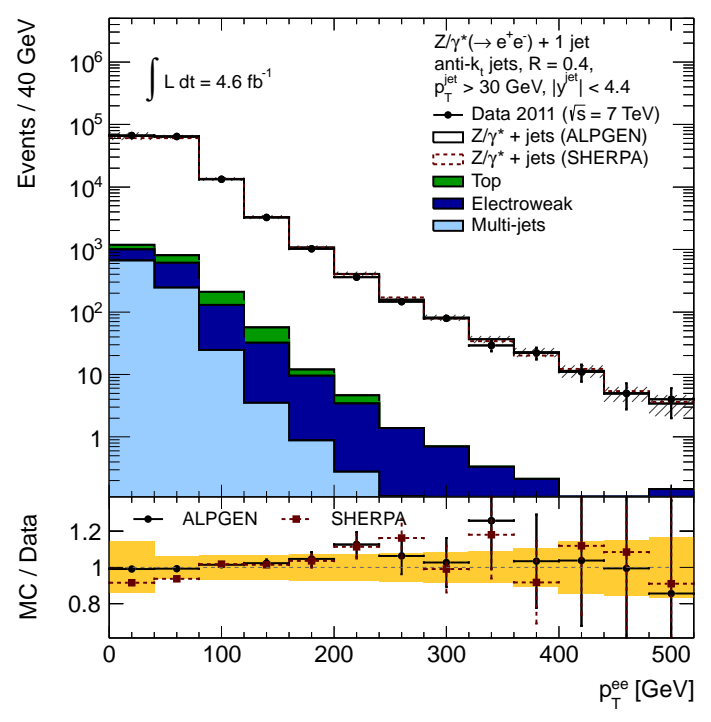

(b) $Z$ boson $p_{T}$

Figure B.6.: Transverse momentum distributions of (a) the leading jet and (b) the $\mathrm{Z}$ boson for events with exactly one jet in the final state in data and simulation. Multi-jets and $t \bar{t}$ backgrounds have been estimated from data. The hatched band incorporates the total statistical and systematic uncertainty on the predictions. The yellow band in the ratio plots reflects the total systematic uncertainty. 


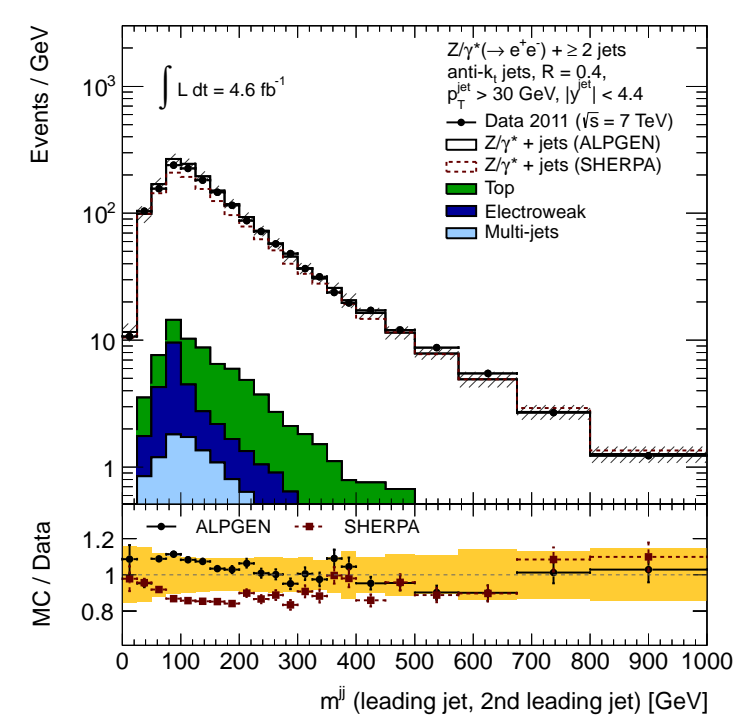

(a) Invariant dijet mass

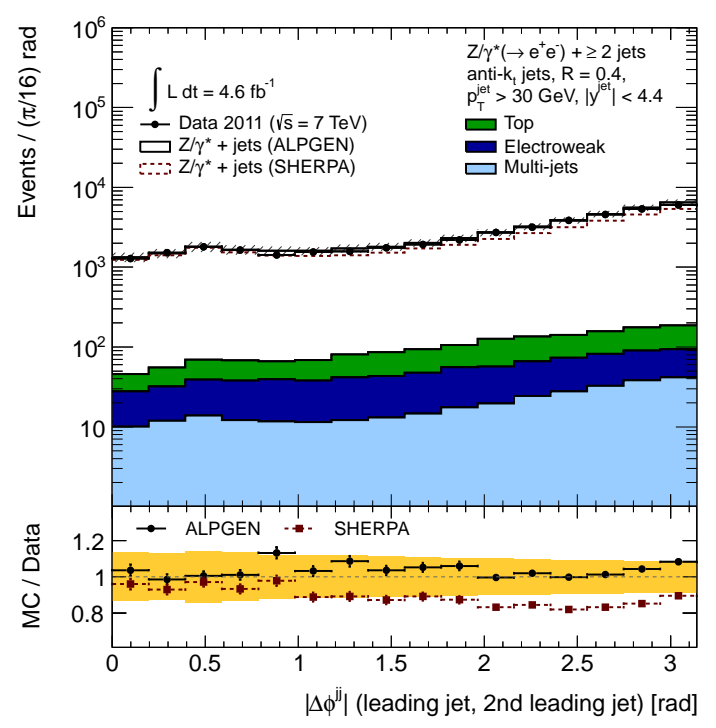

(b) Absolute azimuthal separation

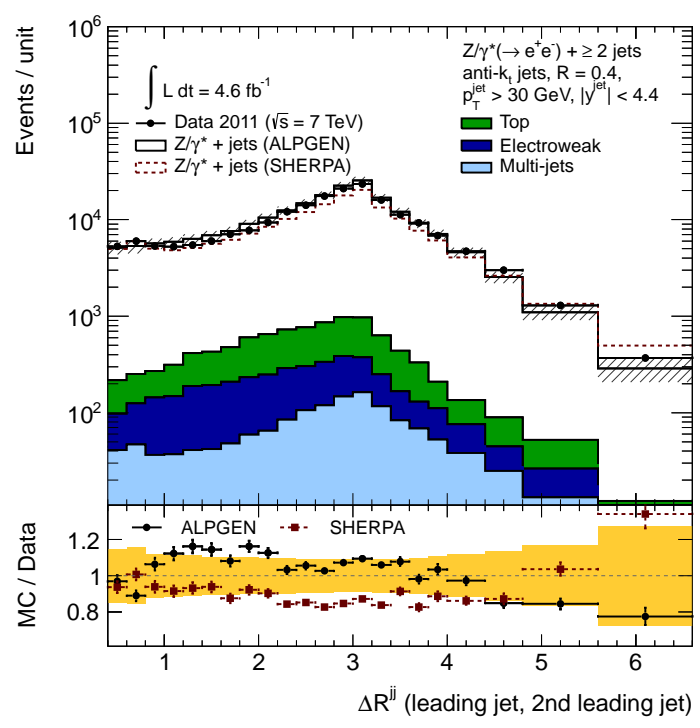

(c) Angular separation in $y-\phi$ space

Figure B.7.: (a) Invariant mass $m^{j j}$ and the angular separations (b) $\left|\Delta \phi^{j j}\right|$ and (c) $\Delta R^{j j}$ of the two leading jets in events with at least two jets in the final state in data and simulation. Multi-jets and $t \bar{t}$ backgrounds have been estimated from data. The hatched band incorporates the total statistical and systematic uncertainty on the predictions. The yellow band in the ratio plots reflects the total systematic uncertainty. 


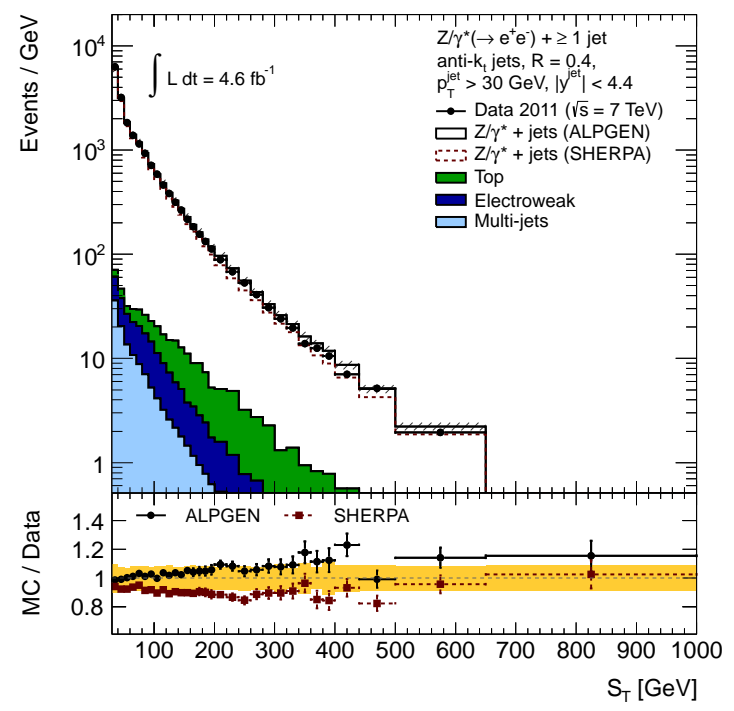

Figure B.8: $S_{\mathrm{T}}$ distribution in events with at least one jet in the final state in data and simulation. Multi-jets and $t \bar{t}$ backgrounds have been estimated from data. The hatched band incorporates the total statistical and systematic uncertainty on the predictions. The yellow band in the ratio plots reflects the total systematic uncertainty. 


\section{B.1.1. Impact of Pile-up}

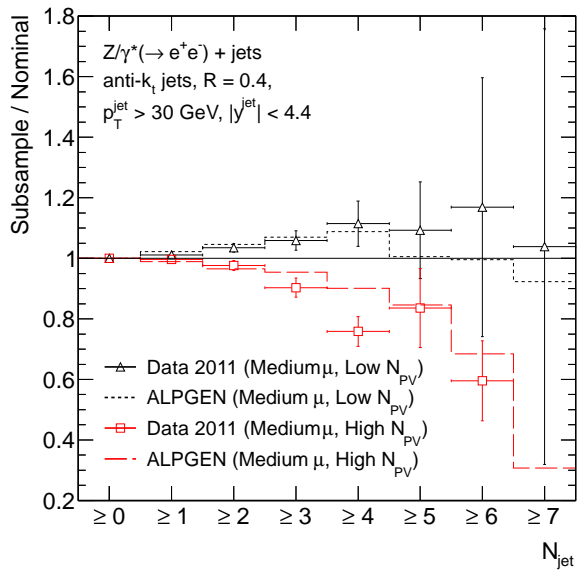

(a) Inclusive jet multiplicity, impact of in-time pile-up

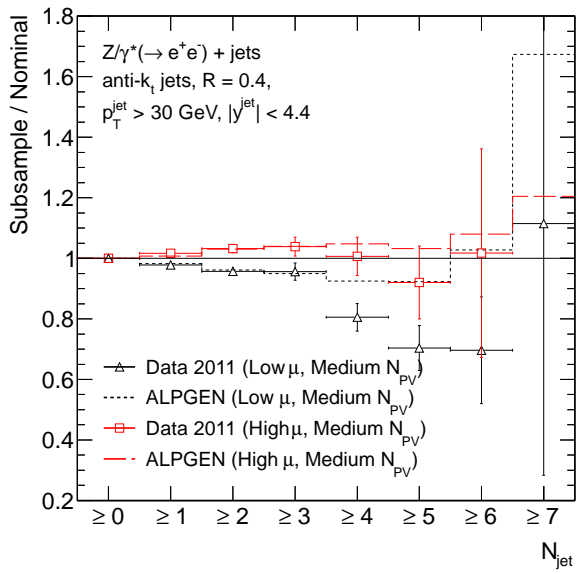

(b) Inclusive jet multiplicity, impact of out-of-time pile-up

Figure B.9.: Ratios of the inclusive jet multiplicity with and without requirements on $\mu$ and $N_{P} V$ in data and simulation to test the impact of (a) in-time pile-up and (b) out-of-time-pile-up. The distributions are divided by the respective number of inclusive $Z / \gamma^{*}(\rightarrow e e)$ events before calculating the ratios.

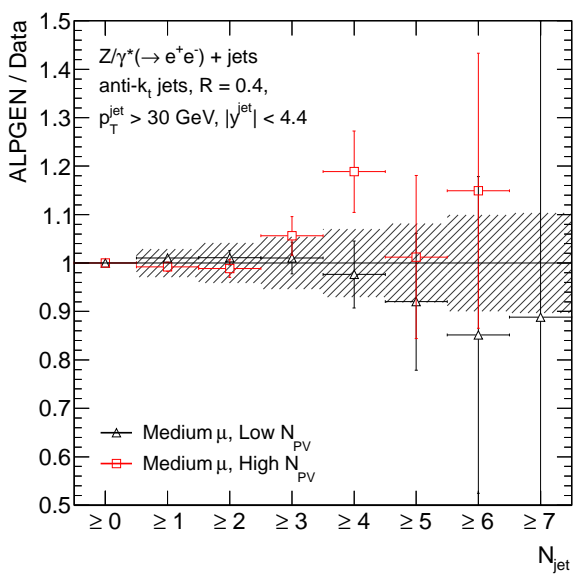

(a) Inclusive jet multiplicity, impact of in-time pile-up

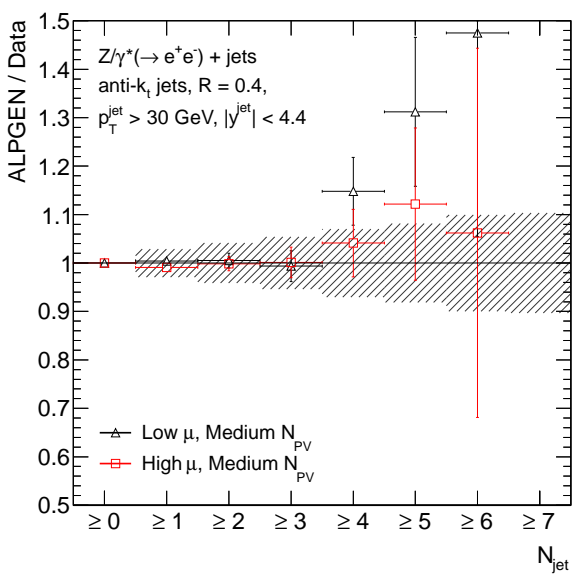

(b) Inclusive jet multiplicity, impact of out-of-time pile-up

Figure B.10.: ALPGEN+HERWIG to data ratio for the inclusive jet multiplicity to test the impact of (a) in-time pile-up and (b) out-of-time pile-up. The hatched bands reflect the pile-up component of the JES uncertainty. 


\section{B.2. Further Unfolding Distributions}

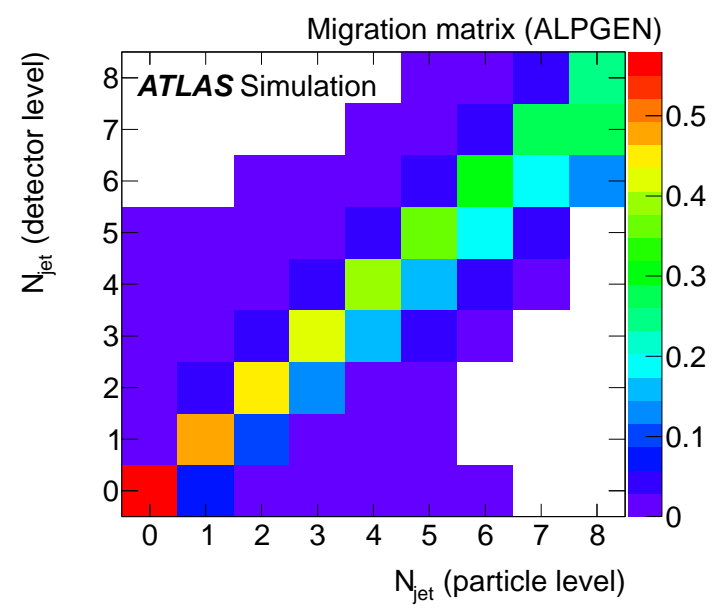

(a) Exclusive jet multiplicity

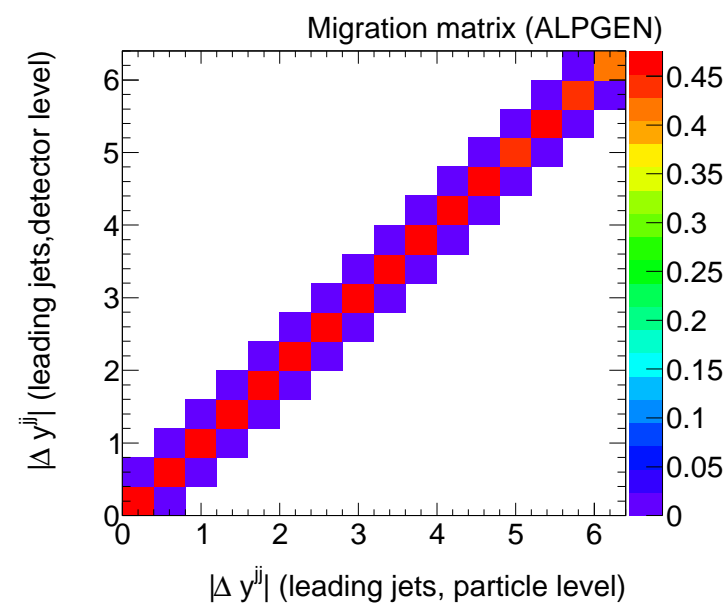

(b) Absolute rapidity difference

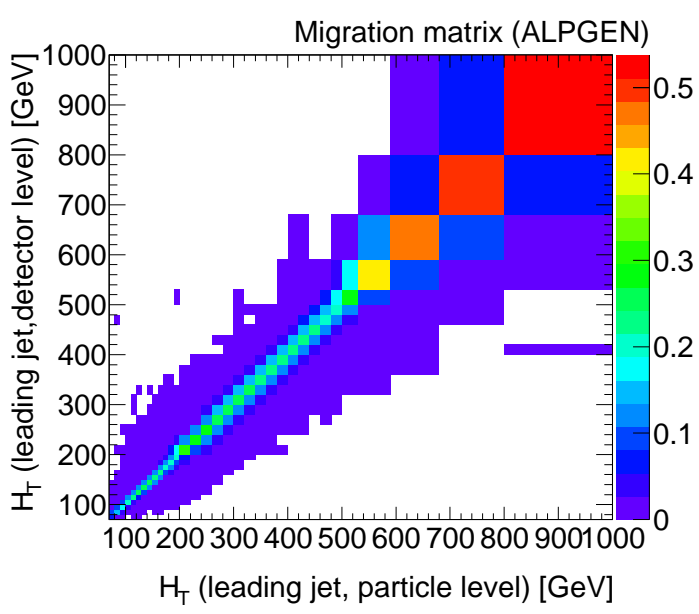

(c) $H_{T}$

Figure B.11.: Migration matrices for (a) the exclusive jet multiplicity, (b) the absolute rapidity difference of the two leading jets $\left|\Delta y^{j j}\right|$ for events with at least two jets in the final state and (c) $H_{T}$ for events with at least one jet in the final state. The migration matrices are determined using ALPGEN+HERWIG MC event samples. 


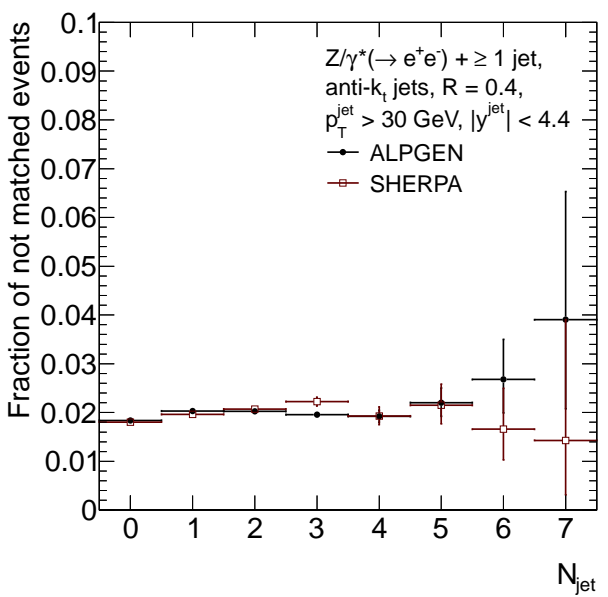

(a) Exclusive jet multiplicity

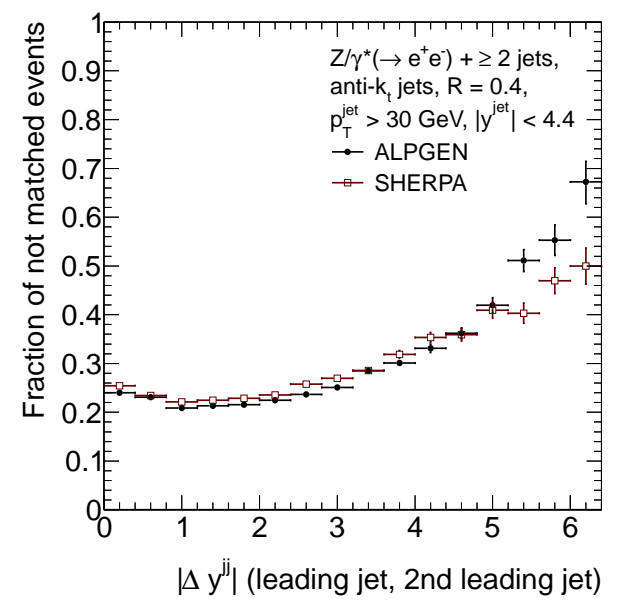

(b) Absolute rapidity difference

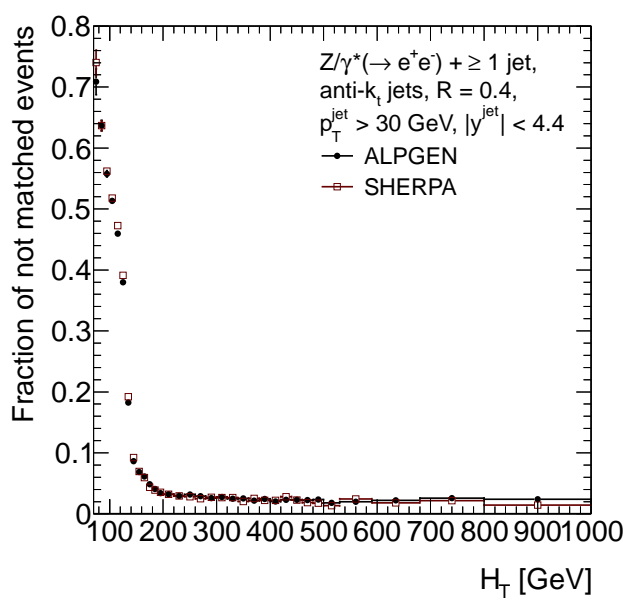

(c) $H_{T}$

Figure B.12.: Correction for not-matched events for (a) the exclusive jet multiplicity, (b) the absolute rapidity difference of the two leading jets $\left|\Delta y^{j j}\right|$ for events with at least two jets in the final state and (c) $H_{T}$ for events with at least one jet in the final state. The correction factors are shown for ALPGEN+HERWIG and SHERPA MC event samples. 


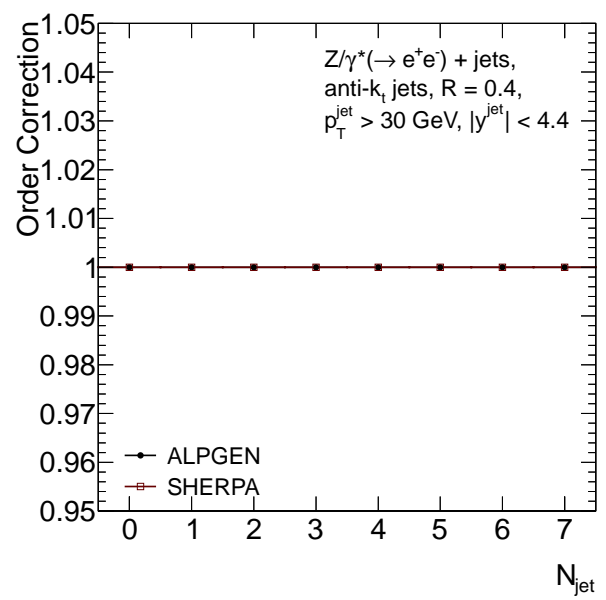

(a) Exclusive jet multiplicity

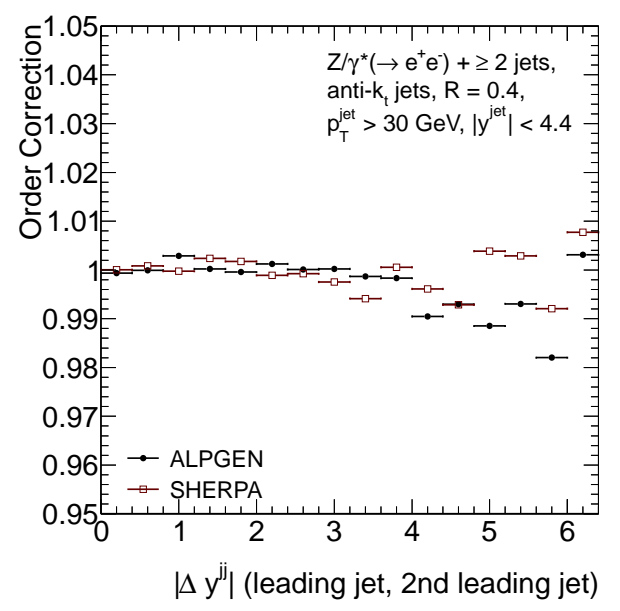

(b) Absolute rapidity difference

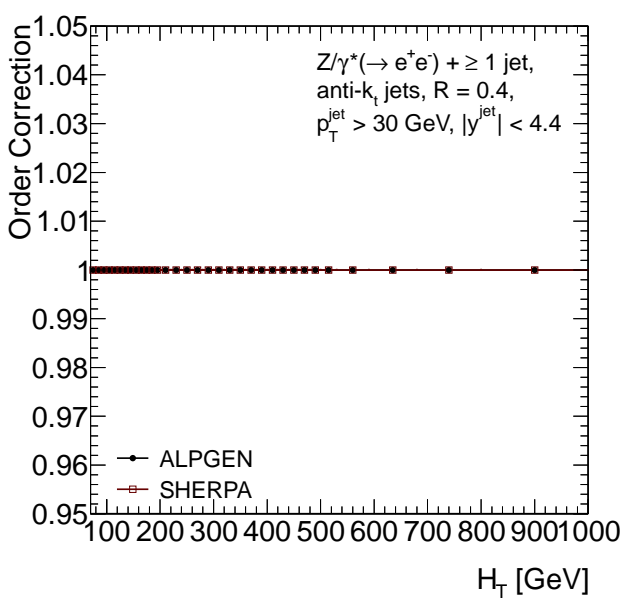

(c) $H_{T}$

Figure B.13.: Order correction for (a) the exclusive jet multiplicity, (b) the absolute rapidity difference of the two leading jets $\left|\Delta y^{j j}\right|$ for events with at least two jets in the final state and (c) $H_{T}$ for events with at least one jet in the final state. The correction factors are shown for ALPGEN+HERWIG and SHERPA MC event samples. 


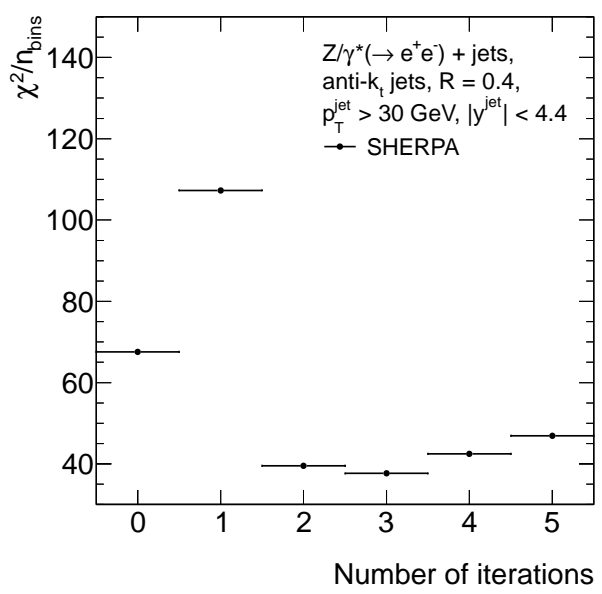

(a) Exclusive jet multiplicity

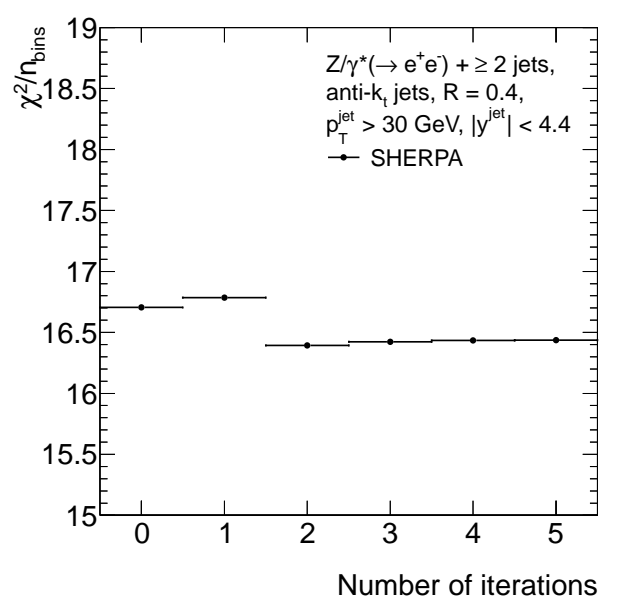

(b) Absolute rapidity difference

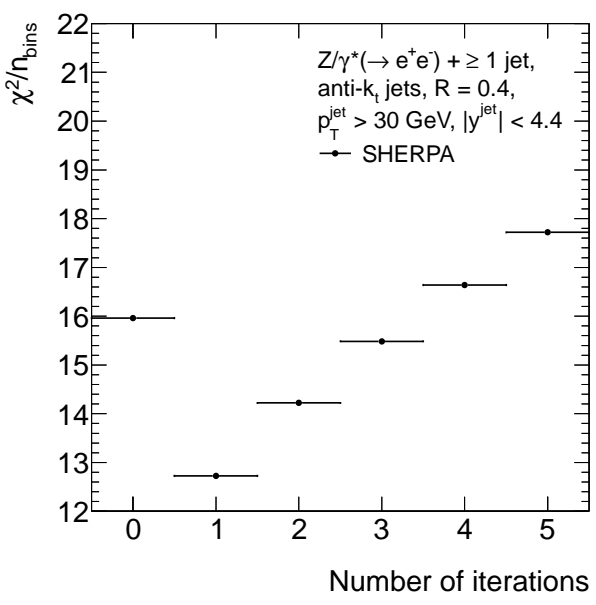

(c) $H_{T}$

Figure B.14.: $\chi^{2}$ divided by the number of bins as a function of the number of iterations for (a) the exclusive jet multiplicity, (b) the absolute rapidity difference of the two leading jets $\left|\Delta y^{j j}\right|$ for events with at least two jets in the final state and (c) $H_{T}$ for events with at least one jet in the final state. The values have been obtained with SHERPA MC event samples. Zero iterations correspond to the result from the Bin-by-Bin method. 


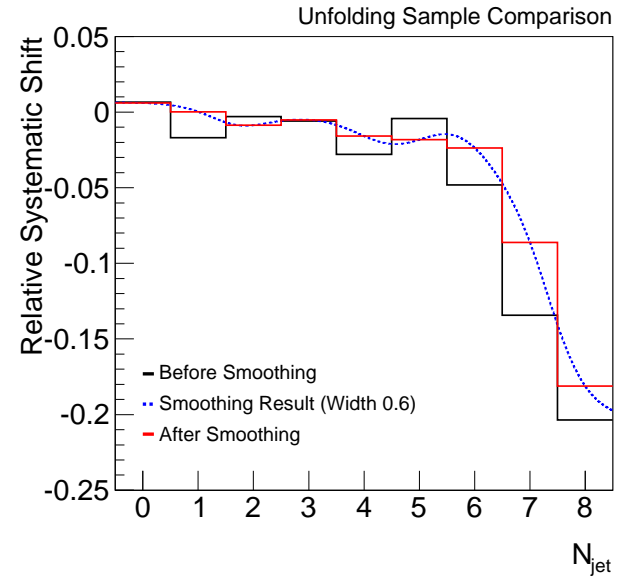

(a) Exclusive jet multiplicity

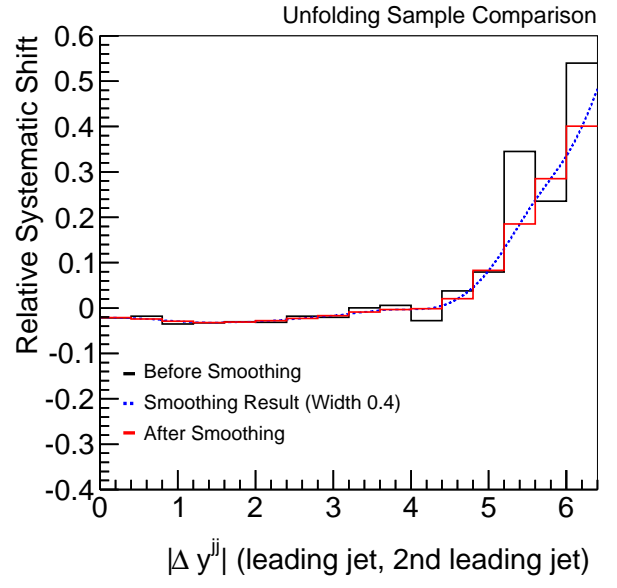

(b) Absolute rapidity difference

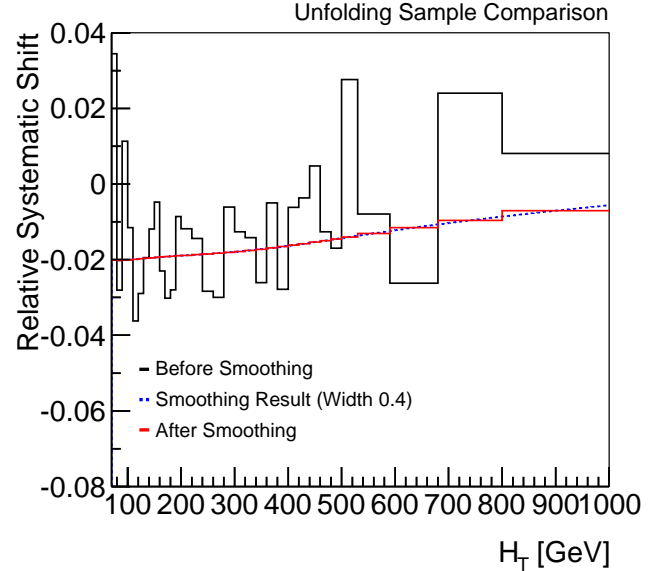

(c) $H_{T}$

Figure B.15.: Relative systematic shifts on the unfolded cross section obtained by comparing the unfolding procedure using ALPGEN+HERWIG or SHERPA for (a) the exclusive jet multiplicity, (b) the absolute rapidity difference of the two leading jets $\left|\Delta y^{j j}\right|$ for events with at least two jets in the final state and (c) $H_{T}$ for events with at least one jet in the final state. The relative shifts are smoothed using a Gaussian kernel. 


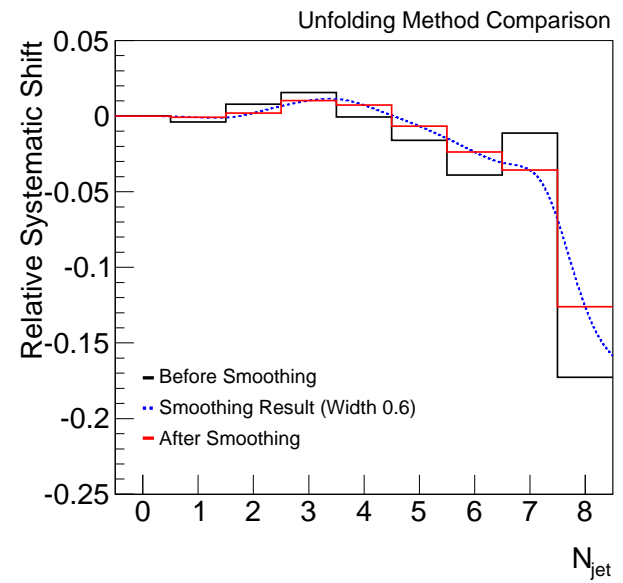

(a) Exclusive jet multiplicity

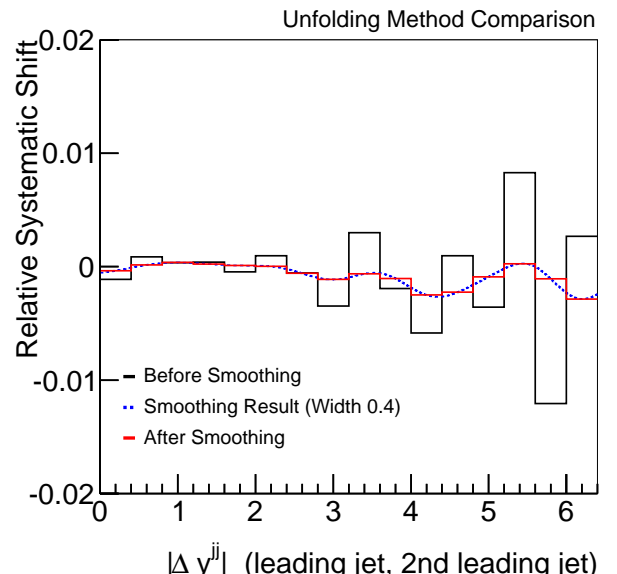

(b) Absolute rapidity difference

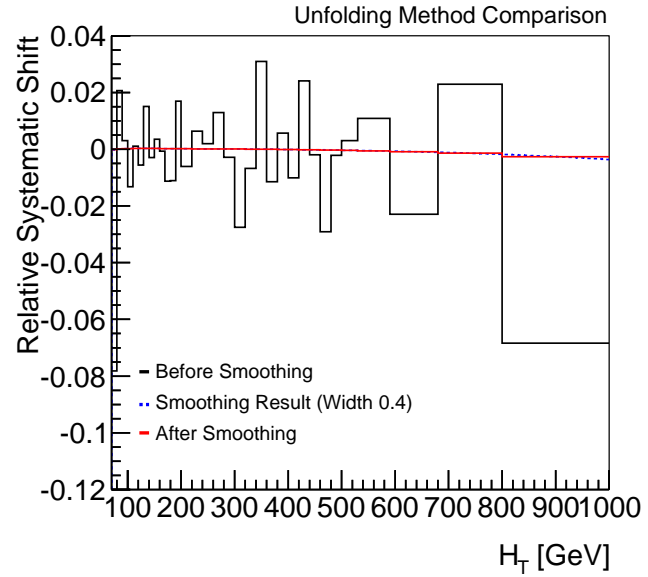

(c) $H_{T}$

Figure B.16.: Relative systematic shifts on the unfolded cross section obtained by comparing the results obtained with iterative (Bayes) method and Bin-by-Bin method for (a) the exclusive jet multiplicity, (b) the absolute rapidity difference of the two leading jets $\left|\Delta y^{j j}\right|$ for events with at least two jets in the final state and (c) $H_{T}$ for events with at least one jet in the final state. The relative shifts are smoothed using a Gaussian kernel. 


\section{B.3. Unfolding Closure Tests}

In this section a sanity check of the iterative (Bayes) method is shown using closure tests with SHERPA MC event samples and ALPGEN + HERWIG event samples reweighted to data. The detector level distributions of both samples are unfolded with the nominal ALPGEN+HERWIG MC event samples and compared to their truth distributions.

\section{B.3.1. ALPGEN+HERWIG Reweighting}

The ALPGEN+HERWIG MC event samples used in this analysis are not fully describing the data. Since ALPGEN+HERWIG is used for the unfolding, the influence of the shape differences between ALPGEN+HERWIG and data have to be checked. The predictions from ALPGEN+HERWIG have been compared to the the measurements on particle level for each distribution and their ratios have been used to calculate reweighting factors for the respective observable. These reweighting factors have been applied on the ALPGEN+HERWIG MC event samples on an event by event basis depending on the truth value. Figure B.17 shows exemplary the cross section plot for the exclusive jet multiplicity before and after reweighting.

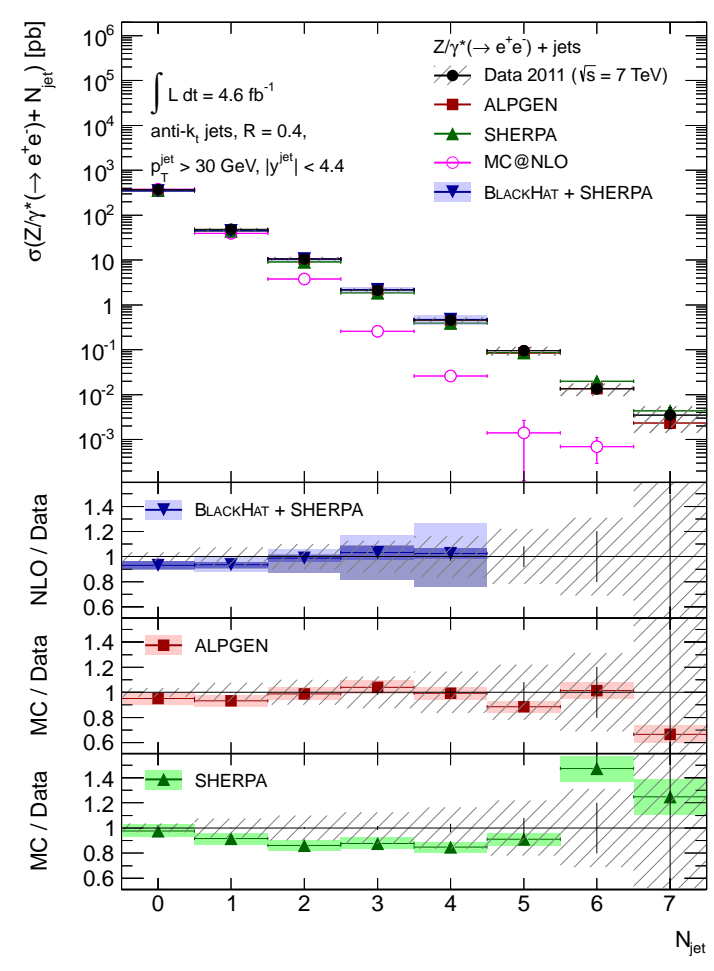

(a) Before reweighting

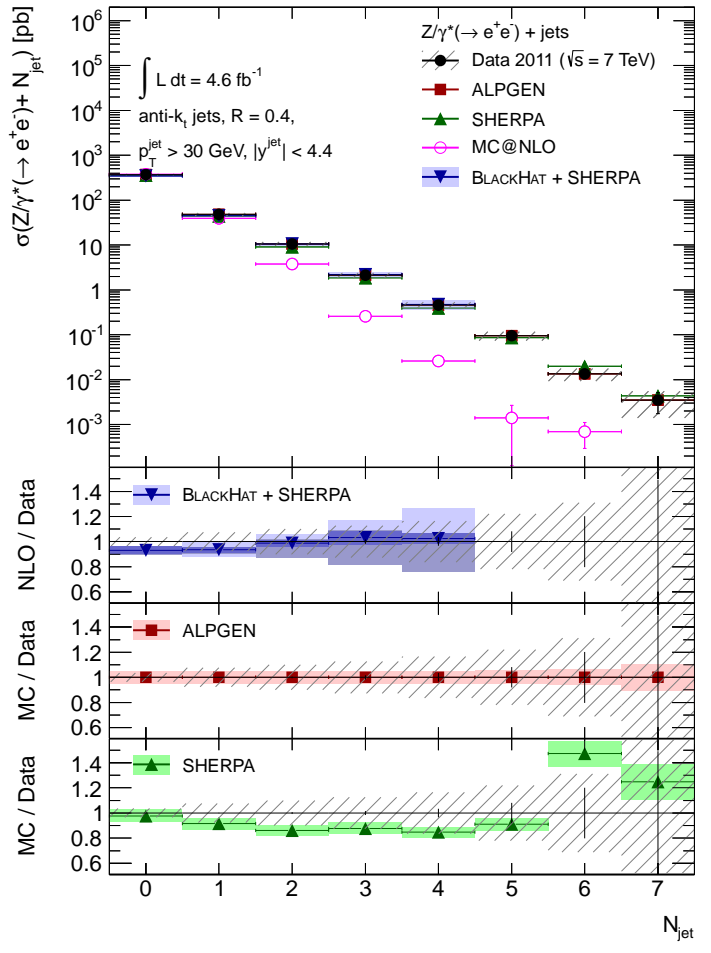

(b) After reweighting

Figure B.17.: Exclusive jet multiplicity (a) before reweighting and (b) after reweighting to data. 


\section{B.3.2. Closure Tests}

Figure B.18 - Fig. B.22 show the progression of the unfolding with increasing number of iterations, indicated by the coloured lines, for both SHERPA and ALPGEN+HERWIG reweighted to data exemplary for the exclusive jet multiplicity, the transverse momentum of the leading jet and the rapidity of the leading jet for events with at least one jet in the final state, the absolute rapidity difference of the two leading jets for events with at least two jets in the final state and $H_{T}$ for events with at least one jet in the final state. For the unfolding of the data, three, three, two, two and one iterations are used for the exclusive jet multiplicity, $p_{\mathrm{T}}^{\text {jet }}$ (leading jet), y(leading jet), $\left|\Delta y^{j j}\right|$ and $H_{\mathrm{T}}$ respectively, as detailed in Sec. 9.2,

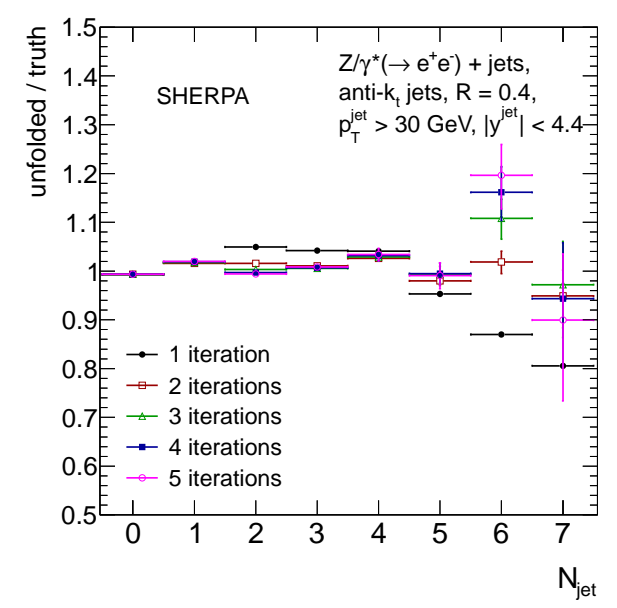

(a) SHERPA

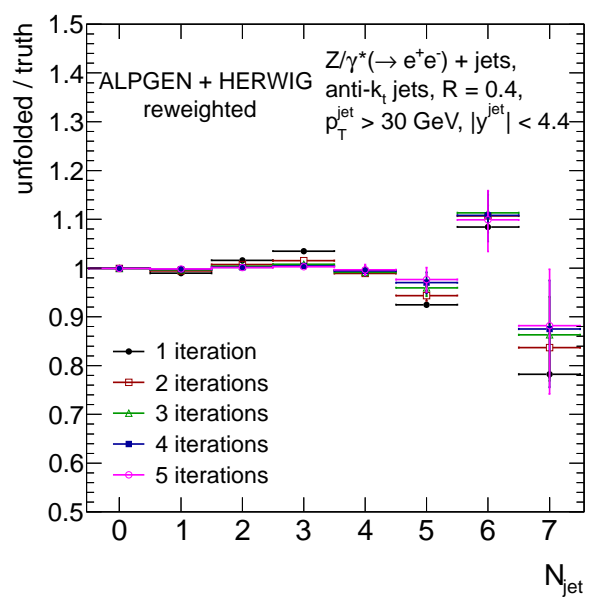

(b) ALPGEN+HERWIG reweighted

Figure B.18.: Closure tests of the exclusive jet multiplicity distribution for (a) SHERPA and (b) ALPGEN+HERWIG reweighted to data.

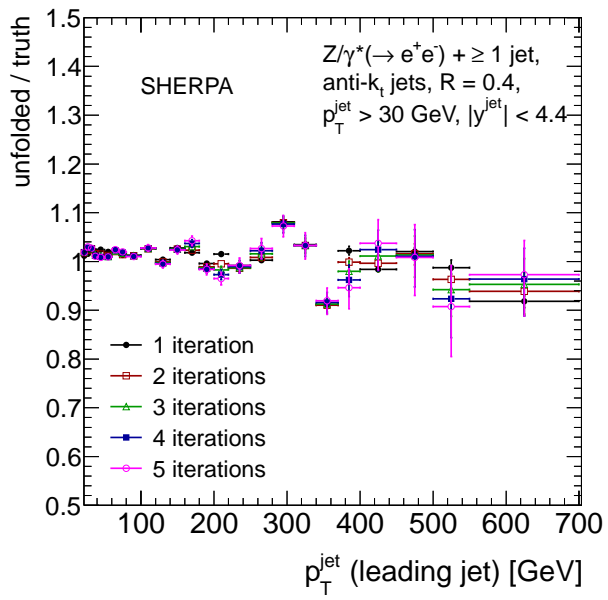

(a) SHERPA

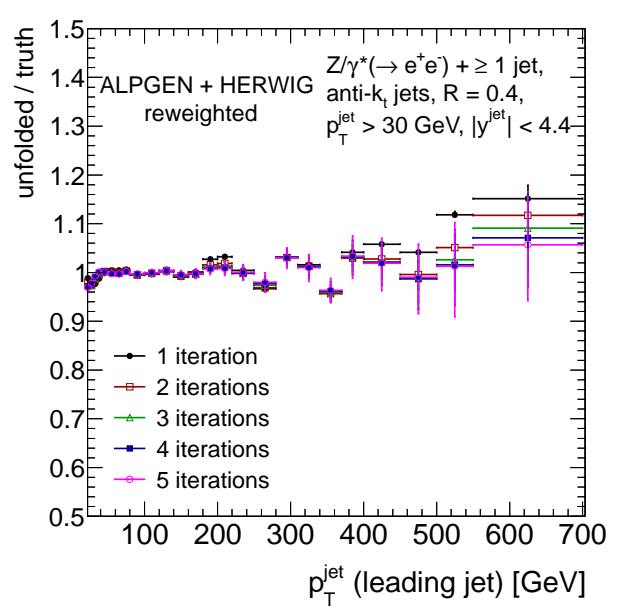

(b) ALPGEN+HERWIG reweighted

Figure B.19.: Closure tests of the transverse momentum distribution of the leading jet for events with at least one jet for (a) SHERPA and (b) ALPGEN+HERWIG reweighted to data. 


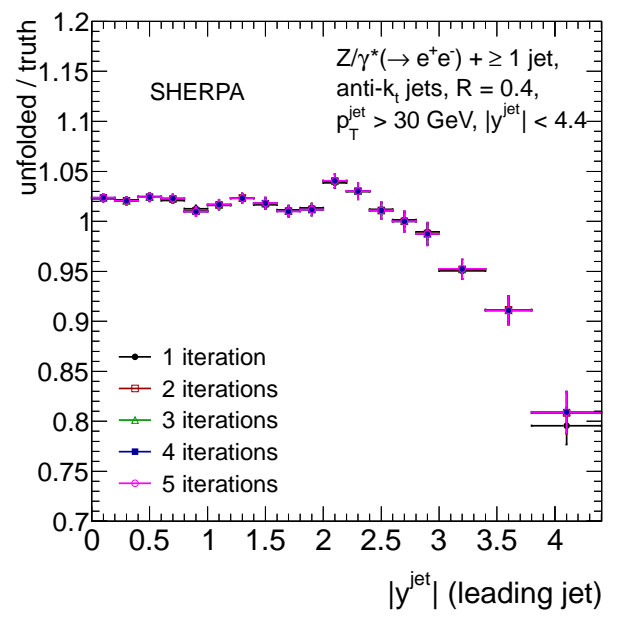

(a) SHERPA

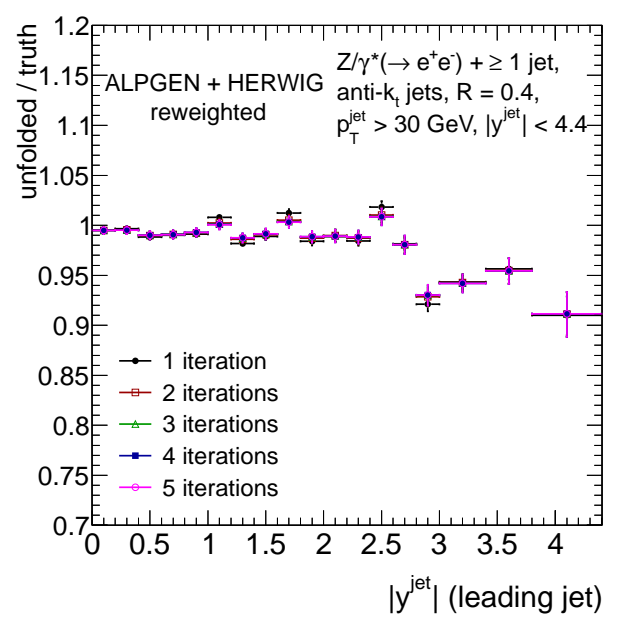

(b) ALPGEN+HERWIG reweighted

Figure B.20.: Closure tests of the rapidity distribution of the leading jet for events with at least one jet for (a) SHERPA and (b) ALPGEN+HERWIG reweighted to data.

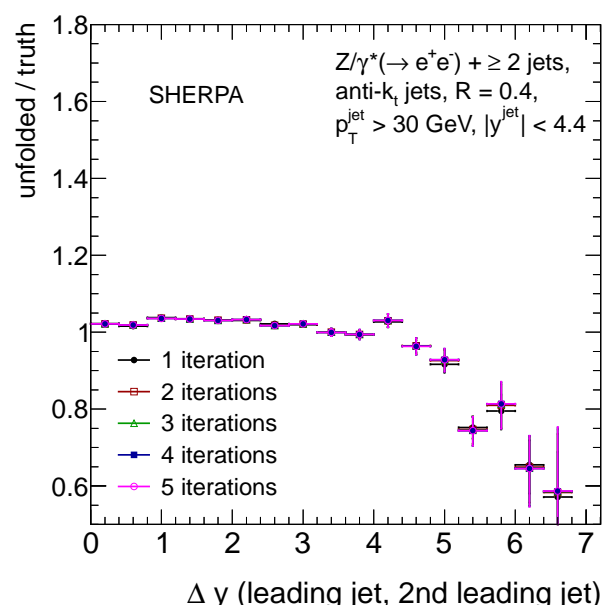

(a) SHERPA

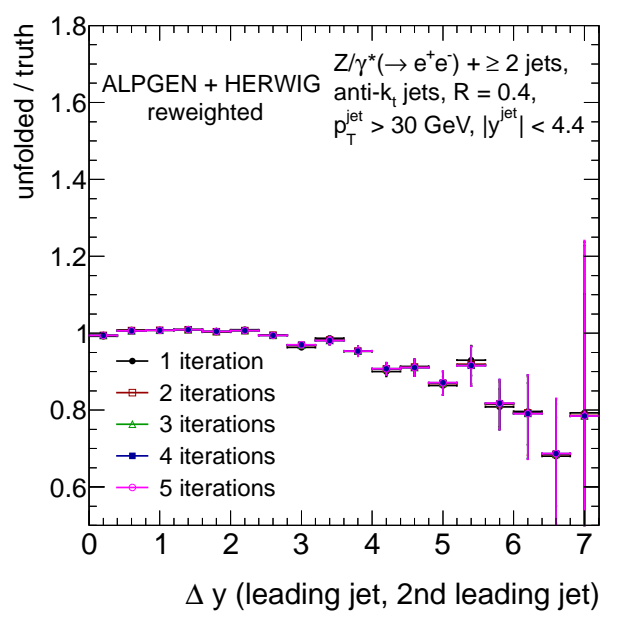

(b) ALPGEN+HERWIG reweighted

Figure B.21.: Closure tests of the absolute rapidity difference of the two leading jets $\left|\Delta y^{j j}\right|$ for events with at least two jets in the final state for (a) SHERPA and (b) ALPGEN+HERWIG reweighted to data. 


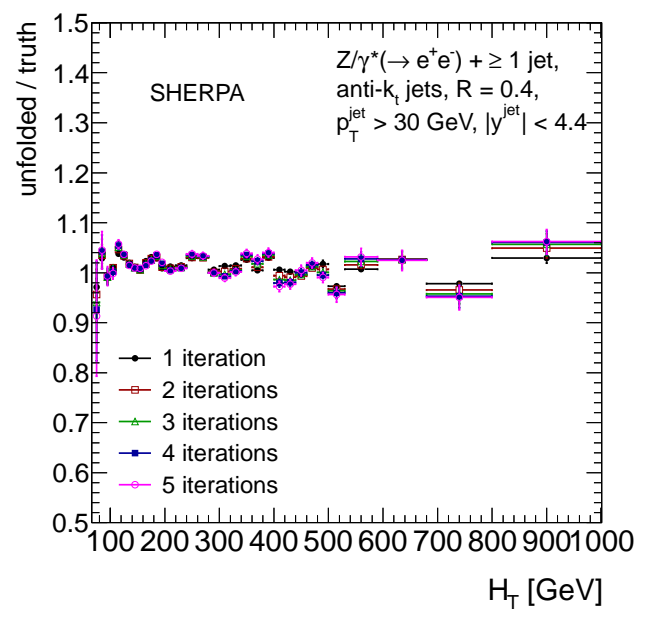

(a) SHERPA

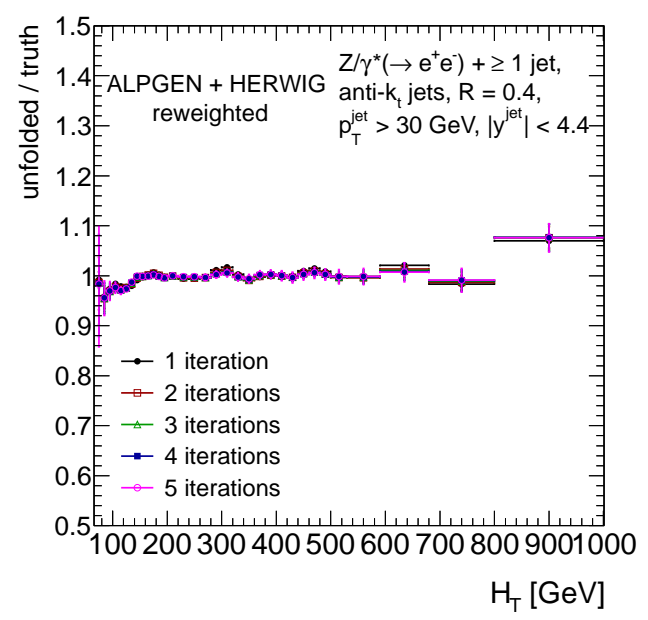

(b) ALPGEN+HERWIG reweighted

Figure B.22.: Closure tests of $H_{T}$ for events with at least one jet in the final state for (a) SHERPA and (b) ALPGEN+HERWIG reweighted to data.

\section{B.4. Cross Section Tables}

\begin{tabular}{|c|c|c|}
\hline \hline$N_{\text {jet }}$ & Data Cross Section $(\mathrm{pb})$ & NLO Cross Section $(\mathrm{pb})$ \\
\hline \hline 0 jets & {$[3.699 \pm 0.004$ (stat) \pm 0.113 (syst) \pm 0.067 (lumi) $] \times 10^{2}$} & $3.442_{-0.112}^{+0.115} \times 10^{2}$ \\
1 jet & {$[4.813 \pm 0.016$ (stat) \pm 0.351 (syst) \pm 0.087 (lumi) $] \times 10^{1}$} & $4.506_{-0.274}^{+0.273} \times 10^{1}$ \\
2 jets & {$[1.060 \pm 0.007$ (stat) \pm 0.104 (syst) \pm 0.019 (lumi) $] \times 10^{1}$} & $1.048_{-0.075}^{+0.075} \times 10^{1}$ \\
3 jets & {$[2.114 \pm 0.035$ (stat) \pm 0.263 (syst) \pm 0.039 (lumi) $] \times 10^{0}$} & $2.185_{-0.283}^{+0.283} \times 10^{0}$ \\
4 jets & {$[4.609 \pm 0.157$ (stat) \pm 0.737 (syst) \pm 0.084 (lumi) $] \times 10^{-1}$} & $4.728_{-1.085}^{+1.087} \times 10^{-1}$ \\
5 jets & {$[9.529 \pm 0.777$ (stat) \pm 1.938 (syst) \pm 0.173 (lumi) $] \times 10^{-2}$} & - \\
6 jets & {$[1.347 \pm 0.273$ (stat) \pm 0.316 (syst) \pm 0.024 (lumi) $] \times 10^{-2}$} & - \\
7 jets & {$[3.472 \pm 1.708$ (stat) \pm 1.162 (syst) \pm 0.062 (lumi) $] \times 10^{-3}$} & - \\
\hline \hline
\end{tabular}

Table B.1.: Cross sections as a function of the exclusive jet multiplicities with respect to the fiducial region measured in data and predicted from NLO fixed-order pQCD calculations from BLACKHAT+SHERPA. 


\begin{tabular}{|c|c|c|}
\hline \hline$N_{\text {jet }}$ & Data Cross Section $(\mathrm{pb})$ & NLO Cross Section $(\mathrm{pb})$ \\
\hline \hline 1 jet & {$\left[6.404 \pm 0.158\right.$ (stat) \pm 0.306 (syst) $\pm 0.116($ lumi) $] \times 10^{-1}$} & $5.778_{-1.862}^{+1.862} \times 10^{-1}$ \\
2 jets & {$[6.793 \pm 0.160$ (stat) \pm 0.372 (syst) \pm 0.123 (lumi) $] \times 10^{-1}$} & $5.867_{-0.822}^{+0.818} \times 10^{-1}$ \\
3 jets & {$[3.134 \pm 0.111$ (stat) \pm 0.241 (syst) \pm 0.057 (lumi) $] \times 10^{-1}$} & $3.052_{-0.588}^{+0.589} \times 10^{-1}$ \\
4 jets & {$[1.169 \pm 0.069$ (stat) \pm 0.138 (syst) \pm 0.021 (lumi) $] \times 10^{-1}$} & $1.187_{-0.392}^{+0.392} \times 10^{-1}$ \\
5 jets & {$[3.481 \pm 0.372$ (stat) \pm 0.624 (syst) \pm 0.063 (lumi) $] \times 10^{-2}$} & - \\
6 jets & {$[5.193 \pm 1.339$ (stat) \pm 1.159 (syst) \pm 0.094 (lumi) $] \times 10^{-3}$} & - \\
7 jets & {$[1.665 \pm 0.798$ (stat) \pm 0.656 (syst) \pm 0.030 (lumi) $] \times 10^{-3}$} & - \\
\hline \hline
\end{tabular}

Table B.2.: Cross sections as a function of the exclusive jet multiplicities with respect to the fiducial region measured in data and predicted from NLO fixed-order pQCD calculations from BLACKHAT+SHERPA with an additional requirement on the transverse momentum of the leading jet of $p_{T}^{\text {jet }}>150 \mathrm{GeV}$.

\begin{tabular}{|c|c|c|}
\hline \hline$N_{\text {jet }}+1 / N_{\text {jet }}$ & Data cross section ratio & NLO cross section ratio \\
\hline \hline 1 jet $/ 0$ jets & {$[1.301 \pm 0.005$ (stat) \pm 0.103 (syst) $] \times 10^{-1}$} & $1.309_{-0.095}^{+0.098} \times 10^{-1}$ \\
2 jets $/ 1$ jet & {$[2.203 \pm 0.017$ (stat) \pm 0.077 (syst) $] \times 10^{-1}$} & $2.325_{-0.275}^{+0.306} \times 10^{-1}$ \\
3 jets $/ 2$ jets & {$[1.994 \pm 0.036$ (stat) \pm 0.061 (syst) $] \times 10^{-1}$} & $2.085_{-0.372}^{+0.423} \times 10^{-1}$ \\
4 jets $/ 3$ jets & {$[2.180 \pm 0.083$ (stat) \pm 0.088 (syst) $] \times 10^{-1}$} & $2.164_{-0.668}^{+0.845} \times 10^{-1}$ \\
5 jets $/ 4$ jets & {$[2.067 \pm 0.183$ (stat) \pm 0.109 (syst) $] \times 10^{-1}$} & - \\
6 jets $/ 5$ jets & {$[1.413 \pm 0.308$ (stat) \pm 0.109 (syst) $] \times 10^{-1}$} & - \\
\hline \hline
\end{tabular}

Table B.3.: Ratio of cross sections for successive exclusive jet multiplicities $\sigma\left(Z / \gamma^{*}+(N+1)\right.$ jets $) / \sigma\left(Z / \gamma^{*}+N\right.$ jets $)$ with respect to the fiducial region measured in data and predicted from NLO fixed-order pQCD calculations from BLACKHAT+SHERPA.

\begin{tabular}{|c|c|c|}
\hline \hline$N_{\text {jet }}+1 / N_{\text {jet }}$ & Data cross section ratio & NLO cross section ratio \\
\hline \hline 2 jets $/ 1$ jet & $1.061 \pm 0.036$ (stat) \pm 0.033 (syst) & $1.015_{-0.350}^{+0.678}$ \\
3 jets $/ 2$ jets & {$[4.614 \pm 0.196$ (stat) \pm 0.186 (syst) $] \times 10^{-1}$} & $5.202_{-1.442}^{+1.878} \times 10^{-1}$ \\
4 jets $/ 3$ jets & {$[3.730 \pm 0.256$ (stat) \pm 0.196 (syst) $] \times 10^{-1}$} & $3.890_{-1.852}^{+2.642} \times 10^{-1}$ \\
5 jets $/ 4$ jets & {$[2.977 \pm 0.363$ (stat) \pm 0.232 (syst) $] \times 10^{-1}$} & - \\
6 jets $/ 5$ jets & {$[1.492 \pm 0.417$ (stat) \pm 0.177 (syst) $] \times 10^{-1}$} & - \\
\hline \hline
\end{tabular}

Table B.4.: Ratio of cross sections for successive exclusive jet multiplicities $\sigma\left(Z / \gamma^{*}+(N+1)\right.$ jets $) / \sigma\left(Z / \gamma^{*}+N\right.$ jets $)$ with respect to the fiducial region measured in data and predicted from NLO fixed-order pQCD calculations from BLACKHAT+SHERPA with an additional requirement on the transverse momentum of the leading jet of $p_{T}^{\text {jet }}>150 \mathrm{GeV}$. 


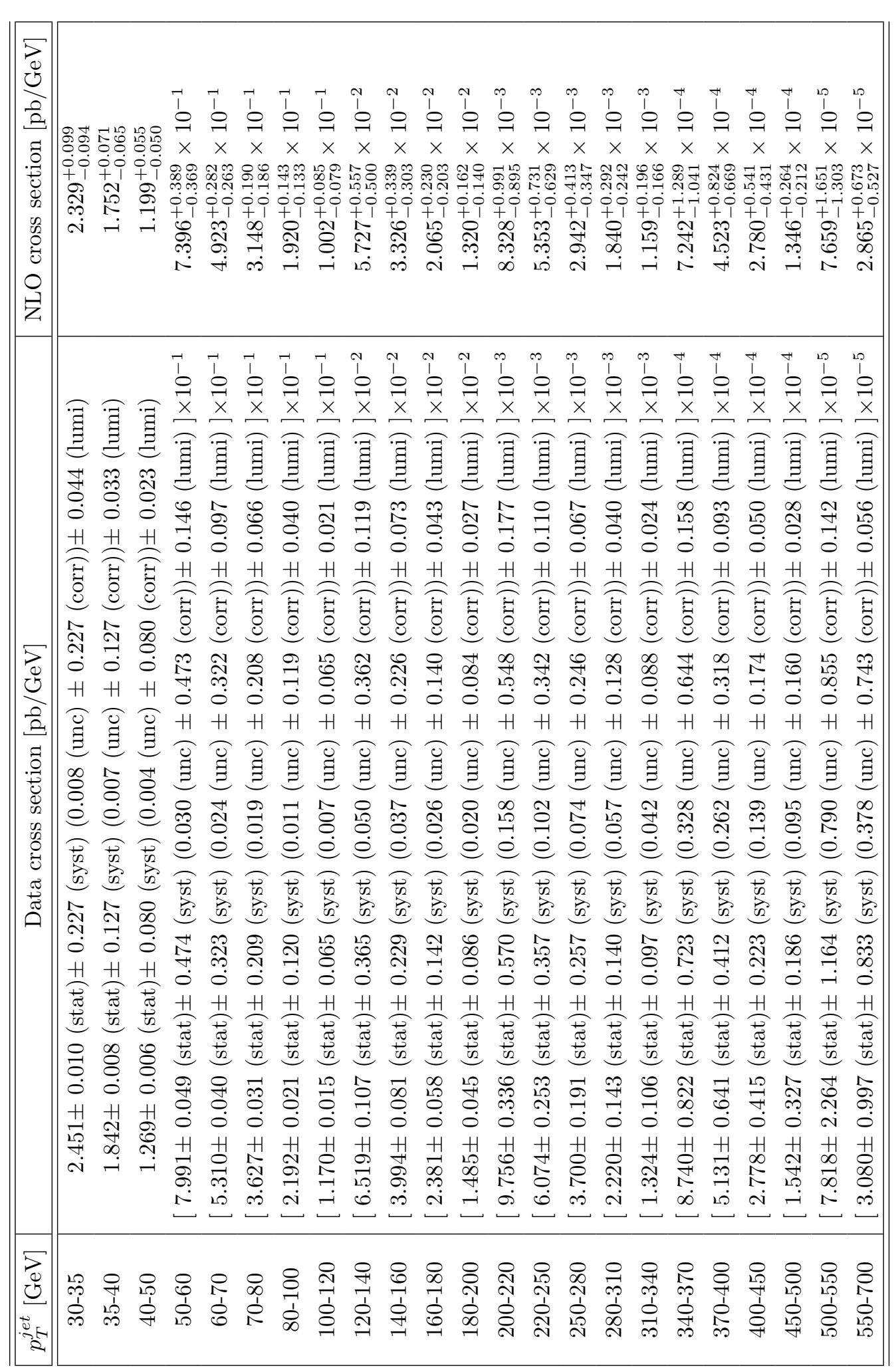

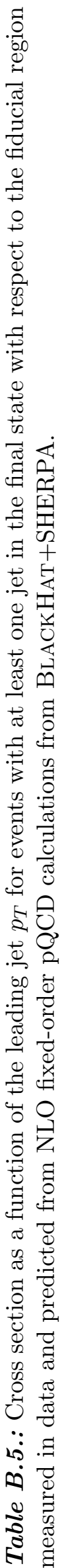




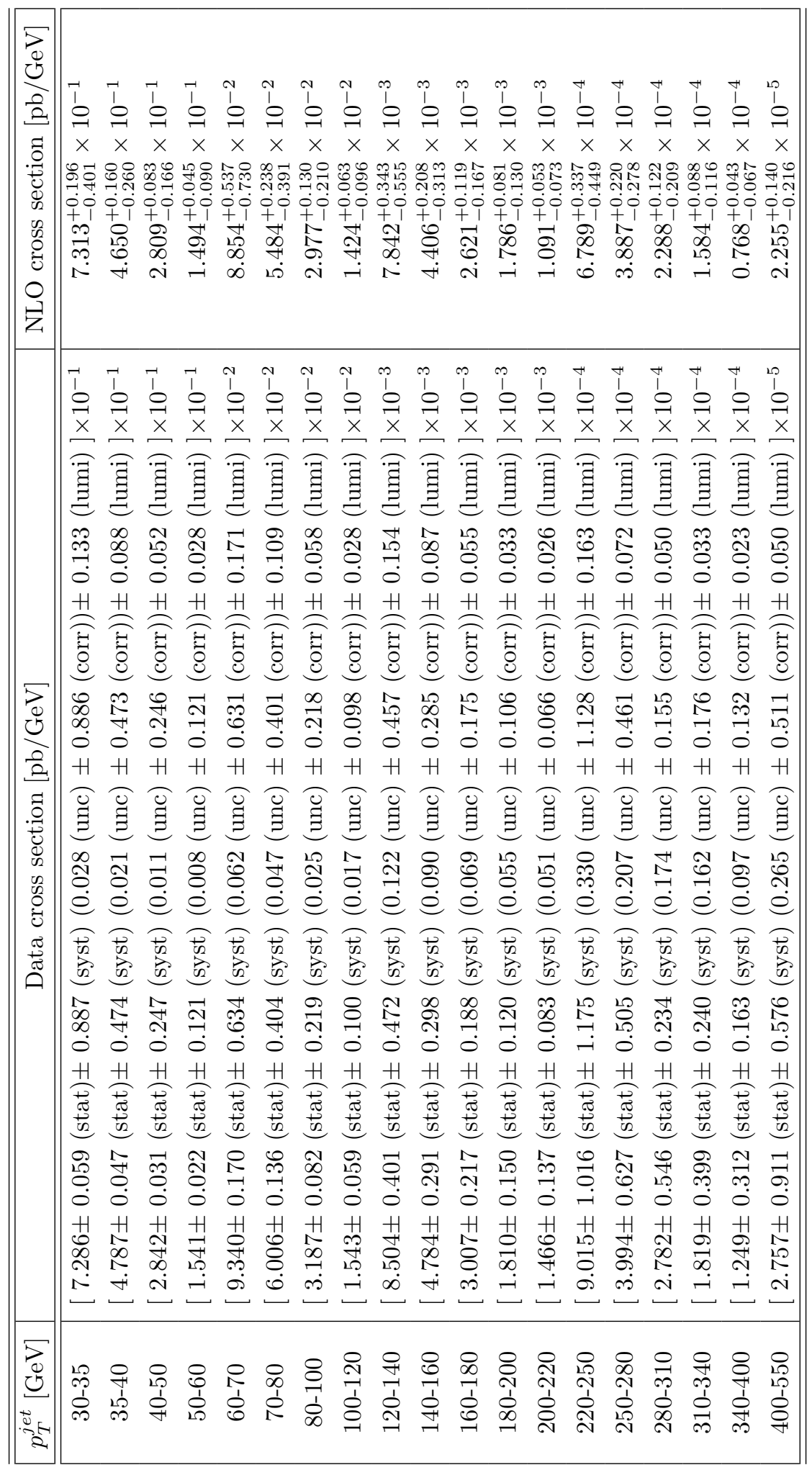



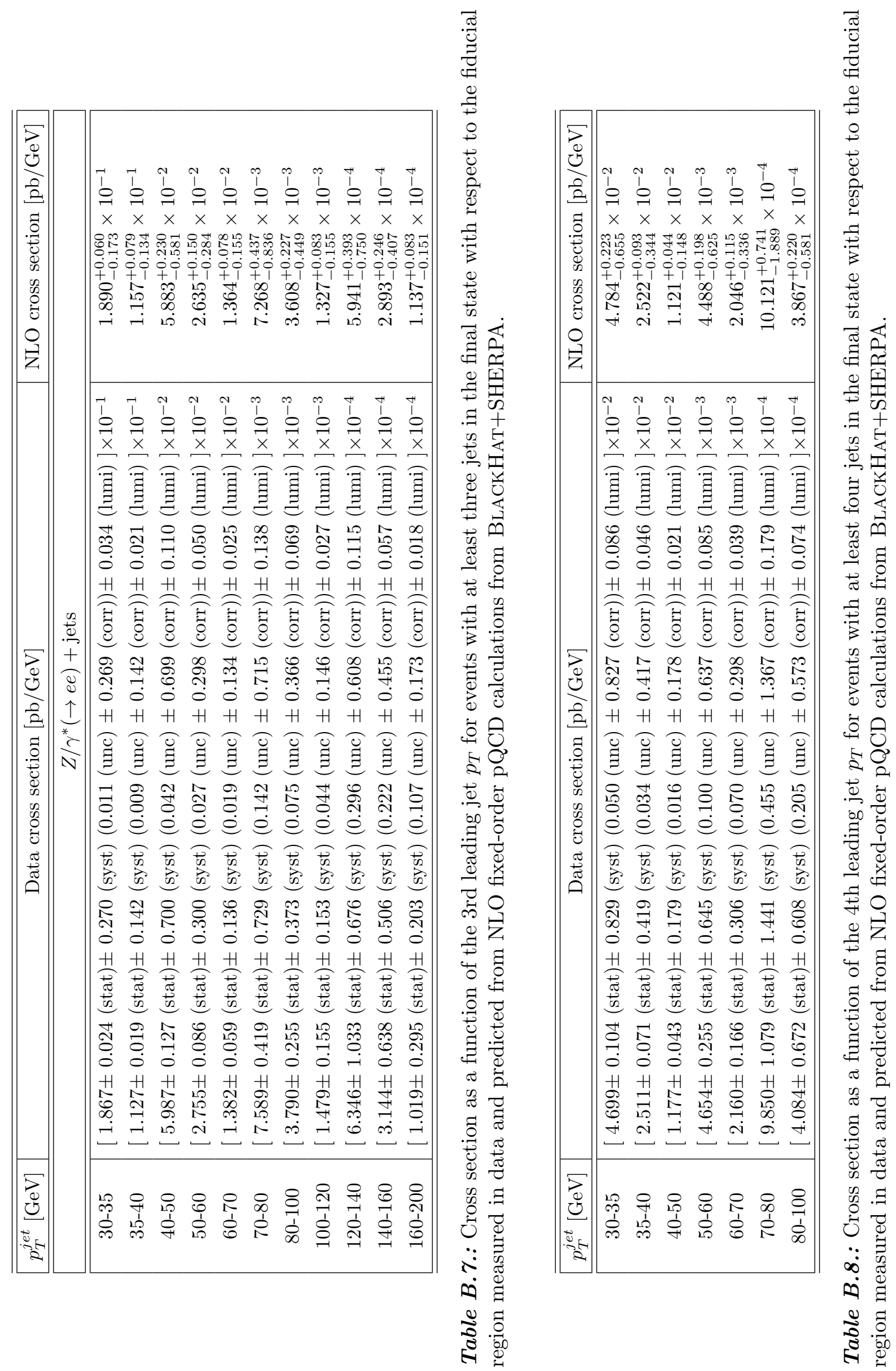


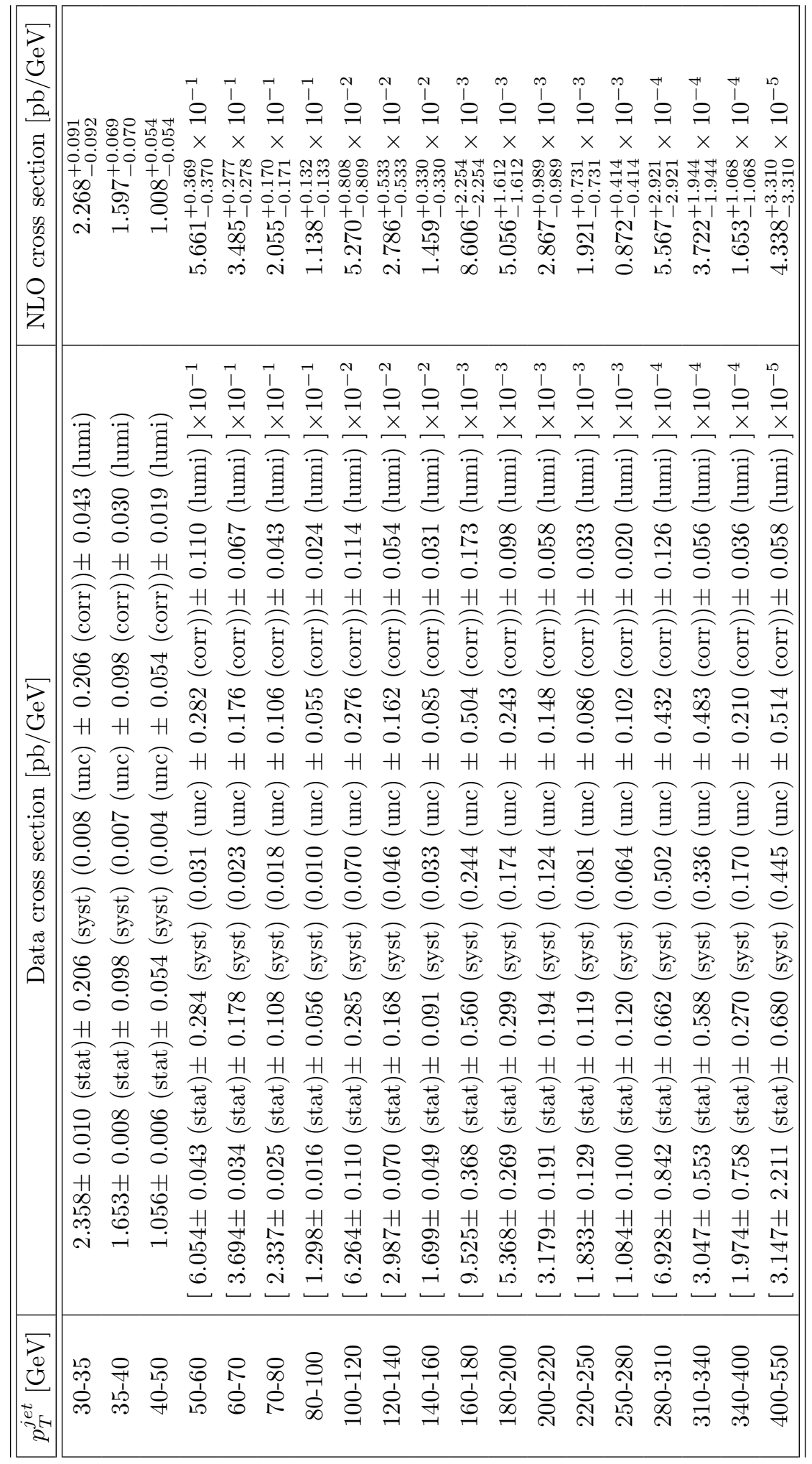




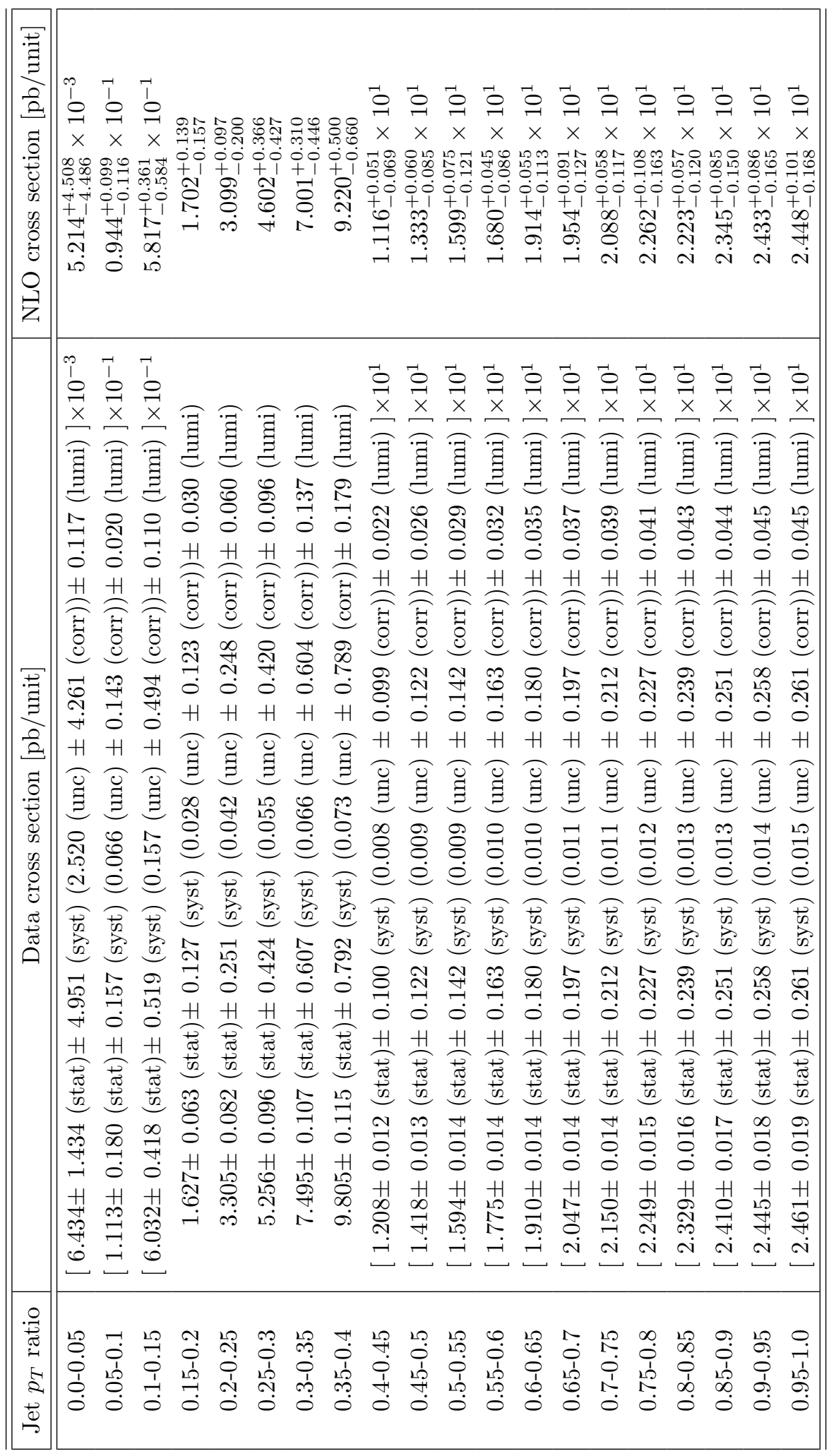

돈

$\stackrel{0}{\pi}$

茎

吾焉

.

告焉

30

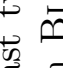

$\underset{\Xi}{\Xi}$

*

돈

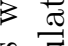

恣

ช

융

in

.

임

ఫ

党

30

云

फे है

.0

苂

E.

$+\overrightarrow{2}$

$\stackrel{\square}{\square}$

ฮี

峲粂

శี

$\because 7$

导

$\approx$ \%

శี छ

.둥

$\mathbb{D}_{\infty}$

की

రి

$\ddot{0} \frac{9}{\dagger}$

คi

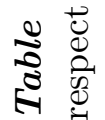




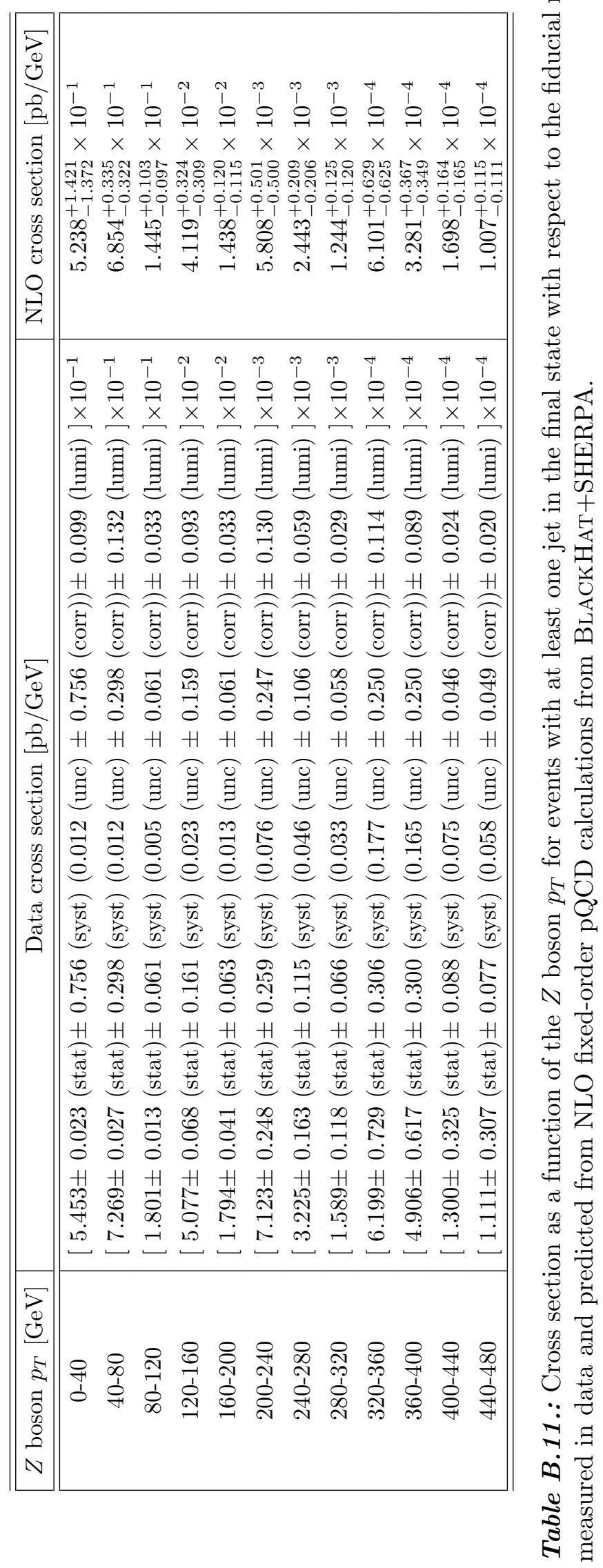



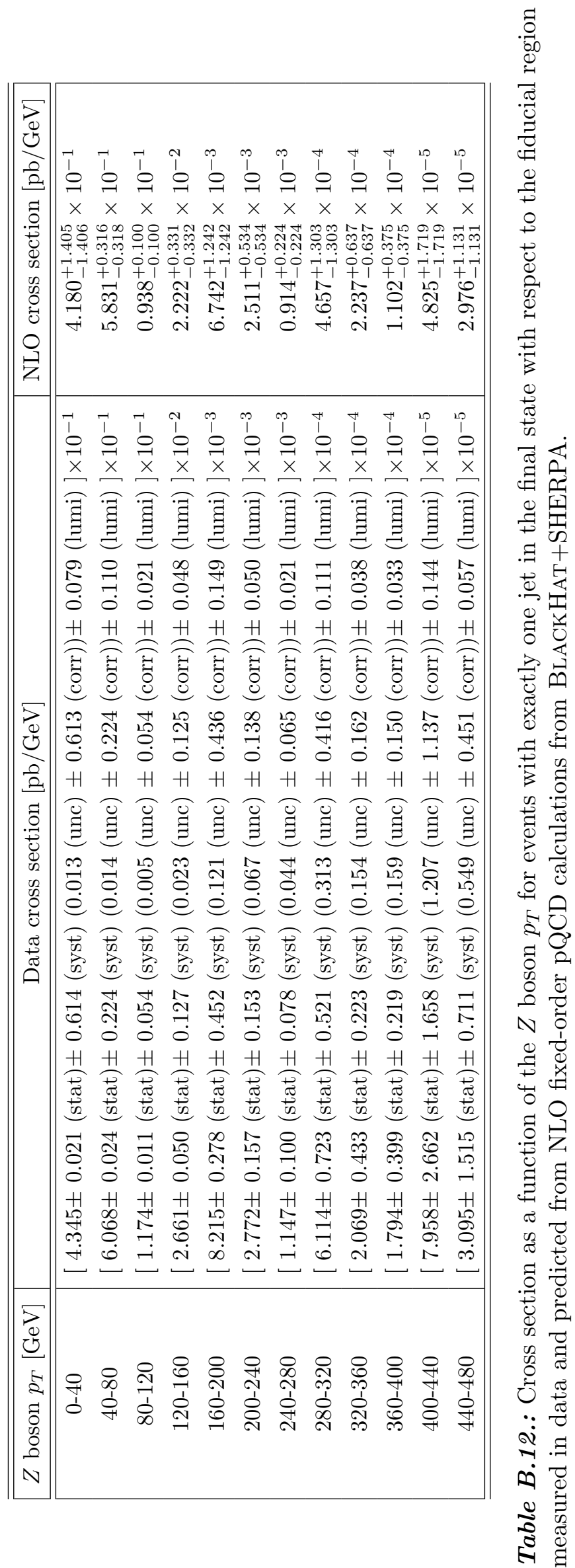


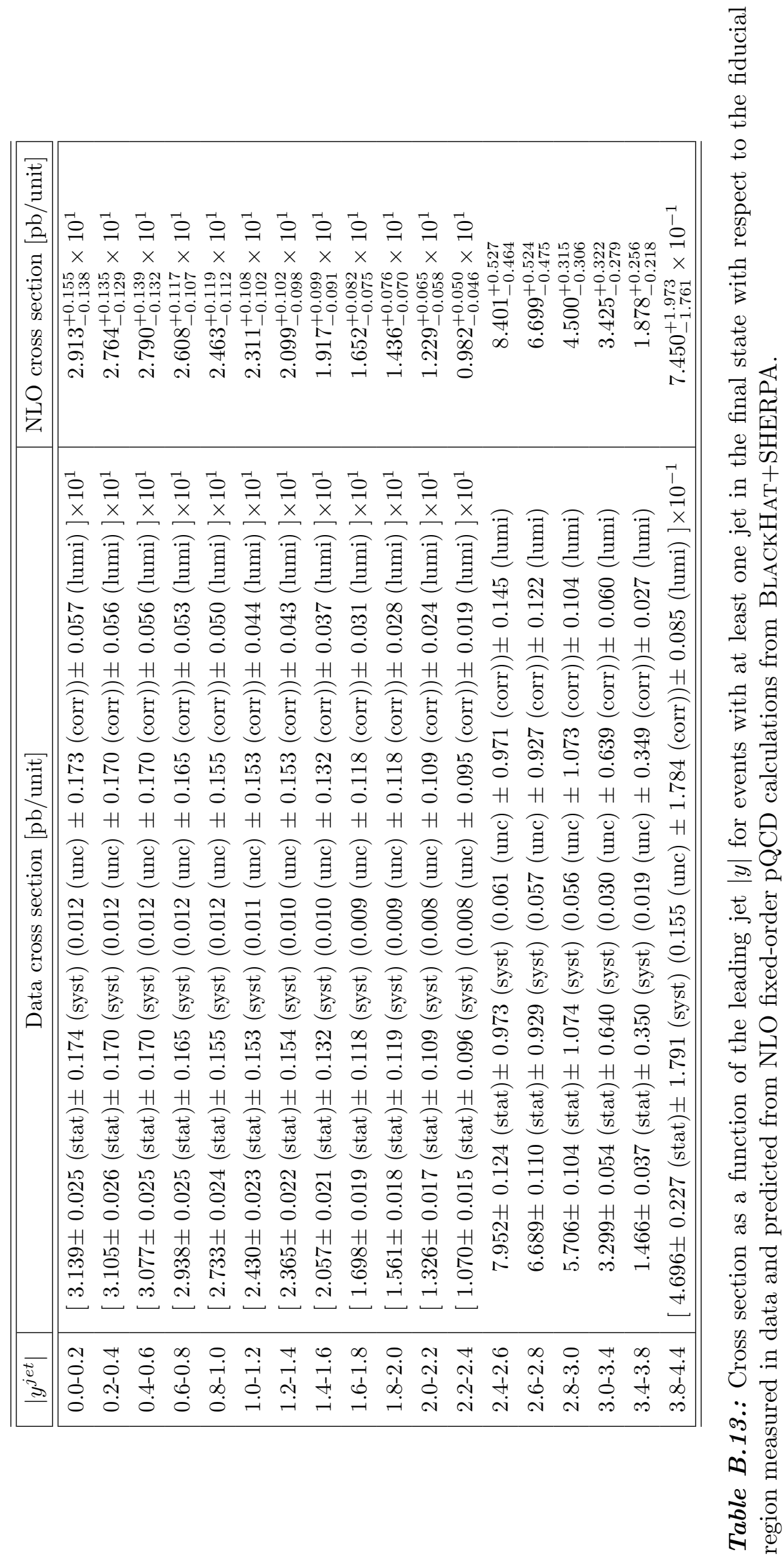




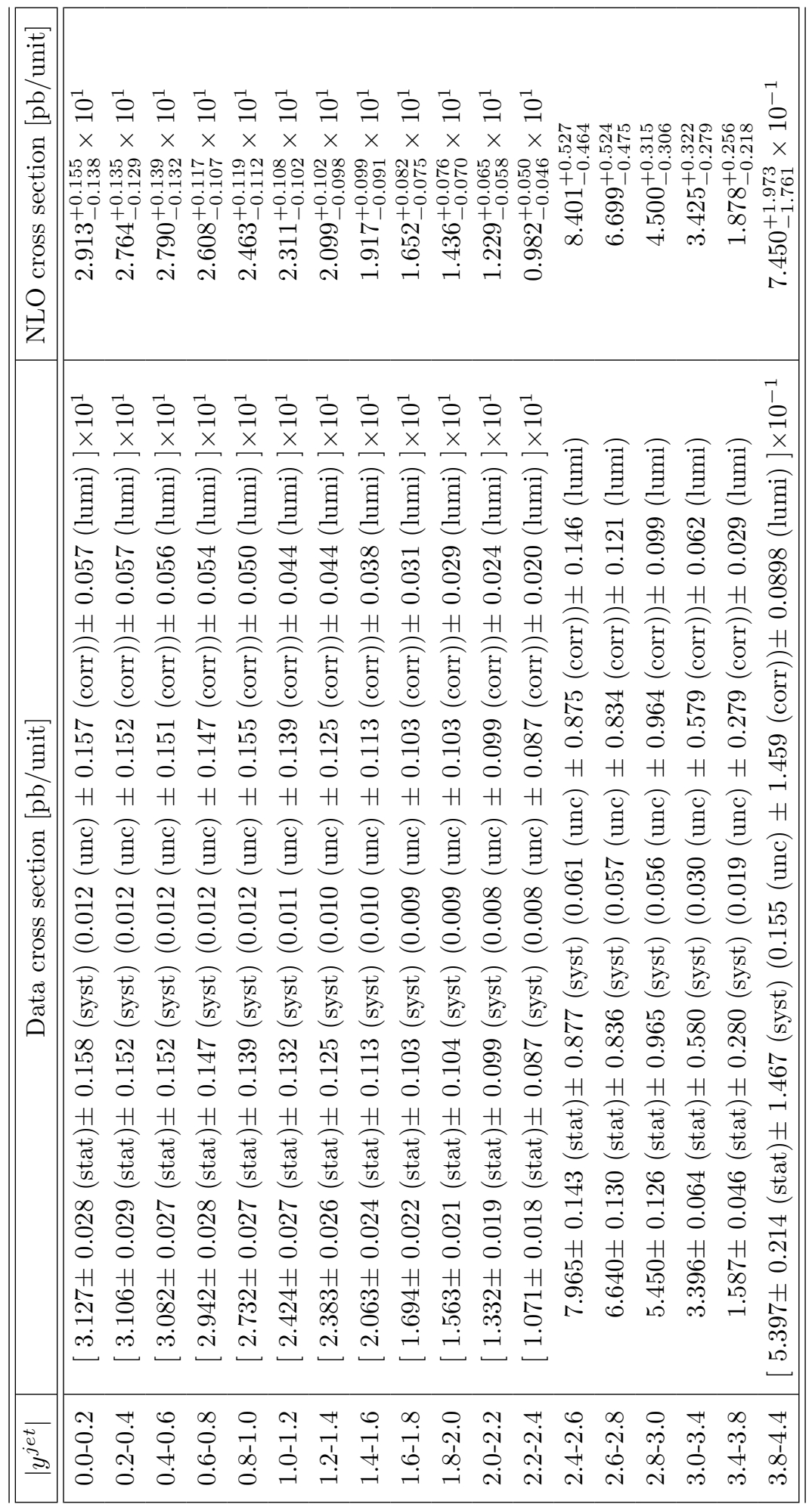

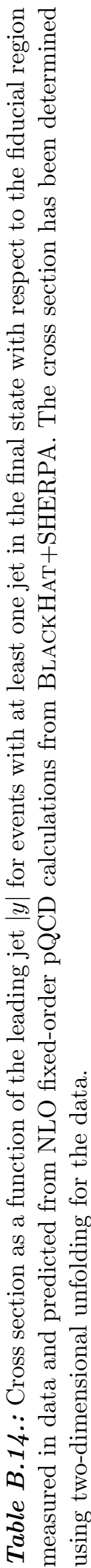




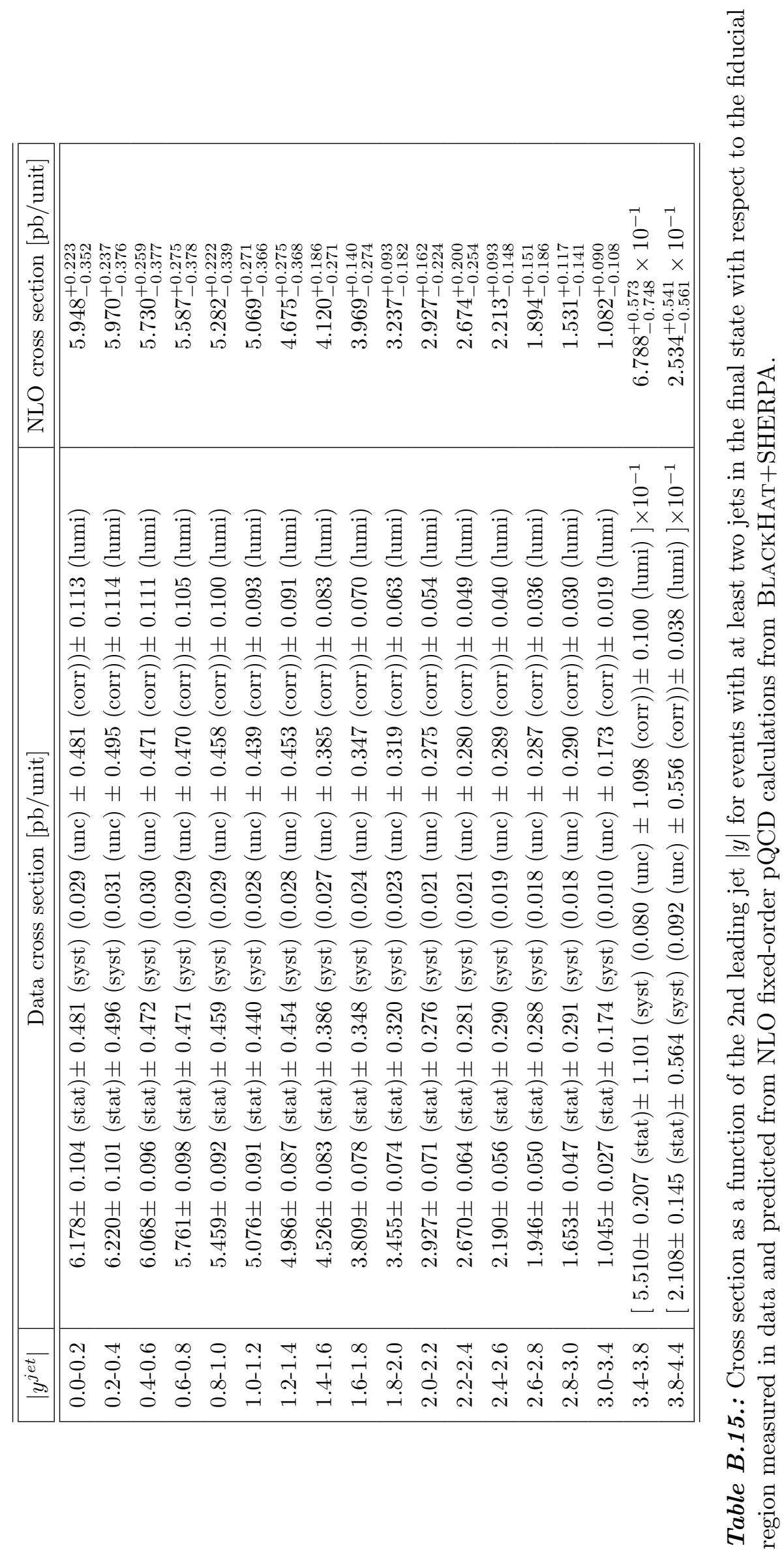



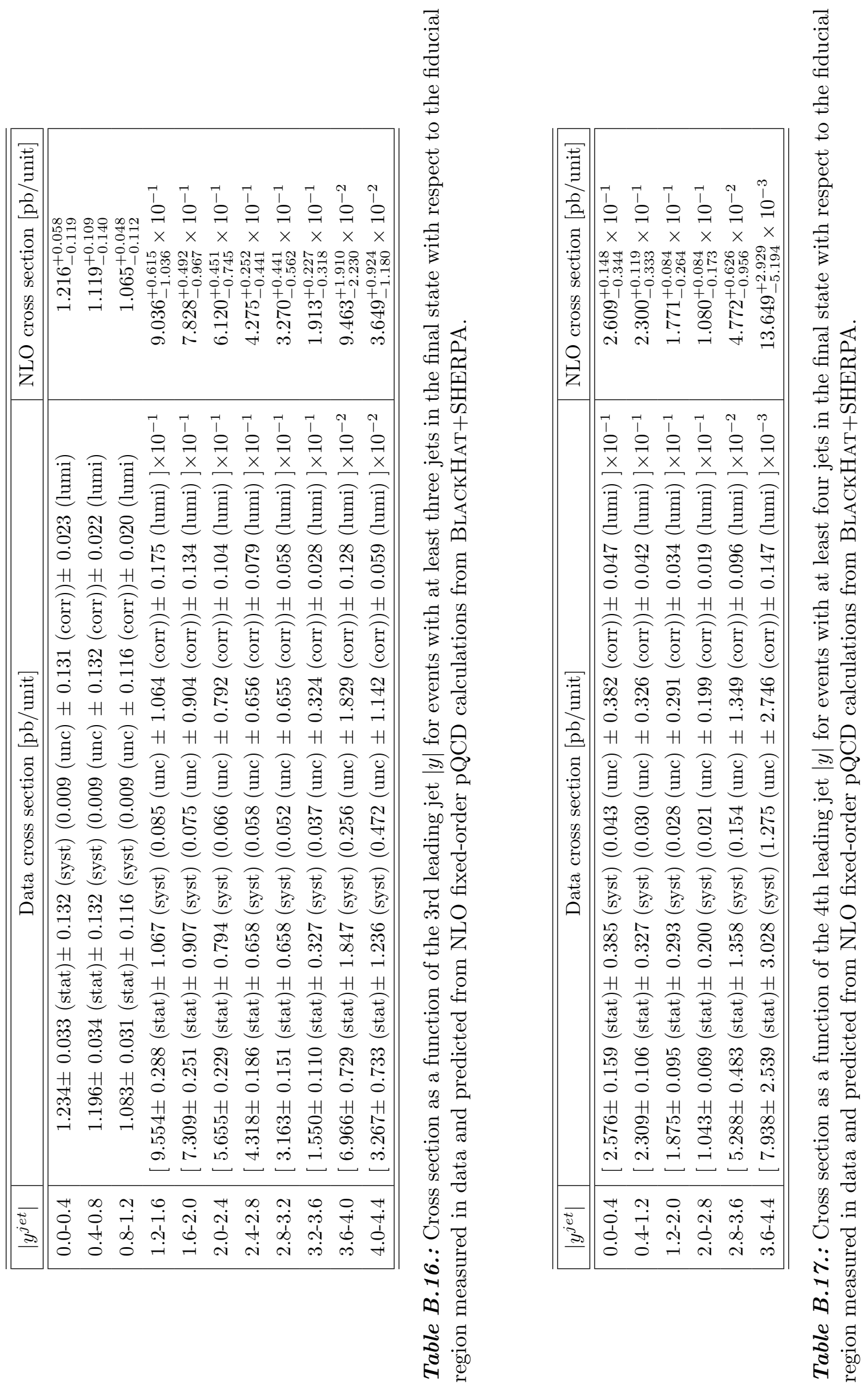

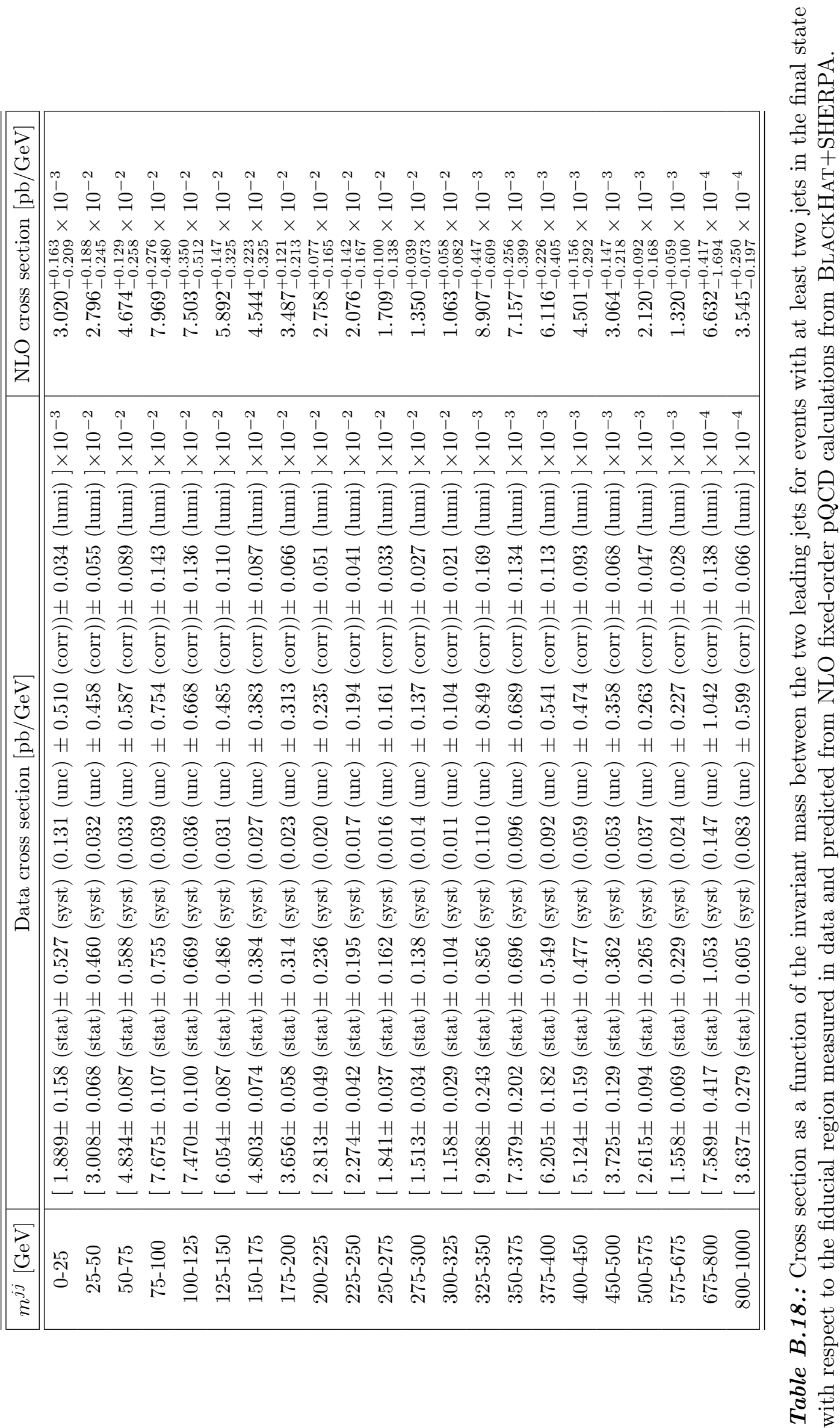


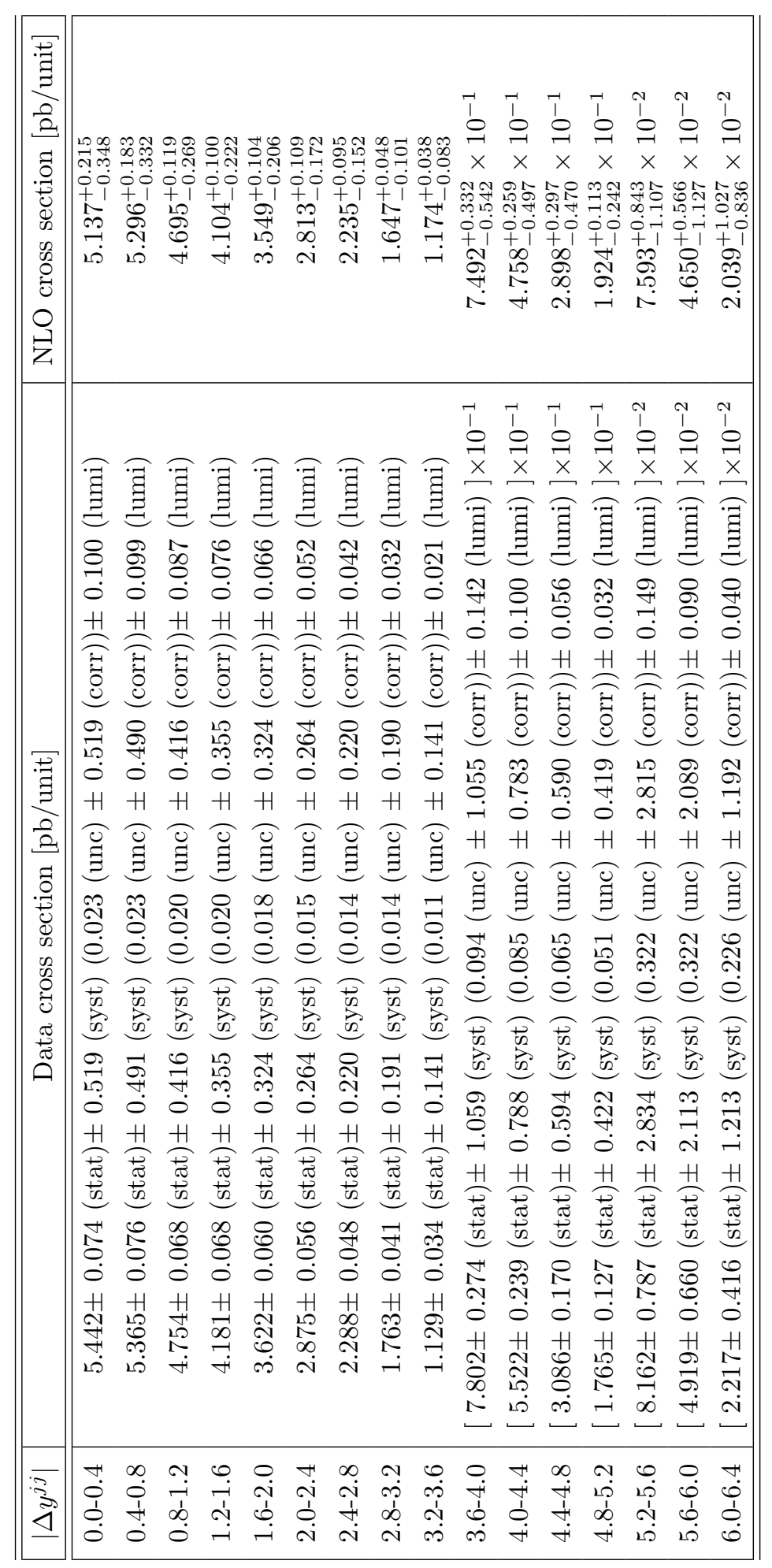

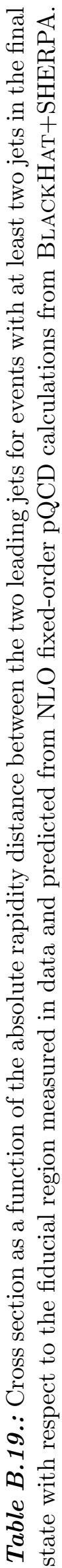




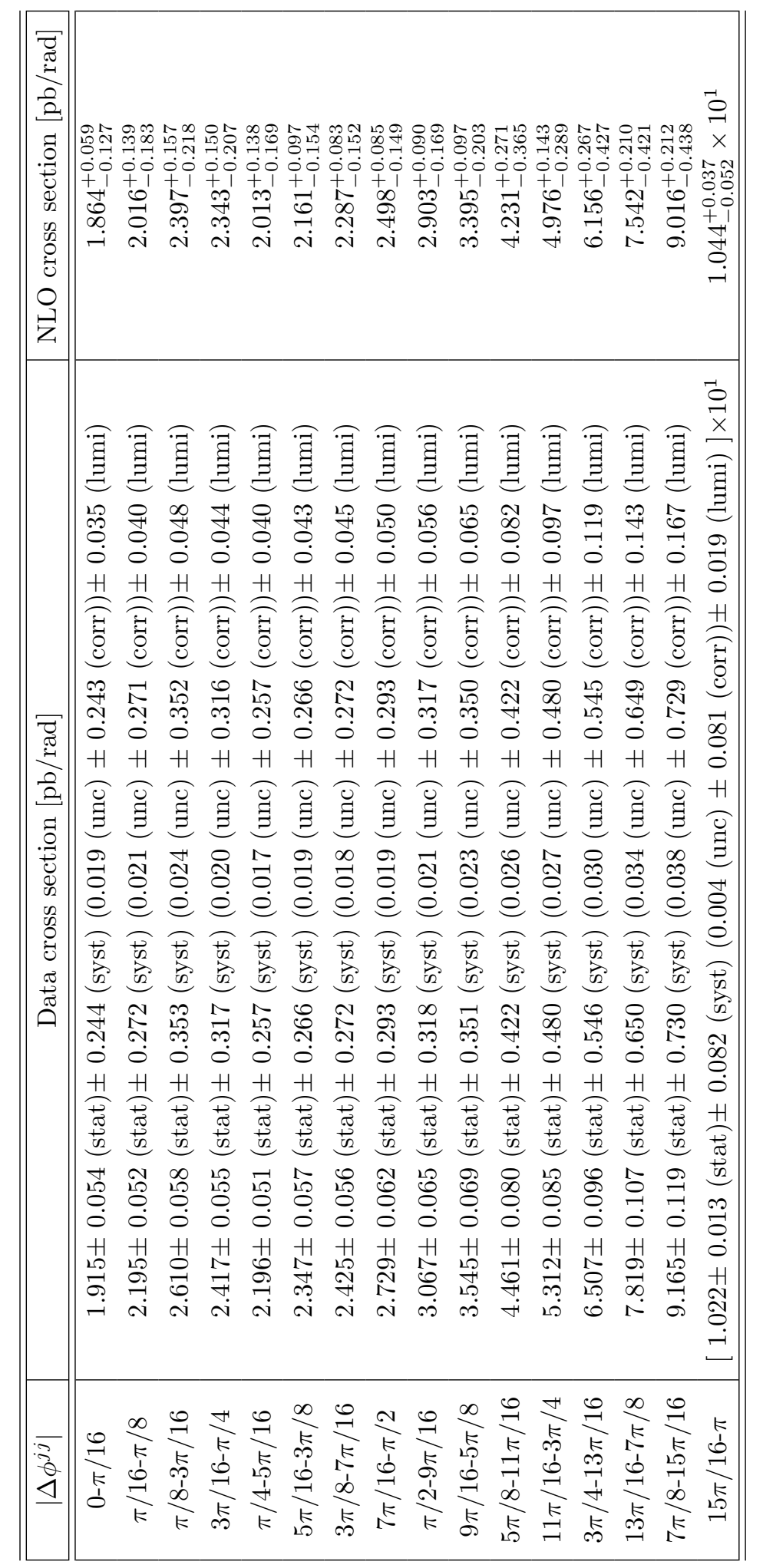

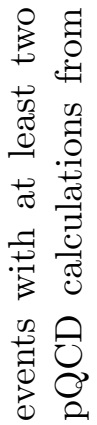

宅

0
.0
0
0
0

$\Leftrightarrow$ 乐

急是

ह

g

:

造

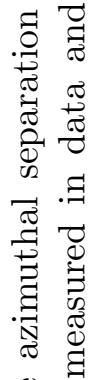

总

ते

욤

눙

규욤

$\because$

$\approx \stackrel{0}{0}$

तै है

.

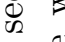

品要

주록

จุ

ต

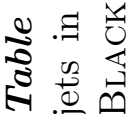




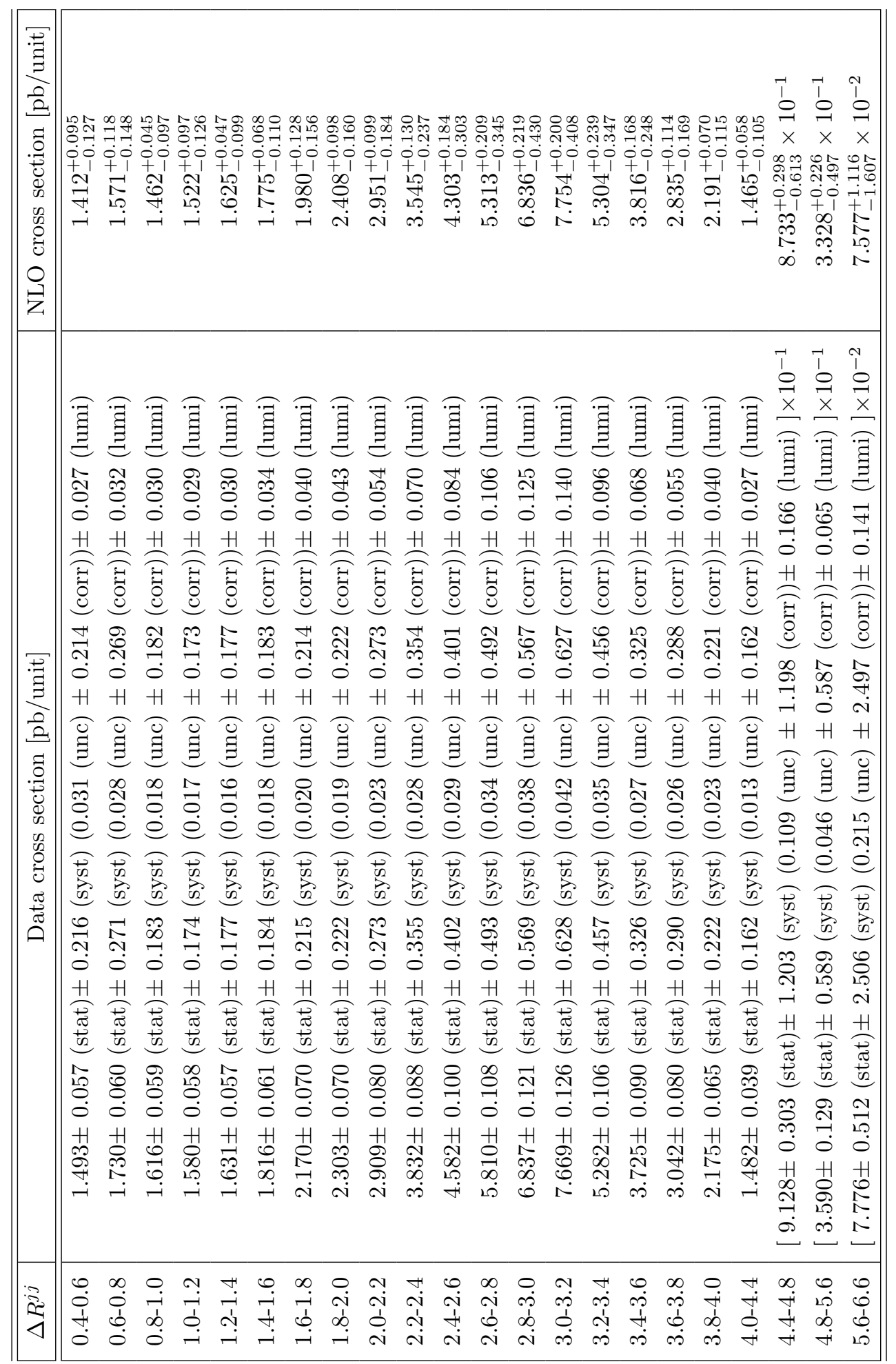

है

羟

.

๘

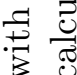

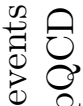

$\dot{0} \dot{0}$

合

-

$\exists$

范

3

ह

$\underset{乛}{ \pm}$

త్ర

:

通

- $\frac{\pi}{\pi}$

$\frac{9}{1} \frac{\pi}{0}$

$. \Xi . \Xi$

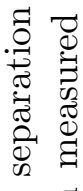

寄 .0

50

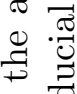

范

도 옴

苞 웅

$\exists$

(1)

के

.

क 0

递要

元

ลे

ข้

․․

要. 


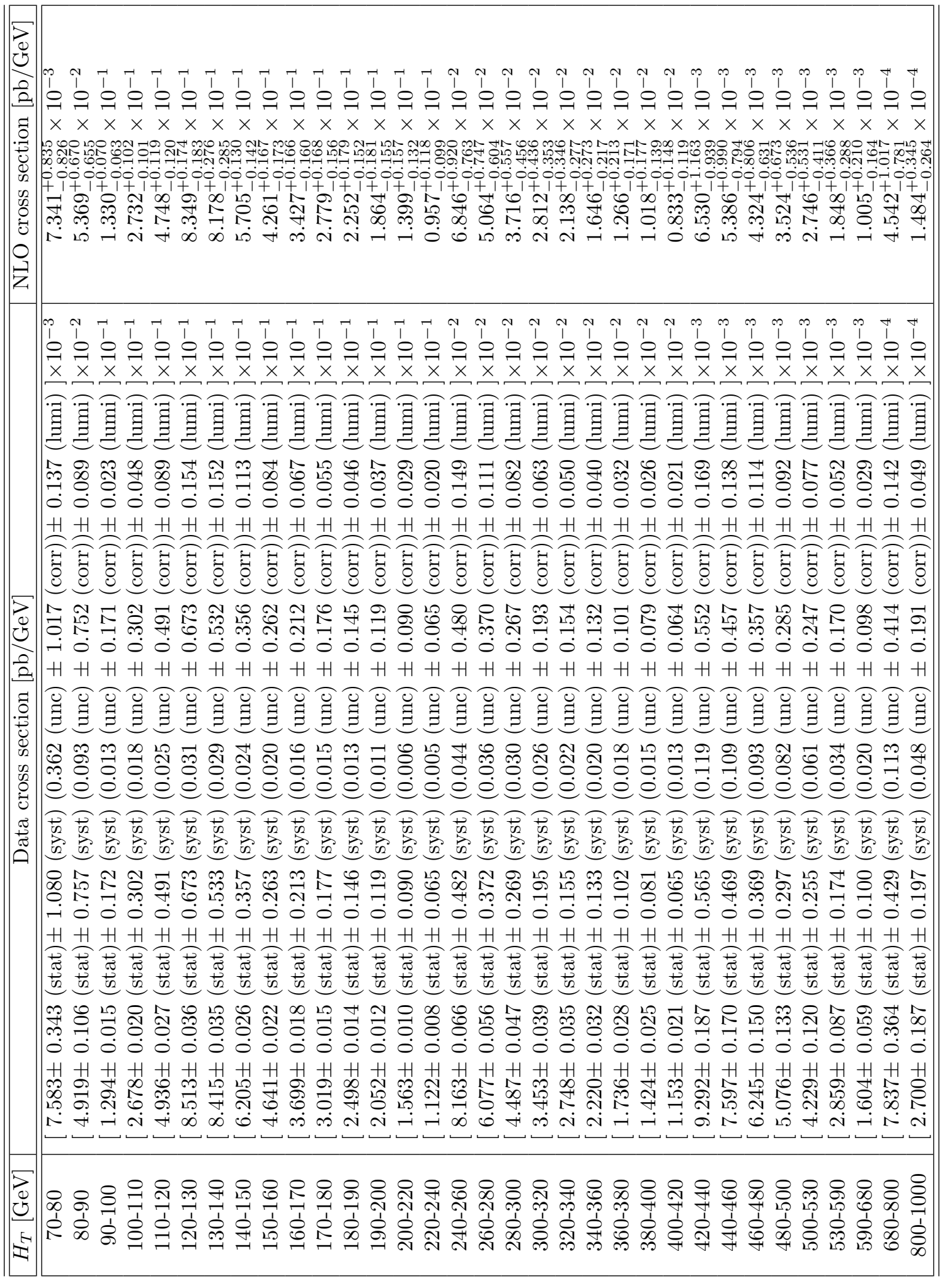




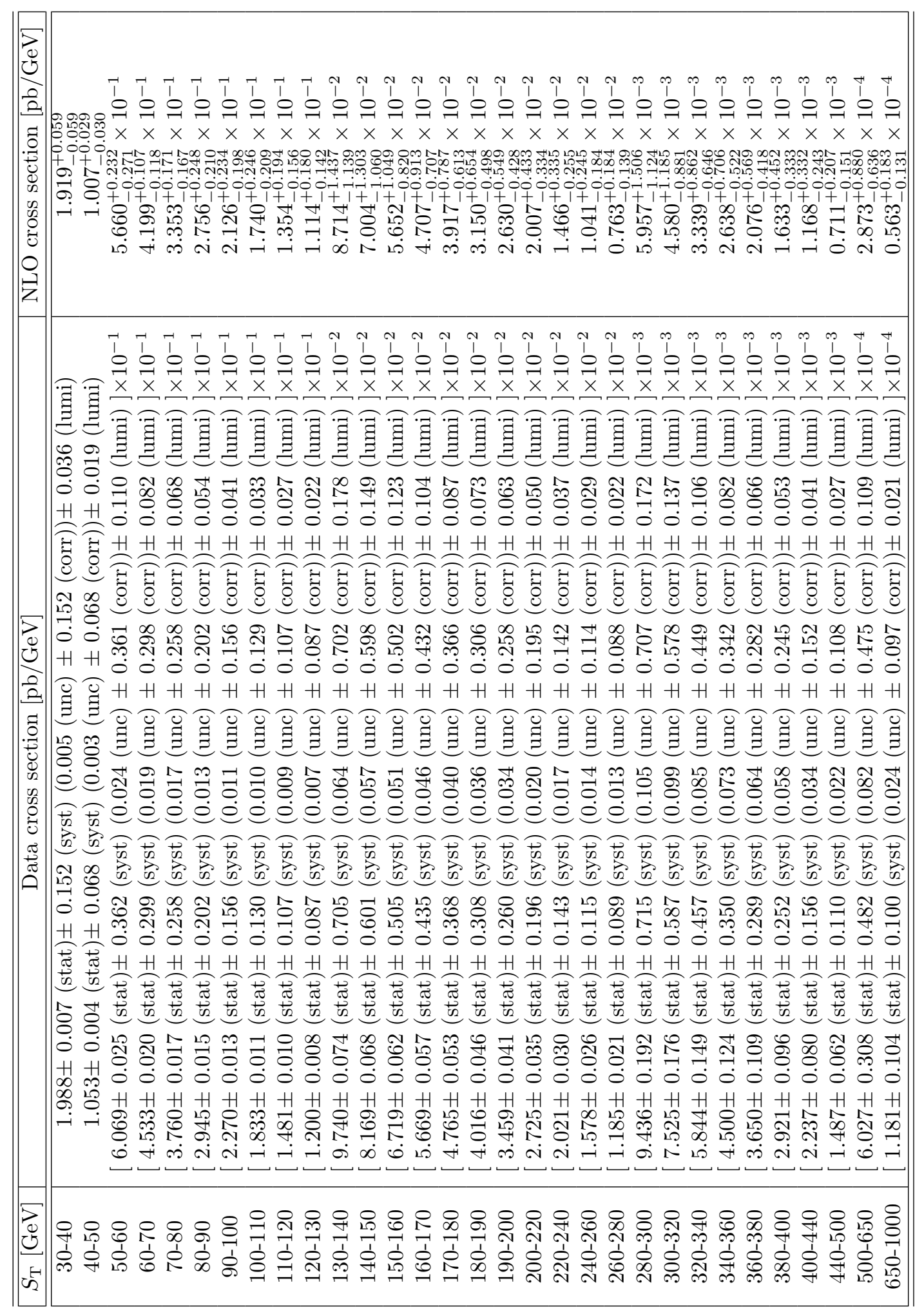




\section{Higgs Boson Production via Weak Boson Fusion}

\section{C.1. Cross Section Tables}

\begin{tabular}{|c|c|c|}
\hline \hline$N_{\text {jet }}$ & Data Cross Section $(\mathrm{pb})$ & NLO Cross Section $(\mathrm{pb})$ \\
\hline \hline 2 jets & {$[5.554 \pm 0.138$ (stat) \pm 0.892 (syst) \pm 0.101 (lumi) $] \times 10^{-1}$} & $4.625_{-0.735}^{+0.747} \times 10^{-1}$ \\
3 jets & {$[1.640 \pm 0.080$ (stat) \pm 0.271 (syst) \pm 0.030 (lumi) $] \times 10^{-1}$} & $1.649_{-0.459}^{+0.459} \times 10^{-1}$ \\
4 jets & {$[3.038 \pm 0.331$ (stat) \pm 0.703 (syst) \pm 0.056 (lumi) $] \times 10^{-2}$} & $3.627_{-1.983}^{+1.985} \times 10^{-2}$ \\
5 jets & {$[7.301 \pm 18.429$ (stat) \pm 1.652 (syst) \pm 0.134 (lumi) $] \times 10^{-3}$} & - \\
6 jets & {$[1.781 \pm 38.218$ (stat) \pm 0.662 (syst) \pm 0.032 (lumi) $] \times 10^{-3}$} & - \\
\hline \hline
\end{tabular}

Table C.1.: Cross sections as a function of the exclusive jet multiplicities after WBF preselection with respect to the fiducial region measured in data and predicted from NLO fixed-order pQCD calculations from BLACKHAT+SHERPA.

\begin{tabular}{|c|c|c|}
\hline \hline$N_{\text {jet }}+1 / N_{\text {jet }}$ & Data cross section ratio & NLO cross section ratio \\
\hline \hline 3 jets $/ 2$ jets & {$[2.953 \pm 0.162$ (stat) \pm 0.146 (syst) $] \times 10^{-1}$} & $3.565_{-1.332}^{+1.828} \times 10^{-1}$ \\
4 jets $/ 3$ jets & {$[1.852 \pm 0.221$ (stat) \pm 0.169 (syst) $] \times 10^{-1}$} & $2.200_{-1.456}^{+2.499} \times 10^{-1}$ \\
5 jets $/ 4$ jets & {$[2.403 \pm 6.070$ (stat) \pm 0.284 (syst) $] \times 10^{-1}$} & - \\
6 jets $/ 5$ jets & {$[2.439 \pm 52.696$ (stat) \pm 0.708 (syst) $] \times 10^{-1}$} & - \\
\hline \hline
\end{tabular}

Table C.2.: Ratio of cross sections for successive exclusive jet multiplicities after WBF preselection with respect to the fiducial region measured in data and predicted from NLO fixed-order pQCD calculations from BLACKHAT+Sherpa. 

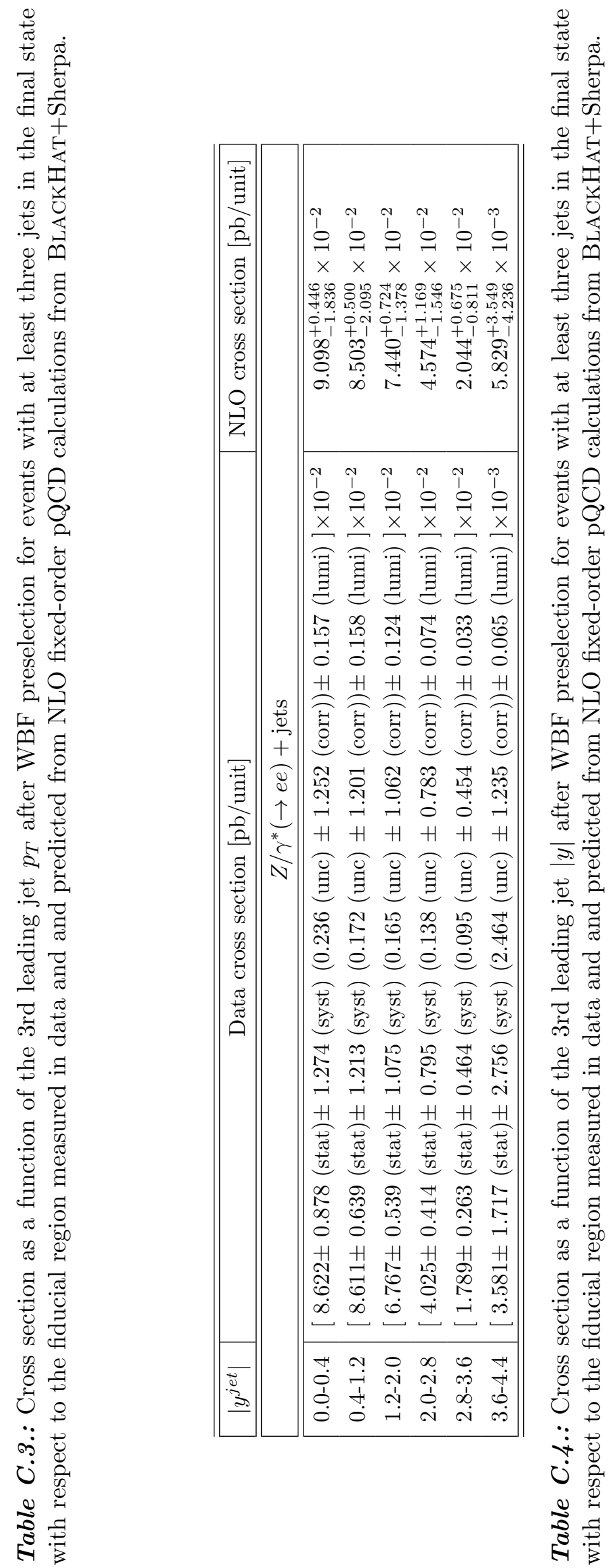


\section{Bibliography}

[1] ATLAS Collaboration, Observation of a new particle in the search for the Standard Model Higgs boson with the ATLAS detector at the LHC, Phys.Lett.B (2012), arXiv:1207.7214 [hep-ex].

[2] CMS Collaboration, Observation of a new boson at a mass of $125 \mathrm{GeV}$ with the CMS experiment at the LHC, Phys.Lett.B (2012), arXiv:1207.7235 [hep-ex].

[3] CDF Collaboration, T. Aaltonen et al., Measurement of inclusive jet cross-sections in $Z / \gamma^{*}\left(\rightarrow e^{+} e^{-)}+\right.$jets production in $p \bar{p}$ collisions at $\sqrt{s}=1.96 \mathrm{TeV}$, Phys.Rev.Lett. 100 (2008) 102001, arXiv:0711.3717 [hep-ex].

[4] D0 Collaboration, V. M. Abazov et al., Measurement of $Z / \gamma^{*}+j e t+X$ angular distributions in $p$ anti-p collisions at $\sqrt{s}=1.96$ TeV, Phys.Lett. B682 (2010) 370-380, arXiv:0907.4286 [hep-ex].

[5] CMS Collaboration, Jet Production Rates in Association with $W$ and $Z$ Bosons in pp Collisions at $\sqrt{s}=7 \mathrm{TeV}$, JHEP 1201 (2012) 010, arXiv:1110.3226 [hep-ex].

[6] ATLAS Collaboration, Measurement of the production cross section for $Z / \gamma^{*}$ in association with jets in $p p$ collisions at $\sqrt{s}=7$ TeV with the ATLAS Detector, ATLAS-CONF-2011-042 (2011).

[7] ATLAS Collaboration, Measurement of the production cross section for $Z / \gamma^{*}$ in association with jets in pp collisions at $\sqrt{s}=7$ TeV with the ATLAS detector, Phys.Rev. D85 (2012) 032009, arXiv:1111.2690 [hep-ex].

[8] ATLAS Collaboration, Measurement of the production cross section of jets in association with a $Z$ boson in pp collisions at $\sqrt{s}=7$ TeV with the ATLAS detector, submitted to JHEP (2013), arXiv:1304.7098 [hep-ex].

[9] M. Rubin, G. P. Salam, and S. Sapeta, Giant QCD K-factors beyond NLO, JHEP 1009 (2010) 084, arXiv:1006.2144 [hep-ph].

[10] E. Gerwick, T. Plehn, S. Schumann, and P. Schichtel, Scaling Patterns for QCD Jets, JHEP 1210 (2012) 162, arXiv:1208.3676 [hep-ph].

[11] M. L. Mangano et al., ALPGEN, a generator for hard multiparton processes in hadronic collisions, JHEP 07 (2003) 001, arXiv:hep-ph/0206293.

[12] T. Gleisberg et al., Event generation with SHERPA 1.1, JHEP 0902 (2009) 007, arXiv:0811.4622 [hep-ph]. 
[13] S. Hoeche, F. Krauss, M. Schoenherr, and F. Siegert, QCD matrix elements + parton showers: The NLO case, JHEP 1304 (2013) 027, arXiv:1207.5030 [hep-ph].

[14] C. Berger, Z. Bern, L. Dixon, F. Febres Cordero, D. Forde, et al., An Automated Implementation of On-Shell Methods for One-Loop Amplitudes, Phys.Rev. D78 (2008) 036003, arXiv:0803.4180 [hep-ph].

[15] C. Berger, Z. Bern, L. J. Dixon, F. Febres Cordero, D. Forde, et al., Next-to-Leading Order QCD Predictions for Z,gamma* +3-Jet Distributions at the Tevatron, Phys.Rev. D82 (2010) 074002, arXiv:1004.1659 [hep-ph].

[16] H. Ita, Z. Bern, L. Dixon, F. Febres Cordero, D. Kosower, et al., Precise Predictions for $Z+4$ Jets at Hadron Colliders, Phys.Rev. D85 (2012) 031501, arXiv:1108.2229 [hep-ph].

[17] A. Salam, Weak and Electromagnetic Interactions, Conf.Proc. C680519 (1968) $367-377$.

[18] S. Glashow, Partial Symmetries of Weak Interactions, Nucl.Phys. 22 (1961) 579-588.

[19] S. Weinberg, A Model of Leptons, Phys.Rev.Lett. 19 (1967) 1264-1266.

[20] D. Gross and F. Wilczek, Asymptotically Free Gauge Theories. 1, Phys.Rev. D8 (1973) 3633-3652.

[21] H. D. Politzer, Asymptotic Freedom: An Approach to Strong Interactions, Phys.Rept. 14 (1974) 129-180.

[22] J. Beringer et al., Review of Particle Physics, Phys. Rev. D 86 (2012) 010001.

[23] The ALEPH, DELPHI, L3, OPAL, SLD Collaborations, the LEP Electroweak Working Group, the SLD Electroweak and Heavy Flavour Groups, Precision Electroweak Measurements on the Z Resonance, Phys. Rept. 427 (2006) 257, hep-ex/0509008.

[24] C. Bennett, D. Larson, J. Weiland, N. Jarosik, G. Hinshaw, et al., Nine-Year Wilkinson Microwave Anisotropy Probe (WMAP) Observations: Final Maps and Results, arXiv:1212.5225 [astro-ph.C0].

[25] Planck Collaboration, P. Ade et al., Planck 2013 results. I. Overview of products and scientific results, arXiv:1303.5062 [astro-ph.CO].

[26] J. Davis, Raymond, D. S. Harmer, and K. C. Hoffman, Search for neutrinos from the sun, Phys.Rev.Lett. 20 (1968) 1205-1209.

[27] Super-Kamiokande Collaboration, Y. Fukuda et al., Evidence for oscillation of atmospheric neutrinos, Phys.Rev.Lett. 81 (1998) 1562-1567, arXiv:hep-ex/9807003 [hep-ex]. 
[28] SNO Collaboration, Q. Ahmad et al., Measurement of the rate of $\nu_{e}+d \rightarrow p+p+e^{-}$interactions produced by B-8 solar neutrinos at the Sudbury Neutrino Observatory, Phys.Rev.Lett. 87 (2001) 071301, arXiv:nucl-ex/0106015 [nucl-ex].

[29] S. P. Martin, A Supersymmetry primer, arXiv:hep-ph/9709356 [hep-ph].

[30] G. Altarelli, A QCD primer, arXiv:hep-ph/0204179.

[31] F. Englert and R. Brout, Broken Symmetry and the Mass of Gauge Vector Mesons, Phys.Rev.Lett. 13 (1964) 321-323.

[32] P. W. Higgs, Broken symmetries, massless particles and gauge fields, Phys.Lett. 12 (1964) 132-133.

[33] P. W. Higgs, Broken Symmetries and the Masses of Gauge Bosons, Phys.Rev.Lett. 13 (1964) 508-509.

[34] G. Guralnik, C. Hagen, and T. Kibble, Global Conservation Laws and Massless Particles, Phys.Rev.Lett. 13 (1964) 585-587.

[35] P. W. Higgs, Spontaneous Symmetry Breakdown without Massless Bosons, Phys.Rev. 145 (1966) 1156-1163.

[36] T. Kibble, Symmetry breaking in non Abelian gauge theories, Phys.Rev. 155 (1967) 1554-1561.

[37] J. F. Gunion et al., The Higgs hunter's guide, Front.Phys. 80 (2000) 1-448.

[38] M. Dobbs and J. B. Hansen, The HepMC C++ Monte Carlo event record for High Energy Physics, Comput.Phys.Commun. 134 (2001) 41-46.

[39] R. Feynman, Photon-hadron interactions. Addison Wesley, 1973.

[40] R. K. Ellis, H. Georgi, M. Machacek, H. D. Politzer, and G. G. Ross, Perturbation Theory and the Parton Model in QCD, Nucl.Phys. B152 (1979) 285.

[41] J. Pumplin, D. Stump, J. Huston, H. Lai, P. M. Nadolsky, et al., New generation of parton distributions with uncertainties from global QCD analysis, JHEP 0207 (2002) 012, arXiv:hep-ph/0201195 [hep-ph].

[42] A. Martin, W. Stirling, R. Thorne, and G. Watt, Parton distributions for the LHC, Eur.Phys.J. C63 (2009) 189-285, arXiv:0901.0002 [hep-ph].

[43] A. Martin, W. Stirling, R. Thorne, and G. Watt, Uncertainties on $\alpha_{s}$ in global PDF analyses and implications for predicted hadronic cross sections, Eur.Phys.J. C64 (2009) 653-680, arXiv:0905.3531 [hep-ph].

[44] A. Martin, W. Stirling, R. Thorne, and G. Watt, Heavy-quark mass dependence in global PDF analyses and 3- and 4-flavour parton distributions, Eur.Phys.J. C70 (2010) 51-72, arXiv:1007.2624 [hep-ph]. 
[45] A. D. Martin, R. G. Roberts, W. J. Stirling, and R. S. Thorne, Uncertainties of predictions from parton distributions. 1: Experimental errors, Eur. Phys. J. C28 (2003) 455-473, arXiv: hep-ph/0211080.

[46] A. Martin, R. Roberts, W. Stirling, and R. Thorne, Uncertainties of predictions from parton distributions. 2. Theoretical errors, Eur.Phys.J. C35 (2004) 325-348, arXiv:hep-ph/0308087 [hep-ph].

[47] D. Stump et al., Uncertainties of predictions from parton distribution functions. 1. The Lagrange multiplier method, Phys. Rev. D65 (2001) 014012, arXiv:hep-ph/0101051.

[48] J. Pumplin et al., Uncertainties of predictions from parton distribution functions. 2. The Hessian method, Phys. Rev. D65 (2001) 014013, arXiv:hep-ph/0101032.

[49] J. M. Campbell, J. Huston, and W. Stirling, Hard Interactions of Quarks and Gluons: A Primer for LHC Physics, Rept.Prog.Phys. 70 (2007) 89, arXiv:hep-ph/0611148 [hep-ph].

[50] J. C. Collins and D. E. Soper, The Theorems of Perturbative QCD, Ann.Rev.Nucl.Part.Sci. 37 (1987) 383-409.

[51] T. Kinoshita, Mass singularities of Feynman amplitudes, J.Math.Phys. 3 (1962) 650-677.

[52] T. Lee and M. Nauenberg, Degenerate Systems and Mass Singularities, Phys.Rev. 133 (1964) B1549-B1562.

[53] ATLAS Collaboration, Luminosity Determination in pp Collisions at $\sqrt{s}=7$ TeV Using the ATLAS Detector at the LHC, Eur.Phys.J. C71 (2011) 1630, arXiv:1101.2185 [hep-ex].

[54] T. Sjostrand and M. van Zijl, A Multiple Interaction Model for the Event Structure in Hadron Collisions, Phys.Rev. D36 (1987) 2019.

[55] T. Sjostrand and P. Z. Skands, Multiple interactions and the structure of beam remnants, JHEP 0403 (2004) 053, arXiv:hep-ph/0402078 [hep-ph].

[56] ATLAS Collaboration, Underlying event characteristics and their dependence on jet size of charged-particle jet events in pp collisions at $\sqrt{s}=7$ TeV with the ATLAS detector, Phys.Rev. D86 (2012) 072004, arXiv:1208.0563 [hep-ex].

[57] T. Sjostrand et al., Pythia 6.3 physics and manual, arXiv:hep-ph/0308153.

[58] G. Altarelli and G. Parisi, Asymptotic Freedom in Parton Language, Nucl.Phys. B126 (1977) 298.

[59] Y. L. Dokshitzer, Calculation of the Structure Functions for Deep Inelastic Scattering and $e+e-$ Annihilation by Perturbation Theory in Quantum Chromodynamics., Sov.Phys.JETP 46 (1977) 641-653. 
[60] V. Gribov and L. Lipatov, Deep inelastic e p scattering in perturbation theory, Sov.J.Nucl.Phys. 15 (1972) 438-450.

[61] T. Sjostrand, A Model for Initial State Parton Showers, Phys.Lett. B157 (1985) 321.

[62] M. Bengtsson and T. Sjostrand, Coherent Parton Showers Versus Matrix Elements: Implications of PETRA - PEP Data, Phys.Lett. B185 (1987) 435.

[63] M. Bengtsson and T. Sjostrand, A Comparative Study of Coherent and Noncoherent Parton Shower Evolution, Nucl.Phys. B289 (1987) 810.

[64] B. Andersson, G. Gustafson, G. Ingelman, and T. Sjostrand, Parton Fragmentation and String Dynamics, Phys. Rept. 97 (1983) 31-145.

[65] H.-U. Bengtsson and T. Sjostrand, The Lund Monte Carlo for Hadronic Processes: Pythia Version 4.8, Comput. Phys. Commun. 46 (1987) 43.

[66] T. D. Gottschalk, A Realistic Model for $e^{+} e^{-}$Annihilation Including Parton Bremsstrahlung Effects, Nucl.Phys. B214 (1983) 201.

[67] T. D. Gottschalk, An improved description of hadronization in the QCD cluster model for $e^{+} e^{-}$annihilation, Nucl.Phys. B239 (1984) 349.

[68] B. Webber, A QCD Model for Jet Fragmentation Including Soft Gluon Interference, Nucl.Phys. B238 (1984) 492.

[69] T. D. Gottschalk and D. A. Morris, A new model for hadronization and $e^{+} e^{-}$ annihilation, Nucl.Phys. B288 (1987) 729.

[70] UA1 Collaboration, G. Arnison et al., Experimental Observation of Lepton Pairs of Invariant Mass Around 95-GeV/ $\mathrm{c}^{2}$ at the CERN SPS Collider, Phys.Lett. B126 (1983) 398-410.

[71] C. Anastasiou, L. J. Dixon, K. Melnikov, and F. Petriello, High precision QCD at hadron colliders: Electroweak gauge boson rapidity distributions at NNLO, Phys.Rev. D69 (2004) 094008, arXiv:hep-ph/0312266 [hep-ph].

[72] R. Gavin, Y. Li, F. Petriello, and S. Quackenbush, FEWZ 2.0: A code for hadronic $Z$ production at next-to-next-to-leading order, Comput.Phys.Commun. 182 (2011) 2388-2403, arXiv:1011.3540 [hep-ph].

[73] R. Gavin, Y. Li, F. Petriello, and S. Quackenbush, W Physics at the LHC with FEWZ 2.1, arXiv:1201.5896 [hep-ph].

[74] ATLAS Collaboration, Measurement of the inclusive $W^{ \pm}$and $Z /$ gamma cross sections in the electron and muon decay channels in pp collisions at $\sqrt{s}=7 \mathrm{TeV}$ with the ATLAS detector, Phys.Rev. D85 (2012) 072004, arXiv:1109.5141 [hep-ex]. 
[75] A. Denner, S. Dittmaier, T. Kasprzik, and A. Mueck, Electroweak corrections to dilepton + jet production at hadron colliders, JHEP 1106 (2011) 069. arXiv:1103.0914 [hep-ph]. Relative electroweak corrections for the transverse momentum of the $\mathrm{Z}$ boson come from private communication.

[76] V. Sudakov, Vertex parts at very high-energies in quantum electrodynamics, Sov.Phys.JETP 3 (1956) 65-71.

[77] S. Ellis, R. Kleiss, and W. J. Stirling, W's, Z's and Jets, Phys.Lett. B154 (1985) 435.

[78] F. A. Berends et al., Multi-jet production in $W, Z$ events at p $\bar{p}$ colliders, Phys.Lett. B224 (1989) 237.

[79] F. A. Berends, H. Kuijf, B. Tausk, and W. Giele, On the production of a $W$ and jets at hadron colliders, Nucl.Phys. B357 (1991) 32-64.

[80] C. Englert, T. Plehn, P. Schichtel, and S. Schumann, Establishing Jet Scaling Patterns with a Photon, JHEP 1202 (2012) 030, arXiv:1108.5473 [hep-ph].

[81] E. Gerwick, T. Plehn, and S. Schumann, Understanding Jet Scaling and Jet Vetos in Higgs Searches, Phys.Rev.Lett. 108 (2012) 032003, arXiv:1108.3335 [hep-ph].

[82] ATLAS Collaboration, Measurement of the cross-section for $b^{-}$jets produced in association with a $Z$ boson at $\sqrt{s}=7$ TeV with the ATLAS detector, Phys.Lett. B706 (2012) 295-313, arXiv:1109.1403 [hep-ex].

[83] A. D. Linde, Dynamical Symmetry Restoration and Constraints on Masses and Coupling Constants in Gauge Theories, JETP Lett. 23 (1976) 64-67.

[84] A. D. Linde, Symmetry Behavior in External Fields, Phys.Lett. B62 (1976) 435.

[85] S. Weinberg, Mass of the Higgs Boson, Phys.Rev.Lett. 36 (1976) 294-296.

[86] N. Cabibbo, L. Maiani, G. Parisi, and R. Petronzio, Bounds on the Fermions and Higgs Boson Masses in Grand Unified Theories, Nucl.Phys. B158 (1979) 295-305.

[87] G. Altarelli and G. Isidori, Lower limit on the Higgs mass in the standard model: An Update, Phys.Lett. B337 (1994) 141-144.

[88] J. Casas, J. Espinosa, and M. Quiros, Improved Higgs mass stability bound in the standard model and implications for supersymmetry, Phys.Lett. B342 (1995) 171-179, arXiv:hep-ph/9409458 [hep-ph].

[89] J. Casas, J. Espinosa, and M. Quiros, Standard model stability bounds for new physics within LHC reach, Phys.Lett. B382 (1996) 374-382, arXiv: hep-ph/9603227 [hep-ph].

[90] T. Hambye and K. Riesselmann, SM Higgs mass bounds from theory, arXiv:hep-ph/9708416 [hep-ph]. 
[91] ALEPH, CDF, D0, DELPHI, L3, OPAL, SLD Collaborations, the LEP Electroweak Working Group, the Tevatron Electroweak Working Group, and the SLD Electroweak and Heavy Flavour Groups, Precision Electroweak Measurements and Constraints on the Standard Model, CERN-PH-EP-2010-095 (2010), arXiv:1012.2367 [hep-ex]. At this time, the latest results come from http://lepewwg.web.cern.ch/LEPEWWG/plots/winter2012/.

[92] LEP Working Group for Higgs boson searches, ALEPH Collaboration, DELPHI Collaboration, L3 Collaboration, OPAL Collaboration, R. Barate et al., Search for the standard model Higgs boson at LEP, Phys.Lett. B565 (2003) 61-75, arXiv:hep-ex/0306033 [hep-ex].

[93] Tevatron New Physics Higgs Working Group, CDF Collaboration, D0 Collaboration, Updated Combination of CDF and DO Searches for Standard Model Higgs Boson Production with up to $10.0 \mathrm{fb}^{-1}$ of Data, arXiv:1207.0449 [hep-ex].

[94] LHC Higgs Cross Section Working Group Collaboration, S. Dittmaier et al., Handbook of LHC Higgs Cross Sections: 1. Inclusive Observables, arXiv:1101.0593 [hep-ph].

[95] ATLAS Collaboration, An update of combined measurements of the new Higgs-like boson with high mass resolution channels, ATLAS-CONF-2012-170 (2012).

[96] CMS Collaboration, Combination of standard model Higgs boson searches and measurements of the properties of the new boson with a mass near $125 \mathrm{GeV}$, CMS-PAS-HIG-12-045 (2012).

[97] L. Evans and P. Bryant, LHC Machine, JINST 3 (2008) S08001.

[98] CMS Collaboration, The CMS experiment at the CERN LHC, JINST 3 no. 08, (2008) S08004.

[99] ALICE Collaboration, The ALICE experiment at the CERN LHC, JINST 3 no. 08, (2008) S08002.

[100] LHCb Collaboration, The LHCb Detector at the LHC, JINST 3 no. 08, (2008) S08005.

[101] ATLAS Collaboration, Improved luminosity determination in pp collisions at $\sqrt{s}$ $=7 \mathrm{TeV}$ using the ATLAS detector at the LHC, arXiv:1302.4393 [hep-ex].

[102] ATLAS Collaboration. https: //twiki.cern.ch/twiki/bin/view/AtlasPublic/LuminosityPublicResults, as of $13^{\text {th }}$ May, 2012.

[103] ATLAS Collaboration, The ATLAS Experiment at the CERN Large Hadron Collider, JINST 3 (2008) S08003.

[104] ATLAS Collaboration, Electron performance measurements with the ATLAS detector using the 2010 LHC proton-proton collision data, Eur.Phys.J. C72 (2012) 1909, arXiv:1110.3174 [hep-ex]. 
[105] ATLAS Electromagnetic Barrel Calorimeter Collaboration, Energy linearity and resolution of the ATLAS electromagnetic barrel calorimeter in an electron test-beam, Nucl.Instrum.Meth. A568 (2006) 601-623, arXiv:physics/0608012 [physics].

[106] ATLAS Collaboration, Testbeam studies of production modules of the ATLAS tile calorimeter, Nucl.Instrum.Meth. A606 (2009) 362-394.

[107] ATLAS Collaboration, ATLAS high-level trigger, data-acquisition and controls: Technical Design Report. Technical Design Report. CERN, Geneva, 2003.

[108] ATLAS Collaboration, ATLAS detector and physics performance: Technical Design Report. Technical Design Report. CERN, Geneva, 1999.

[109] G. Corcella et al., HERWIG 6.5: an event generator for Hadron Emission Reactions With Interfering Gluons (including supersymmetric processes), JHEP 01 (2001) 010, arXiv:hep-ph/0011363.

[110] J. Alwall et al., MadGraph 5 : Going Beyond, JHEP 1106 (2011) 128, arXiv:1106.0522 [hep-ph].

[111] S. Catani et al., QCD matrix elements + parton showers, JHEP 0111 (2001) 063, arXiv:hep-ph/0109231 [hep-ph].

[112] M. L. Mangano, M. Moretti, and R. Pittau, Multijet matrix elements and shower evolution in hadronic collisions: $W b \bar{b}+n$ jets as a case study, Nucl. Phys. B632 (2002) 343-362, arXiv:hep-ph/0108069

[113] F. Caravaglios, M. L. Mangano, M. Moretti, and R. Pittau, A new approach to multi-jet calculations in hadron collisions, Nucl. Phys. B539 (1999) 215-232, arXiv:hep-ph/9807570.

[114] S. Frixione et al., Matching NLO QCD computations and parton shower simulations, JHEP 0206 (2002) 029, arXiv:hep-ph/0204244 [hep-ph].

[115] P. Nason, A New method for combining NLO QCD with shower Monte Carlo algorithms, JHEP 0411 (2004) 040, arXiv: hep-ph/0409146 [hep-ph].

[116] S. Frixione, P. Nason, and C. Oleari, Matching NLO QCD computations with Parton Shower simulations: the POWHEG method, JHEP 0711 (2007) 070, arXiv:0709.2092 [hep-ph], * Temporary entry*.

[117] ATLAS Collaboration, ATLAS computing: Technical design report. Technical Design Report. CERN, Geneva, 2005.

[118] GEANT4 Collaboration, S. Agostinelli et al., GEANT4: A Simulation toolkit, Nucl.Instrum.Meth. A506 (2003) 250-303.

[119] J. Allison, K. Amako, J. Apostolakis, H. Araujo, P. Dubois, et al., Geant4 developments and applications, IEEE Trans.Nucl.Sci. 53 (2006) 270. 
[120] E. Richter-Was, D. Froidevaux, and L. Poggioli, ATLFAST 2.0 a fast simulation package for ATLAS,.

[121] R. Brun and F. Rademakers, ROOT: An object oriented data analysis framework, Nucl.Instrum.Meth. A389 (1997) 81-86.

[122] UA5 Collaboration, G. Alner et al., The UA5 high energy $\bar{p} p$ simulation program, Nucl.Phys. B291 (1987) 445.

[123] J. Butterworth et al., Photoproduction of multi - jet events at HERA: A Monte Carlo simulation, J.Phys. G19 (1993) 1657-1663.

[124] J. Butterworth, J. R. Forshaw, and M. Seymour, Multiparton interactions in photoproduction at HERA, Z.Phys. C72 (1996) 637-646, arXiv:hep-ph/9601371 [hep-ph].

[125] P. Golonka and Z. Was, PHOTOS Monte Carlo: A Precision tool for QED corrections in $Z$ and $W$ decays, Eur.Phys.J. C45 (2006) 97-107, arXiv:hep-ph/0506026 [hep-ph].

[126] S. Schumann and F. Krauss, A Parton shower algorithm based on Catani-Seymour dipole factorisation, JHEP 0803 (2008) 038, arXiv:0709.1027 [hep-ph].

[127] S. Hoeche, F. Krauss, S. Schumann, and F. Siegert, QCD matrix elements and truncated showers, JHEP 0905 (2009) 053, arXiv:0903.1219 [hep-ph].

[128] J.-C. Winter, F. Krauss, and G. Soff, A Modified cluster hadronization model, Eur.Phys.J. C36 (2004) 381-395, arXiv:hep-ph/0311085 [hep-ph].

[129] D. Yennie, S. C. Frautschi, and H. Suura, The infrared divergence phenomena and high-energy processes, Annals Phys. 13 (1961) 379-452.

[130] B. P. Kersevan et al., The Monte Carlo event generator AcerMC version 2.0 with interfaces to PYTHIA 6.2 and HERWIG 6.5, arXiv:hep-ph/0405247 [hep-ph].

[131] G. D'Agostini, A multidimensional unfolding method based on Bayes' theorem, Nucl. Instrum. Meth. A 362 (1995) 487-498.

[132] A. Hoecker and V. Kartvelishvili, SVD approach to data unfolding, Nucl.Instrum.Meth. A372 (1996) 469-481, arXiv:hep-ph/9509307 [hep-ph].

[133] B. Hoffman, Mathematik inverser Probleme. B. G. Teubner Stuttgart - Leipzig, 1999.

[134] T. Adye, Unfolding algorithms and tests using RooUnfold, arXiv:1105.1160 [physics.data-an].

[135] G. D'Agostini, Improved iterative Bayesian unfolding, ArXiv e-prints (2010), arXiv:1010.0632 [physics.data-an].

[136] ATLAS Collaboration, Expected Performance of the ATLAS Experiment Detector, Trigger and Physics, arXiv:0901.0512 [hep-ex]. 
[137] ATLAS Collaboration, Calorimeter clustering algorithms: Description and performance, ATL-LARG-PUB-2008-002 (2008).

[138] ATLAS Collaboration, Expected electron performance in the ATLAS experiment, ATL-PHYS-PUB-2011-006 (2011).

[139] ATLAS Collaboration, Calibrated $Z \rightarrow e^{+} e^{-}$mass with 2011 data, ATL-COM-PHYS-2011-1637 (2011).

[140] CDF Collaboration, D. Acosta et al., First measurements of inclusive $W$ and $Z$ cross sections from Run II of the Tevatron collider, Phys.Rev.Lett. 94 (2005) 091803, arXiv:hep-ex/0406078 [hep-ex].

[141] D0 Collaboration, V. Abazov et al., Measurement of the shape of the boson rapidity distribution for $p \bar{p} \rightarrow Z /$ gamma $^{*} \rightarrow e^{+} e^{-}+X$ events produced at $\sqrt{s}=1.96 \mathrm{TeV}$, Phys.Rev. D76 (2007) 012003, arXiv:hep-ex/0702025 [hep-ex].

[142] ATLAS Collaboration, Identification efficiency measurement for electrons with transverse energy between 7 and 50 GeV, ATL-COM-PHYS-2011-1669 (2011).

[143] M. Cacciari, G. P. Salam, and G. Soyez, The Anti-k(t) jet clustering algorithm, JHEP 0804 (2008) 063, arXiv:0802.1189 [hep-ph].

[144] ATLAS Liquid Argon EMEC/HEC Collaboration, Hadronic calibration of the ATLAS liquid argon end-cap calorimeter in the pseudorapidity region $1.6<|\eta|<1.8$ in beam tests, Nucl.Instrum.Meth. A531 (2004) 481-514, arXiv:physics/0407009 [physics].

[145] ATLAS Collaboration, Jet energy scale and its systematic uncertainty in proton-proton collisions at $\sqrt{s}=7$ TeV with ATLAS 2011 data, ATLAS-CONF-2013-004 (2013).

[146] ATLAS Collaboration, G. Aad et al., Expected Performance of the ATLAS Experiment - Detector, Trigger and Physics, arXiv:0901.0512 [hep-ex].

[147] ATLAS Collaboration, Jet energy measurement with the ATLAS detector in proton-proton collisions at $\sqrt{s}=7 \mathrm{TeV}$, Eur.Phys.J. C73 (2013) 2304, arXiv:1112.6426 [hep-ex]

[148] ATLAS Collaboration, Probing the measurement of jet energies with the ATLAS detector using $Z+$ jet events from proton-proton collisions at $\sqrt{s}=7 \mathrm{TeV}$, ATLAS-CONF-2012-053 (2012).

[149] ATLAS Collaboration, Probing the measurement of jet energies with the ATLAS detector using photon + jet events in proton-proton collisions at sqrts $=7 \mathrm{TeV}$, ATLAS-CONF-2012-063 (2012).

[150] ATLAS Collaboration, TeV-scale jet energy calibration using multijet events including close-by jet effects at the ATLAS experiment, ATLAS-CONF-2013-003 (2013). 
[151] ATLAS Collaboration, In situ jet pseudorapidity intercalibration of the ATLAS detector using dijet events in $\sqrt{s}=7$ TeV proton-proton 2011 data, ATLAS-CONF-2012-124 (2012).

[152] ATLAS Collaboration, Selection of jets produced in proton-proton collisions with the ATLAS detector using 2011 data, ATLAS-CONF-2012-020 (2012).

[153] ATLAS Collaboration, Jet energy resolution and selection efficiency relative to track jets from in-situ techniques with the ATLAS Detector Using Proton-Proton Collisions at a Center of Mass Energy $\sqrt{s}=7$ TeV, ATLAS-CONF-2010-054 (2010).

[154] ATLAS Collaboration, Jet energy resolution in proton-proton collisions at $\sqrt{s}=7$ TeV recorded in 2010 with the ATLAS detector, Eur.Phys.J. C73 (2013) 2306, arXiv:1210.6210 [hep-ex].

[155] ATLAS Collaboration, Performance of the Electron and Photon Trigger in p-p Collisions at $\sqrt{s}=7$ TeV, ATLAS-CONF-2011-114 (2011).

[156] ATLAS Collaboration, Performance of the ATLAS Electron and Photon Trigger in p-p Collisions at $\sqrt{s}=7$ TeV in 2011, ATLAS-CONF-2012-048 (2012).

[157] ATLAS Collaboration, Updated Luminosity Determination in pp Collisions at $\sqrt{s}=7$ TeV using the ATLAS Detector, ATLAS-CONF-2011-011 (2011).

[158] ATLAS Collaboration, Measurement of the cross section for jets produced in association with $Z$ bosons, ATL-COM-PHYS-2011-141 (2011).

[159] ATLAS Collaboration, First tuning of HERWIG/JIMMY to ATLAS data, ATL-PHYS-PUB-2010-014 (2010).

[160] ATLAS Collaboration, New ATLAS event generator tunes to 2010 data, ATL-PHYS-PUB-2011-008 (2011).

[161] A. Sherstnev and R. Thorne, Parton Distributions for LO Generators, Eur.Phys.J. C55 (2008) 553-575, arXiv:0711.2473 [hep-ph].

[162] N. Davidson, G. Nanava, T. Przedzinski, E. Richter-Was, and Z. Was, Universal Interface of TAUOLA Technical and Physics Documentation, Comput.Phys.Commun. 183 (2012) 821-843, arXiv:1002.0543 [hep-ph].

[163] ATLAS Collaboration, Charged particle multiplicities in $p$ p interactions at $\sqrt{s}=$ 0.9 and $7 \mathrm{TeV}$ in a difractive limited phase-space measured with the ATLAS detector at the LHC and new PYTHIA6 tune, ATLAS-CONF-2010-031 (2010).

[164] I. W. Stewart and F. J. Tackmann, Theory Uncertainties for Higgs and Other Searches Using Jet Bins, Phys.Rev. D85 (2012) 034011, arXiv:1107.2117 [hep-ph].

[165] ATLAS Collaboration, Study of jets produced in association with a $W$ boson in $p p$ collisions at $\sqrt{s}=7$ TeV with the ATLAS detector, Phys.Rev. D85 (2012) 092002, arXiv: 1201.1276 [hep-ex]. 
[166] M. Aliev, H. Lacker, U. Langenfeld, S. Moch, P. Uwer, et al., HATHOR: HAdronic Top and Heavy quarks crOss section calculatoR, Comput.Phys.Commun. 182 (2011) 1034-1046, arXiv:1007.1327 [hep-ph].

[167] ATLAS Collaboration, Measurement of the cross section for jets produced in association with $Z$ bosons, ATL-COM-PHYS-2012-735 (2012).

[168] ATLAS Collaboration, Measurement of hard double-parton interactions in $W(\rightarrow l \nu)+2$ jet events at $\sqrt{s}=7$ TeV with the ATLAS detector, New J.Phys. 15 (2013) 033038, arXiv:1301.6872 [hep-ex].

[169] ATLAS Collaboration, Search for the Standard Model Higgs boson in the H to $\tau^{+} \tau^{-}$decay mode in $\sqrt{s}=7$ TeV pp collisions with ATLAS, JHEP 1209 (2012) 070, arXiv: 1206.5971 [hep-ex]

[170] A. Banfi, Event shapes at hadron colliders, PoS DIS2010 (2010) 099, arXiv:1101.0148 [hep-ph].

[171] ATLAS Collaboration, Measurement of the cross-section for $W$ boson production in association with b-jets in pp collisions at $\sqrt{s}=7 \mathrm{TeV}$ with the ATLAS detector, arXiv:1302.2929 [hep-ex]. 


\section{List of Figures}

2.1. Particle content of the Standard Model of particles physics . . . . . . . . 3

2.2. Schematic view of a hadronic collision event. . . . . . . . . . . . . . 10

2.3. CTEQ6M parton distribution functions for a momentum scale of $Q=$ $100 \mathrm{GeV} \ldots \ldots \ldots \ldots \ldots \ldots \ldots$

2.4. Schematic view of a hard scattering process. . . . . . . . . . . 12

2.5. Expected cross section and event rates for various physics processes in $p \bar{p}$ collisions and $p p$-collisions. . . . . . . . . . . . . . . . 14

2.6. Luminosity-weighted distribution of the mean number of interactions per bunch crossing for the data taken in 2011 and 2012 with the ATLAS experiment at the LHC. . . . . . . . . . . . . . . . . . . . . . . . . . . . . . . . . .

2.7. Drell-Yan process . . . . . . . . . . . . . . . 17

2.8. Feynman diagrams for the production of a jet in association with a $Z$ boson via the Compton process and the $q \bar{q}$ annihilation. . . . . . . . . . . . 18

2.9. NLO Feynman diagrams for the production of $Z$ bosons in association with multiple jets. . . . . . . . . . . . . . . . 18

2.10. Relative electroweak corrections for $p_{T}^{Z}$ at the LHC . . . . . . . . . . 19

2.11. The LO and NLO distributions for $p_{T}^{Z}, p_{T}$ of the leading jet and the scalar sum of the transverse momenta of all jets $H_{T}$ obtained with MCFM. . . . . 19

2.12. Leading order Feynman diagrams for the production of two jets in association with a $Z$ boson originating from single-parton interaction and double-parton interaction. . . . . . . . . . . . . . .

2.13. (a) Measured cross section as a function of the inclusive jet multiplicity and (b) measured inclusive differential cross section as a function of the transverse momentum of the jets for $Z / \gamma^{*}\left(\rightarrow e^{+} e^{-}\right)+$jets. . . . . . . . . 22 22

2.14. Chimney plot . . . . . . . . . . . . . . . 23

2.15. Branching ratios of the SM Higgs boson, together with their corresponding theoretical uncertainties. . . . . . . . . . . . . . . . .

2.16. Production cross section for the SM Higgs boson as a function of its mass with its corresponding theoretical uncertainties at a centre-of-mass energy of $7 \mathrm{TeV}$ in $p p$-collisions. . . . . . . . . . . . . . . . 25

2.17. LO Feynman diagrams for the dominant Higgs boson production mechanisms at the LHC. . . . . . . . . . . . . . . . .

2.18. Total production cross section of the SM Higgs boson as a function of its mass for different centre-of-mass energies in pp-collisions. . . . . . . . . . 26

2.19. Observed local probability $p_{0}$ for the individual channels and the combination for the (a) ATLAS experiment and (b) the CMS experiment. . . . . . 27

3.1. The Large Hadron Collider accelerator with its main experiments. . . . . . 29 
3.2. Total integrated luminosity in pp-collisions for (a) 2010 and (b) 2011 versus day, delivered by the LHC and recorded by ATLAS. . . . . . . . . . . . . . 30

3.3. Schematic view of the ATLAS detector. . . . . . . . . . . . . . . 31

3.4. Magnet system of the ATLAS detector including the solenoid and the aircore toroids. . . . . . . . . . . . . . . . 32

3.5. Schematic view of the ATLAS inner detector. . . . . . . . . . . . 33

3.6. (a) Functional overview of the ATLAS trigger/DAQ system and (b) the event rates and processing times at the different decision stages . . . . . 35

5.1. Connections between causes and effects. . . . . . . . . . . . . 42

5.2. Comparison of (a) the pull distributions for the first bin and (b) the width of the pull distributions for the different migration matrices. . . . . . . . 44

5.3. Comparison of (a) the pull distributions and (b) the width of the pull distributions for the old and the new uncertainty calculation for the migration matrix with large migration $S_{1} \ldots \ldots \ldots \ldots$

5.4. Comparison of (a) the pull distributions and (b) the width of the pull distributions for the old and the new uncertainty calculation for the migration matrix with medium migration $S_{2} \ldots \ldots \ldots \ldots$

5.5. Comparison of the width of the pull distributions for (a) the old uncertainty calculation and (b) the new uncertainty calculation for the fixed migration matrix and the randomly generated migration matrices. . . . . . . . . 46

6.1. Performance of the electron energy measurement: Electron energy scale . . 49

6.2. Performance of the electron energy measurement: Electron energy resolution 49

6.3. Identification efficiency for electrons passing the loose ++ criteria in 2011 data with a transverse energy between $7 \mathrm{GeV}$ and $50 \mathrm{GeV} \ldots \ldots . . .50$

6.4. Overview of the jet reconstruction in ATLAS. . . . . . . . . . . . 51

6.5. Overview of the ATLAS jet calibration scheme used for the 2011 dataset. . 52

6.6. (a) Jet transverse momentum distribution and (b) $\phi$ distribution of fake jets before and after applying the different jet quality criteria for 2011 data. 52

6.7. Jet quality selection efficiency for anti- $k_{T}$ jets with $R=0.4$ in the region

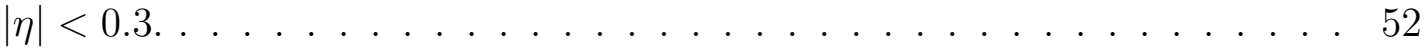

6.8. Performance of the jet energy measurement in 2010: Fractional jet energy scale systematic uncertainty as a function of the jet transverse momentum

6.9. Performance of the jet energy measurement in 2010: Fractional jet transverse momentum resolution. . . . . . . . . . . . . . . . . 54

6.10. Performance of the jet energy measurement in 2011: Fractional jet energy scale systematic uncertainty as a function of the jet transverse momentum.

6.11. Performance of the jet energy measurement in 2011: Fractional jet energy scale systematic uncertainty as a function of the jet transverse momentum for 2010 and $2011 \ldots \ldots \ldots \ldots \ldots$. . . . . . . . . . . . . . . . . . .

7.1. Schematic view of jet production and reconstruction. . . . . . . . . 58

7.2. (a) Average number of interactions per bunch crossing and (b) number of primary vertices in data taken in 2011 and ALPGEN+HERWIG after reweighting. . . . . . . . . . . . . . . . 6 . 63 
7.3. Result of the maximum likelihood fit to the dielectron mass distribution in an extended mass range using two templates, the selected multi-jet events in data and the combined simulated signal events plus non-multi-jet background events. . . . . . . . . . . . . . . 67

7.4. Shape comparisons of (a) the exclusive jet multiplicity distribution and (b) the transverse momentum distribution of the leading jet for the $e e, e \mu$ and $\mu \mu$ channel in $t \bar{t}$ production simulated with MC@NLO. . . . . . . . . . .

7.5. Comparison between data and MC predictions for (a) the exclusive jet multiplicity distribution and (b) the transverse momentum distribution of

7.6. Theoretical uncertainties on the NLO fixed-order pQCD predictions for (a) the exclusive jet multiplicity, (b) the transverse momentum of the leading jet for events with at least one jet in the final state, (c) the rapidity of the leading jet for events with at least one jet in the final state, (d) the absolute rapidity difference of the two leading jets $\left|\Delta y^{j j}\right|$ for events with at least two jets in the final state and (e) $H_{T}$ for events with at least one jet in the final state.

8.1. (a) Dielectron mass for $Z$ candidates with at least one jet in the event and

(b) inclusive jet multiplicity in data and simulation. . . . . . . . . . . . 74

8.2. (a) Transverse momentum distribution and (b) rapidity distribution of all jets in data and simulation.

8.3. Inclusive jet multiplicity in data and simulation. . . . . . . . . . . . . . 75

8.4. (a) Transverse momentum distribution and (b) rapidity distribution of all jets in data and simulation.

8.5. Bin-by-bin correction factors for (a) the inclusive jet multiplicity and (b) the jet transverse momentum distribution for events with at least one jet in the final state.

8.6. Bayesian unfolding: Correction for not-matched events. . . . . . . . . . . 78

8.7. Bayesian unfolding: Migration matrix.

8.8. Comparison between the results obtained with the iterative (Bayes) method and with the bin-by-bin method for the inclusive jet transverse momentum distribution for events with at least one jet in the final state. . . . . . . . .

8.9. Different contributions to the combined relative systematic uncertainty for the measured cross section as a function of (a) the inclusive jet multiplicity and (b) the inclusive jet transverse momentum distribution with at least one jet in the final state.

8.10. (a) Fragmentation correction, (b) UE correction and (c) combined correction for $p_{T}^{\text {jet }}$ in events with at least one jet in the final state. . . . . . . . . 84

8.11. QED FSR correction for $p_{T}^{\text {jet }}$ in events with at least one jet in the final state. 85

8.12. (a) Measured cross section as a function of the inclusive jet multiplicity and (b) the ratio of cross sections for successive inclusive jet multiplicities. 
8.13. Differential cross section as a function of (a) the transverse momentum $p_{\mathrm{T}}^{\text {jet }}$ for events with at least one jet in the final state, (b) $p_{\mathrm{T}}^{\text {jet }}$ of the leading jet for events with at least one jeThe measured values are represented by the black dots, the error bars indicate the statistical uncertainty and the hatched band incorporates the total statistical and systematic uncertainty added in quadrature. The measurements are compared to predictions from $\mathrm{ME}+\mathrm{PS}$ generators, as well as to NLO pQCD predictions from BLACKHAT+SHERPA. The shaded bands represent the theoretical uncertainties.t in the final state and (c) $p_{\mathrm{T}}^{\text {jet }}$ of the 2 nd leading jet for events with at least two jets in the final state.

8.14. Differential cross section as a function of (a) the absolute rapidity $\left|y^{\text {jet }}\right|$ for events with at least one jet in the final state, (b) $\left|y^{\text {jet }}\right|$ of the leading jet for events with at least one jet in the final state and (c) $p_{\mathrm{T}}^{\text {jet }}$ of the 2 nd leading jet for events with at least two jets in the final state.

8.15. Differential cross section as a function of the invariant mass $m^{j j}$ of the two leading jets for events with at least two jets in the final state.

8.16. Differential dijet cross section as a function of (a) the absolute rapidity difference of the two leading jets $\left|\Delta y^{j j}\right|$, (b) the absolute azimuthal separation of the two leading jets $\left|\Delta \phi^{j j}\right|$ and (c) the angular separation in $y$ - $\phi$ space of the two leading jets $\Delta R^{j j}$ for events with at least two jets in the final

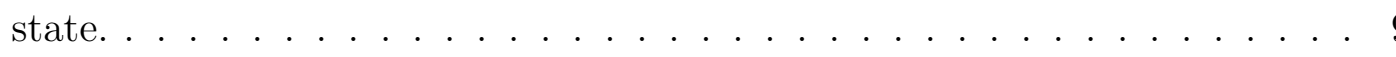

9.1. $Z$ boson rapidity distribution in data and $\mathrm{MC}$ event samples. . . . . . . . 96

9.2. (a) Dielectron mass for $Z$ candidates with at least one jet in the event and

(b) inclusive jet multiplicity in data and simulation. . . . . . . . .

9.3. (a) Transverse momentum distribution of the leading jet in events with at least one jet in the final state, (b) rapidity distribution of the leading jet in events with at least one jet in the final state, (c) $\left|\Delta y^{j j}\right|$ of the two leading jets in events with at least two jets in the final state and (d) $H_{\mathrm{T}}$ in events with at least one jet in the final state in data and simulation. . . . . . . . .

9.4. (a) $\Delta_{\text {jets }}^{n}$ and (b) $\Delta_{\text {jets }}$ distribution in events with exactly two jets in the final state in data and simulation. . . . . . . . . . . . . . . . . . . . 101

9.5. Ratios of (a),(b) the transverse momentum and (c),(d) the rapidity distributions of all jets with and without requirements on $\mu$ and $N_{P V}$ in data and simulation to test the impact of (a),(c) in-time pile-up and (b),(d) out-of-time pile-up. . . . . . . . . . . . . . . . . . 103

9.6. Double ratio between the ratios from ALPGEN+HERWIG and data for (a),(b) the transverse momentum and (c),(d) the rapidity distributions of all jets to test the impact of (a),(c) in-time pile-up and (b),(d) out-of-time pile-up. . . . . . . . . . . . . . . . . . . . . . 104

9.7. Bayesian unfolding: Migration matrices for (a) the transverse momentum of the leading jet and (b) the absolute rapidity of the leading jet for events with at least one jet in the final state. . . . . . . . . . . . . . . . 106

9.8. Bayesian unfolding: Correction for not-matched events for (a) the transverse momentum of the leading jet and (b) the absolute rapidity of the leading jet for events with at least one jet in the final state. . . . . . . . . . 106 
9.9. Bayesian unfolding: Order correction for (a) the transverse momentum of the leading jet and (b) the absolute rapidity of the leading jet for events with at least one jet in the final state. . . . . . . . . . . . . . . . . . . 107

9.10. Bayesian unfolding: $\chi^{2}$ divided by the number of bins as a function of the number of iterations for (a) the transverse momentum of the leading jet and (b) the absolute rapidity of the leading jet for events with at least one jet in the final state. . . . . . . . . . . . . . . . . . . 108

9.11. Different contributions to the combined relative JES uncertainty for the measured cross section as a function of (a) the exclusive jet multiplicity, (b) the transverse momentum of the leading jet for events with at least one jet in the final state, (c) the absolute rapidity of the leading jet for events with at least one jet in the final state, (d) the absolute rapidity difference of the two leading jets $\left|\Delta y^{j j}\right|$ for events with at least two jets in the final state and (e) $H_{T}$ for events with at least one jet in the final state. . . . . . 111

9.12. Relative systematic shifts on the unfolded cross section obtained by comparing the unfolding procedure using ALPGEN+HERWIG or SHERPA for (a) the transverse momentum of the leading jet and (b) the absolute rapidity of the leading jet for events with at least one jet in the final state. 113

9.13. Relative systematic shifts on the unfolded cross section obtained by comparing the results obtained with iterative (Bayes) method and bin-by-bin method for (a) the transverse momentum of the leading jet and (b) the absolute rapidity of the leading jet for events with at least one jet in the final state. . . . . . . . . . . . . . . . . . 114

9.14. Different contributions to the combined relative systematic uncertainty for the measured cross section as a function of (a) the exclusive jet multiplicity, (b) the transverse momentum of the leading jet for events with at least one jet in the final state, (c) the absolute rapidity of the leading jet for events with at least one jet in the final state, $(d)$ the absolute rapidity difference of the two leading jets $\left|\Delta y^{j j}\right|$ for events with at least two jets in the final state and (e) $H_{T}$ for events with at least one jet in the final state. . . . . . 116

9.15. Correlation matrices for the measured cross sections . . . . . . . . . . . . 117

9.16. (a) Fragmentation correction, (b) UE correction and (c) combined fragmentation and UE correction for the exclusive jet multiplicity. . . . . . . . 119

9.17. (a) Fragmentation correction, (b) UE correction and (c) combined fragmentation and UE correction for the transverse momentum of the leading jet for events with at least one jet in the final state. . . . . . . . . . . . 120

9.18. (a) Fragmentation correction, (b) UE correction and (c) combined fragmentation and UE correction for the rapidity of the leading jet for events with at least one jet in the final state. . . . . . . . . . . . . . . . . . . . . 121

9.19. Combined fragmentation and UE correction for (a) the absolute rapidity difference of the two leading jets for events with at least two jets in the final state and (b) $H_{T}$ in events with at least one jet in the final state. . . . 122 
9.20. QED FSR correction as a function of (a) the exclusive jet multiplicity, (b) the transverse momentum of the leading jet for events with at least one jet in the final state, (c) the rapidity of the leading jet for events with at least one jet in the final state, (d) the absolute rapidity difference of the two leading jets $\left|\Delta y^{j j}\right|$ for events with at least two jets in the final state and (e) $H_{T}$ for events with at least one jet in the final state. . . . . . . . 123

9.21. (a) Measured cross section as a function of the inclusive jet multiplicity and (b) the ratio of cross sections for successive inclusive jet multiplicities. 125

9.22. Measured cross section as a function of the exclusive jet multiplicity for events selected with (a) the standard $Z / \gamma^{*}(\rightarrow e e)+$ jets selection and (b) with an additional requirement on the transverse momentum of the leading jet of $p_{T}^{\text {jet }}>150 \mathrm{GeV} \ldots \ldots \ldots \ldots \ldots \ldots$. . . . . . . . . . . . .

9.23. Ratios of cross sections for successive exclusive jet multiplicities for events selected with (a) the standard $Z / \gamma^{*}(\rightarrow e e)+$ jets selection and (b) with an additional requirement on the transverse momentum of the leading jet of $p_{T}^{j e t}>150 \mathrm{GeV} \ldots \ldots \ldots \ldots \ldots \ldots \ldots \ldots$

9.24. Differential cross section as a function of (a) the transverse momentum $p_{\mathrm{T}}^{\text {jet }}$ of the leading jet for events with at least one jet in the final state, (b) $p_{\mathrm{T}}^{\text {jet }}$ of the 2nd leading jet for events with at least two jets in the final state, (c) $p_{\mathrm{T}}^{\text {jet }}$ of the $3 \mathrm{rd}$ leading jet for events with at least three jets in the final state and $(\mathrm{d}) p_{\mathrm{T}}^{\text {jet }}$ of the 4 th leading jet for events with at least four jets in the final state. . . . . . . . . . . . . . . . . . 130

9.25. Differential cross section as a function of (a) $p_{\mathrm{T}}^{\text {jet }}$ for events with exactly one jet in the final state and (b) the ratio of the transverse momentum of the 2nd leading jet to that of the leading jet for events with at least two jets in the final state. . . . . . . . . . . . . . . 131

9.26. Differential cross section as a function of the $Z$ boson transverse momentum for events with (a) at least one jet in the final state and (b) exactly one jet in the final state. . . . . . . . . . . . . . . . . 131

9.27. Average number of jets as a function the transverse momentum of the $Z$ boson in data and predicted by the generators ALPGEN+HERWIG and SHERPA.

9.28. Absolute azimuthal separation $\left|\Delta \phi^{j j}\right|$ of the two leading jets for different bins of $p_{T}^{e e}$, (a) $0 \mathrm{GeV}<p_{T}^{e e}<40 \mathrm{GeV}$ and (b) $160 \mathrm{GeV}<p_{T}^{e e}<200 \mathrm{GeV}$ and the ratio of the transverse momentum of the 2 nd leading jet to that of the leading jet for different bins of $p_{T}^{e e}$, (c) $0 \mathrm{GeV}<p_{T}^{e e}<40 \mathrm{GeV}$ and (d) $160 \mathrm{GeV}<p_{T}^{e e}<200 \mathrm{GeV}$ in events with at least two jets in the final state in data and simulation. . . . . . . . . . . . . . . . 133

9.29. Differential cross section as a function of $Z$ boson transverse momentum for events with at least one jet in the final state. . . . . . . . . . . 134

9.30. Differential cross section as a function of the transverse momentum of (a) the leading jet and (b) the $Z$ boson for events with at least one jet in the final state. . . . . . . . . . . . . . . . . . . . . 135

9.31. Differential cross section as a function of $p_{\mathrm{T}}^{\text {jet }}$ of the 4 th leading jet for events with at least four jets in the final state. . . . . . . . . . . . . 135 
9.32. Differential cross section as a function of (a) the absolute rapidity $\left|y^{\text {jet }}\right|$ of the leading jet for events with at least one jet in the final state, (b) $\left|y^{j e t}\right|$ of the 2nd leading jet for events with at least two jets in the final state, (c) $\left|y^{j e t}\right|$ of the 3rd leading jet for events with at least three jets in the final state and (d) $\left|y^{\text {jet }}\right|$ of the 4th leading jet for events with at least four jets in the final state. . . . . . . . . . . . . . . . . 137

9.33. Absolute rapidity distribution of the leading jet in data and simulation. . . 138

9.34. Two-dimensional Bayesian unfolding: Two-dimensional migration matrix for the absolute rapidity of the leading jet for events with at least one jet in the final state.

9.35. Two-dimensional Bayesian unfolding: (a) Correction for not-matched events and (b) order correction for the absolute rapidity of the leading jet for events with at least one jet in the final state. . . . . . . . . . . . . 139

9.36. Two-dimensional Bayesian unfolding: Closure tests for the absolute rapidity of the leading jet for events with at least one jet in the final state. . . . 139

9.37. Two-dimensional Bayesian unfolding: $\chi^{2}$ divided by the number of bins as a function of the number of iterations for the absolute rapidity of the leading jet for events with at least one jet in the final state. . . . . . . . . . . 140

9.38. Two-dimensional Bayesian unfolding: Relative systematic shifts on the unfolded cross section obtained by comparing the unfolding procedure using ALPGEN+HERWIG or SHERPA for the absolute rapidity of the leading jet for events with at least one jet in the final state. . . . . . . . . . . . . 140

9.39. Different contributions to the combined relative systematic uncertainty for the measured cross section as a function of the absolute rapidity of the leading jet for events with at least one jet in the final state. . . . . . . . . 141

9.40. Differential cross section as a function of the absolute rapidity of the leading jet for events with at least one jet in the final state, determined using twodimensional unfolding. . . . . . . . . . . . . . . . .

9.41. Differential cross section as a function of the invariant mass $m^{j j}$ of the two leading jets for events with at least two jets in the final state.

9.42. Differential dijet cross section as a function of (a) the absolute rapidity difference of the two leading jets $\left|\Delta y^{j j}\right|$, (b) the absolute azimuthal separation of the two leading jets $\left|\Delta \phi^{j j}\right|$ and (c) the angular separation in $y$ - $\phi$ space of the two leading jets $\Delta R^{j j}$ for events with at least two jets in the final state . . . . . . . . . . . . . . . . . . . . 143

9.43. Differential cross section as a function of (a) $H_{T}$ and (b) $S_{T}$ for events with at least one jet in the final state. . . . . . . . . . . . . . . . . . . . . . 144

9.44. Average number of jets as a function of $H_{T}$ in data and predicted by the generators ALPGEN+HERWIG and SHERPA.

9.45. Absolute azimuthal separation $\left|\Delta \phi^{j j}\right|$ of the two leading jets for different bins of $H_{T}$, (a) $140 \mathrm{GeV}<H_{T}<150 \mathrm{GeV}$ and (b) $300 \mathrm{GeV}<H_{T}<$ $320 \mathrm{GeV}$ and ratio of the transverse momentum of the 2nd leading jet to that of the leading jet for different bins of $p_{\mathrm{T}}^{Z}$, (c) $140 \mathrm{GeV}<H_{T}<150 \mathrm{GeV}$ and (d) $300 \mathrm{GeV}<H_{T}<320 \mathrm{GeV}$ in events with at least two jets in the final state in data and simulation. . . . . . . . . . . . . . . . . . 146

9.46. Differential cross section as a function of (a) $H_{T}$ and (b) $S_{T}$ for events with at least one jet in the final state. 
10.1. Leading-order Feynman diagram for the Higgs boson production via vector boson fusion . . . . . . . . . . . . . . . . . . . . . . . 149

10.2. Collinear mass distribution for events passing $H \rightarrow \tau \tau$ selection in the WBF category. . . . . . . . . . . . . . . . . 150

10.3. Leading-order Feynman diagram for QCD and EW $Z+2$ jets production . 150

10.4. (a) Inclusive jet multiplicity and (b) exclusive jet multiplicity for $Z / \gamma^{*}(\rightarrow e e)+$ jets events with at least two jets in the final state passing the WBF preselection $\left(m^{j j}>350 \mathrm{GeV}\right.$ and $\left.\left|\Delta y^{j j}\right|>3.0\right)$ in data and simulation. . . . . . . . 152

10.5. (a) Transverse momentum distribution and (b) rapidity distribution of the 3rd leading jet for $Z / \gamma^{*}(\rightarrow e e)+$ jets events with at least two jets in the final state passing the WBF preselection in data and simulation. . . . . . 153

10.6. (a) 3rd jet veto efficiency and (b) 3rd central jet veto efficiency for $Z / \gamma^{*}(\rightarrow e e)+$ jets events with at least three jets in the final state passing the WBF preselection in data and predicted by the generators ALPGEN+HERWIG and SHERPA. . . . . . . . . . . . . . . . . . 154

10.7. (a) $\eta^{*}$ distribution and (b) $Z^{*}$ distribution for $Z / \gamma^{*}(\rightarrow e e)+$ jets events with at least three jets in the final state passing the WBF preselection in data and simulation. . . . . . . . . . . . . . . 155

10.8. (a) Measured cross section as a function of the exclusive jet multiplicity and (b) the ratio of cross sections for successive exclusive jet multiplicities for events with at least two jets in the final state passing the WBF preselection.156

10.9. Differential cross section as a function of (a) the transverse momentum $p_{\mathrm{T}}^{\text {jet }}$ and (b) the absolute rapidity $\left|y^{j e t}\right|$ of the 3rd leading jet for events with at least three jets in the final state passing the WBF preselection. . . . . . . . 157

A.1. Transverse momentum distribution of (a) the leading jet and (b) the 2nd leading jet and rapidity distribution of (c) the leading jet and $(\mathrm{d})$ the 2 nd leading jet in data and simulation. . . . . . . . . . . . . 163

A.2. (a) Invariant mass $m^{j j}$ and the angular separations (b) $\left|\Delta y^{j j}\right|$, (c) $\left|\Delta \phi^{j j}\right|$ and (d) $\Delta R^{j j}$ (d) of the two leading jets in events with at least two jets in the final state in data and simulation. . . . . . . . . . . . . . . . 164

A.3. Transverse momentum distribution of (a) the leading jet and (b) the 2nd leading jet in data and simulation. . . . . . . . . . . . . . 165

A.4. Rapidity distribution of (a) the leading jet and (b) the 2nd leading jet in data and simulation. . . . . . . . . . . . . . . 165

A.5. (a) Invariant mass $m^{j j}$ and the angular separations (b) $\left|\Delta y^{j j}\right|$, (c) $\left|\Delta \phi^{j j}\right|$ and (d) $\Delta R^{j j}$ of the two leading jets in events with at least two jets in the final state in data and simulation. . . . . . . . . . . . . 166

B.1. Exclusive jet multiplicity with (a) the standard selection and (b) with an additional requirement on the transverse momentum of the leading jet of $p_{T}^{\text {jet }}>150 \mathrm{GeV}$ in data and simulation. . . . . . . . . . . . . 171

B.2. Transverse momentum distribution of (a) the 2nd leading jet, (b) the 3rd leading jet and (c) the 4 th leading jet in data and simulation. . . . . . . 172

B.3. Rapidity distribution of (a) the 2 nd leading jet, (b) the 3rd leading jet and (c) the 4th leading jet in data and simulation. 
B.4. $\phi$ distribution of (a) the leading jet, (b) the 2nd leading jet, (c) the 3rd leading jet and $(\mathrm{d})$ the 4 th leading jet in data and simulation. . . . . . . 174

B.5. (a) $R\left(p_{T} 2 / p_{T} 1\right)$ distribution of the leading and 2 nd leading jet in events with at least two jets in the final state and (b) transverse momentum distribution of the $Z$ boson in events with at least one jet in the final state in data and simulation. . . . . . . . . . . . . . . . 175

B.6. Transverse momentum distributions of (a) the leading jet and (b) the Z boson for events with exactly one jet in the final state in data and simulation.175

B.7. (a) Invariant mass $m^{j j}$ and the angular separations (b) $\left|\Delta \phi^{j j}\right|$ and (c) $\Delta R^{j j}$ of the two leading jets in events with at least two jets in the final state in data and simulation. . . . . . . . . . . . . . . 176

B.8. $S_{\mathrm{T}}$ distribution in events with at least one jet in the final state in data and simulation. . . . . . . . . . . . . . . . . . . 177

B.9. Ratios of the inclusive jet multiplicity with and without requirements on $\mu$ and $N_{P} V$ in data and simulation to test the impact of (a) in-time pile-up and (b) out-of-time-pile-up. . . . . . . . . . . . . . . . . . 178

B.10.ALPGEN+HERWIG to data ratio for the inclusive jet multiplicity to test the impact of (a) in-time pile-up and (b) out-of-time pile-up. . . . . . . . . 178

B.11.Bayesian unfolding: Migration matrices for (a) the exclusive jet multiplicity, (b) the absolute rapidity difference of the two leading jets $\left|\Delta y^{j j}\right|$ for events with at least two jets in the final state and (c) $H_{T}$ for events with at least one jet in the final state.

B.12.Bayesian unfolding: Correction for not-matched events for (a) the exclusive jet multiplicity, (b) the absolute rapidity difference of the two leading jets $\left|\Delta y^{j j}\right|$ for events with at least two jets in the final state and (c) $H_{T}$ for events with at least one jet in the final state. . . . . . . . . . . . . . . 180

B.13.Bayesian unfolding: Order correction for (a) the exclusive jet multiplicity, (b) the absolute rapidity difference of the two leading jets $\left|\Delta y^{j j}\right|$ for events with at least two jets in the final state and (c) $H_{T}$ for events with at least one jet in the final state. . . . . . . . . . . . . . . . . . . . . . . 181

B.14.Bayesian unfolding: $\chi^{2}$ divided by the number of bins as a function of the number of iterations for (a) the exclusive jet multiplicity, (b) the absolute rapidity difference of the two leading jets $\left|\Delta y^{j j}\right|$ for events with at least two jets in the final state and (c) $H_{T}$ for events with at least one jet in the final state. . . . . . . . . . . . . . . . . . . . . . . 182

B.15. Relative systematic shifts on the unfolded cross section obtained by comparing the unfolding procedure using ALPGEN+HERWIG or SHERPA for (a) the exclusive jet multiplicity, (b) the absolute rapidity difference of the two leading jets $\left|\Delta y^{j j}\right|$ for events with at least two jets in the final state and (c) $H_{T}$ for events with at least one jet in the final state. . . . . . . . 183

B.16. Relative systematic shifts on the unfolded cross section obtained by comparing the results obtained with iterative (Bayes) method and Bin-by-Bin method for (a) the exclusive jet multiplicity, (b) the absolute rapidity difference of the two leading jets $\left|\Delta y^{j j}\right|$ for events with at least two jets in the final state and (c) $H_{T}$ for events with at least one jet in the final state. . . 184

B.17.Exclusive jet multiplicity (a) before reweighting and (b) after reweighting to data. 
B.18. Bayesian unfolding: Closure tests of the exclusive jet multiplicity distribution for (a) SHERPA and (b) ALPGEN+HERWIG reweighted to data. . . 186

B.19.Bayesian unfolding: Closure tests of the transverse momentum distribution of the leading jet for events with at least one jet for (a) SHERPA and (b) ALPGEN+HERWIG reweighted to data. . . . . . . . . . . . . . . . 186

B.20. Bayesian unfolding: Closure tests of the rapidity distribution of the leading jet for events with at least one jet for (a) SHERPA and (b) ALPGEN+HERWIG reweighted to data. . . . . . . . . . . . . . . . . . . 187

B.21. Bayesian unfolding: Closure tests of the absolute rapidity difference of the two leading jets $\left|\Delta y^{j j}\right|$ for events with at least two jets in the final state for (a) SHERPA and (b) ALPGEN+HERWIG reweighted to data. . . . . . 187

B.22. Bayesian unfolding: Closure tests of $H_{T}$ for events with at least one jet in the final state for (a) SHERPA and (b) ALPGEN+HERWIG reweighted to data. 


\section{List of Tables}

2.1. Standard Model gauge bosons and their properties, along with their forces and the corresponding theory. . . . . . . . . . . . 4

2.2. Properties of the leptons. . . . . . . . . . . . . . . 5

2.3. Properties and quantum numbers of the different quarks. . . . . . . . . . 5

2.4. Different decay modes of the $Z$ boson with their corresponding branching ratios. . . . . . . . . . . . . . . . 17

3.1. Operational conditions of the LHC for 2010 and 2011 data taking in $p p$ collisions at $\sqrt{s}=7 \mathrm{TeV} \ldots \ldots \ldots \ldots \ldots$

6.1. Electron identification criteria for the central region of the detector with $|\eta|<2.47 \ldots \ldots \ldots \ldots \ldots \ldots \ldots$

7.1. Summary of $Z / \gamma^{*}(\rightarrow e e)$ and jet selection criteria. . . . . . . . . . 58

7.2. Trigger chains for the various data taking periods in 2010 and 2011 with their corresponding integrated luminosities. . . . . . . . . . . . . 60 60

7.3. Primary vertex reweighting factors for MC event samples in 2010 . . . . . . 63

7.4. Details and settings of MC event samples used for the analysis with the full dataset of $2010 \ldots \ldots$. . . . . . . . . . . . . . . . . . 64

7.5. $Z / \gamma^{*}(\rightarrow e e)+$ jets MC events samples generated with PYTHIA and HERWIG+JIMMY used for the determination of the correction factors for nonperturbative effects and systematic studies. . . . . . . . . . . . 64 64

7.6. Details and settings of MC event samples used for the analysis with the full dataset of 2011. . . . . . . . . . . . . . . . . . . . . 65

8.1. Number of events expected from MC event sample and observed in data for several inclusive jet multiplicities.

8.2. Different components of the total systematic uncertainty on the measured cross section for several inclusive jet multiplicities.

8.3. Cross section as a function of the inclusive jet multiplicity with respect to the fiducial region measured in data and predicted from NLO fixed-order pQCD calculations from BLACKHAT+SHERPA.

8.4. Ratio of cross sections for successive inclusive jet multiplicities $\sigma\left(Z / \gamma^{*}+\geq N_{\text {jet }}+1\right.$ jets $) / \sigma\left(Z / \gamma^{*}+\geq N\right.$ jets $)$ with respect to the fiducial region measured in data and predicted from NLO fixed-order pQCD calculations from BlACKHAT+SHERPA. . . . . . . . . . . . . .

9.1. Number of events predicted from MC simulation and observed in data for the different jet multiplicities. . . . . . . . . . . . . . . . 97

9.2. Different components of the total systematic uncertainty on the measured cross section for several inclusive jet multiplicities. . . . . . . . . . . . . . 114 
9.3. Cross sections as a function of the inclusive jet multiplicities with respect to the fiducial region measured in data and predicted from NLO fixed-order pQCD calculations from BlACKHAT+SHERPA. . . . . . . . . . . . . 125

9.4. Ratio of cross sections for successive inclusive jet multiplicities $\sigma\left(Z / \gamma^{*}+\geq N+1\right.$ jets $) / \sigma\left(Z / \gamma^{*}+\geq N\right.$ jets $)$ with respect to the fiducial region measured in data and predicted from NLO fixed-order pQCD calculations from BLACKHAT+SHERPA. . . . . . . . . . . . . . . . . 126

9.5. Results of a linear fit of the exclusive cross-section ratio $R_{(n+1) / n}$ with the standard selection, and results of a fit of the exclusive cross-section ratio $R_{(n+1) / n}$ with a pattern expected for Poisson distributed exclusive jet multiplicity for events with an additional requirement on the transverse momentum of the leading jet of $p_{T}^{\text {jet }}>150 \mathrm{GeV} \ldots \ldots \ldots$. . . . . .

10.1. Number of events predicted from MC simulation and observed in data for the different jet multiplicities for events with at least two jets in the final state passing the WBF preselection. . . . . . . . . . . . . 152

A.1. Cross section as a function of $p_{T}^{\text {jet }}$ for events with at least one jet in the final state with respect to the fiducial region measured in data and predicted from NLO fixed-order pQCD calculations from BLACKHAT+SHERPA. . . 167

A.2. Cross section as a function of the leading jet $p_{T}$ for events with at least one jet in the final state with respect to the fiducial region measured in data and predicted from NLO fixed-order pQCD calculations from BLACKHAT+SHERPA . . . . . . . . . . . . . . . . . . . 167

A.3. Cross section as a function of the 2 nd leading jet $p_{T}$ for events with at least two jets in the final state with respect to the fiducial region measured in data and predicted from NLO fixed-order pQCD calculations from BLACKHAT+SHERPA . . . . . . . . . . . . . . . . . . 167

A.4. Cross section as a function of $\left|y^{\text {jet }}\right|$ for events with at least one jet in the final state with respect to the fiducial region measured in data and predicted from NLO fixed-order pQCD calculations from BLACKHAT+SHERPA. . .

A.5. Cross section as a function of the leading jet $|y|$ for events with at least one jet in the final state with respect to the fiducial region measured in data and predicted from NLO fixed-order pQCD calculations from BLACKHAT+SHERPA. . . . . . . . . . . . . . . . . . .

A.6. Cross section as a function of the 2nd leading jet $|y|$ for events with at least one jet in the final state with respect to the fiducial region measured in data and predicted from NLO fixed-order pQCD calculations from BlACKHAT+SHERPA. . . . . . . . . . . . . . . . . . . 168

A.7. Cross section as a function of the invariant mass between the two leading jets for events with at least two jets in the final state with respect to the fiducial region measured in data and predicted from NLO fixed-order pQCD calculations from BLACKHAT+SHERPA. . . . . . . . . . . . . . . 169

A.8. Cross section as a function of the absolute rapidity distance between the two leading jets for events with at least two jets in the final state with respect to the fiducial region measured in data and predicted from NLO fixed-order pQCD calculations from BLACKHAT+SHERPA. . . . . . . . . 169 
A.9. Cross section as a function of the absolute azimuthal separation between the two leading jets for events with at least two jets in the final state with respect to the fiducial region measured in data and predicted from NLO fixed-order pQCD calculations from BLACKHAT+SHERPA.

A.10.Cross section as a function of the angular separation in $y$ - $\phi$ space between the two leading jets for events with at least two jets in the final state with respect to the fiducial region measured in data and predicted from NLO fixed-order pQCD calculations from BLACKHAT+SHERPA. . . . . . . . . 170

B.1. Cross sections as a function of the exclusive jet multiplicities with respect to the fiducial region measured in data and predicted from NLO fixed-order pQCD calculations from BLACKHAT+SHERPA. . . . . . . . . . . .

B.2. Cross sections as a function of the exclusive jet multiplicities with respect to the fiducial region measured in data and predicted from NLO fixedorder pQCD calculations from BLACKHAT+SHERPA with an additional requirement on the transverse momentum of the leading jet of $p_{T}^{\text {jet }}>150 \mathrm{GeV}$.189

B.3. Ratio of cross sections for successive exclusive jet multiplicities $\sigma\left(Z / \gamma^{*}+(N+1)\right.$ jets $) / \sigma\left(Z / \gamma^{*}+N\right.$ jets $)$ with respect to the fiducial region measured in data and predicted from NLO fixed-order pQCD calculations from BLACKHAT+SHERPA. . . . . . . . . . . . . . . . 189

B.4. Ratio of cross sections for successive exclusive jet multiplicities $\sigma\left(Z / \gamma^{*}+(N+1)\right.$ jets $) / \sigma\left(Z / \gamma^{*}+N\right.$ jets $)$ with respect to the fiducial region measured in data and predicted from NLO fixed-order pQCD calculations from BLACKHAT+SHERPA with an additional requirement on the transverse momentum of the leading jet of $p_{T}^{\text {jet }}>150 \mathrm{GeV}$. . . . . . . . 1

B.5. Cross section as a function of the leading jet $p_{T}$ for events with at least one jet in the final state with respect to the fiducial region measured in data and predicted from NLO fixed-order pQCD calculations from BLACKHAT+SHERPA . . . . . . . . . . . . . . . . . . . . . . . . . . 190

B.6. Cross section as a function of the 2 nd leading jet $p_{T}$ for events with at least two jets in the final state with respect to the fiducial region measured in data and predicted from NLO fixed-order pQCD calculations from BLACKHAT+SHERPA. . . . . . . . . . . . . . . . . 19

B.7. Cross section as a function of the 3rd leading jet $p_{T}$ for events with at least three jets in the final state with respect to the fiducial region measured in data and predicted from NLO fixed-order pQCD calculations from BLACKHAT+SHERPA. . . . . . . . . . . . . . . . . [

B.8. Cross section as a function of the 4 th leading jet $p_{T}$ for events with at least four jets in the final state with respect to the fiducial region measured in data and predicted from NLO fixed-order pQCD calculations from BLACKHAT+SHERPA . . . . . . . . . . . . . . . . . . . . . 192

B.9. Cross section as a function of the leading jet $p_{T}$ for events with exactly one jet in the final state with respect to the fiducial region measured in data and predicted from NLO fixed-order pQCD calculations from BLACKHAT+SHERPA. . . . . . . . . . . . . . . . . . . . . . 193 
B.10.Cross section as a function of the jet $p_{T}$ ratio of the two leading jets for events with at least two jets in the final state with respect to the fiducial region measured in data and predicted from NLO fixed-order pQCD calculations from BLACKHAT+SHERPA. . . . . . . . . . . . . . . . . . 194

B.11.Cross section as a function of the $Z$ boson $p_{T}$ for events with at least one jet in the final state with respect to the fiducial region measured in data and predicted from NLO fixed-order pQCD calculations from BLACKHAT+SHERPA . . . . . . . . . . . . . . . . . . . . . 195

B.12. Cross section as a function of the $Z$ boson $p_{T}$ for events with exactly one jet in the final state with respect to the fiducial region measured in data and predicted from NLO fixed-order pQCD calculations from BLACKHAT+SHERPA . . . . . . . . . . . . . . . . . . . . . 196

B.13. Cross section as a function of the leading jet $|y|$ for events with at least one jet in the final state with respect to the fiducial region measured in data and predicted from NLO fixed-order pQCD calculations from BLACKHAT+SHERPA (one-dimensional unfolding) . . . . . . . . . . . . . 197

B.14.Cross section as a function of the leading jet $|y|$ for events with at least one jet in the final state with respect to the fiducial region measured in data and predicted from NLO fixed-order pQCD calculations from BLACKHAT+SHERPA (two-dimensional unfolding) . . . . . . . . . . . . . . . . 198

B.15. Cross section as a function of the 2nd leading jet $|y|$ for events with at least two jets in the final state with respect to the fiducial region measured in data and predicted from NLO fixed-order pQCD calculations from BLACKHAT+SHERPA . . . . . . . . . . . . . . . . . . . . . . . . 199

B.16.Cross section as a function of the 3rd leading jet $|y|$ for events with at least three jets in the final state with respect to the fiducial region measured in data and predicted from NLO fixed-order pQCD calculations from BLACKHAT+SHERPA. . . . . . . . . . . . . . . . . . . . . . . . . 200

B.17. Cross section as a function of the 4 th leading jet $|y|$ for events with at least four jets in the final state with respect to the fiducial region measured in data and predicted from NLO fixed-order pQCD calculations from BLACKHAT+SHERPA . . . . . . . . . . . . . . . . . . . . . . . 200

B.18. Cross section as a function of the invariant mass between the two leading jets for events with at least two jets in the final state with respect to the fiducial region measured in data and predicted from NLO fixed-order pQCD calculations from BLACKHAT+SHERPA. . . . . . . . . . . . . . . . . 201

B.19. Cross section as a function of the absolute rapidity distance between the two leading jets for events with at least two jets in the final state with respect to the fiducial region measured in data and predicted from NLO fixed-order pQCD calculations from BLACKHAT+SHERPA. . . . . . . . . 202

B.20. Cross section as a function of the absolute azimuthal separation between the two leading jets for events with at least two jets in the final state with respect to the fiducial region measured in data and predicted from NLO fixed-order pQCD calculations from BLACKHAT+SHERPA. . . . . . . . . 203 
B.21. Cross section as a function of the angular separation in $y$ - $\phi$ space between the two leading jets for events with at least two jets in the final state with respect to the fiducial region measured in data and predicted from NLO fixed-order pQCD calculations from BLACKHAT+SHERPA. . . . . . . . . 204

B.22. Cross section as a function of $H_{T}$ for events with at least one jet in the final state with respect to the fiducial region measured in data and predicted from NLO fixed-order pQCD calculations from BLACKHAT+SHERPA. . . 205

B.23. Cross section as a function of $S_{T}$ for events with at least one jet in the final state with respect to the fiducial region measured in data and predicted from NLO fixed-order pQCD calculations from BLACKHAT+SHERPA. . . 206

C.1. Cross sections as a function of the exclusive jet multiplicities after WBF preselection with respect to the fiducial region measured in data and predicted from NLO fixed-order pQCD calculations from BLACKHAT+SHERPA.207

C.2. Ratio of cross sections for successive exclusive jet multiplicities after WBF preselection with respect to the fiducial region measured in data and predicted from NLO fixed-order pQCD calculations from BLACKHAT+Sherpa. 207

C.3. Cross section as a function of the 3 rd leading jet $p_{T}$ after WBF preselection for events with at least three jets in the final state with respect to the fiducial region measured in data and and predicted from NLO fixed-order pQCD calculations from BLACKHAT+Sherpa. . . . . . . . . . . . . . . . 208

C.4. Cross section as a function of the 3rd leading jet $|y|$ after WBF preselection for events with at least three jets in the final state with respect to the fiducial region measured in data and and predicted from NLO fixed-order pQCD calculations from BlACKHAT+Sherpa. . . . . . . . . . . . . . 208 



\section{Acknowledgements}

I would like to express my deepest gratitude to my supervisor Prof. Dr. Arnulf Quadt for giving me the opportunity to work for the ATLAS collaboration at CERN in Geneva, for his valuable guidance and advice, his stimulating ideas and the inspiring discussions. He has provided me with an excellent atmosphere for doing research and opened to me a lot of great opportunities for my scientific career.

I am indebted to Prof. Dr. Jonathan Butterworth for his willingness to be the cocorrector of this thesis. I am also indebted to the other committee members, Prof. Dr. Ariane Frey, Prof. Dr. Florentin Wörgötter, Prof. Dr. Fred Wolf and PD Dr. Jörn Große-Knetter.

I would like to express my very great appreciation to Dr. Ulrike Blumenschein for her enthusiasm, her encouragement, the guidance and support. Many good and decisive ideas were based on various fruitful discussions with her.

I am particularly grateful for the assistance given by Prof. Dr. Steffen Schumann and Dr. Erik Gerwick from the theoretical point of view.

I wish to acknowledge the help provided by Dr. Oleg Brandt, Dr. Erik Gerwick, Boris Lemmer, Prof. Dr. Yvonne Peters and Dr. Jens Weingarten for taking the time to read my thesis and alert me to errors.

My special thanks are extended to the ATLAS collaboration, especially to my colleagues from the ATLAS $Z+$ jets group, Takayuki Kanno, Evelyn Meoni, Estel Perez, Marisa Sandhoff and Jean-Baptiste Sauvan.

Many thanks to my flatmates and colleagues, especially Stefanie Adomeit, Sarah Beranek, Johannes Erdmann, Andrea Knue, Boris Lemmer, Celia Robinson and Jaroslava Schovancova, who made my stay at CERN in Geneva to a pleasurable experience.

I would also like to extend my thanks to all my colleagues from Göttingen for their patience, support and the interesting discussions during coffee breaks.

Particular thanks goes to the Dorothea Schlözer program from the University of Göttingen for the financial support during the whole time of my thesis. Furthermore, I would like to thank the German National Academic Foundation for their opportunity to take part in their program, which gives me the possibility to know many interesting people. Many thanks to the "For Women in Science Programme" from the Christiane Nüsslein-VolhardFoundation, the German L'Oreal foundation and the German UNESCO commission for their encouragement to finalize my thesis with a small child during the last year.

I would also like to thank my friends, Mira Prior, Britta Weinhausen and Stephanie Wiedigen, for great parties and many funny events. They always brought me back down to earth.

Many thanks to the childminder, Tanja Heberling, for her flexibility during the final phase of my thesis.

Finally, special thanks to my family, my parents Elke and Dr. Heinz-Volker Fiekas, my husband Jakob and my little sweet daughter Luisa, for their love and support during good and bad times. After a long day of working and a night with little sleep, Luisas smiling 
gave me a lot of strength in the final phase of my thesis. 


\section{Curriculum Vitae}

\section{Katharina Bierwagen}

Vor der Laakenbreite 8

37075 Göttingen

Tel.: +495513056804

E-mail: katharina.bierwagen@phys.uni-goettingen.de

\section{Personal Details}

Date of birth: August 20, 1985

Place of birth: Preetz

Nationality: German

\section{Education}

Georg-August-University of Göttingen, Germany

Dr. rer. nat. (Ph.D.) in particle physics (2010 to date)

Dissertation: "Physics with jets in association with a $Z$ boson in pp-collisions with the ATLAS detector at the Large Hadron Collider"

Advisor: Prof. Dr. Arnulf Quadt

Georg-August-University of Göttingen, Germany

Diplom (M.S.) in particle physics (2007 - 2009)

Thesis: "Feasibility study for the measurement of the inclusive and differential

$Z / \gamma^{*}(\rightarrow e e)+$ jets cross section in $p p$-collisions with the ATLAS experiment at the LHC" Advisor: Prof. Dr. Arnulf Quadt

Vordiplom (B.S.) in physics (2005-2007)

Gymnasium Elmschenhagen, Kiel, Germany

Secondary school emphasizing mathematics and science (1996 - 2005)

Grund- und Hauptschule Raisdorf, Schwentinental, Germany

Primary school (1992 - 1996) 


\section{Honors and Awards}

- For Women in Science Award - scholarship of the German Commission for UNESCO, L'Oréal Germany and the Christiane Nüsslein-Volhard-Foundation, Award Amount: $20,000 €(2012)$

- Ph.D. student scholarship of the German National Academic Foundation (1/2011 to date)

- Dorothea Schloezer Ph.D. student scholarship of the Georg-August-University of Göttingen (1/2010 to date)

- Scholarship of the German National Academic Foundation (10/2009 - 4/2010)

\section{Teaching Experience}

Georg-August-University of Göttingen, Germany

- Supervision of bachelor and master students (2010 to date)

- Advanced lab course in particle physics (10/2010 - 4/2011)

- Introduction into nuclear and particle physics (10/2008 - 4/2009, 10/2010 - 4/2011)

- Physics 4: quantum physics, atomic physics and molecule physics (4/2009 - 10/2009, $4 / 2010-10 / 2010)$

- e-learning project in physics at university (10/2007 - 10/2008)

\section{Research Experience}

CERN, Geneva, Switzerland

Internship within the Ph.D. program (3/2011 - 2/2012)

Deutsches Elektronen-Synchrotron, Hamburg, Germany

Summer student $(7 / 2008-9 / 2008)$

Topic: "Tracking Performance Analysis for the ZEUS-Detector"

Laser Laboratorium Göttingen e.V., Germany

Lab course (4/2007 - 5/2007)

Topic: "End-pumped solid-state laser for generation of complex radiation fields"

Christian-Albrechts-University of Kiel, Faculty of Engineering, Germany

Internship in material science $(3 / 2004)$ 